\title{
Iridium-Catalyzed Enantioselective Allylic Substitution with Aqueous Solutions of Nucleophiles
}

\author{
Tobias Sandmeier, F. Wieland Goetzke, Simon Krautwald and Erick M. Carreira*
}

ETH Zürich, Vladimir-Prelog-Weg 3, HCI, 8093 Zürich, Switzerland

\section{Supporting Information}

\section{Table of Contents}

1. Materials and Methods S2

2. General Procedures S4

3. Optimization Studies S5

4. Mechanistic investigations $\quad$ S8

5. Synthesis and Characterization of Products $\quad \mathrm{S12}$

6. X-Ray Crystallographic Data S78

7. References $\quad 586$

8. NMR Spectra $\quad \mathbf{S 8 7}$

9. SFC Traces $\quad \mathbf{S 1 5 7}$

10. HPLC Traces $\quad$ S203

11. GC Traces $\quad$ S208 


\section{Materials and Methods}

\subsection{General Methods}

All non-aqueous reactions were performed in flame-dried glassware sealed with a rubber septum under an atmosphere of $\mathrm{N}_{2}$ unless stated otherwise. Reactions were stirred magnetically and monitored by analytical thin layer chromatography (TLC). TLC was performed on Merck silica gel $60 \mathrm{~F}_{254}$ TLC glass plates and visualized with $254 \mathrm{~nm}$ light and aqueous potassium permanganate, aqueous ceric ammonium nitrate or ethanolic $p$-anisaldehyde followed by heating. Crude products were absorbed onto Celite and purified by flash column chromatography on Nacalai Tesque, Inc. Silica Gel 60 (spherical, neutral), 150-325 mesh, 42-105 $\mu \mathrm{m}$ particle size and analytical grade hexane, ethyl acetate, dichloromethane toluene and methanol as eluents at $0.3-0.5$ bar overpressure. Reverse-phase flash chromatography was carried out using reverse phase silica gel $90 \mathrm{C}_{18}$, Fluka ${ }^{\circledR}$, analytical grade acetonitrile and deionized water. Concentration under reduced pressure (in vасио) was performed by rotary evaporation at $40{ }^{\circ} \mathrm{C}$ at the appropriate pressure unless otherwise noted. The yields given refer to the purified products, unless otherwise stated.

Note: As some of the products described herein were found to be labile with regard to epimerization or decomposition, the appropriate choice of a silica gel for chromatographic purifications was crucial. Brands from other suppliers generally lead to lower diastereomeric rations and/or yields.

\subsection{Solvents and Reagents}

All chemicals and solvents were obtained from commercial suppliers and used without further purification unless otherwise noted. The ligands $(R)-\mathbf{L}^{\mathbf{1}},(S)-\mathbf{L}^{\mathbf{1}}$ and $\mathbf{P N ^ { 2 }}$ were prepared according to previously reported procedures. All allylic alcohols were prepared by addition of vinylmageniusm bromide or chloride to the corresponding aldehyde. Analytical data were in agreement with previously reported values ${ }^{3-8}$. The amine catalysts $(R)-\alpha, \alpha-B i s[3,5$-bis(trifluoromethyl)phenyl]-2pyrrolidinemethanol trimethylsilyl ether $(\boldsymbol{R})$-A1 and $(S)$ - $\alpha, \alpha$-Bis[3,5-bis(trifluoromethyl)phenyl]-2pyrrolidinemethanol trimethylsilyl ether $(\boldsymbol{S})$-A1 were purchased from Sigma Aldrich. $[\operatorname{Ir}(\operatorname{cod}) \mathrm{Cl}]_{2}$ was purchased from Combi-Blocks, Dichloroacetaldehyde hydrate and dibenzenesulfonimide from Tokyo Chemical Industry (TCI), dimethylphosphate from Acros and zinc bromide, dichloroacetic acid, chloroacetaldehyde $\left(50 \mathrm{wt} \%\right.$ in $\mathrm{H}_{2} \mathrm{O}$ ), and glutaraldehyde $\left(50 \mathrm{wt} \%\right.$ in $\left.\mathrm{H}_{2} \mathrm{O}\right)$ as well as the hydrazine derivatives were purchased from Sigma Aldrich. Benzhydrylamine was purchased from Acros and freshly distilled under reduced pressure prior to use. 1,2-Dichloroethane used for the biphasic allylic substitution reactions was purchased from Sigma Aldrich and used without further purification. Dichloromethane, THF, acetonitrile and toluene were dried using a LC Technology Solutions SP-1 solvent system under dry $\mathrm{N}_{2}$. Pure $\mathrm{NEt}_{3}$ was obtained by distillation from $\mathrm{CaH}_{2}$ under an atmosphere of dry $\mathrm{N}_{2}$. Deuterated solvents were purchased from Armar Chemicals, Döttingen, Switzerland.

\subsection{Analytics}

NMR Spectroscopy. Nuclear magnetic resonance (NMR) data were recorded on a Bruker AV400, Bruker DRX400, Bruker AV500, Bruker DRX500 spectrometer at $298 \mathrm{~K}$. Chemical shifts $(\delta)$ are reported in ppm with the solvent resonance employed as the internal standard (chloroform at $7.26 \mathrm{ppm}$ for ${ }^{1} \mathrm{H}$-NMR and $77.16 \mathrm{ppm}$ for ${ }^{13} \mathrm{C}$-NMR spectroscopy; methanol at $3.31 \mathrm{ppm}$ for ${ }^{1} \mathrm{H}-\mathrm{NMR}$ and 49.0 for ${ }^{13} \mathrm{C}$-NMR spectroscopy). Signals are reported as $\mathrm{s}=$ singlet, $\mathrm{d}=$ doublet, $\mathrm{t}=$ triplet, $\mathrm{q}=$ quartet, $\mathrm{m}=$ multiplet or unresolved, $\mathrm{br}=$ broad signal, coupling constant(s) in $\mathrm{Hz}$, integration. For diastereomeric mixtures only signals belonging to the major diastereomer are reported. ${ }^{13} \mathrm{C}$ NMR spectra were recorded with broadband ${ }^{1} \mathrm{H}$-decoupling. 
Infrared Spectroscopy. Infrared spectra (IR) were measured neat as thin films on a Perkin-Elmer UATR Two spectrometer. Only major peaks are reported as absorption maxima $\left(\mathrm{n}, \mathrm{cm}^{-1}\right)$.

Mass Spectrometry. Mass spectrometric (MS) analyses were performed by the mass spectrometry service of the Laboratorium für Organische Chemie at ETH Zürich by Mr. Louis Bertschi, Mr. Oswald Greter, Mr. Rolf Häfliger and Daniel Wirz under the direction of Dr. X. Zhang and Dr. Bertran Gerrits. High resolution electron spray ionization (ESI) mass spectral data were obtained on a Bruker Daltonics Maxis (UHR-TOF) instrument, electron impact ionization (EI) data on a Waters Micromass Autospec Ultima instrument at $70 \mathrm{eV}$ and matrix assisted laser desorption ionization (MALDI) data on a Bruker Solarix-MALDI-FTICR-MS instrument.

Specific Rotation. Specific rotations $(\alpha)$ were measured on a Jasco P-2000 digital polarimeter at the sodium D line with a $10 \mathrm{~cm}$ cell length. Data are reported as follows: $[\alpha]^{\mathrm{T}}$ in parentheses concentration $\left(\mathrm{c}=1.00\right.$ corresponds to $10.0 \mathrm{mg} \cdot \mathrm{mL}^{-1}$ ), and solvent. The temperature $(\mathrm{T})$ at which the measurement was made is given as the superscript number $\left({ }^{\circ} \mathrm{C}\right)$.

Chiral separations. Supercritical fluid chromatography (SFC) was performed on a Jasco 2080 Plus system with a diode array detector under the conditions given for each measurement. Normal phase high performance liquid chromatography (HPLC) was performed on a Dionex ultimate 3000 HPLC system (Thermo-Fischer) with a diode array detector under the conditions given for each measurement. Gas chromatography (GC) was performed on an Agilent Intuvo 9000 GC System with an FI detector under the conditions given for each measurement. Racemic samples were obtained by following the general procedures detailed bellow using the achiral PN ligand instead of $(\boldsymbol{R})-\mathbf{L},(\boldsymbol{S})$-L.

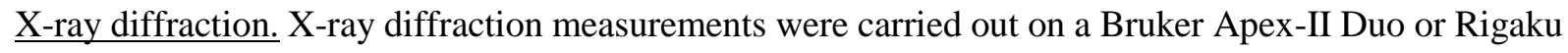
Oxford XtaLAB Synergy-S system by Dr. Niels Trapp and Mr. Michael Solar from the Small Molecule Crystallography Center (SMoCC) at the Department of Chemistry and Applied Biosciences at ETH Zürich. The data obtained was deposited at the Cambridge Crystallographic Data Centre. For details see section 5 .

Melting Points. Melting points were determined on a Büchi M-560 melting point apparatus. 


\section{General Procedures}

\section{A) Allylation of aqueous heteroatom nucleophiles}

A stock solution of the iridium precursor and the chiral phosphoramidate ligand was prepared by stirring a solution [ $\left.\{\operatorname{Ir}(\operatorname{cod}) \mathrm{Cl}\}_{2}\right](15.6 \mathrm{mg}, 22.5 \mu \mathrm{mol}, 0.09$ equiv) and $(\boldsymbol{R})-\mathbf{L}(45.6 \mathrm{mg}, 89.8 \mu \mathrm{mol}, 0.36$ equiv) in 1,2-dichloroethane $(1.5 \mathrm{~mL})$ under an atmosphere of nitrogen for $15 \mathrm{~min}$. A portion of the resulting dark red solution $(0.5 \mathrm{~mL}, 0.06$ equiv. [Ir]) was added to a screw-capped glass vial containing allylic alcohol 1 ( $0.25 \mathrm{mmol}, 1.0$ equiv). The aqueous nucleophile (1.1-2.0 equiv.) and the Brønsted acid promoter was (1.15-2.8 equiv.) were added. The vessel was purged with nitrogen, fitted with a teflonlined cap, and the resulting solution was stirred at room temperature for the period of time indicated. The reaction mixture was either directly purified by column chromatography $(\mathbf{2 j - 2 o}, \mathbf{2 p})$ or quenched by addition of saturated aqueous $\mathrm{NaHCO}_{3}$, followed by phase separation, extraction of the aqueous phase with EtOAc, drying over $\mathrm{Na}_{2} \mathrm{SO}_{4}$, filtration and flash chromatography (2a-2i, 2q).

For details, see the experimental procedures for the individual compounds.

\section{B) Dual-catalytic allylation of chloroacetaldehyde and bromoacetaldehyde}

A stock solution of the iridium precursor and the chiral phosphoramidate ligand was prepared by stirring a solution [ $\left.\{\operatorname{Ir}(\operatorname{cod}) \mathrm{Cl}\}_{2}\right](15.6 \mathrm{mg}, 22.5 \mu \mathrm{mol}, 0.09$ equiv) and $(\boldsymbol{R})-\mathbf{L}(45.6 \mathrm{mg}, 89.8 \mu \mathrm{mol}, 0.36$ equiv) in 1,2-dichloroethane $(1.5 \mathrm{~mL})$ under an atmosphere of nitrogen for $15 \mathrm{~min}$. A portion of the resulting dark red solution $(0.5 \mathrm{~mL}, 0.06$ equiv. [Ir]) was added to a screw-capped glass vial containing allylic alcohol 1 ( $0.250 \mathrm{mmol}, 1.0$ equiv). Chloroacetaldehyde (50\% wt in water, $64 \mu \mathrm{L}, 0.500 \mathrm{mmol}, 2.0$ equiv) was added, followed by $\mathrm{MgSO}_{4}(60.0 \mathrm{mg}, 0.5 \mathrm{mmol}, 2$ equiv), and the mixture was stirred under an atmosphere of nitrogen for $5 \mathrm{~min}$. To the resulting dark red suspension were added the amine catalyst ((S)-A) (15.0 mg, $25.0 \mu \mathrm{mol}, 0.1$ equiv) and dimethylphosphate ( $24.0 \mathrm{mg}, 0.19 \mathrm{mmol}, 0.75$ equiv). The vessel was purged with nitrogen, fitted with a teflon-lined cap, and the resulting solution was stirred at room temperature for the period of time indicated. The mixture was then diluted with hexane $(0.5 \mathrm{~mL})$, loaded directly onto a column and eluted with a mixture of hexane/ethyl acetate/acetic acid. Due to the volatility of the products, residual acetic acid was removed by co-evaporation with cyclohexane instead of high vacuum. The product diastereomers were not separable under these conditions; thus, reported yields refer to the diastereomeric mixture of isolated products.

The thus obtained aldehyde products were reduced to the corresponding primary alcohol (general procedure $\mathbf{C}$ ) prior to SFC analysis.

Bromoacetaldehyde was allylated following the same procedure with the following modifications: $(\boldsymbol{R})$ L, bromoacetaldehyde ( $50 \%$ wt in water, $149 \mathrm{mg}, 0.500 \mathrm{mmol}, 2.0$ equiv), dibenzenesulfonimide ( $37.0 \mathrm{mg}, 0.125 \mathrm{mmol}, 0.500$ equiv.) and $\mathrm{Na}_{2} \mathrm{SO}_{4}(60.0 \mathrm{mg}$ ).

Note: These $\alpha$-halogenated aldehydes epimerized readily on silica, or upon aqueous workups. Hence determination of diastereomeric ratios of crude products was not possible. In all cases NMR-analysis of a reaction aliquot indicated higher diastereomeric ratios (between 15:1 to >20:1) than the isolated products.

\section{C) Reduction of aldehyde products with sodium borohydride}

To a solution of the aldehyde in $\mathrm{CH}_{2} \mathrm{Cl}_{2} / \mathrm{MeOH}(2: 1,0.1 \mathrm{M})$ at $0^{\circ} \mathrm{C}$ was added sodium borohydride (2.0 equiv) and the resulting suspension was stirred at $0^{\circ} \mathrm{C}$ until complete consumption of the starting 
material was observed by TLC. The reaction was then quenched by addition of saturated aqueous $\mathrm{NH}_{4} \mathrm{Cl}$ and diluted with dichloromethane. Water was added until the white precipitate had dissolved completely and the phases were separated. The aqueous phase was extracted with dichloromethane $(2 x)$. The combined organic phases were dried over $\mathrm{Na}_{2} \mathrm{SO}_{4}$, filtered and concentrated. The crude primary alcohols were used for SFC analysis without further purification. Analytically pure samples and corresponding yields were obtained after flash chromatography (hexane/EtOAc).

\section{Optimization Studies}

Supplementary Table 1: Screening of reaction conditions for methylamine. ${ }^{a}$

\begin{tabular}{|c|c|c|c|c|c|}
\hline Entry & $\begin{array}{c}\mathrm{H}_{2} \mathrm{NMe} \text {, } \\
\text { equiv. }\end{array}$ & solvent & acidic promoter (eq.) & yield $^{b}$ & $\mathrm{ee}^{c}$ \\
\hline 1 & 2 & DCE & $\left(\mathrm{PhSO}_{2}\right)_{2} \mathrm{NH}(0.75)$ & $0 \%$ & - \\
\hline 2 & 1.1 & DCE & $\left(\mathrm{PhSO}_{2}\right)_{2} \mathrm{NH}(1.3)$ & $24 \%^{b}$ & $91 \%$ \\
\hline 3 & 1 & DCE & maleic acid (1.05) & $0 \%$ & - \\
\hline 4 & 1 & DCE & $\mathrm{Zn}(\mathrm{OTf})_{3}(1.2)$ & $0 \%$ & - \\
\hline 5 & 1 & DCE & $\mathrm{Zn}(\mathrm{OTf})_{3}(0.5)$ & $6 \%$ & - \\
\hline 6 & 1.1 & DCE & $\mathrm{ArCO}_{2} \mathrm{H}(1.3)$ & $32 \%$ & $98 \%$ \\
\hline 6 & 1.1 & toluene & $\mathrm{ArCO}_{2} \mathrm{H}(1.3)$ & $14 \%^{b}$ & - \\
\hline 7 & 1.1 & acetone & $\mathrm{ArCO}_{2} \mathrm{H}(1.3)$ & $16 \%$ & $2 \%$ \\
\hline 6 & 2 & DCE & $\mathrm{ArCO}_{2} \mathrm{H}(2.3)$ & $44 \%$ & $>99 \%$ \\
\hline
\end{tabular}

${ }^{a}$ reactions carried out on $0.15 \mathrm{mmol}$ scale, for experimental details see page $\mathrm{S} 4 .{ }^{b}$ determined by ${ }^{1} \mathrm{H}-\mathrm{NMR}$ analysis with 1,4-dinitrobenzene as internal standard. $\mathrm{Ar}=3,5$-dichlorophenyl. ${ }^{c}$ determined by SFC analysis of the bis-acetylated product on a chiral stationary phase 
Supplementary Table 2: Screening of reaction conditions for hydroxylamine. ${ }^{a}$

\begin{tabular}{|c|c|c|c|c|c|}
\hline & & $\begin{array}{l}\mathrm{I}_{2} \mathrm{~N}^{-\mathrm{OH}} \\
\% \text { in water }\end{array}$ & 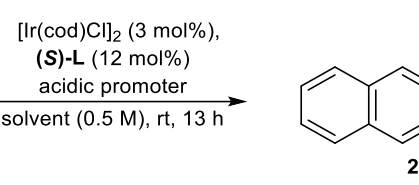 & $\mathrm{N}^{-}$ & \\
\hline Entry & $\begin{array}{c}\mathrm{H}_{2} \mathrm{NOH}, \\
\text { equiv. }\end{array}$ & solvent & acidic promoter (eq.) & yield & $\mathrm{ee}^{d}$ \\
\hline 1 & 2 & DCE & $\left(\mathrm{PhSO}_{2}\right)_{2} \mathrm{NH}(0.75)$ & $0 \%$ & - \\
\hline 2 & 1.1 & DCE & $\left(\mathrm{PhSO}_{2}\right)_{2} \mathrm{NH}(1.3)$ & $57 \%^{b}$ & $93 \%$ \\
\hline 3 & 1.1 & DCE & $\mathrm{ArCO}_{2} \mathrm{H}(1.3)$ & $0 \%$ & - \\
\hline 4 & 1.1 & toluene & $\left(\mathrm{PhSO}_{2}\right)_{2} \mathrm{NH}(1.3)$ & $38 \%^{c}$ & - \\
\hline 5 & 1.1 & dioxane & $\left(\mathrm{PhSO}_{2}\right)_{2} \mathrm{NH}(1.3)$ & $16 \%^{c}$ & - \\
\hline 6 & 1.1 & EtOAc & $\left(\mathrm{PhSO}_{2}\right)_{2} \mathrm{NH}(1.3)$ & $55 \%^{b}$ & $80 \%$ \\
\hline 7 & 1.1 & dioxane & $\left(\mathrm{PhSO}_{2}\right)_{2} \mathrm{NH}(1.3)$ & $0 \%$ & - \\
\hline 8 & 1.1 & DCE & $\left(\mathrm{PhSO}_{2}\right)_{2} \mathrm{NH}(\mathbf{1 . 1 5})$ & $64 \%^{b}$ & $94 \%$ \\
\hline
\end{tabular}

${ }^{a}$ reactions carried out on $0.15 \mathrm{mmol}$ scale, for experimental details see page $\mathrm{S} 4,{ }^{b}$ isolated yields.

${ }^{c}$ determined by $1 \mathrm{H}-\mathrm{NMR}$ analysis with 1,4-dinitrobenzene as internal standard. $\mathrm{Ar}=3,5$-dichlorophenyl

${ }^{d}$ determined by SFC analysis of the bis-acetylated product on a chiral stationary phase

Supplementary Table 3: Screening of acidic promotors for chloroacetaldehyde. ${ }^{a}$

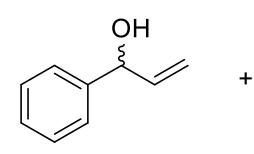

$1 \mathrm{~b}$

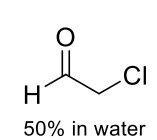

$3 a$

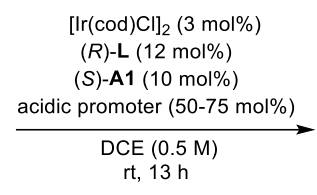

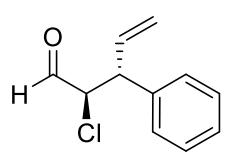

4b

\begin{tabular}{cccc}
\hline Entry & Acidic promoter (mol\%) & conversion $^{b}$ & d.r. $^{b}$ \\
\hline 1 & $\mathrm{Cl}_{2} \mathrm{HCCO}_{2} \mathrm{H}(75)$ & $86 \%$ & $8: 1$ \\
2 & $\mathrm{Cl}_{3} \mathrm{CCO}_{2} \mathrm{H}(50)$ & $84 \%$ & $8: 1$ \\
$\mathbf{3}$ & $(\mathbf{M e O})_{2} \mathbf{P O}_{2} \mathbf{H ~ ( 7 5 )}$ & $\mathbf{8 0 \%}$ & $>\mathbf{2 0 : 1}$ \\
4 & $\left(\mathrm{PhO}_{2} \mathrm{PO}_{2} \mathrm{H}(75)\right.$ & $85 \%$ & $16: 1$ \\
5 & $\left(\mathrm{PhSO}_{2}\right) \mathrm{NH}(50)$ & $92 \%$ & $17: 1$ \\
\hline
\end{tabular}

${ }^{a}$ reactions carried out on $0.25 \mathrm{mmol}$ scale, for experimental details see general procedure $\mathrm{B}^{b}$ determined by ${ }^{1} \mathrm{H}-\mathrm{NMR}$ analysis of a reaction aliquot. 
Supplementary Table 4: Screening additives for chloroacetaldehyde. ${ }^{a}$

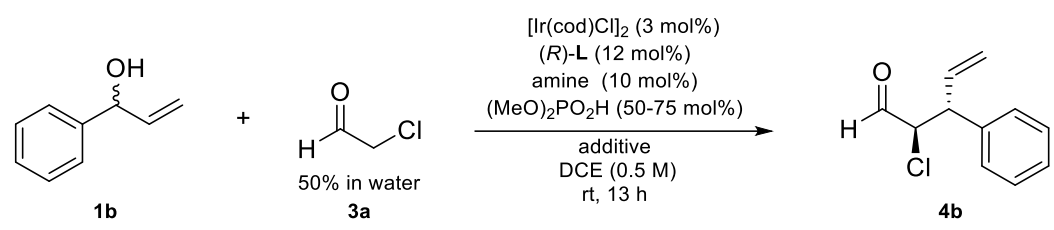

\begin{tabular}{|c|c|c|c|c|c|c|}
\hline Entry & 3a (equiv.) & $\begin{array}{c}(\mathrm{MeO})_{2} \mathrm{P}(\mathrm{O}) \mathrm{OH} \\
(\mathrm{mol} \%)\end{array}$ & additive & amine & conversion $^{b}$ & d.r. ${ }^{b}$ \\
\hline 1 & 2 & 75 & - & $(S)-\mathbf{A 1}$ & $80 \%$ & $>20: 1$ \\
\hline 2 & & & & $(R)-\mathbf{A} \mathbf{1}$ & $71 \%$ & $1: 1$ \\
\hline 3 & 1.5 & 75 & - & $(S)-\mathbf{A 1}$ & $81 \%$ & $18: 1$ \\
\hline 4 & 4 & 75 & - & $(S)-\mathbf{A 1}$ & $86 \%$ & $>20: 1$ \\
\hline 5 & 2 & 100 & - & $(S)-\mathbf{A 1}$ & $82 \%$ & $10: 1$ \\
\hline 6 & 2 & 75 & $4 \AA ̊$ mol sieves & $(S)-\mathbf{A 1}$ & $0 \%$ & - \\
\hline 7 & 3 & 75 & $\mathrm{NaCl}(30 \mathrm{mg})$ & $(S)-\mathbf{A 1}$ & $91 \%$ & $15: 1$ \\
\hline 8 & 2 & 75 & $\mathrm{MgSO}_{4}(30 \mathrm{mg})$ & $(S)$-A1 & $93 \%$ & $14: 1$ \\
\hline 9 & 2 & 75 & $\mathrm{MgSO}_{4}(60 \mathrm{mg})$ & $(S)-\mathrm{A} 1$ & $>98 \%$ & $17: 1$ \\
\hline 10 & 2 & 75 & $\mathrm{Na}_{2} \mathrm{SO}_{4}(30 \mathrm{mg})$ & $(S)-\mathbf{A 1}$ & $77 \%$ & $13: 1$ \\
\hline 11 & 2 & 75 & $\mathrm{Na}_{2} \mathrm{SO}_{4}(60 \mathrm{mg})$ & $(S)-\mathbf{A 1}$ & $76 \%$ & $10: 1$ \\
\hline
\end{tabular}

Supplementary Table 5: Screening of solvents promotors for bromoacetaldehyde. ${ }^{a}$

\begin{tabular}{|c|c|c|c|c|}
\hline \multirow[b]{2}{*}{ Entry } & $\underset{\xi}{\mathrm{OH}}$ & \multicolumn{2}{|c|}{$\begin{array}{c}\text { Ilr(cod)Cl] }]_{2}(3 \mathrm{~mol} \%) \\
(\mathrm{S})-\mathrm{L}(12 \mathrm{~mol} \%) \\
\text { amine }(10 \mathrm{~mol} \%) \\
\mathrm{Cl}_{2} \mathrm{HCCO}_{2} \mathrm{H}(75 \mathrm{~mol} \%) \\
\begin{array}{c}\text { solvent }(0.5 \mathrm{M}) \\
\mathrm{rt}, 14 \mathrm{~h}\end{array}\end{array}$} & \\
\hline & solvent & amine & conversion $^{b}$ & d.r..$^{b}$ \\
\hline 1 & DCE & $(R)-\mathbf{A 1}$ & $93 \%$ & $8: 1$ \\
\hline 2 & DCE & $(S)-\mathbf{A 1}$ & $88 \%$ & $1: 1.3$ \\
\hline 3 & toluene & $(R)-\mathbf{A 1}$ & $20 \%$ & $8: 1$ \\
\hline 4 & EtOAC & $(R)-\mathbf{A 1}$ & $0 \%$ & - \\
\hline 5 & acetone & $(R)-\mathbf{A 1}$ & $0 \%$ & - \\
\hline 6 & 1,4-dioxane & $(R)-\mathbf{A 1}$ & $0 \%$ & - \\
\hline
\end{tabular}

${ }^{a}$ reactions carried out on $0.25 \mathrm{mmol}$ scale, for experimental details see general procedure $\mathrm{B} .{ }^{b}$ determined by ${ }^{1} \mathrm{H}-$ NMR analysis of a reaction aliquot. 


\section{Mechanistic Investigations}

Supplementary Figure 1: Water as a competing nucleophile. A ${ }^{18} \mathrm{O}-$ labeling. B Allylic substitutions with a Boc-protected allylic alcohl. C Racemization of an enantiopure starting material.<smiles>C=CC(O)c1ccc2ccccc2c1</smiles><smiles>C=CC(OC(C)(C)C)c1ccc2ccccc2c1</smiles><smiles>C=C[C@@H](O)c1ccc2ccccc2c1</smiles>

$>99 \%$ ee

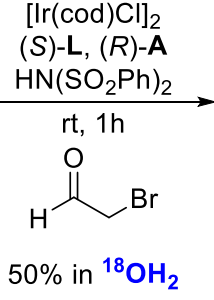<smiles>C=CC(O)c1ccc2ccccc2c1</smiles><smiles>c1ccccc1</smiles>
detected by El-HRMS ca $8 \%{ }^{18} \mathrm{O}$ incoroporation<smiles>C=CC(C=O)C(=O)C(Br)c1ccc2ccccc2c1</smiles><smiles>CCCCCCCCCCCCCCCCCCCC</smiles>

2.3

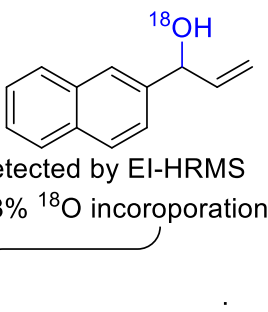<smiles>C=C[C@H](O)c1ccc2ccccc2c1</smiles>

0.7 $>99 \%$ ee<smiles>C=CC(OCC)c1ccc2ccccc2c1</smiles>

7

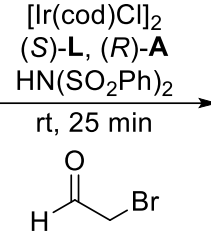

$50 \%$ in water
$[\mathrm{Ir}(\operatorname{cod}) \mathrm{Cl}]_{2}$

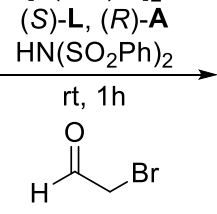

$50 \%$ in water

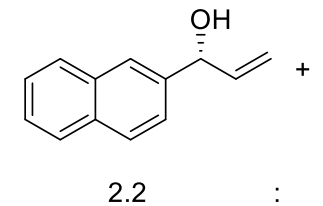

$87 \%$ ee<smiles>C=CC(C=O)C(Br)c1ccc2ccccc2c1</smiles>

$>99 \%$ ee

- absolute configurations determined using chiral SFC analyis by comparing to reference compounds 
Supplementary Figure 2: High resolution EI mass spectrometry of ${ }^{18} \mathrm{O}$-labeled material.

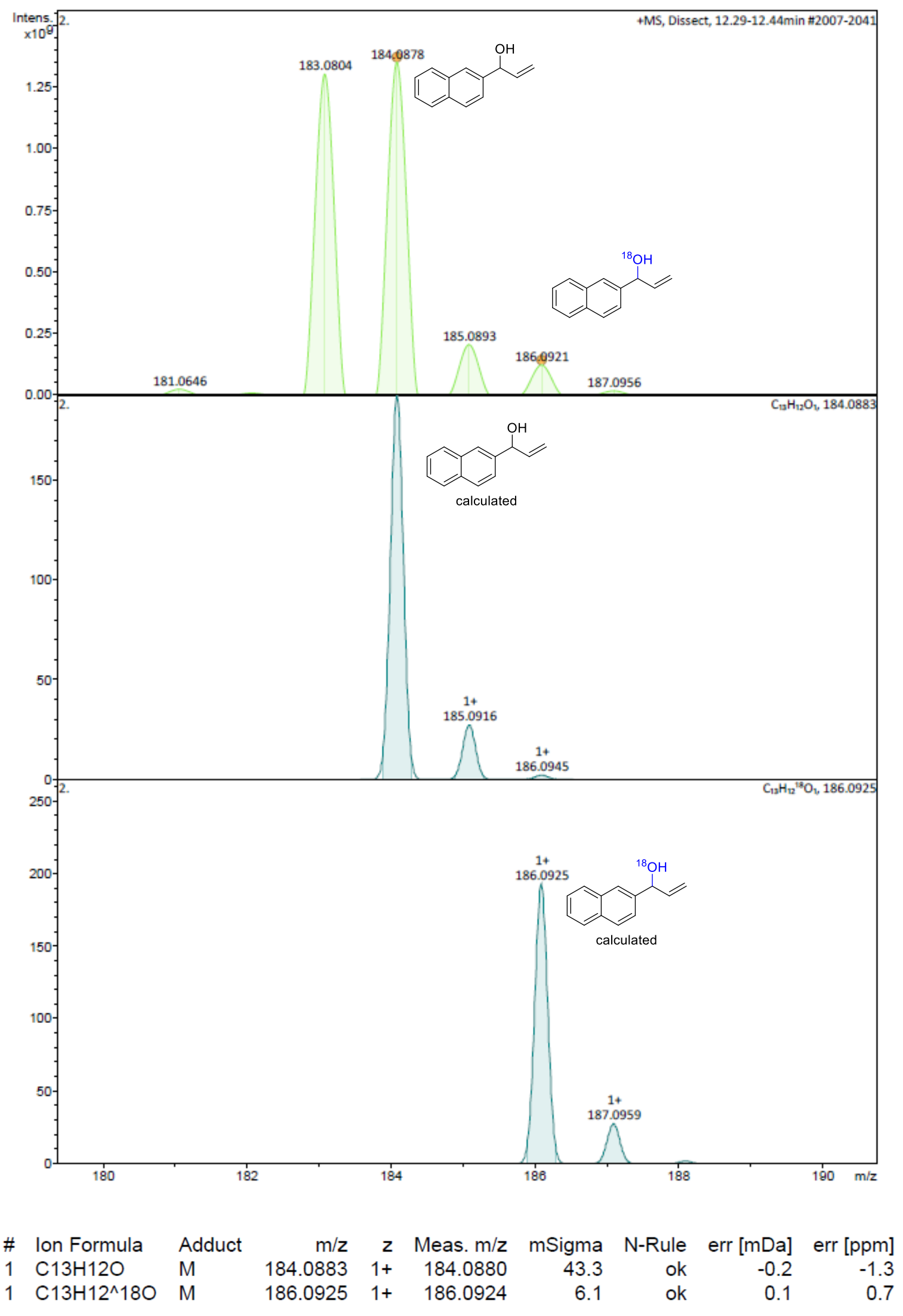


Supplementary Figure 3: SFC data for assignement of absolute configuration of reisolated Boccarbonate

TS: reisolated Boc carbonate from B (supplementary figure 1)

JH: enantioenriched Boc carbonate made via enzymatic resolution, absolute configuration known
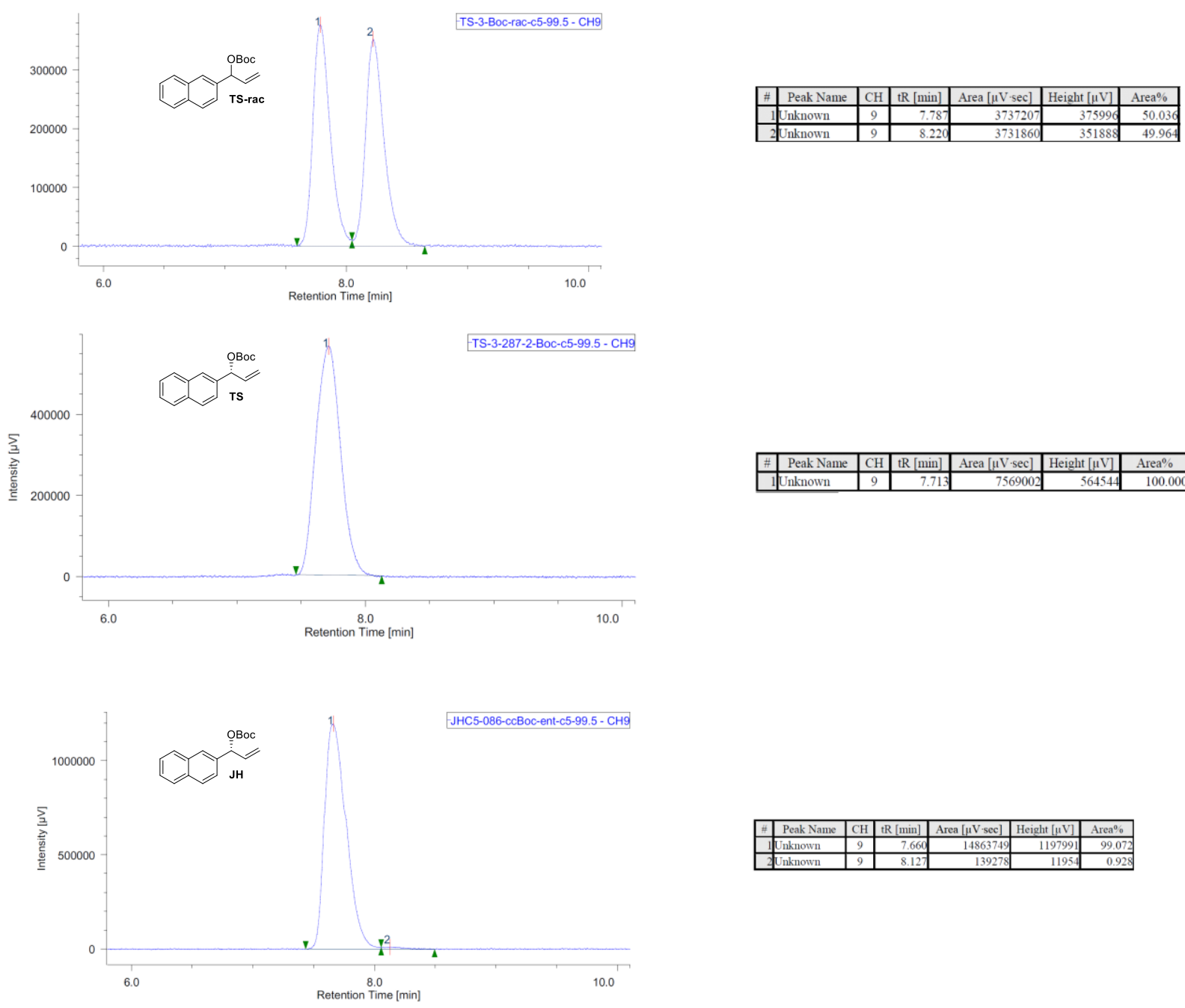
Supplementary Figure 4: SFC data for assignement of absolute configurations of reisolated allylic alcohols

SM: enantioenriched allylic alcohol made via enzymatic resolution, absolute configuration know used in $\mathbf{C}$ (supplementary figure 1)

RS1: reisolated allylic alcohol from $B$

RS2: reisolated allylic alcohol from $\mathbf{C}$
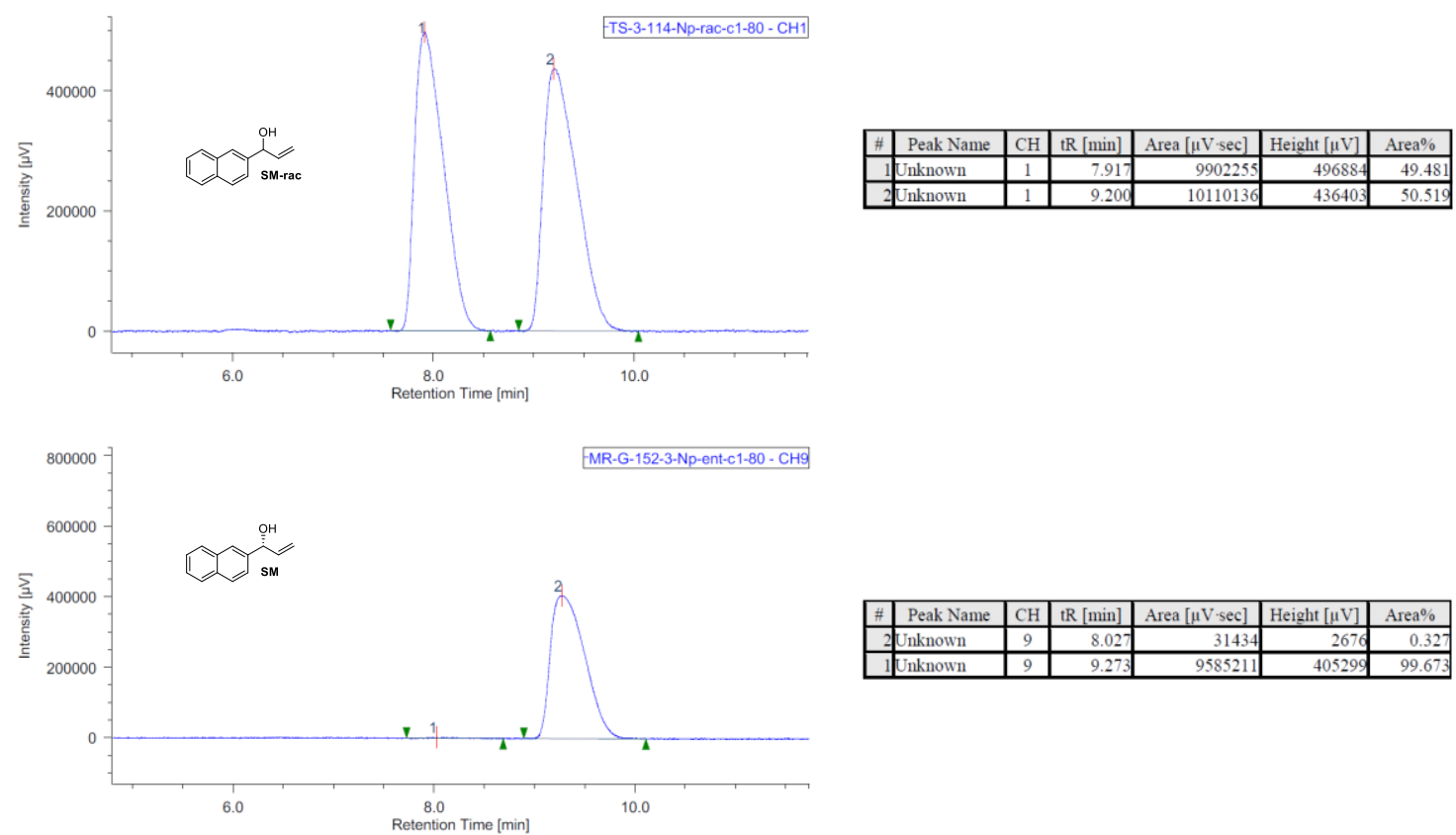

\begin{tabular}{|r|c|c|r|r|r|r|}
\hline$\#$ & Peak Name & $\mathrm{CH}$ & $\mathrm{tR}[\mathrm{min}]$ & Area $[\mu \mathrm{V} \cdot \mathrm{sec}]$ & Height $[\mu \mathrm{V}]$ & \multicolumn{1}{|c|}{ Area\% } \\
\hline 2 & Unknnown & 9 & 8.02 & 31434 & 267 & 0.327 \\
\hline & Unknown & 9 & 9.273 & 9585211 & 40529 & 99.673 \\
\hline
\end{tabular}
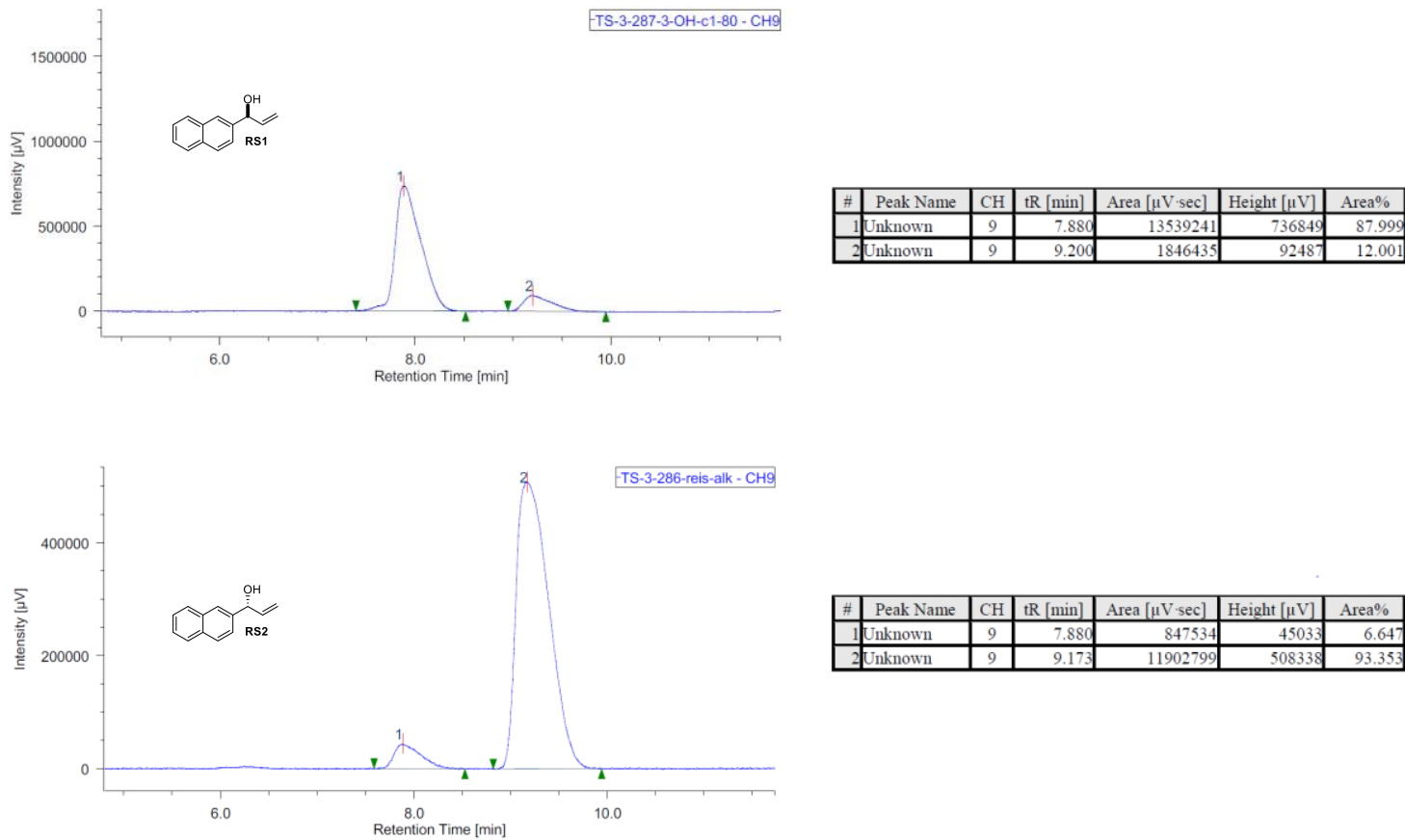


\section{Synthesis and Characterization of Products}

Supplementary Table 6: Allylic alcohols used in this study.<smiles>C=CC(O)c1ccc2ccccc2c1</smiles><smiles>C=CC(O)c1ccccc1</smiles><smiles>C=CC(O)c1ccc(I)cc1</smiles><smiles>C=CC(O)c1ccc(F)cc1</smiles><smiles>C=CC(O)c1ccc(C(C)=O)cc1</smiles><smiles>C=CC(O)c1cccc(C(F)(F)F)c1</smiles><smiles>C=CC(O)c1ccc(OC)cc1</smiles><smiles>C=CC(O)c1cccs1</smiles>

1h<smiles>C=CC(O)c1ccc2c(c1)OCO2</smiles><smiles>C=CC(O)c1cccc(O[Ga](OC(C)(C)C)C(C)(C)C)c1</smiles><smiles>C=Cc1cc(OC)ccc1OCO</smiles><smiles>C=Cc1ccccc1O</smiles>

$\mathrm{OMe} \mathrm{OH}$<smiles>C=CC(O)c1c(OC)cc(C(C)=O)cc1OC</smiles>

$1 \mathrm{~m}$
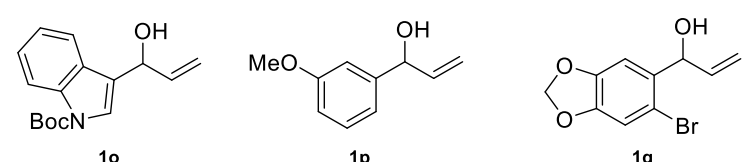<smiles>C=CC(O)c1cccc(Br)c1</smiles><smiles>C=CC(O)c1c[nH]c2ccccc12</smiles>

1s<smiles>C=CC(O)c1ccc(C=O)cc1</smiles>

Supplementary Table 7: Additional examples<smiles>C=C[C@H](NO)c1ccc(C(C)=O)cc1</smiles>

$9 \%, 94 \%$ ee<smiles>C=C[C@@H](NN)C1CCCCC1</smiles>

$2 \mathbf{t}$

$22 \%, 97 \%$ ee<smiles>C=C[C@H](NO)c1ccc(OC)cc1</smiles>

$54 \%, 92 \%$ ee<smiles>C=CC(CO[18O])NN</smiles>

$2 \mathbf{u}$

$<10 \%$ ee

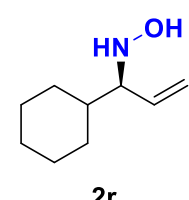

$19 \%,>99 \%$ ee<smiles>C=C[C@H](O)CN1C(=O)c2ccccc2C1=O</smiles>

2s $<10 \%$ ee 
(S)-N'-(1-(naphthalen-2-yl)allyl)benzohydrazide (2a)<smiles>C=CC(NC(=O)c1ccccc1)c1ccc2ccccc2c1</smiles>

The corresponding compound was prepared following general procedure A using $(\boldsymbol{S})$-L, alcohol 1a, hydrazine ( $51 \%$ in water, $17.3 \mathrm{mg}, 275 \mu \mathrm{mol}, 1.1$ equiv.) and dibenzenesulfonimide (97 mg, $325 \mu \mathrm{mol}$, 1.3 equiv.). The reaction stirred at room temperature for $13 \mathrm{~h}$. The reaction was quenched by addition of saturated aqueous $\mathrm{NaHCO}_{3}(2 \mathrm{~mL})$ and the phases were separated. The aqueous phase was extracted with dichloromethane $(2 \times 2 \mathrm{~mL})$ and the combined organic phases were dried over $\mathrm{Na}_{2} \mathrm{SO}_{4}$, filtered and concentrated.

The crude mixture was taken up in dichloromethane $(1.25 \mathrm{~mL})$ and cooled to $0{ }^{\circ} \mathrm{C}$. Hünig's base (175 $\mu \mathrm{L}, 1.00 \mathrm{mmol}, 4.0$ equiv.) and benzoic anhydride (62.2 $\mathrm{mg}, 275 \mu \mathrm{mol}, 1.1$ equiv.) were added sequentially and stirring was continued at the same temperature for $10 \mathrm{~min}$. The cooling bath was removed and the reaction mixture was stirred at room temperature for $3 \mathrm{~h}$. The reaction was quenched by addition of saturated aqueous $\mathrm{NaHCO}_{3}(2 \mathrm{~mL})$ and the phases were separated. The aqueous phase was extracted with ethyl acetate $(2 \times 2 \mathrm{~mL})$ and the combined organic phases were dried over $\mathrm{Na}_{2} \mathrm{SO}_{4}$, filtered and concentrated. Purification by flash chromatography afforded the desired product as an amorphous solid (46 mg, 61\% yield).

Note: The alkylated hydrazine intermediate was found to be very prone to oxidize to the corresponding azo-compound, which underwent 1,3-allylic shifts and numerous decomposition reactions. Hence, workup and acylation were performed quickly, to minimize exposure to air

${ }^{1} \mathbf{H}$ NMR $\left(400 \mathrm{MHz}, \mathrm{CDCl}_{3}\right) \delta 7.90-7.81(\mathrm{~m}, 4 \mathrm{H}), 7.67-7.61(\mathrm{~m}, 2 \mathrm{H}), 7.58(\mathrm{dd}, J=8.5,1.7 \mathrm{~Hz}, 1 \mathrm{H})$, 7.55 (br s, $1 \mathrm{H}), 7.51-7.45(\mathrm{~m}, 3 \mathrm{H}), 7.38(\mathrm{~m}, 2 \mathrm{H}), 6.12(\mathrm{ddd}, J=17.5,10.2,7.5 \mathrm{~Hz}, 1 \mathrm{H}), 5.41(\mathrm{dt}, J=$ 17.1, $1.3 \mathrm{~Hz}, 1 \mathrm{H}), 5.27$ (dt, $J=10.3,1.1 \mathrm{~Hz}, 1 \mathrm{H}), 5.27$ (br s, 1H) 4.87 (d, $J=7.5 \mathrm{~Hz}, 1 \mathrm{H}$ ).

${ }^{13} \mathrm{C}$ NMR $\left(101 \mathrm{MHz}, \mathrm{CDCl}_{3}\right) \delta 167.5,138.2,137.8,133.6,133.3,133.0,132.0,128.8,128.6,128.1$, $127.8,126.97,126.96,126.3,126.2,125.8,118.0,67.9$.

IR (neat) 3280, 3058, 2989, 1636, 1528, 1455, 1346, 925, 818, 748, 693, $478 \mathrm{~cm}^{-1}$.

HRMS (ESI): $m / z$ calcd for $\mathrm{C}_{20} \mathrm{H}_{18} \mathrm{~N}_{2} \mathrm{NaO}[\mathrm{M}+\mathrm{Na}]^{+} 325.1311$ found 325.1312 .

SFC Daicel Chiralpak IB, 15\% MeOH, $2.0 \mathrm{~mL} / \mathrm{min} ., 25{ }^{\circ} \mathrm{C}$, $94 \%$ ee $\left(\mathrm{t}_{\mathrm{R}}(1\right.$, minor enantiomer $)=$ $11.21 \mathrm{~min}, \mathrm{t}_{\mathrm{R}}(2$, major enantiomer $\left.)=13.93 \mathrm{~min}\right)$.

$[\boldsymbol{\alpha}]_{\mathbf{D}}^{25}=-74.8\left(\mathrm{c}=1.0 \mathrm{CHCl}_{3}\right)$. 


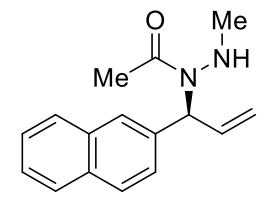

The corresponding compound was prepared following general procedure $\mathbf{A}$ using $(\boldsymbol{S})-\mathbf{L}$, alcohol 1a, methylhydrazine (50\% in water, $25.3 \mathrm{mg}, 275 \mu \mathrm{mol}, 1.1$ equiv.) and and 3,5-dichlorobenzoic acid (62 mg, $325 \mu \mathrm{mol}, 1.3$ equiv.). The reaction was stirred at room temperature for $12 \mathrm{~h}$ and 1,4dinitrobenzene was added as an internal standard. The yield of the reaction was determined by ${ }^{1} \mathrm{H}$ NMR spectroscopy of an aliquot of the reaction mixture $(38.7 \mathrm{mg}, 182 \mu \mathrm{mol}, 73 \%)$. The reaction was quenched addition of saturated aqueous $\mathrm{NaHCO}_{3}(2 \mathrm{~mL})$ and the phases were separated. The aqueous phase was extracted with dichloromethane $(2 \times 2 \mathrm{~mL})$ and the combined organic phases were dried over $\mathrm{Na}_{2} \mathrm{SO}_{4}$, filtered and concentrated.

The crude mixture was taken up in dichloromethane $(1.25 \mathrm{~mL})$ and cooled to $0{ }^{\circ} \mathrm{C}$. Hünig's base (175 $\mu \mathrm{L}, 1.00 \mathrm{mmol}, 4.0$ equiv.) and acetic anhydride ( $35 \mu \mathrm{L}, 371 \mu \mathrm{mol}, 1.5$ equiv.) were added sequentially and stirring was continued at the same temperature for $10 \mathrm{~min}$. The cooling bath was removed and the reaction mixture was stirred at room temperature for $3 \mathrm{~h}$. The reaction was quenched by addition of saturated aqueous $\mathrm{NaHCO}_{3}(2 \mathrm{~mL})$ and the phases were separated. The aqueous phase was extracted with ethyl acetate $(2 \times 2 \mathrm{~mL})$ and the combined organic phases were dried over $\mathrm{Na}_{2} \mathrm{SO}_{4}$, filtered and concentrated. Purification by flash chromatography afforded the desired product as an crystalline, off-white solid (31 mg, 52\% yield). The NMR spectra show a mixture of rotamers (1:1.3 in $\mathrm{CDCl}_{3}, 1: 7$ in Methanol- $\left.d_{4}\right)$. Peaks of the major rotamer are reported.

Note: The alkylated methylhydrazine intermediate was found to be very prone to oxidize to the corresponding azo-compound, which underwent 1,3-allylic shifts and numerous decomposition reactions. Hence, workup and acylation were performed quickly, to minimize exposure to air

${ }^{1}$ H NMR (400 MHz, Methanol- $\left.d_{4}\right) \delta 7.91-7.81(\mathrm{~m}, 4 \mathrm{H}), 7.58(\mathrm{dd}, J=8.5,1.8 \mathrm{~Hz}, 1 \mathrm{H}), 7.51-7.45(\mathrm{~m}$, $2 \mathrm{H}), 6.11$ (ddd, $J=17.1,10.2,8.0 \mathrm{~Hz}, 1 \mathrm{H}), 5.38(\mathrm{dt}, J=17.1,1.3 \mathrm{~Hz}, 1 \mathrm{H}), 5.22(\mathrm{ddd}, J=10.2,1.5,0.7$ $\mathrm{Hz}, 1 \mathrm{H}), 4.87$ (d, $J=7.8 \mathrm{~Hz}, 1 \mathrm{H}), 3.16(\mathrm{~s}, 3 \mathrm{H}), 2.16(\mathrm{~s}, 3 \mathrm{H})$.

${ }^{13}$ C NMR (101 MHz, Methanol- $\left.d_{4}\right) \delta 176.8,139.4,138.9,134.9,134.6,129.2,128.9,128.7,128.0$, $127.2,127.1,126.9,118.2,66.3,33.2,21.2$.

IR (neat) $3455,3253,3056,2979,1646,1382,892,858,821,754,479 \mathrm{~cm}^{-1}$.

HRMS (ESI): $m / z$ calcd for $\mathrm{C}_{16} \mathrm{H}_{19} \mathrm{~N}_{2} \mathrm{O}[\mathrm{M}+\mathrm{H}]^{+} 255.1492$ found 255.1488 .

SFC Daicel Chiralpak IB, $15 \%, 2.0 \mathrm{~mL} / \mathrm{min} ., 25{ }^{\circ} \mathrm{C}, 98 \%$ ee $\left(\mathrm{t}_{\mathrm{R}}(1\right.$, major enantiomer $)=9.55 \mathrm{~min}, \mathrm{t}_{\mathrm{R}}(2$, minor enantiomer) $=14.15 \mathrm{~min}$ ).

$[\boldsymbol{\alpha}]_{\mathbf{D}}^{23}=34.8\left(\mathrm{c}=1.0 \mathrm{CHCl}_{3}\right)$. 
(S)-N'-methyl- $N$-(1-(naphthalen-2-yl)allyl)acetohydrazide (2c)<smiles>C=CC(NN(C)C)c1ccc2ccccc2c1</smiles>

The corresponding compound was prepared following general procedure A using $(\boldsymbol{S})$ - $\mathbf{L}$, alcohol 1a, 1,1dimethylhydrazine (50\% in water, $33.1 \mathrm{mg}, 275 \mu \mathrm{mol}, 1.1$ equiv.) and 3,5-dichlorobenzoic acid (86.0 $\mathrm{mg}, 450 \mu \mathrm{mol}, 1.8$ equiv.). The reaction was stirred at room temperature for $13 \mathrm{~h}$ and then loaded directly onto a column and eluted with hexane/ethyl acetate (3:1) to afford the product as a colorless oil (29 mg, $51 \%$ yield).

${ }^{1} \mathbf{H}$ NMR $\left(500 \mathrm{MHz}, \mathrm{CDCl}_{3}\right) \delta 7.86-7.81(\mathrm{~m}, 4 \mathrm{H}), 7.55(\mathrm{dd}, J=8.5,1.5 \mathrm{~Hz}, 1 \mathrm{H}), 7.50-7.44(\mathrm{~m}, 2 \mathrm{H})$, 6.04 (ddd, $J=17.3,10.2,7.1 \mathrm{~Hz}, 1 \mathrm{H}), 5.33$ (ddd, $J=17.2,1.6,1.1 \mathrm{~Hz}, 1 \mathrm{H}), 5.18$ (ddd, $J=10.2,1.6,0.9$ $\mathrm{Hz}, 1 \mathrm{H}), 4.67(\mathrm{~d}, J=7.1 \mathrm{~Hz}, 1 \mathrm{H}), 2.54(\mathrm{~s}, 6 \mathrm{H}), 2.31$ (bs, 1H).

${ }^{13} \mathrm{C}$ NMR $\left(126 \mathrm{MHz}, \mathrm{CDCl}_{3}\right) \delta 140.3,139.9,133.7,133.1,128.3,128.0,127.8,126.2,126.0,125.81$, $125.75,115.8,65.4,48.2$.

IR (neat) 3056, 2979, 2946, 2843, 2767, 1508, 1467, 1017, 919, 816, 819, 749, 478cm.

HRMS (ESI): $m / z$ calcd for $\mathrm{C}_{15} \mathrm{H}_{19} \mathrm{~N}_{2}[\mathrm{M}+\mathrm{H}]^{+} 227.1543$ found 227.1542 .

SFC Daicel OB-H, 5\% MeOH, $2.0 \mathrm{~mL} / \mathrm{min} ., 25^{\circ} \mathrm{C}, 97 \%$ ee $\left(\mathrm{t}_{\mathrm{R}}(1\right.$, major enantiomer $)=8.10 \mathrm{~min}, \mathrm{t}_{\mathrm{R}}(2$, minor enantiomer) $=11.79 \mathrm{~min}$ ).

$[\boldsymbol{\alpha}]_{\mathrm{D}}^{\mathbf{2 6}}=136.8\left(\mathrm{c}=0.25 \mathrm{CHCl}_{3}\right)$. 


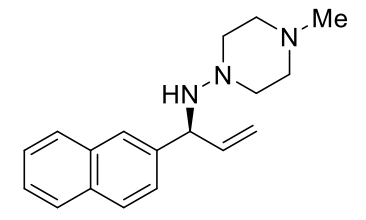

The corresponding compound was prepared following general procedure $\mathbf{A}$ using $(\boldsymbol{S})$-L, alcohol 1a, $1-$ aminopiperazine (50\% in water, $63.3 \mathrm{mg}, 275 \mu \mathrm{mol}, 1.1$ equiv.) and 3,5-dichlorobenzoic acid ( $86.0 \mathrm{mg}$, $450 \mu \mathrm{mol}, 1.8$ equiv.). The reaction was stirred at room temperature for $13 \mathrm{~h}$ and then loaded directly onto a column and eluted with $\mathrm{CH}_{2} \mathrm{Cl}_{2} / \mathrm{MeOH} / \mathrm{NH}_{3}(7 \mathrm{M}$ in $\mathrm{MeOH})(25 / 1 / 0.26)$ to afford the product as a colorless oil ( $44 \mathrm{mg}, 63 \%$ yield).

2k was treated with methyl chloroformate (5.9 equiv.), sodium bicarbonate (6.0 equiv.) in chloroform $(0.1 \mathrm{M})$ at $60{ }^{\circ} \mathrm{C}$ to afford the corresponding carbonate, prior to separation on SFC.

Note: Compound $2 \mathbf{k}$ was found to oxidize readily to the corresponding hydrazone in $\mathrm{CDCl}_{3}$. Therefore, the ${ }^{13} \mathrm{C}$ NMR spectrum of $\mathbf{2 k}$ contains the hydrazone as a trace impurity.

${ }^{1} \mathbf{H}$ NMR $\left(500 \mathrm{MHz}, \mathrm{CDCl}_{3}\right) \delta 7.83-7.76(\mathrm{~m}, 4 \mathrm{H}), 7.51-7.40(\mathrm{~m}, 3 \mathrm{H}), 6.02(\mathrm{ddd}, J=17.1,10.2$, $6.9 \mathrm{~Hz}, 1 \mathrm{H}), 5.25(\mathrm{dt}, J=17.1,1.4 \mathrm{~Hz}, 1 \mathrm{H}), 5.13(\mathrm{ddd}, J=10.2,1.6,1.0 \mathrm{~Hz}, 1 \mathrm{H}), 4.65(\mathrm{~d}, J=6.9 \mathrm{~Hz}$, $1 \mathrm{H}), 2.84(\mathrm{~s}, 4 \mathrm{H}), 2.56(\mathrm{~s}, 4 \mathrm{H}), 2.31(\mathrm{~s}, 3 \mathrm{H})$.

${ }^{13}$ C NMR $\left(125 \mathrm{MHz}, \mathrm{CDCl}_{3}\right) \delta 140.1,139.9,133.6,133.0,128.2,128.0,127.8,126.3,126.1,126.0$, $125.8,115.9,64.9,55.6,54.9,45.4$.

IR (neat) 3399, 3055, 2937, 2839, 2796, 1600, 1508, 1459, 1364, 1285, 1274, 1155, 1012, 920, 856, $819,747,478 \mathrm{~cm}^{-1}$.

HRMS (ESI): $m / z$ calcd for $\mathrm{C}_{18} \mathrm{H}_{24} \mathrm{~N}_{3}[\mathrm{M}+\mathrm{H}]^{+} 282.1965$ found 282.1965 .

SFC (of the corresponding methyl-carbonate) Daicel OB-H, 2\% MeOH, $2.0 \mathrm{~mL} / \mathrm{min} ., 40{ }^{\circ} \mathrm{C},>99 \%$ ee $\left(t_{R}(1\right.$, major enantiomer $)=15.67 \mathrm{~min}, t_{R}(2$, minor enantiomer $\left.)=25.01 \mathrm{~min}\right)$.

$[\boldsymbol{\alpha}]_{\mathbf{D}}^{23}=24.2\left(\mathrm{c}=1.0 \mathrm{CHCl}_{3}\right)$. 
<smiles>C=C[C@H](Nn1cnnc1)c1ccc2ccccc2c1</smiles>

The corresponding compound was prepared following general procedure A using $(\boldsymbol{S})$-L, alcohol 1a, 1aminopiperazine (50\% in water, $63.3 \mathrm{mg}, 275 \mu \mathrm{mol}, 1.1$ equiv.) and 3,5-dichlorobenzoic acid $(86.0 \mathrm{mg}$, $450 \mu \mathrm{mol}, 1.8$ equiv.). The reaction was stirred at room temperature for $13 \mathrm{~h}$ and then loaded directly onto a column and eluted with $\mathrm{CH}_{2} \mathrm{Cl}_{2} / \mathrm{MeOH} / \mathrm{NH}_{3}(7 \mathrm{M}$ in $\mathrm{MeOH})(20 / 1 / 0.25)$ to afford the product as a colorless oil (41 mg, $66 \%$ yield).

Note: Compound $2 \mathrm{l}$ was found to oxidize readily to the corresponding hydrazone in $\mathrm{CDCl}_{3}$. Therefore, the ${ }^{13} \mathrm{C}$ NMR spectrum of $\mathbf{2 l}$ contains the hydrazone as a trace impurity.

${ }^{1} \mathbf{H}$ NMR $\left(500 \mathrm{MHz}, \mathrm{CDCl}_{3}\right) \delta 8.15(\mathrm{~s}, 2 \mathrm{H}), 7.89-7.76(\mathrm{~m}, 3 \mathrm{H}), 7.72(\mathrm{~d}, J=1.9 \mathrm{~Hz}, 1 \mathrm{H}), 7.54-7.44$ (m, 3H), 6.07 (ddd, $J=17.4,10.2,7.3 \mathrm{~Hz}, 1 \mathrm{H}), 5.35-5.23(\mathrm{~m}, 2 \mathrm{H}), 4.83(\mathrm{~d}, J=7.4 \mathrm{~Hz}, 1 \mathrm{H})$.

${ }^{13} \mathrm{C}$ NMR $\left(125 \mathrm{MHz}, \mathrm{CDCl}_{3}\right) \delta 143.2,136.2,135.5,133.5,133.4,129.2,128.1,127.9,127.1,126.8$, $126.8,124.7,119.6,70.3$.

IR (neat) 3203, 3083, 3057, 2928, 1701, 1569, 1509, 1271, 1069, 934, 858, 821, 750, 630, $478 \mathrm{~cm}^{-1}$.

HRMS (ESI): $m / z$ calcd for $\mathrm{C}_{15} \mathrm{H}_{15} \mathrm{~N}_{4}[\mathrm{M}+\mathrm{H}]^{+} 251.1291$ found 251.1295.

SFC Daicel OB-H, 10\% MeOH, $2.0 \mathrm{~mL} / \mathrm{min} ., 25^{\circ} \mathrm{C}$, >99\% ee $\left(\mathrm{t}_{\mathrm{R}}(1\right.$, minor enantiomer $)=26.87 \mathrm{~min}$, $\mathrm{t}_{\mathrm{R}}(2$, major enantiomer $\left.)=31.6 \mathrm{~min}\right)$.

$[\boldsymbol{\alpha}]_{\mathbf{D}}^{24}=-13.6\left(\mathrm{c}=1.0 \mathrm{CHCl}_{3}\right)$.

Melting point $163^{\circ} \mathrm{C}$. 
( $S$ )- $N$-methyl-1-(naphthalen-2-yl)prop-2-en-1-aminium chloride (2f)<smiles>C=CC(O)c1ccc2ccccc2c1</smiles>

The corresponding compound was prepared following general procedure $\mathbf{A}$ using $(\boldsymbol{S})-\mathbf{L}$, alcohol $\mathbf{1 a}$, methylamine ( $40 \%$ in water, $42.1 \mu \mathrm{L}, 500 \mu \mathrm{mol}, 2.0$ equiv.) and 3,5-dichlorobenzoic acid $(110 \mathrm{mg}$, $575 \mu \mathrm{mol}, 2.3$ equiv.). The reaction was stirred at room temperature for $12 \mathrm{~h}$ and then loaded directly onto a column and eluted with $\mathrm{CH}_{2} \mathrm{Cl}_{2} / \mathrm{MeOH} / \mathrm{NH}_{3}(7 \mathrm{M}$ in $\mathrm{MeOH})(19 / 1 / 0.2)$ to afford the recovered starting material as a crystalline solid $(21,45 \%$ yield). The more polar fractions containing the amine were purified by reverse phase flash chromatography (reverse phase silica gel $90 \mathrm{C}_{18}$, Fluka ${ }^{\circledR}$ Analytical, $\mathrm{MeCN} / \mathrm{H}_{2} \mathrm{O} / \mathrm{HCl}$ (aq. 37\%) $=80 / 20 / 1$ ) to afford the ammonium salt of the product as a crystalline solid (24 mg, 41\% yield).

$2 \mathrm{~m}$ was acylated using acetyl chloride (1.1 equiv.) and triethylamine (1.5 equiv) in dichloromethane $(0.1 \mathrm{M})$ at $0^{\circ} \mathrm{C}$ prior to separation of SFC.

${ }^{1}$ H NMR (500 MHz, Methanol- $\left.d_{4}\right) \delta 8.04-7.98(\mathrm{~m}, 2 \mathrm{H}), 7.96-7.90(\mathrm{~m}, 2 \mathrm{H}), 7.61-7.54(\mathrm{~m}, 3 \mathrm{H}), 6.30$ (ddd, $J=17.0,10.4,8.1 \mathrm{~Hz}, 1 \mathrm{H}), 5.64-5.58(\mathrm{~m}, 2 \mathrm{H}), 4.99(\mathrm{~d}, J=8.1 \mathrm{~Hz}, 1 \mathrm{H}), 2.68(\mathrm{~s}, 3 \mathrm{H})$.

${ }^{13}$ C NMR $\left(126 \mathrm{MHz}\right.$, Methanol- $\left.d_{4}\right) \delta 135.0,134.7,133.5,133.2,130.6,129.2,129.0,128.9,128.3$, $128.1,125.5,123.2,67.2,31.8$.

IR (neat) 3402, 2946, 2700, 2486, 1600, 1463, 1421, 939, 822, 750, $479 \mathrm{~cm}^{-1}$.

HRMS (ESI): $m / z$ calcd for $\mathrm{C}_{14} \mathrm{H}_{16} \mathrm{~N}[\mathrm{M}+\mathrm{H}]^{+} 198.1277$ found 198.1275 .

SFC (of the acylated product) Daicel Chiralpak IB, $5 \%, 2.0 \mathrm{~mL} / \mathrm{min} ., 25{ }^{\circ} \mathrm{C}$, >99\% ee $\left(\mathrm{t}_{\mathrm{R}}(1\right.$, major enantiomer $)=12.63 \mathrm{~min}, \mathrm{t}_{\mathrm{R}}(2$, minor enantiomer $\left.)=13.67 \mathrm{~min}\right)$.

$[\boldsymbol{\alpha}]_{\mathbf{D}}^{26}=+81.9\left(\mathrm{c}=1.0 \mathrm{CHCl}_{3}\right)$. Melting point $180^{\circ} \mathrm{C}$.

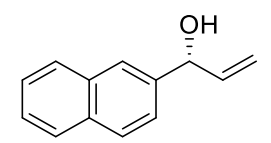

${ }^{1} \mathbf{H}$ NMR $\left(400 \mathrm{MHz}, \mathrm{CDCl}_{3}\right) \delta 7.87-7.80(\mathrm{~m}, 4 \mathrm{H}), 7.52-7.44(\mathrm{~m}, 3 \mathrm{H}), 6.13$ (ddd, $J=17.0,10.3$, $6.0 \mathrm{~Hz}, 1 \mathrm{H}), 5.42(\mathrm{dt}, J=17.0,1.4 \mathrm{~Hz}, 1 \mathrm{H}), 5.41-5.36(\mathrm{~m}, 1 \mathrm{H}), 5.25(\mathrm{~d}, J=10.3 \mathrm{~Hz}, 1 \mathrm{H}), 2.03(\mathrm{~d}, J=$ $3.8 \mathrm{~Hz}, 1 \mathrm{H})$.

${ }^{13}$ C NMR $\left(100 \mathrm{MHz}, \mathrm{CDCl}_{3}\right) \delta 140.3,140.1,133.5,133.2,128.5,128.2,127.8,126.3,126.1,125.1$, 124.7, 115.6, 75.6.

IR (neat) 3363, 3056, 2980, 1508, 1260, 1124, 1019, 989, 927, 856, 819, 746, $477 \mathrm{~cm}^{-1}$.

SFC Daicel Chiralcel OJ-H, 20\% MeOH, $2.0 \mathrm{~mL} / \mathrm{min} ., 25{ }^{\circ} \mathrm{C}, 95 \%$ ee $\left(\mathrm{t}_{\mathrm{R}}(1\right.$, minor enantiomer $)=$ $7.94 \mathrm{~min}, \mathrm{t}_{\mathrm{R}}(2$, major enantiomer $\left.)=9.26 \mathrm{~min}\right)$.

$[\boldsymbol{\alpha}]_{\mathbf{D}}^{25}=-2.0\left(\mathrm{c}=0.3 \mathrm{CHCl}_{3}\right)$. 
(S)- $N$-ethyl-1-(naphthalen-2-yl)prop-2-en-1-aminium (2g)<smiles>C=CC(O)c1ccc2ccccc2c1</smiles>

The corresponding compound was prepared following general procedure $\mathbf{A}$ using $(\boldsymbol{S})-\mathbf{L}$, alcohol 1a, ethylamine (50\% in water, $40 \mu \mathrm{L}, 500 \mu \mathrm{mol}, 2.0$ equiv.) and 3,5-dichlorobenzoic acid (134 mg, $700 \mu \mathrm{mol}, 2.8$ equiv.). The reaction was stirred at room temperature for $13 \mathrm{~h}$ and then loaded directly onto a column and eluted with $\mathrm{CH}_{2} \mathrm{Cl}_{2} / \mathrm{MeOH} / \mathrm{NH}_{3}(7 \mathrm{M}$ in $\mathrm{MeOH})(19 / 1 / 0.2)$ to afford the recovered starting material as a crystalline solid $(19 \mathrm{mg}, 41 \%$ yield). The more polar fractions containing the amine were purified by reverse phase flash chromatography (reverse phase silica gel $90 \mathrm{C}_{18}$, Fluka ${ }^{\circledR}$ Analytical, $\mathrm{MeCN} / \mathrm{H}_{2} \mathrm{O} / \mathrm{HCl}$ (aq. 37\%) $=80 / 20 / 1$ ) to afford the ammonium salt of the product as a crystalline solid ( $27 \mathrm{mg}, 44 \%$ yield).

2n was acylated using acetyl chloride (1.1 equiv.) and triethylamine (1.5 equiv) in dichloromethane $(0.1 \mathrm{M})$ at $0^{\circ} \mathrm{C}$ prior to separation of SFC.

${ }^{1} \mathbf{H}$ NMR (400 MHz, Methanol- $\left.d_{4}\right) \delta 8.04(\mathrm{~d}, J=1.8 \mathrm{~Hz}, 1 \mathrm{H}), 8.02-7.98(\mathrm{~m}, 1 \mathrm{H}), 7.97-7.88(\mathrm{~m}, 2 \mathrm{H})$, $7.62(\mathrm{dd}, J=8.6,1.9 \mathrm{~Hz}, 1 \mathrm{H}), 7.59-7.54(\mathrm{~m}, 2 \mathrm{H}), 6.32(\mathrm{ddd}, J=17.0,10.3,8.2 \mathrm{~Hz}, 1 \mathrm{H}), 5.63-5.53$ $(\mathrm{m}, 2 \mathrm{H}), 5.06(\mathrm{~d}, J=8.2 \mathrm{~Hz}, 1 \mathrm{H}), 3.13(\mathrm{dq}, J=12.5,7.3 \mathrm{~Hz}, 1 \mathrm{H}), 2.97(\mathrm{dq}, J=12.5,7.3 \mathrm{~Hz}, 1 \mathrm{H}), 1.34$ (t, $J=7.3 \mathrm{~Hz}, 3 \mathrm{H})$.

${ }^{13}$ C NMR (101 MHz, Methanol- $\left.d_{4}\right) \delta 135.0,134.7,133.8,133.4,130.6,129.2,129.0,128.9,128.3$, $128.1,125.5,122.9,65.9,42.5,11.4$.

IR (neat) 3393, 2944, 2793, 2503, 1601, 1558, 1365, 939, 821, 799, 749, $479 \mathrm{~cm}^{-1}$.

HRMS (ESI): $m / z$ calcd for $\mathrm{C}_{15} \mathrm{H}_{18} \mathrm{~N}[\mathrm{M}+\mathrm{H}]^{+} 212.1434$ found 212.1431.

SFC Daicel Chiralpak IB, 5\%, $2.0 \mathrm{~mL} / \mathrm{min} ., 25{ }^{\circ} \mathrm{C}, 93 \%$ ee $\left(\mathrm{t}_{\mathrm{R}}(1\right.$, minor enantiomer $)=10.69 \mathrm{~min}, \mathrm{t}_{\mathrm{R}}(2$, major enantiomer) $=11.95 \mathrm{~min}$ ).

$[\boldsymbol{\alpha}]_{\mathrm{D}}^{24}=+72.0\left(\mathrm{c}=1.0 \mathrm{CHCl}_{3}\right)$.<smiles>C=CC(O)c1ccc2ccccc2c1</smiles>

SFC Daicel Chiralcel OJ-H, 20\% MeOH, $2.0 \mathrm{~mL} / \mathrm{min} ., 25{ }^{\circ} \mathrm{C}, 97 \%$ ee $\left(\mathrm{t}_{\mathrm{R}}(1\right.$, minor enantiomer $)=$ $7.94 \mathrm{~min}, \mathrm{t}_{\mathrm{R}}(2$, major enantiomer $\left.)=9.26 \mathrm{~min}\right)$. 
<smiles>C=CC(O)c1ccc2ccccc2c1</smiles>

The corresponding compound was prepared following general procedure $\mathbf{A}$ using $(\boldsymbol{S})-\mathbf{L}$, alcohol 1a, dimethylamine (40\% in water, $34 \mathrm{mg}, 500 \mu \mathrm{mol}, 2.0$ equiv.) and 3,5-dichlorobenzoic acid (110 mg, $575 \mu \mathrm{mol}, 2.3$ equiv.). The reaction was stirred at room temperature for $14 \mathrm{~h}$ and then loaded directly onto a column and eluted with $\mathrm{CH}_{2} \mathrm{Cl}_{2} / \mathrm{MeOH} / \mathrm{NH}_{3}(7 \mathrm{M}$ in $\mathrm{MeOH})(19 / 1 / 0.2)$ to afford the recovered starting material as a crystalline solid $(20 \mathrm{mg}, 43 \%$ yield). The more polar fractions containing the amine were purified by reverse phase flash chromatography (reverse phase silica gel $90 \mathrm{C}_{18}$, Fluka ${ }^{\circledR}$ Analytical, $\mathrm{MeCN} / \mathrm{H}_{2} \mathrm{O} / \mathrm{HCl}$ (aq. 37\%) $=80 / 20 / 1$ ) to afford the ammonium salt of the product as a crystalline solid (26 mg, $45 \%$ yield).

${ }^{1}$ H NMR (400 MHz, Methanol- $\left.d_{4}\right) \delta 8.07(\mathrm{~d}, J=1.8 \mathrm{~Hz}, 1 \mathrm{H}), 8.03(\mathrm{~d}, J=8.5 \mathrm{~Hz}, 1 \mathrm{H}), 7.98-7.91(\mathrm{~m}$, $2 \mathrm{H}), 7.65(\mathrm{dd}, J=8.6,1.9 \mathrm{~Hz}, 1 \mathrm{H}), 7.62-7.55(\mathrm{~m}, 2 \mathrm{H}), 6.48(\mathrm{ddd}, J=16.8,10.2,9.5 \mathrm{~Hz}, 1 \mathrm{H}), 5.71(\mathrm{dt}$, $J=16.9,0.8 \mathrm{~Hz}, 1 \mathrm{H}), 5.64(\mathrm{dd}, J=10.3,0.9 \mathrm{~Hz}, 1 \mathrm{H}), 5.05(\mathrm{~d}, J=9.5 \mathrm{~Hz}, 1 \mathrm{H}), 2.97$ (s, 3H), 2.73 (s, $3 \mathrm{H})$.

${ }^{13}$ C NMR (101 MHz, Methanol- $\left.d_{4}\right) \delta 135.2,134.7,132.4,132.3,130.8,130.1,129.3,128.9,128.6$, $128.3,125.8,125.3,75.3,41.87,41.85$.

IR (neat) 3422, 3016, 2953, 2571, 2460, 1477, 1420, 1012, 951, 926, 823, 748, $480 \mathrm{~cm}^{-1}$.

HRMS (EI): $m / z$ calcd for $\mathrm{C}_{15} \mathrm{H}_{17} \mathrm{~N}[\mathrm{M}]^{+} 211.13555$ found 213.13548 .

HPLC Chiralcel OD-H, $1 \% i$-PrOH in hexane $0.5 \mathrm{~mL} / \mathrm{min}$., $25^{\circ} \mathrm{C}, 94 \%$ ee $\left(\mathrm{t}_{\mathrm{R}}(1\right.$, minor $)=12.30 \mathrm{~min}$, $\mathrm{t}_{\mathrm{R}}(2$, major $\left.)=17.05 \mathrm{~min}\right)$.

$[\alpha]_{\mathbf{D}}^{26}=+118.3\left(\mathrm{c}=1.0 \mathrm{CHCl}_{3}\right)$.

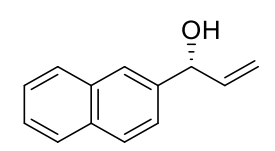

SFC Daicel Chiralcel OJ-H, 20\% MeOH, $2.0 \mathrm{~mL} / \mathrm{min} ., 25{ }^{\circ} \mathrm{C}, 94 \%$ ee $\left(\mathrm{t}_{\mathrm{R}}(1\right.$, minor enantiomer $)=$ $7.94 \mathrm{~min}, \mathrm{t}_{\mathrm{R}}(2$, major enantiomer $\left.)=9.26 \mathrm{~min}\right)$. 


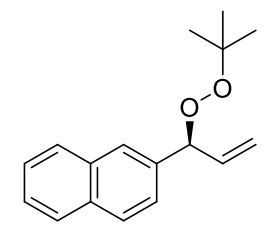

The corresponding compound was prepared following general procedure $\mathbf{A}$ using $(\boldsymbol{S})-\mathbf{L}$, alcohol 1a, $t$ butyl hydroperoxide (70\% in water, $38.0 \mu \mathrm{l}, 274 \mu \mathrm{mol}, 1.1$ equiv.) and (18.6 mg, $62.6 \mu \mathrm{mol}$, 0.25 equiv.). The reaction was stirred at room temperature for $13 \mathrm{~h}$ and then loaded directly onto a column and eluted with $\mathrm{CH}_{2} \mathrm{Cl}_{2} /$ hexane (7/3) to afford the product as a pale yellwo oil $(50 \mathrm{mg}, 66 \%$ yield).

${ }^{1} \mathbf{H}$ NMR $\left(400 \mathrm{MHz}, \mathrm{CDCl}_{3}\right) \delta 7.88-7.81(\mathrm{~m}, 4 \mathrm{H}), 7.53-7.45(\mathrm{~m}, 3 \mathrm{H}), 6.15$ (ddd, $J=17.2,10.4$, $6.7 \mathrm{~Hz}, 1 \mathrm{H}), 5.51(\mathrm{~d}, J=6.7 \mathrm{~Hz}, 1 \mathrm{H}), 5.34(\mathrm{dt}, J=17.3,1.4 \mathrm{~Hz}, 1 \mathrm{H}), 5.32(\mathrm{ddd}, J=10.4,1.1 \mathrm{~Hz}, 1 \mathrm{H})$, $1.28(\mathrm{~s}, 9 \mathrm{H})$.

${ }^{13} \mathrm{C}$ NMR $\left(101 \mathrm{MHz}, \mathrm{CDCl}_{3}\right) \delta 136.9,136.8,133.4,133.3,128.2,128.2,127.8,126.7,126.2,126.1$, 125.5, 118.2, 87.1, 80.7, 26.7.

IR (neat) 3058, 2979, 2930, 1509, 1386, 1363, 1241, 1196, 927, 857, 818, 745, $477 \mathrm{~cm}^{-1}$.

HRMS (ESI): $m / z$ calcd for $\mathrm{C}_{13} \mathrm{H}_{11} \mathrm{O}[\mathrm{M}-t-\mathrm{BuOH}]^{+} 183.0804$ found 183.0803 .

SFC Daicel Chiralcel OJ-H, $2 \% \mathrm{MeOH}, 2.0 \mathrm{~mL} / \mathrm{min} ., 25{ }^{\circ} \mathrm{C}, 90 \%$ ee $\left(\mathrm{t}_{\mathrm{R}}(1\right.$, minor enantiomer $)=$ $8.81 \mathrm{~min}, \mathrm{t}_{\mathrm{R}}(2$, major enantiomer $\left.)=9.55 \mathrm{~min}\right)$.

$[\alpha]_{\mathrm{D}}^{27}=-10.8\left(\mathrm{c}=1.0 \mathrm{CHCl}_{3}\right)$. 


\section{(S)-methyl(1-(naphthalen-2-yl)allyl)sulfane (2j)}<smiles>C=CC(SC)c1ccc2ccccc2c1</smiles>

The corresponding compound was prepared following general procedure $\mathbf{A}$ using $(S)-\mathbf{L}$, alcohol 1a, $t$ butyl hydroperoxide (70\% in water, $38.0 \mu \mathrm{l}, 274 \mu \mathrm{mol}, 1.1$ equiv.) and (18.6 mg, $62.6 \mu \mathrm{mol}$, 0.25 equiv.). The reaction was stirred at room temperature for $10 \mathrm{~h}$ and then quenched by addition of saturated aqueous $\mathrm{Na}_{2} \mathrm{CO}_{3}(2 \mathrm{~mL}) . \mathrm{CH}_{2} \mathrm{Cl}_{2}(2 \mathrm{~mL})$ was added and the phases were separated. The aqueous phase was extracted with dichloromethane $(2 \times 2 \mathrm{~mL})$ and the combined organic phases were dried over $\mathrm{Na}_{2} \mathrm{SO}_{4}$, filtered and concentrated. Purification by flash chromatography (hexane/ethyl acetate $=50 / 1$ to $25 / 1$ ) afforded the product as a pale yellow oil $46 \mathrm{mg}, 86 \%$ yield), containing traces $(\sim 5 \%)$ of the product arising from linear substitution.

${ }^{1} \mathbf{H}$ NMR $\left(400 \mathrm{MHz}, \mathrm{CDCl}_{3}\right) \delta 7.83(\mathrm{dd}, J=9.1,4.1 \mathrm{~Hz}, 3 \mathrm{H}), 7.79-7.77(\mathrm{~m}, 1 \mathrm{H}), 7.54(\mathrm{dd}, J=8.5,1.8$ $\mathrm{Hz}, 1 \mathrm{H}), 7.52-7.45(\mathrm{~m}, 2 \mathrm{H}), 6.20-6.09(\mathrm{~m}, 1 \mathrm{H}), 5.26-5.24(\mathrm{~m}, 1 \mathrm{H}), 5.22-5.21(\mathrm{~m}, 1 \mathrm{H}), 4.49(\mathrm{~d}, J$ $=8.3 \mathrm{~Hz}, 1 \mathrm{H}), 2.04(\mathrm{~s}, 3 \mathrm{H})$.

${ }^{13} \mathrm{C}$ NMR $\left(101 \mathrm{MHz}, \mathrm{CDCl}_{3}\right) \delta 137.9,137.5,133.4,132.9,128.6,128.0,127.8,126.6,126.3,126.1$, $126.0,116.4,54.7,15.2$.

IR (neat) $3055,2915,1678,1508,1124,1018,962,857,814,748,476 \mathrm{~cm}^{-1}$.

HRMS (ESI): $m / z$ calcd for $\mathrm{C}_{14} \mathrm{H}_{14} \mathrm{~S}[\mathrm{M}]^{+} 214.08107$ found 214.08087.

SFC Daicel Chiralpak IB, $1 \% \mathrm{MeOH}, 2.0 \mathrm{~mL} / \mathrm{min} ., 25{ }^{\circ} \mathrm{C}, 80 \%$ ee $\left(\mathrm{t}_{\mathrm{R}}(1\right.$, major enantiomer $)=$ $10.49 \mathrm{~min}, \mathrm{t}_{\mathrm{R}}(2$, minor enantiomer $\left.)=11.15 \mathrm{~min}\right)$.

$[\boldsymbol{\alpha}]_{\mathbf{D}}^{24}=-2.2\left(\mathrm{c}=0.5 \mathrm{CHCl}_{3}\right)$. 
<smiles>C=CC(NO)c1ccc2ccccc2c1</smiles>

The corresponding compound was prepared following general procedure A using $(S)$ - L, alcohol 1a, $N$ hydroxylamine (50\% in water, $17.0 \mu \mathrm{L}, 275 \mu \mathrm{mol}, 1.1$ equiv.) and dibenzenesulfonimide $(97 \mathrm{mg}$, $325 \mu \mathrm{mol}, 1.3$ equiv.). The reaction was stirred at room temperature for $13 \mathrm{~h}$ and then quenched by addition of saturated aqueous $\mathrm{Na}_{2} \mathrm{CO}_{3}(2 \mathrm{~mL})$. Ethyl acetate $(3 \mathrm{~mL})$ was added and the phases were separated. The aqueous phase was extracted with ethyl acetate $(2 \times 3 \mathrm{~mL})$ and the combined organic phases were sequentially washed with water $(3 \mathrm{ml})$ and brine $(3 \mathrm{~mL})$ dried over $\mathrm{Na}_{2} \mathrm{SO}_{4}$, filtered and concentrated. The crude product was purified flash chromatography (hexane/ethyl acetate $=4 / 1$ to $2 / 1$ ) to afford the product as a crystalline solid ( $32 \mathrm{mg}, 64 \%$ yield). The absolute configuration was assigned by X-ray crystallography.

Samples suitable for SFC analysis were obtained by double acylation of $\mathbf{2 a}$ by treatment with triethylamine (3.0 equiv.) and acetyl chloride (2.2 equiv.) in dichloromethane ( $0.24 \mathrm{M})$, followed by column chromatography (hexane/ethyl acetate $=3 / 1$ ).

${ }^{1} \mathbf{H}$ NMR $\left(500 \mathrm{MHz}, \mathrm{CDCl}_{3}\right) \delta 7.85-7.78(\mathrm{~m}, 4 \mathrm{H}), 7.51-7.43(\mathrm{~m}, 3 \mathrm{H}), 6.09$ (ddd, $J=17.4,10.3,7.2$ $\mathrm{Hz}, 1 \mathrm{H}), 5.34$ (dt, $J=17.2,1.3 \mathrm{~Hz}, 1 \mathrm{H}), 5.27$ (ddd, $J=10.4,1.4,0.9 \mathrm{~Hz}, 1 \mathrm{H}), 4.73$ (d, $J=7.2 \mathrm{~Hz}, 1 \mathrm{H})$.

${ }^{13} \mathbf{C}$ NMR $\left(126 \mathrm{MHz}, \mathrm{CDCl}_{3}\right) \delta 137.4,137.3,133.5,133.2,128.5,128.1,127.8,126.9,126.3,126.1$, $125.8,118.1,69.8$.

IR (neat) $3258,3056,2925,1508,1416,1018,991,256,857,819,747,477 \mathrm{~cm}^{-1}$.

HRMS (EI): $m / z$ calcd for $\mathrm{C}_{13} \mathrm{H}_{13} \mathrm{NO}[\mathrm{M}]^{+} 199.09917$ found 199.09880 .

SFC Daicel Chiralpak IB, 5\% MeOH, $2.0 \mathrm{~mL} / \mathrm{min} ., 25^{\circ} \mathrm{C}, 93 \%$ ee $\left(\mathrm{t}_{\mathrm{R}}(1\right.$, minor enantiomer $)=7.15 \mathrm{~min}$, $t_{\mathrm{R}}(2$, major enantiomer $\left.)=8.20 \mathrm{~min}\right)$.

$[\boldsymbol{\alpha}]_{\mathbf{D}}^{26}=+94.2\left(\mathrm{c}=0.2 \mathrm{CHCl}_{3}\right)$.

Melting point (of the corresponding hydrochloride salt) $157^{\circ} \mathrm{C}$. 


\section{(S)-N-(1-(4-iodophenyl)allyl)hydroxylammonium chloride (2l)}<smiles>C=C[C@@H](O)c1ccc(I)cc1</smiles>

The corresponding compound was prepared following general procedure A using $(S)$ - $\mathbf{L}$, alcohol 1c, $N$ hydroxylamine (50\% in water, $17.0 \mu \mathrm{L}, 275 \mu \mathrm{mol}, 1.1$ equiv.) and dibenzenesulfonimide (97 mg, $325 \mu \mathrm{mol}, 1.3$ equiv.). The reaction was stirred at room temperature for $10 \mathrm{~h}$ and then quenched by addition of saturated aqueous $\mathrm{Na}_{2} \mathrm{CO}_{3}(2 \mathrm{~mL})$. Ethyl acetate $(3 \mathrm{~mL})$ was added and the phases were separated. The aqueous phase was extracted with ethyl acetate $(2 \times 3 \mathrm{~mL})$ and the combined organic phases were sequentially washed with water $(3 \mathrm{ml})$ and brine $(3 \mathrm{~mL})$ dried over $\mathrm{Na}_{2} \mathrm{SO}_{4}$, filtered and concentrated. The crude product was purified flash chromatography (hexane/ethyl acetate $=2.5 / 1$ ) followed by reverse phase flash chromatography (reverse phase silica gel $90 \mathrm{C}_{18}$, Fluka ${ }^{\circledR}$ Analytical, $\mathrm{MeCN} / \mathrm{H}_{2} \mathrm{O} / \mathrm{HCl}$ (aq. 37\%) $=80 / 20 / 1$ ) to afford the ammonium salt of the product as a crystalline solid (63 mg, 81\% yield).

${ }^{1}$ H NMR (400 MHz, Methanol- $\left.d_{4}\right) \delta 7.87-7.82(\mathrm{~m}, 2 \mathrm{H}), 7.35-7.30(\mathrm{~m}, 2 \mathrm{H}), 6.26$ (ddd, $J=17.0,10.5$, $7.9 \mathrm{~Hz}, 1 \mathrm{H}), 5.65-5.54(\mathrm{~m}, 2 \mathrm{H}), 5.05(\mathrm{~d}, J=7.7 \mathrm{~Hz}, 1 \mathrm{H})$.

${ }^{13}$ C NMR (101 MHz, Methanol- $\left.d_{4}\right) \delta 139.5,133.7,132.0,131.1,124.4,96.7,68.9$.

IR (neat) 2926, 2767, 2559, 1587, 1487, 1406, 1247, 1063, 1007, 947, $818 \mathrm{~cm}^{-1}$.

HRMS (EI): $m / z$ calcd for $\mathrm{C}_{9} \mathrm{H}_{10} \mathrm{INO}\left[\mathrm{M}-\mathrm{H}_{2} \mathrm{O}\right]^{+} 256.96959$ found 256.96951.

SFC (of the free hydroxylamine) Daicel Chiralpak IA, 20\% MeOH, $2.0 \mathrm{~mL} / \mathrm{min} ., 25{ }^{\circ} \mathrm{C}, 95 \%$ ee $\left(\mathrm{t}_{\mathrm{R}}(1\right.$, minor enantiomer $)=8.56 \mathrm{~min}, \mathrm{t}_{\mathrm{R}}(2$, major enantiomer $\left.)=16.45 \mathrm{~min}\right)$.

$[\boldsymbol{\alpha}]_{\mathrm{D}}^{25}=+53.2(\mathrm{c}=1.0 \mathrm{MeOH})$. 


\section{(S)-N-(1-(4-fluorophenyl)allyl)hydroxylammonium chloride (2m)}<smiles>C=C[C@H](O)c1ccc(F)cc1</smiles>

The corresponding compound was prepared following general procedure $\mathbf{A}$ using $(\boldsymbol{S})$ - $\mathbf{L}$, alcohol $\mathbf{1 d}, \mathrm{N}$ hydroxylamine (50\% in water, $17.0 \mu \mathrm{L}, 275 \mu \mathrm{mol}, 1.1$ equiv.) and dibenzenesulfonimide $(97 \mathrm{mg}$, $325 \mu \mathrm{mol}, 1.3$ equiv.). The reaction was stirred at room temperature for $13 \mathrm{~h}$ and then quenched by addition of saturated aqueous $\mathrm{Na}_{2} \mathrm{CO}_{3}(2 \mathrm{~mL})$. Ethyl acetate $(3 \mathrm{~mL})$ was added and the phases were separated. The aqueous phase was extracted with ethyl acetate $(2 \times 3 \mathrm{~mL})$ and the combined organic phases were sequentially washed with water $(3 \mathrm{ml})$ and brine $(3 \mathrm{~mL})$ dried over $\mathrm{Na}_{2} \mathrm{SO}_{4}$, filtered and concentrated. The crude product was purified flash chromatography (hexane/ethyl acetate $=2.5 / 1$ ) followed by reverse phase flash chromatography (reverse phase silica gel $90 \mathrm{C}_{18}$, Fluka ${ }^{\circledR}$ Analytical, $\mathrm{MeCN} / \mathrm{H}_{2} \mathrm{O} / \mathrm{HCl}($ aq. 37\%) $=80 / 20 / 1$ ) to afford the ammonium salt of the product as a crystalline solid (34 mg, 67\% yield).

${ }^{1}$ H NMR (400 MHz, Methanol- $\left.d_{4}\right) \delta 7.60-7.54(\mathrm{~m}, 2 \mathrm{H}), 7.27-7.19(\mathrm{~m}, 2 \mathrm{H}), 6.28$ (ddd, $J=17.1,10.5$, $7.8 \mathrm{~Hz}, 1 \mathrm{H}), 5.61(\mathrm{dt}, J=10.5,0.9 \mathrm{~Hz}, 1 \mathrm{H}), 5.55(\mathrm{dt}, J=17.1,0.9 \mathrm{~Hz}, 1 \mathrm{H}), 5.08(\mathrm{dt}, J=7.8,1.0 \mathrm{~Hz}$, $1 \mathrm{H})$.

${ }^{13}$ C NMR (101 MHz, Methanol- $\left.d_{4}\right) \delta 164.9(\mathrm{~d}, J=248.3 \mathrm{~Hz}), 132.4(\mathrm{~d}, J=8.6 \mathrm{~Hz}), 131.4,130.0(\mathrm{~d}, J=$ $3.4 \mathrm{~Hz}), 124.2,117.2(\mathrm{~d}, J=22.1 \mathrm{~Hz}), 68.6$.

${ }^{19}$ F NMR $\left(376 \mathrm{MHz}\right.$, Methanol- $\left.d_{4}\right) \delta-114.6$.

IR (neat) 2929, 2766, 1606, 1419, 1233, 1164, 989, 947, $836 \mathrm{~cm}^{-1}$.

HRMS (MALDI): $m / z$ calcd for $\mathrm{C}_{9} \mathrm{H}_{11} \mathrm{FNO}[\mathrm{M}+\mathrm{H}]^{+} 168.0819$ found 168.0819 .

SFC (of the free hydroxylamine) Daicel Chiralpak IA, $20 \% \mathrm{MeOH}, 2.0 \mathrm{~mL} / \mathrm{min} ., 25{ }^{\circ} \mathrm{C}$, $94 \%$ ee $\left(\mathrm{t}_{\mathrm{R}}(1\right.$, minor enantiomer $)=3.46 \mathrm{~min}, \mathrm{t}_{\mathrm{R}}(2$, major enantiomer $\left.)=4.08 \mathrm{~min}\right)$.

$[\boldsymbol{\alpha}]_{\mathbf{D}}^{24}=+54.7(\mathrm{c}=1.0 \mathrm{MeOH})$. 
<smiles>C=C[C@@H](O)c1cccc(C(F)(F)F)c1</smiles>

The corresponding compound was prepared following general procedure A using $(S)-\mathbf{L}$, alcohol $\mathbf{1 f}, N$ hydroxylamine (50\% in water, $17.0 \mu \mathrm{L}, 275 \mu \mathrm{mol}, 1.1$ equiv.) and dibenzenesulfonimide (97 mg, $325 \mu \mathrm{mol}, 1.3$ equiv.). The reaction was stirred at room temperature for $10 \mathrm{~h}$ and then quenched by addition of saturated aqueous $\mathrm{Na}_{2} \mathrm{CO}_{3}(2 \mathrm{~mL})$. Ethyl acetate $(3 \mathrm{~mL})$ was added and the phases were separated. The aqueous phase was extracted with ethyl acetate $(2 \times 3 \mathrm{~mL})$ and the combined organic phases were sequentially washed with water $(3 \mathrm{ml})$ and brine $(3 \mathrm{~mL})$ dried over $\mathrm{Na}_{2} \mathrm{SO}_{4}$, filtered and concentrated. The crude product was purified flash chromatography (hexane/ethyl acetate $=3 / 1$ ) followed by reverse phase flash chromatography (reverse phase silica gel $90 \mathrm{C}_{18}$, Fluka ${ }^{\circledR}$ Analytical, $\mathrm{MeCN} / \mathrm{H}_{2} \mathrm{O} / \mathrm{HCl}($ aq. $37 \%)=80 / 20 / 1$ ) to afford the ammonium salt of the product as a crystalline solid (36 mg, 57\% yield).

${ }^{1}$ H NMR (500 MHz, Methanol- $\left.d_{4}\right) \delta 7.88-7.85(\mathrm{~m}, 1 \mathrm{H}), 7.84-7.78(\mathrm{~m}, 2 \mathrm{H}), 7.71(\mathrm{tt}, J=7.8,0.8 \mathrm{jHz}$, $1 \mathrm{H}), 6.30(\mathrm{ddd}, J=17.1,10.5,7.8 \mathrm{~Hz}, 1 \mathrm{H}), 5.65(\mathrm{dt}, J=10.5,0.8 \mathrm{~Hz}, 1 \mathrm{H}), 5.59$ (dt, $J=17.1,0.9 \mathrm{~Hz}$, $1 \mathrm{H}), 5.23(\mathrm{~d}, J=7.9 \mathrm{~Hz}, 1 \mathrm{H})$.

${ }^{13}$ C NMR (126 MHz, Methanol- $\left.d_{4}\right) \delta 135.4,133.9(\mathrm{q}, J=1.3 \mathrm{~Hz}), 132.5(\mathrm{q}, J=32.6 \mathrm{~Hz}), 131.3,130.9$, 127.7 (q, $J=3.8 \mathrm{~Hz}), 126.9$ (q, $J=3.9 \mathrm{~Hz}), 125.3$ (q, $J=271.6 \mathrm{~Hz}), 124.8$, 68.6.

${ }^{19}$ F NMR $\left(470 \mathrm{MHz}\right.$, Methanol- $\left.d_{4}\right) \delta-64.2$.

IR (neat) 2983, 2937, 2767, 2559, 1771, 1759, 1330, 1247, 1169, 1127, 1076, 805, $702 \mathrm{~cm}^{-1}$.

HRMS (EI): $m / z$ calcd for $\mathrm{C}_{9} \mathrm{H}_{10} \mathrm{INO}[\mathrm{M}-\mathrm{OH}]^{+} 200.06816$ found 200.06764 .

GC (of the free hydroxyl amine) CP-Chirasil-Dex CB $(25 \mathrm{~m} \times 0.25 \mathrm{~mm} \times 0.25 \mu \mathrm{m})$ Temperature: Injector $275{ }^{\circ} \mathrm{C}$, detector $250{ }^{\circ} \mathrm{C}$, oven $120{ }^{\circ} \mathrm{C}$, Gas: $2 \mathrm{~mL} / \mathrm{min} \mathrm{He}, 96 \%$ ee $\left(\mathrm{t}_{\mathrm{R}}(1\right.$, major enantiomer $)=$ $22.64 \mathrm{~min}, \mathrm{t}_{\mathrm{R}}(2$, minor enantiomer $\left.)=25.02 \mathrm{~min}\right)$

$[\boldsymbol{\alpha}]_{\mathbf{D}}^{25}=+60.2(\mathrm{c}=1.0 \mathrm{MeOH})$. 
(S)- $N$-(1-(thiophen-2-yl)allyl)hydroxylammonium chloride (2o)<smiles>C=C[C@@H](O)c1cccs1</smiles>

The corresponding compound was prepared following general procedure A using $(S)$-L, alcohol $1 \mathbf{e}, N$ hydroxylamine (50\% in water, $17.0 \mu \mathrm{L}, 275 \mu \mathrm{mol}, 1.1$ equiv.) and dibenzenesulfonimide (97 mg, $325 \mu \mathrm{mol}, 1.3$ equiv.). The reaction was stirred at room temperature for $10 \mathrm{~h}$ and then quenched by addition of saturated aqueous $\mathrm{Na}_{2} \mathrm{CO}_{3}(2 \mathrm{~mL})$. Ethyl acetate $(3 \mathrm{~mL})$ was added and the phases were separated. The aqueous phase was extracted with ethyl acetate $(2 \times 3 \mathrm{~mL})$ and the combined organic phases were sequentially washed with water $(3 \mathrm{ml})$ and brine $(3 \mathrm{~mL})$ dried over $\mathrm{Na}_{2} \mathrm{SO}_{4}$, filtered and concentrated. The crude product was purified flash chromatography (hexane/ethyl acetate $=2.5 / 1$ ) followed by reverse phase flash chromatography (reverse phase silica gel $90 \mathrm{C}_{18}$, Fluka ${ }^{\circledR}$ Analytical, $\mathrm{MeCN} / \mathrm{H}_{2} \mathrm{O} / \mathrm{HCl}$ (aq. 37\%) $=80 / 20 / 1$ ) to afford the ammonium salt of the product as a crystalline solid (35 mg, 73\% yield).

${ }^{1}$ H NMR (400 MHz, Methanol- $\left.d_{4}\right) \delta 7.67$ (ddd, $\left.J=3.0,1.4,0.5 \mathrm{~Hz}, 1 \mathrm{H}\right), 7.58(\mathrm{ddd}, J=5.1,3.0,0.4 \mathrm{~Hz}$, $1 \mathrm{H}), 7.25(\mathrm{ddd}, J=5.1,1.4,0.4 \mathrm{~Hz}, 1 \mathrm{H}), 6.25(\mathrm{ddd}, J=17.0,10.4,8.0 \mathrm{~Hz}, 1 \mathrm{H}), 5.60(\mathrm{dt}, J=10.4,0.9$ $\mathrm{Hz}, 1 \mathrm{H}), 5.55(\mathrm{dt}, J=17.0,1.0 \mathrm{~Hz}, 1 \mathrm{H}), 5.17(\mathrm{~d}, J=8.2 \mathrm{~Hz}, 1 \mathrm{H})$.

${ }^{13}$ C NMR (101 MHz, Methanol- $\left.d_{4}\right) \delta 134.2,131.3,128.6,127.91,127.88,124.2,64.8$.

IR (neat) 3088, 2920, 2774, 2558, 1582, 1434, 987, 945, 789, $659 \mathrm{~cm}^{-1}$.

HRMS (MALDI): $m / z$ calcd for $\mathrm{C}_{7} \mathrm{H}_{10} \mathrm{NOS}[\mathrm{M}+\mathrm{H}]^{+} 156.0478$ found 159.0478 .

SFC (of the free hydroxylamine) Daicel Chiralpak IA, $20 \% \mathrm{MeOH}, 2.0 \mathrm{~mL} / \mathrm{min} ., 25{ }^{\circ} \mathrm{C}, 97 \%$ ee $\left(\mathrm{t}_{\mathrm{R}}(1\right.$, minor enantiomer $)=5.24 \mathrm{~min}, \mathrm{t}_{\mathrm{R}}(2$, major enantiomer $\left.)=5.79 \mathrm{~min}\right)$.

$[\boldsymbol{\alpha}]_{\mathrm{D}}^{24}=+64.9(\mathrm{c}=1.0 \mathrm{MeOH})$. 
<smiles>C=C[C@@H](O)c1ccc(C(C)=O)cc1</smiles>

The corresponding compound was prepared following general procedure A using $(S)$-L, alcohol $1 \mathbf{e}, N$ hydroxylamine (50\% in water, $17.0 \mu \mathrm{L}, 275 \mu \mathrm{mol}, 1.1$ equiv.) and dibenzenesulfonimide (97 mg, $325 \mu \mathrm{mol}, 1.3$ equiv.). The reaction was stirred at room temperature for $10 \mathrm{~h}$ and then quenched by addition of saturated aqueous $\mathrm{Na}_{2} \mathrm{CO}_{3}(2 \mathrm{~mL})$. Ethyl acetate $(3 \mathrm{~mL})$ was added and the phases were separated. The aqueous phase was extracted with ethyl acetate $(2 \times 3 \mathrm{~mL})$ and the combined organic phases were sequentially washed with water $(3 \mathrm{ml})$ and brine $(3 \mathrm{~mL})$ dried over $\mathrm{Na}_{2} \mathrm{SO}_{4}$, filtered and concentrated. The crude product was purified flash chromatography (hexane/ethyl acetate $=2.5 / 1$ ) followed by reverse phase flash chromatography (reverse phase silica gel $90 \mathrm{C}_{18}$, Fluka ${ }^{\circledR}$ Analytical, $\mathrm{MeCN} / \mathrm{H}_{2} \mathrm{O} / \mathrm{HCl}$ (aq. 37\%) $=80 / 20 / 1$ ) to afford the ammonium salt of the product as a crystalline solid (30 mg, 49\% yield), containing a minor impurity in the aromatic region.

${ }^{1}$ H NMR (400 MHz, Methanol- $\left.d_{4}\right) \delta 8.15-8.08(\mathrm{~m}, 2 \mathrm{H}), 7.65(\mathrm{dt}, J=8.1,0.5 \mathrm{~Hz}, 2 \mathrm{H}), 6.28$ (ddd, $J=$ $17.0,10.4,7.9 \mathrm{~Hz}, 1 \mathrm{H}), 5.67-5.55(\mathrm{~m}, 2 \mathrm{H}), 5.17(\mathrm{~d}, J=7.9 \mathrm{~Hz}, 1 \mathrm{H}), 3.92(\mathrm{~s}, 3 \mathrm{H})$.

${ }^{13}$ C NMR (101 MHz, Methanol- $\left.d_{4}\right) \delta 167.6,138.9,132.7,131.3,131.0,130.2,124.8,68.9,52.9$.

IR (neat) 2952, 2760, 2556, 1722, 1437, 1282, 1248, 1113, 770, $708 \mathrm{~cm}^{-1}$.

HRMS (EI): $m / z$ calcd for $\mathrm{C}_{11} \mathrm{H}_{11} \mathrm{NO}_{2}\left[\mathrm{M}-\mathrm{H}_{2} \mathrm{O}\right]^{+} 189.0784$ found 189.0777 .

SFC (of the free hydroxylamine) Daicel Chiralpak IA, $20 \% \mathrm{MeOH}, 2.0 \mathrm{~mL} / \mathrm{min} ., 25{ }^{\circ} \mathrm{C}$, $94 \%$ ee $\left(\mathrm{t}_{\mathrm{R}}(1\right.$, minor enantiomer $)=8.03 \mathrm{~min}, \mathrm{t}_{\mathrm{R}}(2$, major enantiomer $\left.)=11.64 \mathrm{~min}\right)$.

$[\boldsymbol{\alpha}]_{\mathbf{D}}^{25}=+48.9(\mathrm{c}=1.0 \mathrm{MeOH})$. 


\section{(S)-N-(1-(4-methoxyphenyl)allyl)hydroxylammonium chloride (2q)}<smiles>C=CC(O)(O[Na])c1ccc(OC)cc1</smiles>

The corresponding compound was prepared following general procedure $\mathrm{A}$ using $(\boldsymbol{S})$ - $\mathbf{L}$, alcohol $\mathbf{1 g}, \mathrm{N}$ hydroxylamine (50\% in water, $17.0 \mu \mathrm{L}, 275 \mu \mathrm{mol}, 1.1$ equiv.) and dibenzenesulfonimide ( $97 \mathrm{mg}$, $325 \mu \mathrm{mol}, 1.3$ equiv.). The reaction was stirred at room temperature for $10 \mathrm{~h}$ and then quenched by addition of saturated aqueous $\mathrm{Na}_{2} \mathrm{CO}_{3}(2 \mathrm{~mL})$. Ethyl acetate $(3 \mathrm{~mL})$ was added and the phases were separated. The aqueous phase was extracted with ethyl acetate $(2 \times 3 \mathrm{~mL})$ and the combined organic phases were sequentially washed with water $(3 \mathrm{ml})$ and brine $(3 \mathrm{~mL})$ dried over $\mathrm{Na}_{2} \mathrm{SO}_{4}$, filtered and concentrated. The crude product was purified flash chromatography (hexane/ethyl acetate $=2.5 / 1$ to $2 / 1$ ) followed by reverse phase flash chromatography (reverse phase silica gel $90 \mathrm{C}_{18}$, Fluka ${ }^{\circledR}$ Analytical, $\mathrm{MeCN} / \mathrm{H}_{2} \mathrm{O} / \mathrm{HCl}$ (aq. 37\%) $=80 / 20 / 1$ ) to afford the ammonium salt of the product as a crystalline solid (27 mg, 54\% yield), containing the product arising from linear substitution as a trace ( $3 \%)$ impurity.

${ }^{1}$ H NMR $\left(500 \mathrm{MHz}\right.$, Methanol- $\left.d_{4}\right) \delta 7.44-7.40(\mathrm{~m}, 2 \mathrm{H}), 7.05-7.00(\mathrm{~m}, 2 \mathrm{H}), 6.32-6.21(\mathrm{~m}, 1 \mathrm{H}), 5.58$ $(\mathrm{dt}, J=10.5,1.0 \mathrm{~Hz}, 1 \mathrm{H}), 5.52(\mathrm{dt}, J=17.1,1.0 \mathrm{~Hz}, 1 \mathrm{H}), 4.96(\mathrm{~d}, J=7.8 \mathrm{~Hz}, 1 \mathrm{H}), 3.82(\mathrm{~s}, 3 \mathrm{H})$.

${ }^{13}$ C NMR (126 MHz, Methanol- $\left.d_{4}\right) \delta$ 162.4, 131.7, 131.4, 125.5, 123.6, 115.7, 69.2, 55.9.

IR (neat) 2935, 2838, 2769, 1611, 1515, 1463, 1251, 1182, 1031, $832 \mathrm{~cm}^{-1}$.

HRMS (MALDI): $m / z$ calcd for $\mathrm{C}_{10} \mathrm{H}_{14} \mathrm{NO}_{2}[\mathrm{M}+\mathrm{H}]^{+} 180.1019$ found 180.1017 .

SFC (of the free hydroxylamine) Daicel Chiralpak IA, $20 \% \mathrm{MeOH}, 2.0 \mathrm{~mL} / \mathrm{min} ., 25{ }^{\circ} \mathrm{C}, 92 \%$ ee $\left(\mathrm{t}_{\mathrm{R}}(1\right.$, major enantiomer $)=5.37 \mathrm{~min}, \mathrm{t}_{\mathrm{R}}(2$, minor enantiomer $\left.)=6.09 \mathrm{~min}\right)$.

$[\boldsymbol{\alpha}]_{\mathbf{D}}^{25}=+34.5(\mathrm{c}=0.5 \mathrm{MeOH})$. 


\section{(S)-N-(1-cyclohexylallyl)hydroxylamine (2r)}<smiles>C=CC(NO)C1CCCCC1</smiles>

The corresponding compound was prepared following general procedure A using $(S)-\mathbf{L}$, alcohol $\mathbf{1 g}, \mathrm{N}$ hydroxylamine (50\% in water, $17.0 \mu \mathrm{L}, 275 \mu \mathrm{mol}, 1.1$ equiv.) and dibenzenesulfonimide (97 mg, $325 \mu \mathrm{mol}, 1.3$ equiv.). The reaction was stirred at room temperature for $10 \mathrm{~h}$ and then quenched by addition of saturated aqueous $\mathrm{Na}_{2} \mathrm{CO}_{3}(2 \mathrm{~mL})$. Ethyl acetate $(3 \mathrm{~mL})$ was added and the phases were separated. The aqueous phase was extracted with ethyl acetate $(2 \times 3 \mathrm{~mL})$ and the combined organic phases were sequentially washed with water $(3 \mathrm{ml})$ and brine $(3 \mathrm{~mL})$ dried over $\mathrm{Na}_{2} \mathrm{SO}_{4}$, filtered and concentrated. The crude product was purified flash chromatography (hexane/ethyl acetate $=2 / 1$ ) to afford the product as a crystalline solid (14 $\mathrm{mg}, 19 \%$ yield).

${ }^{1} \mathbf{H}$ NMR $\left(400 \mathrm{MHz}, \mathrm{CDCl}_{3}\right) \delta 5.72(\mathrm{ddd}, J=17.1,10.4,8.5 \mathrm{~Hz}, 1 \mathrm{H}), 5.26-5.16(\mathrm{~m}, 2 \mathrm{H}), 3.18(\mathrm{dd}, J$ $=8.5,6.2 \mathrm{~Hz}, 1 \mathrm{H}), 1.82-1.60(\mathrm{~m}, 5 \mathrm{H}), 1.50(\mathrm{tdt}, J=11.9,6.2,3.1 \mathrm{~Hz}, 1 \mathrm{H}), 1.31-1.11(\mathrm{~m}, 3 \mathrm{H}), 1.08$ $-0.91(\mathrm{~m}, 2 \mathrm{H})$.

${ }^{13}$ C NMR (101 MHz, $\left.\mathrm{CDCl}_{3}\right) \delta 137.2,118.6,70.9,39.3,30.1,29.0,26.7,26.42,26.37$.

IR (neat) $3247,3151,3075,2923,2854,1452,995,916 \mathrm{~cm}^{-1}$.

HRMS (ESI): $m / z$ calcd for $\mathrm{C}_{9} \mathrm{H}_{18} \mathrm{NO}[\mathrm{M}+\mathrm{H}]^{+} 156.1383$ found 156.1382 .

SFC (of the doubly-benzolyated product) Daicel Chiralpak AS-H, 5\% MeOH, $2.0 \mathrm{~mL} / \mathrm{min} ., 25{ }^{\circ} \mathrm{C}$, $>99 \%$ ee $\left(t_{R}(1\right.$, minor enantiomer $)=5.97 \mathrm{~min}, t_{R}(2$, major enantiomer $\left.)=7.23 \mathrm{~min}\right)$.

$[\boldsymbol{\alpha}]_{\mathrm{D}}^{24}=+79.5\left(\mathrm{c}=0.2 \mathrm{CHCl}_{3}\right)$. 


\section{(S)-N'-(1-cyclohexylallyl)benzohydrazide (2t)}<smiles>C=CC(NC(=O)c1ccccc1)C1CCCCC1</smiles>

The corresponding compound was prepared following general procedure $\mathbf{A}$ using $(\boldsymbol{S})$-L, alcohol 1a, hydrazine ( $51 \%$ in water, $17.3 \mathrm{mg}, 275 \mu \mathrm{mol}, 1.1$ equiv.) and dibenzenesulfonimide (97 mg, $325 \mu \mathrm{mol}$, 1.3 equiv.). The reaction stirred at room temperature for $13 \mathrm{~h}$. The reaction was quenched by addition of saturated aqueous $\mathrm{NaHCO}_{3}(2 \mathrm{~mL})$ and the phases were separated. The aqueous phase was extracted with dichloromethane $(2 \times 2 \mathrm{~mL})$ and the combined organic phases were dried over $\mathrm{Na}_{2} \mathrm{SO}_{4}$, filtered and concentrated.

The crude mixture was taken up in dichloromethane $(1.25 \mathrm{~mL})$ and cooled to $0{ }^{\circ} \mathrm{C}$. Hünig's base (175 $\mu \mathrm{L}, 1.00 \mathrm{mmofzzl}, 4.0$ equiv.) and benzoic anhydride $(62.2 \mathrm{mg}, 275 \mu \mathrm{mol}, 1.1$ equiv.) were added sequentially and stirring was continued at the same temperature for $10 \mathrm{~min}$. The cooling bath was removed and the reaction mixture was stirred at room temperature for $3 \mathrm{~h}$. The reaction was quenched by addition of saturated aqueous $\mathrm{NaHCO}_{3}(2 \mathrm{~mL})$ and the phases were separated. The aqueous phase was extracted with ethyl acetate $(2 \times 2 \mathrm{~mL})$ and the combined organic phases were dried over $\mathrm{Na}_{2} \mathrm{SO}_{4}$, filtered and concentrated. Purification by flash chromatography (hexane/ethyl acetate $=9 / 1$ to 5/1) afforded the desired product as an amorphous solid (14 mg, 22\% yield).

Note: The alkylated hydrazine intermediate was found to be very prone to oxidize to the corresponding azo-compound, which underwent 1,3-allylic shifts and numerous decomposition reactions. Hence, workup and acylation were performed quickly, to minimize exposure to air

${ }^{1} \mathbf{H}$ NMR $\left(400 \mathrm{MHz}, \mathrm{CDCl}_{3}\right) \delta 7.77-7.73(\mathrm{~m}, 2 \mathrm{H}), 7.56-7.47(\mathrm{~m}, 1 \mathrm{H}), 7.47-7.39(\mathrm{~m}, 2 \mathrm{H}), 5.74$ (ddd, $J=17.1,10.3,9.0 \mathrm{~Hz}, 1 \mathrm{H}), 5.27-5.17(\mathrm{~m}, 2 \mathrm{H}), 3.33(\mathrm{dd}, J=9.0,6.2 \mathrm{~Hz}, 1 \mathrm{H}), 1.88-1.71(\mathrm{~m}, 4 \mathrm{H})$, $1.70-1.54(\mathrm{~m}, 2 \mathrm{H}), 1.33-1.12(\mathrm{~m}, 4 \mathrm{H}), 1.12-0.97(\mathrm{~m}, 1 \mathrm{H})$.

${ }^{13} \mathrm{C}$ NMR $\left(101 \mathrm{MHz}, \mathrm{CDCl}_{3}\right) \delta 166.9,136.4,132.6,132.6,128.9,127.1,120.0,70.1,40.3,29.9,28.8$, $26.6,26.24,26.22$.

IR (neat) 3280, 3066, 2924, 2852, 1633, 1578, 1536, 1450, 1317, 922, $693 \mathrm{~cm}^{-1}$.

HRMS (ESI): $m / z$ calcd for $\mathrm{C}_{16} \mathrm{H}_{22} \mathrm{~N}_{2} \mathrm{NaO}[\mathrm{M}+\mathrm{Na}]^{+} 281.1624$ found 281.1627 .

SFC Daicel Chiralpak OJ-H, $1 \% \mathrm{MeOH}, 2.0 \mathrm{~mL} / \mathrm{min} ., 25{ }^{\circ} \mathrm{C}, 97 \%$ ee $\left(\mathrm{t}_{\mathrm{R}}(1\right.$, minor enantiomer $)=$ $16.67 \mathrm{~min}, \mathrm{t}_{\mathrm{R}}(2$, major enantiomer $\left.)=21.81 \mathrm{~min}\right)$.

$[\boldsymbol{\alpha}]_{\mathbf{D}}^{26}=19.5\left(\mathrm{c}=4.0 \mathrm{CHCl}_{3}\right)$. 


\section{(2R,3R)-2-chloro-3-(naphthalen-2-yl)pent-4-enal (4a)}<smiles>C=C[C@@H](c1ccc2ccccc2c1)C(Cl)C=O</smiles>

The corresponding compound was prepared following general procedure B using $(\boldsymbol{R})-\mathbf{L},(\boldsymbol{S})$-A1 and alcohol 1a. After stirring at room temperature for 24 hours, the reaction mixture was loaded directly onto a column and eluted with hexane/ethyl acetate/acetic acid (30/1/0.4) to afford the product as a colorless oil (50 mg, $82 \%$ yield). ${ }^{1} \mathrm{H}$ NMR analysis of the isolated product showed a d.r. of 10:1.

${ }^{1} \mathbf{H}$ NMR $\left(400 \mathrm{MHz}, \mathrm{CDCl}_{3}\right) \delta 9.44(\mathrm{~d}, J=3.7 \mathrm{~Hz}, 1 \mathrm{H}), 7.89-7.81(\mathrm{~m}, 3 \mathrm{H}), 7.72(\mathrm{~d}, J=1.8 \mathrm{~Hz}, 1 \mathrm{H})$, $7.55-7.46(\mathrm{~m}, 2 \mathrm{H}), 7.38(\mathrm{dd}, J=8.5,1.8 \mathrm{~Hz}, 1 \mathrm{H}), 6.12(\mathrm{ddd}, J=17.0,10.4,8.1 \mathrm{~Hz}, 1 \mathrm{H}), 5.29(\mathrm{dt}, J$ $=10.4,1.1 \mathrm{~Hz}, 1 \mathrm{H}), 5.27(\mathrm{dt}, J=17.0,1.1 \mathrm{~Hz}, 1 \mathrm{H}), 4.54(\mathrm{dd}, J=8.1,3.7 \mathrm{~Hz}, 1 \mathrm{H}), 4.11(\mathrm{t}, J=8.1 \mathrm{~Hz}$, $1 \mathrm{H})$.

${ }^{13} \mathrm{C}$ NMR $\left(101 \mathrm{MHz}, \mathrm{CDCl}_{3}\right) \delta 194.0,136.0,135.4,133.5,132.9,128.7,128.0,127.9,127.8,126.5$, $126.3,126.4,119.1,65.9,52.3$.

IR (neat) 3021, 2977, 2925, 1732, 1634, 1600, 1508, 1400, 1373, 1272, 1107, 1059, 992, 927, 856, 818, $773,748,666,617,477 \mathrm{~cm}^{-1}$.

HRMS (MALDI): $\mathrm{m} / z$ calcd for $\mathrm{C}_{15} \mathrm{H}_{13} \mathrm{ClNaO}[\mathrm{M}+\mathrm{Na}]^{+} 267.0547$ found 267.0548.

SFC Daicel Chiralpak IA, 10\% MeOH, $2.0 \mathrm{~mL} / \mathrm{min} ., 25{ }^{\circ} \mathrm{C}$, >99\% ee $\left(\mathrm{t}_{\mathrm{R}}(1\right.$, major enantiomer, major diastereomer $)=12.04 \mathrm{~min}, \mathrm{t}_{\mathrm{R}}(2$, minor enantiomer, major diastereomer $)=13.87 \mathrm{~min}, \mathrm{t}_{\mathrm{R}}(3$, major enantiomer, minor diastereomer $)=14.94 \mathrm{~min}, \mathrm{t}_{\mathrm{R}}(4$, minor enantiomer, minor diastereomer $)=$ $16.63 \mathrm{~min})$.

$[\boldsymbol{\alpha}]_{\mathbf{D}}^{24}=-86.9\left(\mathrm{c}=1.0 \mathrm{CHCl}_{3}\right)$. 


\section{(2R,3R)-2-chloro-3-phenylpent-4-enal (4b)}<smiles>C=CC(C(=O)Cl)c1ccccc1</smiles>

The corresponding compound was prepared following general procedure B using $(\boldsymbol{R})-\mathbf{L},(\boldsymbol{S})$-A1 and alcohol 1b. After stirring at room temperature for 14 hours, the reaction mixture was loaded directly onto a column and eluted with hexane/ethyl acetate/acetic acid (30/1/0.4) to afford the product as a colorless oil (36 mg, $74 \%$ yield). ${ }^{1} \mathrm{H}$ NMR analysis of the isolated product showed a d.r. of 10:1.

${ }^{1} \mathbf{H}$ NMR $\left(400 \mathrm{MHz}, \mathrm{CDCl}_{3}\right) \delta 9.39(\mathrm{~d}, J=3.7 \mathrm{~Hz}, 1 \mathrm{H}), 7.39-7.23(\mathrm{~m}, 5 \mathrm{H}), 6.03(\mathrm{ddd}, J=17.1,10.3$, $8.0 \mathrm{~Hz}, 1 \mathrm{H}), 5.27-5.18(\mathrm{~m}, 2 \mathrm{H}), 4.42(\mathrm{dd}, J=8.2,3.7 \mathrm{~Hz}, 1 \mathrm{H}), 3.93(\mathrm{t}, J=8.2 \mathrm{~Hz}, 1 \mathrm{H})$.

${ }^{13}$ C NMR $\left(101 \mathrm{MHz}, \mathrm{CDCl}_{3}\right) \delta 194.0,138.0,136.0,128.9,128.7,127.1,118.9,65.9,52.2$.

IR (neat) 3064, 3031, 2845, 1733, 1494, 1454, 1280, 1113, 1061, 1031, 992, 928, 757, 700, $525 \mathrm{~cm}^{-1}$.

HRMS (MALDI): $\mathrm{m} / z$ calcd for $\mathrm{C}_{11} \mathrm{H}_{11} \mathrm{ClNaO}[\mathrm{M}+\mathrm{Na}]^{+} 217.0391$ found 217.0391 .

SFC Daicel Chiralpak IA, $5 \% \mathrm{MeOH}, 2.0 \mathrm{~mL} / \mathrm{min} ., 25{ }^{\circ} \mathrm{C}$, >99\% ee $\left(\mathrm{t}_{\mathrm{R}}(1\right.$, major enantiomer, major diastereomer $)=8.57, \min t_{R}(2$, minor enantiomer, minor diastereomer $)=9.19 \mathrm{~min}, t_{R}(3$, major enantiomer, minor diastereomer $)=9.75 \mathrm{~min}, \mathrm{t}_{\mathrm{R}}(4$, minor enantiomer, major diastereomer $\left.)=10.33 \mathrm{~min}\right)$.

$[\boldsymbol{\alpha}]_{\mathbf{D}}^{24}=-74.3\left(\mathrm{c}=0.5 \mathrm{CHCl}_{3}\right)$. 


\section{(2R,3R)-2-chloro-3-(4-fluorophenyl)pent-4-enal (4c)}<smiles>C=CC(c1ccc(F)cc1)C(Cl)C=O</smiles>

The corresponding compound was prepared following general procedure B using $(\boldsymbol{R})-\mathbf{L},(\boldsymbol{S})$-A1 and alcohol 1d. After stirring at room temperature for 26 hours, the reaction mixture was loaded directly onto a column and eluted with hexane/ethyl acetate/acetic acid (30/1/0.4) to afford the product as a colorless oil (34 mg, $64 \%$ yield). ${ }^{1} \mathrm{H}$ NMR analysis of the isolated product showed a d.r. of 7:1.

${ }^{1} \mathbf{H}$ NMR $\left(400 \mathrm{MHz}, \mathrm{CDCl}_{3}\right) \delta 9.38(\mathrm{~d}, J=3.5 \mathrm{~Hz}, 1 \mathrm{H}), 7.25-7.19(\mathrm{~m}, 2 \mathrm{H}), 7.08-7.01(\mathrm{~m}, 2 \mathrm{H}), 6.00$ (ddd, $J=17.0,10.3,7.9 \mathrm{~Hz}, 1 \mathrm{H}$ ), 5.25 (app. dt, $J=10.3,1.0 \mathrm{~Hz}, 1 \mathrm{H}$ ), 5.20 (app. dt, $J=17.0,1.0 \mathrm{~Hz}$, $1 \mathrm{H}), 4.39$ (dd, $J=7.9,3.5 \mathrm{~Hz}, 1 \mathrm{H}), 3.95(\mathrm{t}, J=7.9 \mathrm{~Hz}, 1 \mathrm{H})$.

${ }^{13}$ C NMR $\left(101 \mathrm{MHz}, \mathrm{CDCl}_{3}\right) \delta 194.0,162.3(\mathrm{~d}, J=246.5 \mathrm{~Hz}), 135.9,133.7(\mathrm{~d}, J=3.4 \mathrm{~Hz}), 130.4(\mathrm{~d}, J$ $=8.1 \mathrm{~Hz}), 119.0,115.8(\mathrm{~d}, J=21.4 \mathrm{~Hz}), 66.0,51.2$.

${ }^{19}$ F NMR $\left(376 \mathrm{MHz}, \mathrm{CDCl}_{3}\right) \delta-114.6$.

IR (neat) 3084, 2981, 2940, 1734, 1604, 1510, 1414, 1226, 1161, 930, 833, 650, $531 \mathrm{~cm}^{-1}$.

HRMS (MALDI): $m / z$ calcd for $\mathrm{C}_{11} \mathrm{H}_{10} \mathrm{ClFNaO}[\mathrm{M}+\mathrm{Na}]^{+} 235.0296$ found 235.0296.

SFC (of the corresponding carbamate) Daicel Chiralpak IA, $15 \% \mathrm{MeOH}, 2.0 \mathrm{~mL} / \mathrm{min} ., 25^{\circ} \mathrm{C},>99 \%$ ee $\left(t_{R}(1\right.$, major enantiomer, major diastereomer $)=16.37 \mathrm{~min}, t_{R}(2$, major enantiomer, minor diastereomer $)$ $=18.20 \mathrm{~min}, \mathrm{t}_{\mathrm{R}}(3$, minor enantiomer, major diastereomer $)=20.75 \mathrm{~min}, \mathrm{t}_{\mathrm{R}}(4$, minor enantiomer, minor diastereomer) $=23.51 \mathrm{~min})$.

$[\boldsymbol{\alpha}]_{\mathrm{D}}^{24}=-61.4\left(\mathrm{c}=1.0 \mathrm{CHCl}_{3}\right)$. 
<smiles>C=CC(CO)C(Cl)c1ccc(CO)cc1</smiles>

The corresponding compound was prepared following general procedure B using $(\boldsymbol{R})$-L, $(\boldsymbol{S})$-A and alcohol $1 \mathrm{t}$ and dimethylphosphate (0.75 equiv). After stirring at room temperature for 28 hours, the reaction mixture was loaded directly onto a column and eluted with hexane/ethyl acetate/acetic acid (30/1/0.4 to 10/1/0.1). The labile dialdehyde was directly reduced following general procedure $\mathbf{C}$ to afford the corresponding diol as a slightly yellow oil (23 mg, $41 \%$ yield). ${ }^{1} \mathrm{H}$ NMR analysis of the purified dialdehyde showed a d.r. of 5:1.

${ }^{1} \mathbf{H}$ NMR $\left(400 \mathrm{MHz}, \mathrm{CDCl}_{3}\right) \delta 7.37-7.33(\mathrm{~m}, 2 \mathrm{H}), 7.26-7.22(\mathrm{~m}, 2 \mathrm{H}), 6.01$ (ddd, $J=17.0,10.2,8.7$ $\mathrm{Hz}, 1 \mathrm{H}), 5.25-5.13(\mathrm{~m}, 2 \mathrm{H}), 4.69(\mathrm{~s}, 2 \mathrm{H}), 4.32(\mathrm{ddd}, J=8.6,6.4,3.7 \mathrm{~Hz}, 1 \mathrm{H}), 3.91$ (d, $J=11.5 \mathrm{~Hz}$, $1 \mathrm{H}), 3.82-3.66(\mathrm{~m}, 2 \mathrm{H}), 2.00(\mathrm{~s}, 1 \mathrm{H}), 1.57(\mathrm{~s}, 1 \mathrm{H})$.

${ }^{13} \mathrm{C} \mathrm{NMR}\left(126 \mathrm{MHz}, \mathrm{CDCl}_{3}\right) \delta 140.0,139.9,137.6,128.6,127.5,117.9,67.0,65.32,65.25,53.8$.

IR (neat) 339, 3081, 2926, 2874, 1738, 1366. 1216, 1032, 1017, $702 \mathrm{~cm}^{-1}$.

HRMS (MALDI): $\mathrm{m} / z$ calcd for $\mathrm{C}_{12} \mathrm{H}_{15} \mathrm{ClO}_{2}[\mathrm{M}+]^{+} 226.07551$ found 226.07560 .

SFC Daicel Chiralpak AS-H, $15 \% \mathrm{MeOH}, 2.0 \mathrm{~mL} / \mathrm{min} ., 25^{\circ} \mathrm{C}$, >99\% ee $\left(\mathrm{t}_{\mathrm{R}}(1\right.$, major enantiomer, major diastereomer $)=13.26 \mathrm{~min}, \mathrm{t}_{\mathrm{R}}(2$, minor enantiomer, major diastereomer $\left.)=15.25 \mathrm{~min}\right)$.

$[\alpha]_{\mathrm{D}}^{25}=-117.1\left(\mathrm{c}=0.1 \mathrm{CHCl}_{3}\right)$. 


\section{(2R,3R)-2-chloro-3-(4-methoxyphenyl)pent-4-enal (4e)}<smiles>C=CC(c1ccc(OC)cc1)C(Cl)Cl</smiles>

The corresponding compound was prepared following general procedure $\mathbf{B}$ using $(\boldsymbol{R})$-L, $(\boldsymbol{S})$-A and alcohol $1 \mathrm{~g}$ and dimethylphosphate $(15.8 \mathrm{mg}, 0.125 \mathrm{mmol}, 0.5$ equiv). After stirring at room temperature for 16 hours, the reaction mixture was loaded directly onto a column and eluted with hexane/ethyl acetate/acetic acid (30/1/0.4) to afford the product as a colorless oil (37 mg, $65 \%$ yield). ${ }^{1} \mathrm{H}$ NMR analysis of the isolated product showed a d.r. of 10:1.

${ }^{1} \mathbf{H}$ NMR $\left(400 \mathrm{MHz}, \mathrm{CDCl}_{3}\right) \delta 9.40(\mathrm{~d}, J=3.7 \mathrm{~Hz}, 1 \mathrm{H}), 7.22-7.16(\mathrm{~m}, 2 \mathrm{H}), 6.94-6.88(\mathrm{~m}, 2 \mathrm{H}), 6.04$ $(\mathrm{ddd}, J=17.0,10.3,7.9 \mathrm{~Hz}, 1 \mathrm{H}), 5.28-5.18(\mathrm{~m}, 2 \mathrm{H}), 4.41(\mathrm{dd}, J=7.9,3.7 \mathrm{~Hz}, 1 \mathrm{H}), 3.92(\mathrm{t}, J=7.9 \mathrm{~Hz}$, $1 \mathrm{H}), 3.83(\mathrm{~s}, 3 \mathrm{H})$.

${ }^{13} \mathrm{C}$ NMR $\left(101 \mathrm{MHz}, \mathrm{CDCl}_{3}\right) \delta 194.3,159.2,136.3,129.9,129.8,118.5,114.3,66.3,55.4,51.3$.

IR (neat) 2936, 2958, 2837, 1732, 1611, 1512, 1305, 1249, 1180, 1110, 1033, 993, 929, 829, 652, $539 \mathrm{~cm}^{-1}$.

HRMS (MALDI): $m / z$ calcd for $\mathrm{C}_{12} \mathrm{H}_{13} \mathrm{ClNaO}_{2}[\mathrm{M}+\mathrm{Na}]^{+} 204.0496$ found 204.0496.

SFC Daicel Chiralpak IB, $1 \% \mathrm{MeOH}, 2.0 \mathrm{~mL} / \mathrm{min} ., 25{ }^{\circ} \mathrm{C}$, >99\% ee $\left(\mathrm{t}_{\mathrm{R}}(1\right.$, minor enantiomer, minor diastereomer $)=22.60 \mathrm{~min}, \mathrm{t}_{\mathrm{R}}(2$, major enantiomer, major diastereomer $)=23.43 \mathrm{~min}, \mathrm{t}_{\mathrm{R}}(3$, minor enantiomer, major diastereomer $)=25.01 \mathrm{~min}, \mathrm{t}_{\mathrm{R}}(4$, major enantiomer, minor diastereomer $)=$ $31.16 \mathrm{~min})$.

$[\boldsymbol{\alpha}]_{\mathrm{D}}^{24}=-77.6\left(\mathrm{c}=1.0 \mathrm{CHCl}_{3}\right)$. 
<smiles>C=CC(c1ccc2c(c1)OCO2)C(Cl)C=O</smiles>

The corresponding compound was prepared following general procedure $\mathbf{B}$ using $(\boldsymbol{R})-\mathbf{L},(\boldsymbol{S})$-A and alcohol $1 \mathbf{i}$ and dimethylphosphate ( $15.8 \mathrm{mg}, 0.125 \mathrm{mmol}, 0.5$ equiv). After stirring at room temperature for 18 hours, the reaction mixture was loaded directly onto a column and eluted with hexane/ethyl acetate/acetic acid (30/1/0.4) to afford the product as a colorless oil (46 mg, $77 \%$ yield). ${ }^{1} \mathrm{H}$ NMR analysis of the isolated product showed a dr of 12:1.

${ }^{1} \mathbf{H}$ NMR $\left(400 \mathrm{MHz}, \mathrm{CDCl}_{3}\right) \delta 9.37(\mathrm{~d}, J=3.7 \mathrm{~Hz}, 1 \mathrm{H}), 6.78(\mathrm{~d}, J=8.0 \mathrm{~Hz}, 1 \mathrm{H}), 6.75(\mathrm{~d}, J=1.8 \mathrm{~Hz}$, $1 \mathrm{H}), 6.69$ (dd, $J=8.0,1.8 \mathrm{~Hz}, 1 \mathrm{H}), 5.98(\mathrm{ddd}, J=17.0,10.3,8.0 \mathrm{~Hz}, 1 \mathrm{H}), 5.96(\mathrm{~s}, 2 \mathrm{H}), 5.26-5.17$ (m, $2 \mathrm{H}), 4.35(\mathrm{dd}, J=8.0,3.7 \mathrm{~Hz}, 1 \mathrm{H}) 3.86(\mathrm{t}, J=8.0 \mathrm{~Hz}, 1 \mathrm{H})$.

${ }^{13}$ C NMR $\left(101 \mathrm{MHz}, \mathrm{CDCl}_{3}\right) \delta 194.1,148.1,147.2,136.1,131.6,122.2,118.7,108.9,108.6,101.3$, $66.2,51.7$.

IR (neat) 3082, 2979, 2898, 1733, 1504, 1489, 1443, 1245, 1102, 1102, 1039, 931, 861, 811, $678 \mathrm{~cm}^{-1}$

HRMS (MALDI): $\mathrm{m} / z$ calcd for $\mathrm{C}_{12} \mathrm{H}_{12} \mathrm{ClO}[\mathrm{M}+\mathrm{H}]^{+} 239.0469$ found 239.0470

SFC Daicel Chiralpak IA, $5 \% \mathrm{MeOH}, 2.0 \mathrm{~mL} / \mathrm{min} ., 25{ }^{\circ} \mathrm{C}$, >99\% ee $\left(\mathrm{t}_{\mathrm{R}}(1\right.$, major enantiomer, major diastereomer $)=17.51 \min t_{R}(2$, minor enantiomer, minor diastereomer $)=22.24 \mathrm{~min}, t_{R}(3$, minor enantiomer, major diastereomer $)=24.62 \mathrm{~min}, \mathrm{t}_{\mathrm{R}}(4$, major enantiomer, minor diastereomer $)=$ $25.75 \mathrm{~min})$

$[\alpha]_{\mathrm{D}}^{25}=-63.6\left(\mathrm{c}=1.0 \mathrm{CHCl}_{3}\right)$ 
<smiles>C=C[C@H](c1cccc(B2OC(C)(C)C(C)(C)O2)c1)C(Cl)C=O</smiles>

The corresponding compound was prepared following general procedure $\mathbf{B}$ using $(\boldsymbol{R})$-L, $(\boldsymbol{S})$-A and alcohol $\mathbf{1 j}$. After stirring at room temperature for 8 hours, the reaction mixture was loaded directly onto a column and eluted with hexane/ethyl acetate/acetic acid (30/1/0.4 to 4/1/0.05) to afford the product as a colorless oil (43 mg, $55 \%$ yield). ${ }^{1} \mathrm{H}$ NMR analysis of the isolated product showed a d.r. of 15:1.

${ }^{1} \mathbf{H}$ NMR $\left(400 \mathrm{MHz}, \mathrm{CDCl}_{3}\right) \delta 9.42(\mathrm{~d}, J=3.9 \mathrm{~Hz}, 1 \mathrm{H}), 7.76-7.72(\mathrm{~m}, 1 \mathrm{H}), 7.68-7.65(\mathrm{~m}, 1 \mathrm{H}), 7.39$ $-7.33(\mathrm{~m}, 2 \mathrm{H}), 6.05(\mathrm{ddd}, J=17.0,10.3,8.3 \mathrm{~Hz}, 1 \mathrm{H}), 5.22(\mathrm{dt}, J=10.3,0.8 \mathrm{~Hz}, 1 \mathrm{H}), 5.20(\mathrm{dt}, J=17.0$, $1.0 \mathrm{~Hz}, 1 \mathrm{H}), 4.46(\mathrm{dd}, J=8.8,4.0 \mathrm{~Hz}, 1 \mathrm{H}), 3.93(\mathrm{t}, J=8.6 \mathrm{~Hz}, 1 \mathrm{H}), 1.38(\mathrm{~s}, 12 \mathrm{H})$.

${ }^{13} \mathbf{C}$ NMR $\left(101 \mathrm{MHz}, \mathrm{CDCl}_{3}\right) \delta 193.6,137.5,136.0,134.8,134.28,134.28,131.4,128.3,119.0,84.1$, $65.7,52.5,25.0$.

IR (neat) 2780, 1735, 1430, 1359, 1389, 1144, 852, $710 \mathrm{~cm}^{-1}$.

HRMS (ESI): $m / z$ calcd for $\mathrm{C}_{17} \mathrm{H}_{22} \mathrm{BClNaO}_{3}[\mathrm{M}+\mathrm{Na}]^{+} 343.1246$ found 343.1249 .

SFC (of the corresponding carbamate) Daicel Chiralpak IA, $10 \% \mathrm{MeOH}, 1.5 \mathrm{~mL} / \mathrm{min}$., $40{ }^{\circ} \mathrm{C},>99 \%$ ee $\left(t_{R}(1\right.$, major enantiomer, minor diastereomer $)=23.30 \mathrm{~min}, \mathrm{t}_{\mathrm{R}}(2$, minor enantiomer, minor diastereomer $)$ $=24.25 \mathrm{~min}, \mathrm{t}_{\mathrm{R}}(3$, minor enantiomer, major diastereomer $)=25.46 \mathrm{~min}, \mathrm{t}_{\mathrm{R}}(4$, major enantiomer, major diastereomer) $=27.21 \mathrm{~min})$.

$[\boldsymbol{\alpha}]_{\mathbf{D}}^{30}=-45.4\left(\mathrm{c}=1.0 \mathrm{CHCl}_{3}\right)$. 
<smiles>C=CC(c1cc(OC)ccc1OC)C(Cl)Cl</smiles>

The corresponding compound was prepared following general procedure $\mathbf{B}$ using $(\boldsymbol{R})$-L, $(\boldsymbol{S})$-A and alcohol $1 \mathbf{k}$. After stirring at room temperature for 5 hours, the reaction mixture was loaded directly onto a column and eluted with hexane/ethyl acetate/acetic acid (30/1/0.4) to afford the product as a colorless oil (40 mg, $63 \%$ yield). ${ }^{1} \mathrm{H}$ NMR analysis of the isolated product showed a d.r. of 5:1.

${ }^{1} \mathbf{H}$ NMR $\left(400 \mathrm{MHz}, \mathrm{CDCl}_{3}\right) \delta 9.36(\mathrm{~d}, J=3.9 \mathrm{~Hz}, 1 \mathrm{H}), 6.84-6.77(\mathrm{~m}, 3 \mathrm{H}), 6.14-6.04(\mathrm{~m}, 1 \mathrm{H})$, $5.24(\mathrm{dt}, J=7.0,1.1 \mathrm{~Hz}, 1 \mathrm{H}), 5.21(\mathrm{~d}, J=0.9 \mathrm{~Hz}, 1 \mathrm{H}), 4.64(\mathrm{dd}, J=8.0,3.9 \mathrm{~Hz}, 1 \mathrm{H}), 4.24-$ $4.18(\mathrm{~m}, 1 \mathrm{H}), 3.79$ (s, 3H), 3.77 (s, 3H).

${ }^{13} \mathrm{C}$ NMR $\left(101 \mathrm{MHz}, \mathrm{CDCl}_{3}\right)$ 193.5, 153.7, 151.3, 135.1, 127.4, 119.0, 116.6, 112.8, 111.9, 64.8, 56.0, 55.8, 47.3.

IR (neat) 3001, 2940, 2836, 1733, 1499, 1465, 1281, 1221, 1179. 1046, 1025, 929, 804, $714 \mathrm{~cm}^{-1}$.

HRMS (EI): $m / z$ calcd for $\mathrm{C}_{13} \mathrm{H}_{15} \mathrm{ClO}_{3}[\mathrm{M}]^{+} 254.0702$ found 254.0705 .

SFC Daicel Chiralpak IA, $1 \% \mathrm{MeOH}, 2.0 \mathrm{~mL} / \mathrm{min} ., 25{ }^{\circ} \mathrm{C}$, >99\% ee $\left(\mathrm{t}_{\mathrm{R}}(1\right.$, major enantiomer, major diastereomer $)=25.15 \mathrm{~min}, \mathrm{t}_{\mathrm{R}}(2$, minor enantiomer, major diastereomer $)=30.00 \mathrm{~min}, \mathrm{t}_{\mathrm{R}}(3$, major enantiomer, minor diastereomer $)=32.76 \mathrm{~min}, \mathrm{t}_{\mathrm{R}}(4$, minor enantiomer, minor diastereomer $)=$ $38.87 \mathrm{~min})$

$[\boldsymbol{\alpha}]_{\mathrm{D}}^{30}=-139.4\left(\mathrm{c}=1.0 \mathrm{CHCl}_{3}\right)$. 


\section{(2R,3R)-2-chloro-3-(o-tolyl)pent-4-enal (4i)}<smiles>C=CC(c1ccccc1C)C(Cl)C=O</smiles>

The corresponding compound was prepared following general procedure B using $(\boldsymbol{R})$-L, $(\boldsymbol{S})$-A and alcohol 11. After stirring at room temperature for 5 hours, the reaction mixture was loaded directly onto a column and eluted with hexane/ethyl acetate/acetic acid (30/1/0.4) to afford the product as a colorless oil (37 mg, $61 \%$ yield). ${ }^{1} \mathrm{H}$ NMR analysis of the isolated product showed a d.r. of 7:1.

${ }^{1} \mathbf{H}$ NMR $\left(400 \mathrm{MHz}, \mathrm{CDCl}_{3}\right) \delta 9.48(\mathrm{~d}, J=4.2 \mathrm{~Hz}, 1 \mathrm{H}), 7.29-7.18(\mathrm{~m}, 4 \mathrm{H}), 5.92(\mathrm{ddd}, J=17.1,10.2$, $8.1 \mathrm{~Hz}, 1 \mathrm{H}), 5.23(\mathrm{dt}, J=10.3,1.0 \mathrm{~Hz}, 1 \mathrm{H}), 5.18(\mathrm{dt}, J=17.1,1.1 \mathrm{~Hz}, 1 \mathrm{H}), 4.47(\mathrm{dd}, J=9.9,4.2 \mathrm{~Hz}$, $1 \mathrm{H}), 4.25-4.15(\mathrm{~m}, 1 \mathrm{H}), 2.41(\mathrm{~s}, 3 \mathrm{H})$.

${ }^{13}$ C NMR $\left(101 \mathrm{MHz}, \mathrm{CDCl}_{3}\right) \delta 193.2,136.6,136.5,135.8,131.0,127.4,127.0,126.7,119.07,65.2$, 47.6, 19.9.

IR (neat) 3022, 2978, 1735, 1492, 1463, 1050, 991, 929, 756, 726, $665 \mathrm{~cm}^{-1}$.

HRMS (EI): $m / z$ calcd for $\mathrm{C}_{12} \mathrm{H}_{13} \mathrm{ClO}[\mathrm{M}]^{+} 208.650$ found 208.0649 .

SFC Daicel Chiralpak IA, $1 \% \mathrm{MeOH}, 2.0 \mathrm{~mL} / \mathrm{min} ., 25{ }^{\circ} \mathrm{C}$, > $>9 \%$ ee $\left(\mathrm{t}_{\mathrm{R}}(1\right.$, major enantiomer, minor diastereomer $)=20.53 \mathrm{~min}, \mathrm{t}_{\mathrm{R}}(2$, minor enantiomer, major diastereomer $)=22.28 \mathrm{~min}, \mathrm{t}_{\mathrm{R}}(3$, minor enantiomer, minor diastereomer $)=24.28 \mathrm{~min}, \mathrm{t}_{\mathrm{R}}(4$, major enantiomer, major diastereomer $)=$ $25.78 \mathrm{~min})$

$[\boldsymbol{\alpha}]_{\mathbf{D}}^{29}=-75.3\left(\mathrm{c}=1.0 \mathrm{CHCl}_{3}\right)$. 
Methyl 4-((3R,4R)-4-chloro-5-oxopent-1-en-3-yl)-3,5-dimethoxybenzoate (4j)<smiles>C=C[C@H](C(=O)O)c1c(OC)cc(C(C)=O)cc1OC</smiles>

The corresponding compound was prepared following general procedure $\mathbf{B}$ using $(\boldsymbol{R})$ - $\mathbf{L},(\boldsymbol{S})$-A and alcohol $\mathbf{2 m}$. After stirring at room temperature for 5 hours, the reaction mixture was loaded directly onto a column and eluted with hexane/ethyl acetate/acetic acid (30/1/0.4) to afford the product as a colorless oil (67 mg, $86 \%$ yield). ${ }^{1} \mathrm{H}$ NMR analysis of the isolated product showed a d.r. of 2:1.

${ }^{1} \mathbf{H}$ NMR $\left(400 \mathrm{MHz}, \mathrm{CDCl}_{3}\right) 9.41(\mathrm{~d}, J=4.7 \mathrm{~Hz}, 1 \mathrm{H}), 7.26(\mathrm{~s}, 2 \mathrm{H}), 6.12(\mathrm{ddd}, J=17.1,10.1,8.7 \mathrm{~Hz}$, $1 \mathrm{H}), 5.18(\mathrm{dt}, J=17.2,1.2 \mathrm{~Hz}, 1 \mathrm{H}), 5.11(\mathrm{ddd}, J=10.0,1.4,0.7 \mathrm{~Hz}, 1 \mathrm{H}), 4.87(\mathrm{dd}, J=10.8,4.7 \mathrm{~Hz}$, $1 \mathrm{H}), 4.62(\mathrm{dd}, J=10.8,8.7 \mathrm{~Hz}, 1 \mathrm{H}), 3.91(\mathrm{~s}, 3 \mathrm{H}), 3.89$ (s, 3H), 3.87 (s, 3H).

${ }^{13}$ C NMR (101 MHz, $\left.\mathrm{CDCl}_{3}\right) \delta 193.5,166.7,158.3,133.9,130.7,119.5,118.4,105.6,62.5,56.2,52.4$, 42.9 .

IR (neat) 2950, 2842, 1722, 1585, 1456, 1435, 1414, 1322, 1240, 1126, 998, $769 \mathrm{~cm}^{-1}$.

HRMS (MALDI): $\mathrm{m} / z$ calcd for $\mathrm{C}_{15} \mathrm{H}_{17} \mathrm{ClNaO}_{5}[\mathrm{M}+\mathrm{Na}]^{+} 335.0657$ found 360.0655 .

SFC Daicel Chiralpak IA, $2 \% \mathrm{MeOH}, 1.5 \mathrm{~mL} / \mathrm{min} ., 25{ }^{\circ} \mathrm{C},>99 \%$ ee $\left(\mathrm{t}_{\mathrm{R}}(1\right.$, minor enantiomer, major diastereomer $)=16.98 \mathrm{~min}, \mathrm{t}_{\mathrm{R}}(2$, major enantiomer, major diastereomer $)=18.06 \mathrm{~min}, \mathrm{t}_{\mathrm{R}}(3$, major enantiomer, minor diastereomer $)=20.34 \mathrm{~min}, \mathrm{t}_{\mathrm{R}}(4$, major enantiomer, minor diastereomer $)=$ $21.54 \mathrm{~min})$

$[\boldsymbol{\alpha}]_{\mathbf{D}}^{30}=-68.1\left(\mathrm{c}=1.0 \mathrm{CHCl}_{3}\right)$. 


\section{(2R,3R)-2-chloro-3-(thiophen-3-yl)pent-4-enal (4k)}<smiles>C=CC(c1ccsc1)C(Cl)C=O</smiles>

The corresponding compound was prepared following general procedure $\mathbf{B}$ using $(\boldsymbol{R})$-L, $(\boldsymbol{S})$-A and alcohol $\mathbf{2 h}$. After stirring at room temperature for 19 hours, the reaction mixture was loaded directly onto a column and eluted with hexane/ethyl acetate/acetic acid (30/1/0.4) to afford the product as a colorless oil (37 mg, $73 \%$ yield). ${ }^{1} \mathrm{H}$ NMR analysis of the isolated product showed a dr of 10:1.

${ }^{1} \mathbf{H}$ NMR $\left(400 \mathrm{MHz}, \mathrm{CDCl}_{3}\right) \delta 9.39(\mathrm{~d}, J=3.2 \mathrm{~Hz}, 1 \mathrm{H}), 7.32(\mathrm{dd}, J=5.1,3.0 \mathrm{~Hz}, 1 \mathrm{H}), 7.18-7.12(\mathrm{~m}$, $1 \mathrm{H}), 7.01$ (dd, $J=5.1,1.3 \mathrm{~Hz}, 1 \mathrm{H}), 6.04$ (ddd, $J=17.0,10.4,7.9 \mathrm{~Hz}, 1 \mathrm{H}$ ), 5.26 (app. dt, $J=10.4,1.2 \mathrm{~Hz}$, $1 \mathrm{H}), 5.22$ (app. dt, $J=17.0,1.2 \mathrm{~Hz}, 1 \mathrm{H}), 4.40(\mathrm{dd}, J=6.5,3.1 \mathrm{~Hz}, 1 \mathrm{H}), 4.17(\mathrm{dd}, J=7.9,6.5 \mathrm{~Hz}, 1 \mathrm{H})$.

${ }^{13}$ C NMR $\left(101 \mathrm{MHz}, \mathrm{CDCl}_{3}\right) \delta 194.7,138.0,135.7,127.6,126.1,123.2,118.8,66.4,47.5$.

IR (neat) 3475, 3100, 2978, 2925, 1732, 1638, 1411, 1103, 1055, 992, 927, 839, 783, 715, 685, 663, $618 \mathrm{~cm}^{-1}$.

HRMS (MALDI): $\mathrm{m} / z$ calcd for $\mathrm{C}_{9} \mathrm{H}_{9} \mathrm{ClNaOS}[\mathrm{M}+\mathrm{Na}]^{+} 222.9955$ found 222.9955 .

SFC (of the corresponding carbonate) Daicel Chiralpak IA, $30 \% \mathrm{MeOH}, 2.0 \mathrm{~mL} / \mathrm{min} ., 25^{\circ} \mathrm{C},>99 \%$ ee $\left(t_{R}(1\right.$, major enantiomer, major diastereomer $)=10.21$ min $t_{R}(2$, major enantiomer, minor diastereomer $)$ $=12.70 \mathrm{~min}, \mathrm{t}_{\mathrm{R}}(3$, minor enantiomer, minor diastereomer $)=14.68 \mathrm{~min}, \mathrm{t}_{\mathrm{R}}(4$, minor enantiomer, major diastereomer) $=18.20 \mathrm{~min})$.

$[\boldsymbol{\alpha}]_{\mathrm{D}}^{25}=-74.8\left(\mathrm{c}=1.0 \mathrm{CHCl}_{3}\right)$. 
<smiles>C=CC(c1cn(C(=O)OC(C)(C)C)c2ccccc12)C(Cl)C=O</smiles>

The corresponding compound was prepared following general procedure $\mathbf{B}$ using $(\boldsymbol{R})$-L, $(\boldsymbol{S})$-A and alcohol 2o. After stirring at room temperature for 19 hours, the reaction mixture was loaded directly onto a column and eluted with hexane/ethyl acetate/acetic acid (30/1/0.4) to afford the product as a colorless oil (63 mg, $75 \%$ yield). ${ }^{1} \mathrm{H}$ NMR analysis of the isolated product showed a dr of 20:1.

${ }^{1} \mathbf{H}$ NMR $\left(500 \mathrm{MHz}, \mathrm{CDCl}_{3}\right) \delta 9.45(\mathrm{~d}, J=3.3 \mathrm{~Hz}, 1 \mathrm{H}), 8.16(\mathrm{~d}, J=8.4 \mathrm{~Hz}, 1 \mathrm{H}), 7.53$ (ddd, $J=7.9,1.3$, $0.7 \mathrm{~Hz}, 1 \mathrm{H}), 7.61(\mathrm{bs}, 1 \mathrm{H}), 7.34(\mathrm{ddd}, J=8.4,7.1,1.2 \mathrm{H} \quad$ z, $1 \mathrm{H}), 7.26(\mathrm{ddd}, J=7.9,7.3,1.1 \mathrm{~Hz}$, $1 \mathrm{H}), 6.09$ (ddd, $J=17.0,10.2,7.7 \mathrm{~Hz}, 1 \mathrm{H}), 5.31(\mathrm{dt}, J=9.3,1.1 \mathrm{~Hz}, 1 \mathrm{H}), 5.28(\mathrm{dt}, J=2.5,1.0 \mathrm{~Hz}, 1 \mathrm{H})$, $4.58(\mathrm{dd}, J=6.6,3.2 \mathrm{~Hz}, 1 \mathrm{H}), 4.28(\mathrm{ddq}, J=7.7,6.6,1.0 \mathrm{~Hz}, 1 \mathrm{H}), 1.69$ (s, 9H).

${ }^{13}$ C NMR $\left(126 \mathrm{MHz}, \mathrm{CDCl}_{3}\right) \delta 194.5,149.7,135.4,134.9,129.4,124.9,124.4,122.8,119.3,119.1$, $116.9,115.6,84.2,65.6,43.6,28.3$.

IR (neat) 3054, 2980, 1733, 1566, 1452, 1370, 1310, 1255, 1156, 1091, 766, $746 \mathrm{~cm}^{-1}$.

HRMS (MALDI): $m / z$ calcd for $\mathrm{C}_{18} \mathrm{H}_{20} \mathrm{ClNNaO}_{3}[\mathrm{M}+\mathrm{Na}]^{+} 356.1024$ found 356.1020 .

SFC (of the corresponding carbamate) Daicel Chiralpak AS-H, $5 \% \mathrm{MeOH}, 2.0 \mathrm{~mL} / \mathrm{min} ., 25{ }^{\circ} \mathrm{C},>99 \%$ ee $\left(t_{R}(1\right.$, major enantiomer, minor diastereomer $)=29.29 \mathrm{~min} t_{R}(2$, minor enantiomer, minor diastereomer $)=31.41 \mathrm{~min}, \mathrm{t}_{\mathrm{R}}(3$, major enantiomer, major diastereomer $)=33.63 \mathrm{~min}, \mathrm{t}_{\mathrm{R}}(4$, minor enantiomer, major diastereomer) $=38.31 \mathrm{~min}$ ).

$[\alpha]_{\mathbf{D}}^{29}=-46.7\left(\mathrm{c}=1.0 \mathrm{CHCl}_{3}\right)$. 
<smiles>C=CC(c1ccc2ccccc2c1)C(Cl)(Cl)CO</smiles>

[ $\left.\{\mathrm{Ir}(\mathrm{cod}) \mathrm{Cl}\}_{2}\right](10.4 \mathrm{mg}, 15.0 \mathrm{umol}, 0.06$ equiv) and $(\boldsymbol{R})-\mathbf{L}(30.5 \mathrm{mg}, 60.0 \mathrm{umol}, 0.024$ equiv.) were dissolved in 1,2-dichloroethane $(1.0 \mathrm{~mL})$ under an atmosphere of nitrogen and stirred vigorously for $15 \mathrm{~min}$. A portion of the resulting dark red $\mathrm{Ir} /(\mathrm{P}$,olefin) stock solution $(0.5 \mathrm{~mL}, 0.06$ equiv. [Ir] ) was added to a screw-capped glass vial containing allylic alcohol $1 \mathbf{a}$ (46 mg, $0.25 \mathrm{mmol}, 1.0$ equiv). Freshly distilled diphenylmethylamine ( $4.3 \mu \mathrm{L}, 25 \mu \mathrm{mol}, 0.10$ equiv) was added, followed dichloroacetaldehyde (90\% purity, $73 \mathrm{mg}, 0.50 \mathrm{mmol}, 2.0$ equiv) and Zinc bromide ( $28 \mathrm{mg}$, $0.125 \mathrm{mmol}, 0.50$ equiv). The vessel was purged with nitrogen, fitted with a teflon-lined cap, and the resulting solution was stirred at $40{ }^{\circ} \mathrm{C}$ for the 20 hours. A saturated aqueous solution of $\mathrm{NaHCO}_{3}(1$ $\mathrm{mL})$ and ethyl acetate $(2 \mathrm{~mL})$ were added. The phases were separated and the aqueous phase was extracted with ethyl acetate $(2 \times 2 \mathrm{~mL})$. The combined organic phases were dried over $\mathrm{Na}_{2} \mathrm{SO}_{4}$, filtered and concentrated in vacuo. To a solution of the crude aldehyde in $\mathrm{CH}_{2} \mathrm{Cl}_{2} / \mathrm{MeOH}(2 / 1,2.5 \mathrm{~mL}, 0.1 \mathrm{M})$ at $0{ }^{\circ} \mathrm{C}$ was added sodium borohydride $(37.8 \mathrm{mg}, 1.00 \mathrm{mmol}, 4.0$ equiv). The resulting suspension was stirred at $0{ }^{\circ} \mathrm{C}$ for 1 hour. The reaction was quenched by addition of saturated aqueous $\mathrm{NH}_{4} \mathrm{Cl}(2 \mathrm{~mL})$ solution and diluted with dichloromethane $(2 \mathrm{ml})$ and water $(0.5 \mathrm{~mL})$. The phases were separated and the aqueous phase was extracted with dichloromethane $(2 \times 2 \mathrm{~mL})$. The combined organic phases were dried over $\mathrm{Na}_{2} \mathrm{SO}_{4}$, filtered and concentrated in vacuo. Flash chromatography (hexane/ethyl acetate = $85 / 15$ ) afforded the product as a white, crystalline solid (39 $\mathrm{mg}, 55 \%$ yield).

${ }^{1} \mathbf{H}$ NMR $\left(500 \mathrm{MHz}, \mathrm{CDCl}_{3}\right) \delta 7.90-7.88(\mathrm{~m}, 1 \mathrm{H}), 7.84(\mathrm{dd}, J=13.1,8.1 \mathrm{~Hz}, 3 \mathrm{H}), 7.59(\mathrm{dd}, J=8.5$, $1.8 \mathrm{~Hz}, 1 \mathrm{H}), 7.52-7.46(\mathrm{~m}, 2 \mathrm{H}), 6.53$ (ddd, $J=17.0,10.2,8.5 \mathrm{~Hz}, 1 \mathrm{H}), 5.37$ (ddd, $J=10.2,1.4,0.7 \mathrm{~Hz}$, $1 \mathrm{H}), 5.30(\mathrm{dt}, J=16.9,1.1 \mathrm{~Hz}, 1 \mathrm{H}), 4.38(\mathrm{~d}, J=8.5 \mathrm{~Hz}, 1 \mathrm{H}), 3.93(\mathrm{dd}, J=12.3,6.8 \mathrm{~Hz}, 1 \mathrm{H}), 3.79$ (dd, $J=12.3,8.0 \mathrm{~Hz}, 1 \mathrm{H}), 2.43(\mathrm{t}, J=7.5 \mathrm{~Hz}, 1 \mathrm{H})$.

${ }^{13}$ C NMR $\left(126 \mathrm{MHz}, \mathrm{CDCl}_{3}\right) \delta 135.6,135.5,133.3,133.0,129.3,128.2,128.0,127.7,127.6,126.4$, $126.3,120.1,96.1,70.8,59.2$.

IR (neat) 3566, 3427, 3058, 2932, 1508, 1093, 1017, 928, 819, 748, 719, $477 \mathrm{~cm}^{-1}$.

HRMS (EI): $m / z$ calcd for $\mathrm{C}_{15} \mathrm{H}_{14} \mathrm{Cl}_{2} \mathrm{O}[\mathrm{M}]^{+} 280.0417$ found 280.0417 .

SFC Daicel Chiralpak IB, $10 \% \mathrm{MeOH}, 2.0 \mathrm{~mL} / \mathrm{min} ., 25^{\circ} \mathrm{C},>99 \%$ ee $\left(\mathrm{t}_{\mathrm{R}}(1\right.$, minor enantiomer $)=23.43$ $\min , t_{R}(2$, major enantiomer $\left.)=33.43 \mathrm{~min}\right)$.

$[\boldsymbol{\alpha}]_{\mathrm{D}}^{\mathbf{2 4}}=-81.4\left(\mathrm{c}=1.0 \mathrm{CHCl}_{3}\right)$

Melting point: $199^{\circ} \mathrm{C}$ 


\section{Bromoacetaldehyde hydrate (3c)}

In a $50 \mathrm{~mL}$ round bottom flask equipped with a distillation apparatus, oxalic acid $(11.3 \mathrm{~g}, 125 \mathrm{mmol}$, 1.2 equiv.) and 2-(bromomethyl)-1,3-dioxolane (11.3 ml, $125 \mathrm{mmol}, 1.0$ equiv.) were mixed neat. The collecting flask was charged with water $(5 \mathrm{ml})$ and cooled to $0{ }^{\circ} \mathrm{C}$. The reaction mixture was heated to $120-160^{\circ} \mathrm{C}$ (oilbath) and one fraction was collected $\left(80-95^{\circ} \mathrm{C}\right)$. The bromoacetaldeyhde/water ratio of the collected distillate was determined by ${ }^{1} \mathrm{H}$ NMR analysis using in $\mathrm{D}_{2} \mathrm{O}$ using maleic acid as an internal standard and water was added until a 50\% ratio (wt/wt) was obtained (13.6 g, 53\% yield).

\section{(2S,3S)-2-bromo-3-(naphthalen-2-yl)pent-4-enal (4n)}<smiles>C=CC(C(=O)Br)c1ccc2ccccc2c1</smiles>

The corresponding compound was prepared following general procedure $\mathbf{B}$ using bromoacetaldeyhde (50\% in $\mathrm{H}_{2} \mathrm{O}$ (wt/wt), $123 \mathrm{mg}, 0.500 \mathrm{mmol}, 2.0$ equiv) (S)-L, $(\boldsymbol{R})$-A, alcohol 1a and $\mathrm{Na}_{2} \mathrm{SO}_{4}(60 \mathrm{mg}$ ). After stirring at room temperature for 23 hours, the reaction mixture was loaded directly onto a column and eluted with hexane/ethyl acetate/acetic acid (95/4/1) to afford the product as a colorless oil (54 mg, $75 \%$ yield). ${ }^{1} \mathrm{H}$ NMR analysis of the isolated product showed a d.r. of $>20: 1$.

${ }^{1} \mathbf{H}$ NMR $\left(400 \mathrm{MHz}, \mathrm{CDCl}_{3}\right) \delta 9.44(\mathrm{~d}, J=4.7 \mathrm{~Hz}, 1 \mathrm{H}), 8.00-7.80(\mathrm{~m}, 3 \mathrm{H}), 7.72(\mathrm{~d}, J=1.8 \mathrm{~Hz}, 1 \mathrm{H})$, $7.59-7.47(\mathrm{~m}, 2 \mathrm{H}), 7.36(\mathrm{dd}, J=8.5,1.8 \mathrm{~Hz}, 1 \mathrm{H}), 6.07(\mathrm{ddd}, J=17.3,10.2,8.1 \mathrm{~Hz}, 1 \mathrm{H}), 5.51-5.04$ $(\mathrm{m}, 2 \mathrm{H}), 4.59(\mathrm{dd}, J=9.8,4.7 \mathrm{~Hz}, 1 \mathrm{H}), 4.11(\mathrm{dd}, J=9.7,8.2 \mathrm{~Hz}, 1 \mathrm{H})$.

${ }^{13}$ C NMR $\left(101 \mathrm{MHz}, \mathrm{CDCl}_{3}\right) \delta 191.4,136.5,136.2,133.5,132.9,128.7,128.0,127.8,127.6,126.5$, 126.3, 125.9, 119.1, 57.4, 52.0.

IR (neat) 3083, 2832, 1727, 1570, 1475, 1074, 931, 783, $707 \mathrm{~cm}^{-1}$.

HRMS (EI): $m / z$ calcd for $\mathrm{C}_{15} \mathrm{H}_{13} \mathrm{BrO}[\mathrm{M}]^{+} 288.0150$ found 288.0151 .

HPLC (of the corresponding tosylate) Daicel Chiracel IC, $10 \% \mathrm{i}$-PrOH in hexane, $1.0 \mathrm{~mL} / \mathrm{min} ., 25{ }^{\circ} \mathrm{C}$, $>99 \%$ ee $\left(t_{R}(1\right.$, minor enantiomer, major diastereomer $)=14.59 \mathrm{~min}, \mathrm{t}_{\mathrm{R}}(2$, major enantiomer, major diastereomer $)=15.81 \mathrm{~min}, \mathrm{t}_{\mathrm{R}}(3$, major enantiomer, minor diastereomer $\left.)=19.40 \mathrm{~min}\right), \mathrm{t}_{\mathrm{R}}(4$, minor enantiomer, minor diastereomer) $=20.14 \mathrm{~min}$ ).

$[\alpha]_{\mathrm{D}}^{22}=+78.1\left(\mathrm{c}=1.0, \mathrm{CHCl}_{3}\right)$. 


\section{(2S,3S)-2-bromo-3-phenylpent-4-enal (4o)}<smiles>C=CC(C(=O)O)c1ccccc1</smiles>

The corresponding compound was prepared following general procedure $\mathbf{B}$ using bromoacetaldeyhde (50\% in $\mathrm{H}_{2} \mathrm{O}$ (wt/wt), $123 \mathrm{mg}, 0.500 \mathrm{mmol}, 2.0$ equiv) (S)-L, (R)-A, alcohol $\mathbf{1 b}$ and $\mathrm{Na}_{2} \mathrm{SO}_{4}(60 \mathrm{mg})$. The mixture was stirred at room temperature for 22 hours. Purification by flash chromatography (hexane/EtOAc/AcOH = 94/5/1) afforded the product as a pale yellow oil (43 mg, 72\% yield). ${ }^{1} \mathrm{H}$ NMR analysis of the isolated product showed a d.r. of 16:1.

${ }^{1} \mathbf{H}$ NMR $\left(400 \mathrm{MHz}, \mathrm{CDCl}_{3}\right) \delta 9.38(\mathrm{~d}, J=4.8 \mathrm{~Hz}, 1 \mathrm{H}), 8.06-6.94(\mathrm{~m}, 5 \mathrm{H}), 5.97$ (ddd, $J=16.8,10.5$, $8.1 \mathrm{~Hz}, 1 \mathrm{H}), 5.87-4.80(\mathrm{~m}, 2 \mathrm{H}), 4.45(\mathrm{dd}, J=9.8,4.7 \mathrm{~Hz}, 1 \mathrm{H}), 3.92(\mathrm{dd}, J=9.6,8.3 \mathrm{~Hz}, 1 \mathrm{H})$.

${ }^{13} \mathrm{C}$ NMR $\left(101 \mathrm{MHz}, \mathrm{CDCl}_{3}\right) \delta 191.4,138.9,136.6,128.9,128.3,127,118.8,57.5,51.9$.

IR (neat) $3031,2832,1726,1493,1454,1062,928,759,726,700 \mathrm{~cm}^{-1}$.

HRMS (MALDI): $\mathrm{m} / z$ calcd for $\mathrm{C}_{11} \mathrm{H}_{11} \mathrm{BrNaO}[\mathrm{M}+\mathrm{Na}]^{+} 260.9885$ found 260.9884

SFC Daicel Chiralpak IB, 5\% MeOH, $2.0 \mathrm{~mL} / \mathrm{min}$., $25{ }^{\circ} \mathrm{C}$, >99\% ee $\left(\mathrm{t}_{\mathrm{R}}(1\right.$, both enantiomers, minor diastereomer $)=15.00 \mathrm{~min}, \mathrm{t}_{\mathrm{R}}(2$, major enantiomer, major diastereomer $)=16.44 \mathrm{~min}, \mathrm{t}_{\mathrm{R}}(3$, minor enantiomer, major diastereomer) $=19.62 \mathrm{~min}$ ).

$[\alpha]_{\mathrm{D}}^{22}=+88.1\left(\mathrm{c}=1.0, \mathrm{CHCl}_{3}\right)$. 


\section{(2S,3S)-2-bromo-3-(o-tolyl)pent-4-enal (4p)}<smiles>C=CC(c1ccccc1C)C(Br)C=O</smiles>

The corresponding compound was prepared following general procedure $\mathbf{B}$ using bromoacetaldeyhde (50\% in $\mathrm{H}_{2} \mathrm{O}$ (wt/wt), $123 \mathrm{mg}, 0.500 \mathrm{mmol}, 2.0$ equiv) (S)-L, $(\boldsymbol{R})$-A, alcohol $\mathbf{1 l}$ and $\mathrm{Na}_{2} \mathrm{SO}_{4}(60 \mathrm{mg})$. The mixture was stirred at room temperature for 22 hours. Purification by flash chromatography (hexane/EtOAc/AcOH = 95/4/1) afforded the product as a pale yellow oil (50 mg, 80\% yield). ${ }^{1} \mathrm{H}$ NMR analysis of the isolated product showed a d.r. of 10:1.

${ }^{1} \mathbf{H}$ NMR $\left(400 \mathrm{MHz}, \mathrm{CDCl}_{3}\right) \delta 9.45(\mathrm{~d}, J=4.9 \mathrm{~Hz}, 1 \mathrm{H}), 7.56-6.98(\mathrm{~m}, 4 \mathrm{H}), 5.88$ (ddd, $J=17.0,10.3$, $8.1 \mathrm{~Hz}, 1 \mathrm{H}), 5.28-5.04(\mathrm{~m}, 2 \mathrm{H}), 4.51(\mathrm{dd}, J=10.7,4.9 \mathrm{~Hz}, 1 \mathrm{H}), 4.25(\mathrm{dd}, J=10.7,8.1 \mathrm{~Hz}, 1 \mathrm{H}), 2.40$ $(\mathrm{s}, 3 \mathrm{H})$.

${ }^{13}$ C NMR (101 MHz, $\left.\mathrm{CDCl}_{3}\right) \delta 191.1,137.2,136.5,136.3,131.0,127.4,126.7,126.7,119.0,56.8,47.1$, 19.8 .

IR (neat) 3020, 2831, 1727,1492, 1463, 1049, 927, 757, $726 \mathrm{~cm}^{-1}$.

HRMS (EI): $m / z$ calcd for $\mathrm{C}_{12} \mathrm{H}_{13} \mathrm{BrO}[\mathrm{M}]^{+} 252.0150$ found 252.0154 .

SFC (of the corresponding carbamate) Daicel Chiralcel OJ-H, $15 \% \mathrm{MeOH}, 2.0 \mathrm{~mL} / \mathrm{min} ., 25{ }^{\circ} \mathrm{C},>99 \%$ ee $\left(t_{R}(1\right.$, both enantiomers, minor diastereomer $)=5.47 \mathrm{~min}, t_{R}(2$, major enantiomer, major diastereomer $)=7.60 \mathrm{~min}, \mathrm{t}_{\mathrm{R}}(3$, minor enantiomer, major diastereomer $\left.)=10.53 \mathrm{~min}\right)$.

$[\alpha]_{\mathrm{D}}^{23}=+80.5\left(\mathrm{c}=1.0, \mathrm{CHCl}_{3}\right)$. 


\section{(2S,3S)-2-bromo-3-(3-methoxyphenyl)pent-4-enal (4q)}<smiles>C=CC(C(=O)O)c1cccc(OC)c1</smiles>

The corresponding compound was prepared following general procedure $\mathbf{B}$ using bromoacetaldeyhde (50\% in $\mathrm{H}_{2} \mathrm{O}$ (wt/wt), $123 \mathrm{mg}, 0.500 \mathrm{mmol}, 2.0$ equiv) (S)-L, (R)-A, alcohol $\mathbf{1 p}$ and $\mathrm{Na}_{2} \mathrm{SO}_{4}(60 \mathrm{mg})$. The mixture was stirred at room temperature for 22 hours. Purification by flash chromatography (hexane/EtOAc/AcOH = 93/6/1) afforded the product as a pale yellow oil (56 mg, 83\% yield). ${ }^{1} \mathrm{H}$ NMR analysis of the isolated product showed a d.r. of 17:1.

${ }^{1} \mathbf{H}$ NMR $\left(400 \mathrm{MHz}, \mathrm{CDCl}_{3}\right) \delta 9.35(\mathrm{~d}, J=4.7 \mathrm{~Hz}, 1 \mathrm{H}), 7.32-7.22(\mathrm{~m}, 1 \mathrm{H}), 6.93-6.65(\mathrm{~m}, 3 \mathrm{H}), 5.95$ (ddd, $J=17.2,10.1,8.2 \mathrm{~Hz}, 1 \mathrm{H}), 5.31-5.11(\mathrm{~m}, 2 \mathrm{H}), 4.43(\mathrm{dd}, J=9.7,4.8 \mathrm{~Hz}, 1 \mathrm{H}), 3.87(\mathrm{~d}, J=8.5$, $8.3 \mathrm{~Hz}, 1 \mathrm{H}), 3.80(\mathrm{~s}, 3 \mathrm{H})$.

${ }^{13} \mathrm{C} \mathrm{NMR}\left(101 \mathrm{MHz}, \mathrm{CDCl}_{3}\right) \delta 191.4,160.0,140.4,136.4,129.9,120.5,118.9,114.4,112.8,57.3,55.4$, 52.0 .

IR (neat) 3083, 2939, 1727, 1601, 1586, 1490, 1264, 1045, $701 \mathrm{~cm}^{-1}$.

HRMS (EI): $\mathrm{m} / \mathrm{z}$ calcd for $\mathrm{C}_{12} \mathrm{H}_{13} \mathrm{BrO}_{2}[\mathrm{M}]^{+} 268.0094$ found 268.0095 .

HPLC (of the corresponding carbamate) Daicel Chiracel IC, $5 \% i$-PrOH in hexane, $1.0 \mathrm{~mL} / \mathrm{min} ., 25^{\circ} \mathrm{C}$, $98 \%$ ee $\left(t_{R}(1\right.$, minor enantiomer, major diastereomer $)=9.37 \mathrm{~min}, t_{R}(2$, major enantiomer, major diastereomer $)=9.84 \mathrm{~min}, \mathrm{t}_{\mathrm{R}}(3$, minor enantiomer, minor diastereomer $\left.)=11.73 \mathrm{~min}\right), \mathrm{t}_{\mathrm{R}}(4$, major enantiomer, minor diastereomer) $=14.47 \mathrm{~min}$ ).

$[\alpha]_{\mathrm{D}}^{23}=+78.6\left(\mathrm{c}=1.0, \mathrm{CHCl}_{3}\right)$. 


\section{$(2 S, 3 S)$-2-bromo-3-(6-bromobenzo[d][1,3]dioxol-5-yl)pent-4-enal (4r)}<smiles>C=CC(C(=O)Br)c1cc2c(cc1Br)OCO2</smiles>

The corresponding compound was prepared following general procedure $\mathbf{B}$ using bromoacetaldeyhde (50\% in $\mathrm{H}_{2} \mathrm{O}$ (wt/wt), $123 \mathrm{mg}, 0.500 \mathrm{mmol}, 2.0$ equiv) (S)-L, $(\boldsymbol{R})$ - $\mathbf{A}$, alcohol $\mathbf{2} \mathrm{m}$ and $\mathrm{Na}_{2} \mathrm{SO}_{4}(60 \mathrm{mg})$. The mixture was stirred at room temperature for 27 hours. Purification by flash chromatography (hexane/EtOAc/AcOH = 93/6/1) and reverse-phase chromatography $\left(\mathrm{CH}_{3} \mathrm{CN} / \mathrm{H}_{2} \mathrm{O}=19 / 1\right.$ ) afforded the product as a pale yellow oil (67 mg, $74 \%$ yield). ${ }^{1} \mathrm{H}$ NMR analysis of the isolated product showed a d.r. of 10:1.

${ }^{1} \mathbf{H}$ NMR $\left(400 \mathrm{MHz}, \mathrm{CDCl}_{3}\right) \delta 9.38(\mathrm{~d}, J=4.5 \mathrm{~Hz}, 1 \mathrm{H}), 7.04(\mathrm{~s}, 1 \mathrm{H}), 6.73(\mathrm{~s}, 1 \mathrm{H}), 6.53-5.79(\mathrm{~m}, 2 \mathrm{H})$, $5.86(\mathrm{ddd}, J=16.9,10.5,7.4 \mathrm{~Hz}, 1 \mathrm{H}), 5.76-5.08(\mathrm{~m}, 2 \mathrm{H}), 4.79-4.00(\mathrm{~m}, 2 \mathrm{H})$.

${ }^{13}$ C NMR (101 MHz, $\left.\mathrm{CDCl}_{3}\right) \delta 190.7,148.0,147.8,135.1$ 131.1, 119.7, 115.6, 113.1, 108.4, 102.2, $56.3,49.8$.

IR (neat) 3084, 2900, 1726, 1503, 1479, 1236, 1037, $932 \mathrm{~cm}^{-1}$.

HRMS (EI): $\mathrm{m} / \mathrm{z}$ calcd for $\mathrm{C}_{12} \mathrm{H}_{10} \mathrm{Br}_{2} \mathrm{O}_{3}[\mathrm{M}]^{+} 359.8992$ found 359.8991 .

HPLC (of the corresponding carbamate) Daicel Chiracel IC, $5 \% i$-PrOH in hexane, $1.0 \mathrm{~mL} / \mathrm{min} ., 25^{\circ} \mathrm{C}$, $>99 \%$ ee $\left(t_{R}(1\right.$, major enantiomer, minor diastereomer $)=9.64$ min, $t_{R}(2$, minor enantiomer, minor diastereomer $)=10.22 \mathrm{~min}, \mathrm{t}_{\mathrm{R}}(3$, major enantiomer, major diastereomer $\left.)=11.84 \mathrm{~min}\right), \mathrm{t}_{\mathrm{R}}(4$, minor enantiomer, major diastereomer) $=12.55 \mathrm{~min}$ ).

$[\boldsymbol{\alpha}]_{\mathrm{D}}^{23}=+33.2\left(\mathrm{c}=1.0, \mathrm{CHCl}_{3}\right)$. 


\section{(2S,3S)-2-bromo-3-(3-bromophenyl)pent-4-enal (4s)}<smiles>C=CC(C(=O)Br)c1cccc(Br)c1</smiles>

The corresponding compound was prepared following general procedure $\mathbf{B}$ using bromoacetaldeyhde (50\% in $\mathrm{H}_{2} \mathrm{O}$ (wt/wt), $123 \mathrm{mg}, 0.500 \mathrm{mmol}, 2.0$ equiv) $(\boldsymbol{S})$-L, $(\boldsymbol{R})$-A, alcohol $\mathbf{1 r}$ and $\mathrm{Na}_{2} \mathrm{SO}_{4}(60 \mathrm{mg})$. The mixture was stirred at room temperature for 24 hours. Purification by flash chromatography (hexane/EtOAc/AcOH = 95/4/1) afforded the product as a pale yellow oil (49 $\mathrm{mg}, 62 \%$ yield). ${ }^{1} \mathrm{H}$ NMR analysis of the isolated product showed a d.r. of 19:1.

${ }^{1} \mathbf{H}$ NMR $\left(400 \mathrm{MHz}, \mathrm{CDCl}_{3}\right) \delta 9.36(\mathrm{~d}, \mathrm{~J}=4.5 \mathrm{~Hz}, 1 \mathrm{H}), 7.46-7.40(\mathrm{~m}, 1 \mathrm{H}), 7.38$ (app. t, $J=1.8 \mathrm{~Hz}$, $1 \mathrm{H}$ ), 7.23 (app. t, $J=8.0 \mathrm{~Hz}, 1 \mathrm{H}$ ), 7.16 (app. dt, $J=7.7,1.4 \mathrm{~Hz}, 1 \mathrm{H}$ ), 5.92 (ddd, $J=17.1,10.3,8.1 \mathrm{~Hz}$, $1 \mathrm{H}), 5.52-4.92(\mathrm{~m}, 2 \mathrm{H}), 4.42(\mathrm{dd}, J=9.8,4.4 \mathrm{~Hz}, 1 \mathrm{H}), 3.89(\mathrm{dd}, J=9.0,8.9 \mathrm{~Hz}, 1 \mathrm{H})$.

${ }^{13} \mathrm{C} \mathrm{NMR}\left(101 \mathrm{MHz}, \mathrm{CDCl}_{3}\right) \delta 190.9,141.2,135.9,131.5,130.9,130.4,127.0,122.9,119.5,56.9,51.4$.

IR (neat) $3056,2832,1726,1241,1062,930,818,747 . \mathrm{cm}^{-1}$.

HRMS (EI): $m / z$ calcd for $\mathrm{C}_{11} \mathrm{H}_{10} \mathrm{Br}_{2} \mathrm{O}[\mathrm{M}]^{+} 315.9098$ found 315.9095 .

SFC (of the corresponding carbamate) Daicel Chiralcel AS-H, 7\% MeOH, $2.0 \mathrm{~mL} / \mathrm{min}$., $25{ }^{\circ} \mathrm{C},>99 \%$ ee $\left(t_{R}(1\right.$, minor enantiomer, minor diastereomer $)=27.28 \mathrm{~min}, t_{R}(2$, major enantiomer, minor diastereomer $)=28.75 \mathrm{~min}, \mathrm{t}_{\mathrm{R}}(3$, minor enantiomer, major diastereomer $)=31.64 \mathrm{~min}, \mathrm{t}_{\mathrm{R}}(4$, major enantiomer, major diastereomer) $=34.17 \mathrm{~min}$ ).

$[\boldsymbol{\alpha}]_{\mathrm{D}}^{22}=+56.3\left(\mathrm{c}=1.0, \mathrm{CHCl}_{3}\right)$. 


\section{(2S,3S)-2-bromo-3-(4-iodophenyl)pent-4-enal (4t)}<smiles>C=CC(Br)C(Br)c1ccc(I)cc1</smiles>

The corresponding compound was prepared following general procedure $\mathbf{B}$ using bromoacetaldeyhde (50\% in $\mathrm{H}_{2} \mathrm{O}$ (wt/wt), $123 \mathrm{mg}, 0.500 \mathrm{mmol}, 2.0$ equiv) (S)-L, $(\boldsymbol{R})$-A, alcohol $\mathbf{1 c}$ and $\mathrm{Na}_{2} \mathrm{SO}_{4}(60 \mathrm{mg})$. The mixture was stirred at room temperature for 27 hours. Purification by flash chromatography (hexane/EtOAc/AcOH $=92 / 7 / 1$ ) afforded the product as a pale yellow oil (65 mg, 71\% yield). ${ }^{1} \mathrm{H}$ NMR analysis of the isolated product showed a dr of 13:1.

${ }^{1} \mathbf{H ~ N M R}\left(400 \mathrm{MHz}, \mathrm{CDCl}_{3}\right) \delta 9.37(\mathrm{~d}, J=4.5 \mathrm{~Hz}, 1 \mathrm{H}), 7.69(\mathrm{~d}, \mathrm{~J}=8.5 \mathrm{~Hz}, 2 \mathrm{H}), 6.99(\mathrm{~d}, J=8.4 \mathrm{~Hz}$, 2H), $5.92(\mathrm{ddd}, J=17.0,10.3,8.0 \mathrm{~Hz}, 1 \mathrm{H}), 5.51-4.93(\mathrm{~m}, 2 \mathrm{H}), 4.41(\mathrm{dd}, J=9.7,4.4 \mathrm{~Hz}, 1 \mathrm{H}), 3.87$ $(\mathrm{dd}, J=8.9,8.7 \mathrm{~Hz}, 1 \mathrm{H})$.

${ }^{13}$ C NMR (101 MHz, $\left.\mathrm{CDCl}_{3}\right) \delta 191.1,138.6,138.0,136.2,130.4,119.3,93.3,57.0,51.3$.

IR (neat) 2980, 1770, 1759, 1727, 1485, 1401, 1246, 1063, 1006, 930, $814 \mathrm{~cm}^{-1}$.

HRMS (EI): $\mathrm{m} / \mathrm{z}$ calcd for $\mathrm{C}_{11} \mathrm{H}_{10} \mathrm{BrIO}[\mathrm{M}]^{+} 363.8955$ found 363.8957 .

HPLC (of the corresponding carbamate) Daicel Chiracel IC, $5 \% i$-PrOH in hexane, $1.0 \mathrm{~mL} / \mathrm{min} ., 25{ }^{\circ} \mathrm{C}$, $>99 \%$ ee $\left(t_{R}(1\right.$, major enantiomer, major diastereomer $)=7.83 \mathrm{~min}, t_{R}(2$, minor enantiomer, major diastereomer $)=8.40 \mathrm{~min}, \mathrm{t}_{\mathrm{R}}(3$, major enantiomer, minor diastereomer $\left.)=8.94 \mathrm{~min}\right), \mathrm{t}_{\mathrm{R}}(4$, minor enantiomer, minor diastereomer) $=9.96 \mathrm{~min}$ ).

$[\boldsymbol{\alpha}]_{\mathrm{D}}^{23}=+59.8\left(\mathrm{c}=1.0, \mathrm{CHCl}_{3}\right)$. 


\section{(2S,3S)-2-bromo-3-(4-fluorophenyl)pent-4-enal (4u)}<smiles>C=CC(Br)C(Br)c1ccc(F)cc1</smiles>

The corresponding compound was prepared following general procedure $\mathbf{B}$ using bromoacetaldeyhde (50\% in $\mathrm{H}_{2} \mathrm{O}$ (wt/wt), $123 \mathrm{mg}, 0.500 \mathrm{mmol}, 2.0$ equiv) (S)-L, (R)-A, alcohol $\mathbf{1 d}$ and $\mathrm{Na}_{2} \mathrm{SO}_{4}(60 \mathrm{mg}$ ). The mixture was stirred at room temperature for 25 hours. Purification by flash chromatography (hexane/EtOAc/AcOH = 94/5/1) afforded the product as a pale yellow oil (48 mg, 75\% yield). ${ }^{1} \mathrm{H}$ NMR analysis of the isolated product showed a dr of 14:1.

${ }^{1} \mathbf{H}$ NMR $\left(400 \mathrm{MHz}, \mathrm{CDCl}_{3}\right) \delta 9.37(\mathrm{~d}, J=4.5 \mathrm{~Hz}, 1 \mathrm{H}), 7.20(\mathrm{dd}, J=8.8,5.3 \mathrm{~Hz}, 2 \mathrm{H}), 7.06(\mathrm{t}, J=$ $8.7 \mathrm{~Hz}, 2 \mathrm{H}), 5.95(\mathrm{ddd}, J=17.1,10.3,8.0 \mathrm{~Hz}, 1 \mathrm{H}), 5.53-4.94(\mathrm{~m}, 2 \mathrm{H}), 4.42(\mathrm{dd}, J=9.6,4.5 \mathrm{~Hz}, 1 \mathrm{H})$, $3.93(\mathrm{dd}, J=9.0,8.6 \mathrm{~Hz}, 1 \mathrm{H})$.

${ }^{13}$ C NMR $\left(101 \mathrm{MHz}, \mathrm{CDCl}_{3}\right) \delta 191.3,162.3(\mathrm{~d}, J=246.5 \mathrm{~Hz}), 136.4,134.6(\mathrm{~d}, J=3.4 \mathrm{~Hz}), 130.0(\mathrm{~d}, J$ $=8.1 \mathrm{~Hz}), 119.0,115.8(\mathrm{~d}, \mathrm{~J}=21.6 \mathrm{~Hz}), 57.6,50.9$.

${ }^{19} \mathbf{F}$ NMR $\left(376 \mathrm{MHz}, \mathrm{CDCl}_{3}\right) \delta-114.6$.

IR (neat) 3084, 2726, 1727, 1604, 1510, 1225, 1160, 931, $832 \mathrm{~cm}^{-1}$.

HRMS (EI): $\mathrm{m} / \mathrm{z}$ calcd for $\mathrm{C}_{11} \mathrm{H}_{10} \mathrm{BrFO}[\mathrm{M}]^{+} 255.9879$ found 255.9882.

SFC Daicel Chiralcel AS-H, $1 \% \mathrm{MeOH}, 2.0 \mathrm{~mL} / \mathrm{min} ., 25^{\circ} \mathrm{C}$, >99\% ee $\left(\mathrm{t}_{\mathrm{R}}(1\right.$, major enantiomer, major diastereomer $)=9.91 \mathrm{~min}, \mathrm{t}_{\mathrm{R}}(2$, minor enantiomer, minor diastereomer $)=10.57 \mathrm{~min}, \mathrm{t}_{\mathrm{R}}(3$, major enantiomer, minor diastereomer $)=11.05 \mathrm{~min}), \mathrm{t}_{\mathrm{R}}(2$, minor enantiomer, minor diastereomer $)=10.57$ $\min , t_{R}(4$, minor enantiomer, major diastereomer $\left.)=12.87 \mathrm{~min}\right)$.

$[\alpha]^{23}{ }_{\mathbf{D}}=+59.3\left(\mathrm{c}=1.0, \mathrm{CHCl}_{3}\right)$. 
Methyl 4-((3S,4S)-4-bromo-5-oxopent-1-en-3-yl)benzoate (4v)<smiles>C=CC(C=O)c1ccc(C(=O)OC)cc1</smiles>

The corresponding compound was prepared following general procedure $\mathbf{B}$ using bromoacetaldeyhde (50\% in $\mathrm{H}_{2} \mathrm{O}$ (wt/wt), $123 \mathrm{mg}, 0.500 \mathrm{mmol}, 2.0$ equiv) (S)-L, (R)-A, alcohol $2 \mathrm{e}$ and $\mathrm{Na}_{2} \mathrm{SO}_{4}(60 \mathrm{mg})$. The mixture was stirred at room temperature for 24 hours. Purification by flash chromatography (hexane/EtOAc/AcOH = 95/4/1) afforded the product as a pale yellow oil ( $35 \mathrm{mg}, 47 \%$ yield). ${ }^{1} \mathrm{H}$ NMR analysis of the isolated product showed a dr of 12:1.

${ }^{1} \mathbf{H}$ NMR $\left(400 \mathrm{MHz}, \mathrm{CDCl}_{3}\right) \delta \delta 9.38(\mathrm{~d}, J=4.4 \mathrm{~Hz}, 1 \mathrm{H}), 8.08-7.99(\mathrm{~m}, 2 \mathrm{H}), 7.35-7.27(\mathrm{~m}, 2 \mathrm{H})$, $5.95(\mathrm{ddd}, J=17.0,10.3,8.1 \mathrm{~Hz}, 1 \mathrm{H}), 5.25-5.15(\mathrm{~m}, 2 \mathrm{H}), 4.47(\mathrm{dd}, J=9.9,4.4 \mathrm{~Hz}, 1 \mathrm{H}), 4.00(\mathrm{dd}, J=$ 9.7, $8.1 \mathrm{~Hz}, 1 \mathrm{H}), 3.91(\mathrm{~s}, 3 \mathrm{H})$.

${ }^{13}$ C NMR $\left(101 \mathrm{MHz}, \mathrm{CDCl}_{3}\right) \delta 190.9,166.8,144.00,135.9,130.2,129.7,128.5,119.6,56.7,52.3,51.7$.

IR (neat) 3441, 2952, 1721, 1436, 1281, 1112, $710 \mathrm{~cm}^{-1}$.

HRMS (EI): $m / z$ calcd for $\mathrm{C}_{12} \mathrm{H}_{10} \mathrm{BrO}_{2}[\mathrm{M}]^{+} 264.9859$ found 264.9862 .

SFC Daicel Chiralpak AS-H, $7 \% \mathrm{MeOH}, 2.0 \mathrm{~mL} / \mathrm{min} ., 25^{\circ} \mathrm{C}$, >99\% ee $\left(\mathrm{t}_{\mathrm{R}}(1\right.$, major enantiomer, major

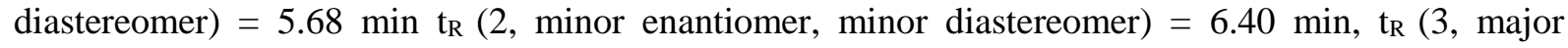
enantiomer, minor diastereomer $)=7.18 \mathrm{~min}, \mathrm{t}_{\mathrm{R}}(4$, minor enantiomer, major diastereomer $\left.)=8.50 \mathrm{~min}\right)$.

$[\boldsymbol{\alpha}]_{\mathrm{D}}^{225}=+35.4\left(\mathrm{c}=1.0, \mathrm{CHCl}_{3}\right)$. 


\section{(2S,3S)-2-bromo-3-(thiophen-3-yl)pent-4-enal (4w)}<smiles>C=CC(c1ccsc1)C(Br)C=O</smiles>

The corresponding compound was prepared following general procedure $\mathbf{B}$ using bromoacetaldeyhde (50\% in $\mathrm{H}_{2} \mathrm{O}$ (wt/wt), $123 \mathrm{mg}, 0.500$ mmol, 2.0 equiv) (S)-L, (R)-A, alcohol $\mathbf{2 h}$ and $\mathrm{Na}_{2} \mathrm{SO}_{4}(60 \mathrm{mg})$. The mixture was stirred at room temperature for 19 hours. Purification by flash chromatography (hexane/EtOAc/AcOH = 95/4/1) afforded the product as a pale yellow oil (45 mg, 73\% yield). ${ }^{1} \mathrm{H}$ NMR analysis of the isolated product showed a dr of 19:1.

${ }^{1} \mathbf{H}$ NMR $\left(400 \mathrm{MHz}, \mathrm{CDCl}_{3}\right) \delta 9.37(\mathrm{~d}, J=4.2 \mathrm{~Hz}, 1 \mathrm{H}), 7.33(\mathrm{dd}, J=5.0,3.0 \mathrm{~Hz}, 1 \mathrm{H}), 7.14(\mathrm{dd}, J=3.1$, $1.3 \mathrm{~Hz}, 1 \mathrm{H}), 7.01(\mathrm{dd}, J=5.0,1.4 \mathrm{~Hz}, 1 \mathrm{H}), 5.99(\mathrm{ddd}, J=17.0,10.3,8.0 \mathrm{~Hz}, 1 \mathrm{H}), 5.56-4.86(\mathrm{~m}, 2 \mathrm{H})$, 4.44 (dd, $J=8.1,4.3 \mathrm{~Hz}, 1 \mathrm{H}), 4.13$ (app. t, $J=8.0 \mathrm{~Hz}, 1 \mathrm{H}$ ).

${ }^{13} \mathbf{C}$ NMR $\left(101 \mathrm{MHz}, \mathrm{CDCl}_{3}\right) \delta 192.0,138.9,136.1,127.2,126.3,122.9,118.9,57.9,47.1$.

IR (neat) 3104, 2979, 2836, 1724, 1062, 990, 929, 838, 783, 682, $659 \mathrm{~cm}^{-1}$.

HRMS (EI): $m / z$ calcd for $\mathrm{C}_{9} \mathrm{H}_{9} \mathrm{BrOS}[\mathrm{M}]^{+} 243.9552$ found 243.9550 .

SFC Daicel Chiralcel AS-H, $1 \% \mathrm{MeOH}, 2.0 \mathrm{~mL} / \mathrm{min} ., 25^{\circ} \mathrm{C}$, >99\% ee $\left(\mathrm{t}_{\mathrm{R}}(1\right.$, major enantiomer, major diastereomer $)=14.45 \mathrm{~min}, \mathrm{t}_{\mathrm{R}}(2$, both enantiomers, minor diastereomer $)=15.35 \mathrm{~min}, \mathrm{t}_{\mathrm{R}}(3$, minor enantiomer, major diastereomer) $=17.30 \mathrm{~min}$ ).

$[\boldsymbol{\alpha}]_{\mathbf{D}}^{22}=+73.1\left(\mathrm{c}=1.0, \mathrm{CHCl}_{3}\right)$. 


\section{(2R,3R)-2-chloro-3-phenylpent-4-en-1-ol (4b-reduced)}<smiles>C=CC(c1ccccc1)C(Cl)CO</smiles>

The corresponding compound was prepared following general procedure $\mathbf{C}$ using aldehyde $\mathbf{4 b}(200 \mathrm{mg}$, $1.03 \mathrm{mmol}$ ). After stirring for 2 hours at $0^{\circ} \mathrm{C}$, the reaction mixture was quenched by addition of saturated aqueous $\mathrm{NH}_{4} \mathrm{Cl}(8 \mathrm{~mL})$. The mixture was allowed to warm to room temperature and water was added until the white precipitate had dissolved completely. The phase were separated and the aqueous phase was extracted with dichloromethane $(2 \times 8 \mathrm{~mL})$. The combined organic phases were washed with water $(10 \mathrm{ml})$ and brine $(10 \mathrm{ml})$, dried over $\mathrm{MgSO}_{4}$, filtered and concentrated in vacuo. The crude product was purified by flash chromatography (hexane/EtOAc $=3 / 1$ ) to afford the product as a colorless viscous oil (155 mg, 77\% yield). ${ }^{1} \mathrm{H}$ NMR analysis of the isolated product showed a d.r. of 10:1.

${ }^{1} \mathbf{H}$ NMR $\left(400 \mathrm{MHz}, \mathrm{CDCl}_{3}\right) \delta 7.38-7.32(\mathrm{~m}, 2 \mathrm{H}), 7.29-7.23(\mathrm{~m}, 3 \mathrm{H}), 6.03$ (ddd, $J=17.0,10.1$, $8.7 \mathrm{~Hz}, 1 \mathrm{H}), 5.25-5.15(\mathrm{~m}, 2 \mathrm{H}), 4.33(\mathrm{ddd}, J=8.7,6.6,3.7 \mathrm{~Hz}, 1 \mathrm{H}), 3.98-3.76(\mathrm{~m}, 2 \mathrm{H}), 3.73(\mathrm{t}, J=$ $8.7 \mathrm{~Hz}, 1 \mathrm{H})$.

${ }^{13}$ C NMR $\left(101 \mathrm{MHz}, \mathrm{CDCl}_{3}\right) \delta 140.4,137.7,128.8,128.3,127.3,117.8,67.1,65.3,54.1$.

IR (neat) 3385, 3083, 3063, 3030, 2942, 1638, 1601, 1494, 1453, 1077, 1033, 992, 924, 760, 701, 521 $\mathrm{cm}^{-1}$.

HRMS (EI): $\mathrm{m} / z$ calcd for $\mathrm{C}_{11} \mathrm{H}_{11} \mathrm{ClO}[\mathrm{M}]^{+} 196.0650$ found 196.0657

SFC Daicel Chiralpak IA, $5 \% \mathrm{MeOH}, 2.0 \mathrm{~mL} / \mathrm{min} ., 25{ }^{\circ} \mathrm{C}$, >99\% ee $\left(\mathrm{t}_{\mathrm{R}}(1\right.$, major enantiomer, major diastereomer $)=8.57$ min $t_{R}(2$, minor enantiomer, minor diastereomer $)=9.19 \mathrm{~min}, t_{R}(3$, major enantiomer, minor diastereomer $)=9.75 \mathrm{~min}, \mathrm{t}_{\mathrm{R}}(4$, minor enantiomer, major diastereomer $\left.)=10.33 \mathrm{~min}\right)$.

$[\boldsymbol{\alpha}]_{\mathrm{D}}^{25}=-93.7\left(\mathrm{c}=0.5 \mathrm{CHCl}_{3}\right)$ 


\section{(2R,3R)-2-chloro-3-phenylpent-4-en-1-yl (4-nitrophenyl)carbamate (4b-carbamate)}

$\mathrm{O}_{2} \mathrm{~N}$<smiles>C=CC(COC(=O)Nc1ccccc1)c1ccccc1</smiles>

To a solution of $(2 R, 3 R)$-2-chloro-3-phenylpent-4-en-1-ol $(30 \mathrm{mg}, 0.15 \mathrm{mmol}, 1.0$ equiv) and triethylamine $(21 \mu \mathrm{l}, 0.15 \mathrm{mmol}, 1.0$ equiv) in dichloromethane $(0.5 \mathrm{~mL})$ was added 1-isocyanato-4nitrobenzene ( $30 \mathrm{mg}, 0.18 \mathrm{mmol}, 1.2$ equiv) and the resulting mixture was stirred at room temperature for 13 hours. The mixture was directly loaded onto a column and eluted with hexane/ethyl acetate (6/1) to afford the title compound as an off-white solid (57 $\mathrm{mg}, 84 \%$ yield) in 12:1 d.r.

${ }^{1} \mathbf{H}$ NMR $\left(400 \mathrm{MHz}, \mathrm{CDCl}_{3}\right) \delta 8.24-8.19(\mathrm{~m}, 2 \mathrm{H}), 7.61-7.54(\mathrm{~m}, 2 \mathrm{H}), 7.38-7.33(\mathrm{~m}, 2 \mathrm{H}), 7.31-$ $7.25(\mathrm{~m}, 3 \mathrm{H}), 7.13(\mathrm{br} \mathrm{s}, 1 \mathrm{H}), 6.15-6.03(\mathrm{~m}, 1 \mathrm{H}), 5.29-5.18(\mathrm{~m}, 2 \mathrm{H}), 4.56(\mathrm{dd}, J=11.6,3.9 \mathrm{~Hz}, 1 \mathrm{H})$, 4.45 (app. td, $J=7.4,3.9 \mathrm{~Hz}, 1 \mathrm{H}), 4.30$ (dd, $J=11.6,7.4 \mathrm{~Hz}, 1 \mathrm{H}), 3.73$ (t, $J=8.2 \mathrm{~Hz}, 1 \mathrm{H})$.

${ }^{13} \mathbf{C}$ NMR $\left(101 \mathrm{MHz}, \mathrm{CDCl}_{3}\right) \delta 152.3,143.6,143.4,139.5,137.0,128.8,128.5,127.6,125.4,118.3$, $118.0,67.7,61.8,54.1$.

IR (neat) 3337, 3085, 3007, 2955, 1744, 1721, 1612, 1599, 1546, 1509, 1414, 1331, 1305, 1211, 1179, $1111,1063,928,852,751,704 \mathrm{~cm}^{-1}$.

HRMS (ESI): $m / z$ calcd for $\mathrm{C}_{18} \mathrm{H}_{21} \mathrm{ClN}_{3} \mathrm{O}_{4}\left[\mathrm{M}+\mathrm{NH}_{4}\right]^{+} 378.1215$, found 378.1210.

$[\alpha]_{\mathbf{D}}^{24}=-45.4\left(\mathrm{c}=1.0, \mathrm{CHCl}_{3}\right)$

Melting point $120^{\circ} \mathrm{C}$. 


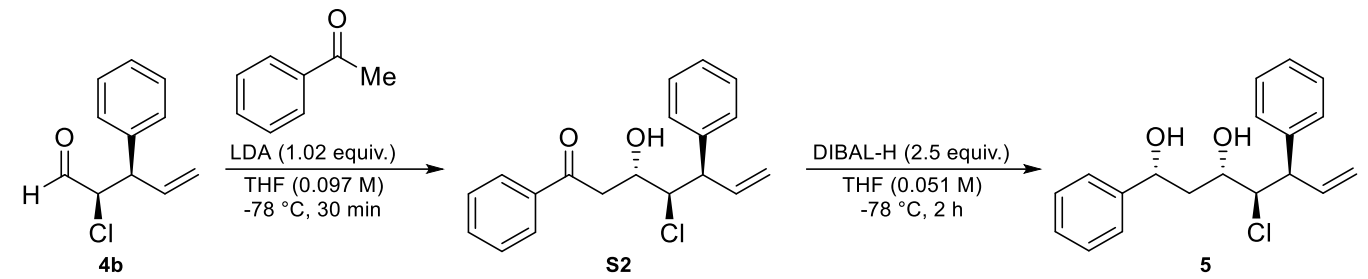

(3S,4R,5R)-4-chloro-3-hydroxy-1,5-diphenylhept-6-en-1-one (S2) ${ }^{9}$<smiles>C=CC(c1ccccc1)C(Cl)[C@H](O)CC(=O)c1ccccc1</smiles>

To a solution of diisopropylamine ( $38.0 \mu \mathrm{L}, 0.269 \mathrm{mmol}, 1.07$ equiv.) in THF (2.60 mL, $0.097 \mathrm{M})$ was cooled to $-78{ }^{\circ} \mathrm{C}$ and $n$-butyllithium $(1.6 \mathrm{M}$ in hexane, $0.160 \mathrm{~mL}, 0.256 \mathrm{mmol}, 1.02$ equiv.) was added dropwise. The resulting solution was stirred at the same temperature and then allowed to warm to $0^{\circ} \mathrm{C}$. After stirring for $15 \mathrm{~min}$ at $0^{\circ} \mathrm{C}$, the pale yellow solution was cooled to $-78{ }^{\circ} \mathrm{C}$ and acetophenone (30.0 $\mu \mathrm{L}, 0.251 \mathrm{mmol}, 1.00$ equiv.) was added in one portion. After stirring for $30 \mathrm{~min}$ at $-78{ }^{\circ} \mathrm{C}$, a solution of $\mathbf{4 b}(54.5 \mathrm{mg}, 0.266 \mathrm{mmol}, 1.06$ equiv. $)$ in THF $(0.5 \mathrm{~mL})$ was added dropwise and stirring was continued for $30 \mathrm{~min}$ at the same temperature. The reaction mixture was quenched by addition of saturated aqueous $\mathrm{NH}_{4} \mathrm{Cl}(3 \mathrm{~mL})$, followed by the addition of EtOAc $(5 \mathrm{~mL})$. The phases were separated and the aqueous phase was extracted with EtOAc $(3 \times 3 \mathrm{~mL})$. The combined organic phases were washed with brine $(10 \mathrm{~mL})$, dried over $\mathrm{Na}_{2} \mathrm{SO}_{4}$, filtered and concentrated in vacuo. ${ }^{1} \mathrm{H}$ NMR analysis of the crude mixture showed a d.r. of 10:1. The crude product was purified by flash chromatography (hexane/EtOAc $=6 / 1)$ to afford the product as a colorless oil $(68.0 \mathrm{mg}, 86 \%$ yield). The configuration of the newly formed stereogenic center was assigned by analogy. ${ }^{9}$

${ }^{1} \mathbf{H}$ NMR $\left(400 \mathrm{MHz}, \mathrm{CDCl}_{3}\right) \delta 7.97(\mathrm{dd}, J=8.4,1.3 \mathrm{~Hz}, 2 \mathrm{H}), 7.62$ (ddt, $\left.J=7.8,6.9,1.3 \mathrm{~Hz}, 1 \mathrm{H}\right), 7.54$ -7.47 (m, 2H), $7.45-7.26(\mathrm{~m}, 5 \mathrm{H}), 6.25(\mathrm{ddd}, J=17.1,10.3,8.0 \mathrm{~Hz}, 1 \mathrm{H}), 5.26-5.19(\mathrm{~m}, 2 \mathrm{H}), 4.37$ $(\mathrm{dd}, J=7.7,5.4 \mathrm{~Hz}, 1 \mathrm{H}), 4.20-4.12(\mathrm{~m}, 2 \mathrm{H}), 3.82(\mathrm{~d}, J=4.9 \mathrm{~Hz}, 1 \mathrm{H}), 3.50(\mathrm{dd}, J=17.8,2.4 \mathrm{~Hz}, 1 \mathrm{H})$, $3.29(\mathrm{dd}, J=17.8,8.6 \mathrm{~Hz}, 1 \mathrm{H})$.

${ }^{13} \mathrm{C}$ NMR $\left(101 \mathrm{MHz}, \mathrm{CDCl}_{3}\right) \delta 201.0,139.5,138.5,136.7,134.0,129.6,128.9,128.4,128.3,127.3$, $116.9,69.1,68.0,51.3,40.8$.

IR (neat) 3499, 3062, 2909, 1678, 1598, 1449, 1211, 1002, 924, 755, 702, $689 \mathrm{~cm}^{-1}$.

$[\boldsymbol{\alpha}]_{\mathrm{D}}^{\mathbf{2 6}}=-65.4\left(\mathrm{c}=1.0, \mathrm{CHCl}_{3}\right)$. 


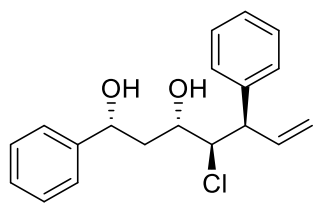

A solution of $(3 S, 4 R, 5 R)$-4-chloro-3-hydroxy-1,5-diphenylhept-6-en-1-one (68 $\mathrm{mg}, 0.22 \mathrm{mmol}$, 1.0 equiv.) in THF $(4.2 \mathrm{ml}, 0.051 \mathrm{M})$ cooled to $-78^{\circ} \mathrm{C}$ and diisobutylaluminum hydride (1.0 $\mathrm{M}$ in THF, $0.54 \mathrm{~mL}, 2.5$ equiv.) was added dropwise. The resulting mixture was stirred at $-78^{\circ} \mathrm{C}$ for $2 \mathrm{~h}$. The reaction mixture was quenched by addition of aqueous $\mathrm{HCl}(1.0 \mathrm{M}, 1.3 \mathrm{~mL})$ and allowed to warm to room temperature. Water $(5 \mathrm{~mL})$ and EtOAc $(5 \mathrm{~mL})$ were added and the phases were separated. The aqueous phase was extracted with EtOAc $(2 \times 5 \mathrm{~mL})$ and the combined organic phases were washed with water $(10 \mathrm{~mL})$ and brine $(10 \mathrm{~mL})$. Drying $\left(\mathrm{Na}_{2} \mathrm{SO}_{4}\right)$, filtration and concentration in vacuo afforded the crude product. ${ }^{1} \mathrm{H}$ NMR analysis of the crude mixture showed a d.r. of 8:1. After flash chromatography (hexane/EtOAc $=6: 1),(1 R, 3 S, 4 R, 5 R)$-4-chloro-1,5-diphenylhept-6-ene-1,3-diol was obtained as a white solid (54 mg, $0.170 \mathrm{mmol}, 79 \%$ yield). The configuration of the newly formed stereogenic center was assigned by analogy. ${ }^{9}$

${ }^{1} \mathrm{H}$ NMR (400 MHz, $\left.\mathrm{CDCl}_{3}\right) \delta 7.41-7.22(\mathrm{~m}, 10 \mathrm{H}), 6.08(\mathrm{ddd}, J=17.0,10.2,8.6 \mathrm{~Hz}, 1 \mathrm{H}), 5.16-5.09$ (m, 2H), 4.89 (dd, $J=9.9,3.3 \mathrm{~Hz}, 1 \mathrm{H}), 4.23(\mathrm{dd}, J=7.1,6.0 \mathrm{~Hz}, 1 \mathrm{H}), 3.95$ (ddd, $J=10.1,6.1,1.8 \mathrm{~Hz}$, 1H), $3.89-3.84$ (m, 1H), 2.15 (ddd, $J=14.6,3.3,1.8 \mathrm{~Hz}, 1 \mathrm{H}), 1.98$ (dt, $J=14.6,10.0 \mathrm{~Hz}, 1 \mathrm{H}$ ).

${ }^{13} \mathrm{C}$ NMR $\left(101 \mathrm{MHz}, \mathrm{CDCl}_{3}\right) \delta 144.1,139.8,138.3,129.0,128.8,128.5,128.1,127.2,125.8,116.9$, $75.2,72.9,69.5,52.5,40.6$.

IR (neat) 3370, 3030, 2926, 1494, 1454, 1066, 922, 760, 736, $700 \mathrm{~cm}^{-1}$.

HRMS (EI): $m / z$ calcd for $\mathrm{C}_{19} \mathrm{H}_{21} \mathrm{ClO}_{2}\left[\mathrm{M}-\mathrm{H}_{2} \mathrm{O}\right]^{+} 298.1120$, found 298.1119.

$[\alpha]_{\mathrm{D}}^{\mathbf{2 6}}=-22.1\left(\mathrm{c}=1.0, \mathrm{CHCl}_{3}\right)$. 


\section{(3S,4R)-3-chloro-4-phenylhex-5-enenitrile (6)}<smiles>C=CC(c1ccccc1)C(Cl)CC#N</smiles>

A solution of $\mathbf{4 b}(80 \mathrm{mg}, 0.41 \mathrm{mmol}, 1.0$ equiv) and 2,6-lutidine ( $0.24 \mathrm{ml}, 2.1 \mathrm{mmol}, 5.1$ equiv. $)$ in dichloromethane $(2.0 \mathrm{ml}, 0.2 \mathrm{M})$ was cooled to $0^{\circ} \mathrm{C}$. Trifluoromethanesulfonic anhydride $(82 \mu \mathrm{l}$, $0.49 \mathrm{mmol}, 1.2$ equiv.) was added dropwise and the solution was stirred at $0{ }^{\circ} \mathrm{C}$ for $40 \mathrm{~min}$. The reaction was quenched with brine $(2 \mathrm{~mL})$ and water $(0.5 \mathrm{~mL})$. The phases were separated and the aqueous phase was extracted with dichloromethane $(2 \times 2 \mathrm{~mL})$. The combined organic phases were dried over $\mathrm{Na}_{2} \mathrm{SO}_{4}$, filtered and concentrated.

The crude product was dissolved in acetonitrile $(2.0 \mathrm{ml}, 0.2 \mathrm{M}) .18$-crown-6 (22 $\mathrm{mg}, 0.083 \mathrm{mmol}$, 0.21 equiv.) was added and the mixture was stirred at room temperature until all solids were dissolved. Potassium cyanide (270 mg, $4.1 \mathrm{mmol}, 10$ equiv.) was added and the reaction was stirred at room temperature for $11 \mathrm{~h}$. The reaction mixture was quenched by addition of saturated aqueous $\mathrm{NaHCO}_{3}$ $(4 \mathrm{~mL})$ and the phases were separated. The aqueous phase was extracted with dichloromethane $(3 \mathrm{x}$ $3 \mathrm{~mL}$ ). The combined organic phases were dried over $\mathrm{Na}_{2} \mathrm{SO}_{4}$, filtered and concentrated in vacuo. ${ }^{1} \mathrm{H}$ NMR analysis of the crude mixture showed a d.r. of 10:1. Purification by flash chromatography (hexane/EtOAc $=7 / 2)$ afforded the desired product as a pale yellow oil $(69 \mathrm{mg}, 82 \%$ yield) with a d.r. $>20: 1$.

${ }^{1} \mathbf{H}$ NMR $\left(400 \mathrm{MHz}, \mathrm{CDCl}_{3}\right) \delta 7.40-7.34(\mathrm{~m}, 2 \mathrm{H}), 7.33-7.25(\mathrm{~m}, 3 \mathrm{H}), 6.00$ (ddd, $J=17.0,10.2$, $8.9 \mathrm{~Hz}, 1 \mathrm{H}), 5.34(\mathrm{dt}, J=17.0,1.1 \mathrm{~Hz}, 1 \mathrm{H}), 5.26(\mathrm{ddd}, J=10.2,1.1,0.6 \mathrm{~Hz}, 1 \mathrm{H}), 4.34$ (ddd, $J=8.2$, $6.3,5.2 \mathrm{~Hz}, 1 \mathrm{H}), 3.71(\mathrm{t}, J=8.5 \mathrm{~Hz}, 1 \mathrm{H}), 2.93-2.80(\mathrm{~m}, 2 \mathrm{H})$.

${ }^{13}$ C NMR $\left(101 \mathrm{MHz}, \mathrm{CDCl}_{3}\right) \delta 138.9,136.5,129.0,128.4,127.8,119.2,116.4,58.2,56.3,26.1$.

IR (neat) 3031, 2980, 2253, 1494, 1455, 1413, 993, 929, 762, 708, $521 \mathrm{~cm}^{-1}$.

HRMS (EI): $\mathrm{m} / z$ calcd for $\mathrm{C}_{12} \mathrm{H}_{12} \mathrm{ClN}[\mathrm{M}]^{+}$205.0654, found 205.0653 .

$[\alpha]_{\mathrm{D}}^{25}=-57.2\left(\mathrm{c}=1.0, \mathrm{CHCl}_{3}\right)$. 
<smiles>O=C1C(OCc2ccccc2)C(Cl)C(c2ccccc2)N1C1CCCCC1</smiles>

To a solution of $\mathbf{4 a}(50 \mathrm{mg}, 0.26 \mathrm{mmol}, 1.0$ equiv.) in dichloromethane $(1.3 \mathrm{~mL}, 0.2 \mathrm{M})$ were added anhydrous magnesium sulfate $(62 \mathrm{mg}, 0.51 \mathrm{mmol})$ and freshly distilled cyclohexylamine $(0.030 \mathrm{~mL}$, $0.26 \mathrm{mmol}, 1.0$ equiv.). After stirring the resulting suspension for $2 \mathrm{~h}$ at room temperature, the solids were removed by filtration. Benzene $(1.3 \mathrm{~mL}, 0.2 \mathrm{M})$ was added and the residual dichloromethane was removed in vacuo. Triethylamine ( $110 \mu 1,0.77 \mathrm{mmol}, 3.0$ equiv.) was added and the reaction mixture was heated to reflux. A solution of 2-(benzyloxy)acetyl chloride ( $56 \mu 1,0.33 \mathrm{mmol}, 1.3$ equiv.) in benzene $(0.3 \mathrm{~mL})$ was added dropwise and the reaction was stirred at reflux for $10 \mathrm{~min}$, before being cooled to room temperature. After stirring at room temperature for $13 \mathrm{~h}$, the reaction was quenched by addition of water $(2 \mathrm{ml})$. The aqueous phase was extracted with dichloromethane $(3 \times 2 \mathrm{~mL})$ and the combined organic phases were dried over $\mathrm{Na}_{2} \mathrm{SO}_{4}$, filtered and concentrated in vacuo. ${ }^{1} \mathrm{H}$ NMR analysis of the crude mixture showed a d.r. of 5.5:1.5:1. The crude mixture was purified by flash chromatography (hexane/EtOAc $=4 / 1)$ to afford the product as a pale yellow oil $(80 \mathrm{mg}, 73 \%$ yield). The configuration of the newly formed stereogenic centers were assigned by analogy. ${ }^{10}$

${ }^{1} \mathbf{H}$ NMR $\left(400 \mathrm{MHz}, \mathrm{CDCl}_{3}\right) \delta 7.48-7.16(\mathrm{~m}, 10 \mathrm{H}), 6.21$ (ddd, $\left.J=17.1,10.2,7.4 \mathrm{~Hz}, 1 \mathrm{H}\right), 5.15(\mathrm{dt}, J$ $=10.3,1.3 \mathrm{~Hz}, 1 \mathrm{H}), 5.11-5.04(\mathrm{~m}, 2 \mathrm{H}), 4.80(\mathrm{~d}, J=11.5,1 \mathrm{H}), 4.55(\mathrm{~d}, J=5.0,1 \mathrm{H}), 4.53(\mathrm{dd}, J=9.8$, $3.9 \mathrm{~Hz}, 1 \mathrm{H}), 4.15(\mathrm{dd}, J=7.5,4.0 \mathrm{~Hz}, 1 \mathrm{H}), 3.50(\mathrm{dd}, J=9.7,5.1 \mathrm{~Hz}, 1 \mathrm{H}), 3.28(\mathrm{tt}, J=11.4,4.1 \mathrm{~Hz}$, $1 \mathrm{H}), 2.06-1.53(\mathrm{~m}, 7 \mathrm{H}), 1.25-1.04(\mathrm{~m}, 3 \mathrm{H})$.

${ }^{13}$ C NMR $\left(101 \mathrm{MHz}, \mathrm{CDCl}_{3}\right) \delta 167.2,138.6,138.3,137.0,129.6,128.7,128.5,128.3,128.2,127.5$, $116.9,80.2,73.0,66.8,60.3,55.5,51.5,31.3,30.4,25.9,25.5,25.3$.

IR (neat) 3030, 2933, 2855, 1751, 1453, 1356, 1214, 1156, 1061, 992, 736, $700 \mathrm{~cm}^{-1}$.

HRMS (MALDI): $\mathrm{m} / z$ calcd for $\mathrm{C}_{26} \mathrm{H}_{30} \mathrm{ClNNaNO}_{2}[\mathrm{M}+\mathrm{Na}]^{+} 446.1857$, found 446.1854 .

$[\alpha]_{\mathrm{D}}^{25}=+66.2\left(\mathrm{c}=1.0, \mathrm{CHCl}_{3}\right)$. 
(S)-1-benzyl-2-((R)-1-phenylallyl)aziridine (8)<smiles>C=C[C@@H](c1ccccc1)C1CN1Cc1ccccc1</smiles>

A solution of $\mathbf{4 b}$-reduced (50.0 $\mathrm{mg}, 0.254 \mathrm{mmol}, 1.0$ equiv.) and 2,6-lutidine (148 $\mu 1,1.27 \mathrm{mmol})$ in dichloromethane $(1.3 \mathrm{~mL}, 0.2 \mathrm{M})$ was cooled to $0^{\circ} \mathrm{C}$ and a solution of trifluoromethanesulfonic anhydride $(52.0 \mu \mathrm{l}, 0.305 \mathrm{mmol}, 1.2$ equiv.) in dichloromethane $(0.3 \mathrm{~mL})$ was added dropwise. The reaction was stirred at room temperature for $30 \mathrm{~min}$ and then transferred via cannula to a separate flask containing a solution of benzylamine $(280 \mu \mathrm{l}, 2.54 \mathrm{mmol}, 10$ equiv. $)$ in dichloromethane $(0.6 \mathrm{~mL})$ at $0^{\circ} \mathrm{C}$. Dichloromethane $(2 \times 0.5 \mathrm{~mL})$ was used to facilitate transfer of the triflate solution. The reaction mixture was allowed to warm to room temperature and stirred for $13 \mathrm{~h}$. The reaction was quenched by addition of saturated aqueous $\mathrm{NaHCO}_{3}(3 \mathrm{~mL})$ followed by addition of dichloromethane $(2 \mathrm{~mL})$. The phases were separated and the aqueous phase was extracted with dichloromethane $(3 \times 3 \mathrm{~mL})$. The combined organic phases were dried over $\mathrm{Na}_{2} \mathrm{SO}_{4}$, filtered and concentrated. The thus obtained crude amine was dissolved in $\mathrm{THF} / \mathrm{H}_{2} \mathrm{O}$ (1:1, $1.7 \mathrm{~mL}$ ). Potassium hydroxide ( $93.0 \mathrm{mg}, 1.65 \mathrm{mmol}, 6.5$ equiv.) was added and the reaction was heated in a sealed flask to $60{ }^{\circ} \mathrm{C}$ for $12 \mathrm{~h}$. After cooling to room temperature, the reaction mixtue was dilluted with water $(2 \mathrm{~mL})$ and extracted with EtOAc $(3 \times 3 \mathrm{~mL})$. The combined organic phases were dried over $\mathrm{Na}_{2} \mathrm{SO}_{4}$, filtered and concentrated in vacuo. ${ }^{1} \mathrm{H} \mathrm{NMR}$ analysis of the crude mixture showed a d.r. of 10:1. Purification by flash chromatography (hexane/EtOAc 4/1 to 2/1) afforded the product as a slightly yellow oil (27 mg, 43\% yield).

${ }^{1} \mathbf{H}$ NMR (400 MHz, $\left.\mathrm{CDCl}_{3}\right) \delta 7.36-7.22(\mathrm{~m}, 10 \mathrm{H}), 6.02$ (ddd, $\left.J=17.0,10.1,8.7 \mathrm{~Hz}, 1 \mathrm{H}\right), 5.19-5.10$ $(\mathrm{m}, 2 \mathrm{H}), 4.40(\mathrm{td}, J=8.3,3.5 \mathrm{~Hz}, 1 \mathrm{H}), 3.86(\mathrm{~d}, J=13.2 \mathrm{~Hz}, 1 \mathrm{H}), 3.78(\mathrm{~d}, J=13.2 \mathrm{~Hz}, 1 \mathrm{H}), 3.71(\mathrm{t}, J=$ $8.4 \mathrm{~Hz}, 1 \mathrm{H}), 3.07$ (dd, $J=12.9,3.5 \mathrm{~Hz}, 1 \mathrm{H}), 2.85$ (dd, $J=13.0,8.4 \mathrm{~Hz}, 1 \mathrm{H})$.

${ }^{13} \mathrm{C}$ NMR $\left(101 \mathrm{MHz}, \mathrm{CDCl}_{3}\right) \delta 140.7,140.0,138.0,128.6,128.6,128.5,128.3,127.2,127.2,117.5$, $65.7,55.4,53.57,53.55$.

IR (neat) 3029, 2979, 1495, 1452, 1374, 1175, 917, 739, 699, $584 \mathrm{~cm}^{-1}$.

HRMS (ESI): $m / z$ calcd for $\mathrm{C}_{18} \mathrm{H}_{20} \mathrm{~N}[\mathrm{M}+\mathrm{H}]^{+} 250.1590$, found 250.1587 .

$[\boldsymbol{\alpha}]_{\mathrm{D}}^{27}=-28.7\left(\mathrm{c}=1.0, \mathrm{CHCl}_{3}\right)$. 


\section{(2S,3S)-2-bromo-3-(naphthalen-2-yl)pent-4-en-1-ol (4n-reduced)}<smiles>C=CC(c1ccc2ccccc2c1)C(Br)CO</smiles>

The corresponding compound was prepared following general procedure $\mathbf{C}$ using aldehyde $4 \mathrm{n}$ (51 $\mathrm{mg}$, $0.18 \mathrm{mmol}$ ) and sodium borohydride. The mixture was stirred at room temperature for $30 \mathrm{~min}$. Purification by flash chromatography (hexane/EtOAc $=3 / 1$ ) afforded the product as a colorless oil (44 mg, 85\% yield). ${ }^{1} \mathrm{H}$ NMR analysis of the isolated product showed a d.r. of $>20: 1$.

${ }^{1} \mathbf{H}$ NMR $\left(400 \mathrm{MHz}, \mathrm{CDCl}_{3}\right) \delta 7.89-7.78(\mathrm{~m}, 3 \mathrm{H}), 7.70(\mathrm{~d}, J=1.7 \mathrm{~Hz}, 1 \mathrm{H}), 7.54-7.43(\mathrm{~m}, 2 \mathrm{H}), 7.39$ (dd, $J=8.5,1.9 \mathrm{~Hz}, 1 \mathrm{H}), 6.11$ (ddd, $J=17.0,10.1,8.7 \mathrm{~Hz}, 1 \mathrm{H}), 5.41-4.96(\mathrm{~m}, 2 \mathrm{H}), 4.55$ (ddd, $J=9.1$, $6.3,3.8 \mathrm{~Hz}, 1 \mathrm{H}), 4.22-3.69(\mathrm{~m}, 3 \mathrm{H}), 2.08(\mathrm{~s}, 1 \mathrm{H})$.

${ }^{13} \mathrm{C}$ NMR $\left(101 \mathrm{MHz}, \mathrm{CDCl}_{3}\right) \delta 138.5,137.9,133.6,132.8,128.5,128.0,127.8,127.1,126.3,126.1$, $126.0,118.0,65.5,61.8,54.5$.

IR (neat) 3401, 3056, 2930, 2726, 1508, 1374, 1272, 1074, 924, 818, $747 \mathrm{~cm}^{-1}$

HRMS (EI): $\mathrm{m} / \mathrm{z}$ calcd for $\mathrm{C}_{15} \mathrm{H}_{14} \mathrm{BrO}[\mathrm{M}-\mathrm{H}]^{+} 289.0223$ found 289.0225 .

$[\boldsymbol{\alpha}]_{\mathbf{D}}^{23}=+89.2\left(\mathrm{c}=1.0, \mathrm{CHCl}_{3}\right)$. 
<smiles>C=CC(COC(=O)Nc1ccc(Br)cc1)c1ccc2ccccc2c1</smiles>

To a solution of (2S,3S)-2-bromo-3-(naphthalen-2-yl)pent-4-en-1-ol (28.9 mg, $99.3 \mu \mathrm{mol}, 1.0$ equiv.) and triethylamine $(27.7 \mu 1,199 \mu \mathrm{mol}, 2.0$ equiv. $)$ in dichloromethane $(2 \mathrm{~mL})$ was added 1-bromo-4isocyanatobenzene $(19.7 \mathrm{mg}, 99.5 \mu \mathrm{mol}, 1.0$ equiv.) and the resulting mixture was stirred at room temperature for 12 hours. The mixture was directly loaded onto a column and eluted with hexane/ethyl acetate (9/1) to afford the title compound as an off-white solid (36 mg, 74\% yield).

${ }^{1} \mathbf{H}$ NMR $\left(400 \mathrm{MHz}, \mathrm{CDCl}_{3}\right) \delta 7.88-7.77(\mathrm{~m}, 3 \mathrm{H}), 7.72-7.68(\mathrm{~m}, 1 \mathrm{H}), 7.51-7.44(\mathrm{~m}, 2 \mathrm{H}), 7.44-$ $7.33(\mathrm{~m}, 3 \mathrm{H}), 7.26(\mathrm{~s}, 1 \mathrm{H}), 7.24(\mathrm{~s}, 1 \mathrm{H}), 6.67(\mathrm{~s}, 1 \mathrm{H}), 6.14(\mathrm{ddd}, J=17.0,10.2,8.5 \mathrm{~Hz}, 1 \mathrm{H}), 5.29-5.24$ $(\mathrm{m}, 1 \mathrm{H}), 5.24-5.21(\mathrm{~m}, 1 \mathrm{H}), 4.63-4.56(\mathrm{~m}, 2 \mathrm{H}), 4.41(\mathrm{dd}, J=13.2,8.1 \mathrm{~Hz}, 1 \mathrm{H}) 3.94(\mathrm{t}, J=8.0 \mathrm{~Hz}$, $1 \mathrm{H})$.

${ }^{13}$ C NMR $\left(101 \mathrm{MHz}, \mathrm{CDCl}_{3}\right) \delta 152.7,137.8,137.4,136.7,133.5,132.8,132.2,128.5,128.0,127.8$, $127.3,126.4,126.2,126.1,120.4,118.3,116.4,67.4,54.4,54.3$.

IR (neat) 3396, 3319, 1710, 1594, 1522, 1491, 1400, 1308, 1214, 1075, 820, $748 \mathrm{~cm}^{-1}$

HRMS (MALDI): $\mathrm{m} / \mathrm{z}$ calcd for $\mathrm{C}_{22} \mathrm{H}_{19} \mathrm{Br}_{2} \mathrm{NaO}_{2}[\mathrm{M}+\mathrm{Na}]^{+} 509.9675$ found 509.9674

$[\boldsymbol{\alpha}]_{\mathrm{D}}^{23}=+34.1\left(\mathrm{c}=1.0, \mathrm{CHCl}_{3}\right)$

Melting point $87^{\circ} \mathrm{C}$ 


\section{(2R,3R)-2-chloro-3-phenylpent-4-en-1-ol (4o-reduced)}<smiles>C=CC(CO)c1ccccc1</smiles>

The corresponding compound was prepared following general procedure $\mathbf{C}$ using aldehyde $\mathbf{4 o}(155 \mathrm{mg}$, $0.648 \mathrm{mmol})$. After stirring for 2.5 hours at $0^{\circ} \mathrm{C}$, the reaction mixture was quenched by addition of saturated aqueous $\mathrm{NH}_{4} \mathrm{Cl}(6 \mathrm{~mL})$. The mixture was allowed to warm to room temperature and water was added until the white precipitate had dissolved completely. The phase were separated and the aqueous phase was extracted with dichloromethane $(2 \times 6 \mathrm{~mL})$. The combined organic phases were washed with water $(10 \mathrm{ml})$ and brine $(10 \mathrm{ml})$, dried over $\mathrm{MgSO}_{4}$, filtered and concentrated in vacuo. The crude product was purified by flash chromatography (hexane/EtOAc $=4 / 1$ ) to afford the product as a colorless viscous oil (149 mg, 95\% yield). ${ }^{1} \mathrm{H}$ NMR analysis of the isolated product showed a d.r. of 13:1.

${ }^{1} \mathbf{H}$ NMR $\left(400 \mathrm{MHz}, \mathrm{CDCl}_{3}\right) \delta 7.36-7.30(\mathrm{~m}, 2 \mathrm{H}), 7.29-7.21(\mathrm{~m}, 3 \mathrm{H}), 6.00$ (ddd, $J=17.0,10.1$, $8.8 \mathrm{~Hz}, 1 \mathrm{H}), 5.21(\mathrm{dt}, J=17.0,1.1 \mathrm{~Hz}, 1 \mathrm{H}), 5.16(\mathrm{ddd}, J=10.1,1.4,0.7 \mathrm{~Hz}, 1 \mathrm{H}), 4.42(\mathrm{ddd}, J=8.9$, 6.4, 3.9 Hz, 1H), $3.93(\mathrm{dd}, J=12.5,3.9 \mathrm{~Hz}, 1 \mathrm{H}), 3.85(\mathrm{dd}, J=12.5,6.4 \mathrm{~Hz}, 1 \mathrm{H}), 3.79$ (t, $J=8.8 \mathrm{~Hz}$, $1 \mathrm{H})$.

${ }^{13} \mathrm{C}$ NMR $\left(101 \mathrm{MHz}, \mathrm{CDCl}_{3}\right) \delta 101 \mathrm{MHz}$, Chloroform-d) $\delta 141.1,138.0,128.7,128.1,127.3,117.7$, $65.5,62.0,54.5$.

IR (neat) 3401, 3056, 2930, 2726, 1508, 1374, 1272, 1074, 924, 818, 747.cm-1.

HRMS (EI): $\mathrm{m} / \mathrm{z}$ calcd for $\mathrm{C}_{11} \mathrm{H}_{13} \mathrm{BrO}[\mathrm{M}]^{+} 240.0145$ found 240.0142 .

$[\alpha]_{\mathrm{D}}^{23}=+72.6\left(\mathrm{c}=1.0, \mathrm{CHCl}_{3}\right)$. 
(4S,5R)-3-(ethoxycarbonyl)-4-hydroxy-5-((S)-1-phenylallyl)-4,5-dihydroisoxazole 2-oxide (9)<smiles>C=CC(c1ccccc1)C1ON([O-])C(C(=O)OCC)C1O</smiles>

A solution of (2S,3S)-2-bromo-3-phenylpent-4-enal $(\mathbf{4 o}, 52 \mathrm{mg}, 0.217 \mathrm{mmol})$ in DCM (Volume: 870 $\mu \mathrm{l})$ was cooled to $-20{ }^{\circ} \mathrm{C}$ and ethyl 2-nitroacetate $(31.4 \mu 1,0.283 \mathrm{mmol})$ and $1 \mathrm{H}$-imidazole $(48.9 \mathrm{mg}$, $0.718 \mathrm{mmol}$ ) were added sequentially. After stirring at $-20{ }^{\circ} \mathrm{C}$ for $20 \mathrm{~h}$, the reaction mixture was directly loaded on silica and purified by flash chromatography (hexane/EtOAc $=3 / 1$ to $2 / 1$ ) to afford the product as a colorless oil $(56 \mathrm{mg}, 88 \%)$. ${ }^{1} \mathrm{H}$ NMR analysis of a reaction aliquot showed a d.r. of 8:1. The configuration of the newly formed stereogenic centers were assigned by analogy. ${ }^{11}$

${ }^{1} \mathbf{H}$ NMR $\left(400 \mathrm{MHz}, \mathrm{CDCl}_{3}\right) \delta 7.42-7.26(\mathrm{~m}, 5 \mathrm{H}), 6.09$ (ddd, $\left.J=17.1,10.4,7.6 \mathrm{~Hz}, 1 \mathrm{H}\right), 5.31-5.20$ $(\mathrm{m}, 3 \mathrm{H}), 4.87$ (dd, $J=7.0,2.7 \mathrm{~Hz}, 1 \mathrm{H}), 4.35(\mathrm{q}, J=7.1 \mathrm{~Hz}, 2 \mathrm{H}), 3.62(\mathrm{t}, J=7.3 \mathrm{~Hz}, 1 \mathrm{H}), 1.36$ (t, $J=7.1$ $\mathrm{Hz}, 3 \mathrm{H})$.

${ }^{13} \mathrm{C}$ NMR $\left(101 \mathrm{MHz}, \mathrm{CDCl}_{3}\right) \delta 159.1,137.8,135.3,129.2,128.4,127.9,119.0,110.5,84.9,75.6,62.3$, $52.2,14.4$.

IR (neat) 3473, 3063, 2983, 1726, 1616, 1420, 1233, 1021, 898, 763, 741, $702 \mathrm{~cm}^{-1}$.

HRMS (ESI): $m / z$ calcd for $\mathrm{C}_{15} \mathrm{H}_{17} \mathrm{NNaO}_{5}[\mathrm{M}+\mathrm{Na}]^{+} 314.0999$, found 314.1005 .

$[\alpha]_{\mathrm{D}}^{29}=+127.2\left(\mathrm{c}=1.0, \mathrm{CHCl}_{3}\right)$. 
<smiles>BrCC1OCC(Br)C1c1ccccc1</smiles>

To a solution of 4o-reduced ( $40.0 \mathrm{mg}, 0.166 \mathrm{mmol}, 1.0$ equiv.) in acetonitrile $(0.42 \mathrm{~mL}, 0.4 \mathrm{M})$ were sequentially added potassium carbonate $\left(68.8 \mathrm{mg}, 0.498 \mathrm{mmol}, 3.0\right.$ equiv.) and $\mathrm{I}_{2}(126 \mathrm{mg}, 0.498 \mathrm{mmol}$, 3.0 equiv.) at $0^{\circ} \mathrm{C}$. The resulting brown suspension was stirred at $0{ }^{\circ} \mathrm{C}$ for $1 \mathrm{~h}$ and then diluted with EtOAc ( $3 \mathrm{~mL}$ ). Saturated aqueous $\mathrm{Na}_{2} \mathrm{~S}_{2} \mathrm{O}_{3}(1 \mathrm{~mL})$ was added to quench excess $\mathrm{I}_{2}$, followed by brine ( 3 $\mathrm{mL})$. The phases were separated and the aqueous phase was extracted with EtOAc $(2 \times 3 \mathrm{~mL})$. The combined organic phases were dried over $\mathrm{Na}_{2} \mathrm{SO}_{4}$, filtered and concentrated. The crude product was purified by flash chromatography (hexane/EtOAC $=20 / 1$ ) to afford the product as a slightly yellow oil (46 mg, 76\% yield). ${ }^{1} \mathrm{H}$ NMR analysis of the isolated product showed a d.r. of 13:1. The relative configuration of the newly formed stereocenter was assigned using NOE NMR spectroscopy.

${ }^{1} \mathbf{H}$ NMR $\left(400 \mathrm{MHz}, \mathrm{CDCl}_{3}\right) \delta 7.44-7.33(\mathrm{~m}, 3 \mathrm{H}), 7.29(\mathrm{dd}, J=6.7,1.8 \mathrm{~Hz}, 2 \mathrm{H}), 4.52-4.45(\mathrm{~m}, 2 \mathrm{H})$, $4.26-4.19$ (m, 1H), 3.96 (ddd, $J=8.4,5.5,4.1 \mathrm{~Hz}, 1 \mathrm{H}), 3.48$ (dd, $J=10.8,4.1 \mathrm{~Hz}, 1 \mathrm{H}), 3.39$ (t, $J=8.3$ $\mathrm{Hz}, 1 \mathrm{H}), 3.29$ (dd, $J=10.8,5.5 \mathrm{~Hz}, 1 \mathrm{H})$.

${ }^{13}$ C NMR (101 MHz, $\left.\mathrm{CDCl}_{3}\right) \delta 136.7,129.3,128.2,127.8,84.0,75.3,61.1,50.6,8.5$.

IR (neat) 3030, 2876, 1455, 1182, 1108, 1062, 1033, 749, $699 \mathrm{~cm}^{-1}$.

HRMS (EI): $m / z$ calcd for $\mathrm{C}_{11} \mathrm{H}_{12} \mathrm{BrIO}[\mathrm{M}]^{+} 365.9111$, found 365.9115 .

$[\alpha]_{\mathbf{D}}^{27}=-1.5\left(\mathrm{c}=1.0, \mathrm{CHCl}_{3}\right)$.

Structural assignment (only diagnostic correlations are shown, for details see NMR spectra)

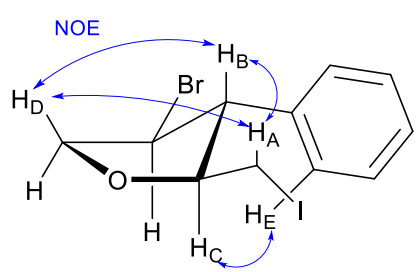


<smiles>C=CC(c1ccccc1)C(O)Sc1ccccc1</smiles>

To a solution of (2S,3S)-2-bromo-3-phenylpent-4-en-1-ol (4o-reduced) ( $20 \mathrm{mg}, 0.083 \mathrm{mmol}, 1.0$ equiv.) in $\mathrm{MeCN}(0.2 \mathrm{~mL})$ was added a solution of sodium hydroxide $(84 \mathrm{mg}, 0.32 \mathrm{mmol}, 3.8$ equiv.) in water $(0.2 \mathrm{~mL})$ and ethanol $(0.4 \mathrm{~mL})$. The resulting mixture was stirred at room temperature for $3 \mathrm{~h}$ until full conversion was observed by TLC. Thiophenol $(13 \mu \mathrm{L}, 0.13 \mathrm{mmol}, 1.5$ equiv. $)$ was added in one portion and the mixture was heated to $40{ }^{\circ} \mathrm{C}$ in a sealed vessel for $23 \mathrm{~h}$. The reaction was diluted with water $(1$ $\mathrm{mL})$ and extracted with diethyl ether $(3 \times 2 \mathrm{~mL})$. The combined organic phases were washed with brine (4 mL), dried over $\mathrm{Na}_{2} \mathrm{SO}_{4}$ and concentrated in vacuo. ${ }^{1} \mathrm{H} \mathrm{NMR}$ analysis of the crude mixture showed a d.r. of 13:1. Purification by flash chromatography (hexane/EtOAc $=10 / 1$ ) gave the desired product as a white solid (18 mg, 80\%).

${ }^{1} \mathbf{H}$ NMR (400 MHz, $\left.\mathrm{CDCl}_{3}\right) \delta 7.33(\mathrm{~m}, 2 \mathrm{H}), 7.31-7.15(\mathrm{~m}, 8 \mathrm{H}), 6.17$ (ddd, $\left.J=17.1,10.3,8.6 \mathrm{~Hz}, 1 \mathrm{H}\right)$, $5.23(\mathrm{ddd}, J=10.3,1.7,0.7 \mathrm{~Hz}, 1 \mathrm{H}), 5.18(\mathrm{dt}, J=17.0,1.3 \mathrm{~Hz}, 1 \mathrm{H}), 3.95(\mathrm{ddd}, J=8.5,7.3,3.2 \mathrm{~Hz}$, $1 \mathrm{H}), 3.43(\mathrm{t}, J=7.9 \mathrm{~Hz}, 1 \mathrm{H}), 3.07(\mathrm{dd}, J=13.8,3.2 \mathrm{~Hz}, 1 \mathrm{H}), 2.80(\mathrm{dd}, J=13.8,8.5 \mathrm{~Hz}, 1 \mathrm{H}), 2.45$ (br $\mathrm{s}, 1 \mathrm{H})$.

${ }^{13}$ C NMR $\left(101 \mathrm{MHz}, \mathrm{CDCl}_{3}\right) \delta 141.1,137.8,135.5,129.8,129.1,128.9,128.2,127.1,126.5,118.0$, $72.3,55.9,39.8$.

IR (neat) 3452, 3060, 2922, 1583, 1481, 1439, 1025, 921, 739, 702, 691, $\mathrm{cm}^{-1}$.

HRMS (ESI): $m / z$ calcd for $\mathrm{C}_{17} \mathrm{H}_{18} \mathrm{NaOS}[\mathrm{M}+\mathrm{Na}]^{+} 293.0971$, found 293.0967 .

$[\alpha]_{\mathrm{D}}^{27}=+29.5\left(\mathrm{c}=1.0, \mathrm{CHCl}_{3}\right)$. 
<smiles>C=CC(O)c1ccccc1</smiles>

To a solution of (2S,3S)-2-bromo-3-phenylpent-4-en-1-ol (40-reduced) (50 mg, $0.21 \mathrm{mmol}, 1.0$ equiv.) in $\mathrm{MeCN}(0.5 \mathrm{~mL})$ was added a solution of sodium hydroxide $(210 \mathrm{mg}, 0.79 \mathrm{mmol}, 3.8$ equiv.) in water $(0.5 \mathrm{~mL})$ and ethanol $(1.0 \mathrm{~mL})$. The resulting mixture was stirred at room temperature for $3 \mathrm{~h}$ until full conversion was observed by TLC. The reaction was diluted with water $(2 \mathrm{~mL})$ and extracted with diethyl ether $(3 \times 3 \mathrm{~mL})$. The combined organic phases were washed with brine $(5 \mathrm{~mL})$, dried over $\mathrm{Na}_{2} \mathrm{SO}_{4}$ and concentrated in vacuo. ${ }^{1} \mathrm{H}$ NMR analysis of the crude mixture showed a d.r. of 13:1. Purification by flash chromatography (pentane/Et $2 \mathrm{O}=93 / 7)$ gave the desired product as a colorless oil $(21 \mathrm{mg}, 64 \%)$. Spectral data were in agreement with those previously reported. ${ }^{12}$

${ }^{1} \mathbf{H}$ NMR (400 MHz, $\left.\mathrm{CD}_{2} \mathrm{Cl}_{2}\right) \delta 7.37$ - $7.32(\mathrm{~m}, 2 \mathrm{H}), 7.30$ - $7.24(\mathrm{~m}, 3 \mathrm{H}), 6.05$ (ddd, $J=17.3,10.4$, $6.7 \mathrm{~Hz}, 1 \mathrm{H}), 5.30-5.12(\mathrm{~m}, 2 \mathrm{H}), 3.32-3.06(\mathrm{~m}, 2 \mathrm{H}), 2.74(\mathrm{dd}, J=5.0,3.7 \mathrm{~Hz}, 1 \mathrm{H}), 2.57(\mathrm{dd}, J=5.1$, $2.3 \mathrm{~Hz}, 1 \mathrm{H})$.

${ }^{13}$ C NMR $\left(101 \mathrm{MHz}, \mathrm{CD}_{2} \mathrm{Cl}_{2}\right) \delta 141.0,138.1,129.2,128.6,127.5,117.2,55.1,52.5,46.3$.

IR (neat) $3083,2983,1493,1453,997,919,871,753,700 \mathrm{~cm}^{-1}$

HRMS (EI): $\mathrm{m} / \mathrm{z}$ calcd for $\mathrm{C}_{11} \mathrm{H}_{12} \mathrm{O}[\mathrm{M}]^{+} 160.0883$ found 160.0886

$[\alpha]_{\mathrm{D}}^{23}=+66.5\left(\mathrm{c}=1.0, \mathrm{CHCl}_{3}\right)$. 


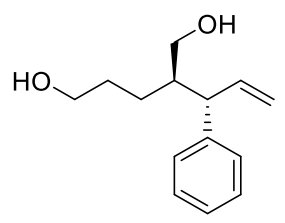

[ $\left.\{\mathrm{Ir}(\operatorname{cod}) \mathrm{Cl}\}_{2}\right](10.4 \mathrm{mg}, 15.0 \mathrm{umol}, 0.06$ equiv) and $(\boldsymbol{R})-\mathbf{L}(30.5 \mathrm{mg}, 60.0 \mathrm{umol}, 0.024$ equiv.) were dissolved in 1,2-dichloroethane $(1.0 \mathrm{~mL})$ under an atmosphere of nitrogen and stirred vigorously for 15 $\mathrm{min}$. A portion of the resulting dark red $\mathrm{Ir} /(\mathrm{P}$,olefin) stock solution $(0.5 \mathrm{~mL}, 0.06$ equiv. [Ir] $)$ was added to a $5 \mathrm{~mL}$ round-bottom flask containing 1-phenylprop-2-en-1-ol (1b) $(33.5 \mathrm{mg}, 250 \mu \mathrm{mol}, 1.0$ equiv.). To this yellow solution were sequentially added, glutaraldehyde (50\% in water, $100 \mathrm{mg}, 500 \mu \mathrm{mol}, 2.0$ equiv.), (S)- $\alpha, \alpha$-Bis[3,5-bis(trifluoromethyl)phenyl]-2-pyrrolidinemethanol trimethylsilyl ether (S)-A1 (19.4 mg, $25.0 \mu \mathrm{mol}, 0.1$ equiv.) and dichloroacetic acid (15.5 $\mu \mathrm{L}, 188 \mu \mathrm{mol}, 0.75$ equiv.). The reaction vessel was purged with nitrogen and sealed. After stirring at room temperature for $13 \mathrm{~h}$, the reaction was diluted with dichloromethane $(1 \mathrm{~mL})$. The resulting yellow solution was washed with saturated aqueous $\mathrm{NaHCO}_{3}(1 \mathrm{~mL})$ and $\mathrm{pH} 7$ buffer $(1 \mathrm{ml})$. The organic phase was dried over $\mathrm{Na}_{2} \mathrm{SO}_{4}$ and filtered. The residual $\mathrm{Na}_{2} \mathrm{SO}_{4}$ and the filter were washed with dichloromethane $(2 \times 0.3 \mathrm{~mL})$. The combined dichloromethane fractions were added dropwise to a solution of lithium aluminum hydride $(2.4 \mathrm{M}$ in THF, $310 \mu \mathrm{L}, 0.744 \mathrm{mmol}, 3.0$ equiv.) in tetrahydrofuran $(0.75 \mathrm{~mL})$ at $0{ }^{\circ} \mathrm{C}$. The reaction was stirred at the same temperature for $1 \mathrm{~h}$ and then quenched by addition of saturated aqueous Rochelle salt solution $(5 \mathrm{~mL})$ and water $(2 \mathrm{~mL})$. The resulting emulsion was diluted with ethyl acetate $(5 \mathrm{~mL})$ and stirred for 10 min until two distinct phases were obtained. The phases were separated and the aqueous phase was extracted with ethyl acetate $(3 \times 5 \mathrm{~mL})$. The combined organic phases were dried over $\mathrm{Na}_{2} \mathrm{SO}_{4}$, filtered and concentrated. ${ }^{1} \mathrm{H}$ NMR analysis of the crude mixture showed a d.r. of $>10: 1$. Purification of the crude material by flash chromatography (EtOAc/hexane $=2 / 1$ to $3 / 1$ ) afforded the product as a crystalline solid (39 mg, 71\% yield). The absolute configuration of $\mathbf{2 0}$ was determined by X-ray diffraction analysis (see section $\mathbf{5}$ for details).

${ }^{1} \mathbf{H}$ NMR $\left(400 \mathrm{MHz}, \mathrm{CDCl}_{3}\right) \delta 7.33-7.27(\mathrm{~m}, 2 \mathrm{H}), 7.23-7.17$ (m, 3H), 6.01 (ddd, $J=16.9,10.1$, $9.3 \mathrm{~Hz}, 1 \mathrm{H}), 5.13-5.05(\mathrm{~m}, 2 \mathrm{H}), 3.68-3.57(\mathrm{~m}, 2 \mathrm{H}), 3.52(\mathrm{dd}, J=11.1,4.5 \mathrm{~Hz}, 1 \mathrm{H}), 3.38(\mathrm{dd}, J=$ $11.1,5.1 \mathrm{~Hz}, 1 \mathrm{H}), 3.32(\mathrm{t}, J=9.2 \mathrm{~Hz}, 1 \mathrm{H}), 1.92-1.81(\mathrm{~m}, 1 \mathrm{H}), 1.84(\mathrm{~s}, 2 \mathrm{H}), 1.71-1.41(\mathrm{~m}, 4 \mathrm{H})$.

${ }^{13} \mathrm{C}$ NMR $\left(101 \mathrm{MHz}, \mathrm{CDCl}_{3}\right) \delta 143.5,140.4,128.8,127.9,126.5,116.0,63.1,62.7,52.3,44.8,29.9$, 24.4.

IR (neat) 3334, 3079, 2933, 1492, 1452, 1050, 994, 915, $701 \mathrm{~cm}^{-1}$.

HRMS (EI): $m / z$ calcd for $\mathrm{C}_{14} \mathrm{H}_{18} \mathrm{O}\left[\mathrm{M}-\mathrm{H}_{2} \mathrm{O}\right]^{+} 202.1358$ found 202.1353 .

SFC Daicel Chiralcel OJ-H, 5\% MeOH, $2.0 \mathrm{~mL} / \mathrm{min}$., $25^{\circ} \mathrm{C}$, >99\% ee $\left(\mathrm{t}_{\mathrm{R}}(1\right.$, major enantiomer, minor diastereomer $)=15.18 \min t_{R}(2$, minor enantiomer, minor diastereomer $)=16.65 \min , t_{R}(3$, major enantiomer, major diastereomer $)=20.08 \mathrm{~min}, t_{R}(4$, minor enantiomer, major diastereomer $)=$ $35.39 \mathrm{~min})$.

$[\boldsymbol{\alpha}]_{\mathbf{D}}^{26}=-82.3\left(\mathrm{c}=1.0 \mathrm{CHCl}_{3}\right)$.

Melting point $173{ }^{\circ} \mathrm{C}$ 


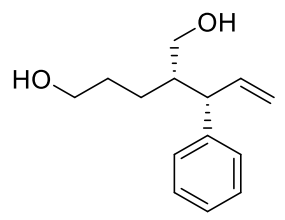

$(S, S)-14$ was prepared following the procedure outline above for $(S, R)-14$ using $(R)-\mathrm{L}$ and $(R)-A 1$.

${ }^{1} \mathbf{H}$ NMR $\left(400 \mathrm{MHz}, \mathrm{CDCl}_{3}\right) \delta 7.33-7.27(\mathrm{~m}, 2 \mathrm{H}), 7.20(\mathrm{td}, J=7.0,1.6 \mathrm{~Hz}, 3 \mathrm{H}), 6.02(\mathrm{dt}, J=17.0$, $9.7 \mathrm{~Hz}, 1 \mathrm{H}), 5.22-4.93(\mathrm{~m}, 2 \mathrm{H}), 3.76-3.67(\mathrm{~m}, 2 \mathrm{H}), 3.55-3.45(\mathrm{~m}, 2 \mathrm{H}), 3.29(\mathrm{t}, J=9.4 \mathrm{~Hz}, 1 \mathrm{H})$, $2.10(\mathrm{~s}, 2 \mathrm{H}), 1.94-1.85(\mathrm{~m}, 1 \mathrm{H}), 1.61-1.39(\mathrm{~m}, 2 \mathrm{H}), 1.34-1.23(\mathrm{~m}, 2 \mathrm{H})$.

${ }^{13}$ C NMR (101 MHz, $\left.\mathrm{CDCl}_{3}\right) \delta 143.3,141.1,128.8,128.0,126.5,115.5,62.9,62.8,52.9,44.6,29.7,24.7$.

IR (neat): 3337, 3079, 2936, 2876, 1452, 1054, 914, $701 \mathrm{~cm}^{-1}$.

HRMS (EI): $m / z$ calcd for $\mathrm{C}_{14} \mathrm{H}_{18} \mathrm{O}\left[\mathrm{M}-\mathrm{H}_{2} \mathrm{O}\right]^{+} 202.1358$ found 202.1354 .

SFC Daicel Chiralcel OJ-H, 5\% MeOH, $2.0 \mathrm{~mL} / \mathrm{min} ., 25^{\circ} \mathrm{C}$, >99\% ee $\left(\mathrm{t}_{\mathrm{R}}(1\right.$, minor enantiomer, major diastereomer $)=15.18 \min t_{R}(2$, major enantiomer, major diastereomer $)=16.65 \mathrm{~min}, \mathrm{t}_{\mathrm{R}}(3$, major enantiomer, minor diastereomer $)=20.08 \mathrm{~min}, \mathrm{t}_{\mathrm{R}}(4$, minor enantiomer, minor diastereomer $)=$ $35.39 \mathrm{~min})$.

$[\boldsymbol{\alpha}]_{\mathbf{D}}^{27}=-81.4\left(\mathrm{c}=1.0 \mathrm{CHCl}_{3}\right)$. 


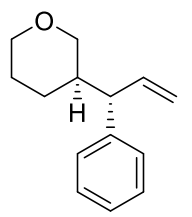

$(S)$-2-((S)-1-phenylallyl)pentane-1,5-diol (20) was dissolved in toluene/1,2-dichloroethane (5/1, 0.08 M). Magnesium sulfate $(23 \mathrm{mg}, 200 \mathrm{mg} / \mathrm{mmol})$ and $p$-toluenesulfonic acid monohydrate $(4.3 \mathrm{mg}$, $23 \mu \mathrm{mol}, 0.2$ equiv.) were added and the reaction vessel was purged with argon and sealed. The resulting suspension was heated to $120^{\circ} \mathrm{C}$ for $16 \mathrm{~h}$. The mixture was allowed to cool to room temperature, loaded directly onto a column and eluted with hexane/ethyl acetate (15/1) to afford the product as a colorless oil (19 mg, $83 \%$ yield) in 14:1 diastereomeric ratio.

${ }^{1} \mathbf{H}$ NMR $\left(400 \mathrm{MHz}, \mathrm{CDCl}_{3}\right) \delta 7.34-7.27(\mathrm{~m}, 2 \mathrm{H}), 7.22-7.14(\mathrm{~m}, 3 \mathrm{H}), 5.93$ (ddd, J = 16.9, 10.1, 9.2 $\mathrm{Hz}, 1 \mathrm{H}), 5.08-5.00(\mathrm{~m}, 2 \mathrm{H}), 4.05(\mathrm{ddd}, \mathrm{J}=11.4,4.1,1.7 \mathrm{~Hz}, 1 \mathrm{H}), 3.88-3.81(\mathrm{~m}, 1 \mathrm{H}), 3.39-3.31(\mathrm{~m}$, $1 \mathrm{H}), 3.18(\mathrm{dd}, \mathrm{J}=11.4,9.7 \mathrm{~Hz}, 1 \mathrm{H}), 2.97(\mathrm{t}, \mathrm{J}=9.6 \mathrm{~Hz}, 1 \mathrm{H}), 1.92(\mathrm{qt}, \mathrm{J}=10.1,3.7 \mathrm{~Hz}, 1 \mathrm{H}), 1.55-1.46$ (m, 3H), $1.09-0.96(\mathrm{~m}, 1 \mathrm{H})$.

${ }^{13} \mathrm{C}$ NMR $\left(101 \mathrm{MHz}, \mathrm{CDCl}_{3}\right) \delta 142.9,140.3,128.7,128.0,126.5,115.5,72.4$ 68.6, 53.9, 39.8, 28.3, 25.8

IR (neat) 3027, 2932, 2846, 1453, 1094, 1034, 914, 760, 701, $680 \mathrm{~cm}^{-1}$.

HRMS (EI): $m / z$ calcd for $\mathrm{C}_{14} \mathrm{H}_{18} \mathrm{O}[\mathrm{M}]^{+} 202.1358$ found 202.1353 .

$[\alpha]_{\mathbf{D}}^{25}=-56.2\left(\mathrm{c}=1.0 \mathrm{CHCl}_{3}\right)$. 


\section{(R)-1-(benzyloxy)-3-((S)-1-phenylallyl)piperidine (22)}

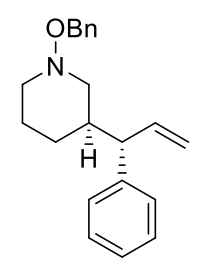

[ $\left.\{\operatorname{Ir}(\operatorname{cod}) \mathrm{Cl}\}_{2}\right](16.6 \mathrm{mg}, 24.0 \mu \mathrm{mol}, 0.06$ equiv.) and $(\boldsymbol{R})-\mathbf{L}(48.7 \mathrm{mg}, 96.0 \mu \mathrm{mol}, 0.024$ equiv.) were dissolved in 1,2-dichloroethane $(1.6 \mathrm{~mL})$ under an atmosphere of nitrogen and stirred vigorously for 15 $\mathrm{min}$. A portion of the resulting dark red $\mathrm{Ir} /(\mathrm{P}$,olefin) stock solution $(0.8 \mathrm{~mL}, 0.06$ equiv. [Ir] $)$ was added to a $5 \mathrm{~mL}$ round-bottom flask containing 1-phenylprop-2-en-1-ol (1b) $(53.7 \mathrm{mg}, 400 \mu \mathrm{mol}, 1.0$ equiv.). To this yellow solution were sequentially added, glutaraldehyde (50\% in water, $160 \mathrm{mg}, 800 \mu \mathrm{mol}, 2.0$ equiv.), (S)- $\alpha, \alpha$-Bis[3,5-bis(trifluoromethyl)phenyl]-2-pyrrolidinemethanol trimethylsilyl ether (A1) ( $23.9 \mathrm{mg}, 40.0 \mu \mathrm{mol}, 0.1$ equiv.) and dichloroacetic acid $(24.8 \mu \mathrm{L}, 300 \mu \mathrm{mol}, 0.75$ equiv.). The reaction vessel was purged with nitrogen and sealed. After stirring at room temperature for $13 \mathrm{~h}$, the reaction was diluted with dichloromethane $(1 \mathrm{~mL})$. The resulting yellow solution was washed with saturated aqueous $\mathrm{NaHCO}_{3}(1 \mathrm{~mL})$ and $\mathrm{pH} 7$ buffer $(1 \mathrm{ml})$. The organic phase was dried over $\mathrm{Na}_{2} \mathrm{SO}_{4}$ and filtered. The residual $\mathrm{Na}_{2} \mathrm{SO}_{4}$ and the filter were washed with dichloromethane $(2 \times 0.3 \mathrm{~mL})$. The organic phase was concentrated to a volume of approximately $0.5 \mathrm{~mL}$. Methanol $(4 \mathrm{~mL})$ and tetrahydrofuran $(0.4 \mathrm{~mL})$ were added to obtain a yellow solution. Activated molecular sieves (4 $\AA, 0.7 \mathrm{~g})$ and $O$-benzylhydroxylamine hydrochloride ( $255 \mathrm{mg}, 1.60 \mathrm{mmol}, 4.0$ equiv.) were added. The resulting suspension was stirred at room temperature for $4 \mathrm{~h}$. Then, acetic acid $(0.37 \mathrm{~mL})$ and sodium cyanoborohydride $(201 \mathrm{mg}, 3.20 \mathrm{mmol}$, 8.0 equiv) were added sequentially and stirring was continued at room temperature for $3 \mathrm{~h}$. Additional acetic acid $(0.37 \mathrm{~mL})$ and sodium cyanoborohydride $(201 \mathrm{mg}, 3.20 \mathrm{mmol}, 8.0$ equiv) were added and stirring was continued for $23 \mathrm{~h}$. The reaction mixture was quenched by addition of aqueous $\mathrm{NaOH}(1$ $\mathrm{M}, 8 \mathrm{~mL})$ and brine $(8 \mathrm{~mL})$. The mixture was stirred for $30 \mathrm{~min}$ at room temperature and the phases were separated. The aqueous phase was extracted with ethyl acetate $(3 \times 10 \mathrm{~mL})$. The combined organic phases were washed with brine $(10 \mathrm{ml})$, dried over $\mathrm{Na}_{2} \mathrm{SO}_{4}$, filtered and concentrated. ${ }^{1} \mathrm{H}$ NMR analysis of the crude mixture showed a d.r. of 5:1. Purification of the crude material by flash chromatography (toluene/hexane $=4 / 1$, then toluene/ $\left.\mathrm{CH}_{2} \mathrm{Cl}_{2}=9 / 1\right)$ afforded the product as a pale yellow oil $(78 \mathrm{mg}, 63 \%$ yield). The enantiomeric excess was determined by reduction of a reaction aliquot with lithium aluminum hydride to afford $\mathbf{2 0}$ and subsequent SFC analysis

NMR-spectra show a complex mixture of rotamers, all peaks are reported.

${ }^{1}$ H NMR (400 MHz, Methanol- $\left.d_{4}\right) \delta 7.41-7.06(\mathrm{~m}, 10 \mathrm{H}), 5.95(\mathrm{dt}, J=16.9,9.7 \mathrm{~Hz}, 1 \mathrm{H}), 5.06-4.93$ (m, 2H), 4.69 (br s, 2H), $3.58-2.88(\mathrm{~m}, 3 \mathrm{H}), 2.15(\mathrm{~m}, 1.7 \mathrm{H}), 1.97-1.18(\mathrm{~m}, 4.3 \mathrm{H}), 0.70(\mathrm{~d}, J=11.3 \mathrm{~Hz}$, $1 \mathrm{H})$.

${ }^{13}$ C NMR (101 MHz, Methanol- $\left.d_{4}\right) \delta 144.4,141.7,139.3,129.6,129.5,129.2,128.8,128.7,127.3$, $115.7,74.8,62.6,57.7,56.9,42.0,29.6,25.6,10.9$.

IR (neat) 2961, 2926, 1955, 1491, 1463, 1438, 1238, 1031, 841, $750 \mathrm{~cm}^{-1}$.

HRMS (ESI): $m / z$ calcd for $\mathrm{C}_{21} \mathrm{H}_{26} \mathrm{NO}[\mathrm{M}+\mathrm{H}]^{+} 308.2009$ found 308.2010.

SFC Daicel Chiralcel OJ-H, 5\% MeOH, $2.0 \mathrm{~mL} / \mathrm{min}$., $25^{\circ} \mathrm{C}$, >99\% ee $\left(\mathrm{t}_{\mathrm{R}}(1\right.$, major enantiomer, minor diastereomer $)=15.18 \min t_{R}(2$, minor enantiomer, minor diastereomer $)=16.65 \mathrm{~min}, t_{R}(3$, major 
enantiomer, major diastereomer $)=20.08 \mathrm{~min}, \mathrm{t}_{\mathrm{R}}(4$, minor enantiomer, major diastereomer $)=$ $35.39 \mathrm{~min})$.

$[\boldsymbol{\alpha}]_{\mathbf{D}}^{25}=-56.2\left(\mathrm{c}=1.0 \mathrm{CHCl}_{3}\right)$. 
<smiles>C=C[C@H](CC[C@@H](CO)c1ccccc1)c1ccccc1</smiles>

[ $\left.\{\mathrm{Ir}(\operatorname{cod}) \mathrm{Cl}\}_{2}\right](10.4 \mathrm{mg}, 15.0 \mathrm{umol}, 0.06$ equiv) and $(\boldsymbol{R})-\mathbf{L}(30.5 \mathrm{mg}, 60.0 \mathrm{umol}, 0.024$ equiv.) were dissolved in 1,2-dichloroethane $(1.0 \mathrm{~mL})$ under an atmosphere of nitrogen and stirred vigorously for 15 $\mathrm{min}$. A portion of the resulting dark red $\mathrm{Ir} /(\mathrm{P}$,olefin) stock solution $(0.5 \mathrm{~mL}, 0.06$ equiv. [Ir] $)$ was added to a $5 \mathrm{~mL}$ round-bottom flask containing 1-phenylprop-2-en-1-ol (1) $(33.5 \mathrm{mg}, 250 \mu \mathrm{mol}, 1.0$ equiv.). To this yellow solution were sequentially added, glutaraldehyde ( $50 \%$ in water, $50 \mathrm{mg}, 250 \mu \mathrm{mol}, 1.0$ equiv.), (S)- $\alpha, \alpha$-Bis[3,5-bis(trifluoromethyl)phenyl]-2-pyrrolidinemethanol trimethylsilyl ether (A1) (19.4 mg, $25.0 \mu \mathrm{mol}, 0.1$ equiv.) and dichloroacetic acid (15.5 $\mu \mathrm{L}, 188 \mu \mathrm{mol}, 0.75$ equiv.). The reaction vessel was purged with nitrogen and sealed. After stirring at room temperature for $15 \mathrm{~h}$, the reaction was diluted with dichloromethane $(1 \mathrm{~mL})$. The resulting yellow solution was washed with saturated aqueous $\mathrm{NaHCO}_{3}(1 \mathrm{~mL})$ and $\mathrm{pH} 7$ buffer $(1 \mathrm{ml})$. The organic phase was dried over $\mathrm{Na}_{2} \mathrm{SO}_{4}$ and filtered. The residual $\mathrm{Na}_{2} \mathrm{SO}_{4}$ and the filter were washed with dichloromethane $(2 \times 0.3 \mathrm{~mL})$. The combined dichloromethane fractions were added dropwise to a solution of lithium aluminum hydride $(2.4 \mathrm{M}$ in THF, $210 \mu \mathrm{L}, 0.744 \mathrm{mmol}, 2.0$ equiv.) in tetrahydrofuran $(0.75 \mathrm{~mL})$ at $0{ }^{\circ} \mathrm{C}$. The reaction was stirred at the same temperature for $1 \mathrm{~h}$ and then quenched by addition of saturated aqueous Rochelle salt solution $(5 \mathrm{~mL})$ and water $(2 \mathrm{~mL})$. The resulting emulsion was diluted with ethyl acetate $(5 \mathrm{~mL})$ and stirred for 10 min until two distinct phases were obtained. The phases were separated and the aqueous phase was extracted with ethyl acetate $(3 \times 5 \mathrm{~mL})$. The combined organic phases were dried over $\mathrm{Na}_{2} \mathrm{SO}_{4}$, filtered and concentrated. ${ }^{1} \mathrm{H}$ NMR analysis of the crude mixture showed a d.r. of $>10: 1$. Purification of the crude material by flash chromatography (EtOAc/hexane $=1 / 2$ to $1 / 1$ ) afforded the product as a colorless solid (51 $\mathrm{mg}, 61 \%$ yield). The absolute configuration of $\mathbf{2 3}$ was assigned in analogy to $\mathbf{2 0}$.

${ }^{1} \mathbf{H}$ NMR $\left(400 \mathrm{MHz}, \mathrm{CDCl}_{3}\right) \delta 7.35$ - $7.26(\mathrm{~m}, 4 \mathrm{H}), 7.23-7.18(\mathrm{~m}, 2 \mathrm{H}), 7.17$ - $7.12(\mathrm{~m}, 4 \mathrm{H}), 5.97$ (ddd, $J=17.0,10.0,9.5 \mathrm{~Hz}, 2 \mathrm{H}), 5.07-4.98(\mathrm{~m}, 4 \mathrm{H}), 3.58(\mathrm{dd}, J=11.5,3.9 \mathrm{~Hz}, 2 \mathrm{H}), 3.51(\mathrm{dd}, J=11.5,4.3$ $\mathrm{Hz}, 2 \mathrm{H}), 3.09$ (t, $J=9.7 \mathrm{~Hz}, 2 \mathrm{H}), 1.92-1.83(\mathrm{~m}, 2 \mathrm{H}), 1.36(\mathrm{~s}, 2 \mathrm{H}), 1.03(\mathrm{dd}, J=7.4,5.5 \mathrm{~Hz}, 2 \mathrm{H})$.

${ }^{13}$ C NMR $\left(101 \mathrm{MHz}, \mathrm{CDCl}_{3}\right) \delta 143.5,141.1,128.9,127.9,126.6,115.6,63.1,53.6,43.2,27.6$.

IR (neat) 3574, 3349, 3061, 2926, 1492, 1452, 1069, 1030, 995, 956, 759, $701 \mathrm{~cm}^{-1}$.

HRMS (MALDI): $m / z$ calcd for $\mathrm{C}_{23} \mathrm{H}_{28} \mathrm{AgO}_{2}[\mathrm{M}+\mathrm{Ag}]^{+} 443.1135$ found 443.1136 .

SFC Daicel Chiracel IC, 5\% MeOH, $2.0 \mathrm{~mL} / \mathrm{min} ., 25{ }^{\circ} \mathrm{C}$, >99\% ee $\left(\mathrm{t}_{\mathrm{R}}(1\right.$, major enantiomer, major diastereomer $)=23.47 \min t_{R}(2$, minor enantiomer, major diastereomer $\left.)=26.85 \mathrm{~min}\right)$. (minor diastereomer was removed via preparative TLC prior to SFC analysis.)

$[\boldsymbol{\alpha}]_{\mathbf{D}}^{26}=-48.3\left(\mathrm{c}=1.0 \mathrm{CHCl}_{3}\right)$. 
<smiles>C=CC1C[C@H]([C@H](C=C)c2ccccc2)C(=O)OC[C@H]1c1ccccc1</smiles>

A $10 \mathrm{~mL}$ Schlenk flask was charged with $(2 R, 4 R)$-2,4-bis((S)-1-phenylallyl)pentane-1,5-diol (35.0 mg, $0.104 \mathrm{mmol}) 4 \AA$ molecular sieves $(20 \mathrm{mg})$ and acetonitrile $(1.2 \mathrm{~mL}, 0.087 \mathrm{M})$. $N$-Methylmorpholine$\mathrm{N}$-oxide (36.6 mg, $0.312 \mathrm{mmol}, 3.0$ equiv.) and tetrapropylammonium perruthenate $(5.5 \mathrm{mg}, 0.016$ mmol, 0.15 equiv.) were added sequentially and the reaction mxiture was stirred for $24 \mathrm{~h}$.Silica gel (500 $\mathrm{mg}$ ) was added and the mixture was concentrated in vacuo to dryness. Purification by flash cromatography (hexane/EtOAc, 20/1 to 10/1) afforded the product as colorless, highly viscous oil (29 $\mathrm{mg}, 84 \%$ yield).

${ }^{1} \mathbf{H}$ NMR $\left(400 \mathrm{MHz}, \mathrm{CDCl}_{3}\right) \delta 7.29-7.18(\mathrm{~m}, 6 \mathrm{H}), 7.11-7.06(\mathrm{~m}, 2 \mathrm{H}), 7.04-6.99(\mathrm{~m}, 2 \mathrm{H}), 6.10$ (ddd, $J=17.4,10.4,7.3 \mathrm{~Hz}, 1 \mathrm{H}), 5.85(\mathrm{ddd}, J=16.9,10.2,9.1 \mathrm{~Hz}, 1 \mathrm{H}), 5.11-5.04(\mathrm{~m}, 3 \mathrm{H}), 4.95(\mathrm{dt}, J=$ $17.2,1.4 \mathrm{~Hz}, 1 \mathrm{H}), 4.28(\mathrm{dd}, J=11.4,4.8 \mathrm{~Hz}, 1 \mathrm{H}), 4.07(\mathrm{dd}, J=11.4,9.1 \mathrm{~Hz}, 1 \mathrm{H}), 3.83(\mathrm{t}, J=7.0 \mathrm{~Hz}$, $1 \mathrm{H}), 3.05(\mathrm{t}, J=9.6 \mathrm{~Hz}, 1 \mathrm{H}), 2.93(\mathrm{td}, J=8.1,6.7 \mathrm{~Hz}, 1 \mathrm{H}), 2.22-2.10(\mathrm{~m}, 1 \mathrm{H}), 1.48-1.35(\mathrm{~m}, 2 \mathrm{H})$.

${ }^{13}$ C NMR (101 MHz, $\left.\mathrm{CDCl}_{3}\right) \delta 173.4,141.3,140.7,138.8$ (2x), 128.9, 128.6 (2x), 127.8, 127.0, 126.9, $116.7,116.2,71.1,52.7,49.9,42.5,35.5,26.8$.

IR (neat) 3028, 2978, 1743, 1453, 1156, 1125, 920, 760, $701 \mathrm{~cm}^{-1}$.

HRMS (ESI): $m / z$ calcd for $\mathrm{C}_{23} \mathrm{H}_{25} \mathrm{O}_{2}[\mathrm{M}+\mathrm{H}]^{+} 333.1849$ found 333.1848 .

$[\boldsymbol{\alpha}]_{\mathrm{D}}^{25}=-44.5\left(\mathrm{c}=1.0 \mathrm{CHCl}_{3}\right)$. 


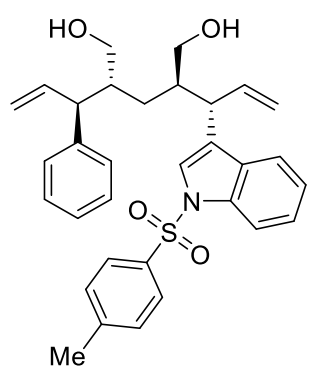

[ $\left.\{\mathrm{Ir}(\mathrm{cod}) \mathrm{Cl}\}_{2}\right](10.4 \mathrm{mg}, 15.0 \mathrm{umol}, 0.06$ equiv) and $(\boldsymbol{R})$-L (30.5 mg, $60.0 \mathrm{umol}, 0.024$ equiv.) were dissolved in 1,2-dichloroethane $(1.0 \mathrm{~mL})$ under an atmosphere of nitrogen and stirred vigorously for 15 min. A portion of the resulting dark red $\mathrm{Ir} /(\mathrm{P}$, olefin) stock solution $(0.5 \mathrm{~mL}, 0.06$ equiv. [Ir] $)$ was added to a glass vial containing 1-(1-tosyl-1H-indol-3-yl)prop-2-en-1-ol (1s) $(82.0 \mathrm{mg}, 250 \mu \mathrm{mol}, 1.0$ equiv.). To this yellow solution were sequentially added, glutaraldehyde ( $50 \%$ in water, $75 \mathrm{mg}, 250 \mu \mathrm{mol}, 1.0$ equiv.), (S)- $\alpha, \alpha$-Bis[3,5-bis(trifluoromethyl)phenyl]-2-pyrrolidinemethanol trimethylsilyl ether (A1) (19.4 mg, $25.0 \mu \mathrm{mol}, 0.1$ equiv.) and dichloroacetic acid $(15.5 \mu \mathrm{L}, 188 \mu \mathrm{mol}, 0.75$ equiv.). The reaction vessel was purged with nitrogen, sealed with a teflon-lined cap and stirred at room temperature for 1.5 h. 1-Phenylprop-2-en-1-ol (1b) (84.0 mg, $625 \mu \mathrm{mol}, 2.5$ equiv.) was added and stirring was continued $18 \mathrm{~h}$. The reaction was diluted with dichloromethane $(1 \mathrm{~mL})$ and washed with saturated aqueous $\mathrm{NaHCO}_{3}(1 \mathrm{~mL})$ and $\mathrm{pH} 7$ buffer $(1 \mathrm{ml})$. The organic phase was dried over $\mathrm{Na}_{2} \mathrm{SO}_{4}$ and filtered. The residual $\mathrm{Na}_{2} \mathrm{SO}_{4}$ and the filter were washed with dichloromethane $(2 \times 0.3 \mathrm{~mL})$. The combined dichloromethane fractions were added dropwise to a solution of lithium aluminum hydride $(2.4 \mathrm{M}$ in THF, $210 \mu \mathrm{L}, 0.744 \mathrm{mmol}, 2.0$ equiv.) in tetrahydrofuran $(0.75 \mathrm{~mL})$ at $0{ }^{\circ} \mathrm{C}$. The reaction was stirred at the same temperature for $1 \mathrm{~h}$ and then quenched by addition of saturated aqueous Rochelle salt solution $(5 \mathrm{~mL})$ and water $(2 \mathrm{~mL})$. The resulting emulsion was diluted with ethyl acetate $(5 \mathrm{~mL})$ and stirred for 10 min until two distinct phases were obtained. The phases were separated and the aqueous phase was extracted with ethyl acetate $(3 \times 5 \mathrm{~mL})$. The combined organic phases were dried over $\mathrm{Na}_{2} \mathrm{SO}_{4}$, filtered and concentrated. ${ }^{1} \mathrm{H}$ NMR analysis of the crude mixture showed a d.r. of $>10: 1$. Purification of the crude material by flash chromatography (EtOAc/hexane $=1 / 2$ to $1 / 1$ ) afforded the product as a colorless solid (58 mg, 44\% yield). The absolute configuration of $\mathbf{2 5}$ was assigned in analogy to $\mathbf{2 0}$.

${ }^{1} \mathbf{H}$ NMR $\left(500 \mathrm{MHz}, \mathrm{CDCl}_{3}\right) \delta 7.98-7.94(\mathrm{~m}, 1 \mathrm{H}), 7.76-7.72(\mathrm{~m}, 2 \mathrm{H}), 7.45(\mathrm{dt}, J=7.9,1.0 \mathrm{~Hz}, 1 \mathrm{H})$, $7.41(\mathrm{~s}, 1 \mathrm{H}), 7.29(\mathrm{~m}, 3 \mathrm{H}), 7.22-7.17(\mathrm{~m}, 4 \mathrm{H}), 7.13-7.10(\mathrm{~m}, 2 \mathrm{H}), 6.00$ (ddd, $J=17.0,10.1,9.5 \mathrm{~Hz}$, 1H), 5.92 (ddd, $J=17.0,10.1,8.9 \mathrm{~Hz}, 1 \mathrm{H}), 5.10-4.98(\mathrm{~m}, 4 \mathrm{H}), 3.61$ (d, $J=4.3 \mathrm{~Hz}, 2 \mathrm{H}), 3.58$ (dd, $J=$ $11.4,3.8 \mathrm{~Hz}, 1 \mathrm{H}$ ), 3.49 (dd, $J=11.4,4.3 \mathrm{~Hz}, 1 \mathrm{H}), 3.44(\mathrm{t}, J=8.8 \mathrm{~Hz}, 1 \mathrm{H}), 3.10$ (t, $J=9.5 \mathrm{~Hz}, 1 \mathrm{H}), 2.34$ (s, 3H), $2.05(\mathrm{~m}, 1 \mathrm{H}), 1.99-1.92(\mathrm{~m}, 1 \mathrm{H}), 1.36(\mathrm{br} \mathrm{s}, 2 \mathrm{H}), 1.26(\mathrm{ddd}, J=14.3,10.0,3.0 \mathrm{~Hz}, 1 \mathrm{H}), 1.12$ (ddd, $J=14.0,10.6,2.8 \mathrm{~Hz}, 1 \mathrm{H}$ ).

${ }^{13} \mathrm{C} \mathrm{NMR}\left(126 \mathrm{MHz}, \mathrm{CDCl}_{3}\right) \delta 144.9,143.3,141.0,139.1,135.43,135.42,130.7,129.9,128.9,127.9$, 127.0, 126.7, 124.8, 123.8, 123.3, 123.2, 120.0, 116.3, 115.6, 113.9, 63.3, 62.5, 53.8, 43.7, 42.8, 41.7, 28.4, 21.7 .

IR (neat) 3572, 3402, 3079, 2925, 1447, 1367, 1173, 1131, 759, 747, 704, 665, 584, 573, $538 \mathrm{~cm}^{-1}$.

HRMS (MALDI): $\mathrm{m} / z$ calcd for $\mathrm{C}_{32} \mathrm{H}_{36} \mathrm{NO}_{4} \mathrm{~S}[\mathrm{M}+\mathrm{H}]^{+} 530.2360$ found 530.2356. 
SFC Daicel Chiralcel OJ-H, $10 \% \mathrm{MeOH}, 2.0 \mathrm{~mL} / \mathrm{min} ., 25{ }^{\circ} \mathrm{C}$, > $99 \%$ ee $\left(\mathrm{t}_{\mathrm{R}}(1\right.$, major enantiomer, major diastereomer $)=19.98 \min t_{R}(2$, minor enantiomer, major diastereomer $\left.)=22.48 \mathrm{~min}\right)$. (minor diastereomer was removed via preparative TLC prior to SFC analysis.)

$[\alpha]_{\mathrm{D}}^{27}=-18.8\left(\mathrm{c}=0.5 \mathrm{CHCl}_{3}\right)$. 


\section{X-Ray Crystallographic Data}

\subsection{General Methods}

Single crystalline samples were measured on the following instruments:

- Bruker Kappa APEX-II Duo diffractometer with sealed tube Mo-K $\alpha$ radiation $(\lambda=0.71073 \AA$ A, 4 bcarbamate).

- Rigaku Oxford Diffraction XtaLAB Synergy-S Dualflex kappa diffractometer equipped with a Dectris Pilatus 300 HPAD detector and using microfocus sealed tube $\mathrm{Cu}-\mathrm{K} \alpha$ radiation with mirror optics $(\lambda=1.54178 \AA$, 2e, 2f, 2k, 4l, 4m-carbamate, 20).

All measurements were carried out at 100K (unless specifically noted) using an Oxford Cryosystems Cryostream 700 or 800 sample cryostat. Data collected on Bruker instruments were integrated using SAINT from the Bruker Apex-II program suite and corrected for absorption effects using the multi-scan method (SADABS). Data collected on the Rigaku instrument were integrated using CrysAlisPro and corrected for absorption effects using a combination of empirical (ABSPACK) and numerical corrections. The structures were solved using SHELXS, ${ }^{17}$ SHELXT $^{18}$ or Superflip ${ }^{19}$ and refined by fullmatrix least-squares analysis (SHELXL) ${ }^{17,18}$ using the program package OLEX2. ${ }^{20}$ All non-hydrogen atoms were refined anisotropically and hydrogen atoms were constrained to ideal geometries and refined with fixed isotropic displacement parameters (in terms of a riding model). CCDC 1880963 (2e), 1880960 (2f), 1880961 (2k), 1880962 (4l), 1880965 (4b-carbamate), 1880964 (4b-carbamate), 1880959 (20) contain the supplementary crystallographic data for this paper, including structure factors and refinement instructions. These data can be obtained free of charge from The Cambridge Crystallographic Data Centre, 12 Union Road, Cambridge CB2 1EZ, UK (fax: +44(1223)-336-033; email: deposit@ccdc.cam.ac.uk), or via https://www.ccdc.cam.ac.uk/getstructures.

\subsection{General Crystallization Methods}

\section{CA) Gas-phase diffusion}

A $5 \mathrm{~mL}$ round bottom flask was charged with a solution of the substrate in solvent A (approximately 0.2 $\mathrm{ml} / \mathrm{mg}$ ). If required, the mixture as briefly sonicated, warmed to $40{ }^{\circ} \mathrm{C}$ or filtered in order to obtain a clear solution. This solution was placed in a $100 \mathrm{~mL}$ glass jar containing $15 \mathrm{~mL}$ of solvent $\mathrm{B}$. The jar was sealed tightly and left standing at the temperature indicated for 1-5 days.

\section{CB) Slow evaporation}

A $1 \mathrm{~mL}$ glass vial was charged with the substrate $(1-5 \mathrm{mg})$ and the solvent indicated below (approximately $0.1 \mathrm{ml} / \mathrm{mg}$ ) was added. If required, the mixture as briefly sonicated, warmed to $40{ }^{\circ} \mathrm{C}$ or filtered, in order to obtain a clear solution. The vial was closed and two needles $(0.9 \mathrm{~mm}$ internal diameter) were pushed through the cap. The solution was left standing at room temperature for 3-7 days. 


\subsection{X-ray Structures and Data}

\section{(S)-N-(1-(naphthalen-2-yl)allyl)-4H-1,2,4-triazol-4-amine (2e)}

$\mathrm{X}$-ray quality single crystals of $\mathbf{2 e}$ were obtained following general procedure $\mathbf{C B}$ with dichloromethane. The data acquired was used to determine the absolute configuration of $2 \mathbf{e}$.

CCDC

Empirical formula

Formula weight

Temperature/K

Crystal system

Space group

$\mathrm{a} / \AA$

$\mathrm{b} / \AA$

c/Å

$\alpha /{ }^{\circ}$

$\beta /{ }^{\circ}$

$\gamma /{ }^{\circ}$

Volume $/ \AA^{3}$

Z

$\rho_{\text {calc }} \mathrm{g} / \mathrm{cm}^{3}$

$\mu / \mathrm{mm}^{-1}$

$\mathrm{F}(000)$

Crystal size $/ \mathrm{mm}^{3}$

Radiation
1880963

$\mathrm{C}_{15} \mathrm{H}_{14} \mathrm{~N}_{4}$

250.30

100.0(1)

monoclinic

$\mathrm{P} 21$

5.55961(5)

$8.22570(5)$

$13.54910(8)$

90

91.2759(7)

90

619.470(7)

2

1.342

0.662

264.0

$0.171 \times 0.132 \times 0.046$

$\mathrm{CuK} \alpha(\lambda=1.54184)$

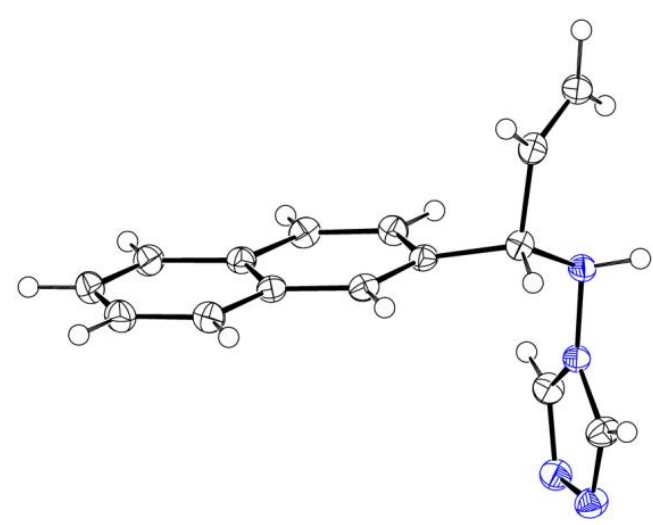

$2 \Theta$ range for data collection/ $/{ }^{\circ} 6.526$ to 159.528

Index ranges

$-6 \leq \mathrm{h} \leq 7,-10 \leq \mathrm{k} \leq 10,-17 \leq 1 \leq 17$

Reflections collected

23694

Independent reflections

$2640\left[R_{\text {int }}=0.0383, R_{\text {sigma }}=0.0183\right]$

Data/restraints/parameters

$2640 / 2 / 175$

Goodness-of-fit on $\mathrm{F}^{2}$

1.052

Final $R$ indexes $[\mathrm{I}>=2 \sigma(\mathrm{I})] \quad \mathrm{R}_{1}=0.0293, \mathrm{wR}_{2}=0.0711$

Final $\mathrm{R}$ indexes [all data] $\quad \mathrm{R}_{1}=0.0298, \mathrm{wR}_{2}=0.0717$

Largest diff. peak/hole / e $\AA^{-3} 0.12 /-0.19$

Flack parameter

$-0.13(13)$ 


\section{(S)- $N$-methyl-1-(naphthalen-2-yl)prop-2-en-1-aminium chloride (2f)}

$\mathrm{X}$-ray quality single crystals of were obtained following general procedure $\mathrm{CA}$ (solvent $\mathrm{A}=$ methanol, solvent $\mathrm{B}=$ diethyl ether) as colorless plates. The data acquired was used to determine the absolute configuration of $\mathbf{2 f}$.

CCDC code

Empirical formula

Formula weight

Temperature/K

Crystal system

Space group

$\mathrm{a} / \AA$

$\mathrm{b} / \AA$

c/Å

$\alpha /{ }^{\circ}$

$\beta /{ }^{\circ}$

$\gamma /{ }^{\circ}$

Volume $/ \AA^{3}$

Z

$\rho_{\text {calc }} \mathrm{g} / \mathrm{cm}^{3}$

$\mu / \mathrm{mm}^{-1}$

$\mathrm{F}(000)$

Crystal size $/ \mathrm{mm}^{3}$

Radiation
1880960

$\mathrm{C}_{14} \mathrm{H}_{16} \mathrm{NCl}$

233.73

100.0(1)

orthorhombic

$\mathrm{P} 2{ }_{1} 2_{1} 2_{1}$

6.95930(10)

$7.46620(10)$

24.7555(2)

90

90

90

1286.28(3)

4

1.207

2.389

496.0

$0.185 \times 0.153 \times 0.056$

$\mathrm{CuK} \alpha(\lambda=1.54184)$

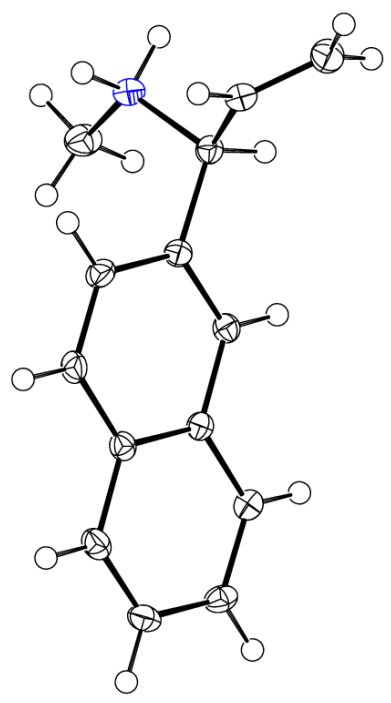

$2 \Theta$ range for data collection $/{ }^{\circ} 7.142$ to 159.412

Index ranges

$-7 \leq \mathrm{h} \leq 8,-9 \leq \mathrm{k} \leq 9,-31 \leq 1 \leq 31$

Reflections collected

29240

Independent reflections

$2762\left[\mathrm{R}_{\mathrm{int}}=0.0453, \mathrm{R}_{\text {sigma }}=0.0190\right]$

Data/restraints/parameters

$2762 / 3 / 152$

Goodness-of-fit on $\mathrm{F}^{2}$

1.079

Final $R$ indexes $[\mathrm{I}>=2 \sigma(\mathrm{I})] \quad \mathrm{R}_{1}=0.0249, \mathrm{wR}_{2}=0.0658$

Final $\mathrm{R}$ indexes [all data] $\quad \mathrm{R}_{1}=0.0254, \mathrm{wR}_{2}=0.0661$

Largest diff. peak/hole / e $\AA^{-3} 0.20 /-0.18$

Flack parameter

$0.002(5)$ 


\section{(S)-N-(1-(naphthalen-2-yl)allyl)hydroxylammonium chloride (2k-HCl)}

Hydroxylamine 2a was taken up in diethyl ether (approx.. $10 \mathrm{ml} / \mathrm{mmol}$ ) and $\mathrm{HCl}(2.0 \mathrm{M}$ in diethyl ether, approx. 2 equiv.) was added. The resulting suspension was concentrated under reduced pressure at 40 ${ }^{\circ} \mathrm{C}$. X-ray quality single crystals of the resulting crystalline solid were obtained following general procedure $\mathrm{CA}$ (solvent $\mathrm{A}=$ methanol, solvent $\mathrm{B}=$ diethyl ether) as colorless plates. The data acquired was used to determine the absolute configuration of $\mathbf{2 a}$.

CCDC code

Empirical formula

Formula weight

Temperature/K

Crystal system

Space group

$\mathrm{a} / \AA$

$\mathrm{b} / \AA$

$\mathrm{c} / \AA$

$\alpha /{ }^{\circ}$

$\beta /{ }^{\circ}$

$\gamma /{ }^{\circ}$

Volume $/ \AA^{3}$

Z

$\rho_{\text {calc }} \mathrm{g} / \mathrm{cm}^{3}$

$\mu / \mathrm{mm}^{-1}$

$\mathrm{F}(000)$

Crystal size $/ \mathrm{mm}^{3}$

Radiation
1880961

$$
\mathrm{C}_{13} \mathrm{H}_{14} \mathrm{ClNO}
$$

235.70

100.0(1)

orthorhombic

$\mathrm{P} 2{ }_{1} 2_{1} 2_{1}$

6.2187(2)

$7.2638(2)$

27.5254(7)

90

90

90

1243.36(6)

4

1.259

2.540

496.0

$0.172 \times 0.087 \times 0.013$

$\mathrm{CuK} \alpha(\lambda=1.54184)$

$2 \Theta$ range for data collection $/{ }^{\circ} 6.422$ to 160.494

Index ranges

$-7 \leq \mathrm{h} \leq 6,-9 \leq \mathrm{k} \leq 9,-34 \leq 1 \leq 34$

Reflections collected

27420

Independent reflections

Data/restraints/parameters

$2678\left[R_{\text {int }}=0.0646, R_{\text {sigma }}=0.0231\right]$

Goodness-of-fit on $\mathrm{F}^{2}$

$2678 / 24 / 171$

Final R indexes $[\mathrm{I}>=2 \sigma(\mathrm{I})]$

1.090

Final $\mathrm{R}$ indexes [all data]

$\mathrm{R}_{1}=0.0667, \mathrm{wR}_{2}=0.2010$

Largest diff. peak/hole / e $\AA^{-3}$

$\mathrm{R}_{1}=0.0729, \mathrm{wR}_{2}=0.2104$

Flack parameter

$0.038(10)$

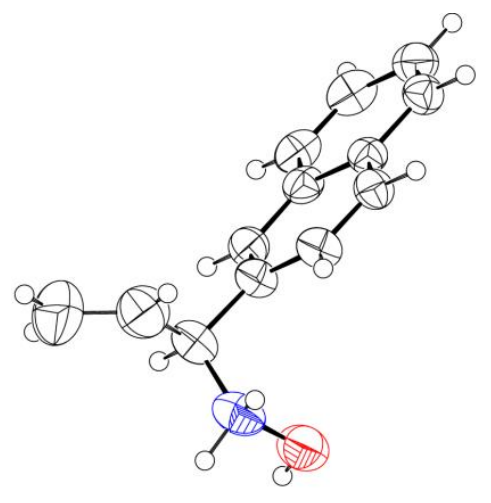




\section{(R)-2,2-dichloro-3-(naphthalen-2-yl)pent-4-en-1-ol (4m)}

$\mathrm{X}$-ray quality single crystals were obtained following general procedure $\mathrm{CA}$ (solvent $\mathrm{A}=$ dichloromethane, solvent $\mathrm{B}=$ hexane) as colorless needles. The acquired data was deemed good enough to confirm the structure of $\mathbf{4 m}$.

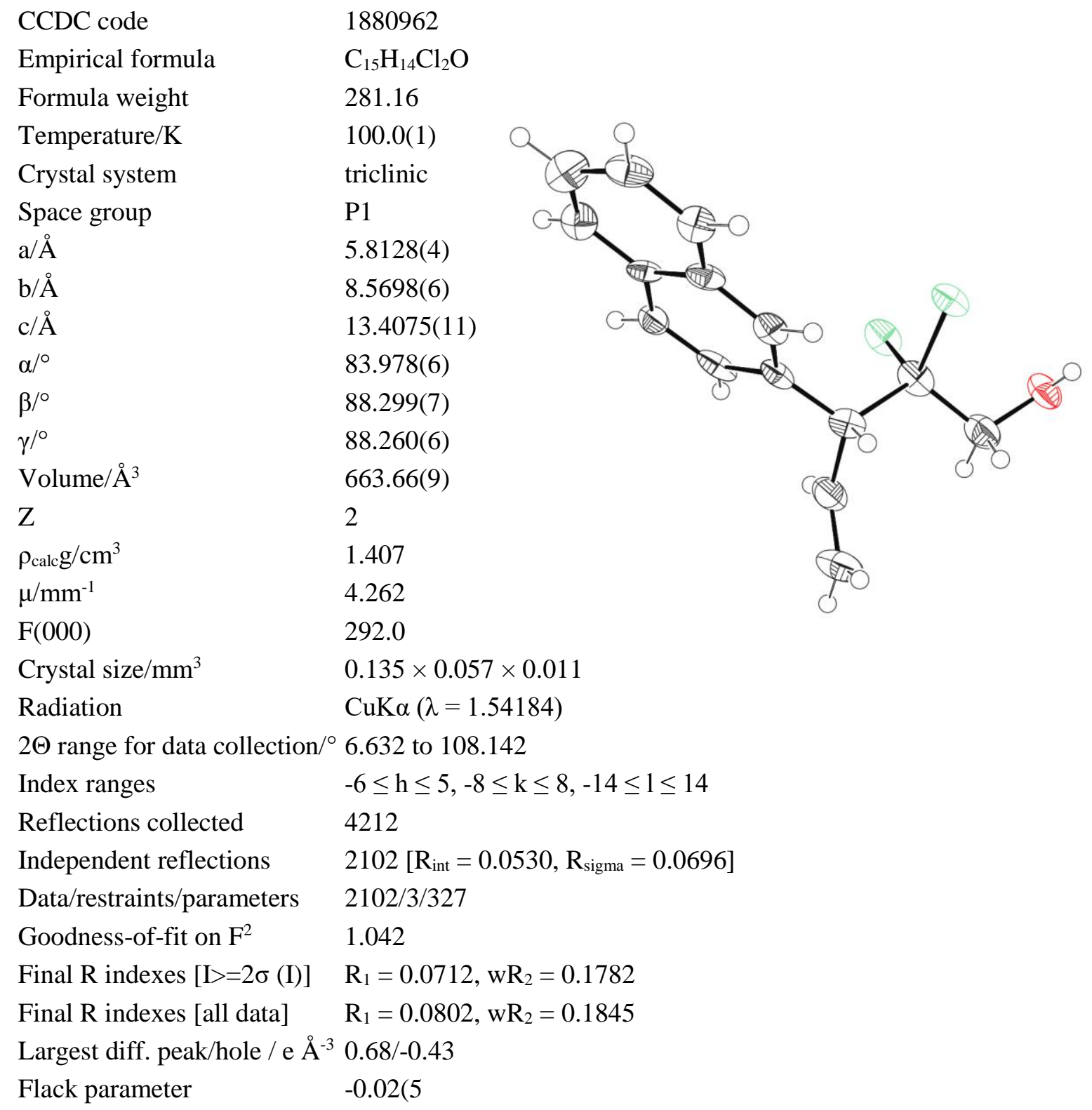




\section{(2R,3R)-2-chloro-3-phenylpent-4-en-1-yl (4-nitrophenyl)carbamate (4b-carbamate)}

X-ray quality single crystals of $\mathbf{4 b}$-carbamate (12:1 d.r. determined by ${ }^{1} \mathrm{H}$ NMR) were obtained following general procedure $\mathbf{C B}$ with toluene. Curiously, the thus obtained colorless plates contained approximately $5-10 \%$ of the minor diastereomer. Therefore, some disorder was observed at the phenyl group and at $\mathrm{C} 16$, leading to partial inversion of stereochemistry at that carbon. As no disorder was detected at $\mathrm{C} 18$, the absolute configurations of both diastereomers of $\mathbf{4 b}$-carbamate could be determined. This data is in agreement with the absolute configuration determined for $\mathbf{4 n}$ using the $(\boldsymbol{S})$ - $\mathbf{L}$ and $(\boldsymbol{R})$-A1 catalyst combination.

CCDC code

Empirical formula

Formula weight

Temperature/K

Crystal system

Space group

$\mathrm{a} / \AA ̊$

$\mathrm{b} / \AA$

$\mathrm{c} / \AA$

$\alpha /{ }^{\circ}$

$\beta /{ }^{\circ}$

$\gamma /{ }^{\circ}$

Volume $/ \AA^{3}$

Z

$\rho_{\text {calc }} \mathrm{g} / \mathrm{cm}^{3}$

$\mu / \mathrm{mm}^{-1}$

$\mathrm{F}(000)$

Crystal size $/ \mathrm{mm}^{3}$

Radiation
1880965

$\mathrm{C}_{18} \mathrm{H}_{17} \mathrm{ClN}_{2} \mathrm{O}_{4}$

360.78

$100.0(2)$

monoclinic

$\mathrm{P} 21_{1}$

7.8468(14)

$8.0192(14)$

$13.739(2)$

90

95.361(4)

90

$860.8(3)$

2

1.392

0.247

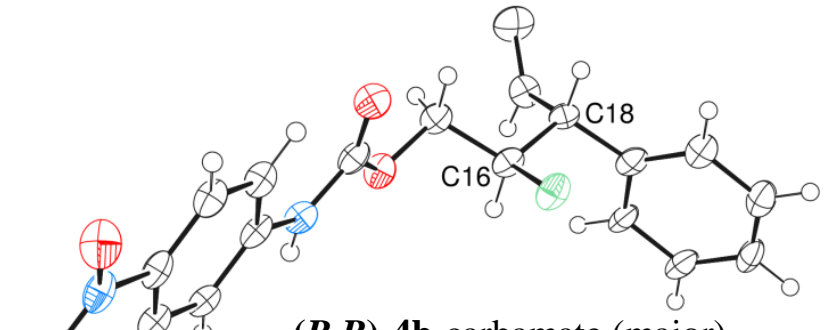

$(\boldsymbol{R}, \boldsymbol{R})-\mathbf{4 b}$-carbamate (major)

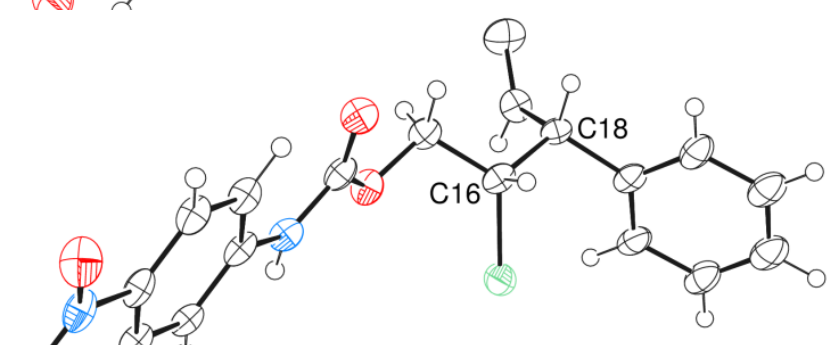

$(S, R)$ - 4b-carbamate (minor)

376.0

$0.24 \times 0.08 \times 0.01$

$2 \Theta$ range for data collection $/{ }^{\circ} 2.978$ to 55.108

Index ranges

$-10 \leq \mathrm{h} \leq 10,-10 \leq \mathrm{k} \leq 10,-17 \leq 1 \leq 17$

Reflections collected

7352

Independent reflections

$3703\left[R_{\text {int }}=0.0506, R_{\text {sigma }}=0.0826\right]$

Data/restraints/parameters

$3703 / 324 / 264$

Goodness-of-fit on $\mathrm{F}^{2}$

0.959

Final $R$ indexes $[I>=2 \sigma(I)] \quad R_{1}=0.0388, w_{2}=0.0739$

Final $R$ indexes [all data] $\quad R_{1}=0.0640, \mathrm{wR}_{2}=0.0816$

Largest diff. peak/hole / e $\AA^{-3} 0.18 /-0.20$

Flack parameter

$0.09(6)$ 
X-ray quality single crystals of $\mathbf{4 n}$-carbamate were obtained following general procedure $\mathbf{C B}$ with toluene. The data acquired was used to determine the absolute configuration of $\mathbf{4 n}$.

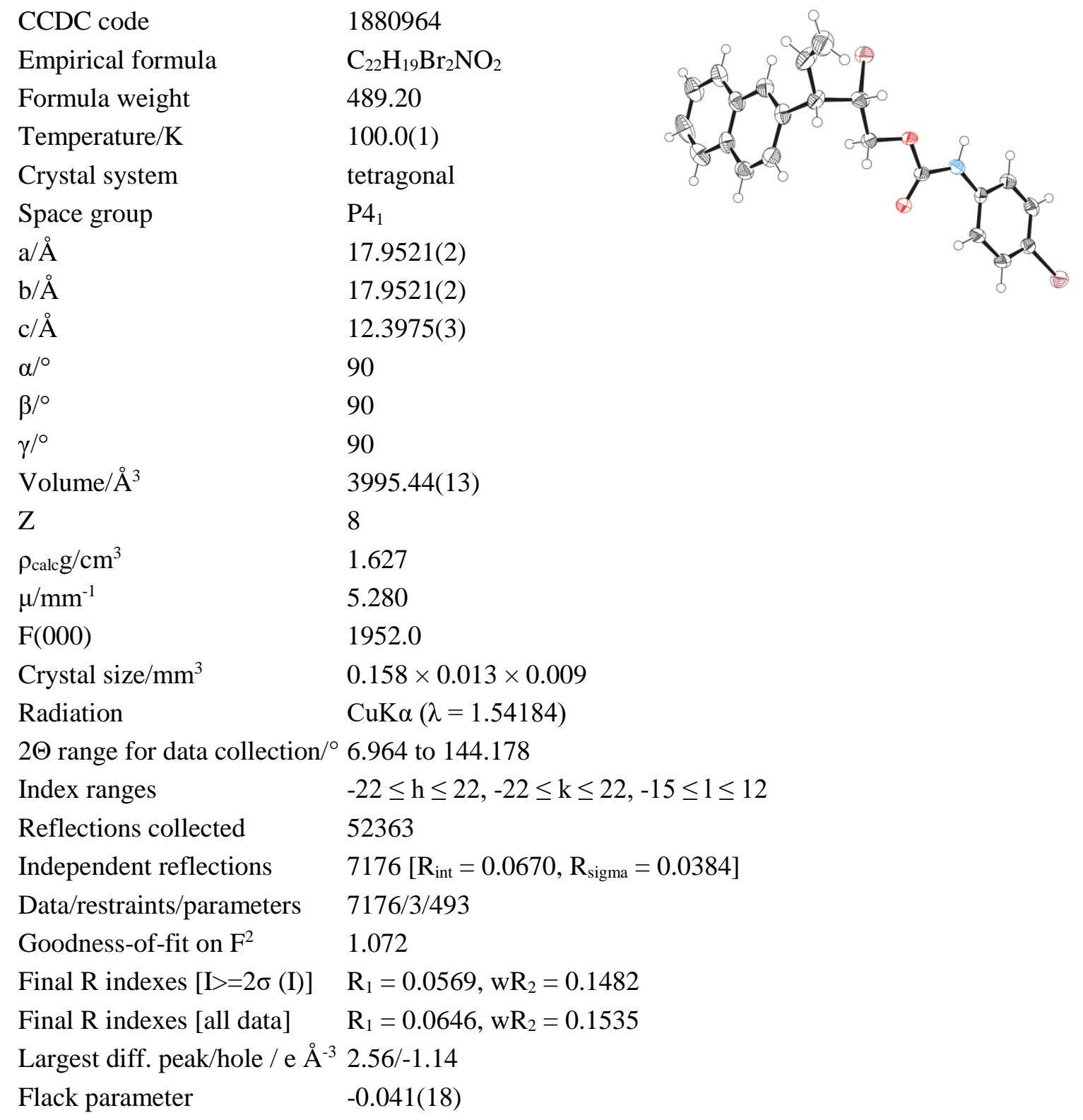




\section{(S)-2-((S)-1-phenylallyl)pentane-1,5-diol (20)}

X-ray quality single crystals were obtained following general procedure CA (solvent $\mathrm{A}=$ diethyl ether, solvent $\mathrm{B}=$ hexane) as colorless plates. The data acquired was used to determine the absolute configuration of 14 .

CCDC code

Empirical formula

Formula weight

Temperature/K

Crystal system

Space group

$\mathrm{a} / \AA$

$\mathrm{b} / \AA$

c/Å

$\alpha /{ }^{\circ}$

$\beta /{ }^{\circ}$

$\gamma /{ }^{\circ}$

Volume $/ \AA^{3}$

Z

$\rho_{\text {calc } g / \mathrm{cm}^{3}}$

$\mu / \mathrm{mm}^{-1}$

$\mathrm{F}(000)$

Crystal size $/ \mathrm{mm}^{3}$

Radiation
1880959

$\mathrm{C}_{14} \mathrm{H}_{20} \mathrm{O}_{2}$

220.30

100.0(1)

monoclinic

$\mathrm{P} 2_{1}$

10.71250(10)

7.90530(10)

15.48200(10)

90

98.7750(10)

90

1295.75(2)

4

1.129

0.580

480.0

$0.232 \times 0.157 \times 0.066$

$\mathrm{CuK} \alpha(\lambda=1.54184)$

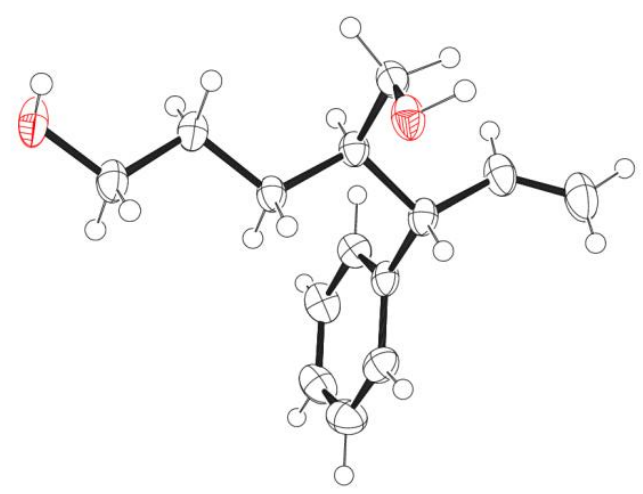

$2 \Theta$ range for data collection $/{ }^{\circ} 5.776$ to 159.158

Index ranges

$-13 \leq \mathrm{h} \leq 13,-10 \leq \mathrm{k} \leq 10,-19 \leq 1 \leq 19$

Reflections collected

49250

Independent reflections

$5561\left[R_{\text {int }}=0.0359, R_{\text {sigma }}=0.0179\right]$

Data/restraints/parameters

$5561 / 5 / 301$

Goodness-of-fit on $\mathrm{F}^{2}$

1.076

Final $\mathrm{R}$ indexes $[\mathrm{I}>=2 \sigma(\mathrm{I})]$

$\mathrm{R}_{1}=0.0320, \mathrm{wR}_{2}=0.0847$

Final $\mathrm{R}$ indexes [all data]

$\mathrm{R}_{1}=0.0334, \mathrm{wR}_{2}=0.0858$

Largest diff. peak/hole / e $\AA^{-3} 0.17 /-0.16$

Flack parameter

$0.00(5)$ 


\section{References}

(1) Hamilton, J. Y.; Sarlah, D.; Carreira, E. M. Org. Synth. 2015, 92, 1-12.

(2) Dfieber, C.; Ariger, M. A.; Moriel, P.; Carreira, E. M. Angew. Chem. Int. Ed. 2007, 46, 3139-3143.

(3) Roggen, M.; Carreira, E. M. Angew. Chem. Int. Ed. 2011, 50, 5568-5571.

(4) Lafrance, M.; Roggen, M.; Carreira, E. M. Angew. Chem. Int. Ed. 2012, 51, 3470-3473.

(5) Roggen, M.; Carreira, E. M. Angew. Chem. Int. Ed. 2012, 51, 8652-8655.

(6) Bartholomäus, R.; Baachmann, J.; Mang, C.; Haustedt, L. O.; Harms, K.; Koert, U. Eur. J. Org. Chem. 2013, 180190.

(7) Krishna, J.; Niharika, P.; Satyanarayana, G.. RSC Adv.2013, 5, 26749-26761.

(8) Barcellos, T.; Tauber, K.; Kroutil, W.; Andrade, L. H. Tetrahedron: Asymmetry. 2011, 22, 1772-1777.

(9) Kang, B.; Mowat, J.; Pinter, T.; Britton, R.. Org. Lett. 2009, 11, 1717-1720.

(10) Dekeukeleire, S.; D’hooghe, M.; Törnroos, K.; De Kimpe, N. J. Org. Chem. 2010, 75, 5934-5340.

(11) Jiang, H.; Elsner, P.; Jensen, K. L.; Falcicchio, A.; Marcos, V.; Jørgensen, K. A. Angew. Chem. Int. Ed. 2009, 48, 6844-6848.

(12) Katsuki, T.; Fukuda, T. Tetrahedron 1999, 55, 649-664.

(13) Brochu, M. P.; Brown, S. P.; MacMillan, D. W. C. J. Am. Chem. Soc. 2004, 126, 4108-4109.

(14) Beeson, T. D.; Macmillan, D. W. C. J. Am. Chem. Soc. 2005, 127, 8826-8828.

(15) Sandmeier, T.; Krautwald, S.; Carreira, E. M. Angew. Chem. Int. Ed. 2016, 54, 14363-14367.

(16) Cozzi, P. G.; Benfatti, F.; Zoli, L. Angew. Chem. Int. Ed. 2019, 48, 1313-1316.

(17) Sheldrick, G. M. Acta Cryst. 2008, A64, 112-122.

(18) Sheldrick, G. M. Acta Cryst. 2015, A71, 3-8.

(19) Palatinus, L.; Prathapa, S. J.; van Smaalen, S. J. Appl. Cryst. 2012, 45, 575-580.

(20) Dolomanov, O. V.; Bourhis, L. J.; Gildea, R. J.; Howard, J. A. K.; Puschmann, H. J. Appl. Cryst. 2009, 42, 339341. 
8. NMR Spectra

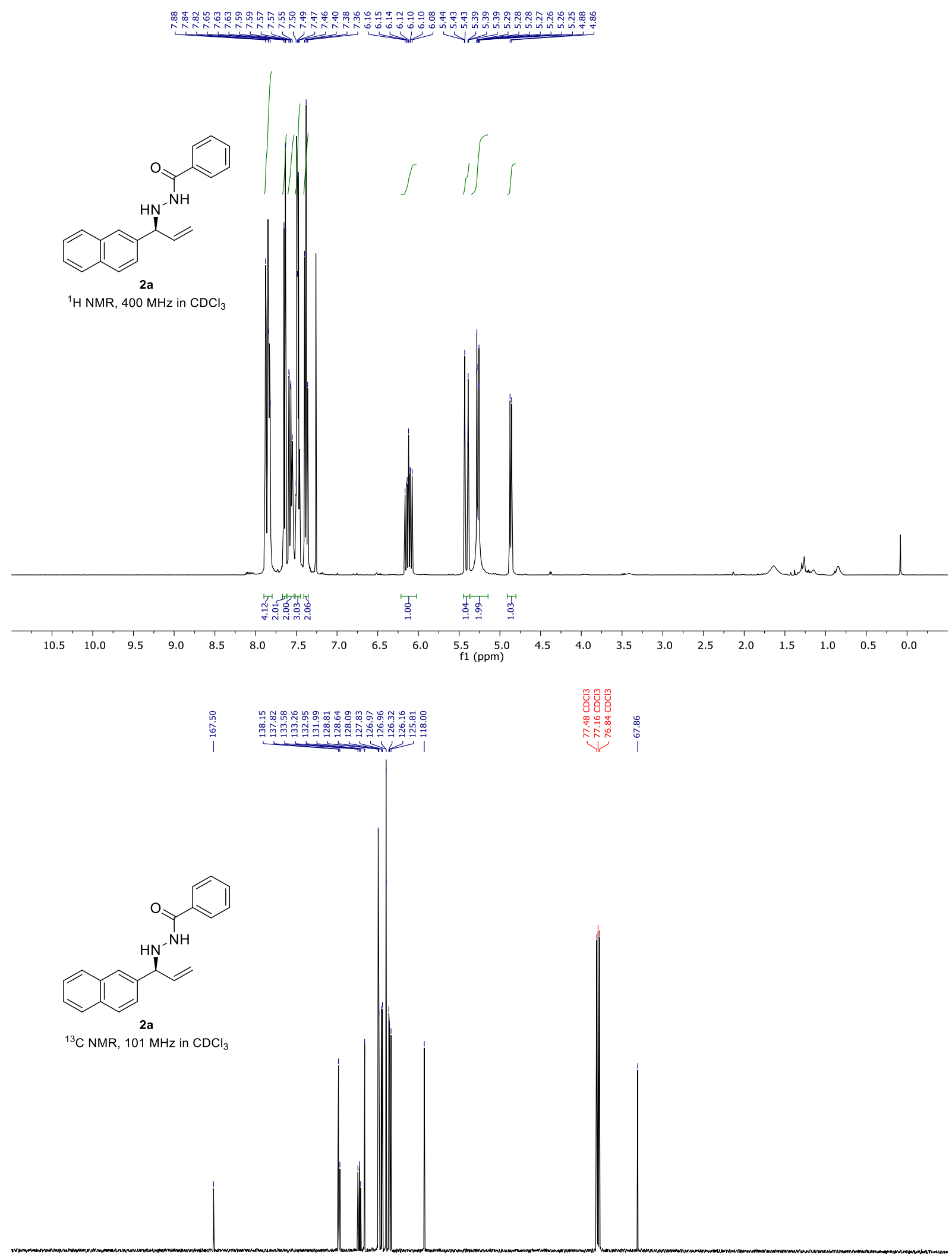

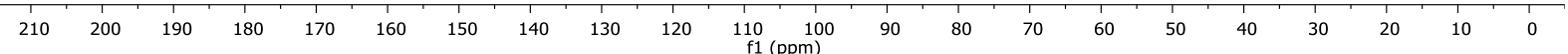



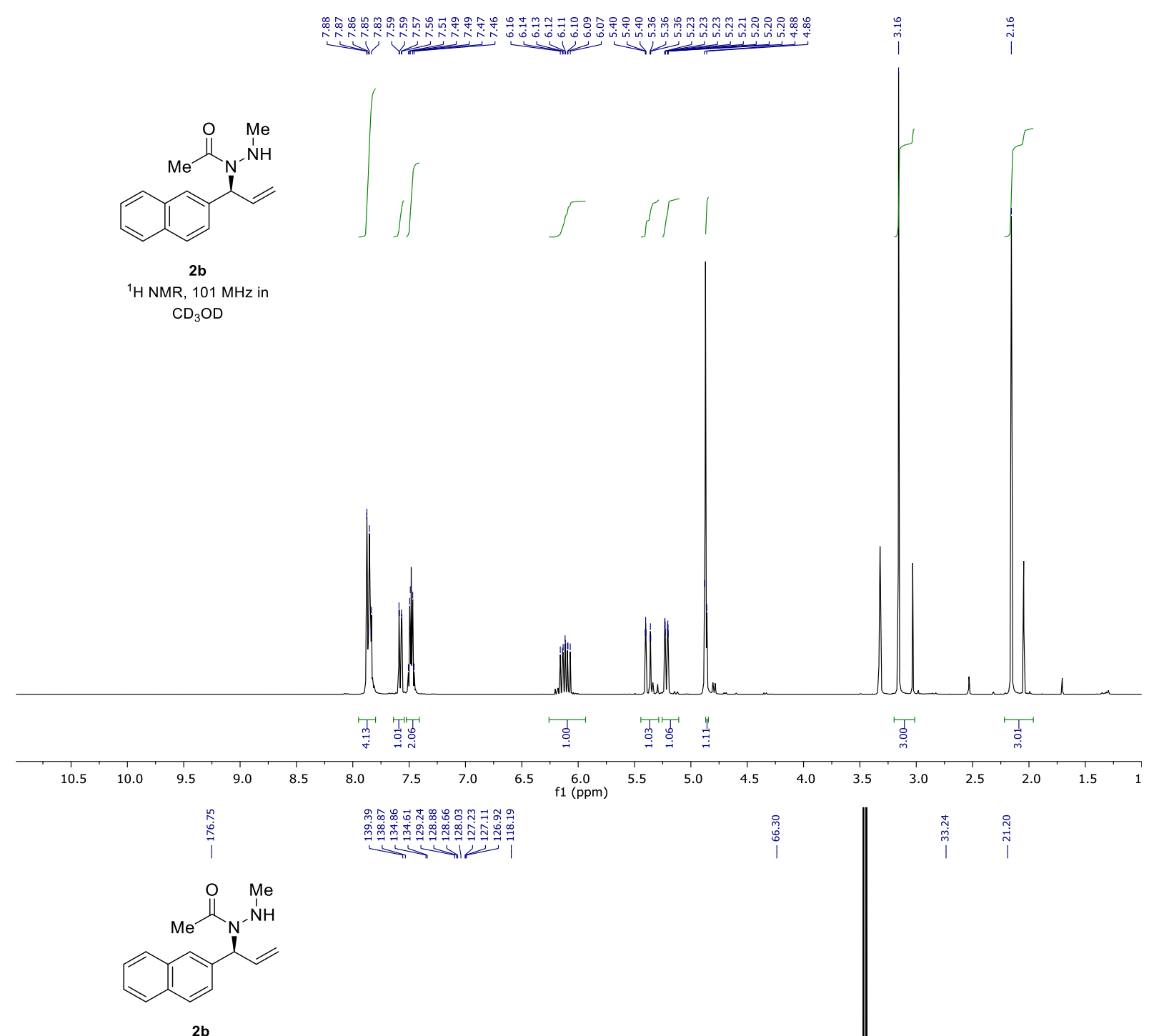

${ }^{13} \mathrm{C} \mathrm{NMR}, 101 \mathrm{MHz}$ in $\mathrm{CD}_{3} \mathrm{OD}$

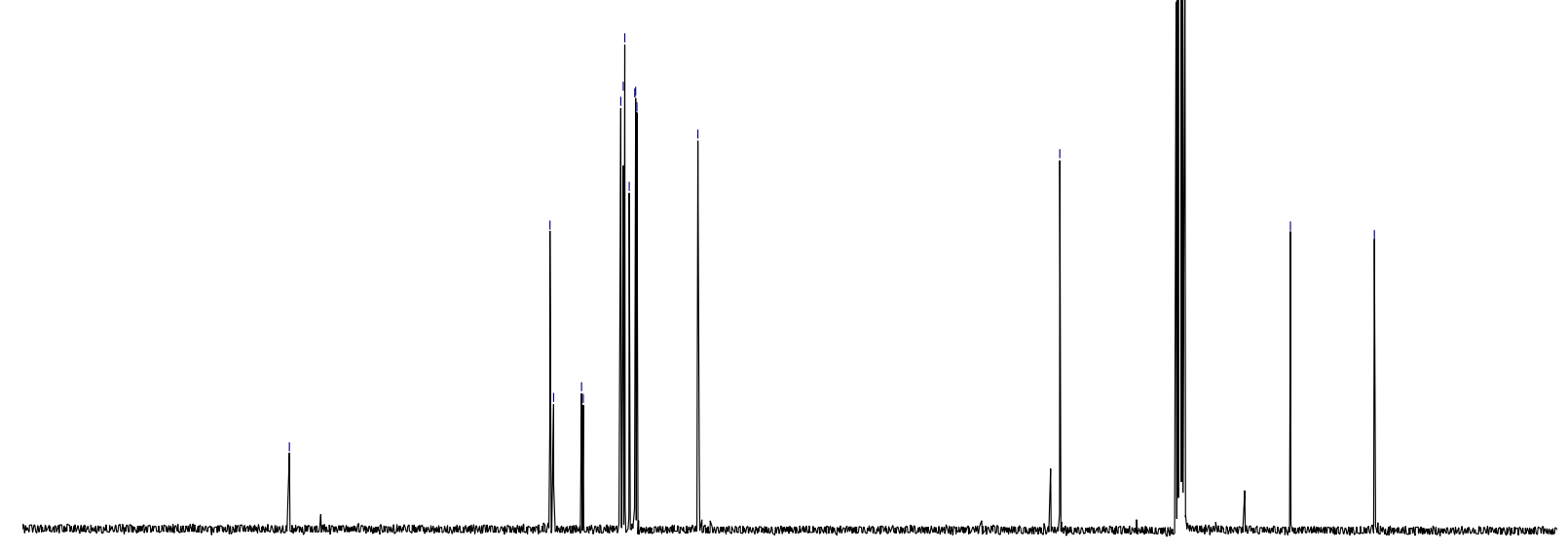

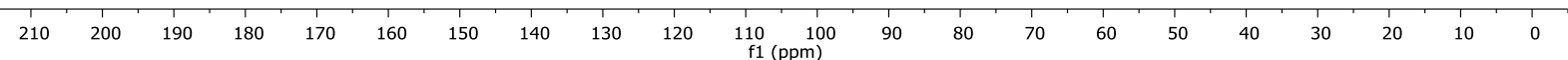




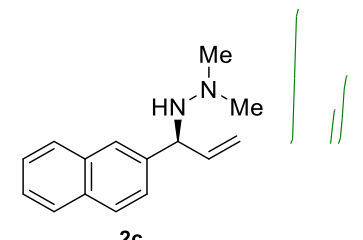

${ }^{1} \mathrm{H}$ NMR, $500 \mathrm{MHz}$ in $\mathrm{CDCl}_{3}$
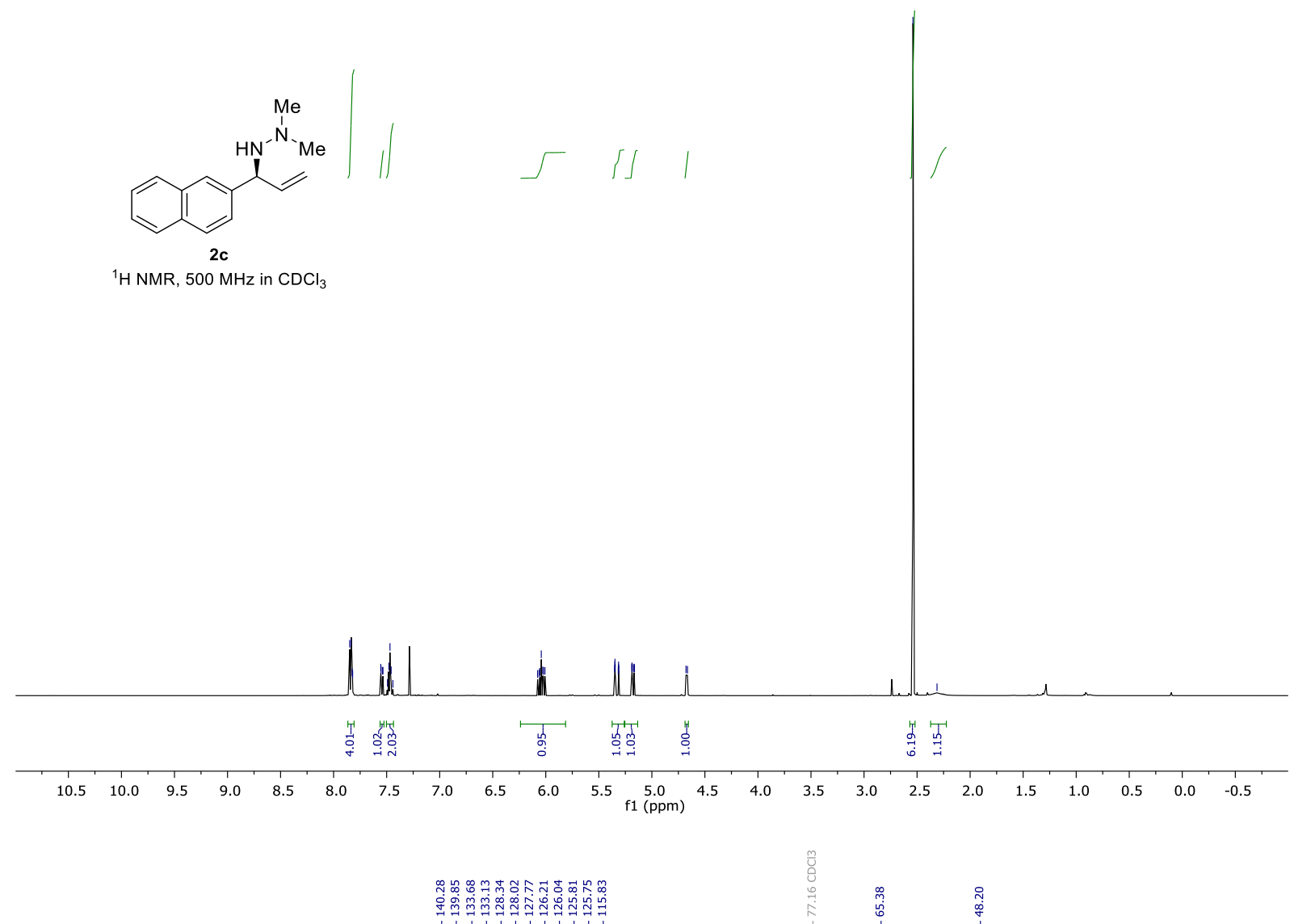

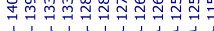
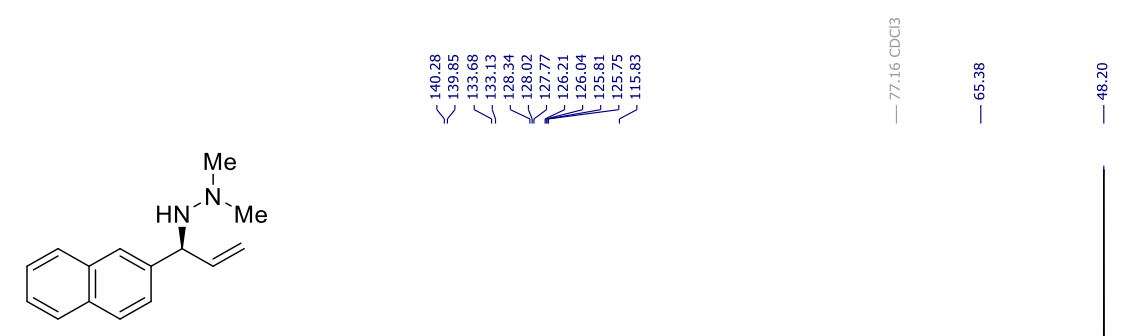

${ }^{13} \mathrm{C} \mathrm{NMR}, 125 \mathrm{MHz}$ in $\mathrm{CDCl}_{3}$

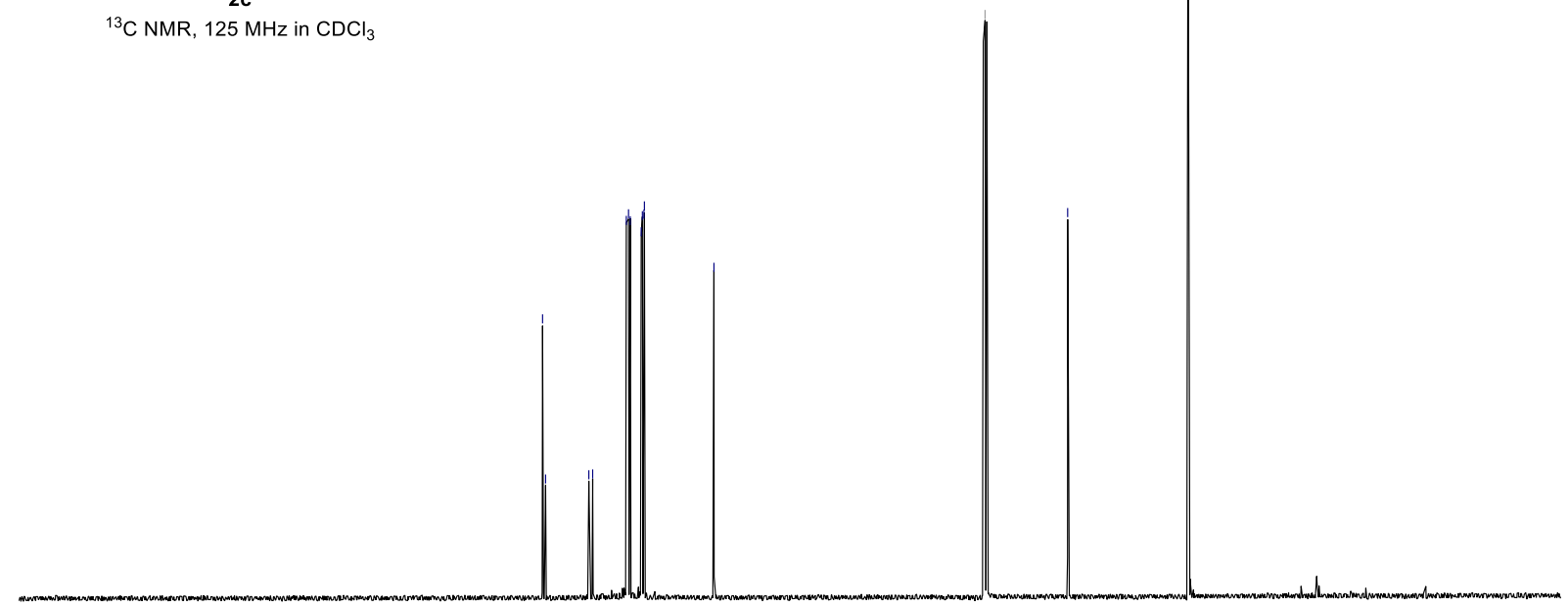

$\begin{array}{lllllllllll}210 & 200 & 190 & 180 & 170 & 160 & 150 & 140 & 130 & 120 & 110 \quad \begin{array}{l}100 \\ \mathrm{f} 1(\mathrm{ppm})\end{array}\end{array}$ 


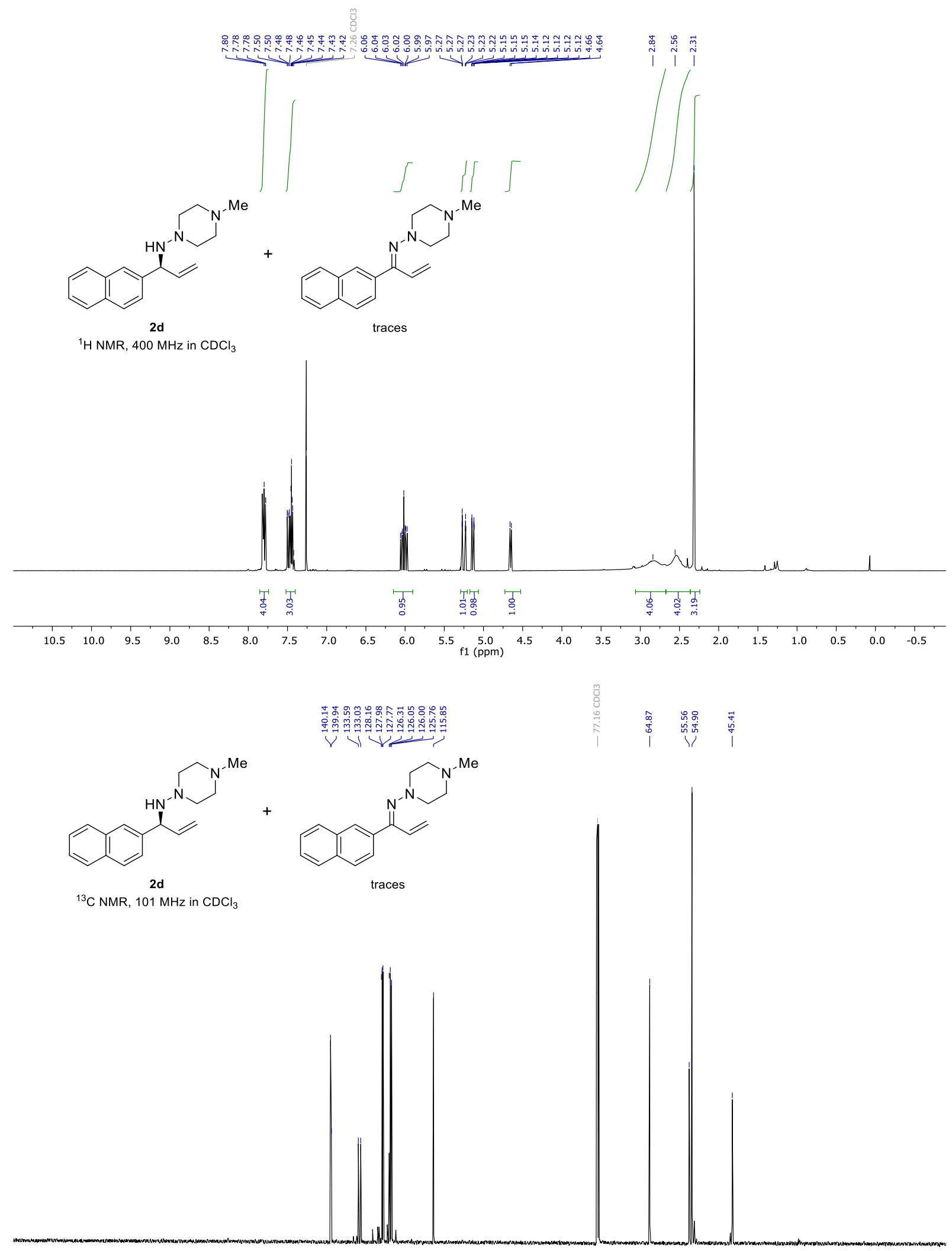

$\begin{array}{llllllllllllllllllllllll}210 & 200 & 190 & 180 & 170 & 160 & 150 & 140 & 130 & 120 & 110 & 100 & 90 & 80 & 70 & 60 & 50 & 40 & 30 & 20 & 10 & 0\end{array}$ 

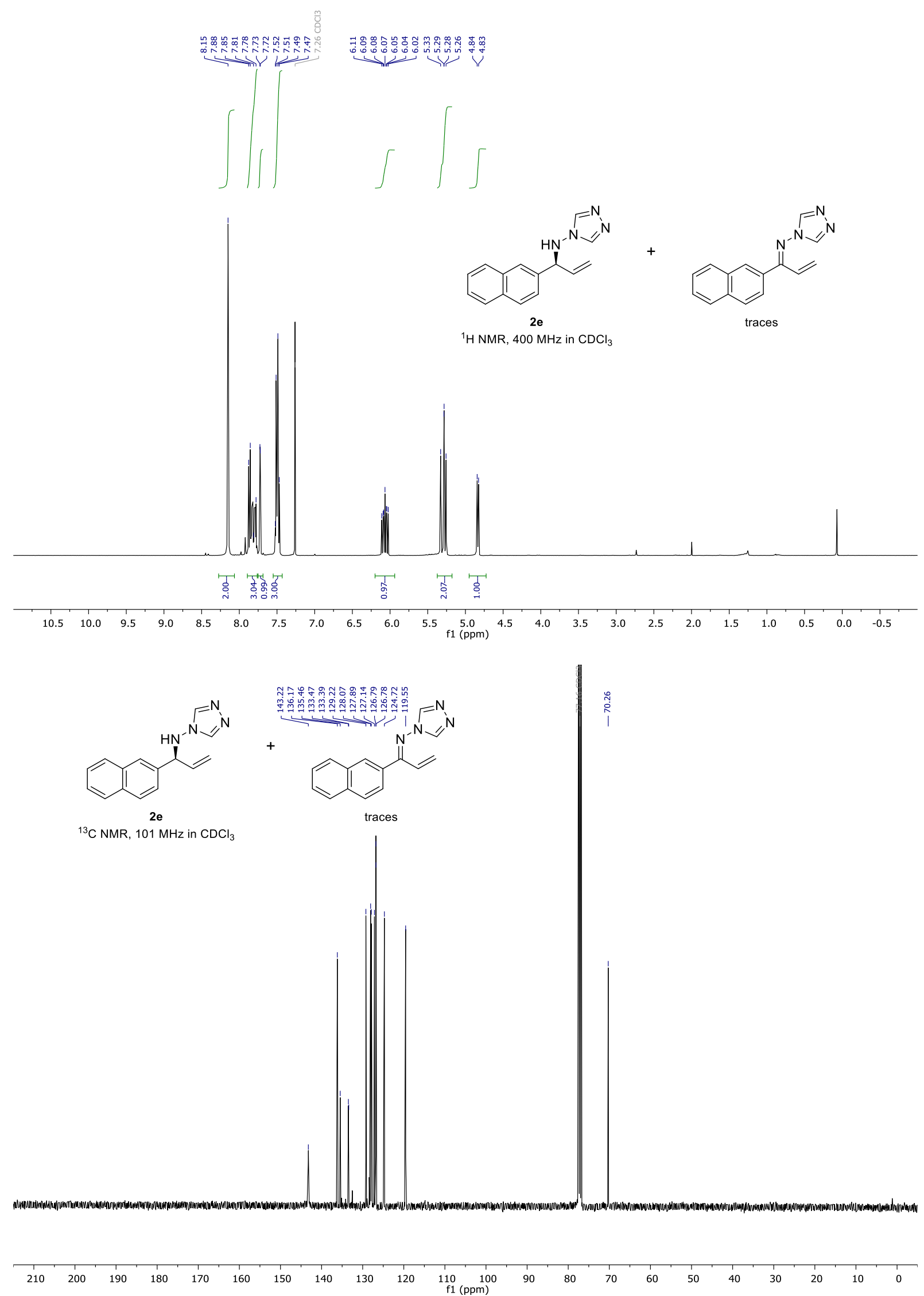

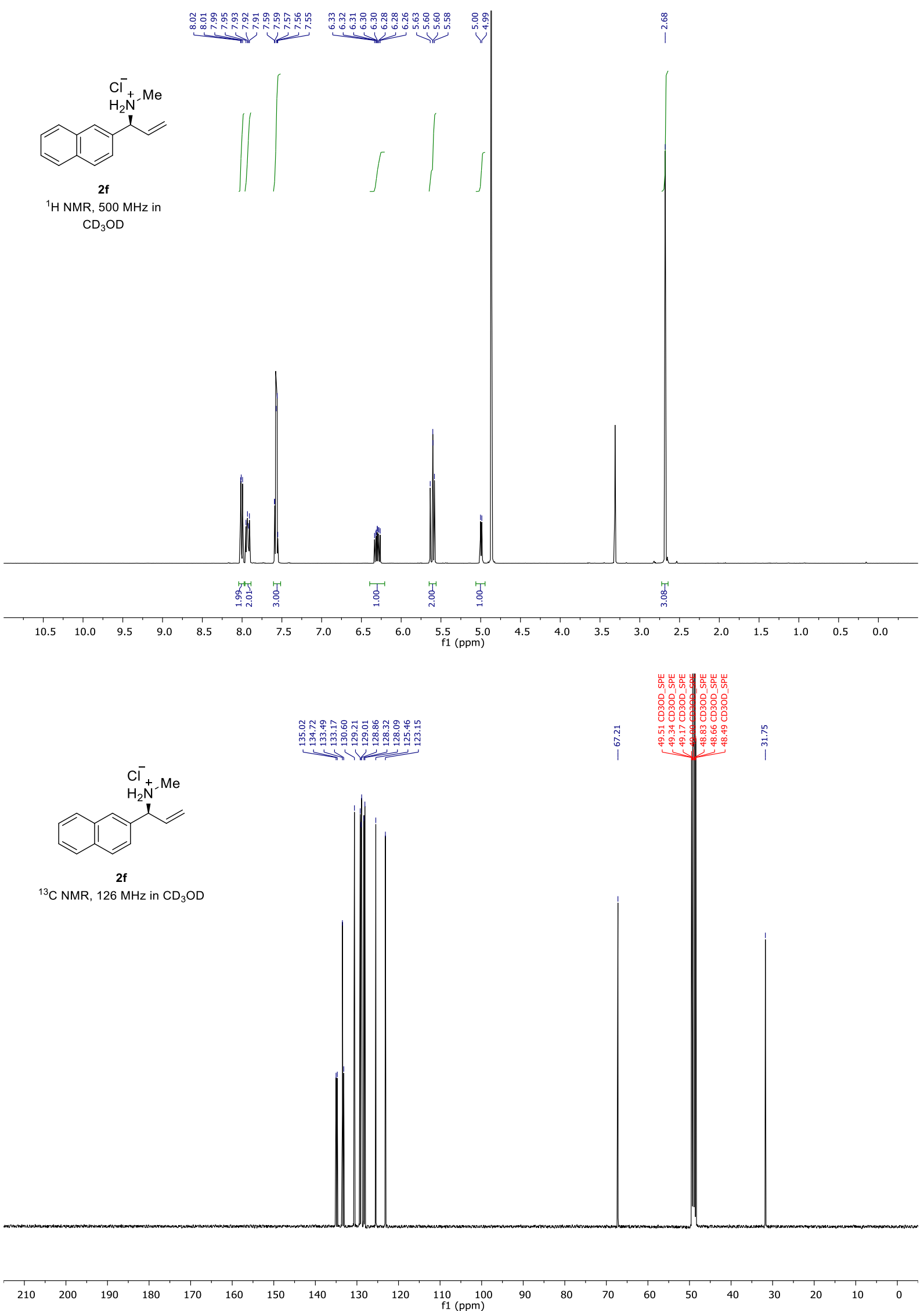

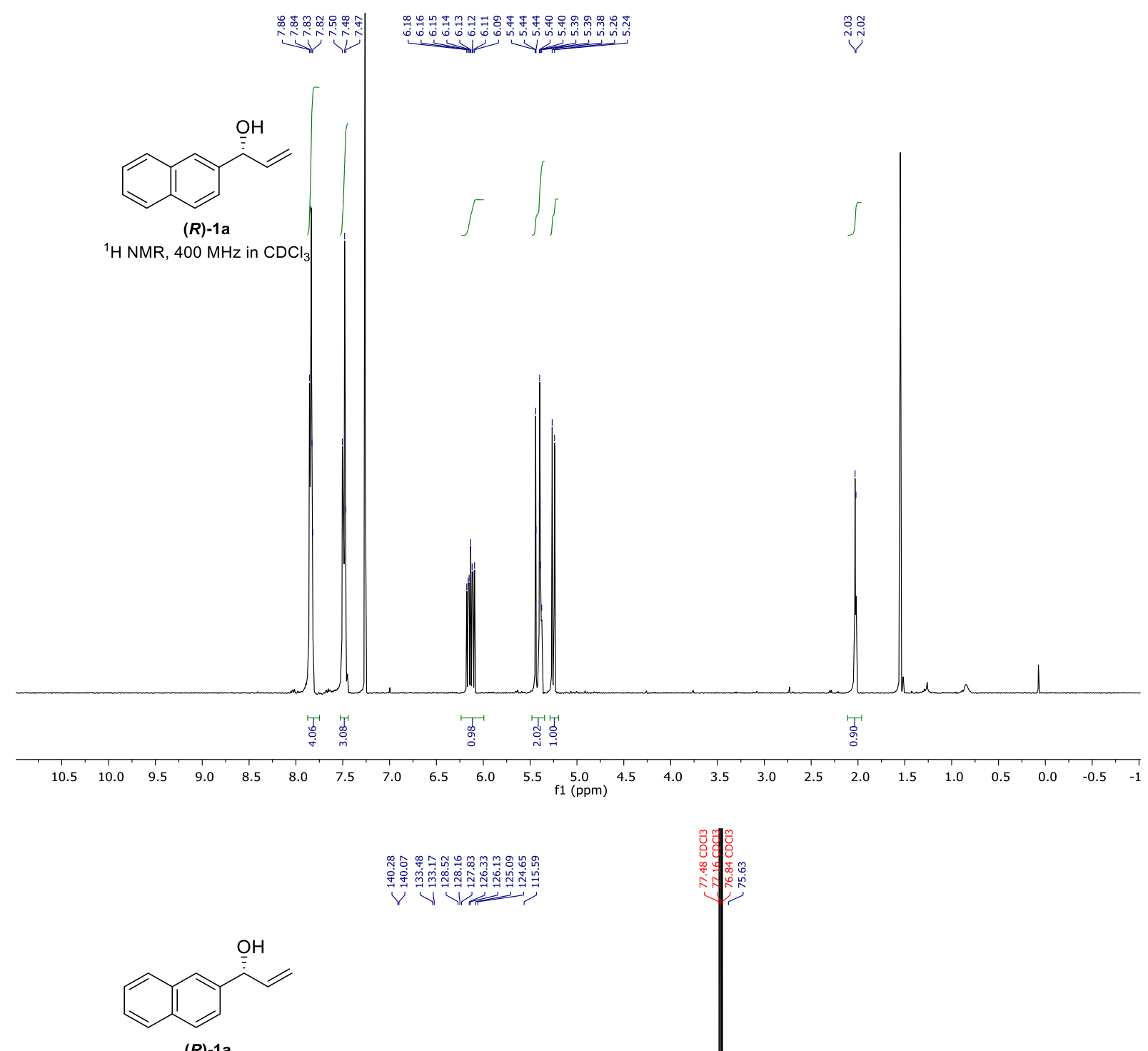

${ }^{13} \mathrm{C} \mathrm{NMR}, 100 \mathrm{MHz}$ in $\mathrm{CDCl}_{3}$
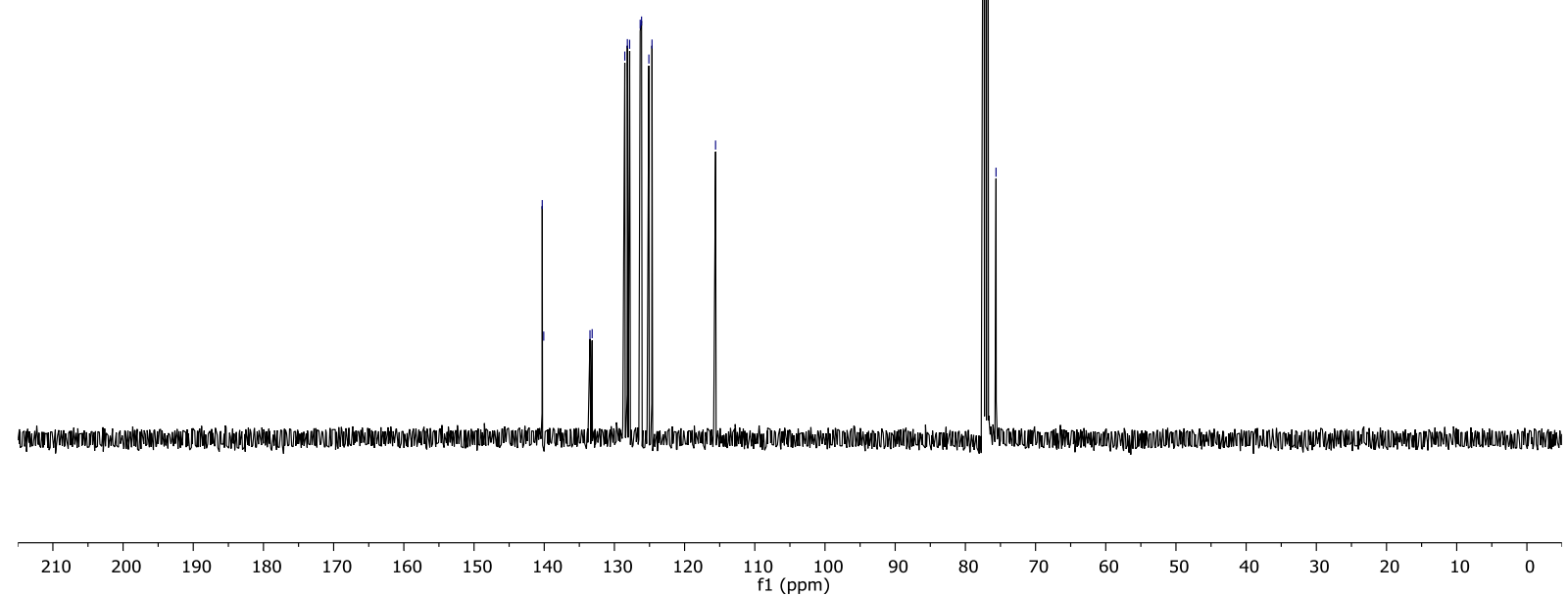

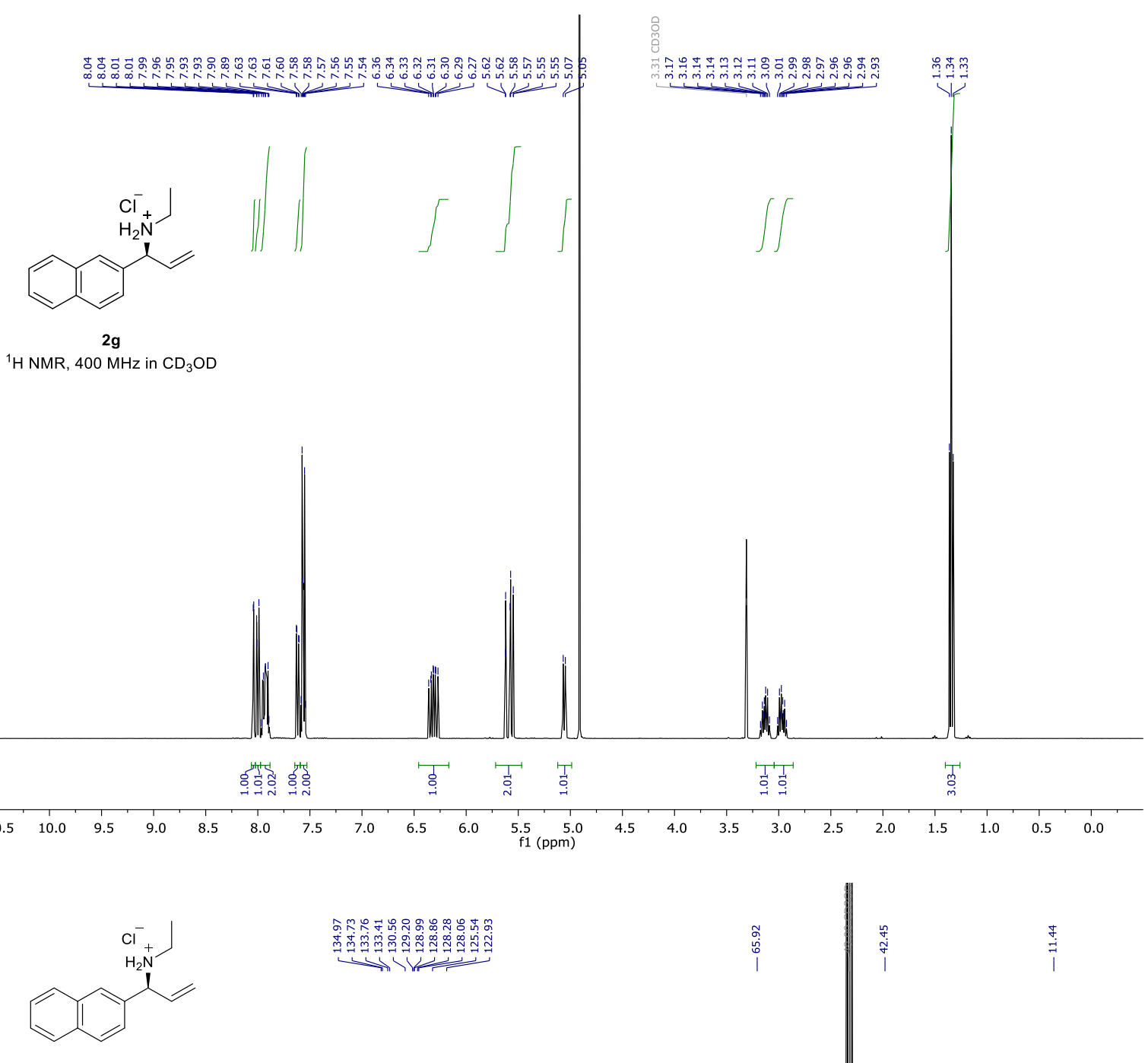

${ }^{13} \mathrm{C} \mathrm{NMR}, 101 \mathrm{MHz}$ in $\mathrm{CD}_{3} \mathrm{OD}$

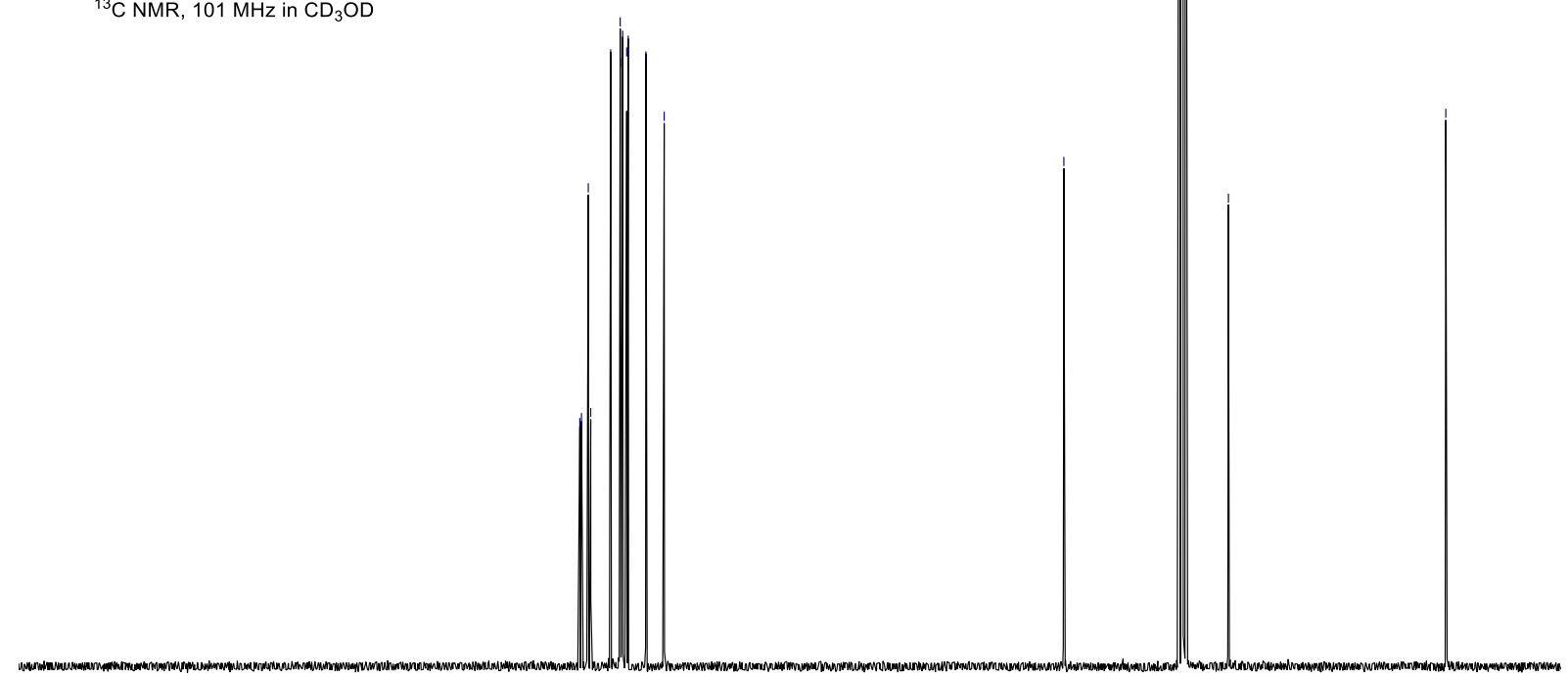

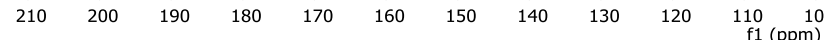



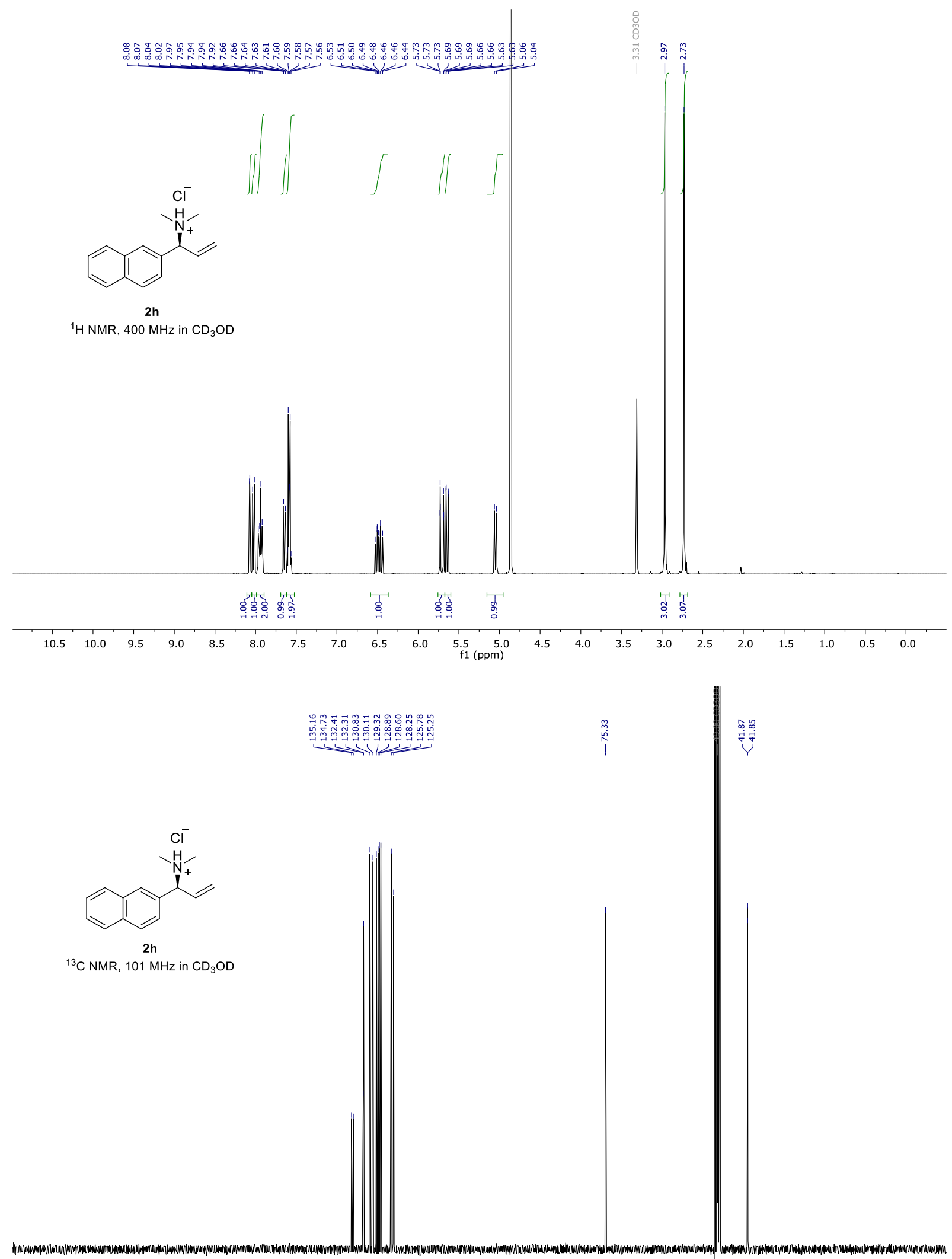

$\begin{array}{lllllllllllllllllllllllll}210 & 200 & 190 & 180 & 170 & 160 & 150 & 140 & 130 & 120 & 110 & 100 & 90 & 80 & 70 & 60 & 50 & 40 & 30 & 20 & 10 & 0\end{array}$ 

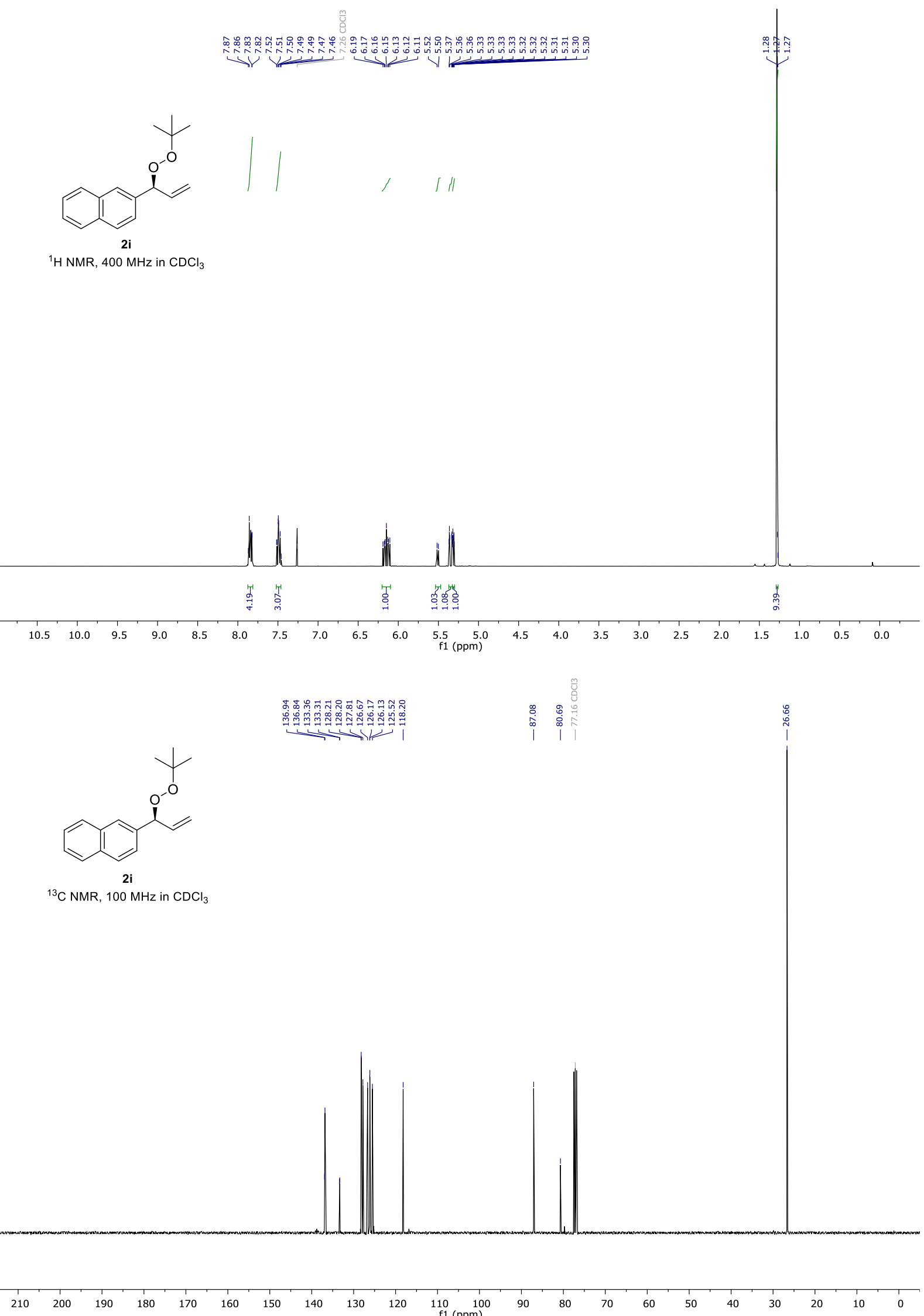

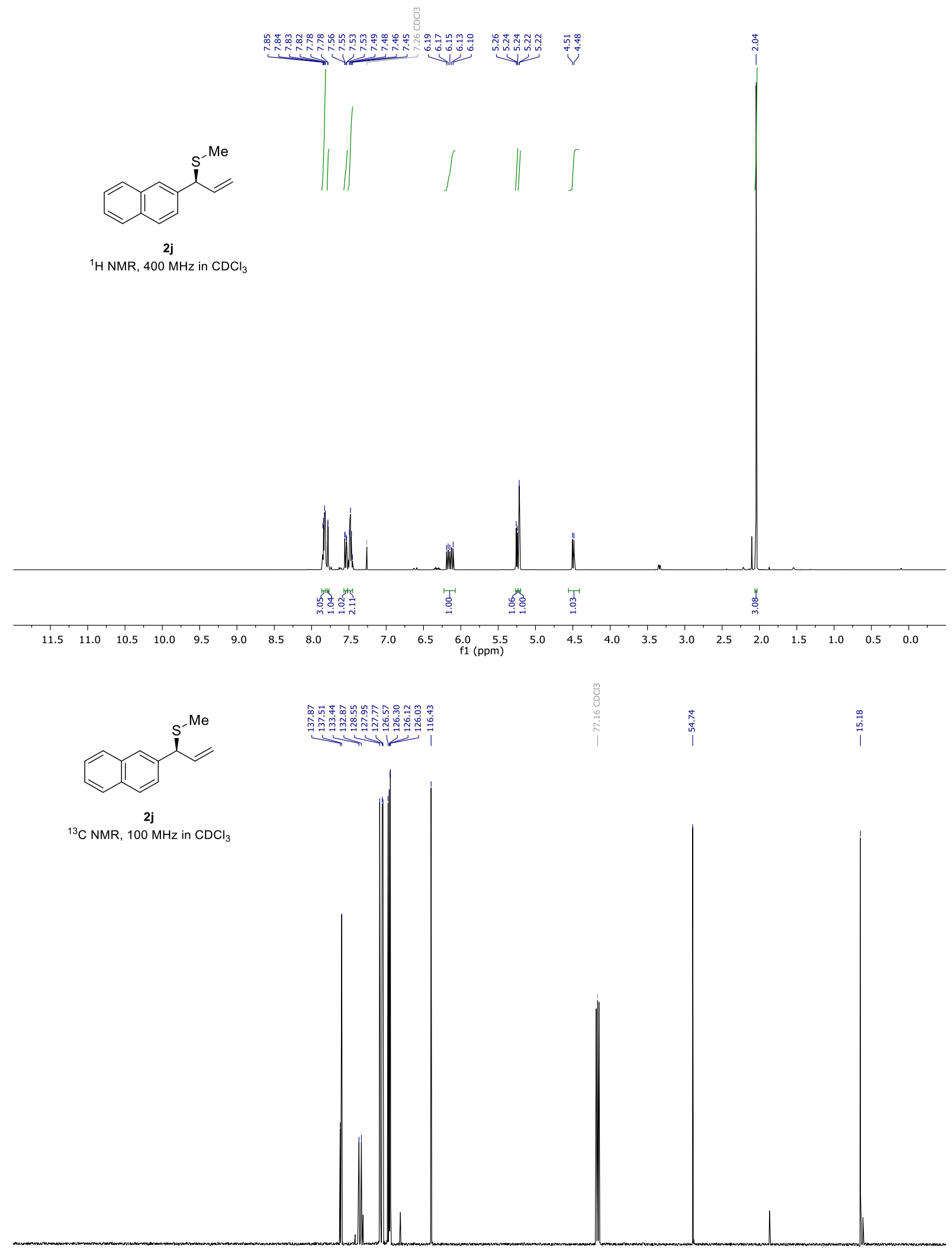

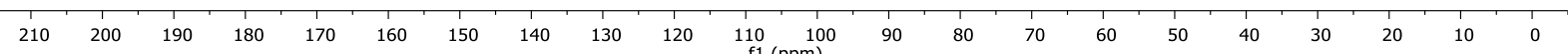



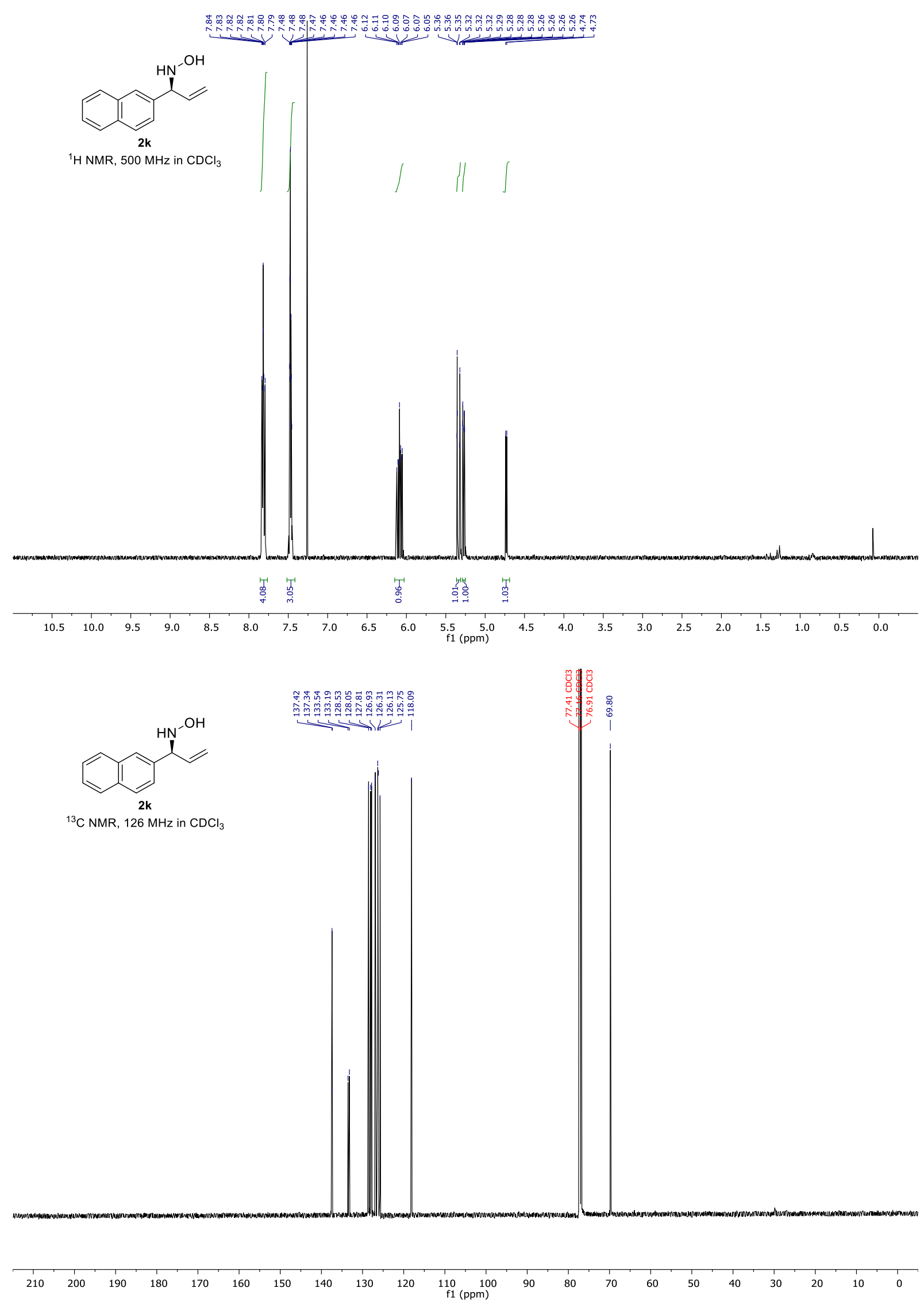


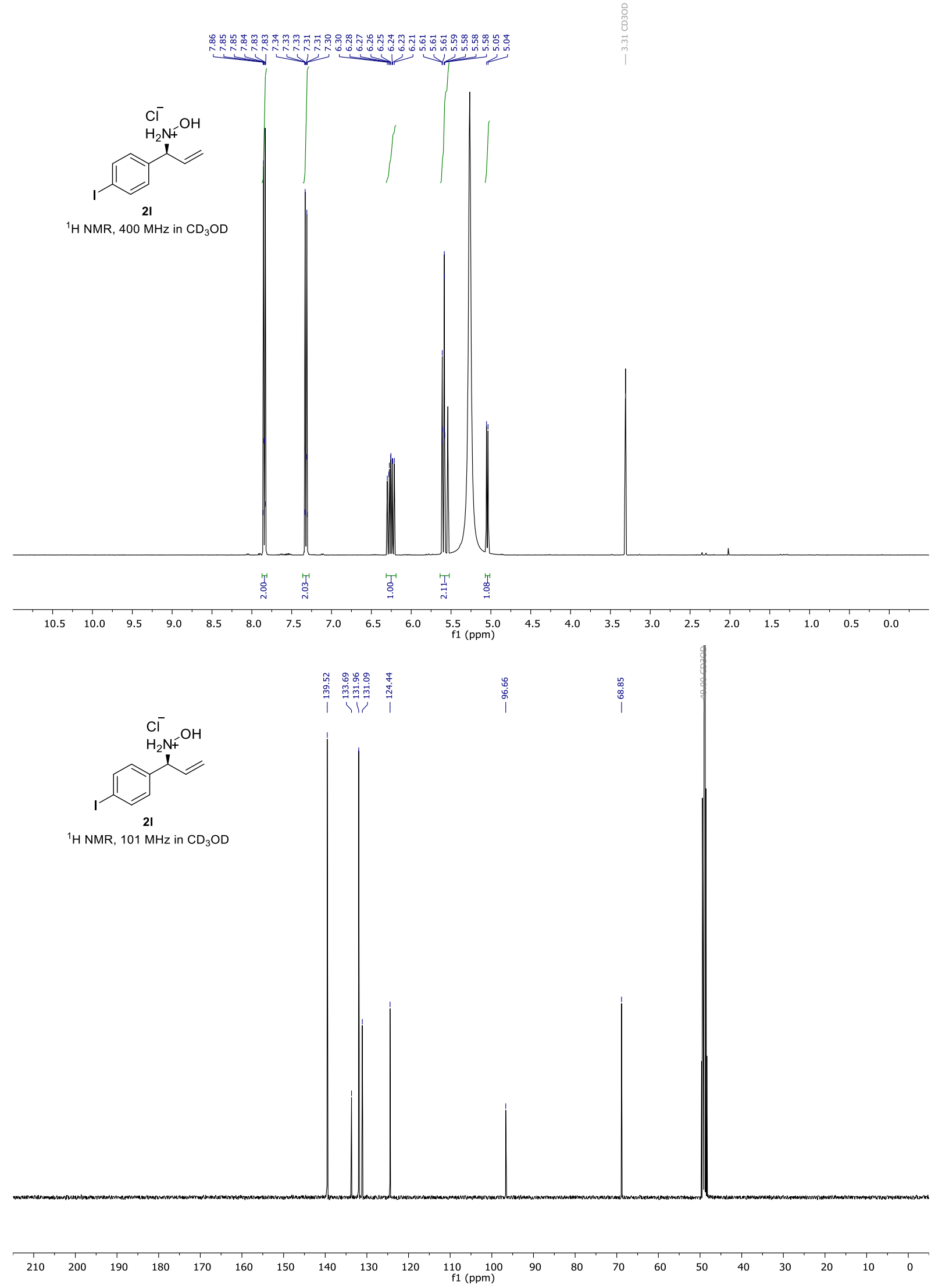



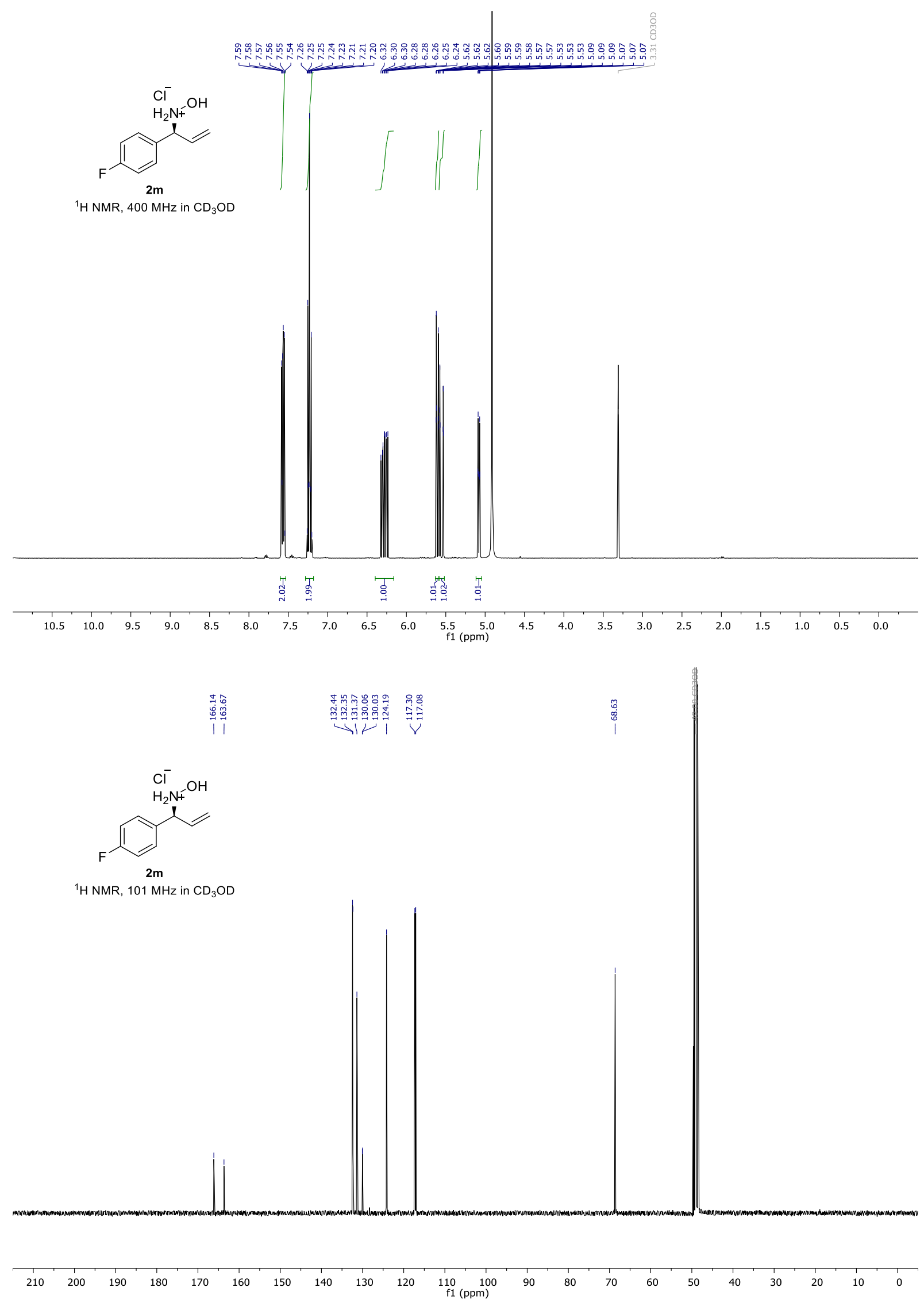


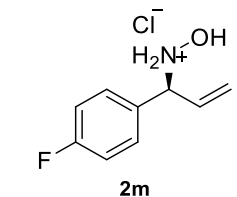

${ }^{19} \mathrm{~F} \mathrm{NMR}, 376 \mathrm{MHz}$ in $\mathrm{CD}_{3} \mathrm{OD}$

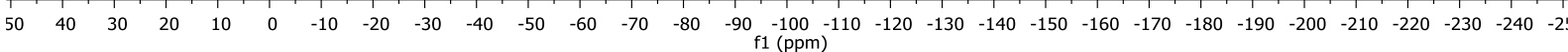



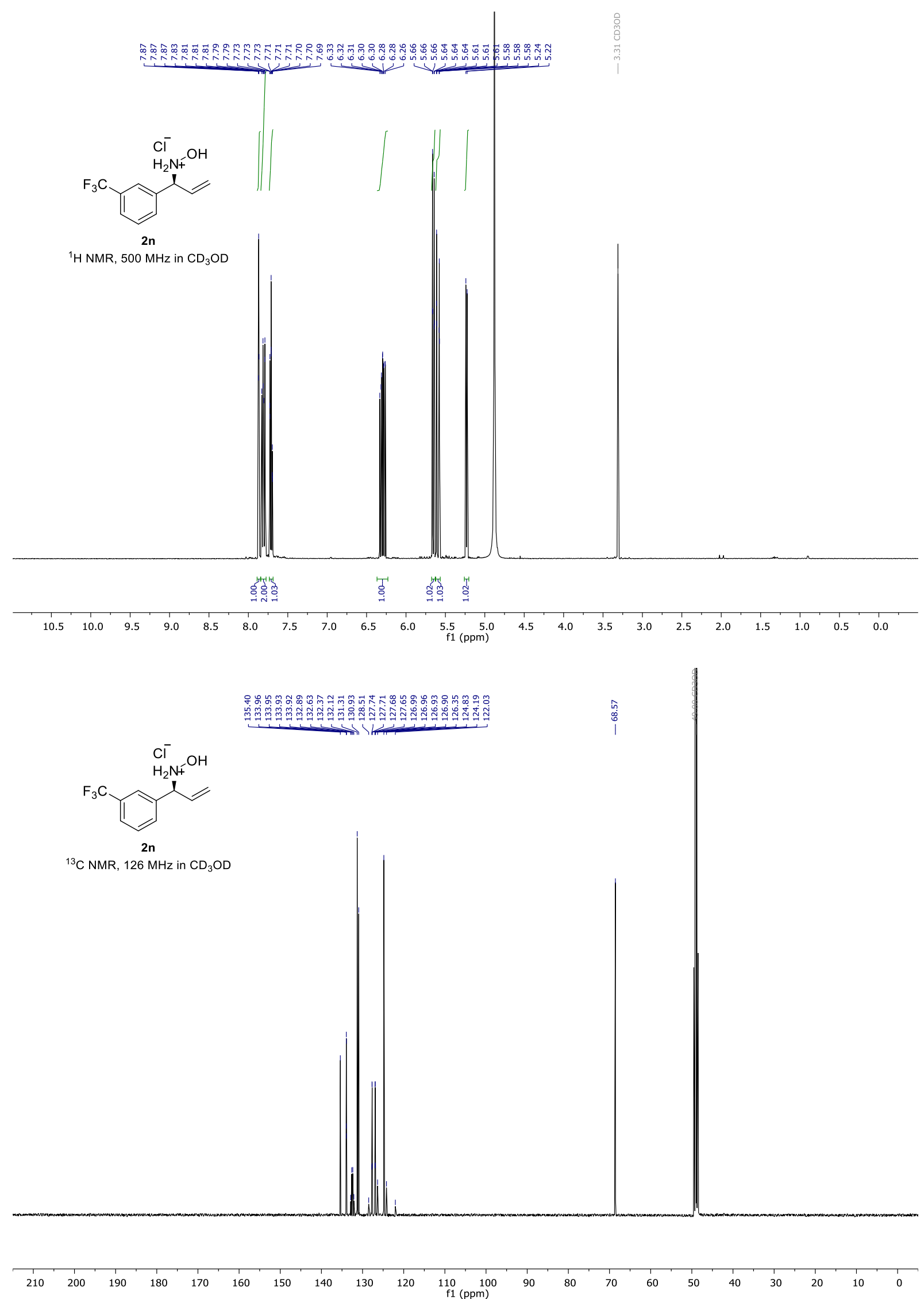


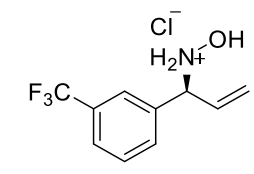

$2 n$

${ }^{19} \mathrm{~F}$ NMR, $470 \mathrm{MHz}$ in $\mathrm{CD}_{3} \mathrm{OD}$

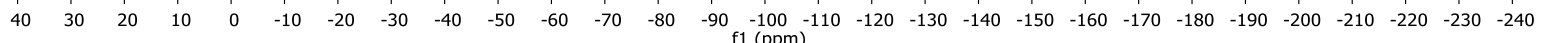



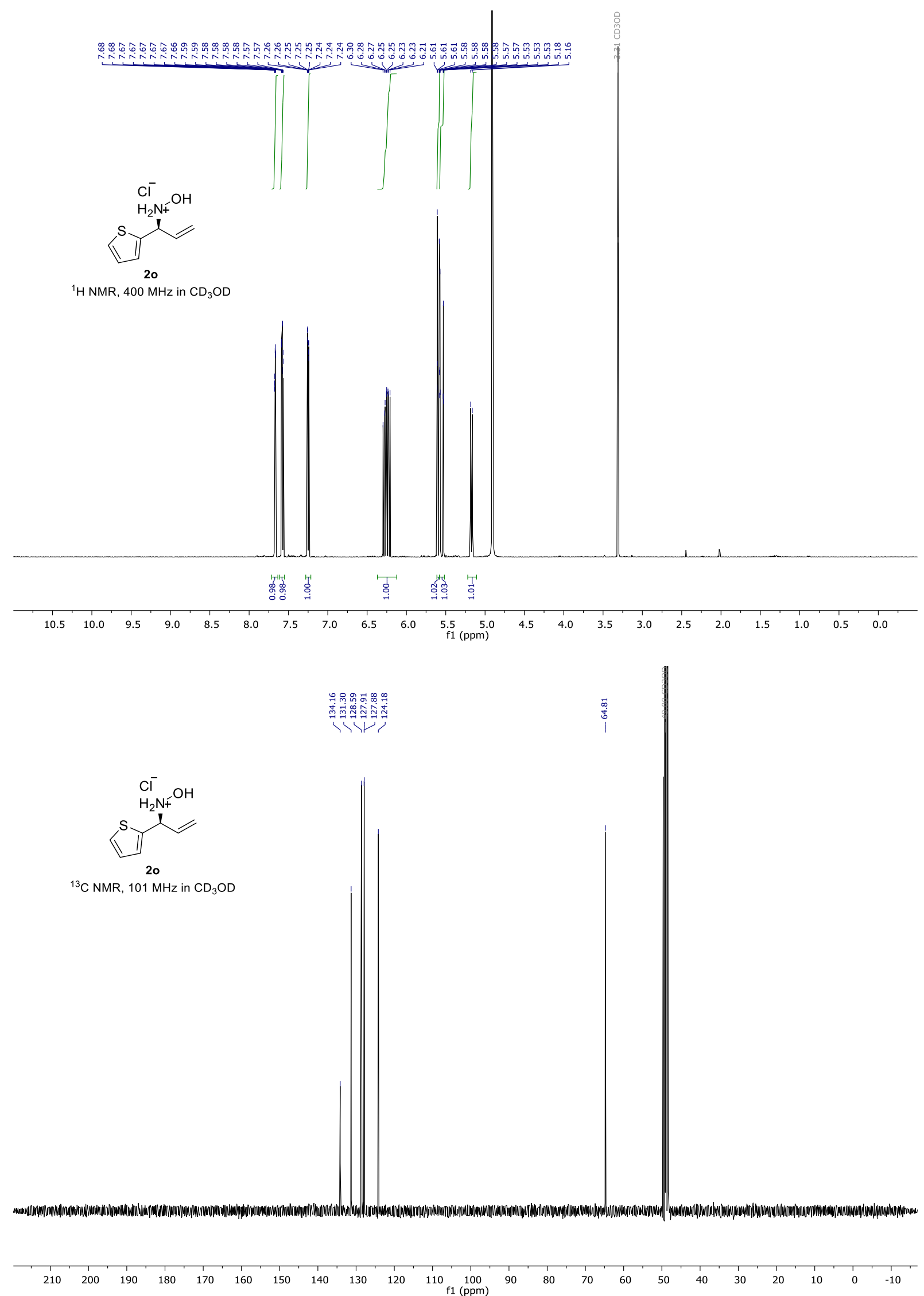


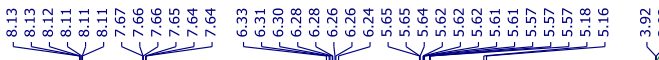

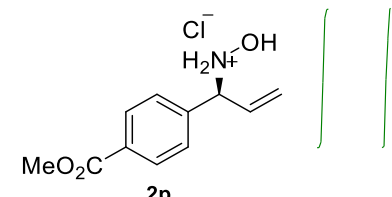

${ }^{1} \mathrm{H}$ NMR, $400 \mathrm{MHz}$ in $\mathrm{CD}_{3} \mathrm{OD}$
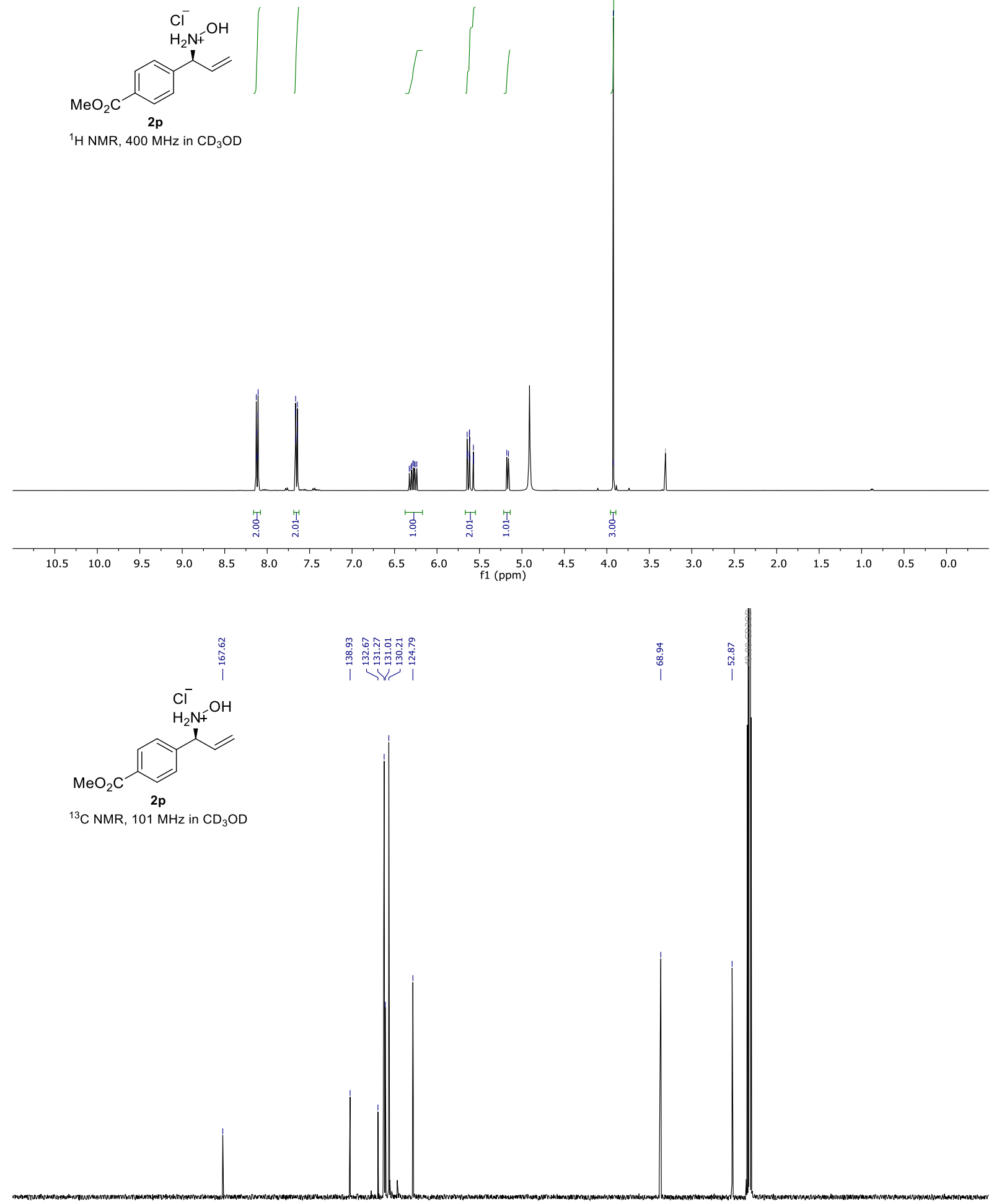

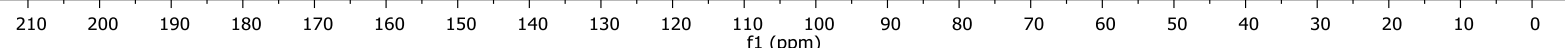



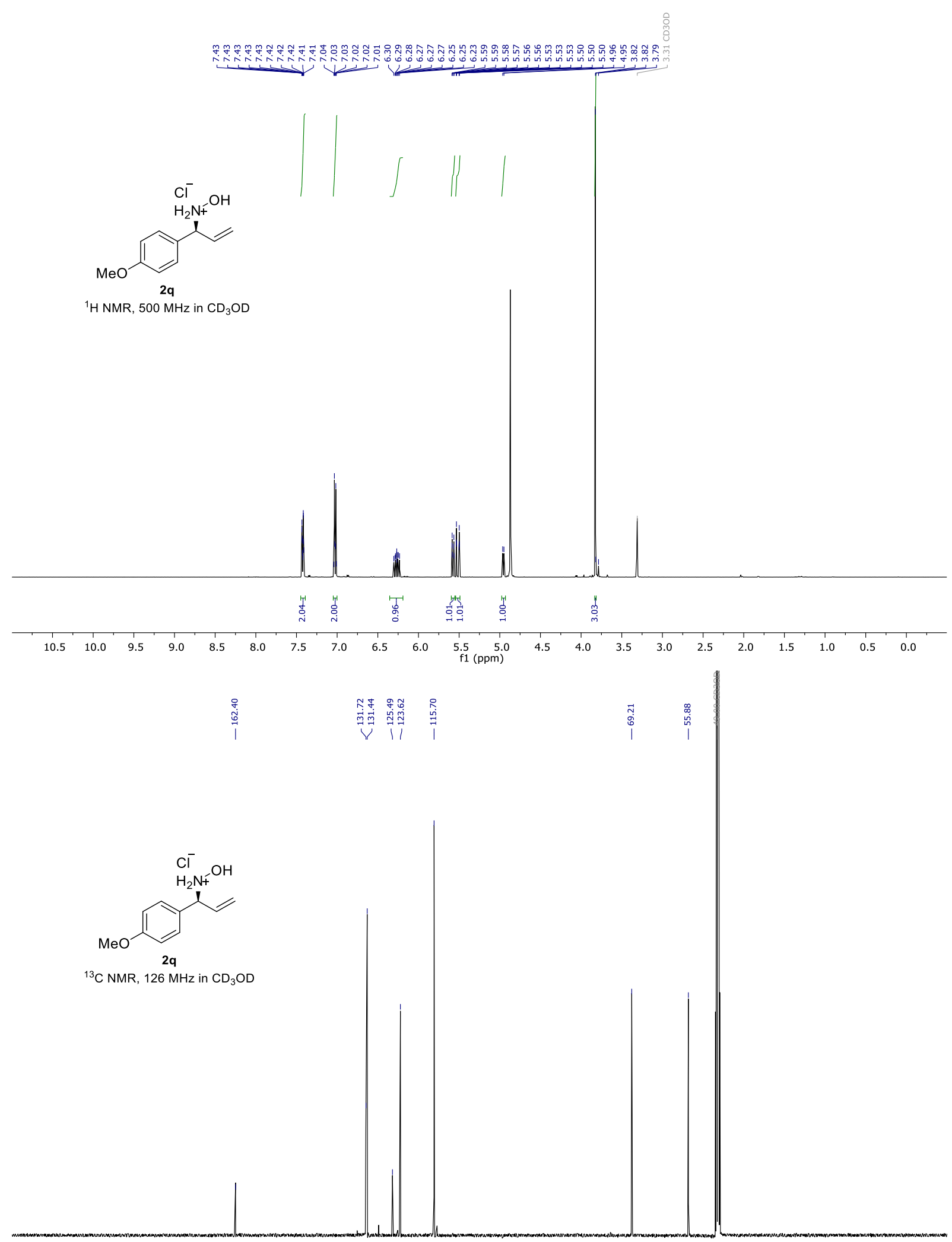

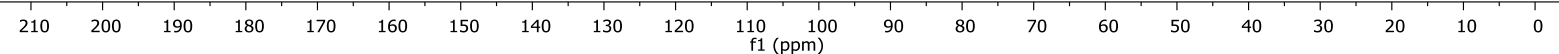



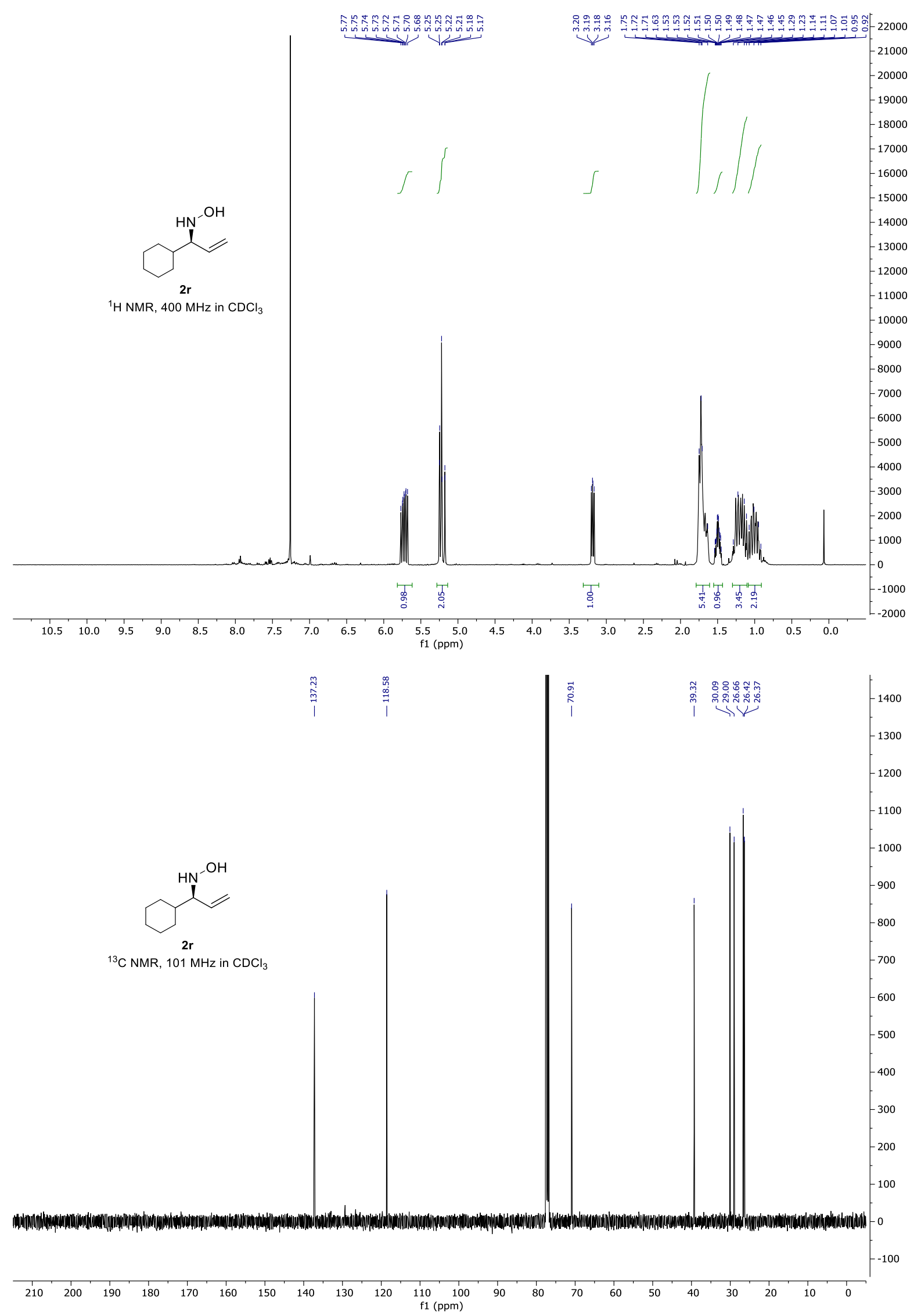


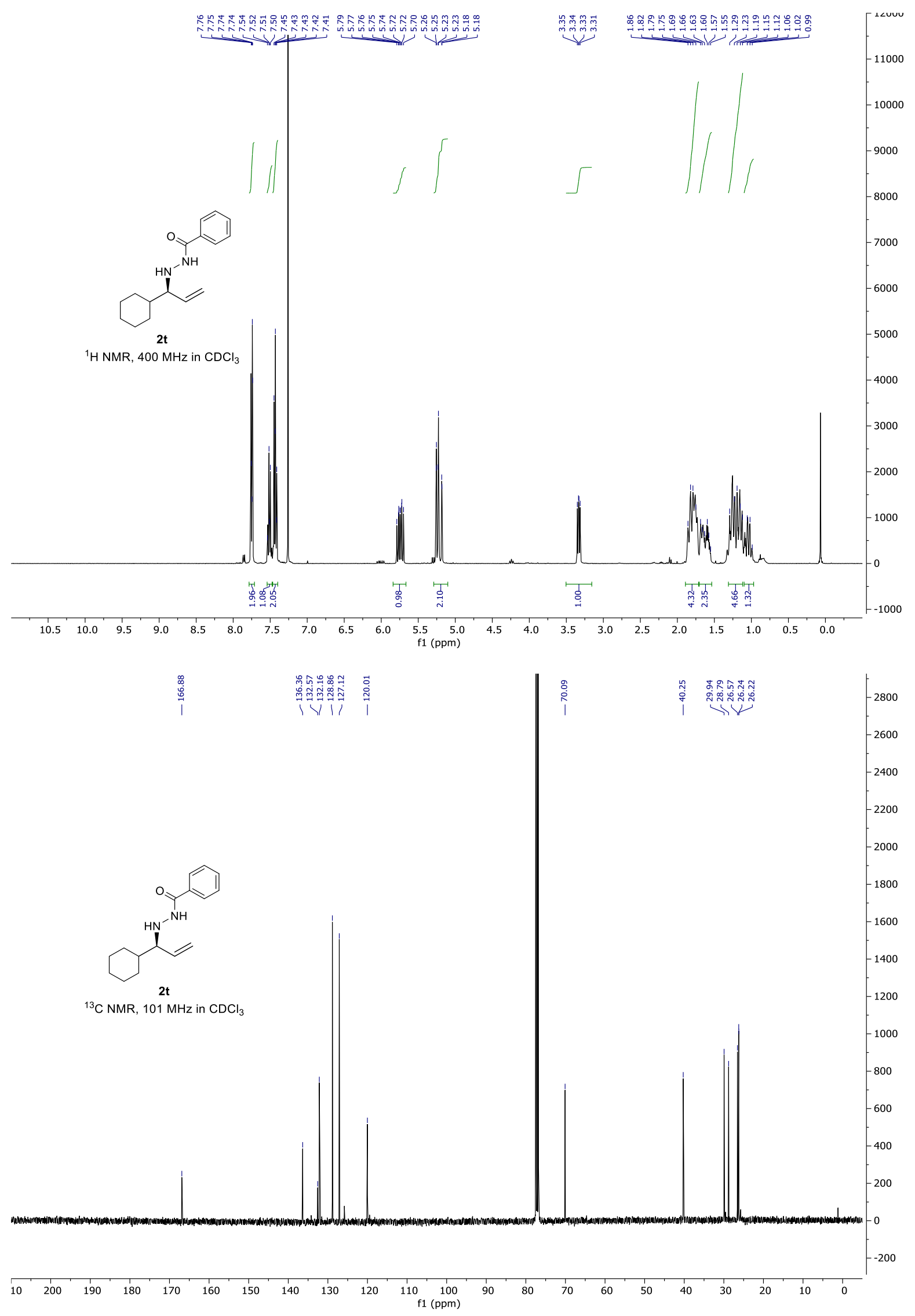



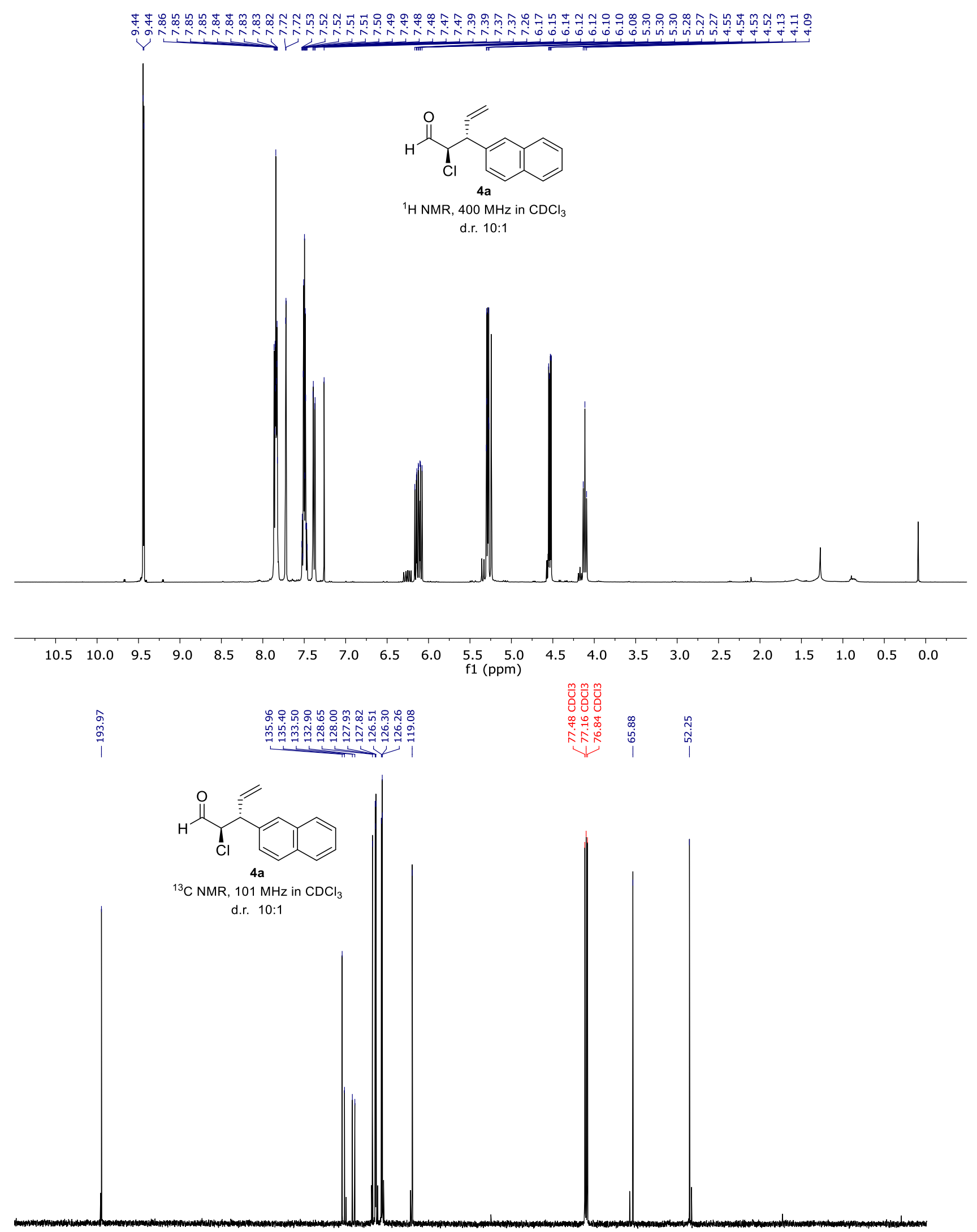

$\begin{array}{lllllllllllllllllllllllll}210 & 200 & 190 & 180 & 170 & 160 & 150 & 140 & 130 & 120 & 110 & 100 & 90 & 80 & 70 & 60 & 50 & 40 & 30 & 20 & 10 & 0\end{array}$

1 (ppm) 


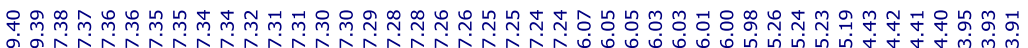

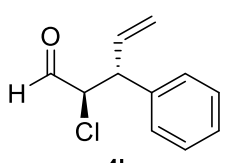

$4 b$

${ }^{1} \mathrm{H}$ NMR, $400 \mathrm{MHz}$ in $\mathrm{CDCl}_{3}$ d.r. $10: 1$
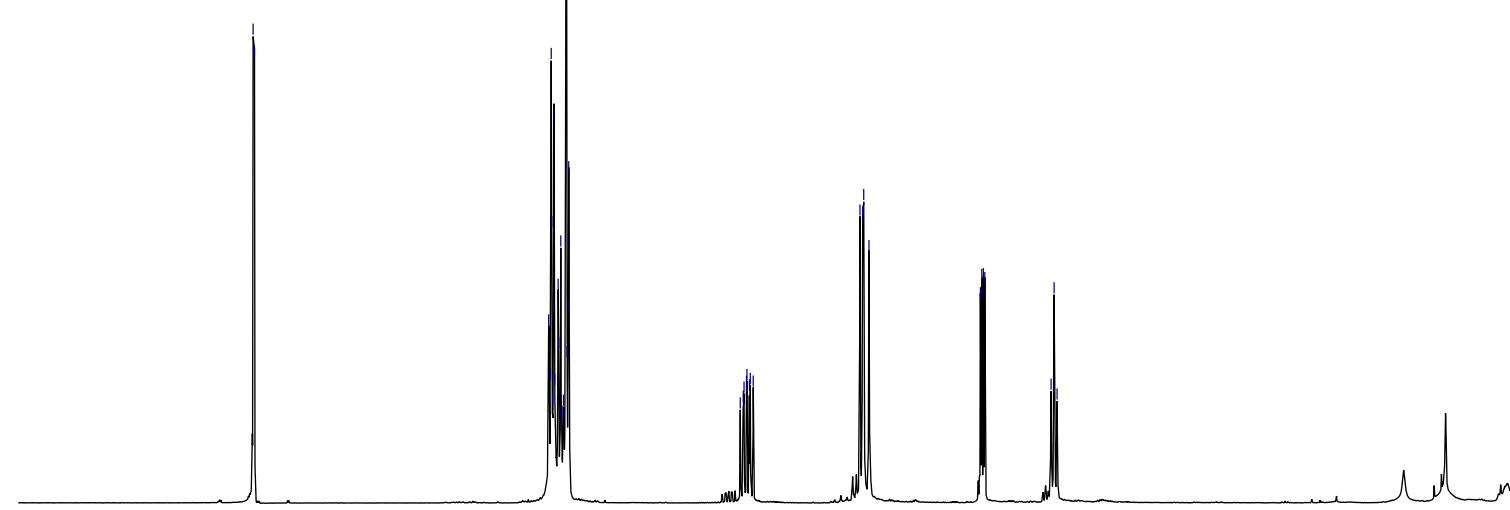

$\begin{array}{lllllllllll}10.5 & 10.0 & 9.5 & 9.0 & 8.5 & 8.0 & 7.5 & 7.0 & 6.5 & 6.0 & \begin{array}{l}5.5 \\ \mathrm{f} 1(\mathrm{ppm})\end{array}\end{array}$
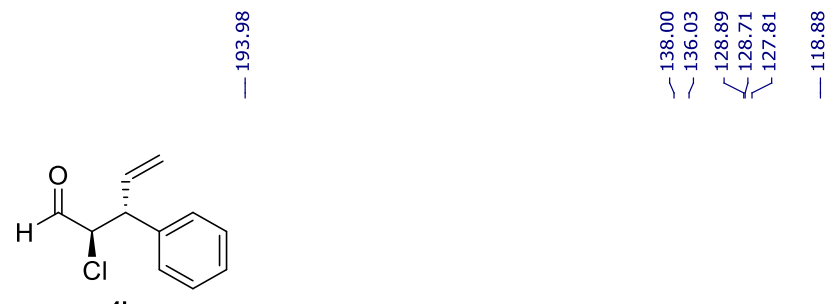

$4 b$

${ }^{1} \mathrm{H}$ NMR, $400 \mathrm{MHz}$ in $\mathrm{CDCl}_{3}$

d.r. $10: 1$
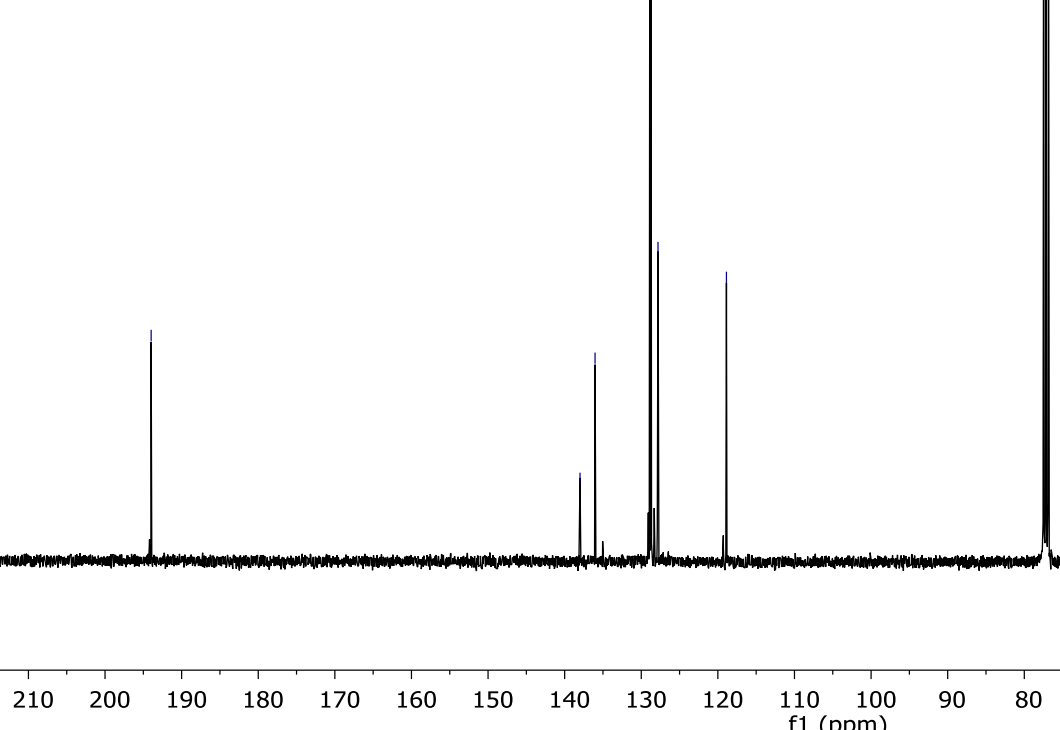

$120 \quad 110 \quad 100$
$\mathrm{f} 1(\mathrm{ppm})$ 

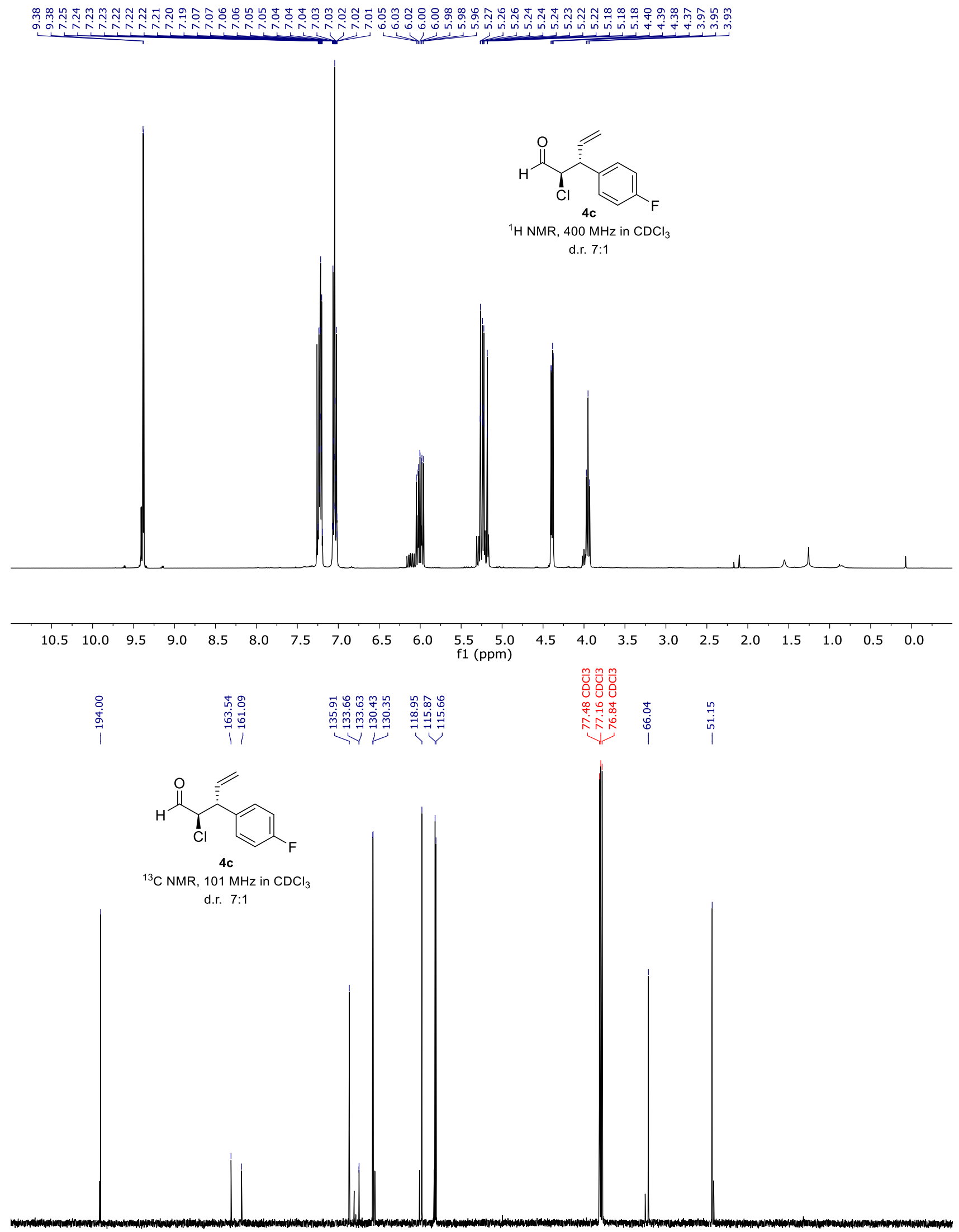

$\begin{array}{llllllllllllllllllllll}210 & 200 & 190 & 180 & 170 & 160 & 150 & 140 & 130 & 120 & 110 & 100 & 90 & 80 & 70 & 60 & 50 & 40 & 30 & 20 & 10 & 0\end{array}$ 


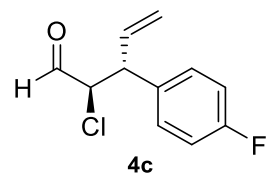

${ }^{19} \mathrm{~F} \mathrm{NMR}, 376 \mathrm{MHz}$ in $\mathrm{CDCl}_{3}$

d.r. $7: 1$ 


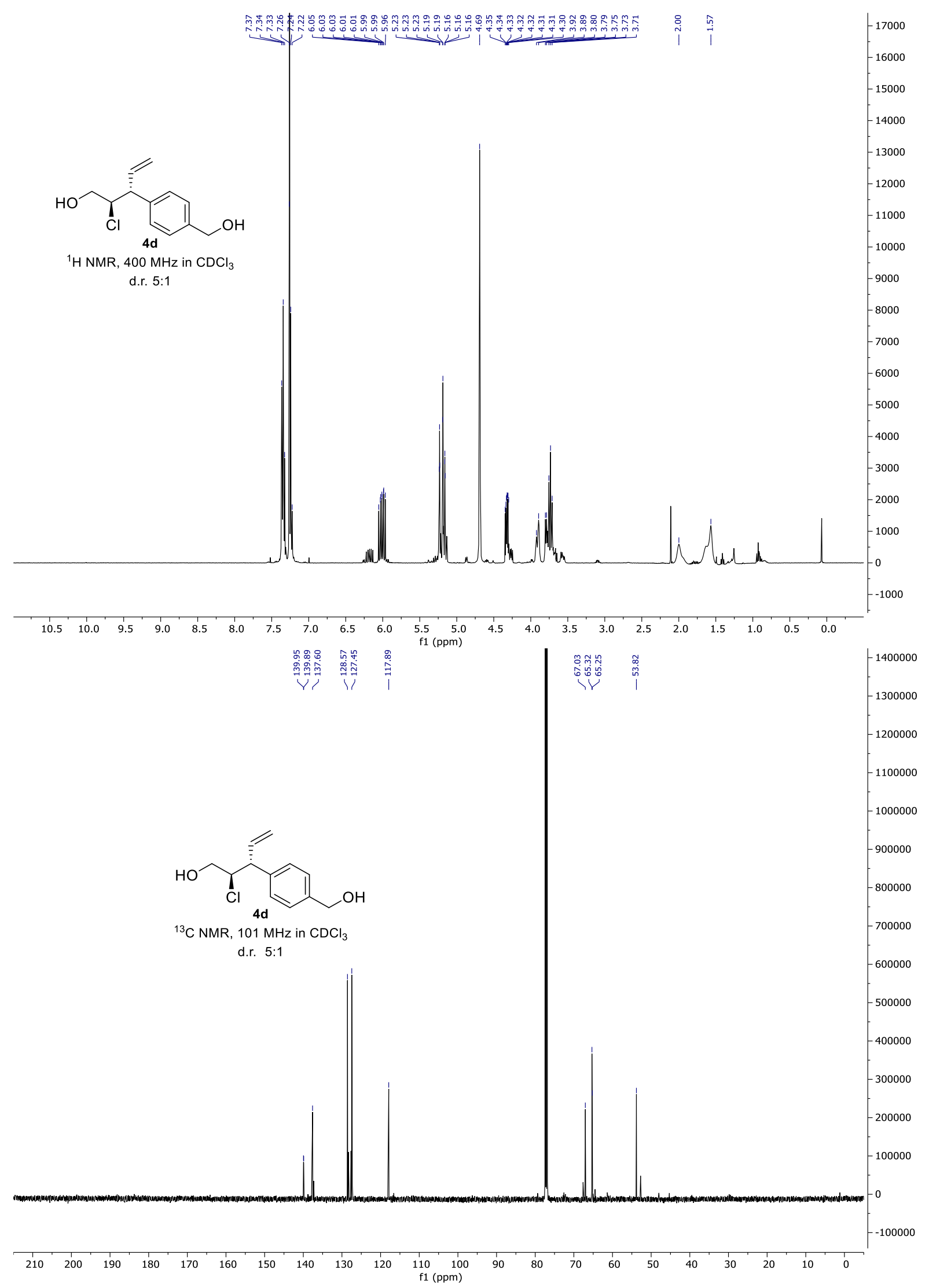




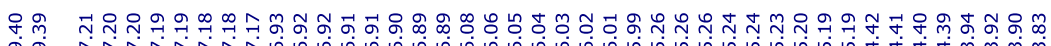

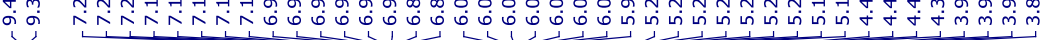

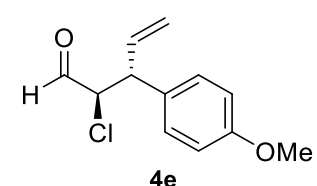

${ }^{1} \mathrm{H} \mathrm{NMR}, 400 \mathrm{MHz}$ in $\mathrm{CDCl}_{3}$ d.r. 10:1

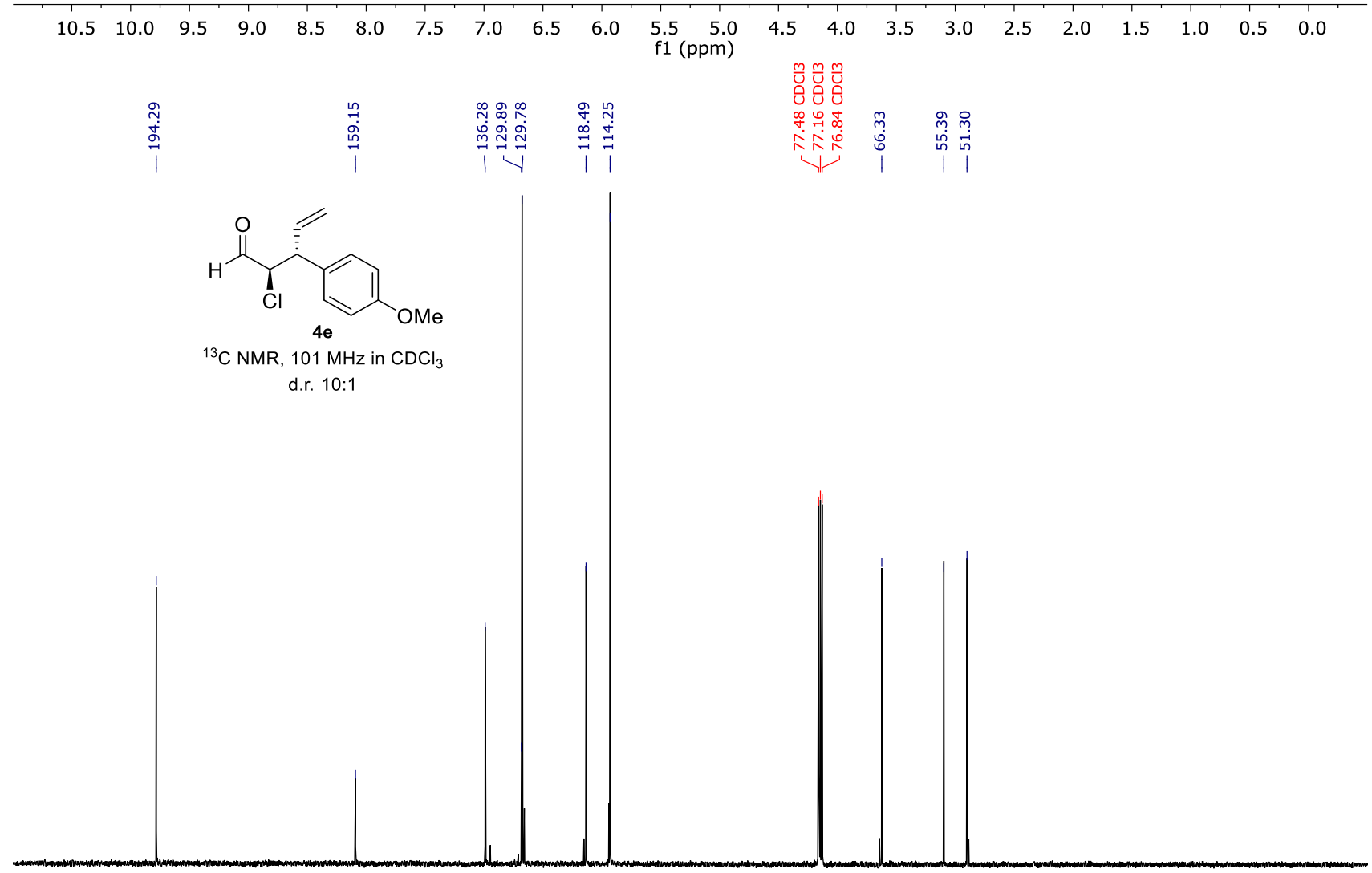

$\begin{array}{lllllllllllllllllllllllllllll}210 & 200 & 190 & 180 & 170 & 160 & 150 & 140 & 130 & 120 & 110 & 100 & 90 & 80 & 70 & 60 & 50 & 40 & 30 & 20 & 10 & 0 & -10\end{array}$ 

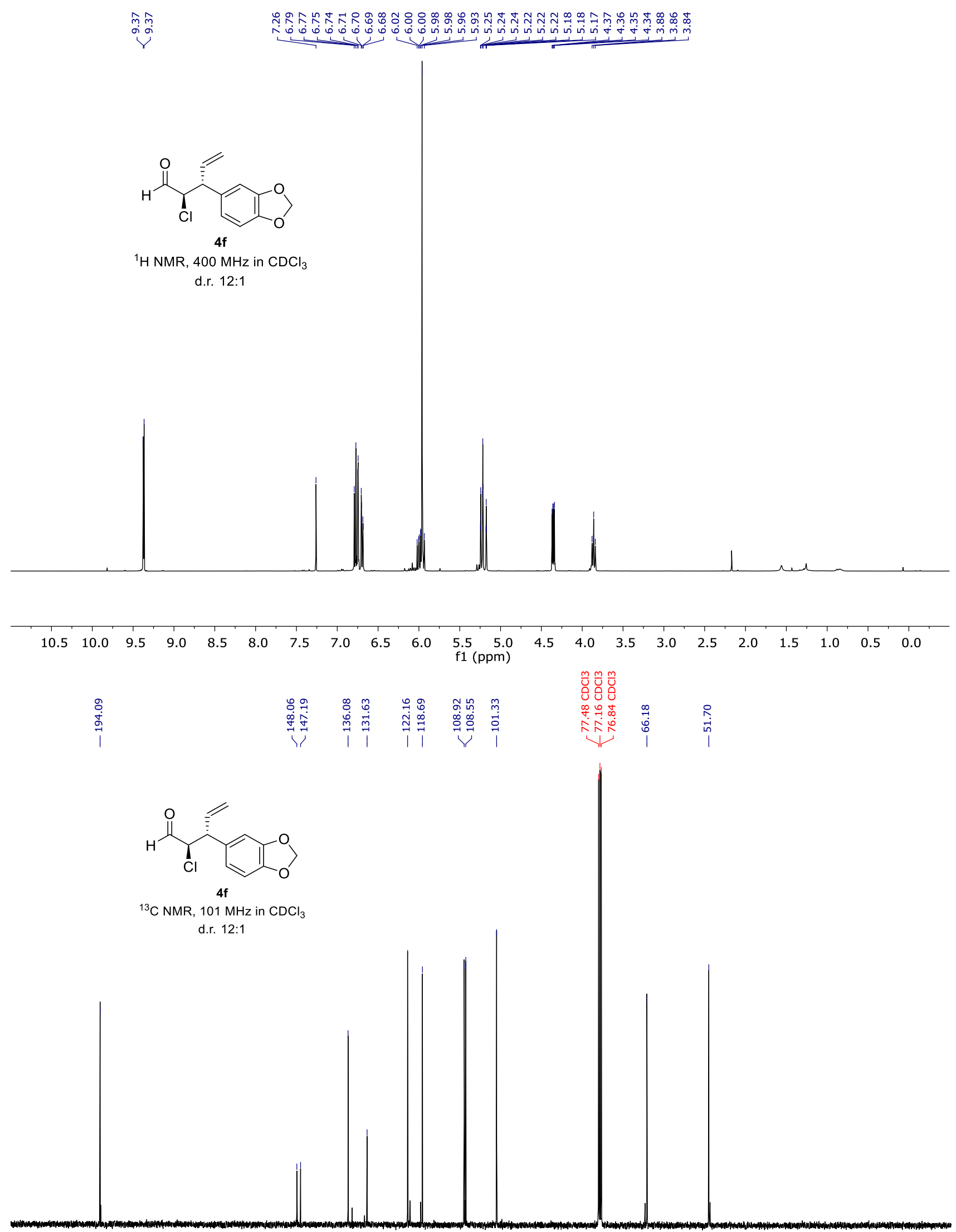

$\begin{array}{llllllllllllllllllllllll}210 & 200 & 190 & 180 & 170 & 160 & 150 & 140 & 130 & 120 & 110 & 100 & 90 & 80 & 70 & 60 & 50 & 40 & 30 & 20 & 10 & 0\end{array}$ f1 (ppm) 


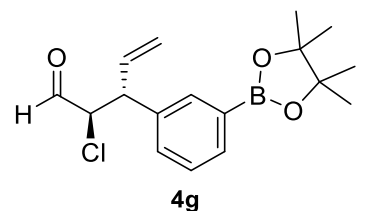

${ }^{1} \mathrm{H} \mathrm{NMR}, 400 \mathrm{MHz}$ in $\mathrm{CDCl}_{3}$ d.r. $15: 1$
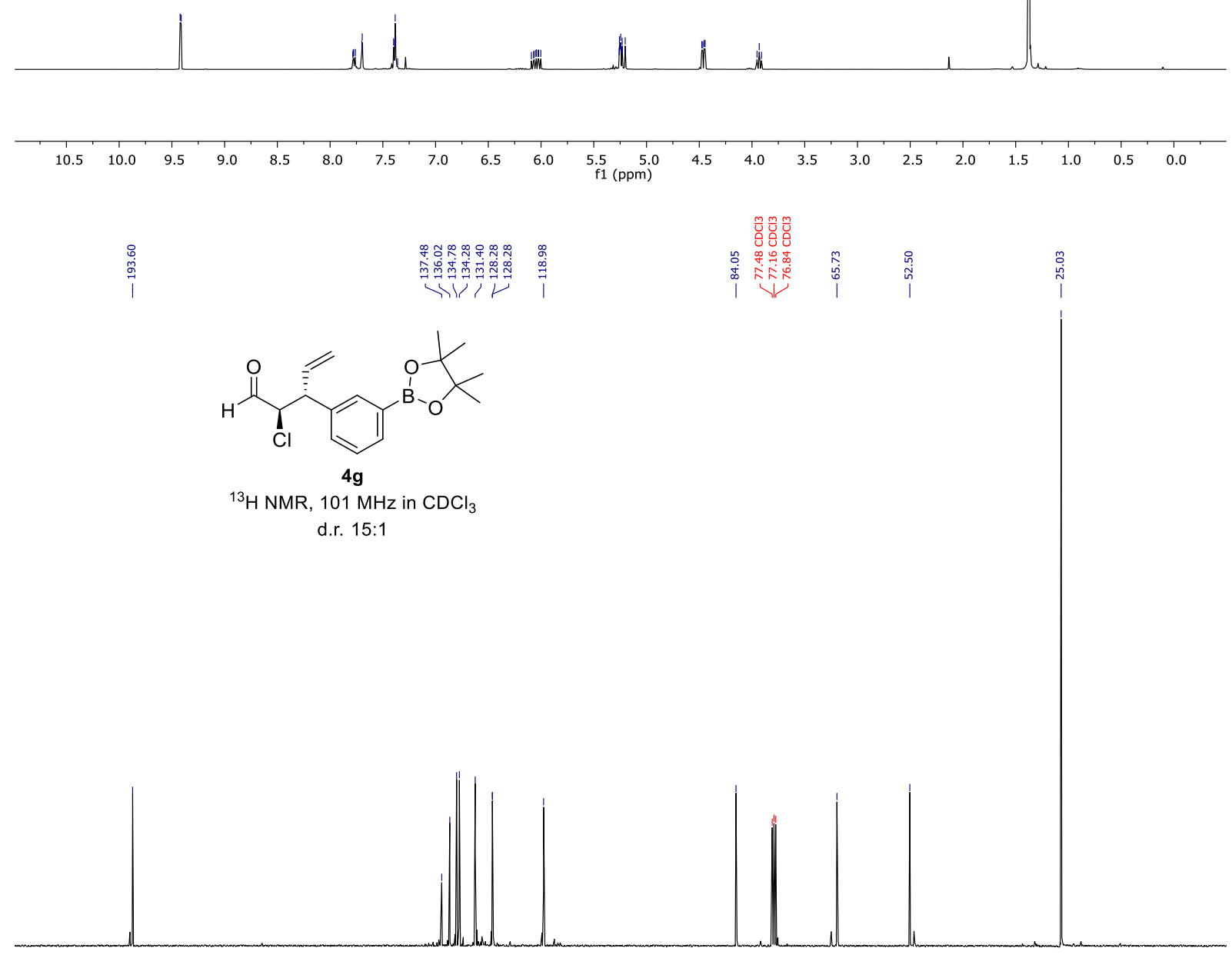

$\begin{array}{lllllllllll}210 & 200 & 190 & 180 & 170 & 160 & 150 & 140 & 130 & 120 & 110 \\ \mathrm{f} 1(\mathrm{ppm}) & 100\end{array}$ 


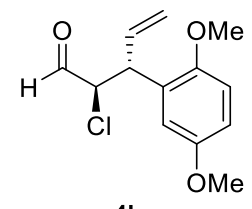

${ }^{1} \mathrm{H} \mathrm{NMR}, 400 \mathrm{MHz}$ in $\mathrm{CDCl}_{3}$

d.r. $5: 1$
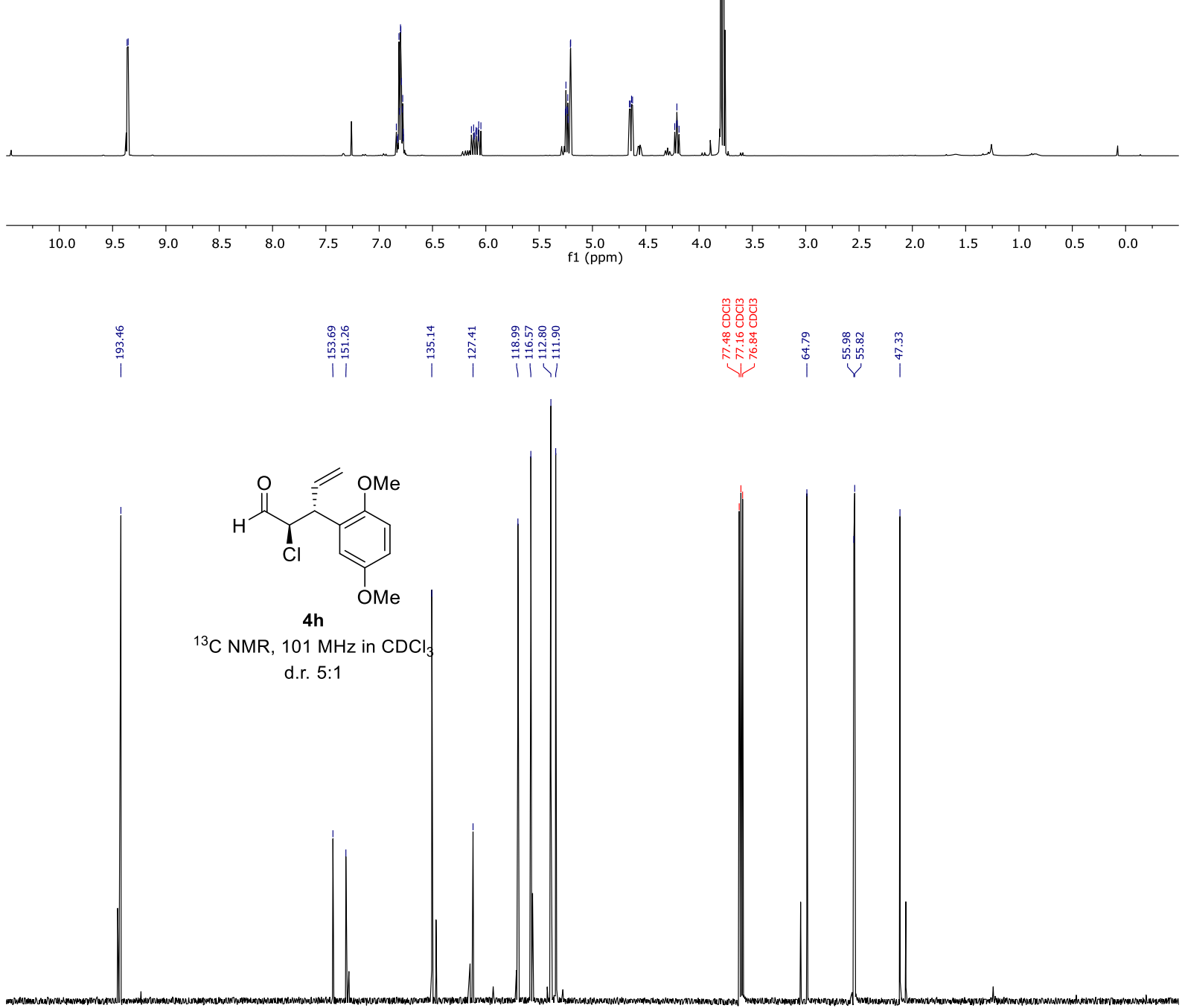

$\begin{array}{lllllllllll}210 & 200 & 190 & 180 & 170 & 160 & 150 & 140 & 130 & 120 & 110 \quad \begin{array}{l}100 \\ \mathrm{f} 1(\mathrm{ppm})\end{array}\end{array}$ 


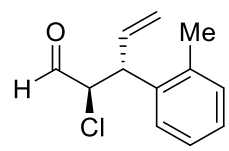

4i

${ }^{1} \mathrm{H}$ NMR, $400 \mathrm{MHz}$ in $\mathrm{CDCl}_{3}$

d.r. $7: 1$
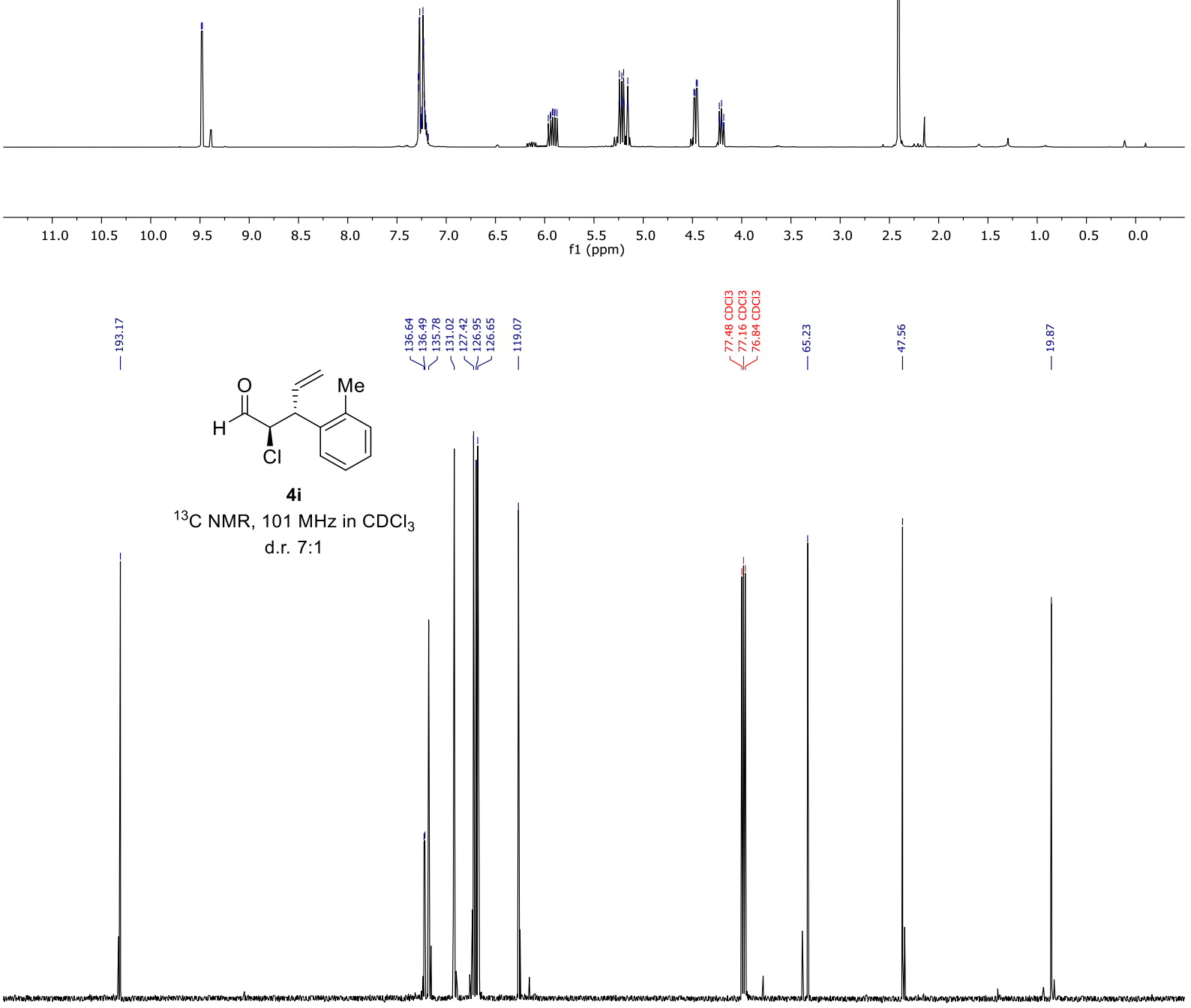

$\begin{array}{lllllllllll}210 & 200 & 190 & 180 & 170 & 160 & 150 & 140 & 130 & 120 & 110 \\ \mathrm{f} 1(\mathrm{ppm}) & 100\end{array}$ 

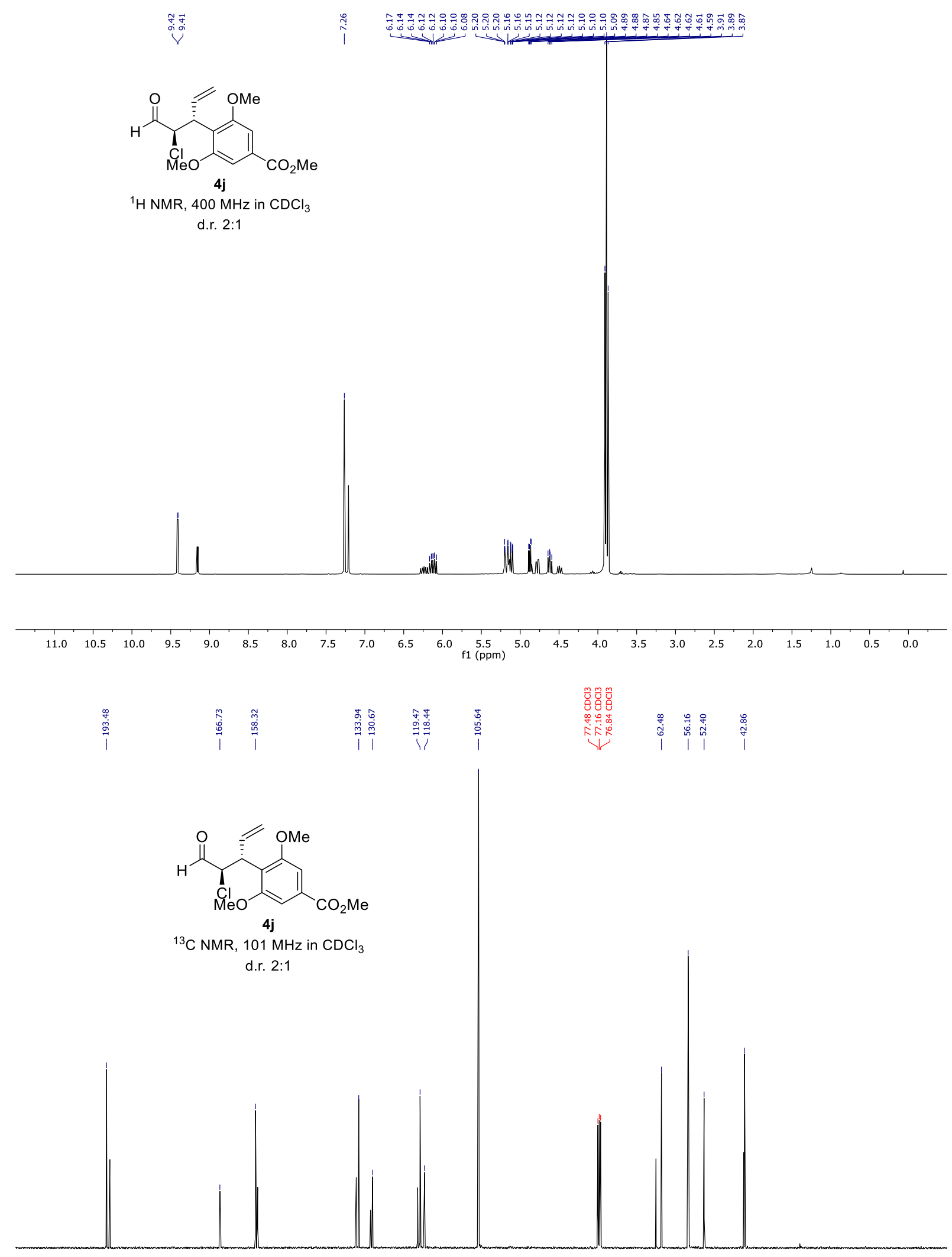

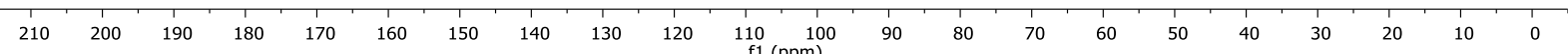



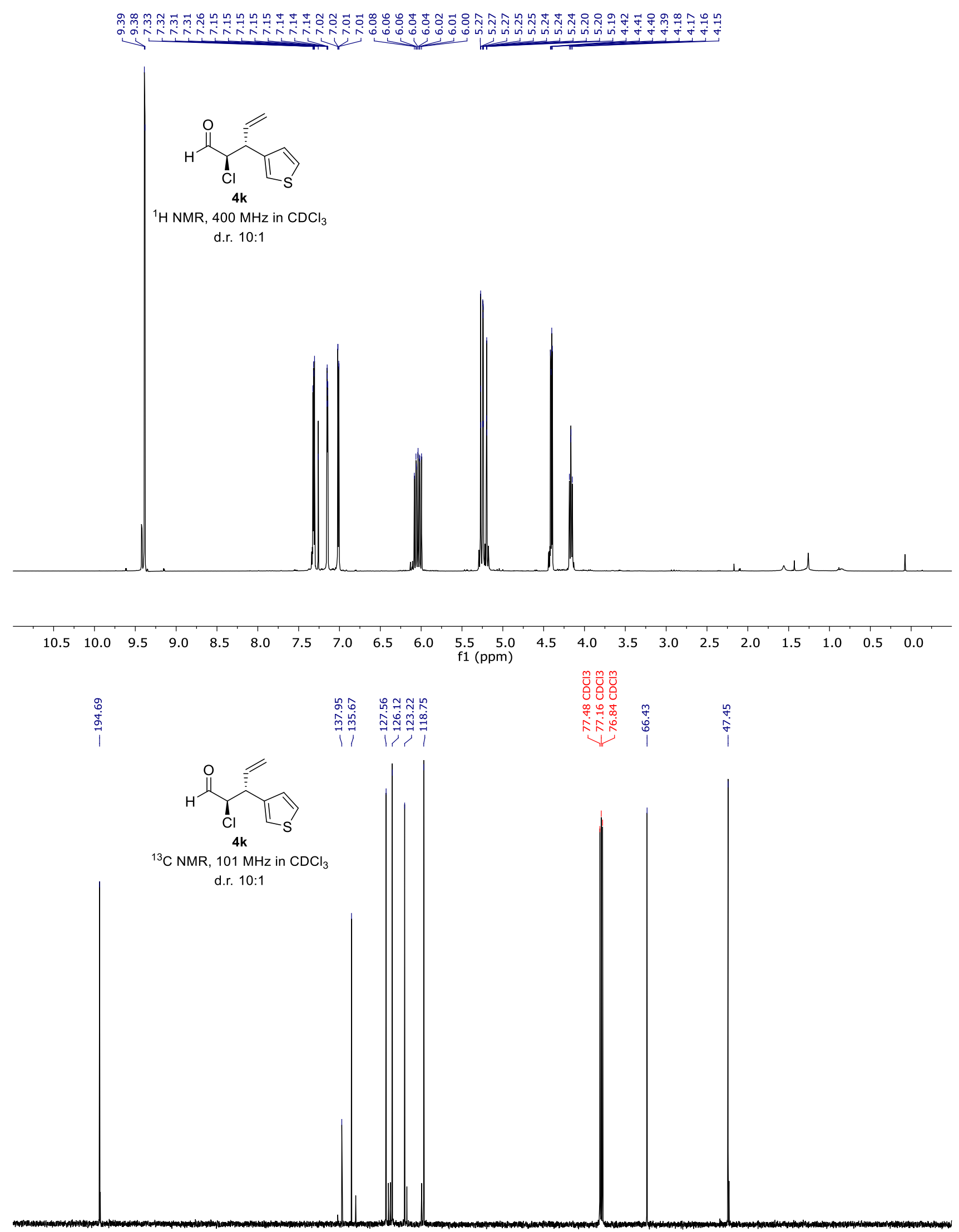

$\begin{array}{lllllllllllllllllllllllll}210 & 200 & 190 & 180 & 170 & 160 & 150 & 140 & 130 & 120 & 110 & 100 & 90 & 80 & 70 & 60 & 50 & 40 & 30 & 20 & 10 & 0\end{array}$ f1 (ppm) 


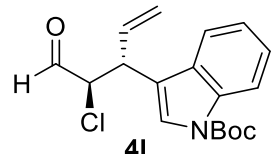

${ }^{1} \mathrm{H} \mathrm{NMR}, 500 \mathrm{MHz}$ in $\mathrm{CDCl}_{3}$ d.r. $20: 1$
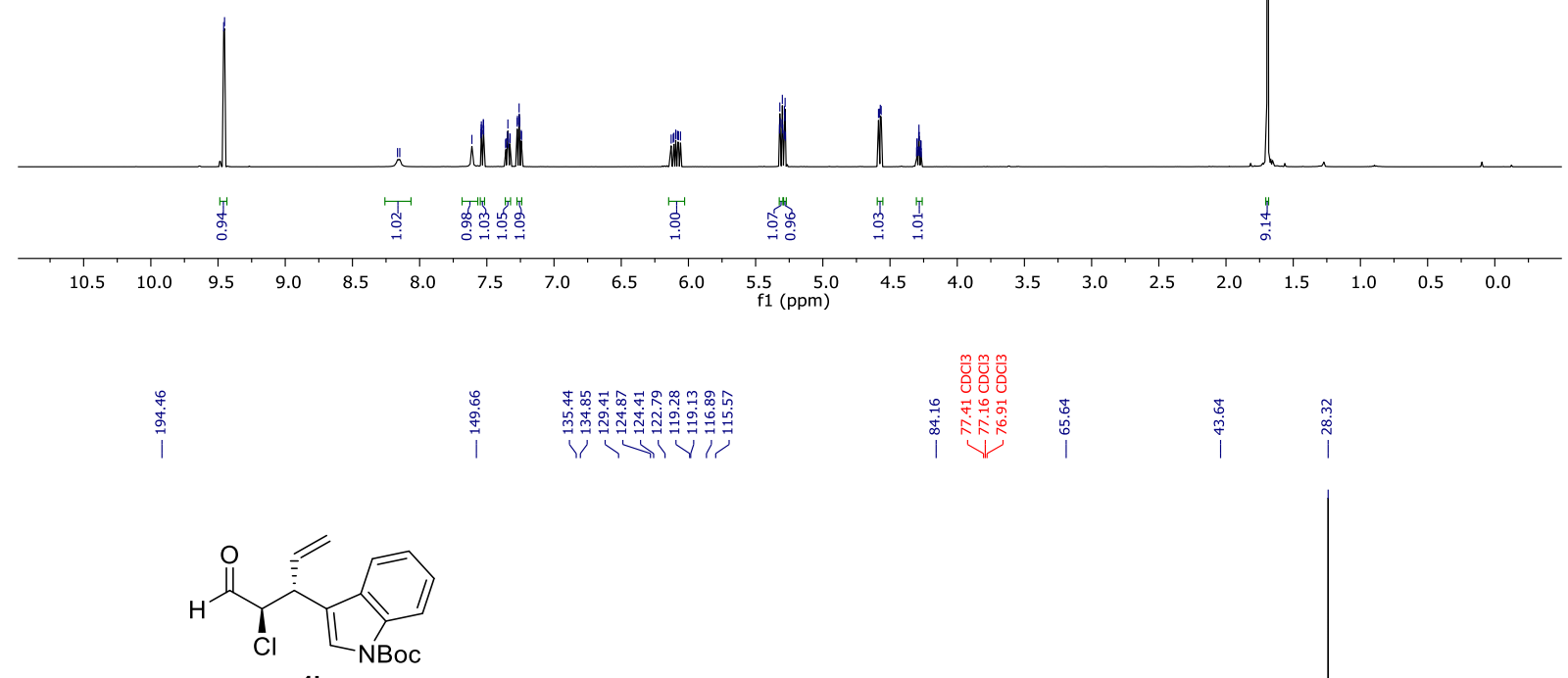

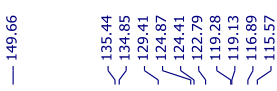

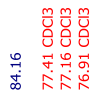

|

${ }^{1} \mathrm{H} \mathrm{NMR}, 126 \mathrm{MHz}$ in $\mathrm{CDCl}_{3}$

d.r. $20: 1$

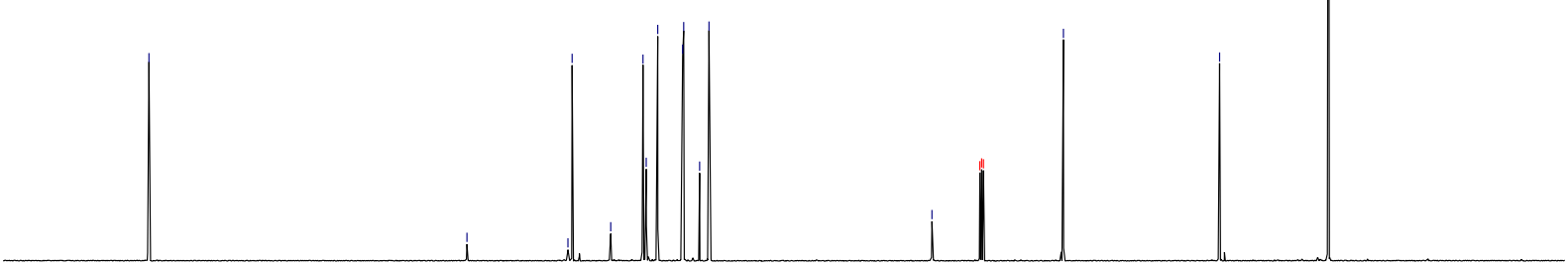

$\begin{array}{lllllllllll}210 & 200 & 190 & 180 & 170 & 160 & 150 & 140 & 130 & 120 & \begin{array}{c}110 \\ \mathrm{f} 1(\mathrm{ppm})\end{array}\end{array}$ 

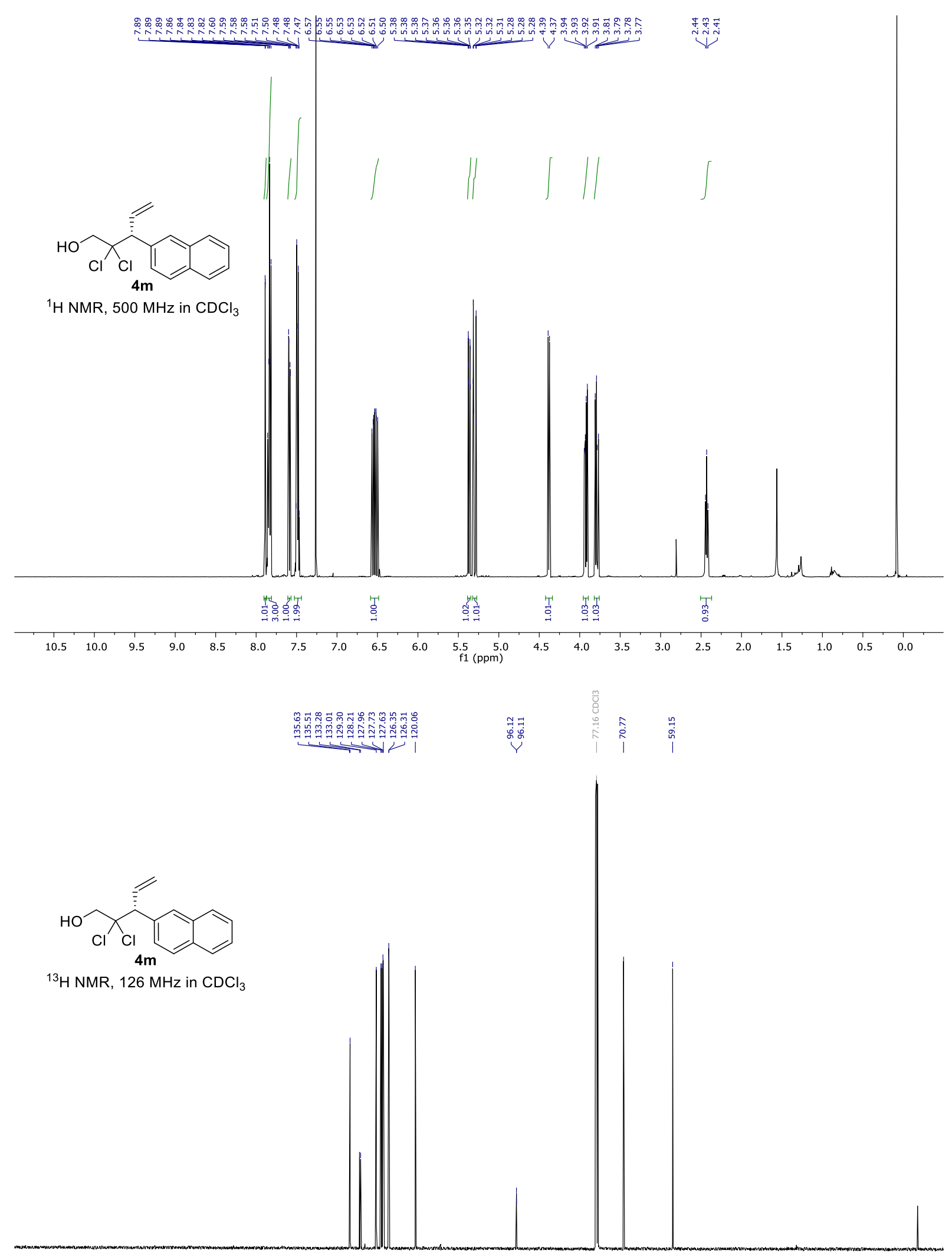

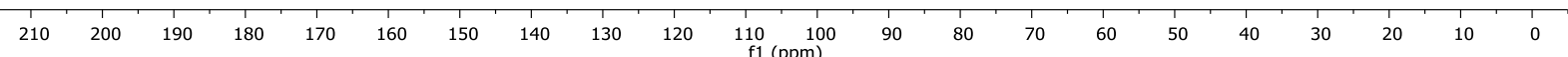



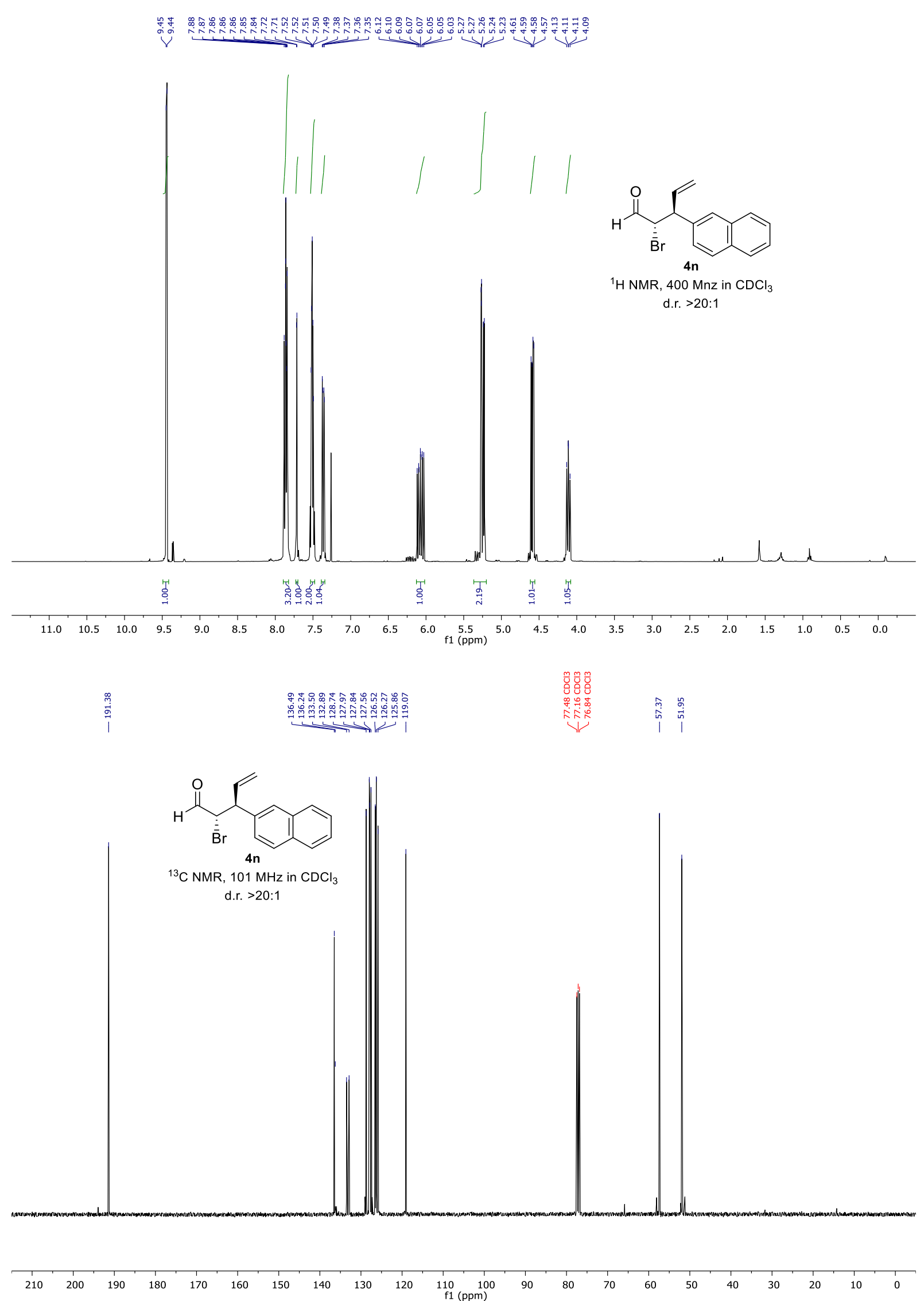

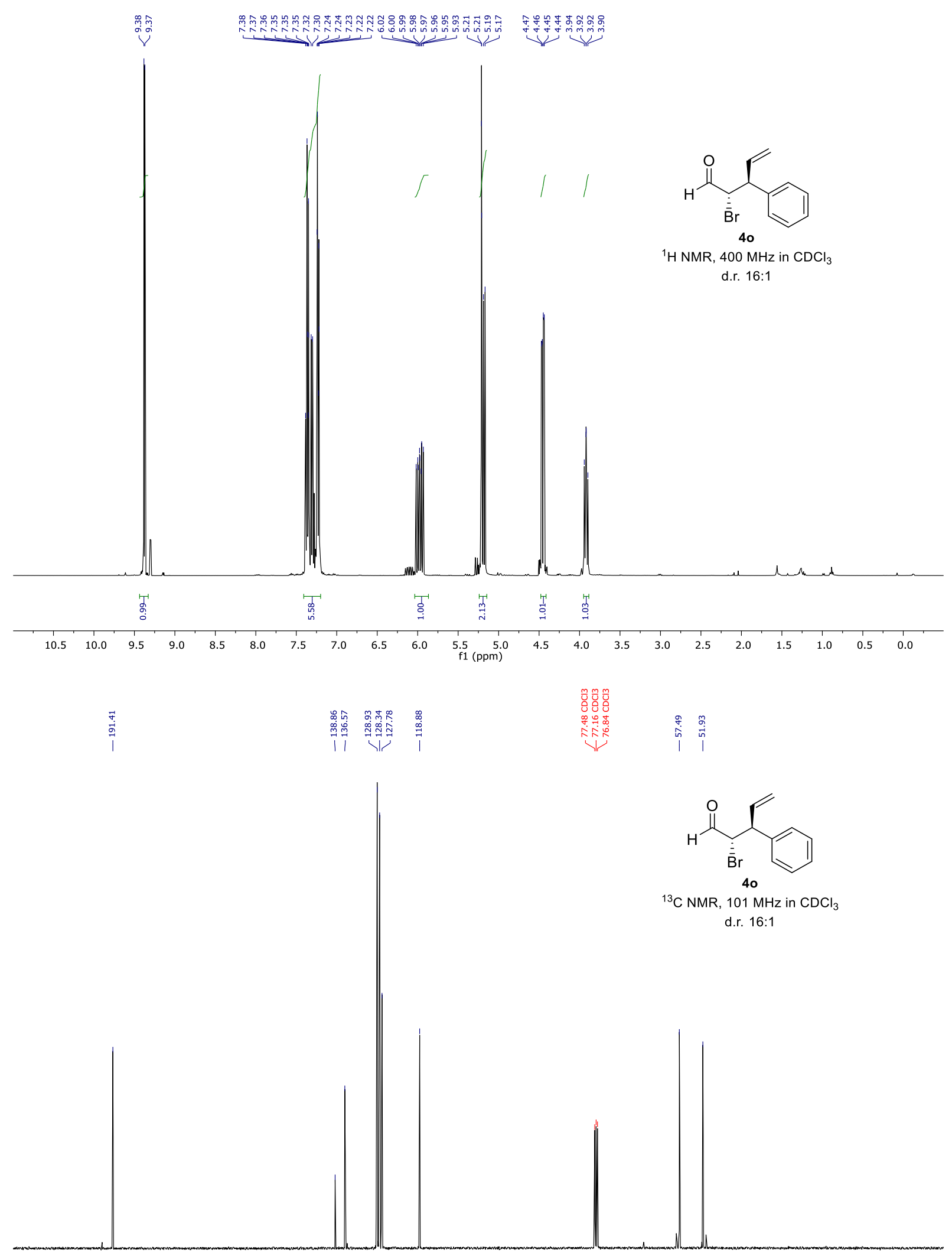

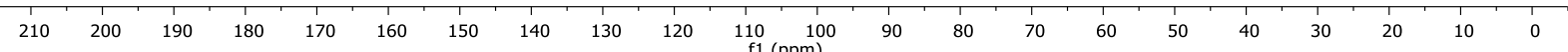




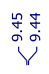

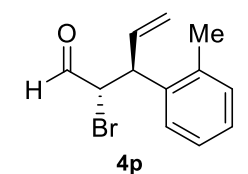

${ }^{1} \mathrm{H}$ NMR, $400 \mathrm{MHz}$ in $\mathrm{CDCl}_{3}$

d.r. $10: 1$

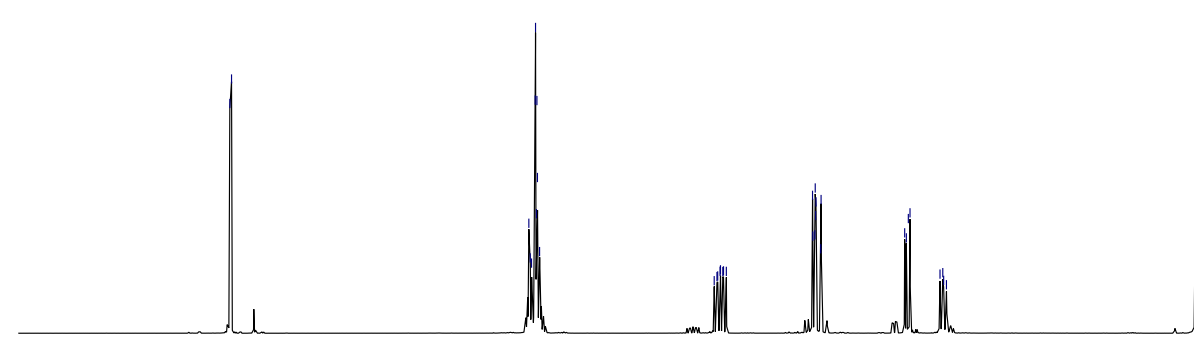

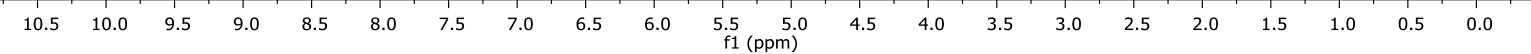
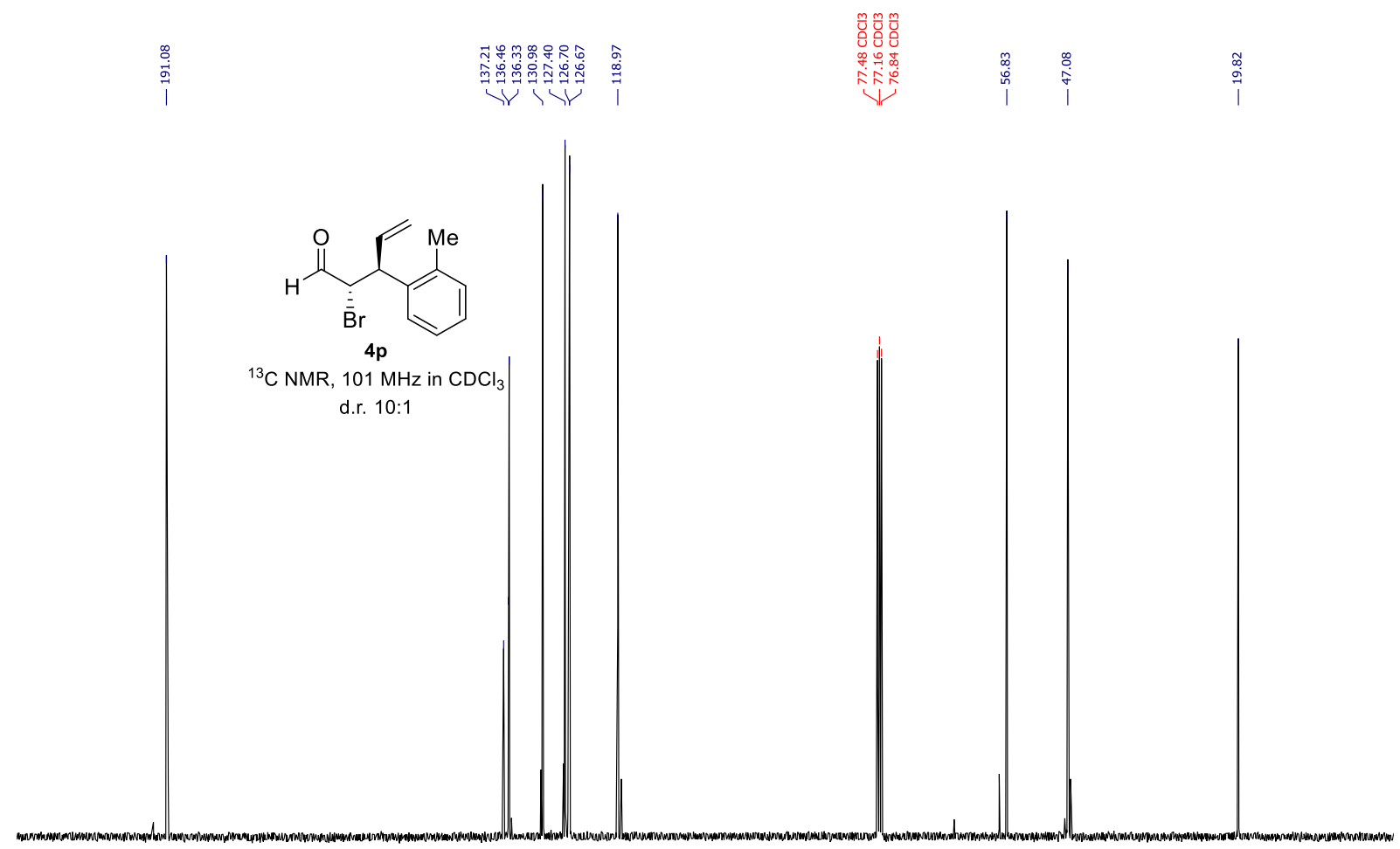

\begin{tabular}{|lllllllllllllllllllllllll}
210 & 200 & 190 & 180 & 170 & 160 & 150 & 140 & 130 & 120 & 110 & 100 & 90 & 80 & 70 & 60 & 50 & 40 & 30 & 20 & 10 & 0
\end{tabular} 

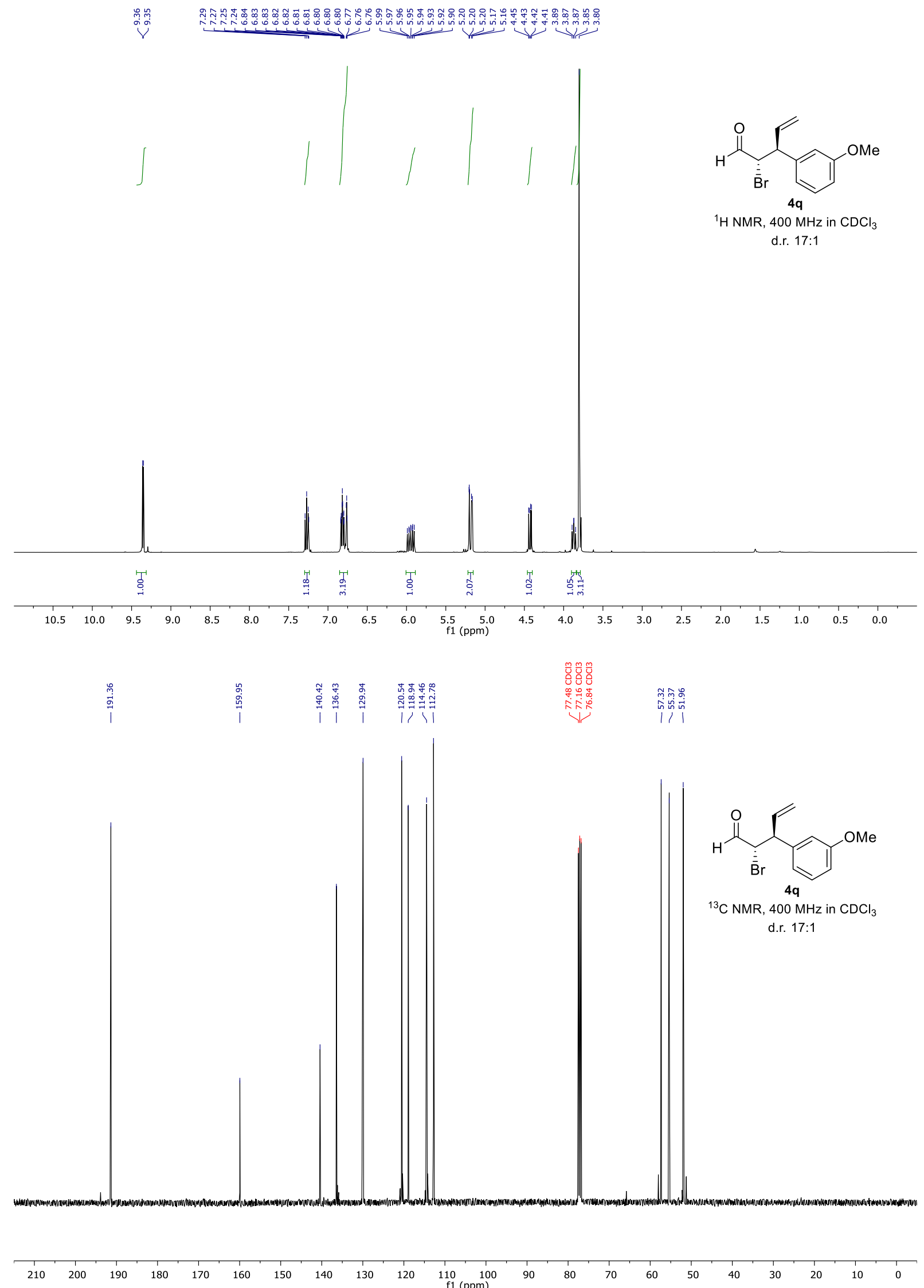


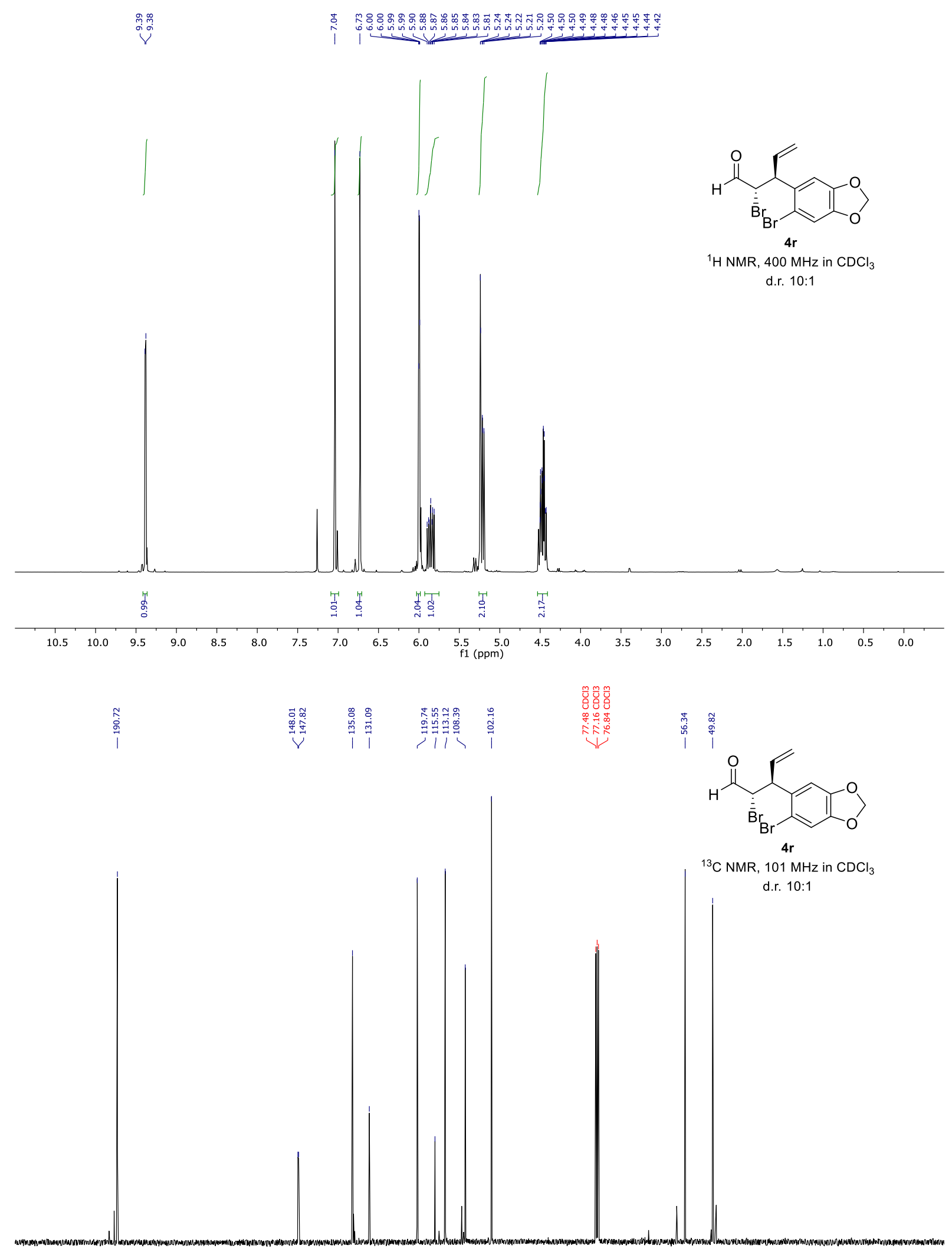

$\begin{array}{lllllllllllllllllllllllll}210 & 200 & 190 & 180 & 170 & 160 & 150 & 140 & 130 & 120 & 110 & 100 & 90 & 80 & 70 & 60 & 50 & 40 & 30 & 20 & 10 & 0\end{array}$ 


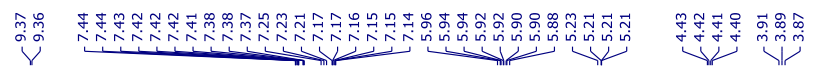
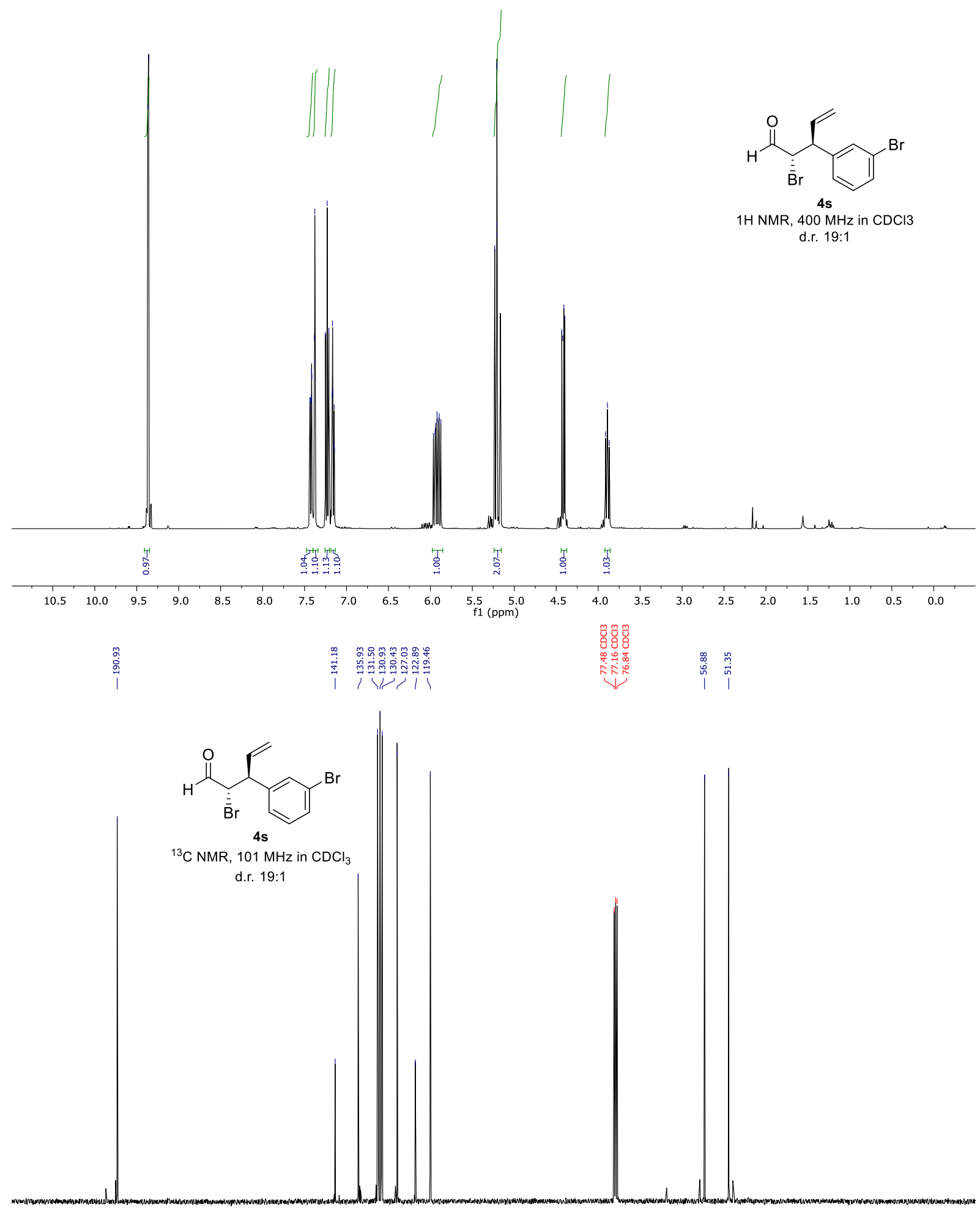

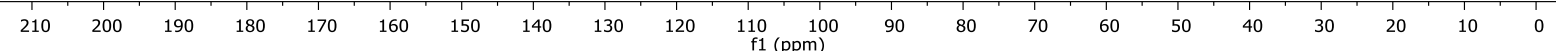



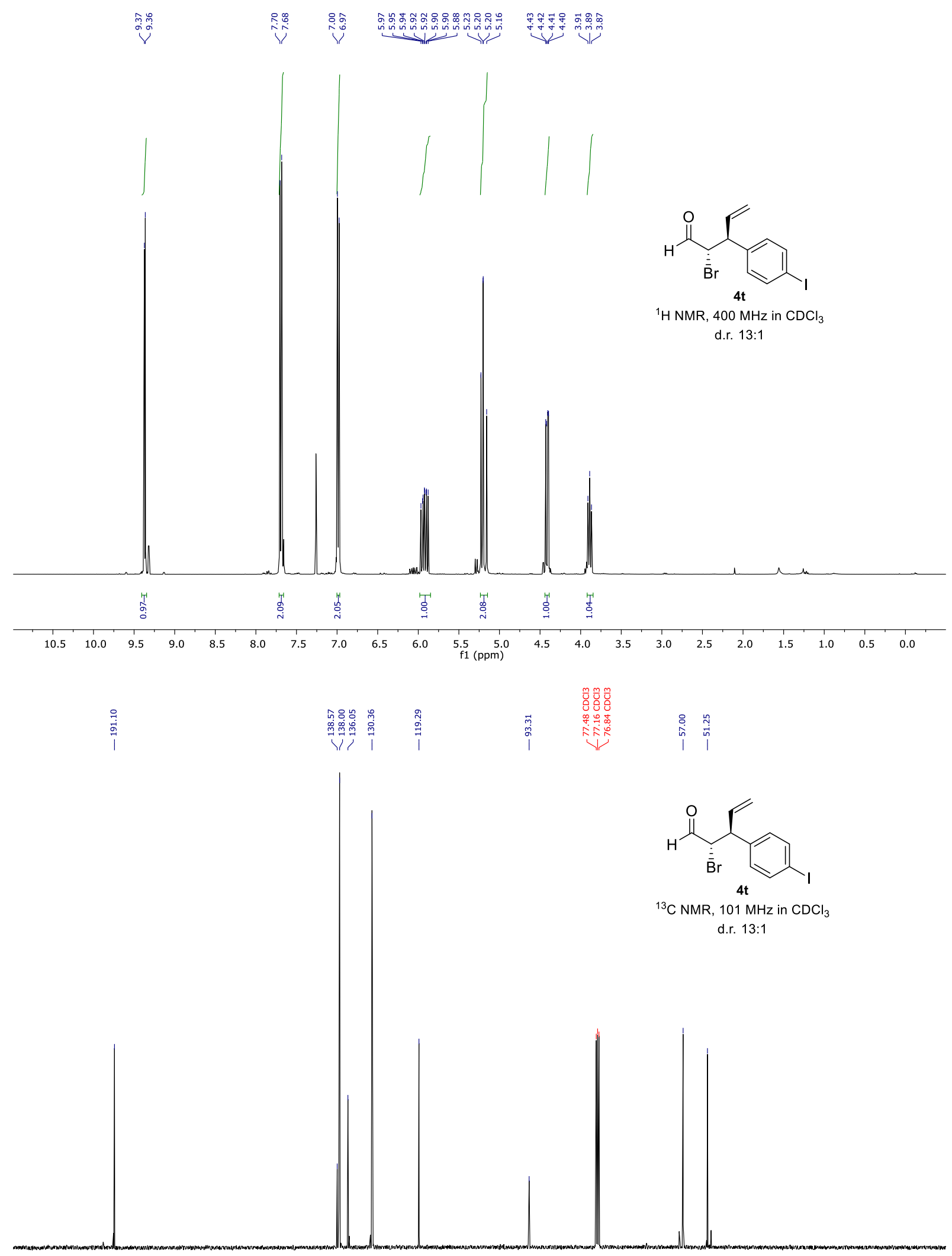

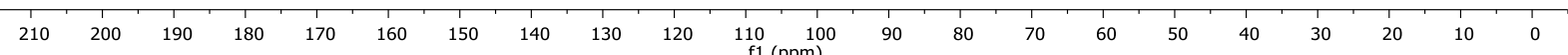



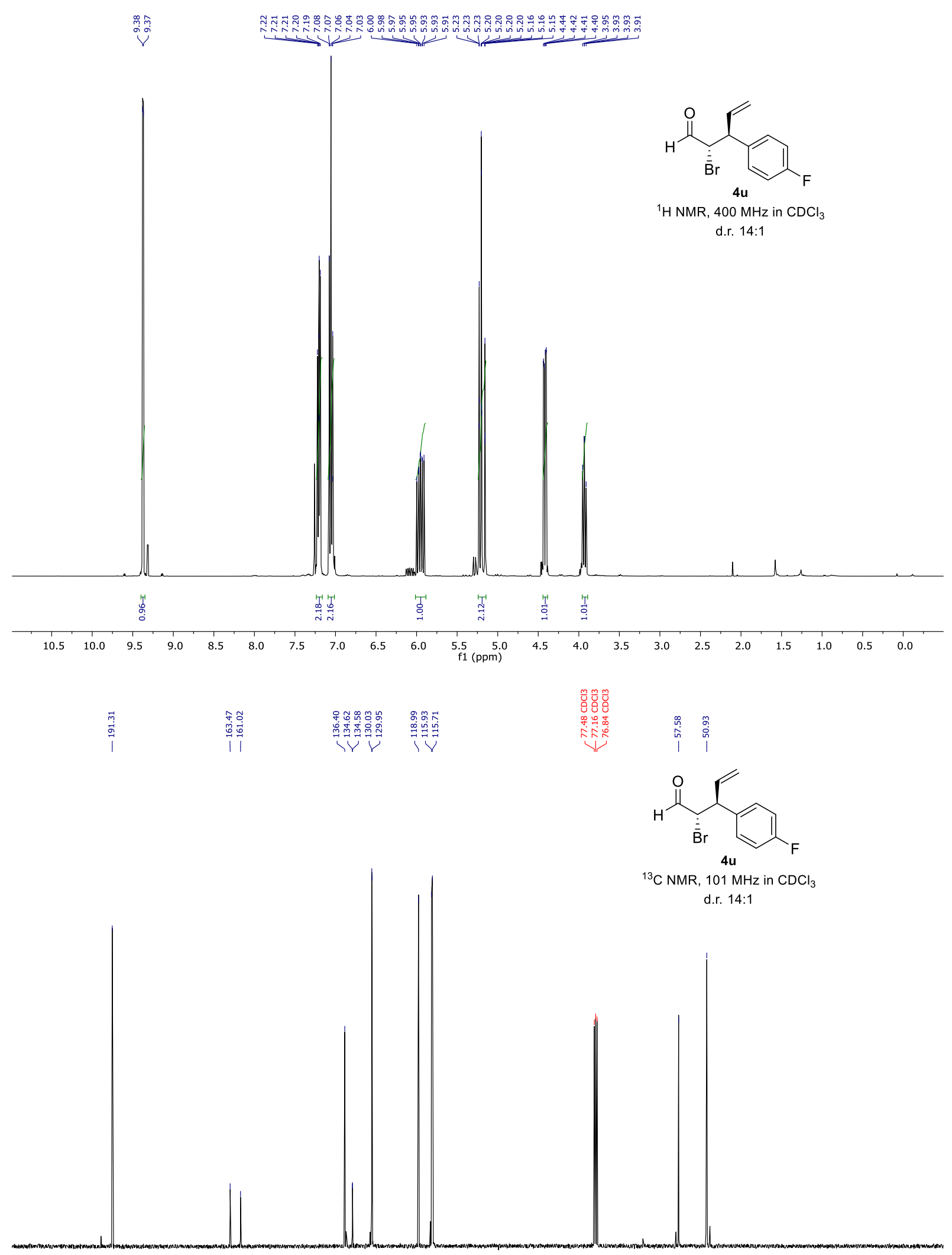

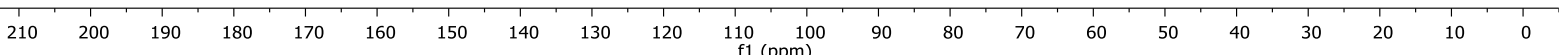




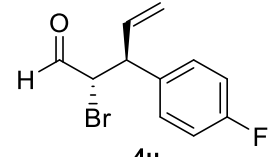

${ }^{19} \mathrm{~F}$ NMR, $376 \mathrm{MHz}$ in $\mathrm{CDCl}_{3}$

d.r. $14: 1$ 


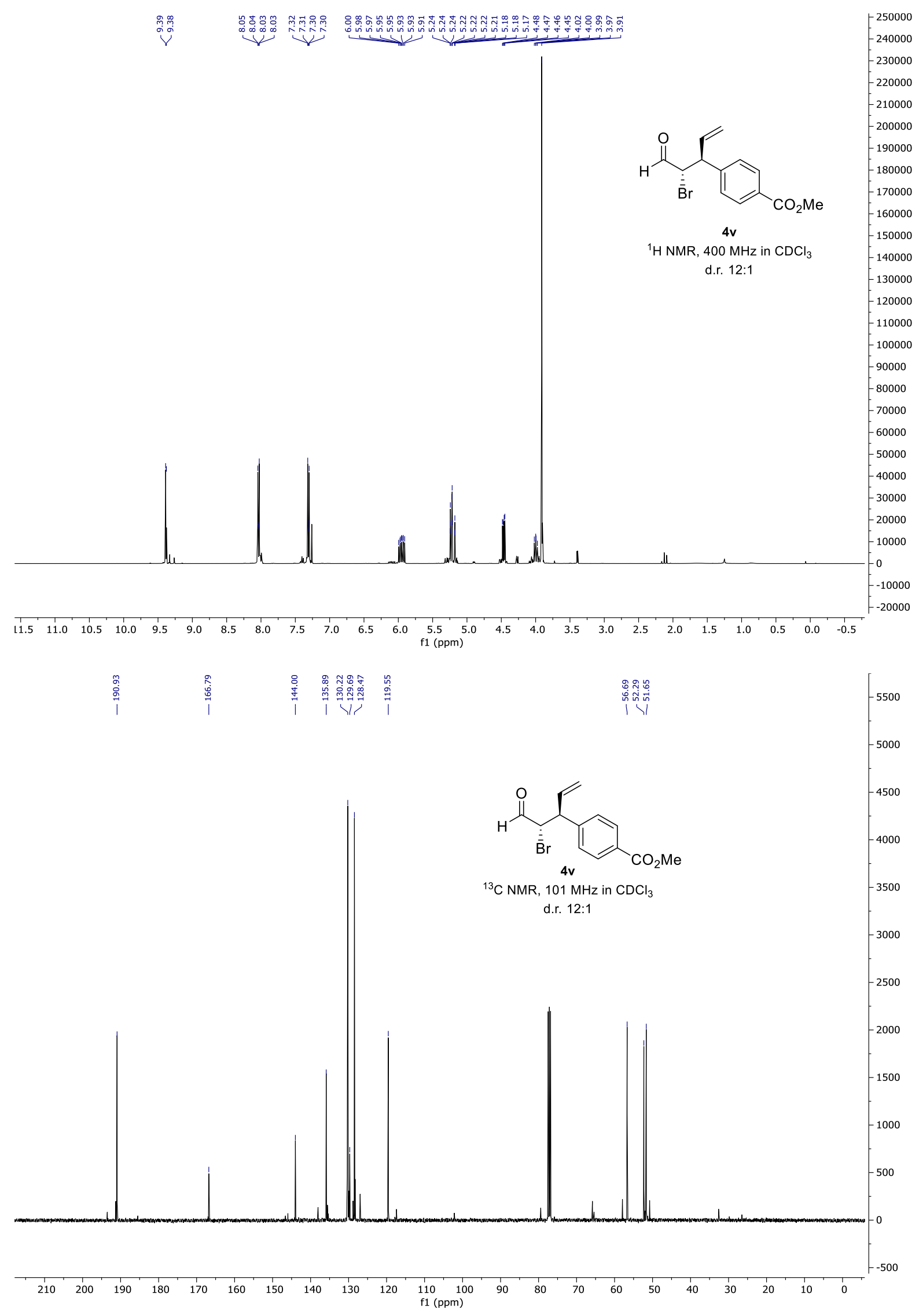




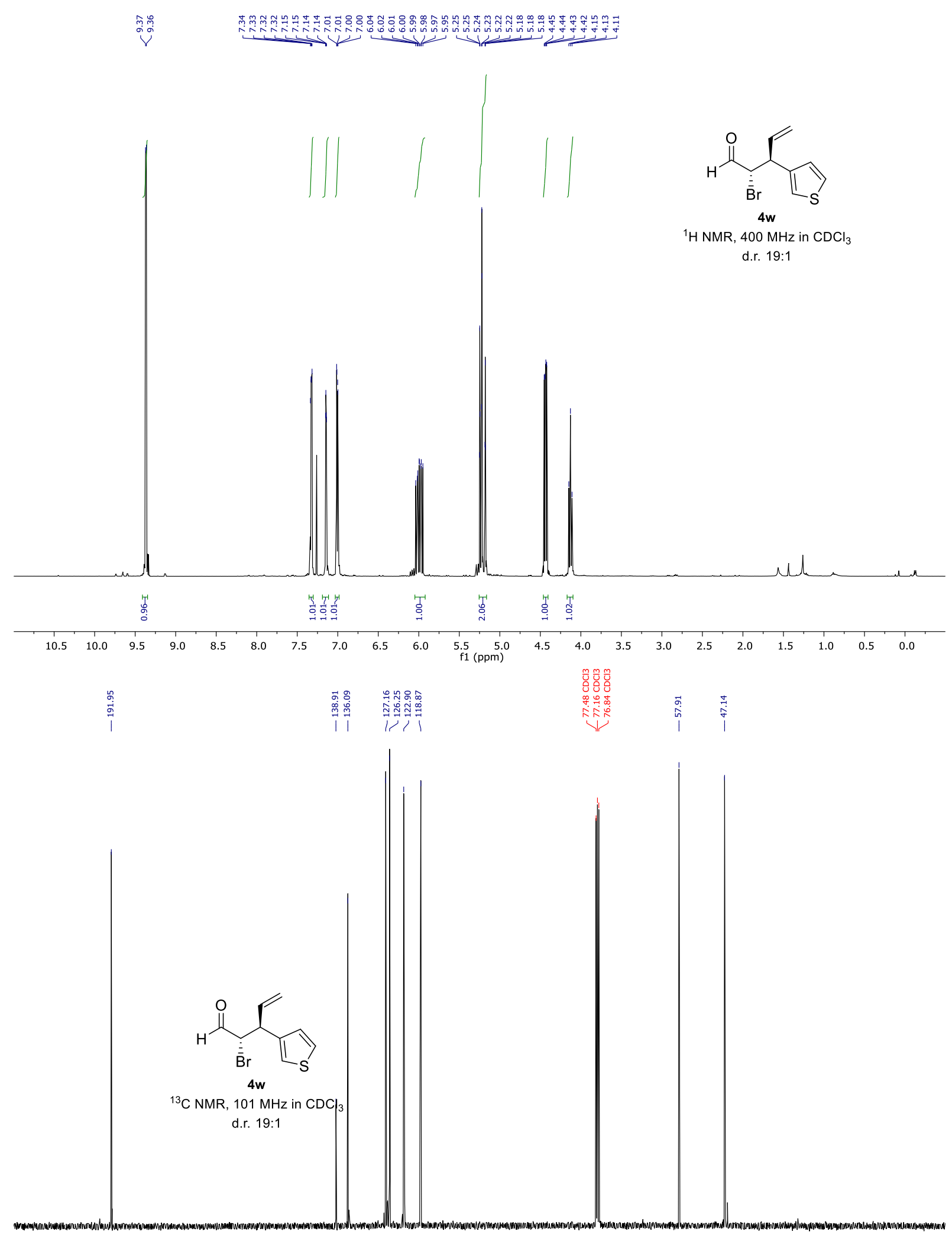

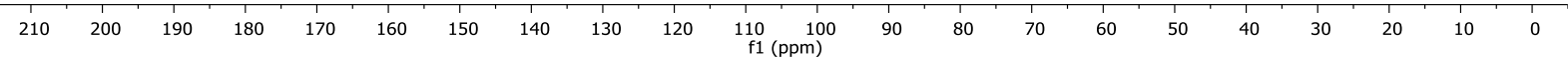




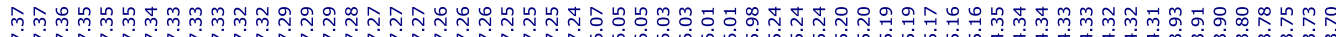

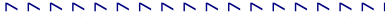

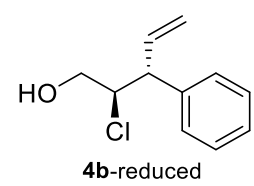

${ }^{1} \mathrm{H} \mathrm{NMR,}, 400 \mathrm{MHz}$ in $\mathrm{CDCl}_{3}$

d.r. $10: 1$

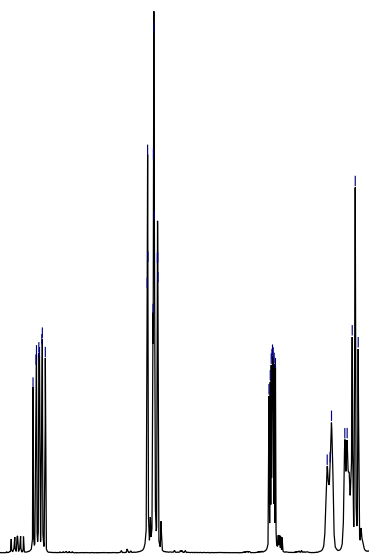

$\begin{array}{lllllllllll}10.5 & 10.0 & 9.5 & 9.0 & 8.5 & 8.0 & 7.5 & 7.0 & 6.5 & 6.0 & 5.5 \\ \mathrm{f} 1(\mathrm{ppm})\end{array}$
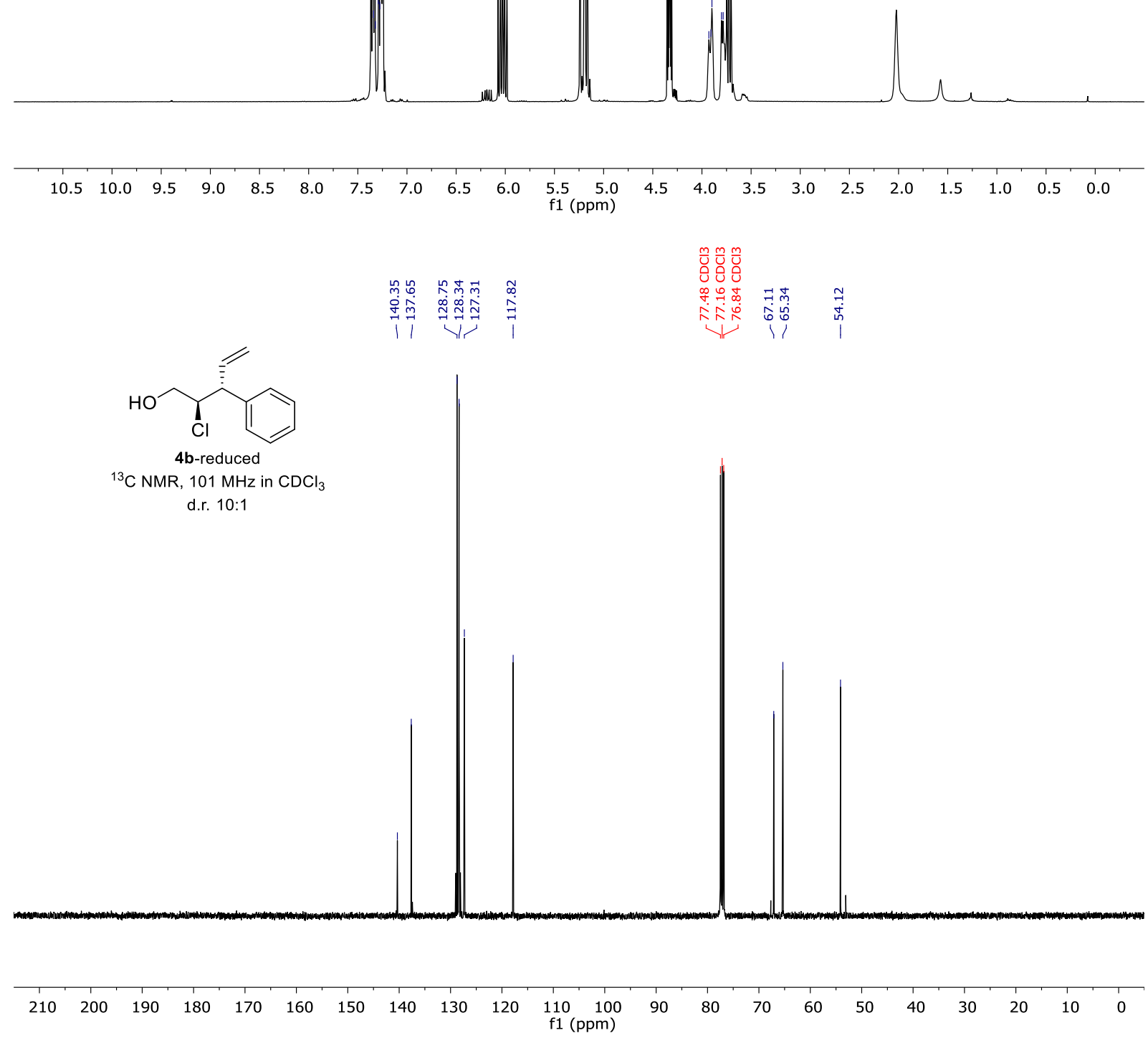

${ }^{13} \mathrm{C}$ NMR, $101 \mathrm{MHz}$ in $\mathrm{CDCl}_{3}$

d.r. $10: 1$ 


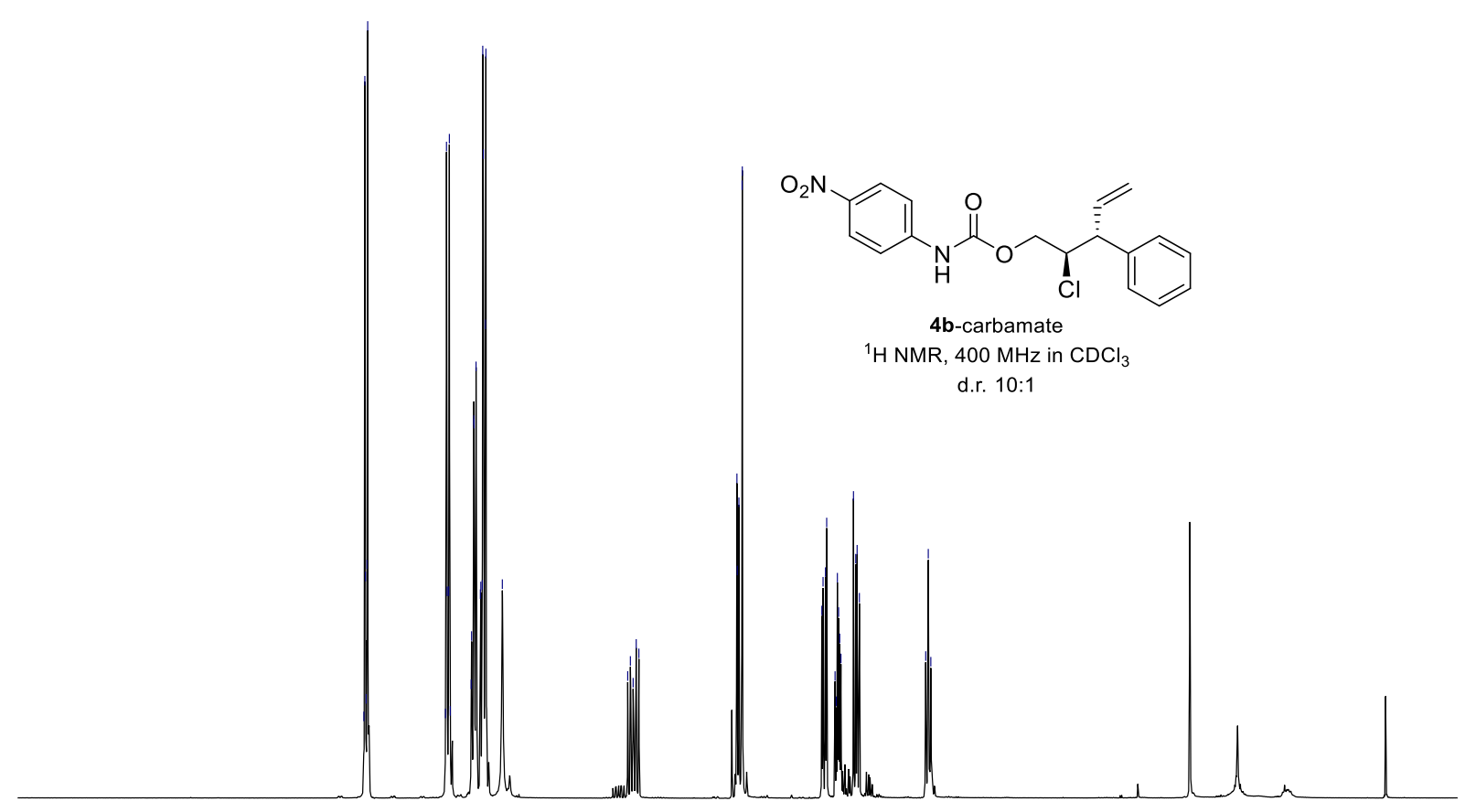

$\begin{array}{llllllllllll}10.5 & 10.0 & 9.5 & 9.0 & 8.5 & 8.0 & 7.5 & 7.0 & 6.5 & 6.0 & \begin{array}{l}5.5 \\ \mathrm{f} 1(\mathrm{ppm})\end{array}\end{array}$

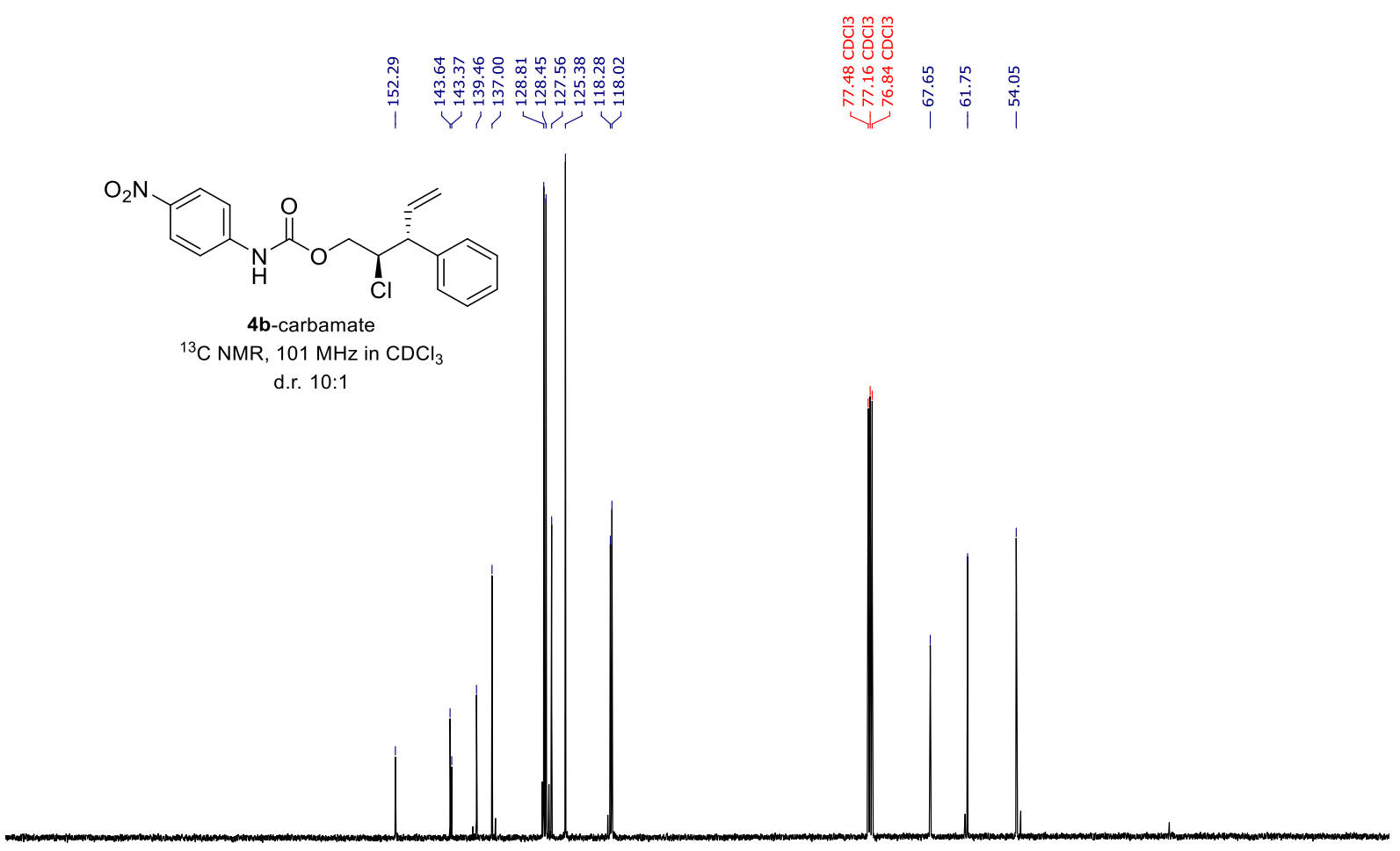

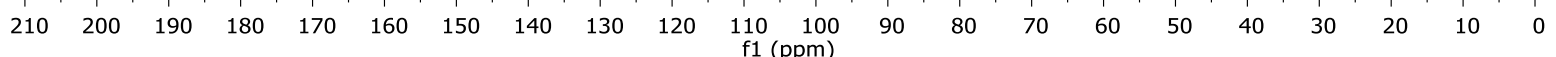



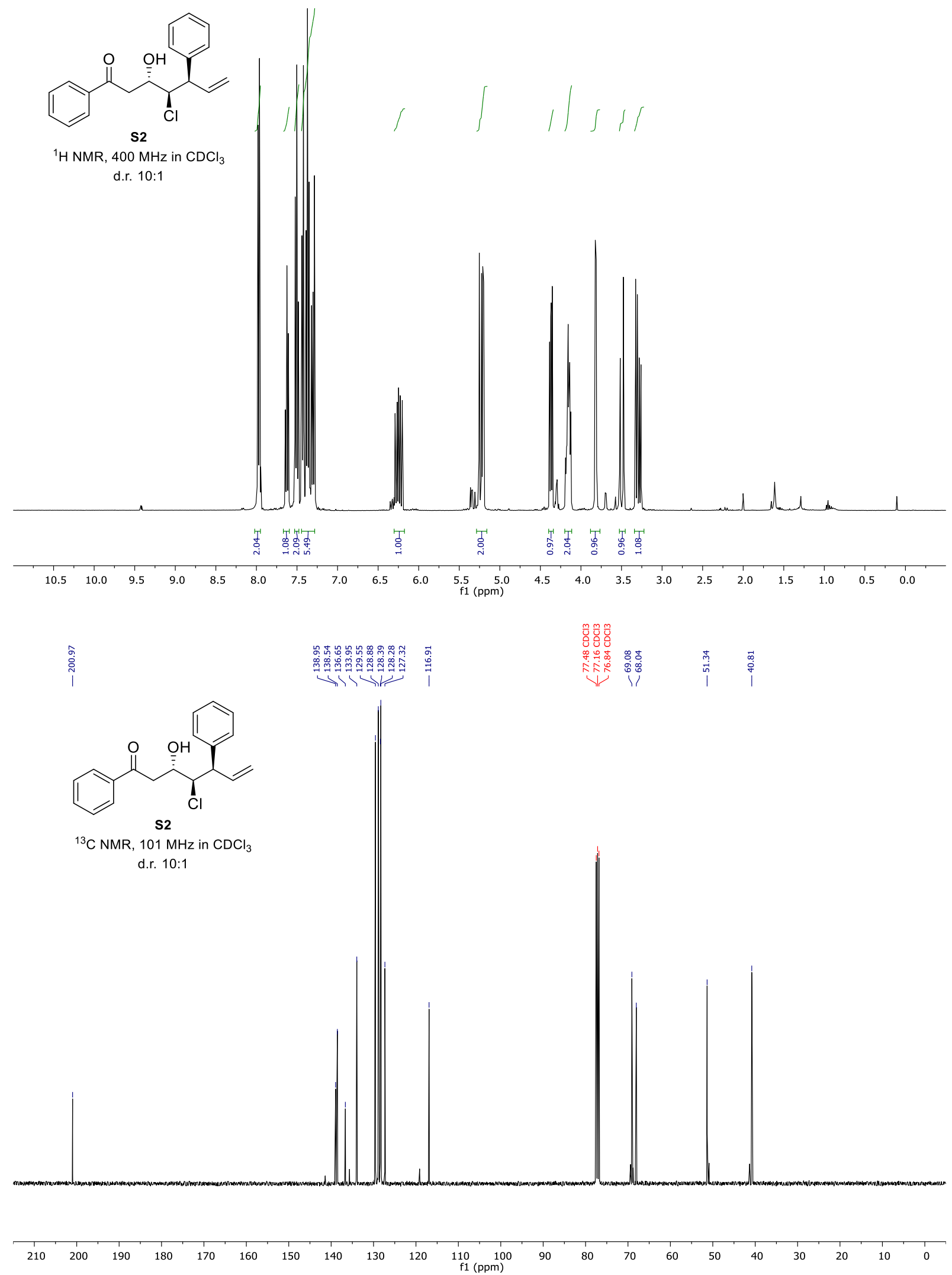


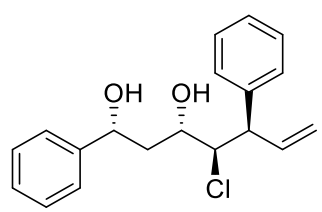

5

${ }^{1} \mathrm{H} \mathrm{NMR}, 400 \mathrm{MHz}$ in $\mathrm{CDCl}_{3}$

d.r. $8: 1$

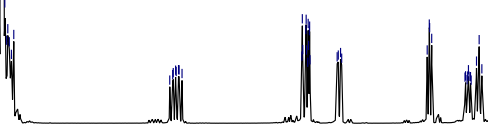

$\begin{array}{llllllllllll}10.5 & 10.0 & 9.5 & 9.0 & 8.5 & 8.0 & 7.5 & 7.0 & 6.5 & 6.0 & 5.5 & 5.0 \\ & & & & & & & & & & & \\ f 1(\mathrm{ppm})\end{array}$

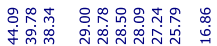

|ᄂ

$m$
0
0
0

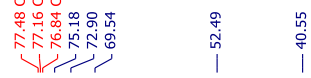

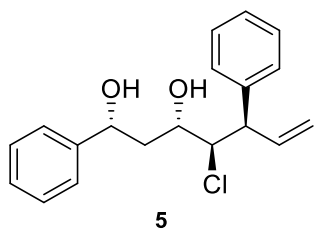

${ }^{13} \mathrm{C} \mathrm{NMR}, 101 \mathrm{MHz}$ in $\mathrm{CDCl}_{3}$

d.r. $8: 1$
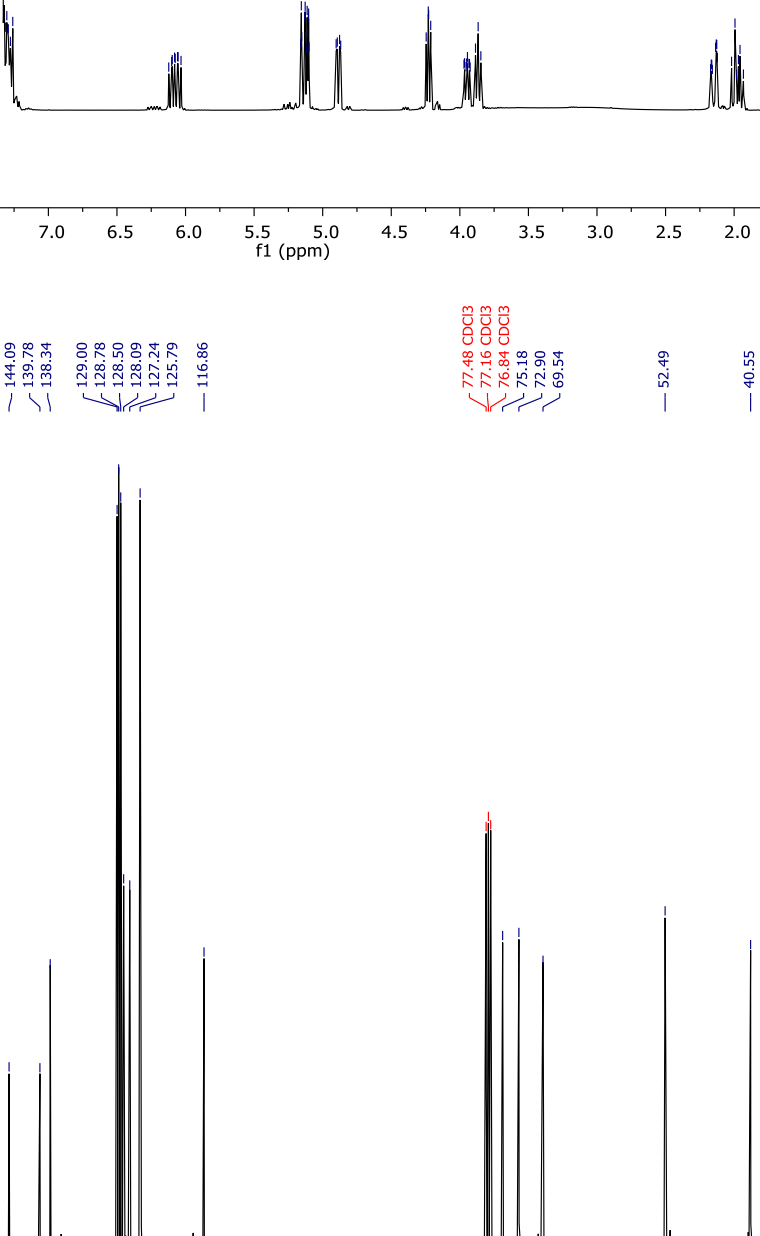

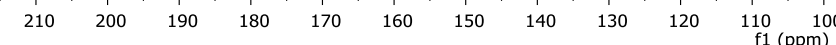




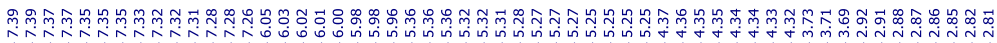
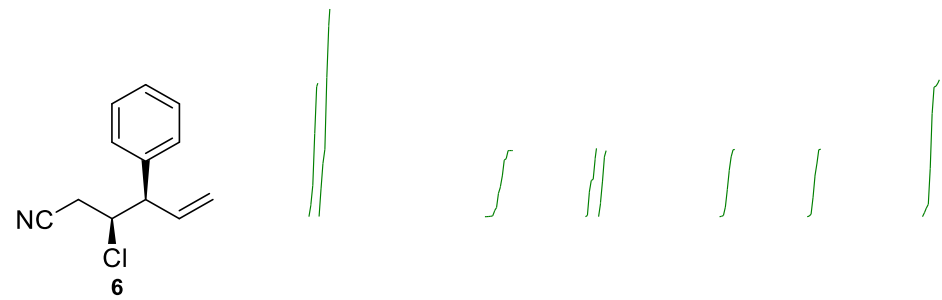

${ }^{1} \mathrm{H}$ NMR, $400 \mathrm{MHz}$ in $\mathrm{CDCl}_{3}$

d.r. $>20: 1$
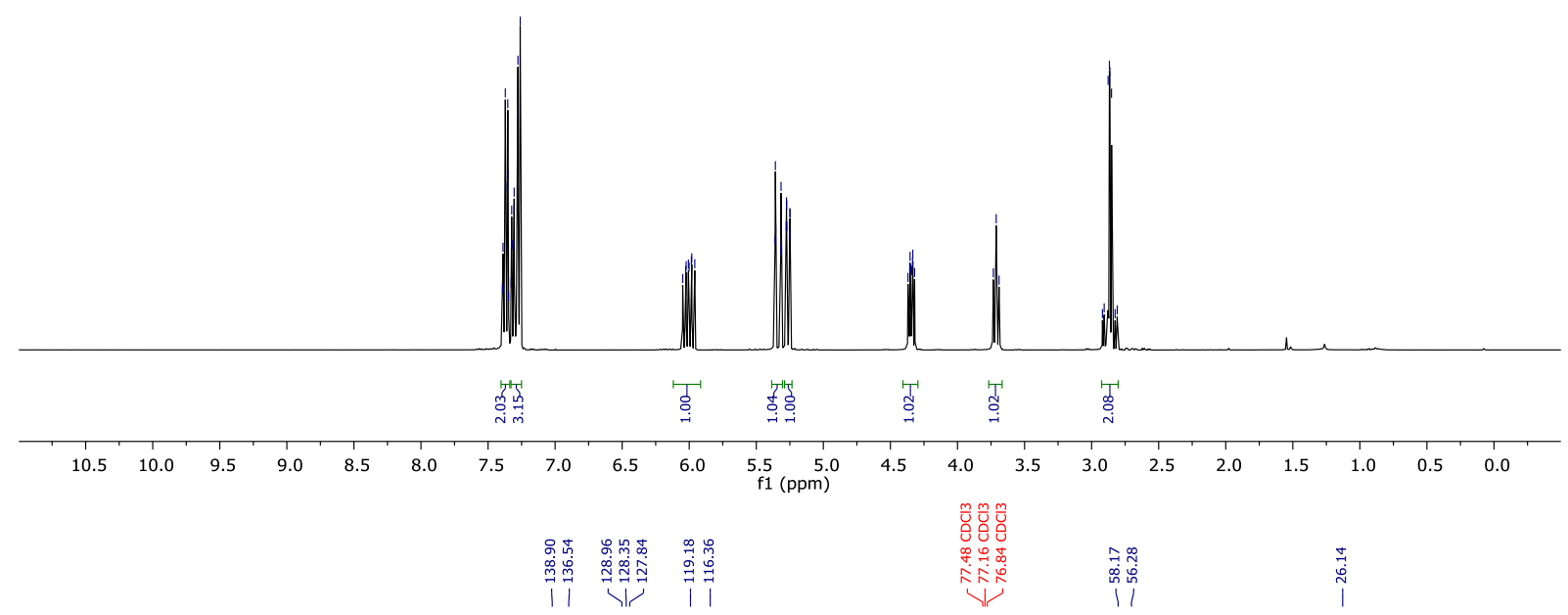

$m$
0
0

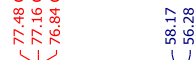

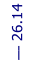

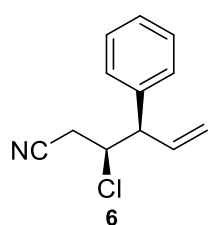

${ }^{13} \mathrm{C} \mathrm{NMR}, 101 \mathrm{MHz}$ in $\mathrm{CDCl}_{3}$

d.r. $>20: 1$

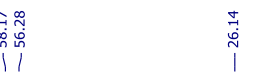

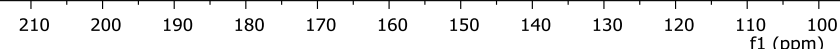




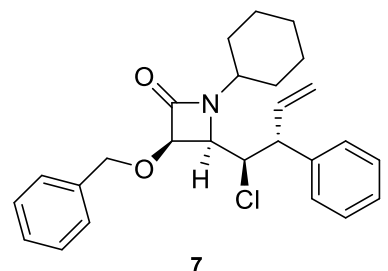

${ }^{1} \mathrm{H} \mathrm{NMR}, 400 \mathrm{MHz}$ in $\mathrm{CDCl}_{3}$ d.r. 1:14:42
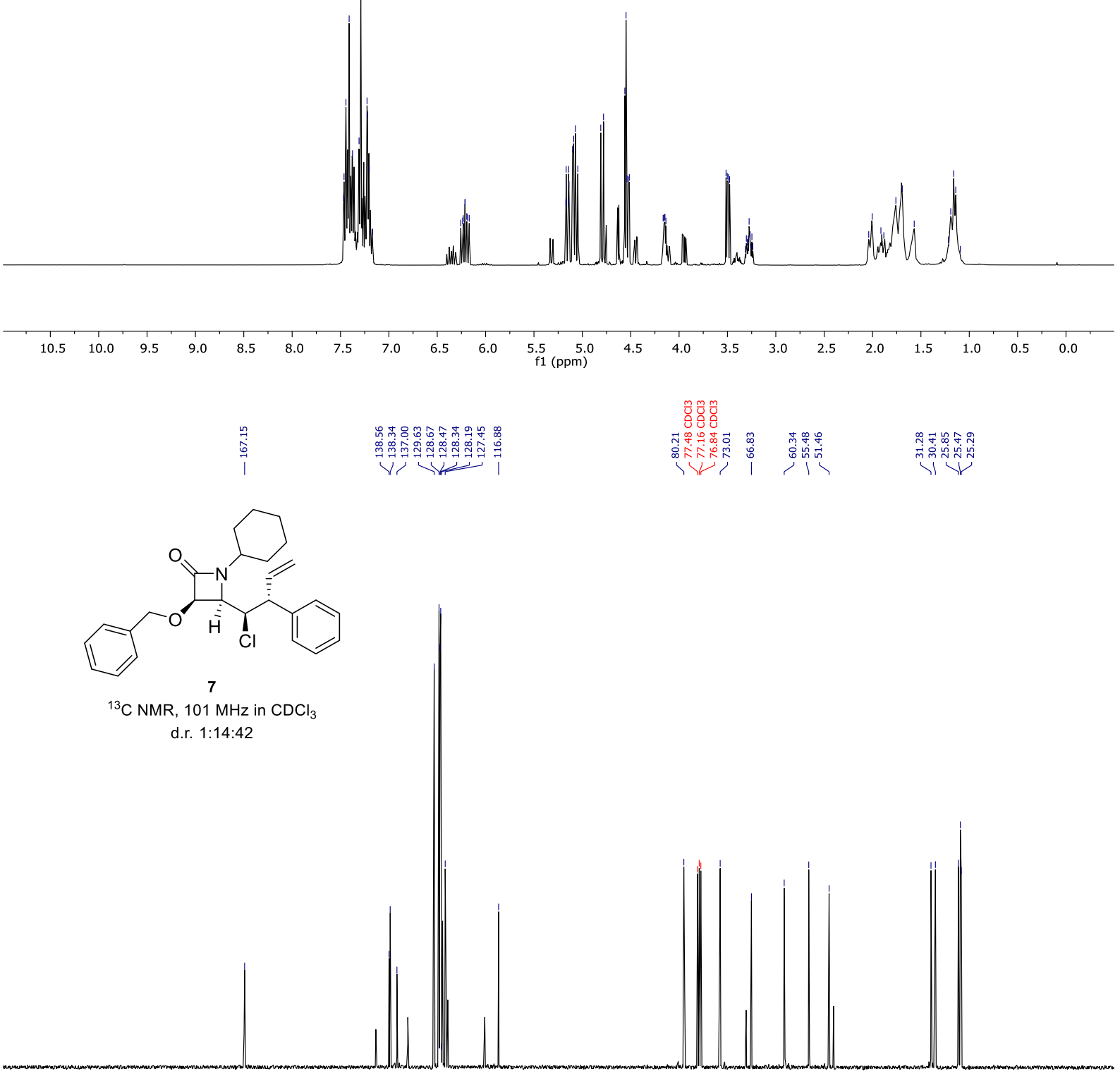

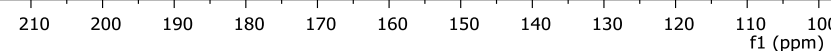




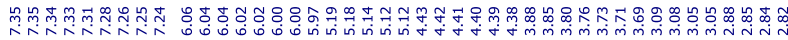

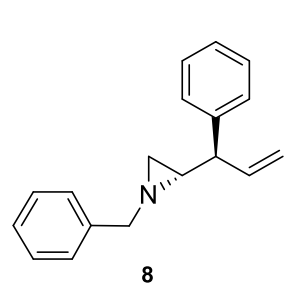

${ }^{1} \mathrm{H} \mathrm{NMR}, 400 \mathrm{MHz}$ in $\mathrm{CDCl}_{3}$ d.r. $1: 19$
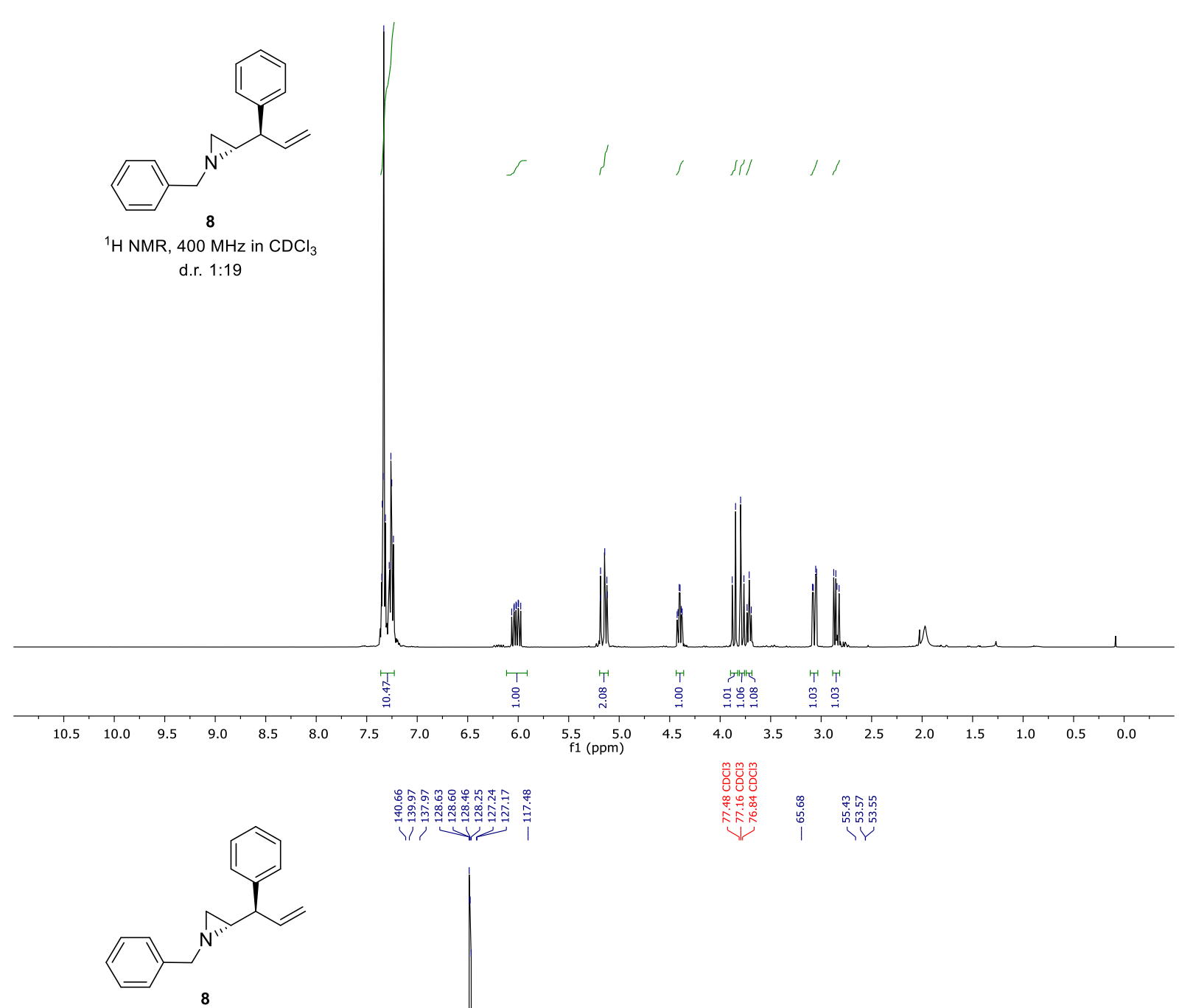

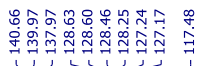

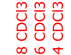

(1)

岤

${ }^{13} \mathrm{C} \mathrm{NMR}, 101 \mathrm{MHz}$ in $\mathrm{CDCl}_{3}$

d.r. $1: 19$

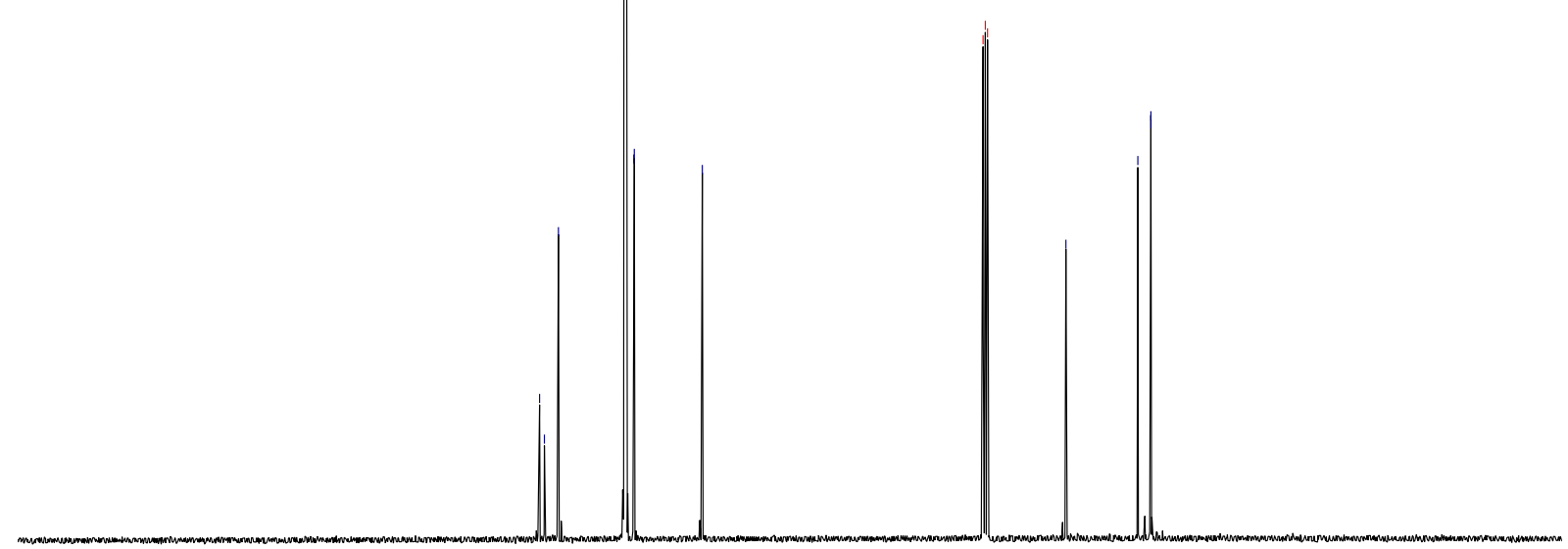

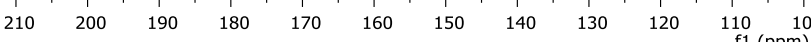




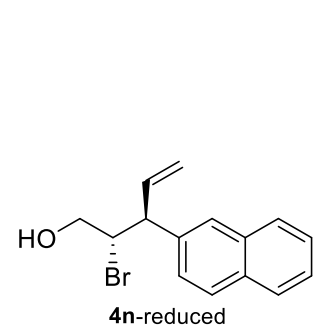

${ }^{1} \mathrm{H} \mathrm{NMR}, 400 \mathrm{MHz}$ in $\mathrm{CDCl}_{3}$

d.r. $>20: 1$
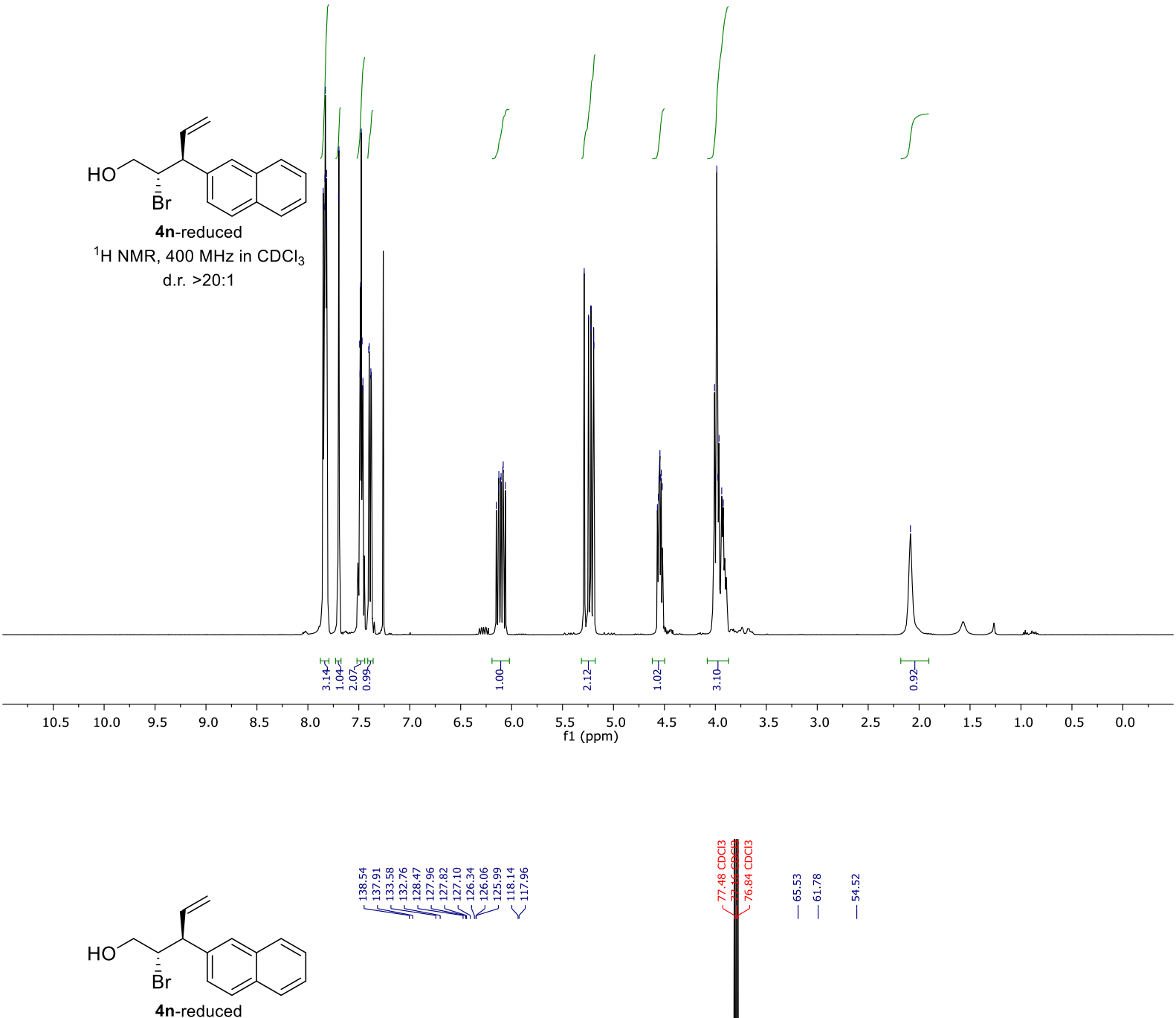

年

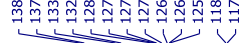

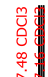

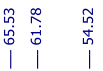

${ }^{13} \mathrm{C} \mathrm{NMR}, 101 \mathrm{MHz}$ in $\mathrm{CDCl}_{3}$

d.r. $>20: 1$

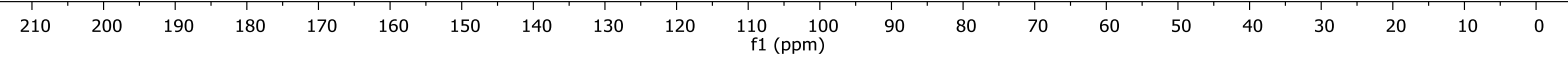




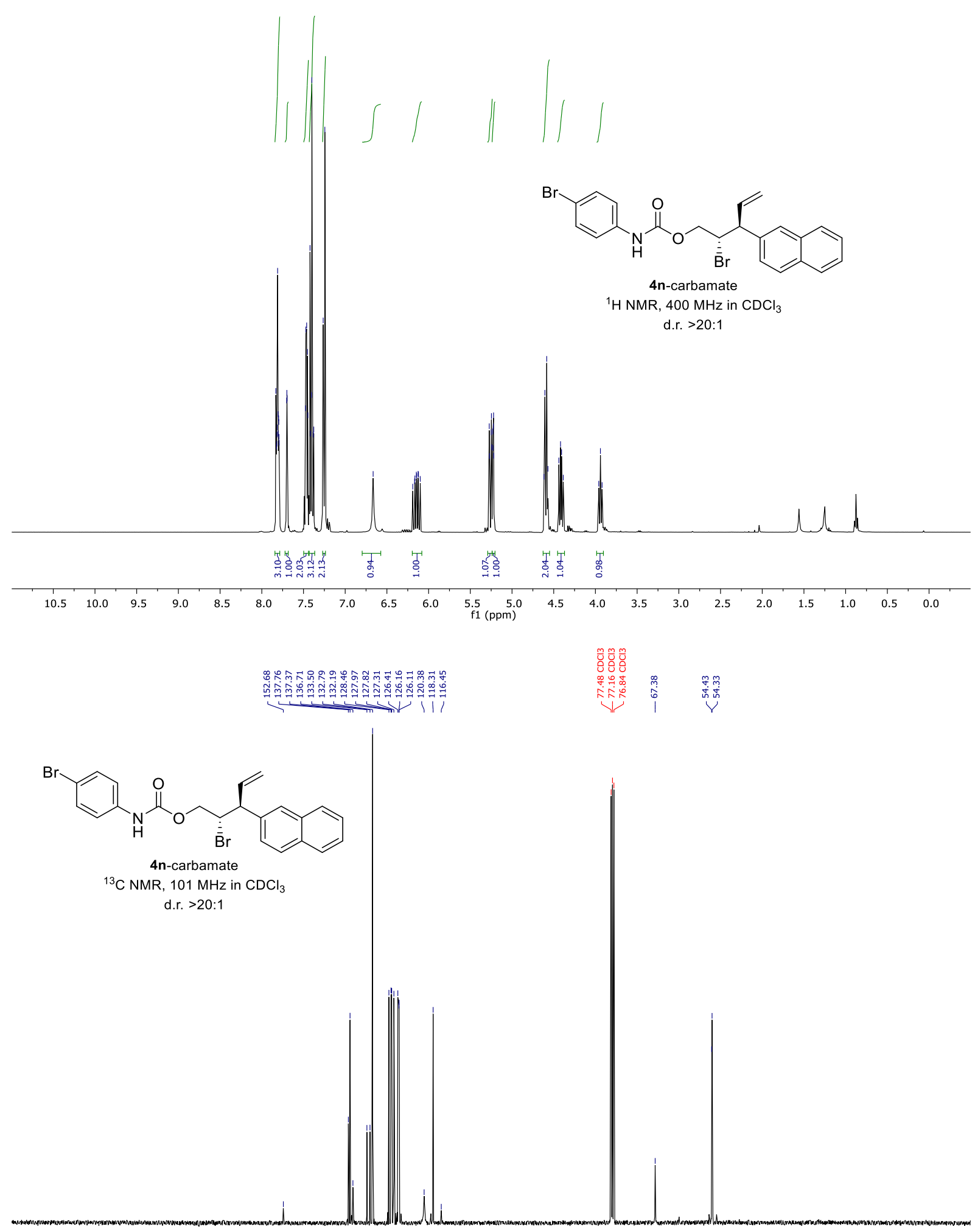

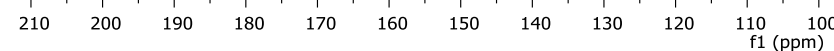




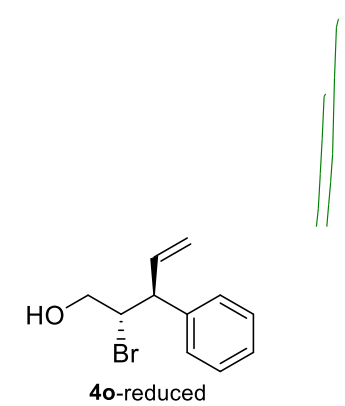

${ }^{1} \mathrm{H} \mathrm{NMR}, 400 \mathrm{MHz}$ in $\mathrm{CDCl}_{3}$

d.r. $13: 1$
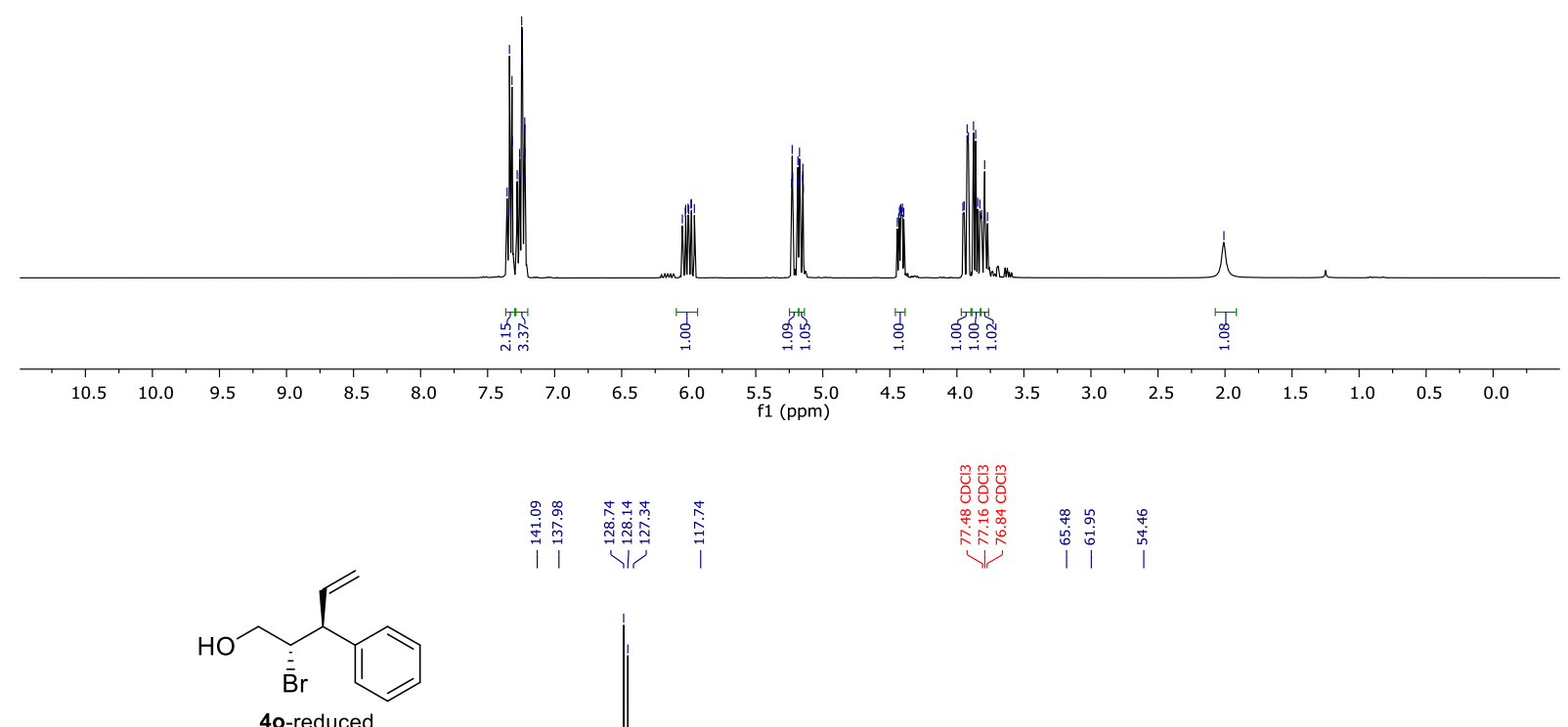

mom

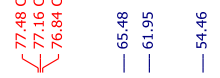

${ }^{13} \mathrm{C} \mathrm{NMR}, 101 \mathrm{MHz}$ in $\mathrm{CDCl}_{3}$

d.r. $13: 1$

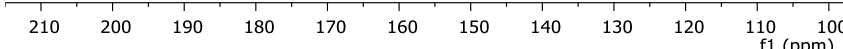



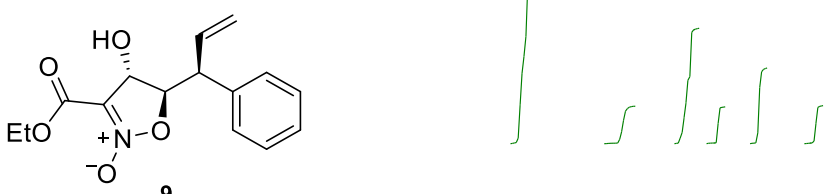

${ }^{1} \mathrm{H} \mathrm{NMR}, 400 \mathrm{MHz}$ in $\mathrm{CDCl}_{3}$

d.r. $8: 1$

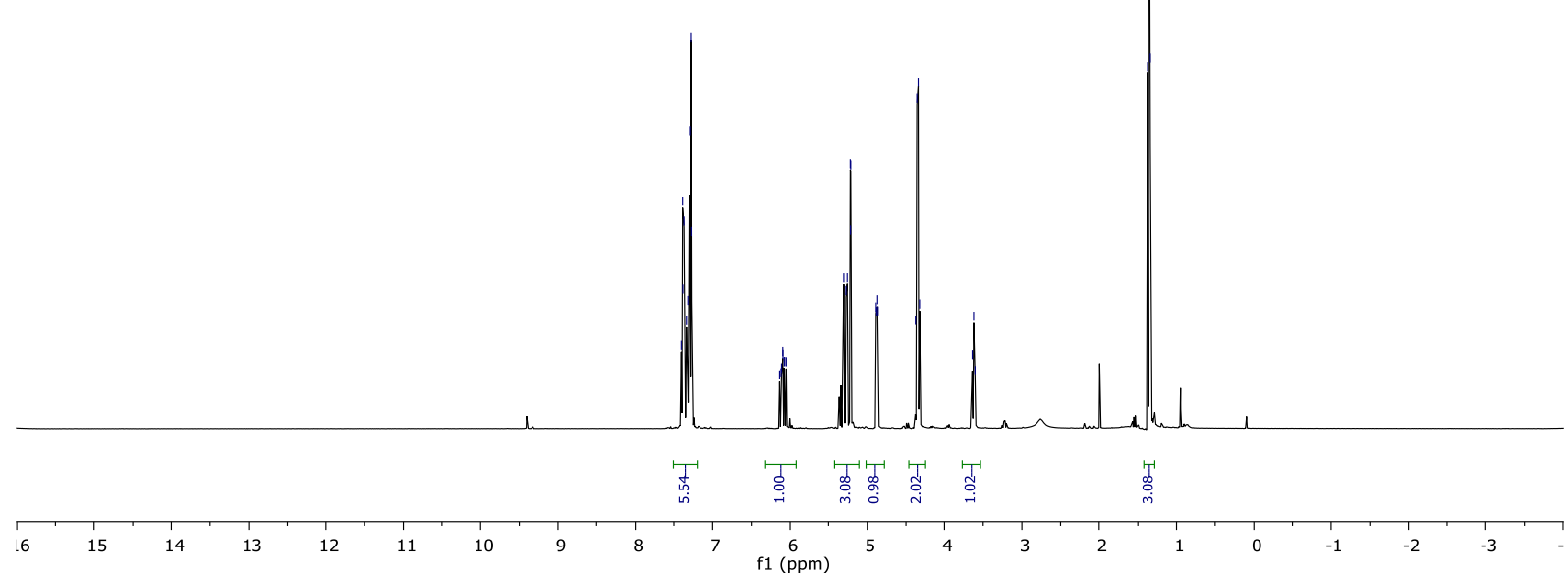

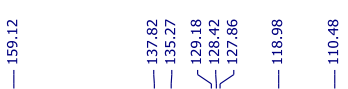

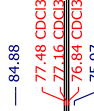

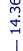

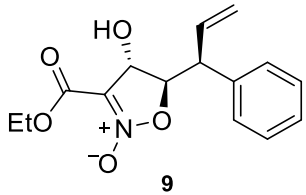

${ }^{13} \mathrm{C} \mathrm{NMR,} 101 \mathrm{MHz}$ in $\mathrm{CDCl}_{3}$ d.r. $8: 1$ 


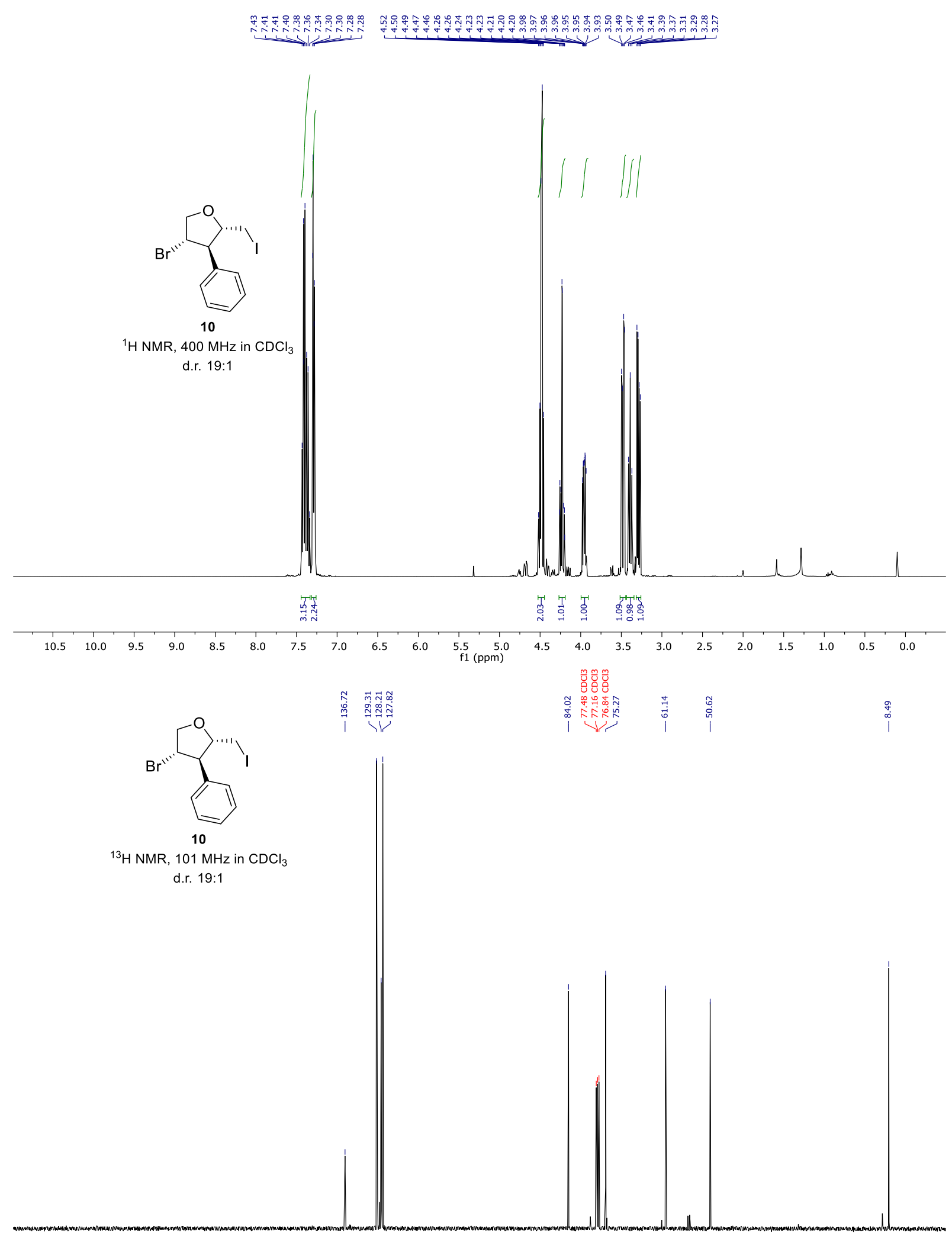

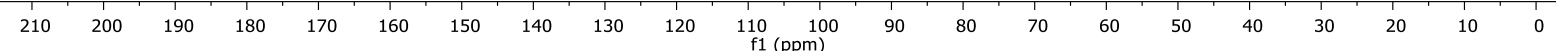



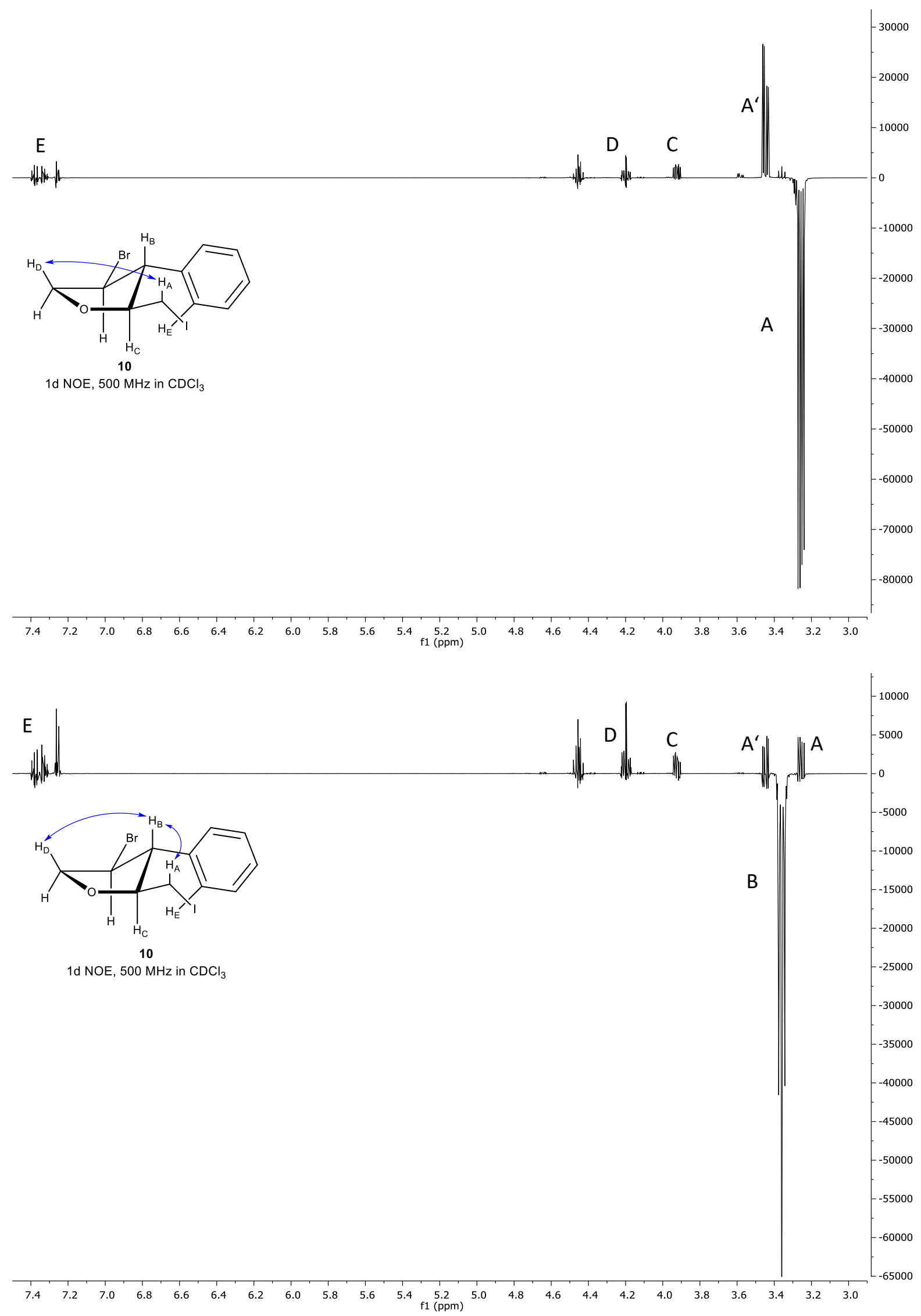

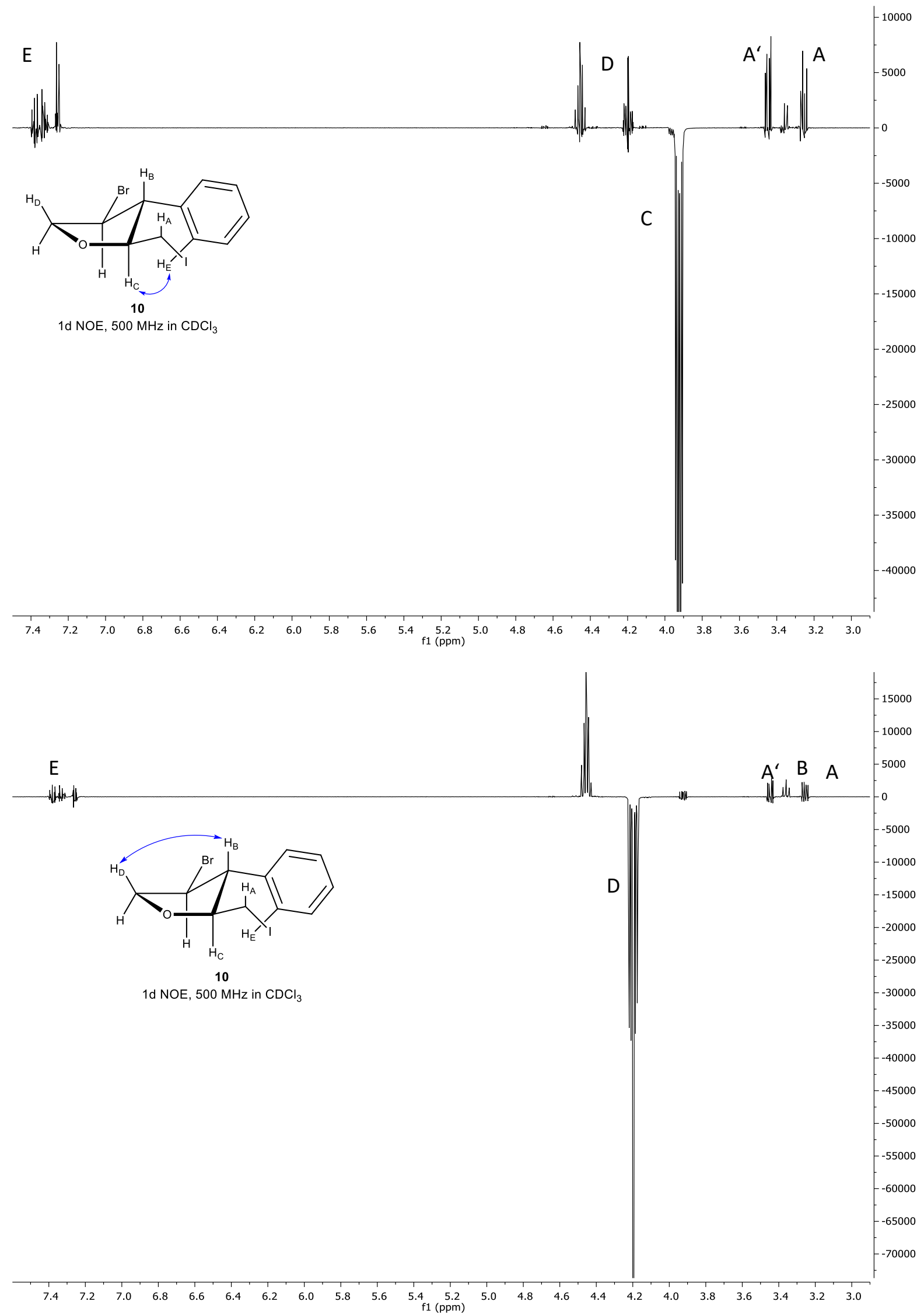


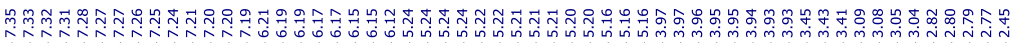

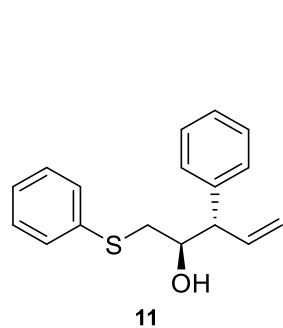

${ }^{1} \mathrm{H} \mathrm{NMR}, 400 \mathrm{MHz}$ in $\mathrm{CDCl}_{3}$ d.r. $13: 1$
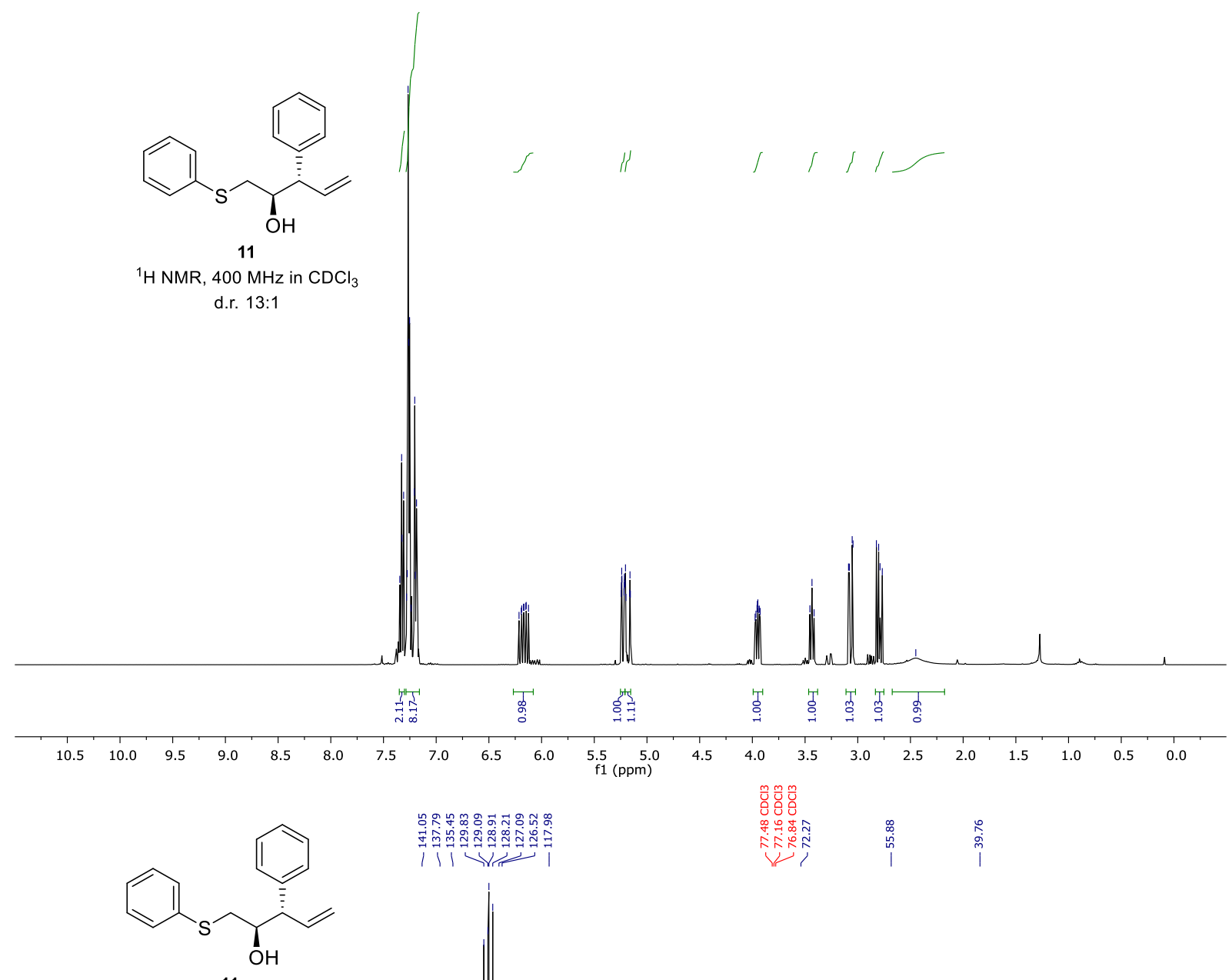

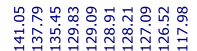

111 V

${ }^{13} \mathrm{C} \mathrm{NMR}, 101 \mathrm{MHz}$ in $\mathrm{CDCl}_{3}$

d.r. $13: 1$

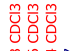

证i

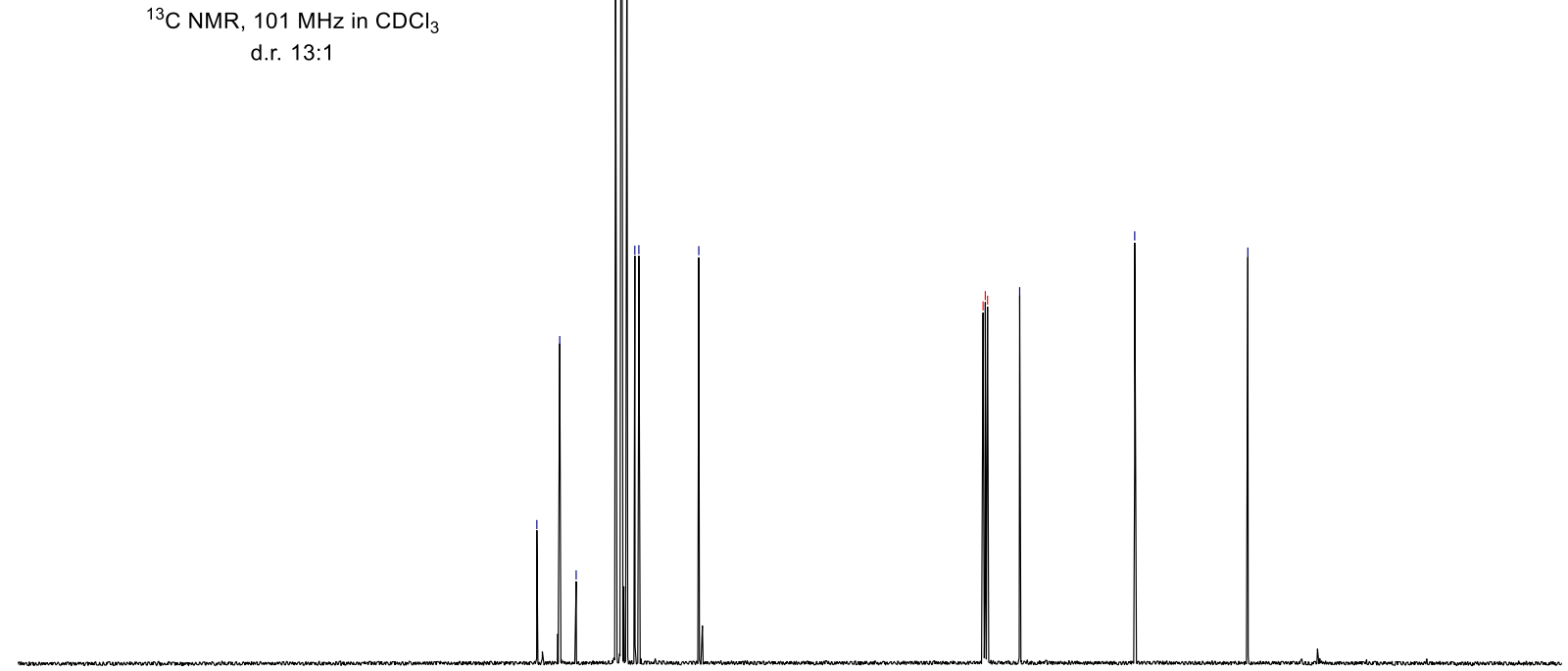

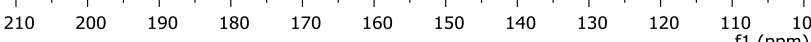




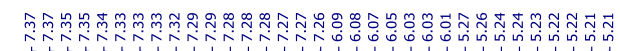

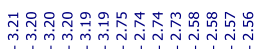

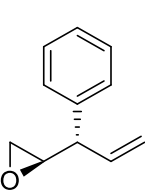

12

${ }^{1} \mathrm{H} \mathrm{NMR}, 400 \mathrm{MHz}$ in $\mathrm{CD}_{2} \mathrm{Cl}_{2}$

d.r. $14: 1$
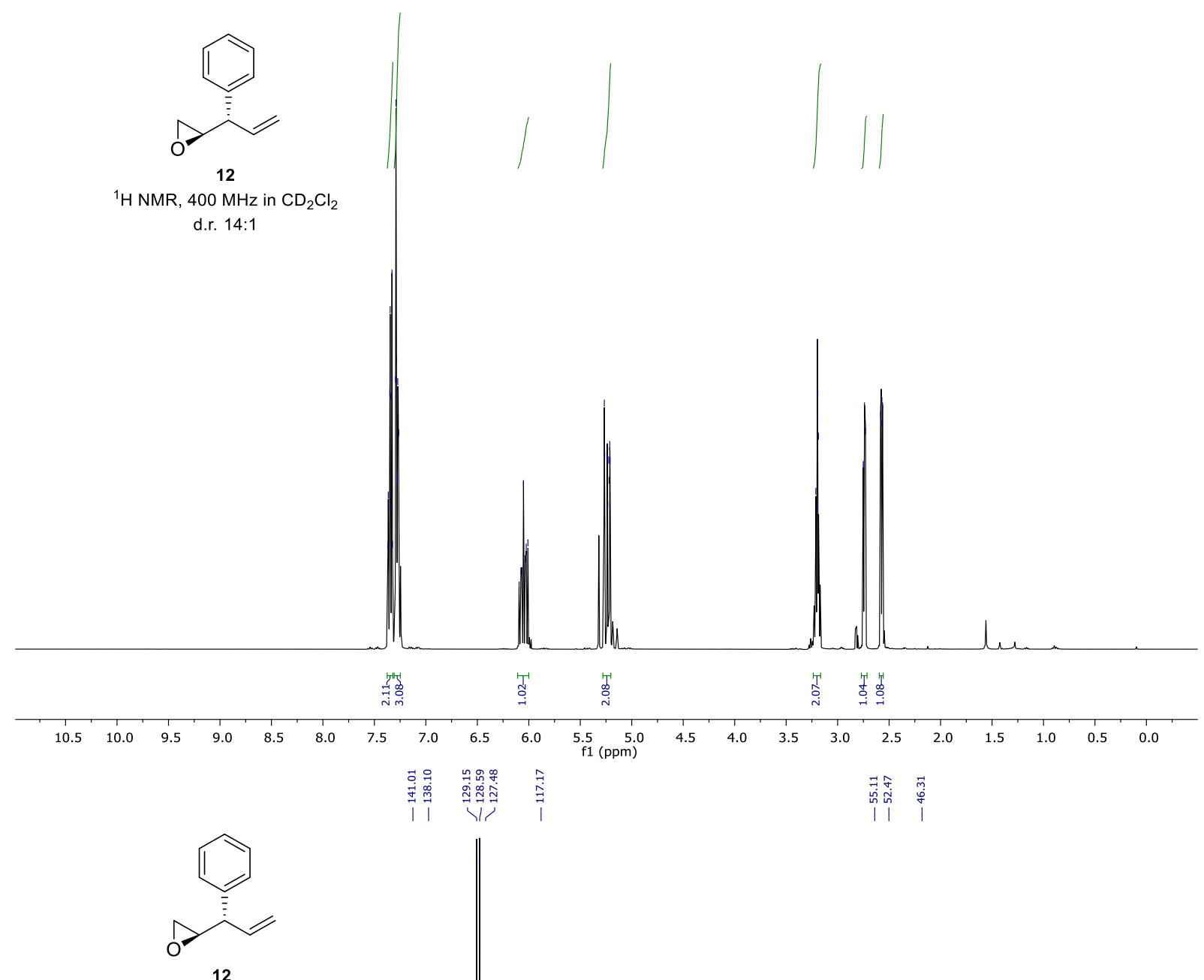

${ }^{13} \mathrm{C} \mathrm{NMR}, 101 \mathrm{MHz}$ in $\mathrm{CD}_{2} \mathrm{Cl}_{2}$

d.r. $14: 1$

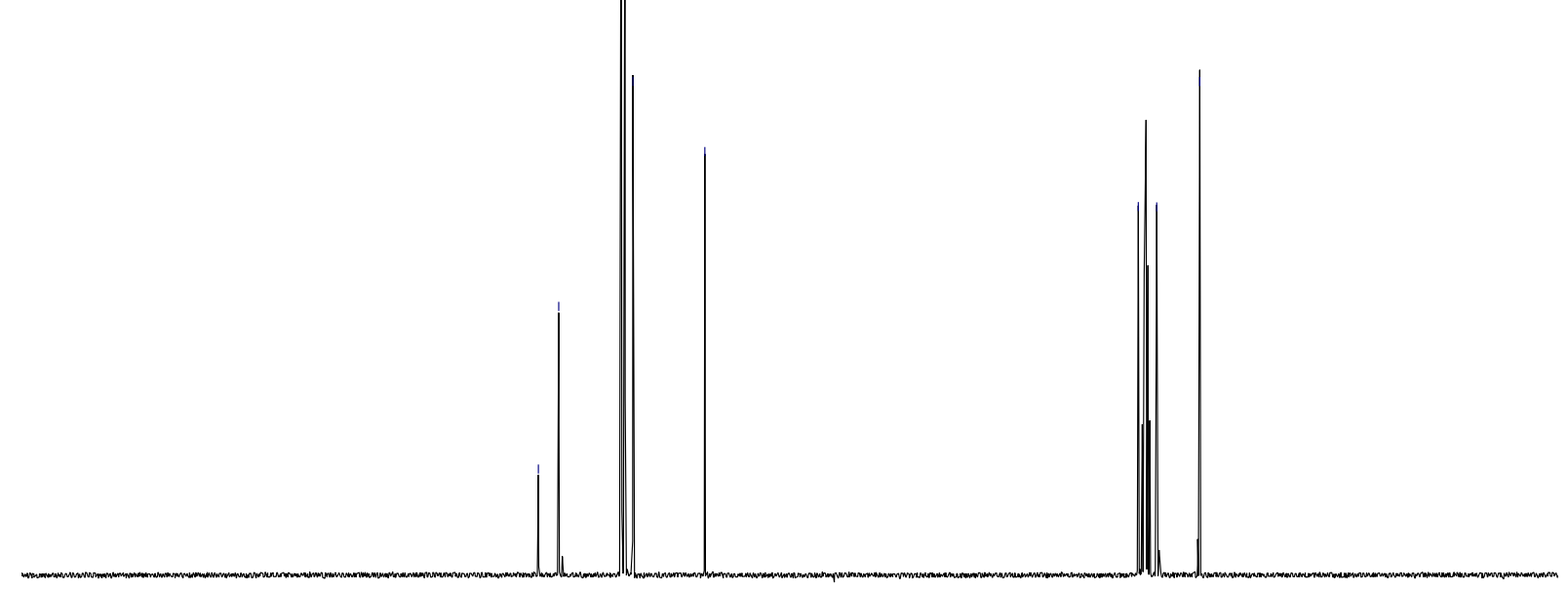

$\begin{array}{lllllllllllllllllllllll}210 & 200 & 190 & 180 & 170 & 160 & 150 & 140 & 130 & 120 & 110 & 100 & 90 & 80 & 70 & 60 & 50 & 40 & 30 & 20 & 10 & 0\end{array}$ 


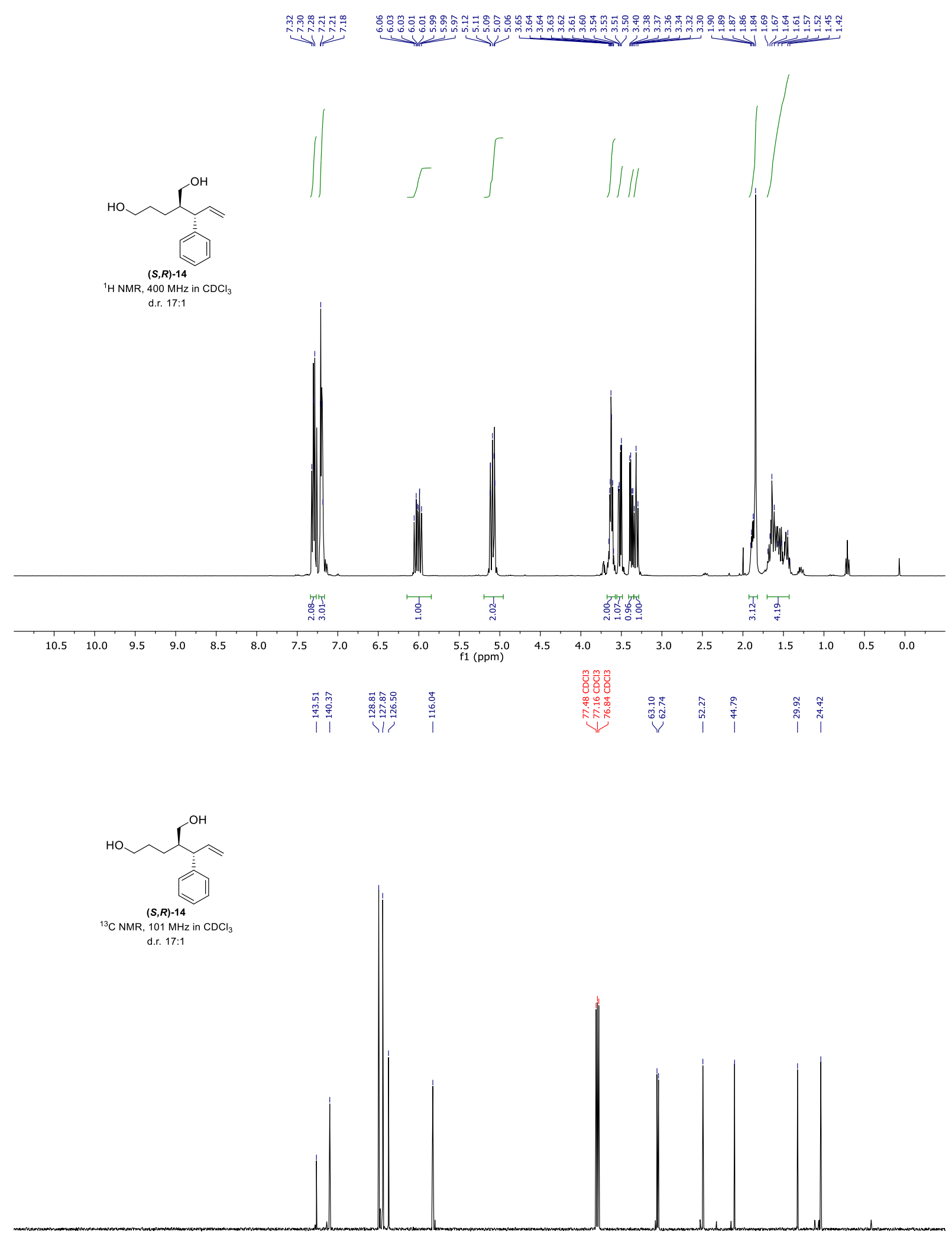

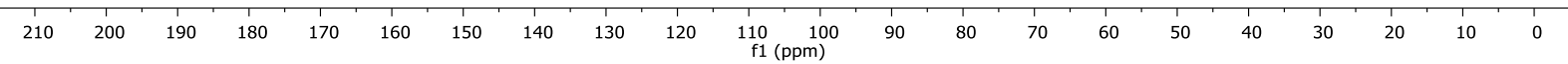



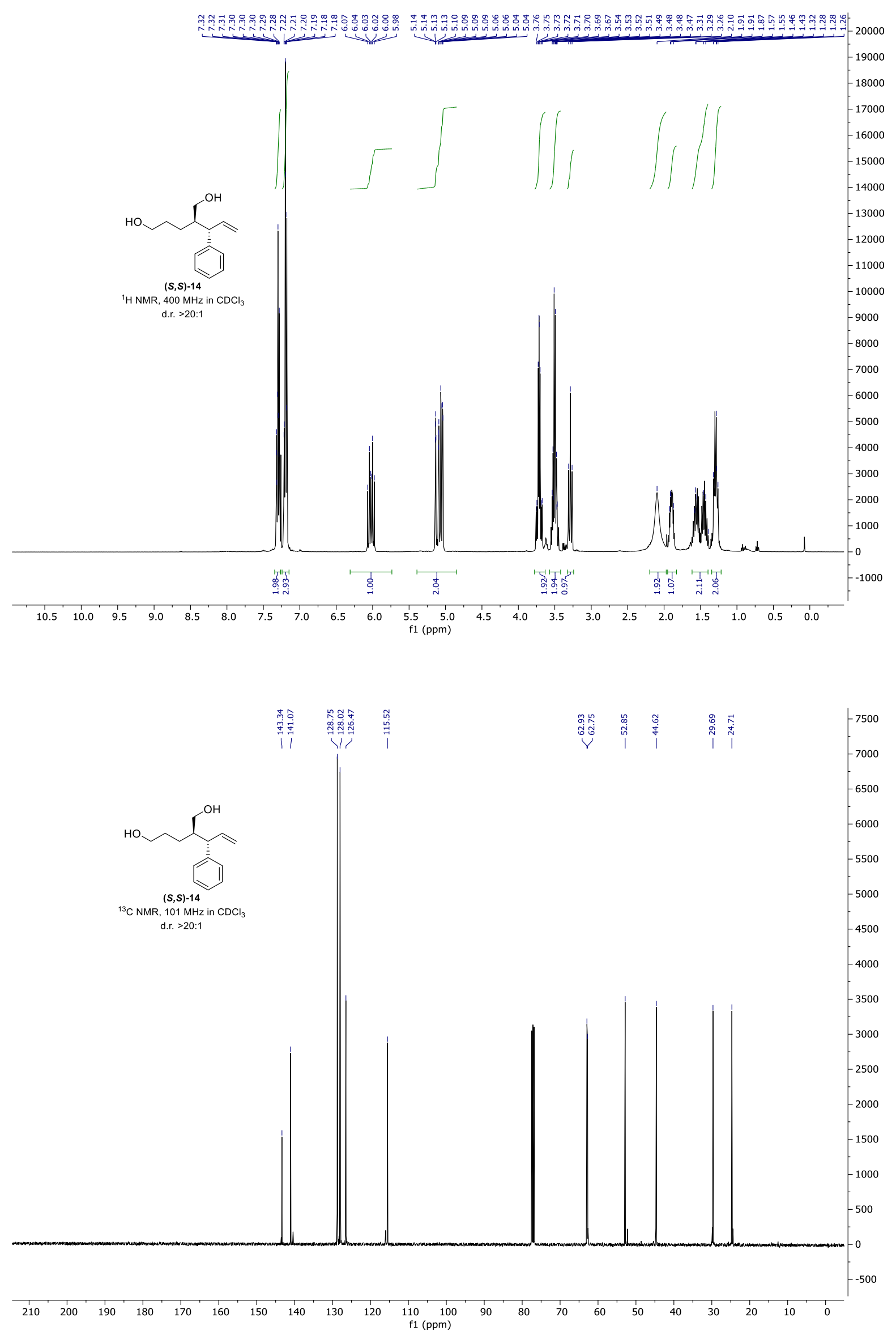


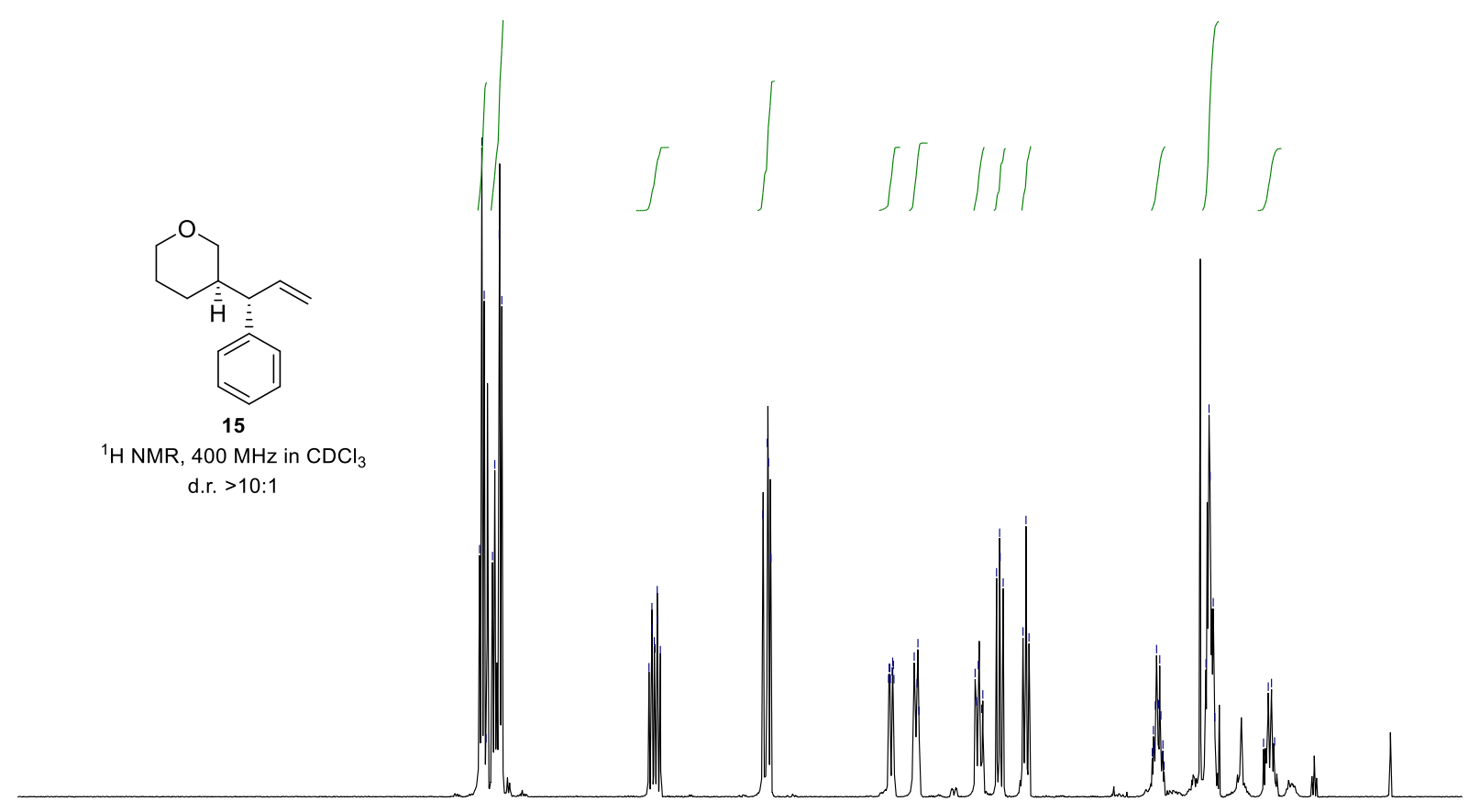

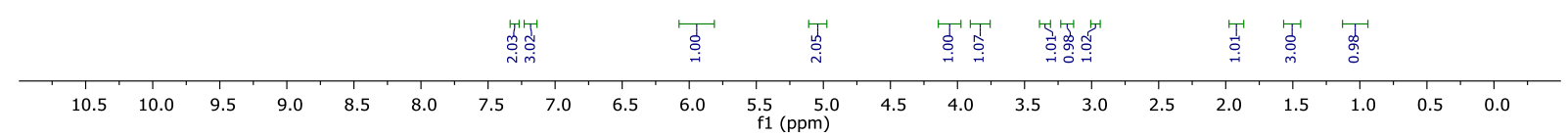
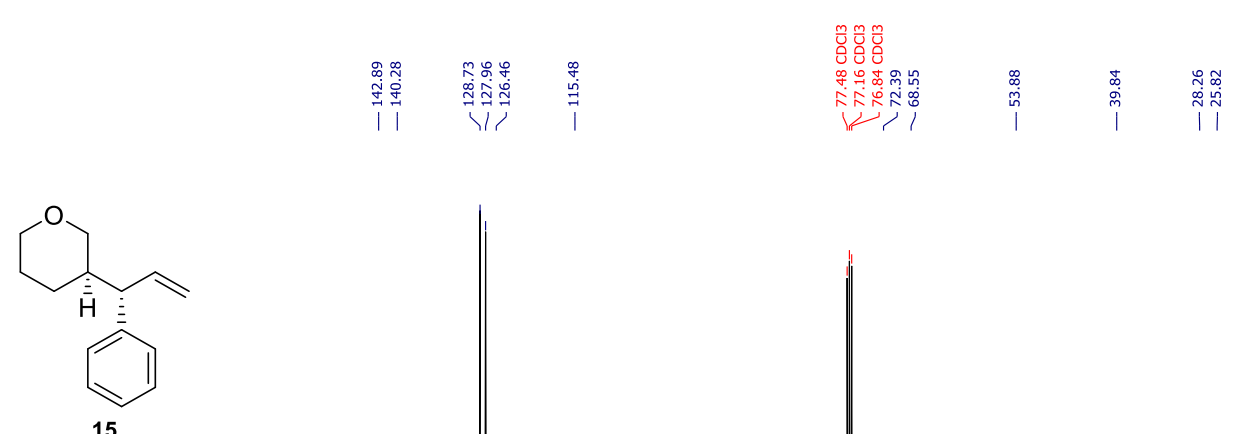

${ }^{13} \mathrm{C} \mathrm{NMR}, 101 \mathrm{MHz}$ in $\mathrm{CDCl}_{3}$

d.r. $>10: 1$

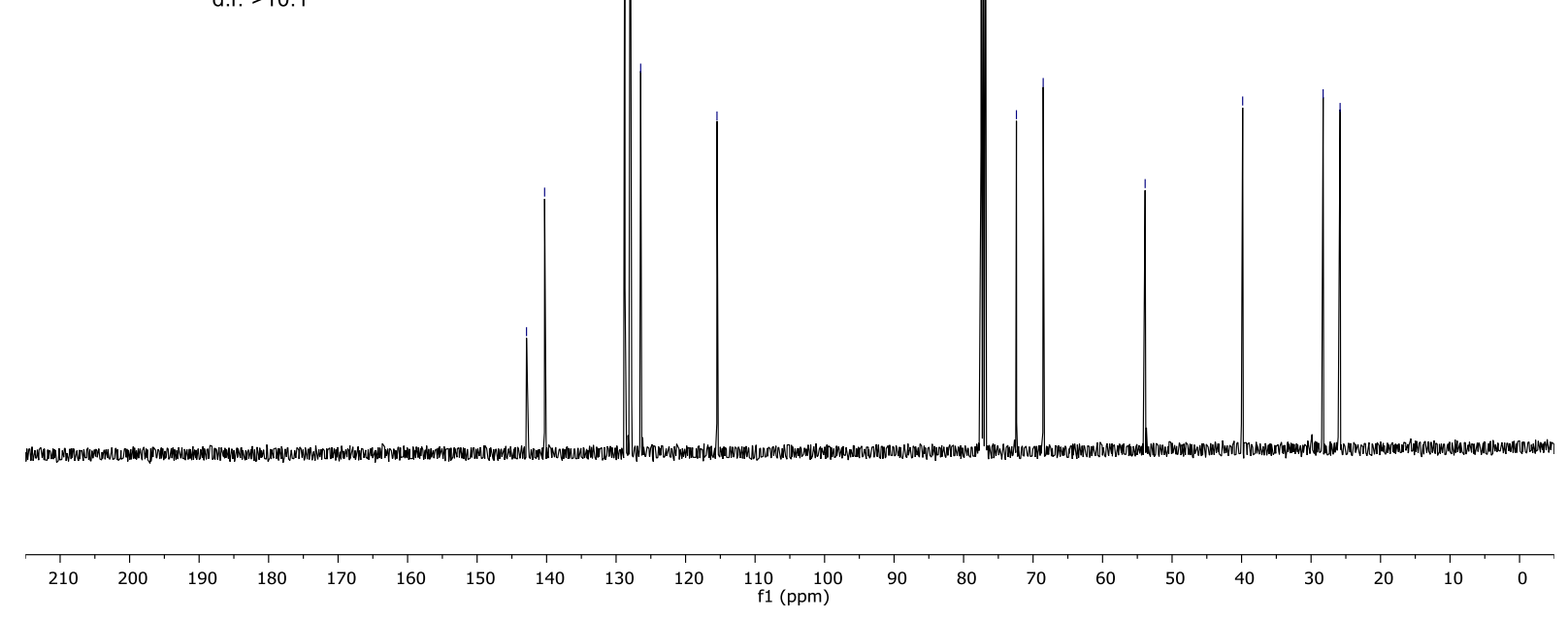



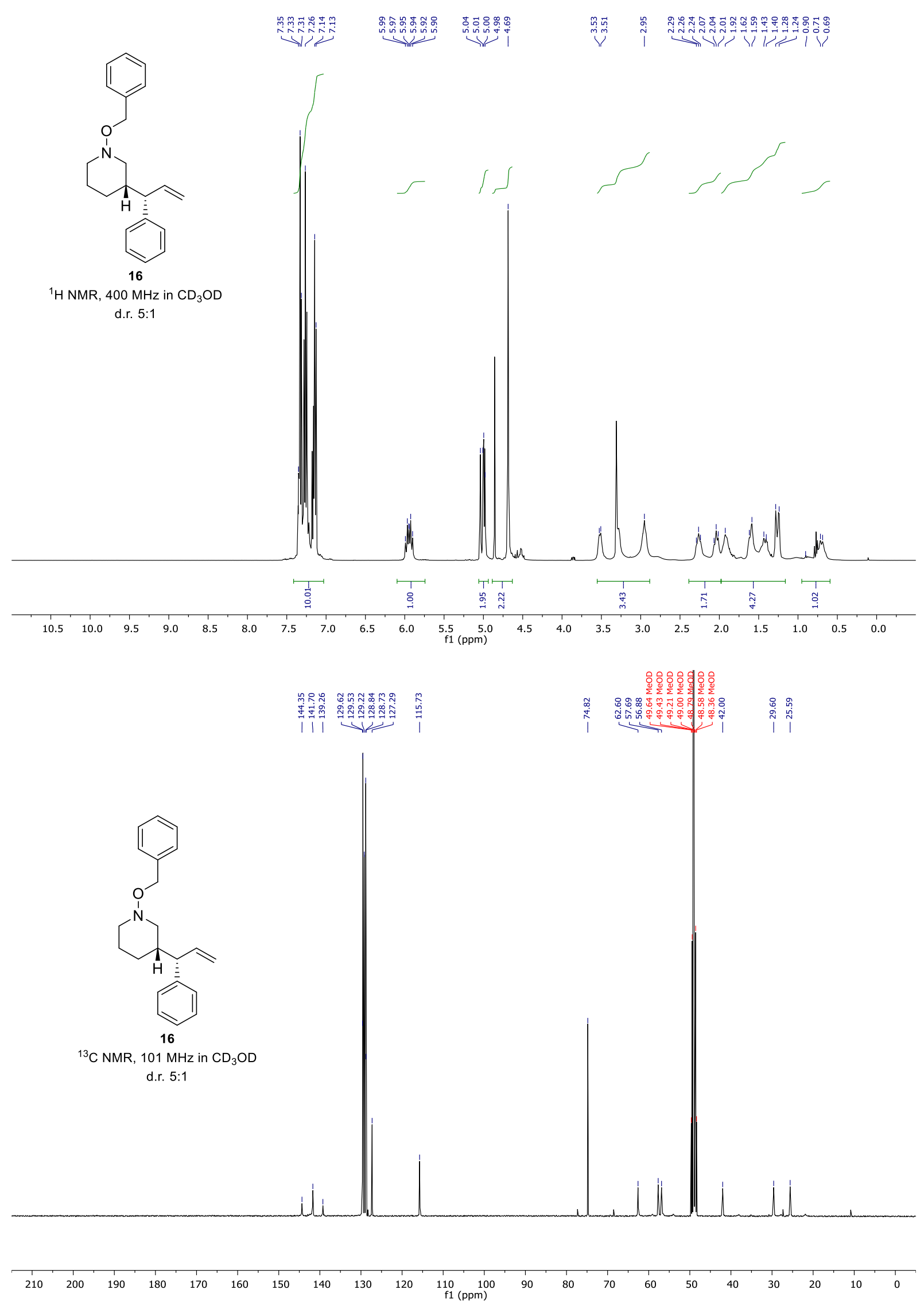


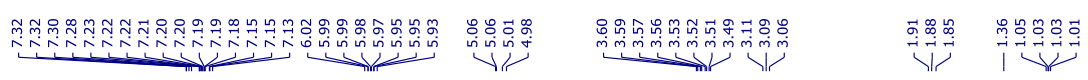

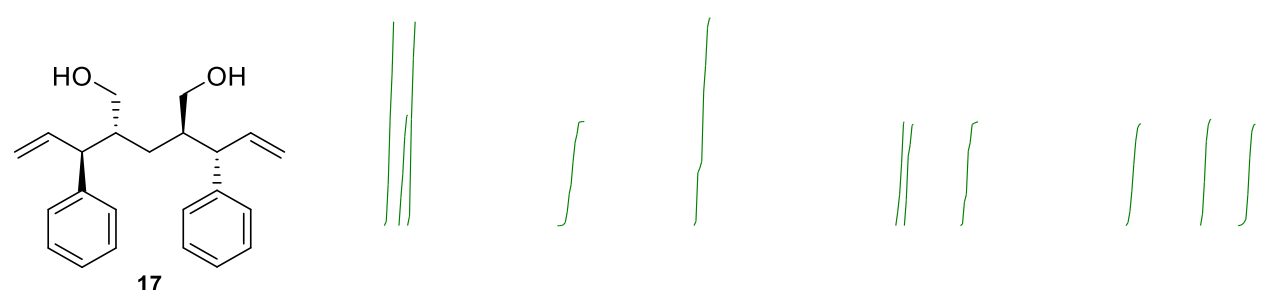

${ }^{1} \mathrm{H} \mathrm{NMR}, 400 \mathrm{MHz}$ in $\mathrm{CDCl}_{3}$

d.r. $>20: 1$

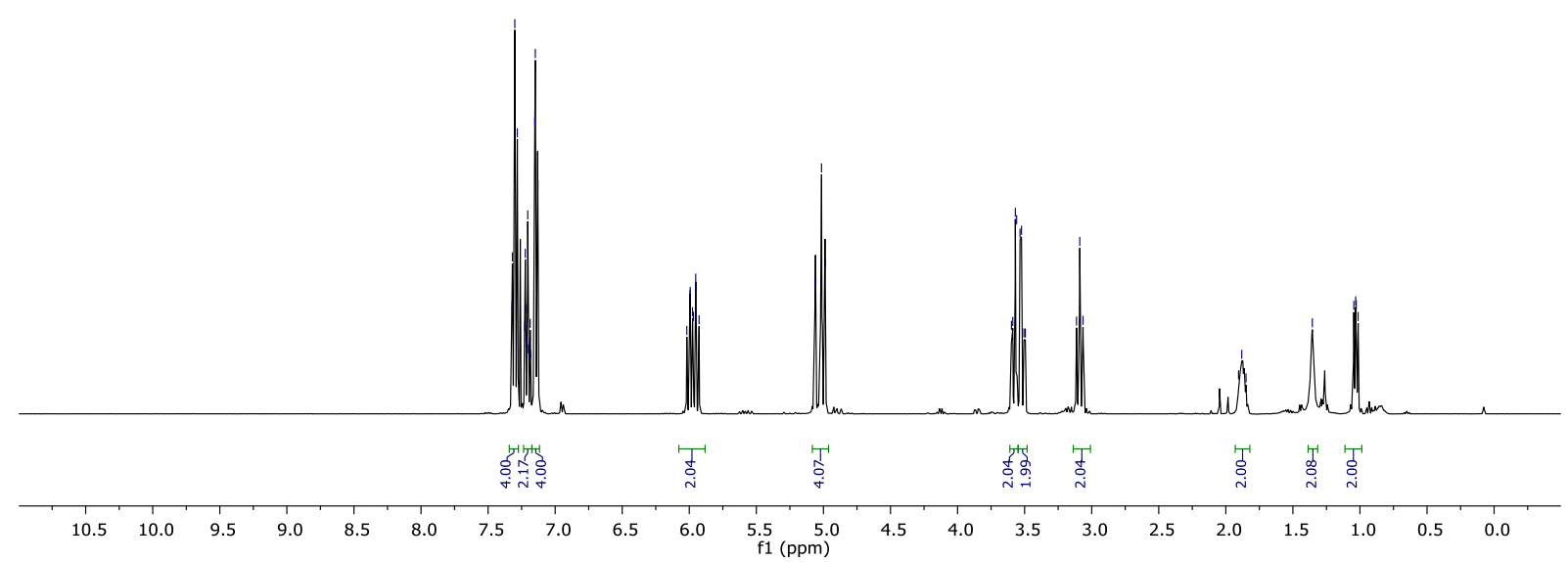

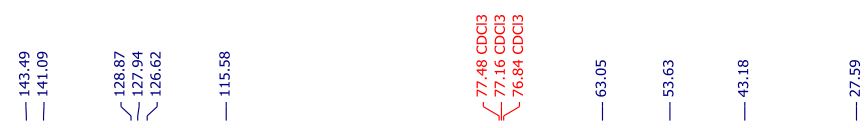<smiles>C=C[C@@H](c1ccccc1)[C@@H](CO)C[C@H](CO)[C@@H](C=C)c1ccccc1</smiles>

${ }^{13} \mathrm{C} \mathrm{NMR,}, 101 \mathrm{MHz}$ in $\mathrm{CDCl}_{3}$ d.r. $>20: 1$

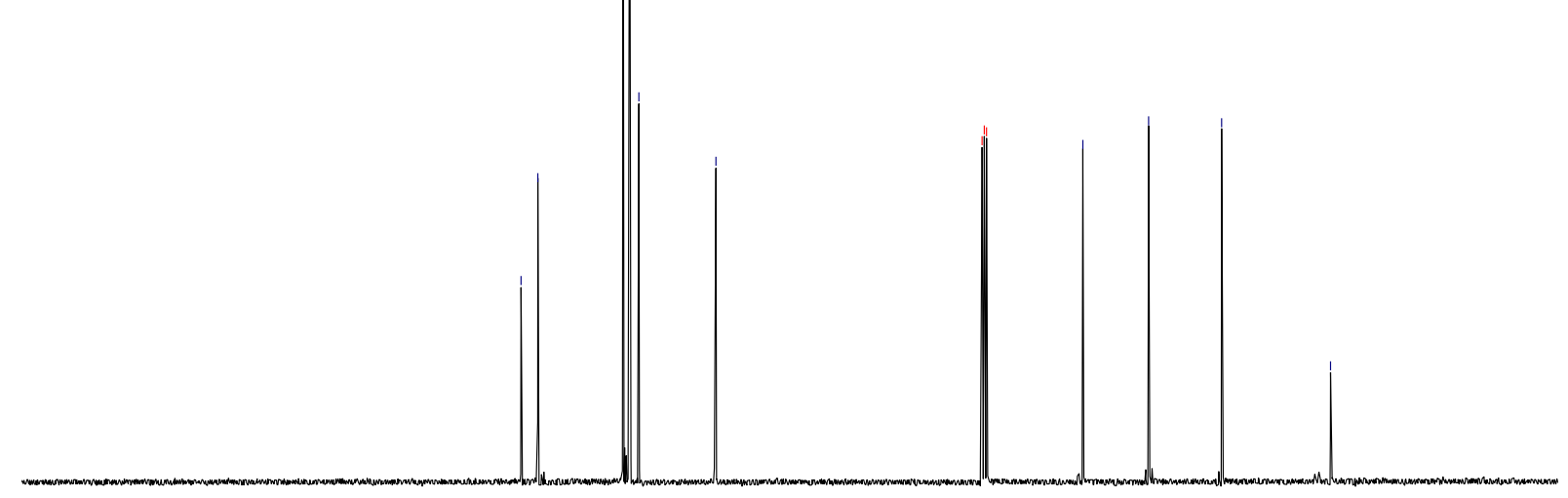

$\begin{array}{llllllllllllllllllllllll}210 & 200 & 190 & 180 & 170 & 160 & 150 & 140 & 130 & 120 & 110 & 100 & 90 & 80 & 70 & 60 & 50 & 40 & 30 & 20 & 10 & 0\end{array}$ 

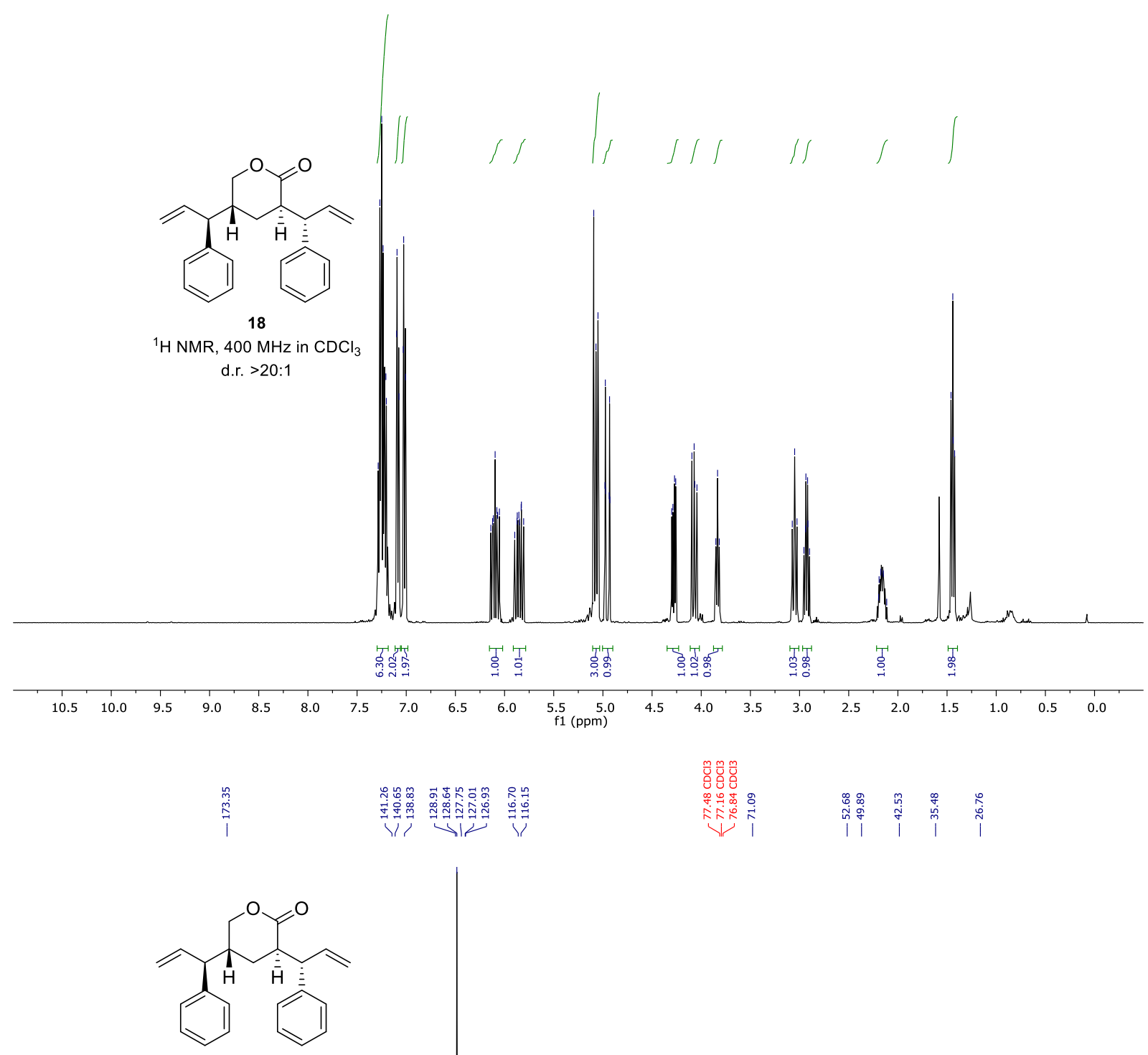

18

${ }^{13} \mathrm{C} \mathrm{NMR}, 101 \mathrm{MHz}$ in $\mathrm{CDCl}_{3}$ d.r. $>20: 1$

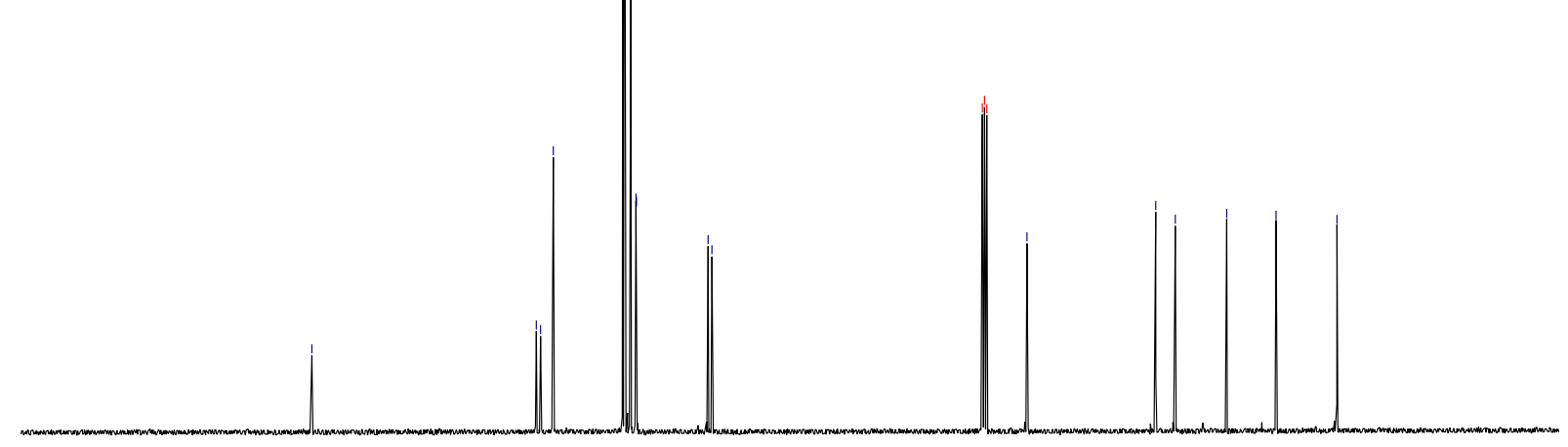

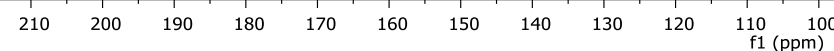




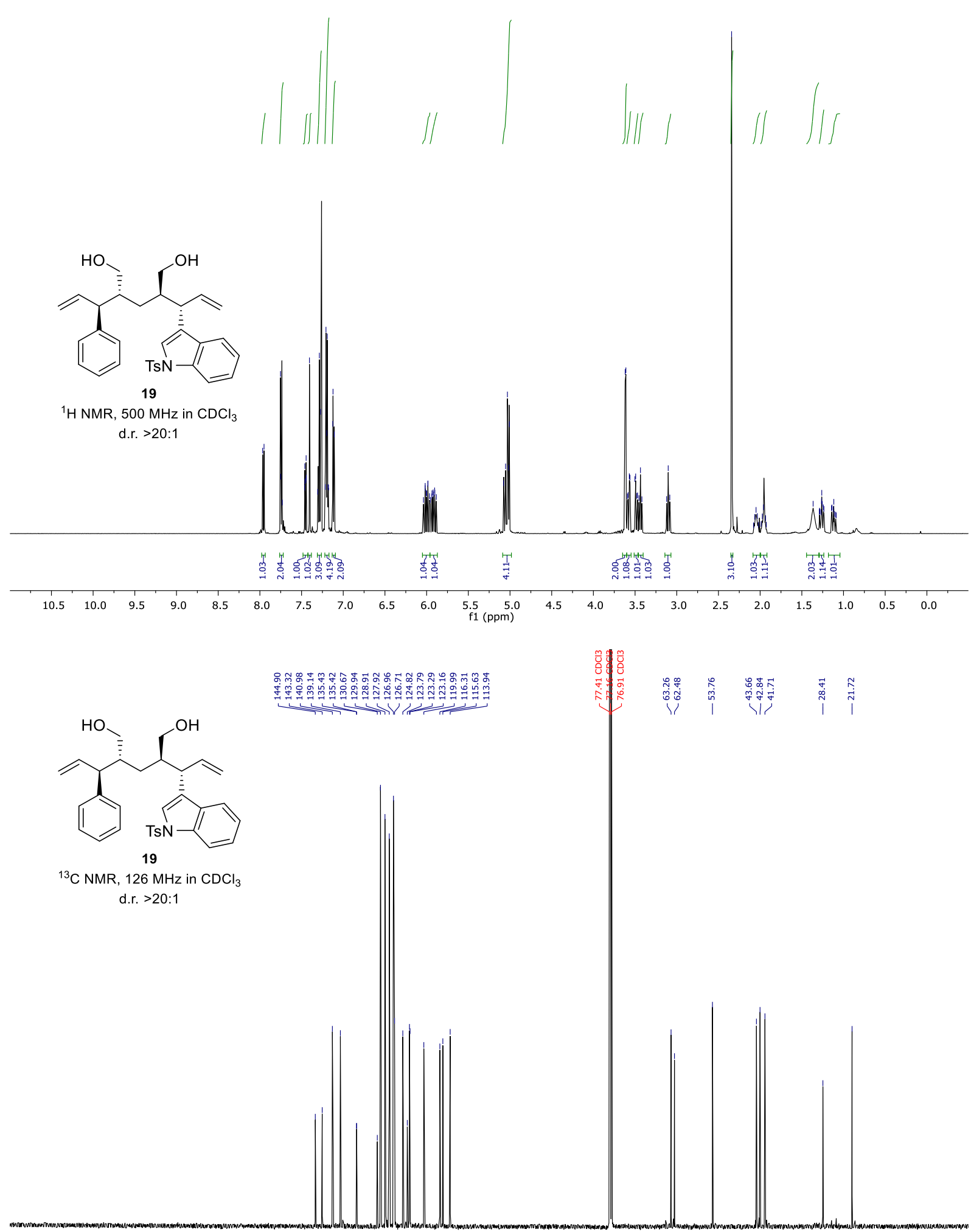

\begin{tabular}{|lllllllllllllllllllllllll}
210 & 200 & 190 & 180 & 170 & 160 & 150 & 140 & 130 & 120 & 110 & 100 & 90 & 80 & 70 & 60 & 50 & 40 & 30 & 20 & 10 & 0
\end{tabular} 


\section{SFC Traces}

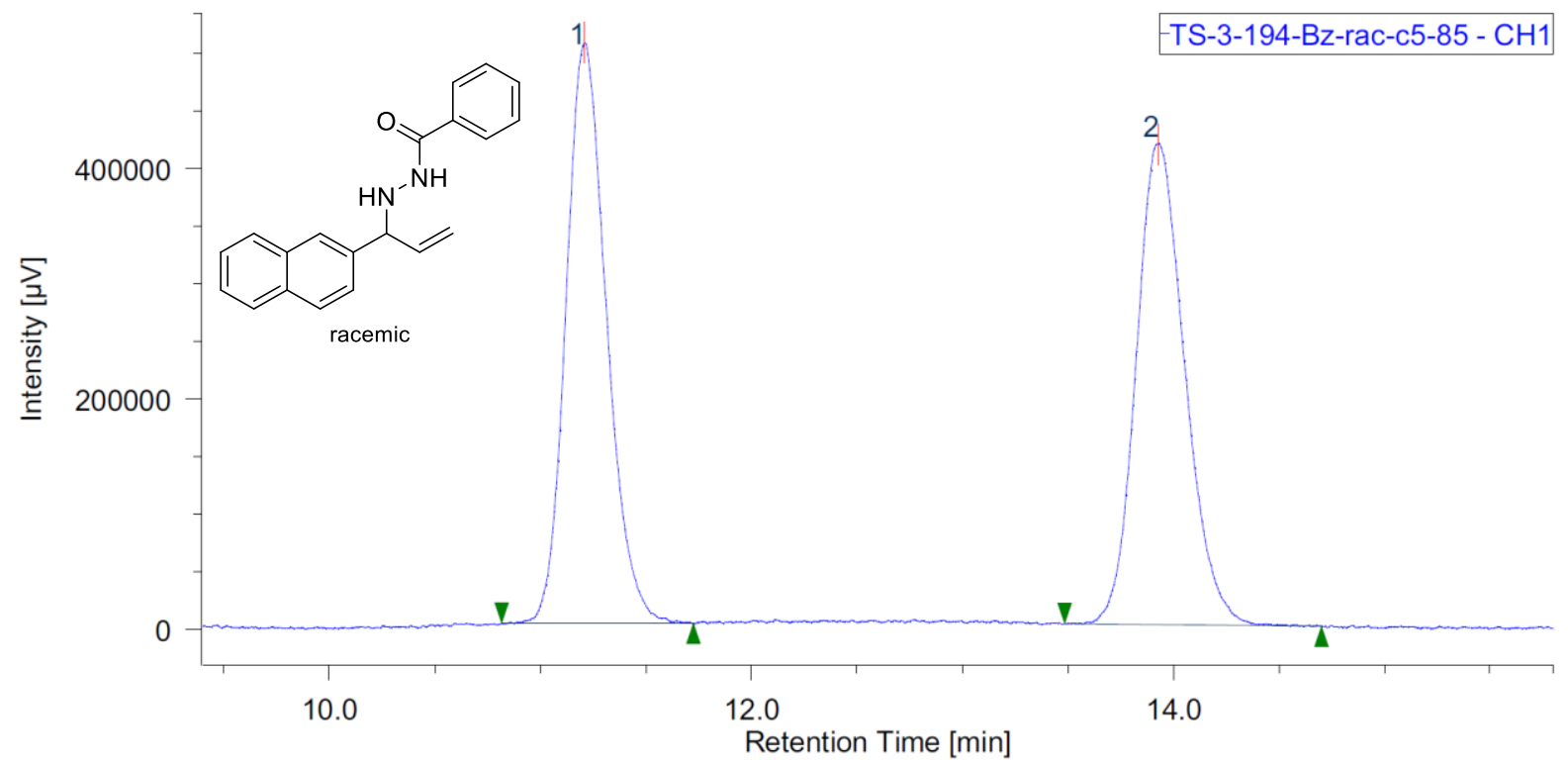

\begin{tabular}{|r|c|c|r|r|r|r|r|r|r|r|r|}
\hline$\#$ & Peak Name & $\mathrm{CH}$ & tR $[\mathrm{min}]$ & Area $[\mu \mathrm{V} \cdot \mathrm{sec}]$ & Height $[\mu \mathrm{V}]$ & \multicolumn{1}{|c|}{ Area $\%$} & Height $\%$ & Quantity & NTP & Resolution & Symmetry Factor \\
\hline 1 & Unknown & 1 & 11.208 & 6720140 & 504539 & 50.124 & 54.762 & N/A & 16748 & 7.131 & 1.184 \\
\hline 2 Unknown & 1 & 13.925 & 6686871 & 416794 & 49.876 & 45.238 & N/A & 17800 & N/A & 1.174 \\
\hline
\end{tabular}

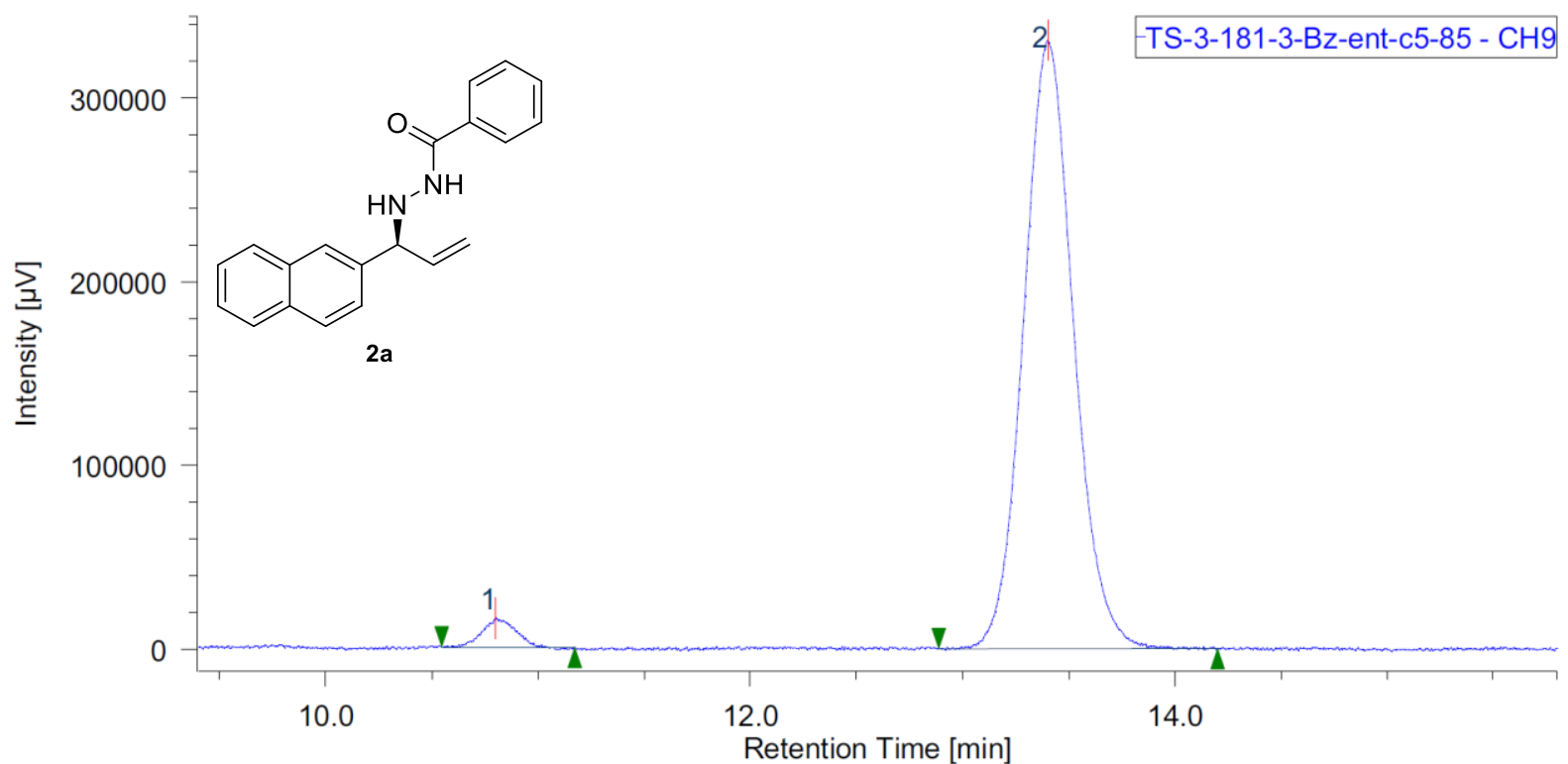

\begin{tabular}{|r|c|c|r|r|r|r|r|r|r|r|r|}
\hline$\#$ & Peak Name & CH & tR $[\mathrm{min}]$ & Area $[\mu \mathrm{V} \cdot \mathrm{sec}]$ & Height $[\mu \mathrm{V}]$ & Area $\%$ & Height $\%$ & Quantity & NTP & Resolution & Symmetry Factor \\
\hline 1 & Unknown & 9 & 10.800 & 170067 & 15720 & 3.058 & 4.534 & N/A & 21864 & 7.307 & 1.112 \\
\hline 2 & Unknown & 9 & 13.400 & 5392025 & 331008 & 96.942 & 95.466 & N/A & 16181 & N/A & 1.094 \\
\hline
\end{tabular}




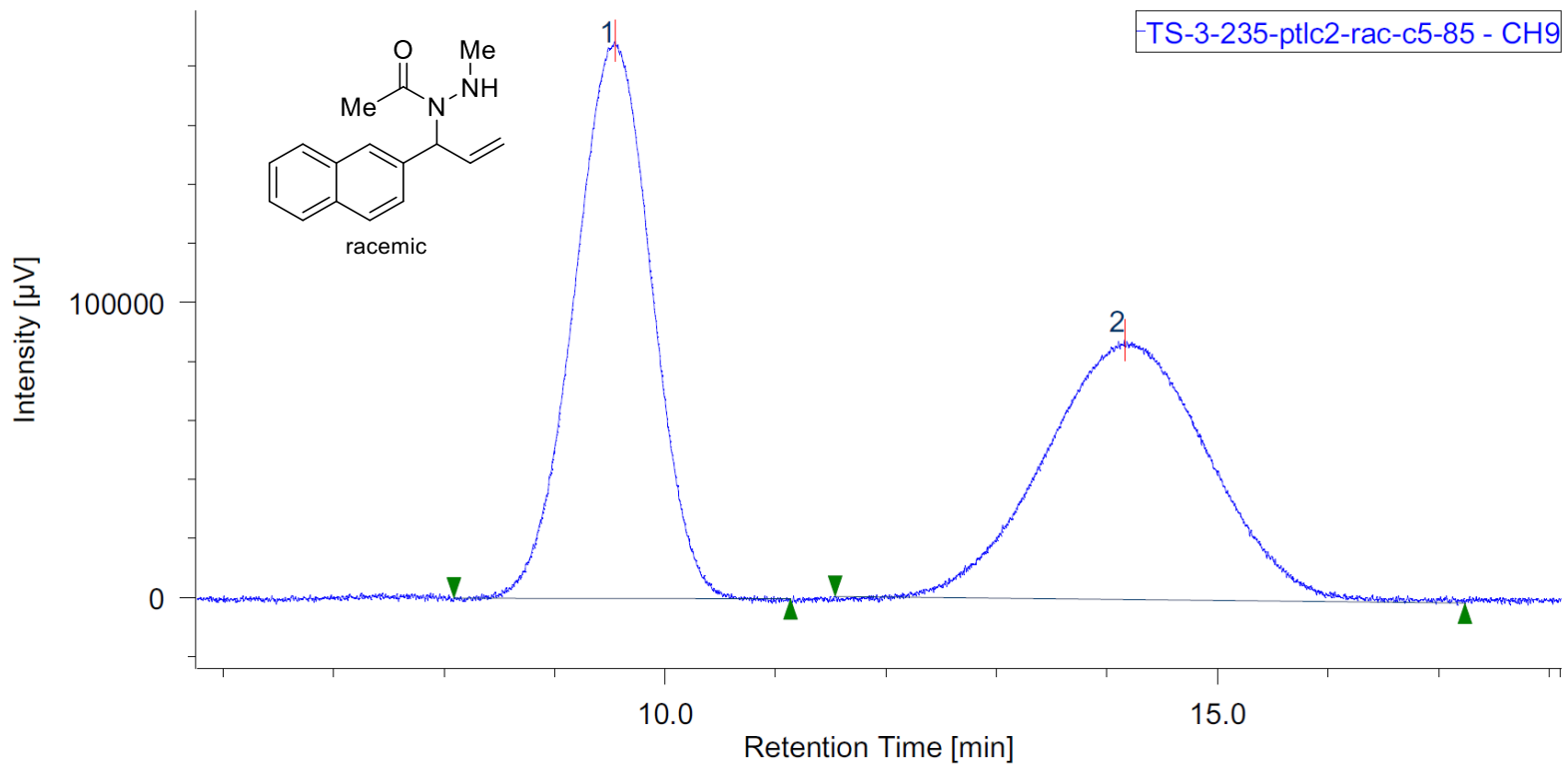

\begin{tabular}{|c|c|c|r|r|r|r|r|r|r|r|r|r|}
\hline$\#$ & Peak Name & $\mathrm{CH}$ & tR $[\mathrm{min}]$ & Area $[\mu \mathrm{V} \cdot \mathrm{sec}]$ & Height $[\mu \mathrm{V}]$ & Area $\%$ & Height $\%$ & Quantity & NTP & Resolution & Symmetry Factor & Warning \\
\hline 1 & Unknown & 9 & 9.547 & 9206854 & 189068 & 50.519 & 68.252 & $\mathrm{~N} / \mathrm{A}$ & 860 & 2.266 & 0.972 & \\
\hline 2 & Unknown & 9 & 14.153 & 9017739 & 87948 & 49.481 & 31.748 & $\mathrm{~N} / \mathrm{A}$ & 416 & $\mathrm{~N} / \mathrm{A}$ & & 1.006 \\
\hline
\end{tabular}

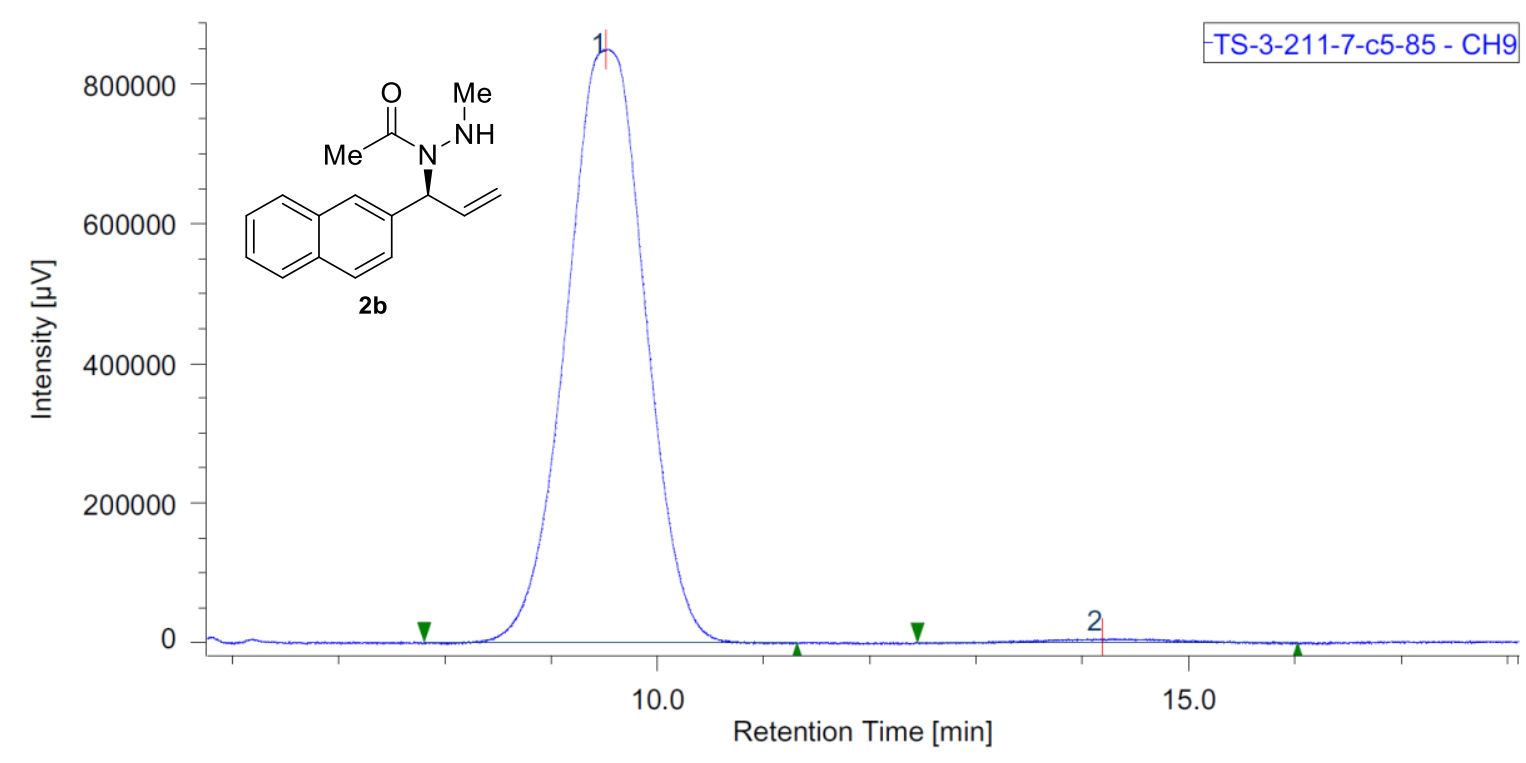

\begin{tabular}{|c|c|c|c|c|c|c|c|c|c|c|c|c|}
\hline$\#$ & Peak Name & $\mathrm{CH}$ & $\mathrm{tR}$ [min] & Area $[\mu \mathrm{V} \cdot \mathrm{sec}]$ & Height $[\mu \mathrm{V}]$ & Area $\%$ & Height $\%$ & Quantity & NTP & Resolution & Symmetry Factor & Warning \\
\hline & Unknown & 9 & 9.513 & 43598378 & 84820 & 99.099 & 99.310 & $\mathrm{~N} / \mathrm{A}$ & 783 & 2.405 & 0.994 & \\
\hline & Unknown & 9 & 14.187 & 396421 & 5893 & 0.901 & 0.690 & $\mathrm{~N} / \mathrm{A}$ & 500 & $\mathrm{~N} / \mathrm{A}$ & 1.047 & \\
\hline
\end{tabular}




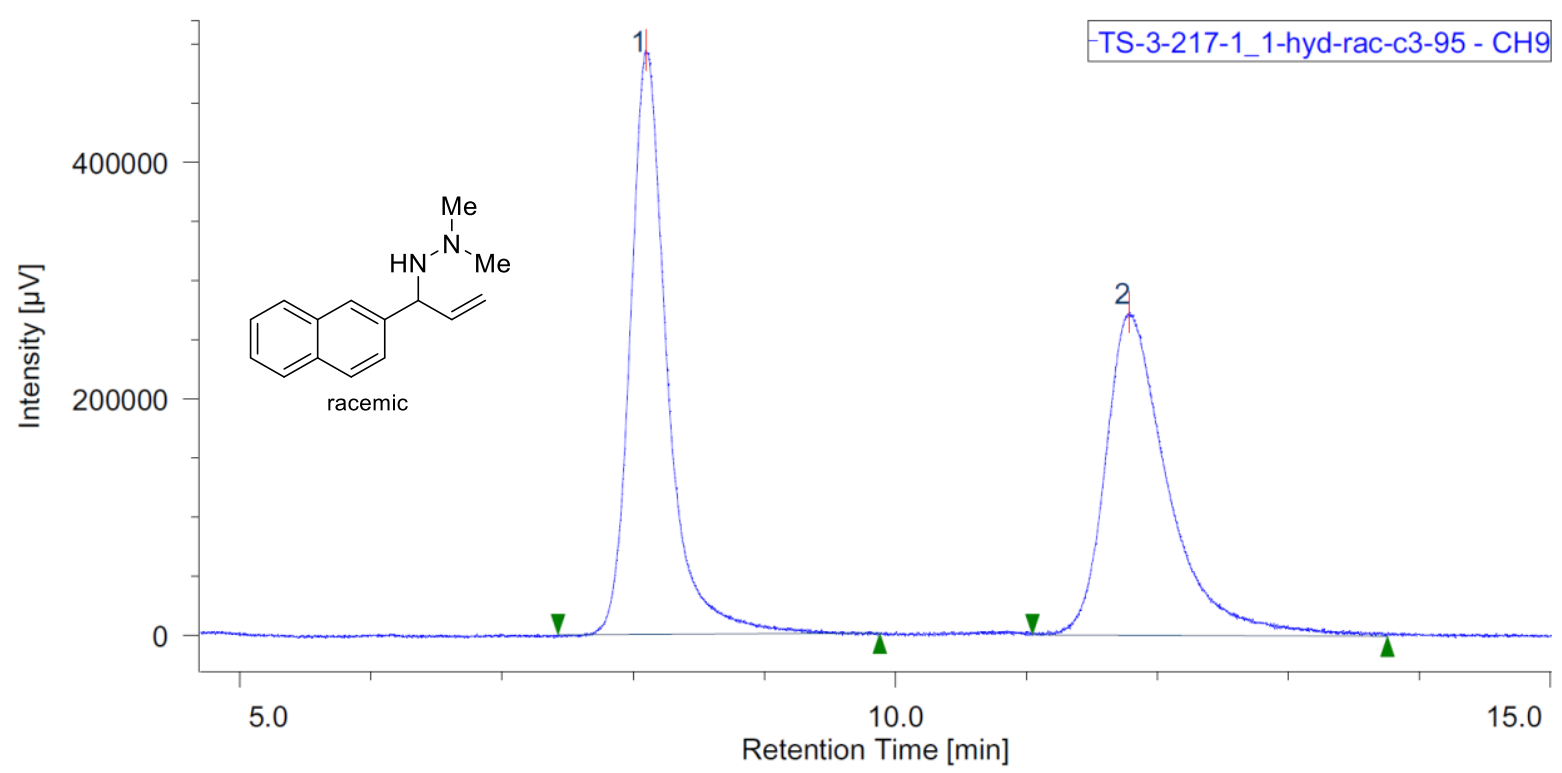

\begin{tabular}{|c|c|c|c|c|c|c|c|c|c|c|}
\hline Peak Name & $\mathrm{CH}$ & tR [min] & Area $[\mu \mathrm{V} \cdot \mathrm{sec}]$ & Height $[\mu \mathrm{V}]$ & Area $\%$ & Height $\%$ & Quantity & NTP & Resolution & Symmetry Factor \\
\hline Unknown & 9 & 8.100 & 9710891 & 493978 & 51.626 & 64.408 & $\mathrm{~N} / \mathrm{A}$ & 4741 & 5.822 & 1.344 \\
\hline 2 Unknown & 9 & 11.787 & 9099150 & 272969 & 48.374 & 35.592 & $\mathrm{~N} / \mathrm{A}$ & 3479 & N/A & 1.642 \\
\hline
\end{tabular}

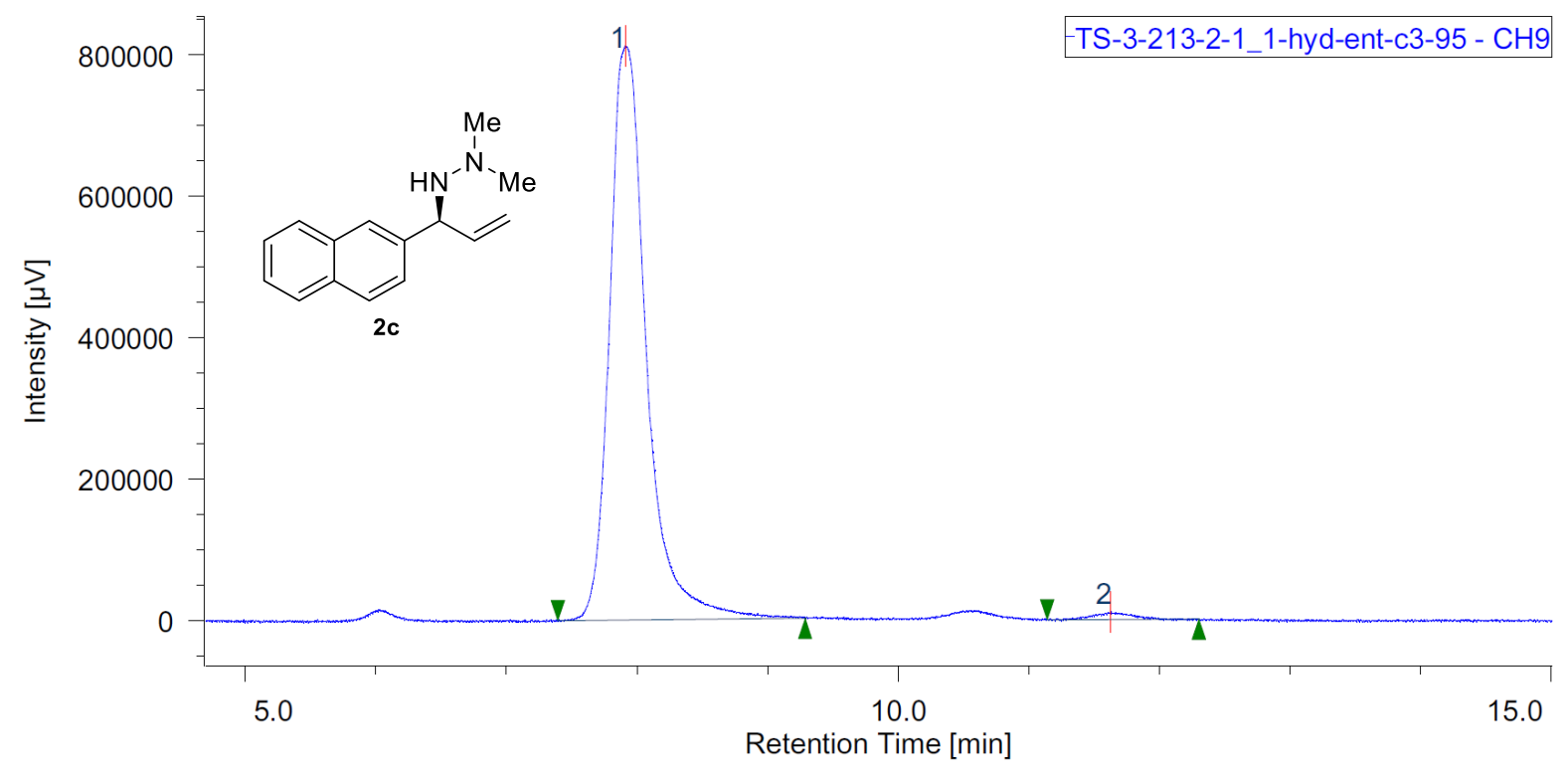

\begin{tabular}{|r|c|c|r|r|r|r|r|r|r|r|r|}
\hline$\#$ & Peak Name & $\mathrm{CH}$ & tR $[\mathrm{min}]$ & Area $[\mu \mathrm{V} \cdot \mathrm{sec}]$ & Height $[\mu \mathrm{V}]$ & \multicolumn{1}{|c|}{ Area\% } & Height $\%$ & Quantity & NTP & Resolution & Symmetry Factor \\
\hline 1 & Unknown & 9 & 7.907 & 15937663 & 811802 & 98.593 & 98.677 & $\mathrm{~N} / \mathrm{A}$ & 4473 & 6.993 & 1.376 \\
\hline 2 & Unknown & 9 & 11.627 & 227393 & 10880 & 1.407 & 1.323 & $\mathrm{~N} / \mathrm{A}$ & 6132 & $\mathrm{~N} / \mathrm{A}$ & 1.133 \\
\hline
\end{tabular}




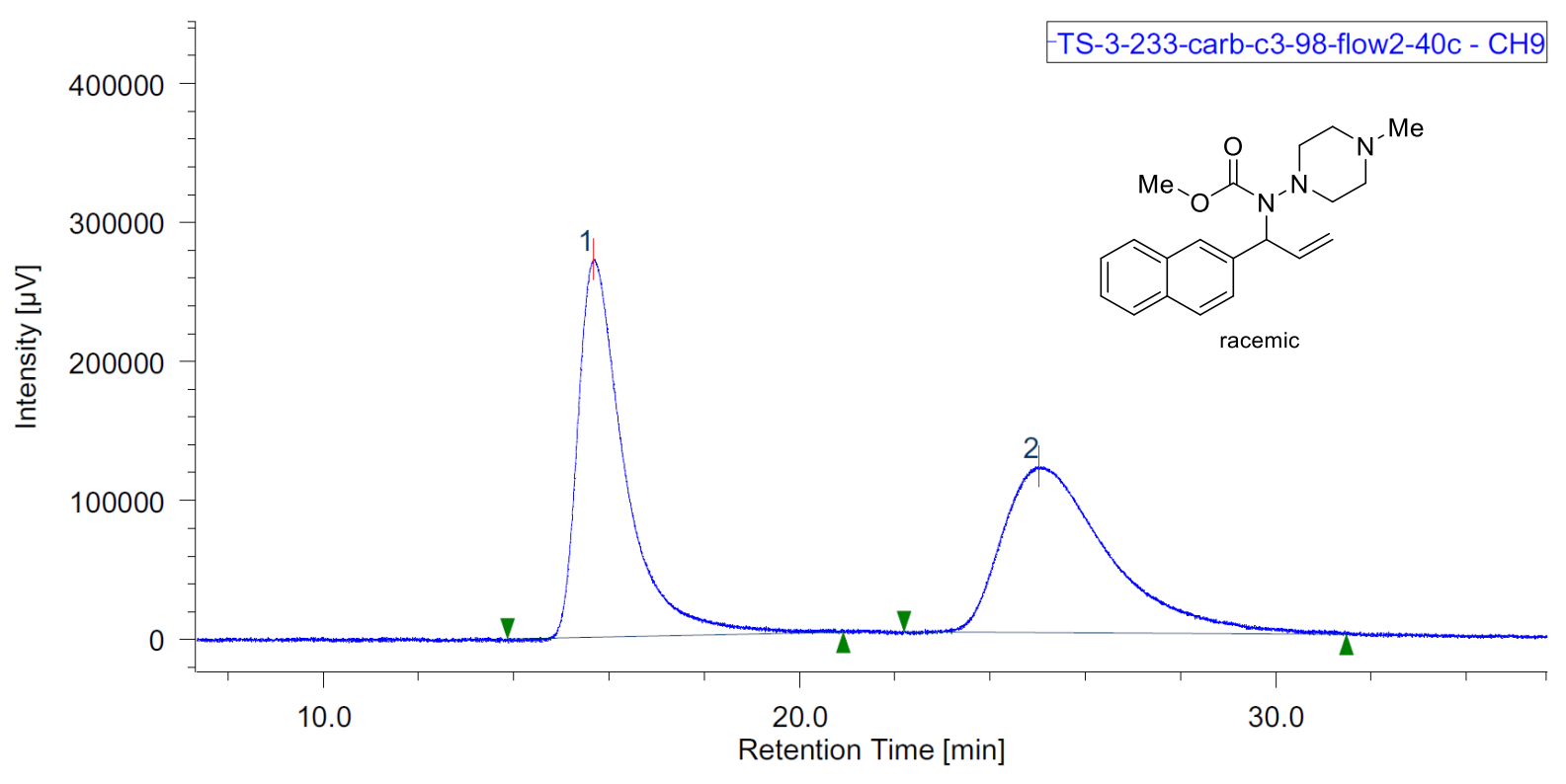

\begin{tabular}{|r|c|c|r|r|r|r|r|r|r|r|r|}
\hline$\#$ & Peak Name & CH & tR $[\mathrm{min}]$ & Area $[\mu \mathrm{V} \cdot \mathrm{sec}]$ & Height $[\mu \mathrm{V}]$ & \multicolumn{1}{|c|}{ Area $\%$} & Height $\%$ & Quantity & NTP & Resolution & Symmetry Factor \\
\hline 1 & Unknown & 9 & 15.667 & 18587595 & 271774 & 50.196 & 69.468 & N/A & 1516 & 3.436 & 2.042 \\
\hline 2 & Unknown & 9 & 25.013 & 18442350 & 119450 & 49.804 & 30.532 & N/A & 677 & N/A & 1.919 \\
\hline
\end{tabular}

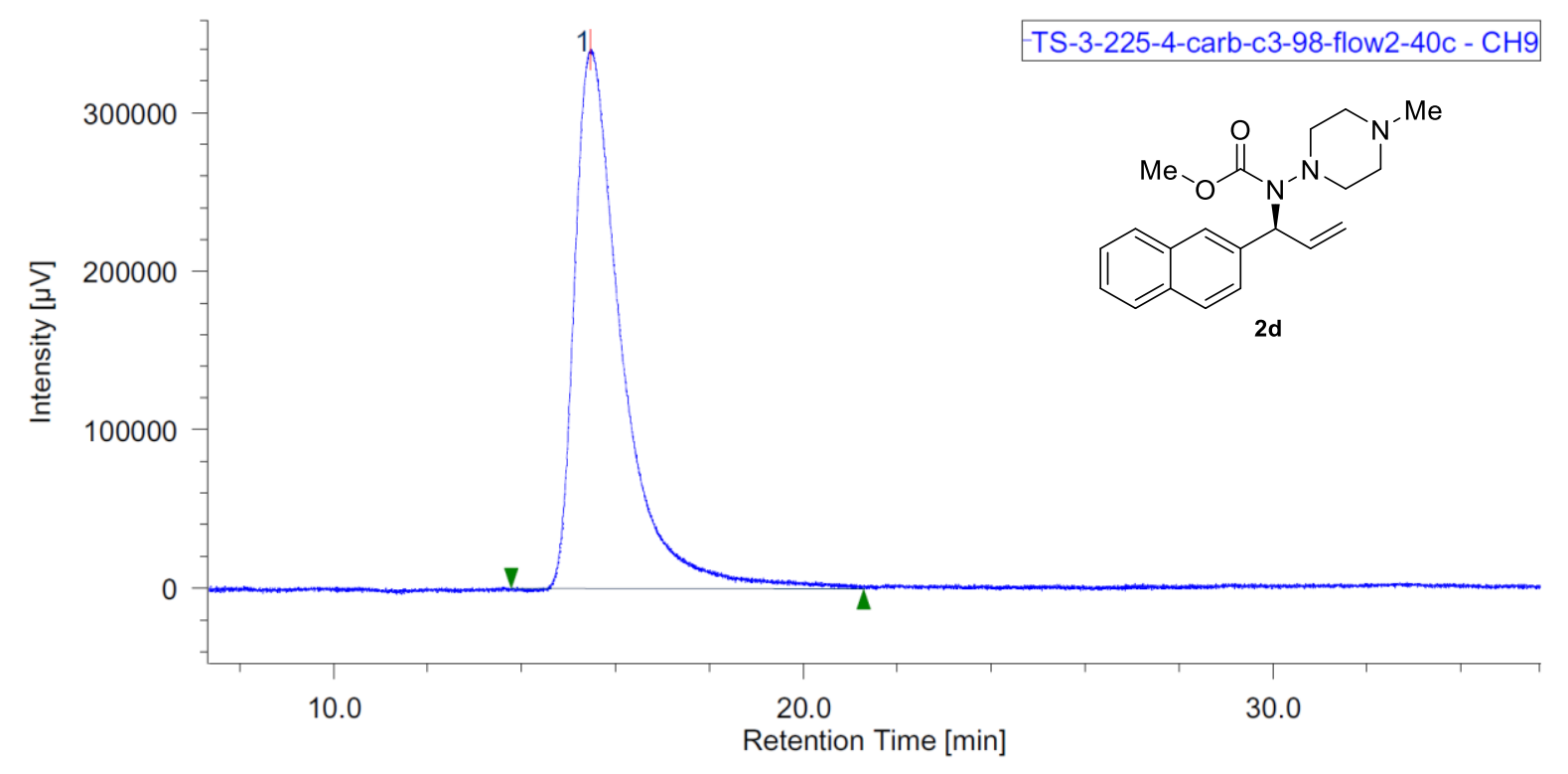

\begin{tabular}{|c|c|c|c|c|c|c|c|c|c|c|c|}
\hline \# & Peak Name & $\mathrm{CH}$ & $\mathrm{tR}$ [min] & Area $[\mu \mathrm{V}$ & Height $[\mu \mathrm{V}]$ & Area $\%$ & Height $\%$ & atity & NTP & tion & ctor \\
\hline & Unknown & 9 & 15.467 & 23849522 & 339996 & 100.000 & 100.000 & $\mathrm{~N} / \mathrm{A}$ & 139 & $\mathrm{~N} / \mathrm{A}$ & 1.9 \\
\hline
\end{tabular}




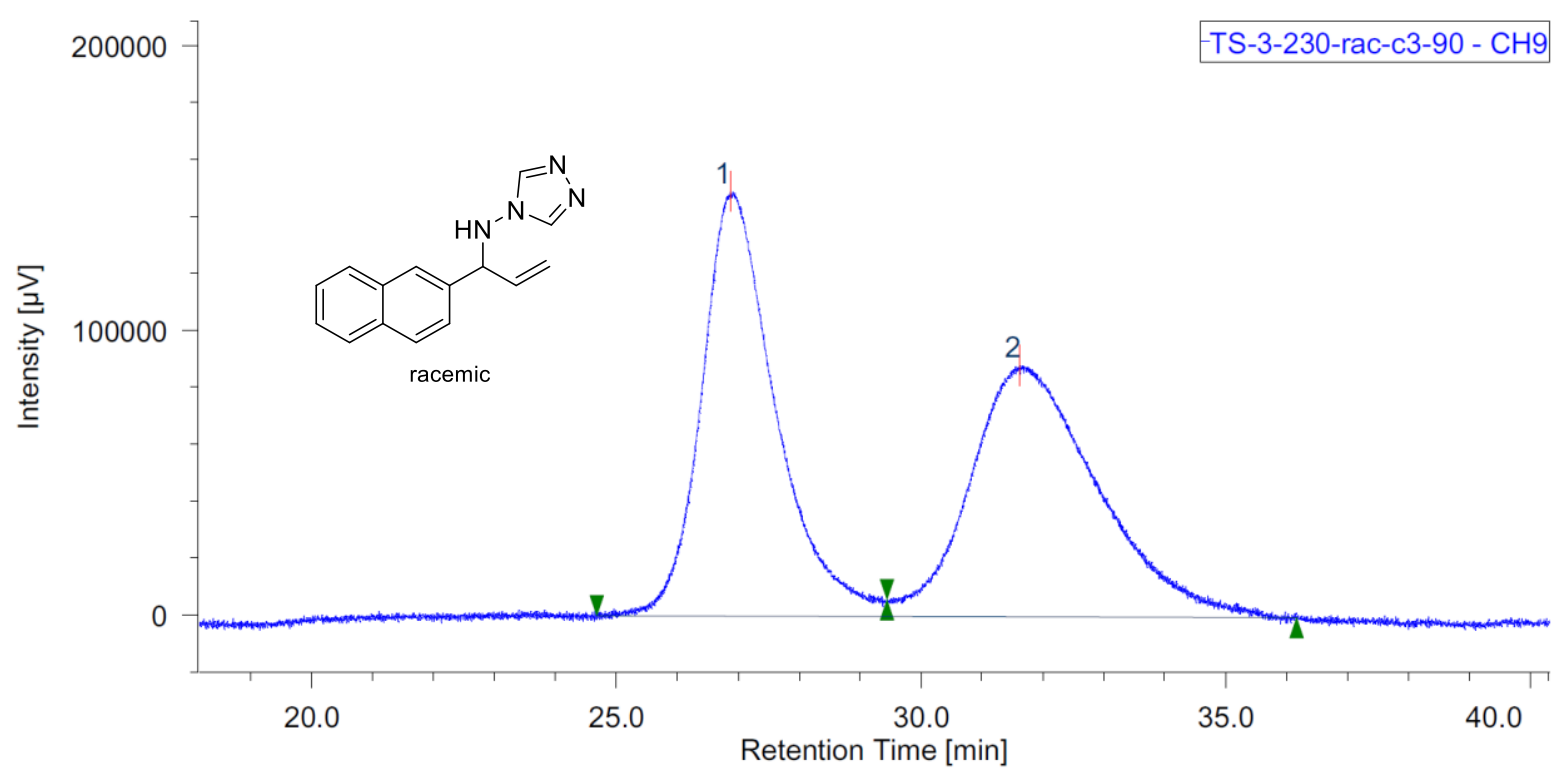

\begin{tabular}{|c|c|c|c|c|c|c|c|c|c|c|}
\hline Peak Name & $\mathrm{CH}$ & $\mathrm{tR}$ [min] & Area $[\mu \mathrm{V} \cdot \mathrm{sec}]$ & Height $[\mu \mathrm{V}]$ & Area\% & Height $\%$ & Quantity & NTP & Resolution & Symmetry Factor \\
\hline Unknown & 9 & 26.873 & 12570964 & 149092 & 49.605 & 62.815 & $\mathrm{~N} / \mathrm{A}$ & 2711 & 1.639 & 1.484 \\
\hline Unknown & 9 & 31.613 & 12771158 & 88257 & 50.395 & 37.185 & $\mathrm{~N} / \mathrm{A}$ & 1147 & $\mathrm{~N} / \mathrm{A}$ & $\mathrm{N} / \mathrm{A}$ \\
\hline
\end{tabular}

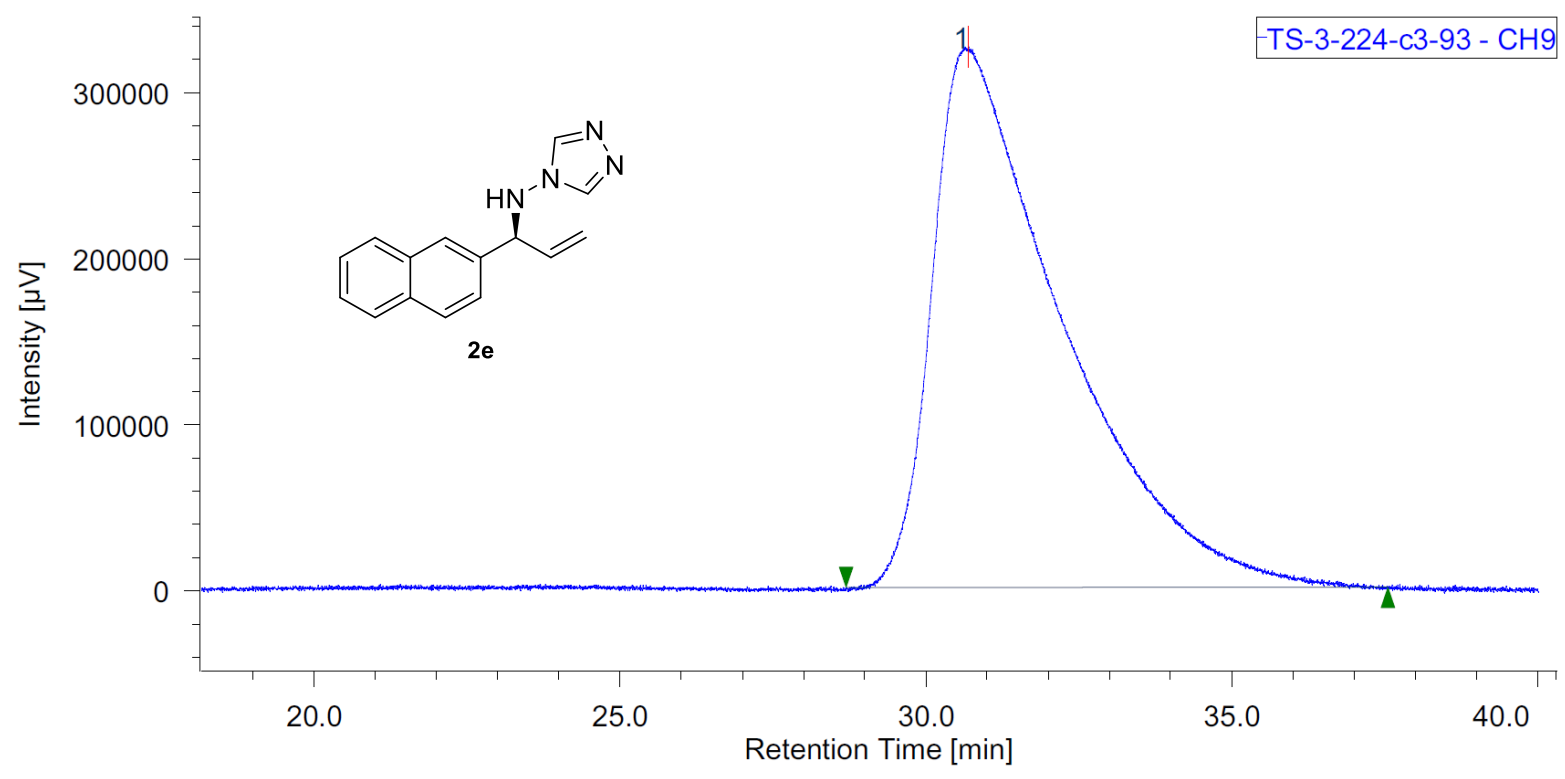

\begin{tabular}{|c|c|c|c|c|c|c|c|c|c|c|}
\hline Pea & $\mathrm{CH}$ & {$[\mathrm{min}]$} & ea $[\mu \mathrm{V} \cdot \mathrm{sec}]$ & eight $[\mu \mathrm{V}]$ & Area\% & Height $\%$ & uantity & NTP & Resolution & $\mathrm{Fa}$ \\
\hline $\mathrm{Ing}$ & 9 & 30.700 & 48206372 & 325509 & 100.000 & 100.000 & $\mathrm{~N} / \mathrm{A}$ & 1129 & $\mathrm{~N} / \mathrm{A}$ & \\
\hline
\end{tabular}




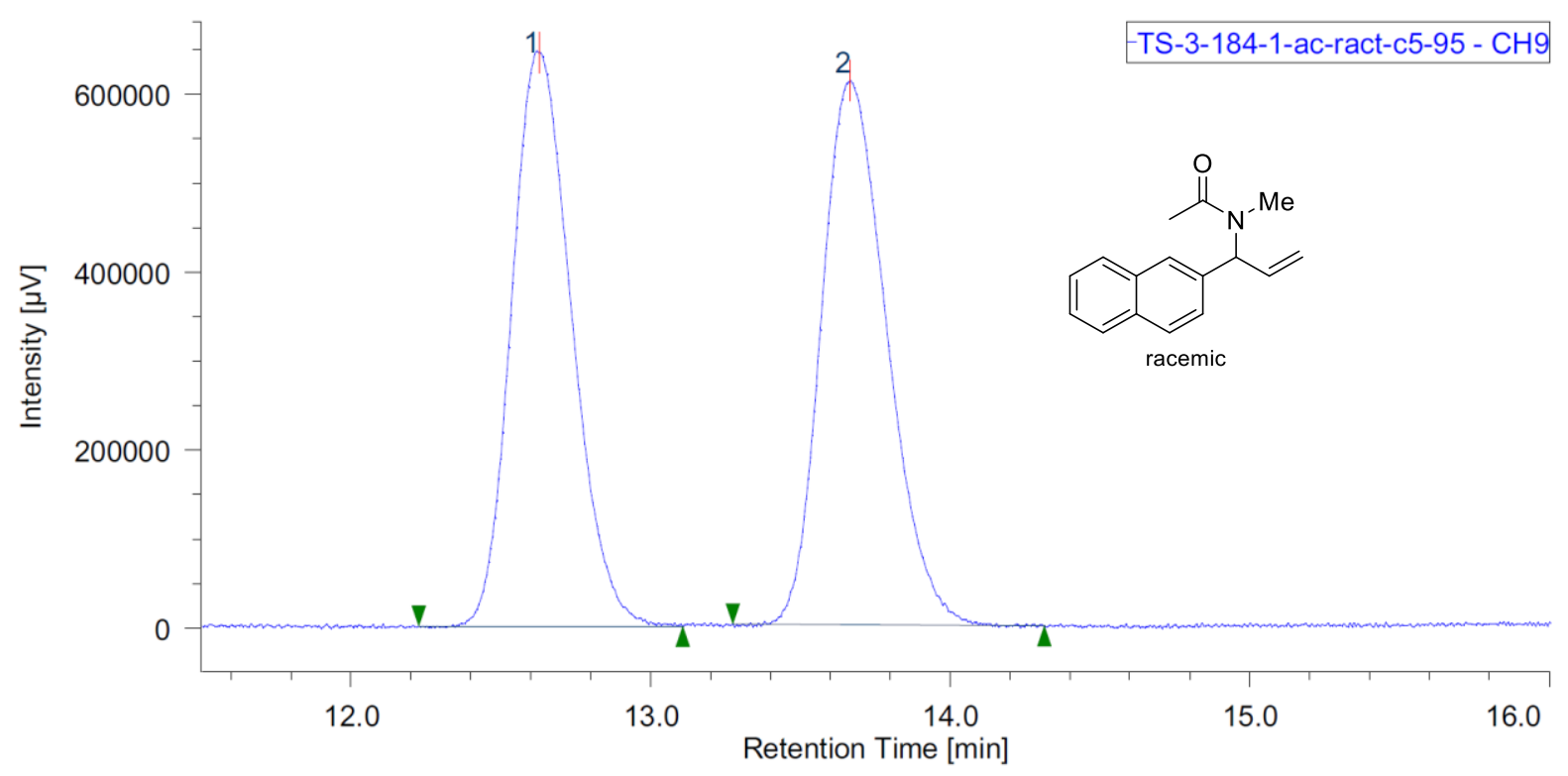

\begin{tabular}{|r|l|c|r|r|r|r|r|r|r|r|r|}
\hline$\#$ & Peak Name & $\mathrm{CH}$ & $\mathrm{tR}[\mathrm{min}]$ & \multicolumn{1}{|c|}{ Area $[\mu \mathrm{V} \cdot \mathrm{sec}]$} & Height $[\mu \mathrm{V}]$ & \multicolumn{1}{|c|}{ Area\% } & Height $\%$ & Quantity & NTP & Resolution & Symmetry Factor \\
\hline 1 & Unknown & 9 & 12.627 & 8957756 & 643724 & 49.201 & 51.312 & $\mathrm{~N} / \mathrm{A}$ & 18932 & 2.717 & 1.177 \\
\hline 2 & Unknown & 9 & 13.667 & 9248539 & 610810 & 50.799 & 48.688 & $\mathrm{~N} / \mathrm{A}$ & 18633 & $\mathrm{~N} / \mathrm{A}$ & 1.228 \\
\hline
\end{tabular}

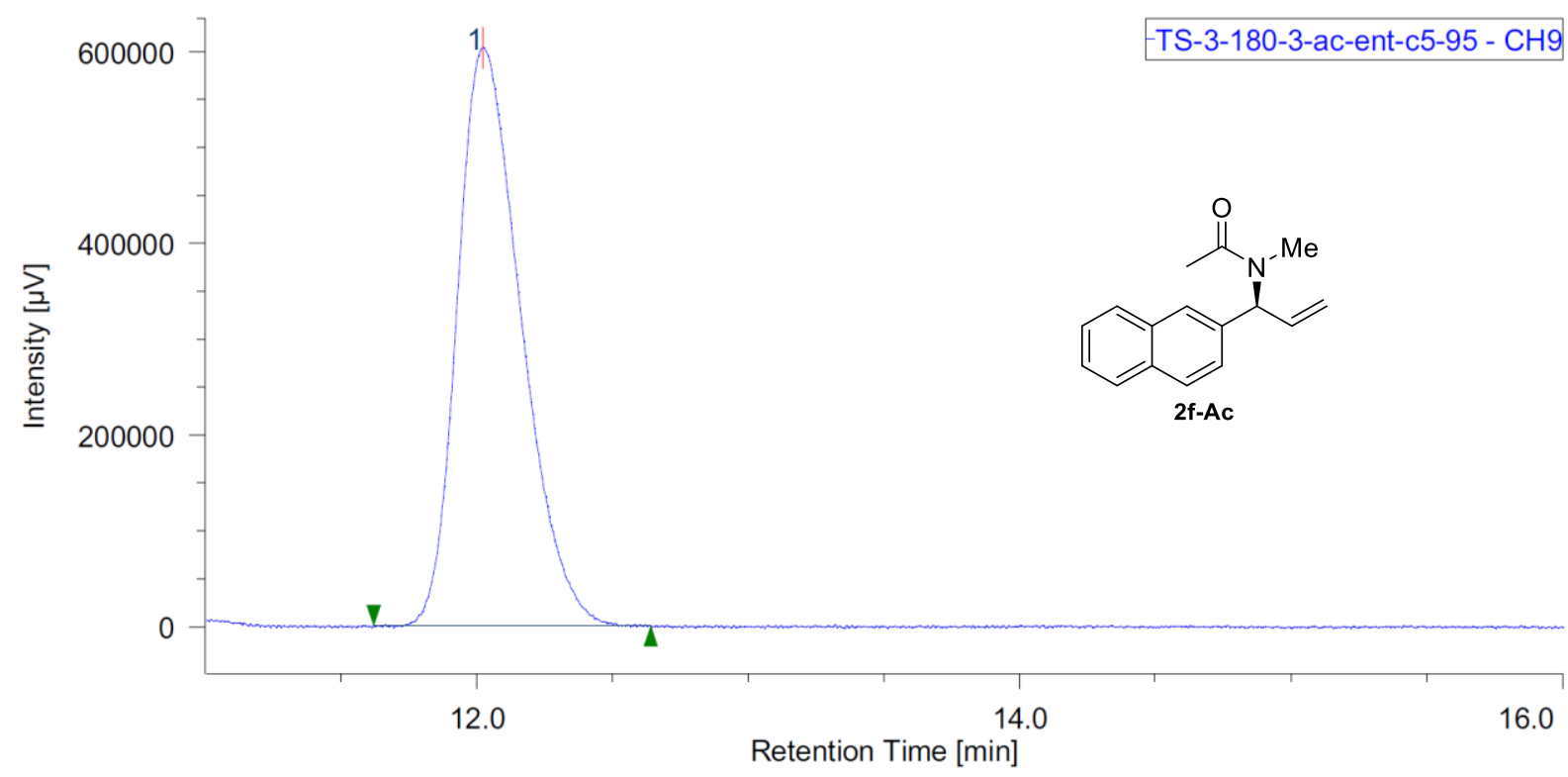

\begin{tabular}{|l|l|c|c|c|c|c|c|c|c|c|c|}
\hline$\#$ & Peak Name & $\mathrm{CH}$ & tR $[\mathrm{min}]$ & Area $[\mu \mathrm{V} \cdot \mathrm{sec}]$ & Height $[\mu \mathrm{V}]$ & Area $\%$ & Height $\%$ & Quantity & NTP & Resolution & Symmetry Factor \\
\hline
\end{tabular}

\begin{tabular}{|l|r|r|r|r|r|r|r|r|r|r|r|}
\hline 1 Unknown & 9 & 12.020 & 9825090 & 602404 & 100.000 & 100.000 & $\mathrm{~N} / \mathrm{A}$ & 12543 & $\mathrm{~N} / \mathrm{A}$ & 1.360 \\
\hline
\end{tabular}




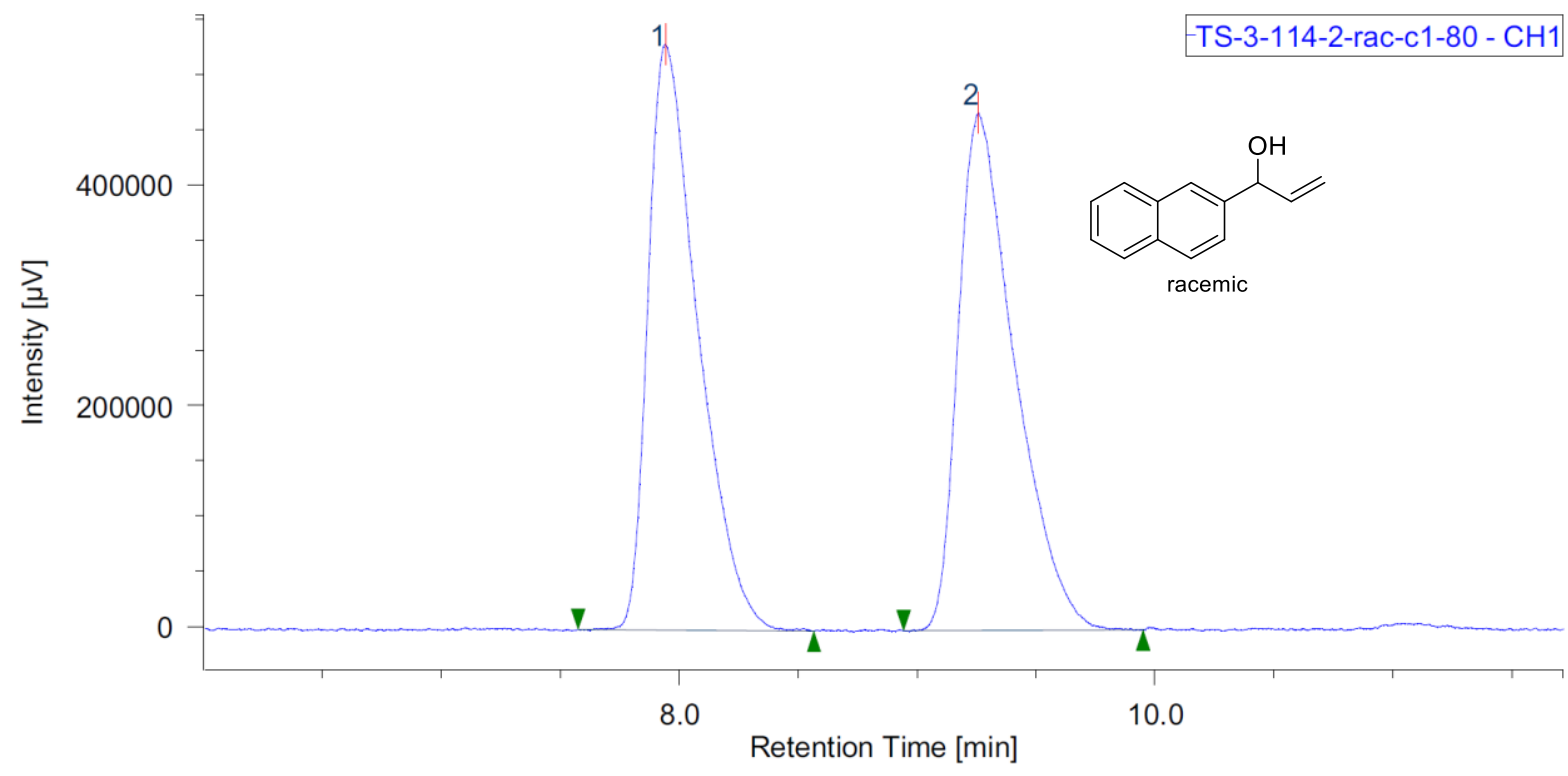

\begin{tabular}{|l|l|c|c|c|c|c|c|c|c|c|c|}
\hline$\#$ & Peak Name & CH & tR $[\mathrm{min}]$ & Area $[\mu \mathrm{V} \cdot \mathrm{sec}]$ & Height $[\mu \mathrm{V}]$ & Area\% & Height $\%$ & Quantity & NTP & Resolution & Symmetry Factor \\
\hline
\end{tabular}

\begin{tabular}{|c|c|c|c|c|c|c|c|c|c|c|}
\hline Unknown & 1 & 7.942 & 7745122 & 530374 & 50.013 & 53.107 & $\mathrm{~N} / \mathrm{A}$ & 7066 & 3.273 & 1.655 \\
\hline & 1 & 9.258 & 7741134 & 468320 & 49.987 & 46.893 & N/A & 7463 & $\mathrm{~N} / \mathrm{A}$ & \\
\hline
\end{tabular}

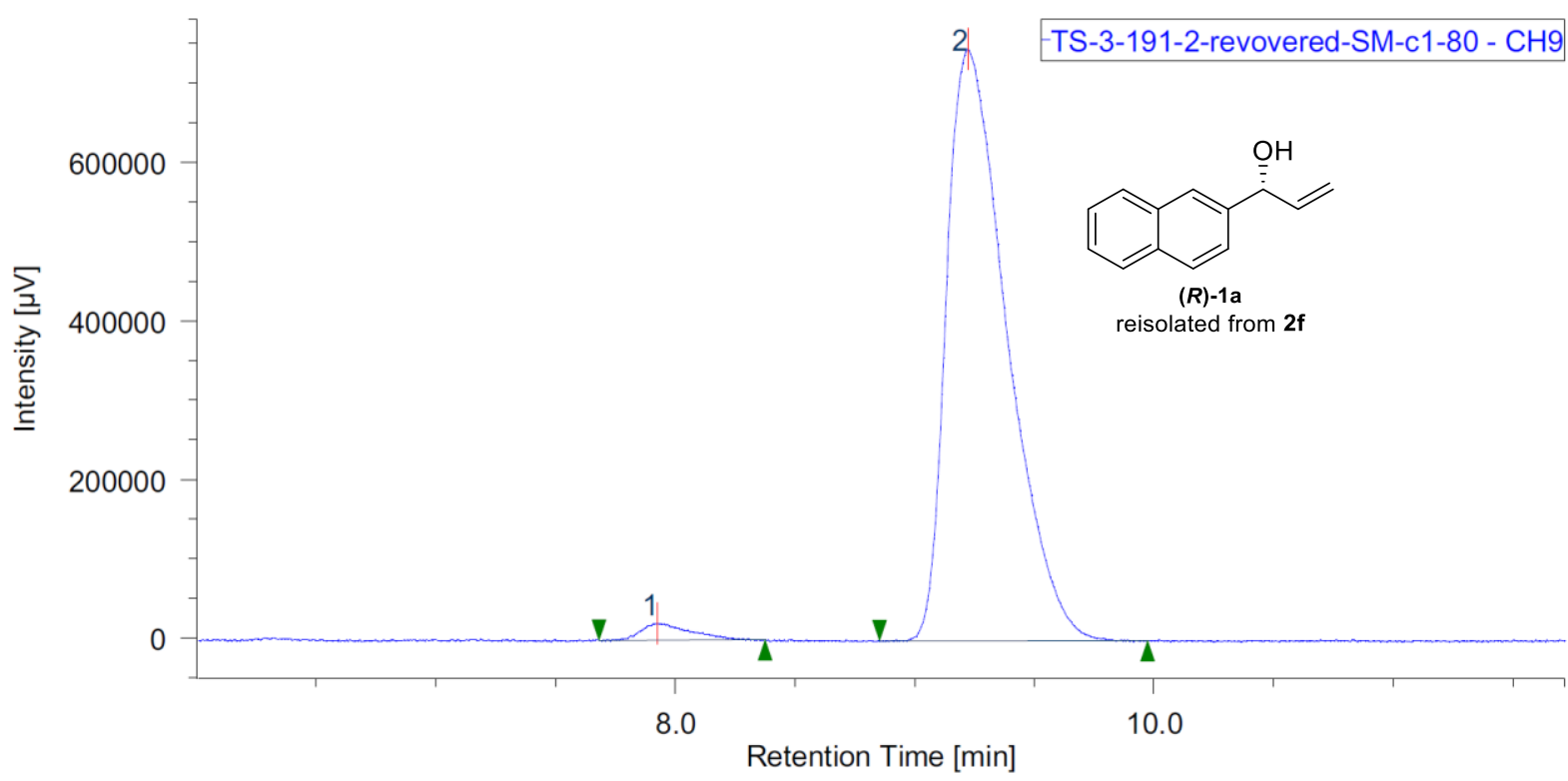

\begin{tabular}{|r|c|c|r|r|r|r|r|r|r|r|r|}
\hline$\#$ & Peak Name & CH & tR $[\mathrm{min}]$ & \multicolumn{1}{|c|}{ Area $[\mu \mathrm{V} \cdot \mathrm{sec}]$} & Height $[\mu \mathrm{V}]$ & \multicolumn{1}{|c|}{ Area\% } & Height $\%$ & Quantity & NTP & Resolution & Symmetry Factor \\
\hline 1 & Unknown & 9 & 7.927 & 307439 & 21085 & 2.295 & 2.749 & N/A & 6838 & 3.087 & 1.591 \\
\hline 2 & Unknown & 9 & 9.220 & 13086904 & 745943 & 97.705 & 97.251 & N/A & 6524 & N/A & 1.650 \\
\hline
\end{tabular}




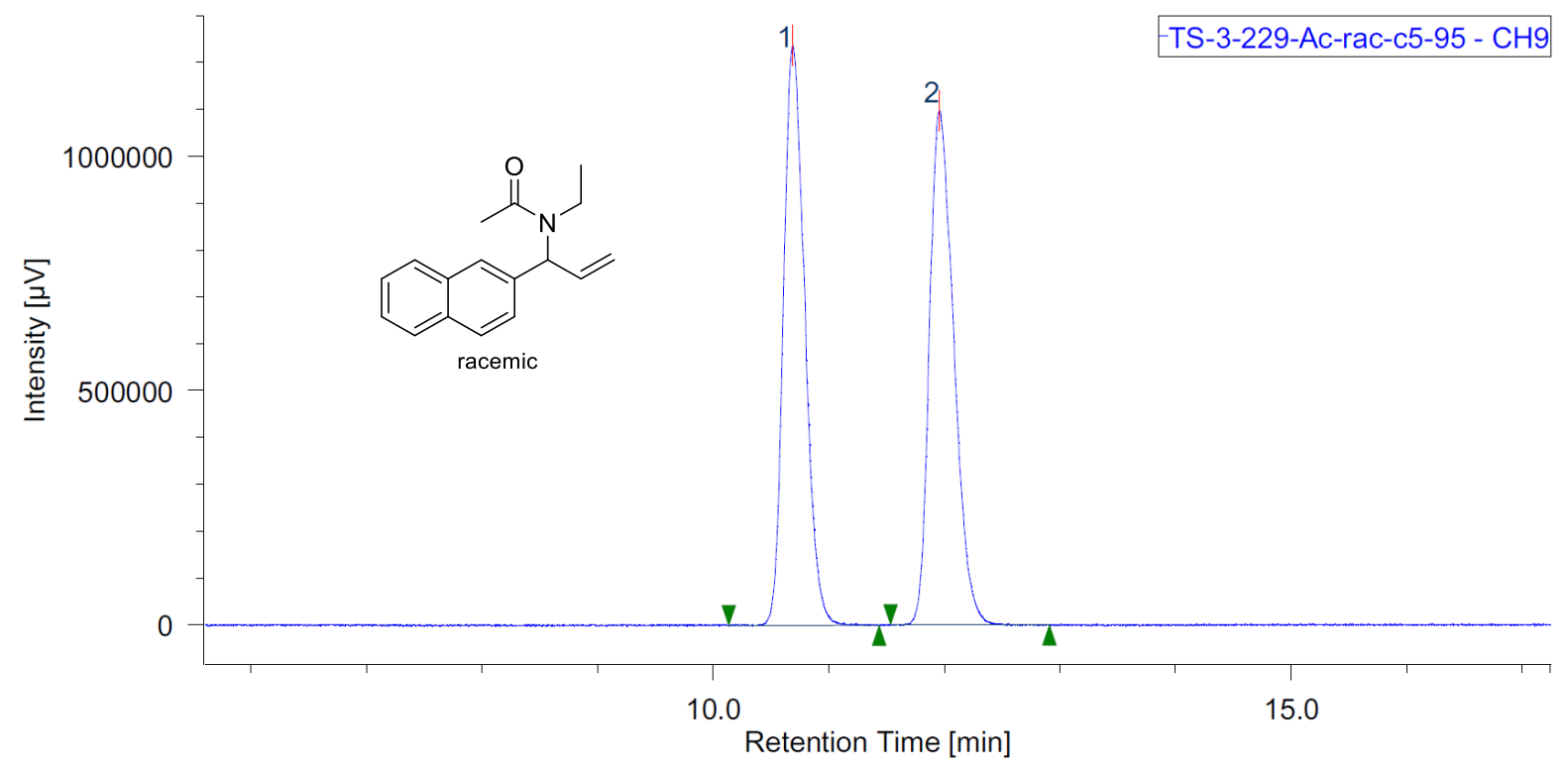

\begin{tabular}{|c|c|c|c|c|c|c|c|c|c|c|c|}
\hline$\#$ & Peak Name & $\mathrm{CH}$ & $\mathrm{tR}[\mathrm{min}]$ & Area $[\mu \mathrm{V} \cdot \mathrm{sec}]$ & Height $[\mu \mathrm{V}]$ & Area $\%$ & Height $\%$ & Quantity & NTP & Resolution & Symmetry Factor \\
\hline & Unknown & 9 & 10.687 & 15894515 & 1236566 & 49.815 & 53.008 & $\mathrm{~N} / \mathrm{A}$ & 15286 & 3.453 & 1.230 \\
\hline & Unknown & 9 & 11.953 & 16012353 & 1096243 & 50.185 & 46.992 & $\mathrm{~N} / \mathrm{A}$ & 15049 & $\mathrm{~N} / \mathrm{A}$ & 1.345 \\
\hline
\end{tabular}

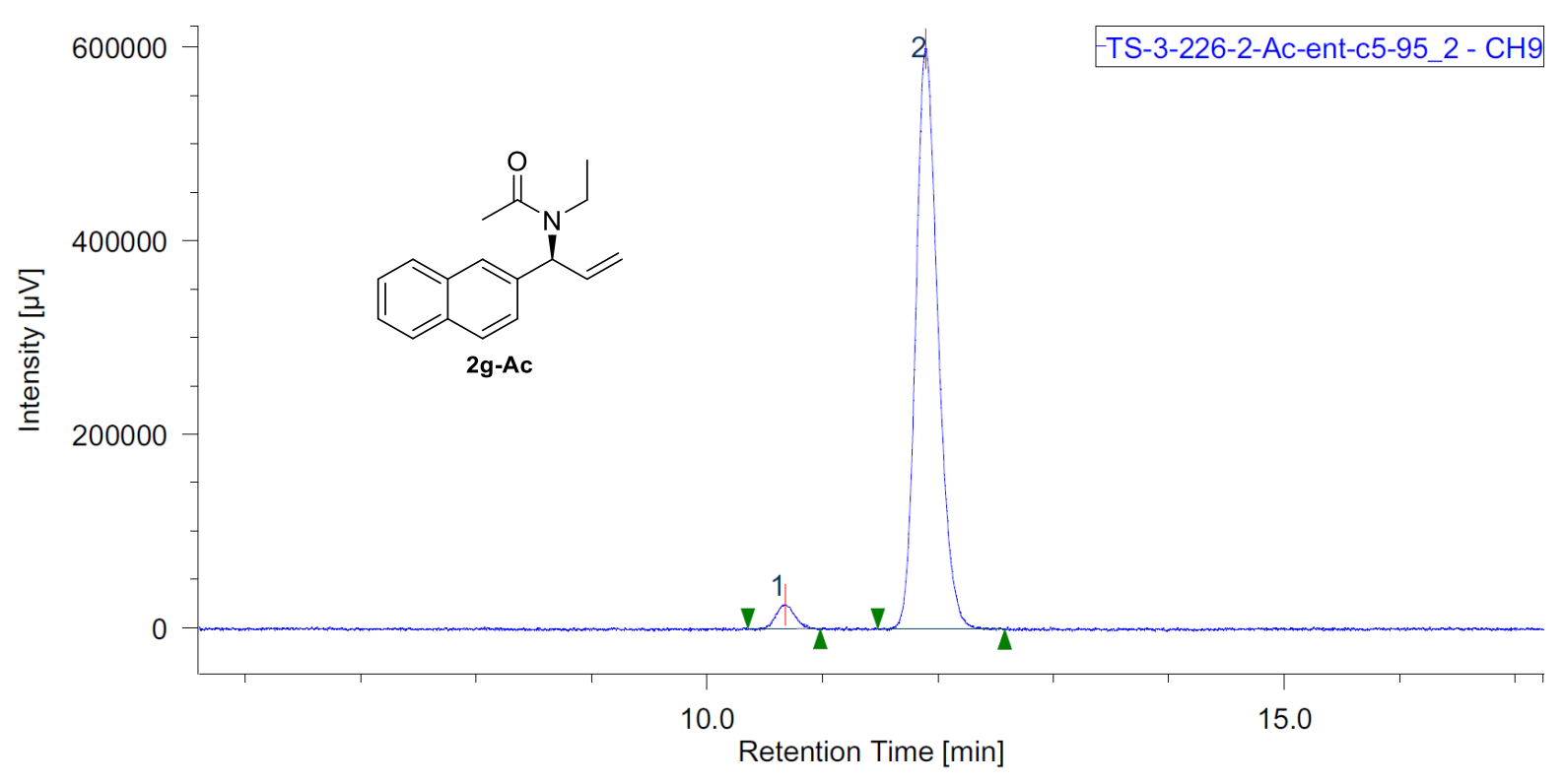

\begin{tabular}{|r|c|c|r|r|r|r|r|r|r|r|r|}
\hline$\#$ & Peak Name & CH & tR $[\mathrm{min}]$ & Area $[\mu \mathrm{V} \cdot \mathrm{sec}]$ & Height $[\mu \mathrm{V}]$ & \multicolumn{1}{|c|}{ Area $\%$} & Height $\%$ & Quantity & NTP & Resolution & Symmetry Factor \\
\hline 1 & Unknown & 9 & 10.673 & 280794 & 24834 & 3.395 & 3.980 & N/A & 20419 & 3.804 & 1.101 \\
\hline 2 & Unknown & 9 & 11.893 & 7988966 & 599150 & 96.605 & 96.020 & N/A & 19092 & N/A & 1.210 \\
\hline
\end{tabular}




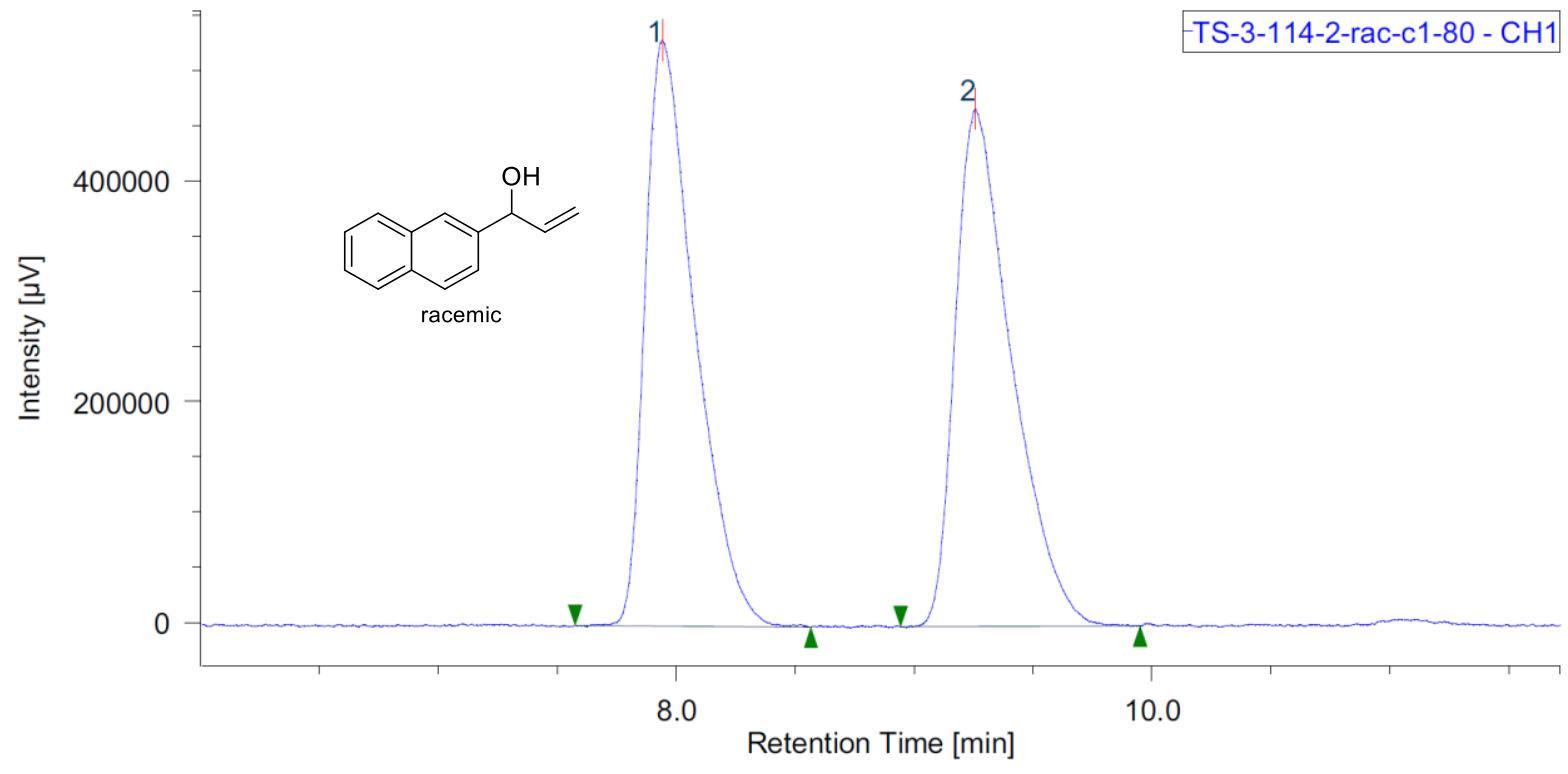

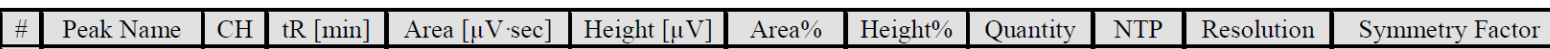

\begin{tabular}{|c|c|c|c|c|c|c|c|c|c|c|}
\hline Unknown & 1 & 7.942 & 7745122 & 530374 & 50.013 & 53.107 & $\mathrm{~N} / \mathrm{A}$ & 7066 & 3.273 & 1.655 \\
\hline & 1 & 9.258 & 7741134 & 468320 & 49.987 & 46.893 & N/A & 7463 & $\mathrm{~N} / \mathrm{A}$ & \\
\hline
\end{tabular}

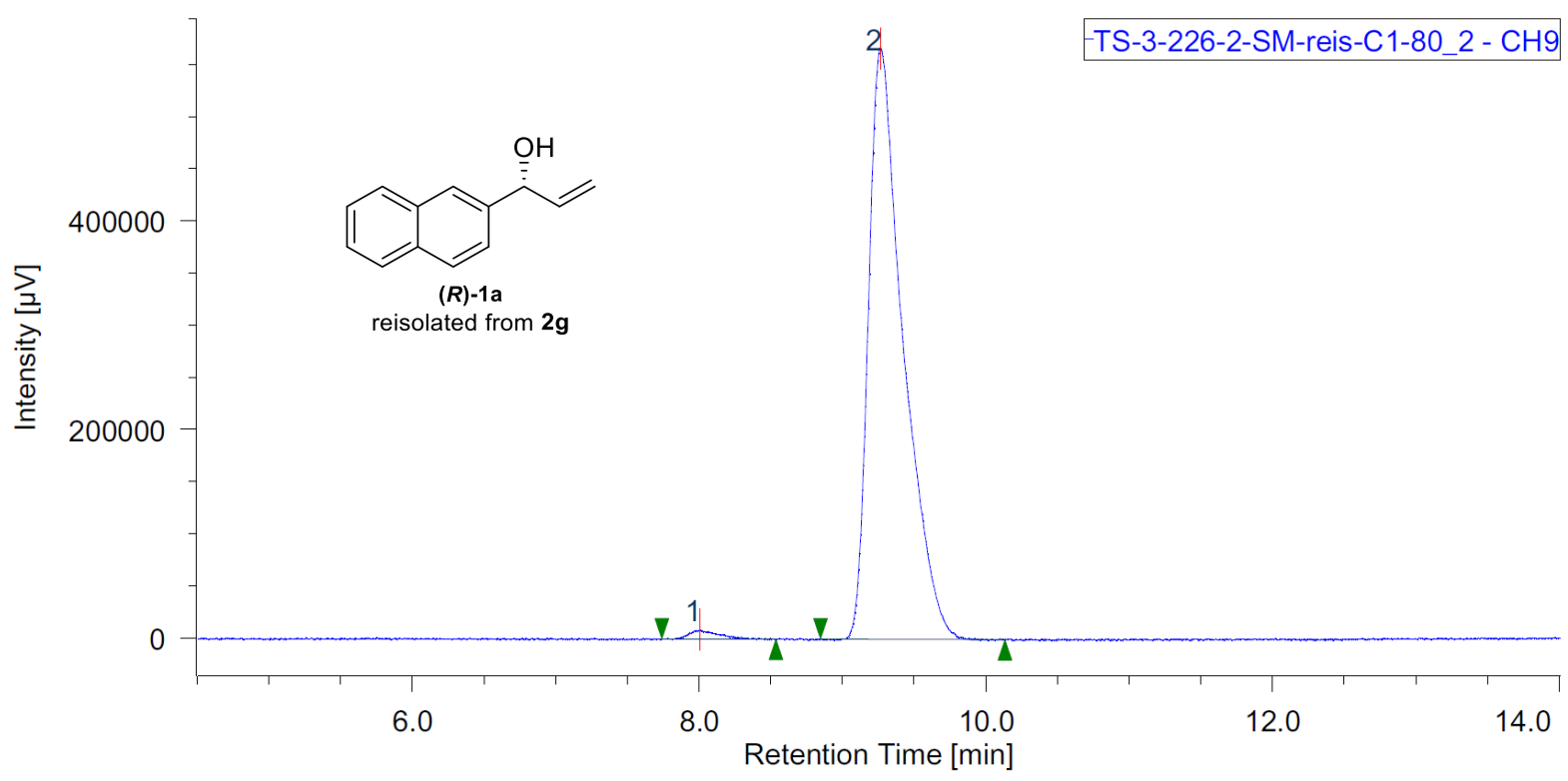

\begin{tabular}{|r|c|c|r|r|r|r|r|r|r|r|r|}
\hline$\#$ & Peak Name & CH & tR [min] & Area $[\mu \mathrm{V} \cdot \mathrm{sec}]$ & Height $[\mu \mathrm{V}]$ & \multicolumn{1}{|c|}{ Area\% } & Height $\%$ & Quantity & NTP & Resolution & Symmetry Factor \\
\hline 2 & Unknown & 9 & 8.007 & 129924 & 9304 & 1.349 & 1.616 & N/A & 9375 & 3.353 & 1.560 \\
\hline 1 & Unknown & 9 & 9.267 & 9503370 & 566367 & 98.651 & 98.384 & N/A & 7689 & N/A & 1.658 \\
\hline
\end{tabular}




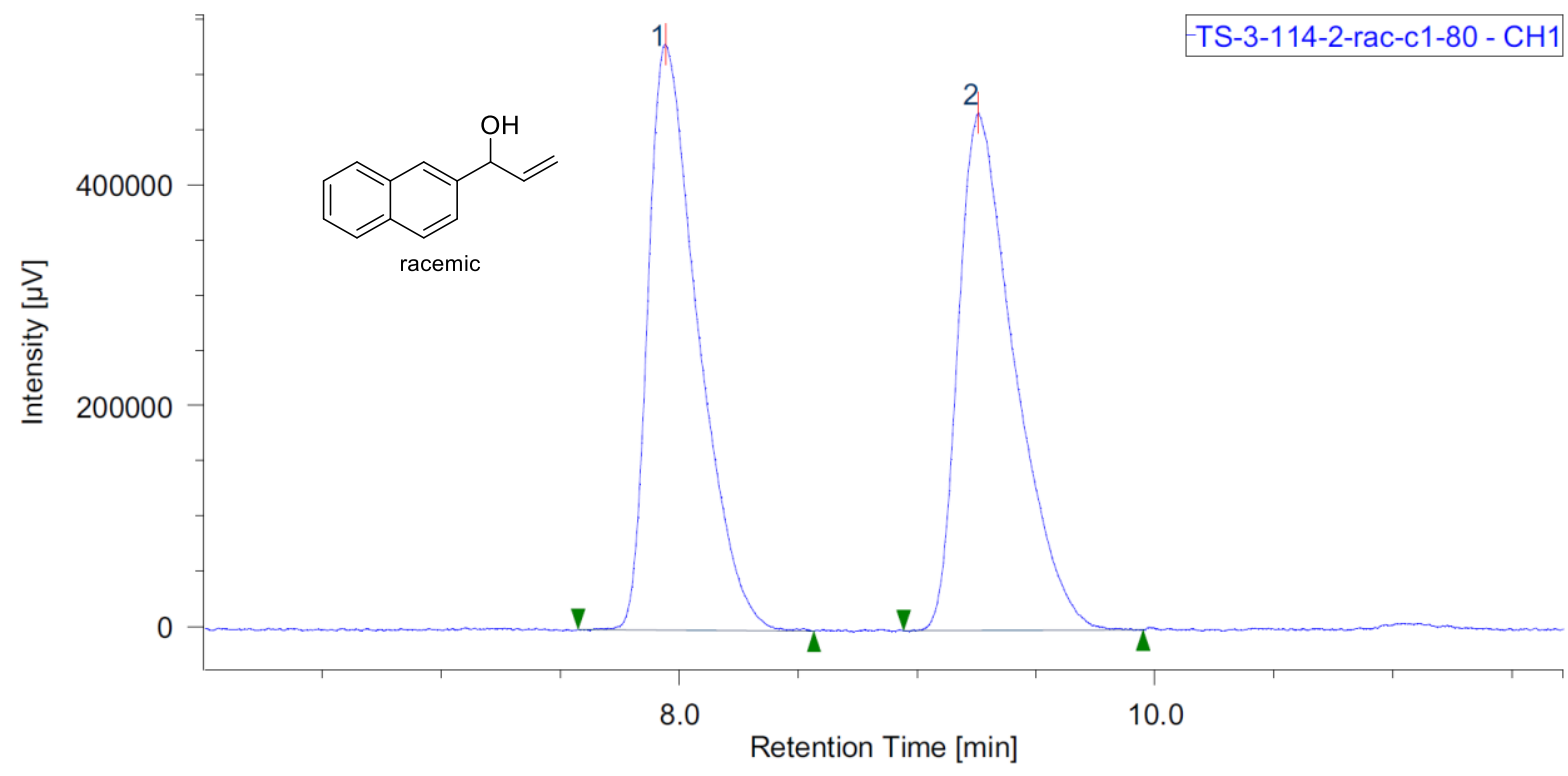

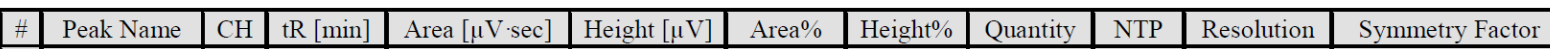

\begin{tabular}{|c|c|c|c|c|c|c|c|c|c|c|}
\hline Unknown & 1 & 7.942 & 7745122 & 530374 & 50.013 & 53.107 & $\mathrm{~N} / \mathrm{A}$ & 7066 & 3.273 & 1.655 \\
\hline & 1 & 9.258 & 7741134 & 468320 & 49.987 & 46.893 & N/A & 7463 & $\mathrm{~N} / \mathrm{A}$ & \\
\hline
\end{tabular}

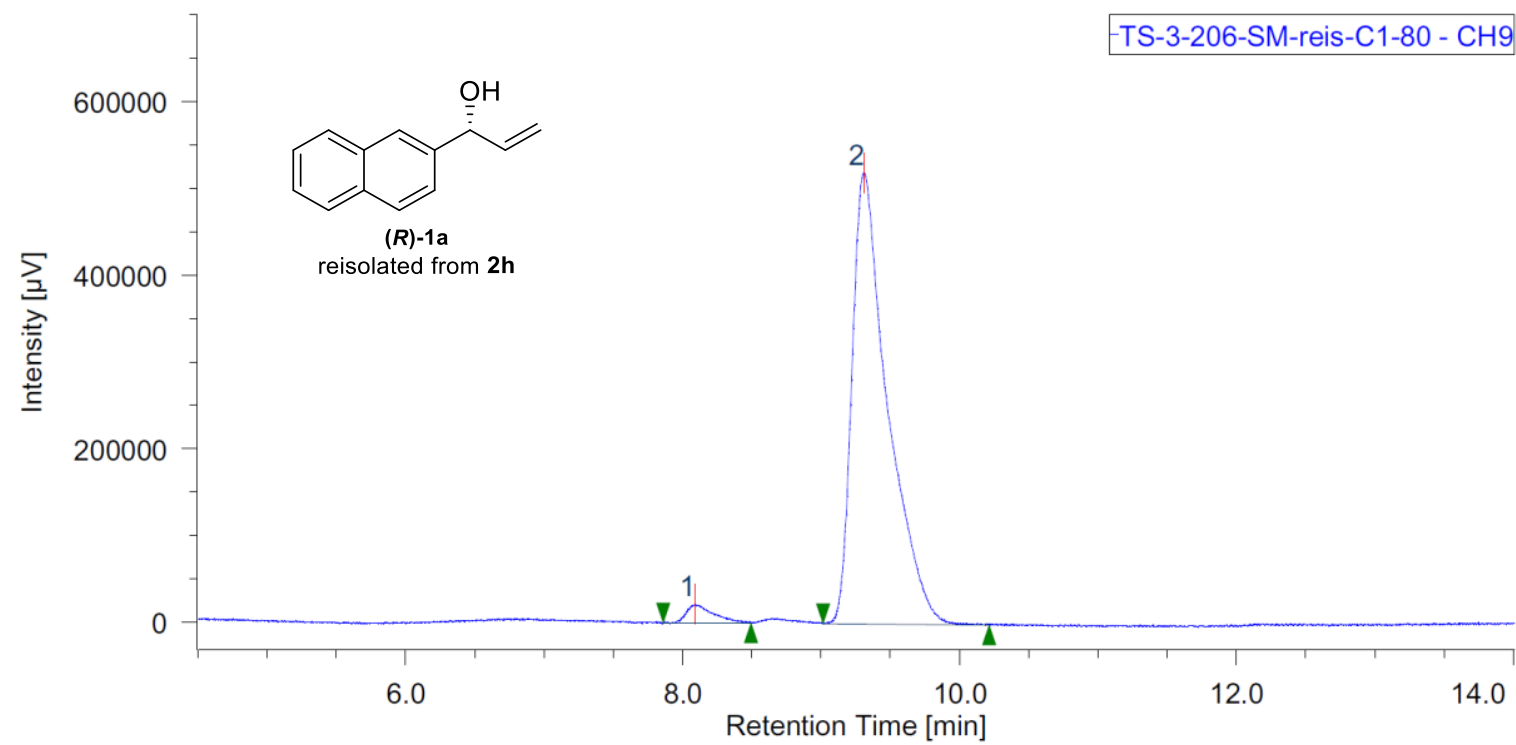

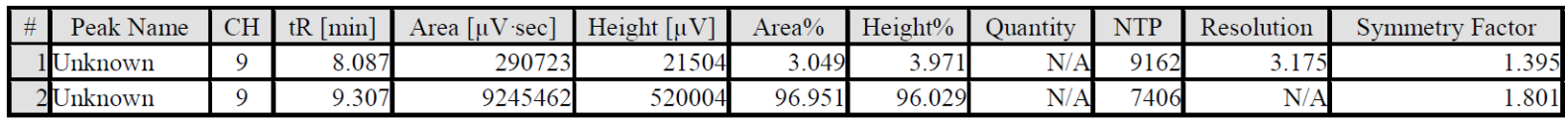




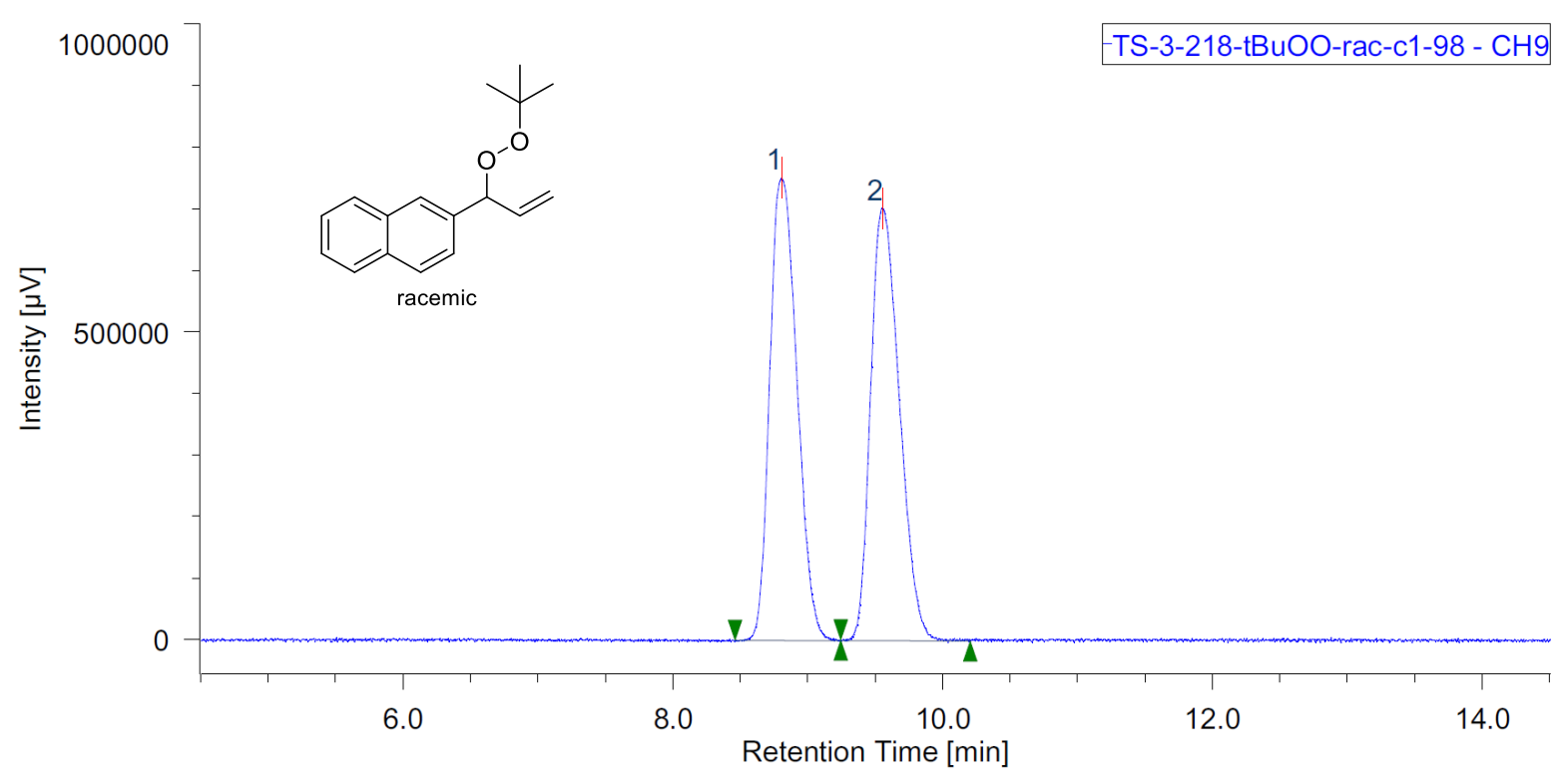

\begin{tabular}{|c|c|c|c|c|c|c|c|c|c|c|}
\hline Peak & $\mathrm{CH}$ & {$[\mathrm{min}]$} & Area $[\mu \mathrm{V} \cdot \mathrm{sec}]$ & Height $[\mu \mathrm{V}]$ & Area\% & Height $\%$ & Quantity & NTP & Resolution & Factor \\
\hline UnkI & $\overline{9}$ & 8.807 & 10530808 & 751016 & 50.267 & 51.686 & $\mathrm{~N} / \mathrm{A}$ & 8743 & 1.93 & 1.2 \\
\hline Unknown & 9 & 9.553 & 10418749 & 702019 & 49.733 & 48.314 & $\mathrm{~N} / \mathrm{A}$ & 9239 & $\mathrm{~N} / \mathrm{A}$ & 1.3 \\
\hline
\end{tabular}

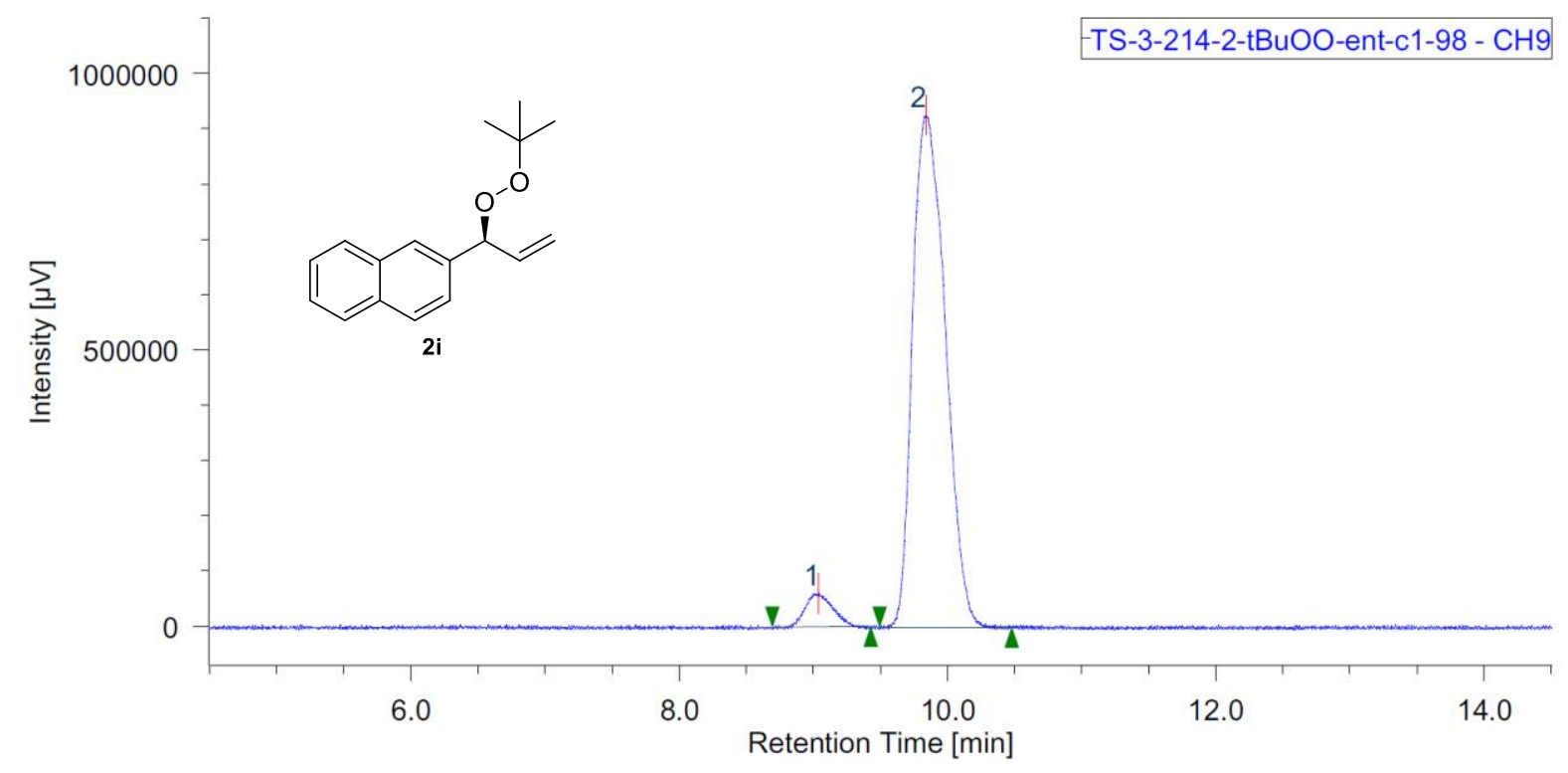

\begin{tabular}{|c|c|c|c|c|c|c|c|c|c|c|}
\hline Peak Name & $\overline{\mathrm{CH}}$ & $\mathrm{tR}$ [min] & Area $[\mu \mathrm{V} \cdot \mathrm{sec}]$ & Height $[\mu \mathrm{V}]$ & Area $\%$ & Height $\%$ & Quantity & NTP & Resolution & Symmetry Factor \\
\hline Unknown & 9 & 9.040 & 853524 & 60553 & 5.170 & 6.135 & $\mathrm{~N} / \mathrm{A}$ & 8718 & 1.884 & 1.098 \\
\hline 2 Unknown & 9 & 9.840 & 15654317 & 926530 & 94.830 & 93.865 & $\mathrm{~N} / \mathrm{A}$ & 7192 & $\mathrm{~N} / \mathrm{A}$ & 1.292 \\
\hline
\end{tabular}




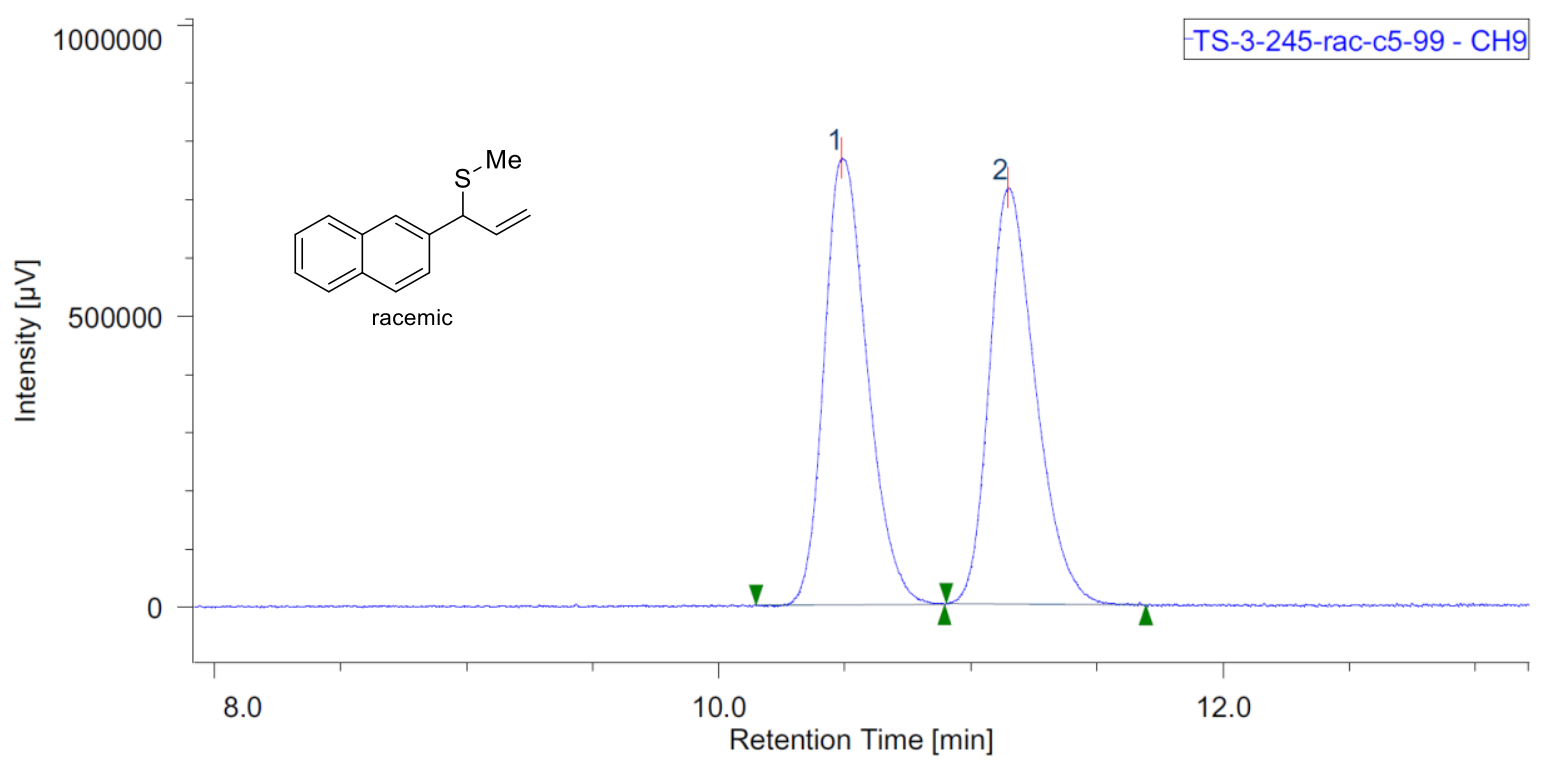

\begin{tabular}{|r|c|c|r|r|r|r|r|r|r|r|r|}
\hline$\#$ & Peak Name & CH & tR $[\mathrm{min}]$ & Area $[\mu \mathrm{V}$ sec] & Height $[\mu \mathrm{V}]$ & \multicolumn{1}{|c|}{ Area\% } & Height $\%$ & Quantity & NTP & Resolution & Symmetry Factor \\
\hline 1 & Unknown & 9 & 10.487 & 9126714 & 766635 & 50.060 & 51.747 & N/A & 18341 & 2.067 & 1.290 \\
\hline 2 & Unknown & 9 & 11.147 & 9104912 & 714874 & 49.940 & 48.253 & N/A & 18190 & N/A & 1.325 \\
\hline
\end{tabular}

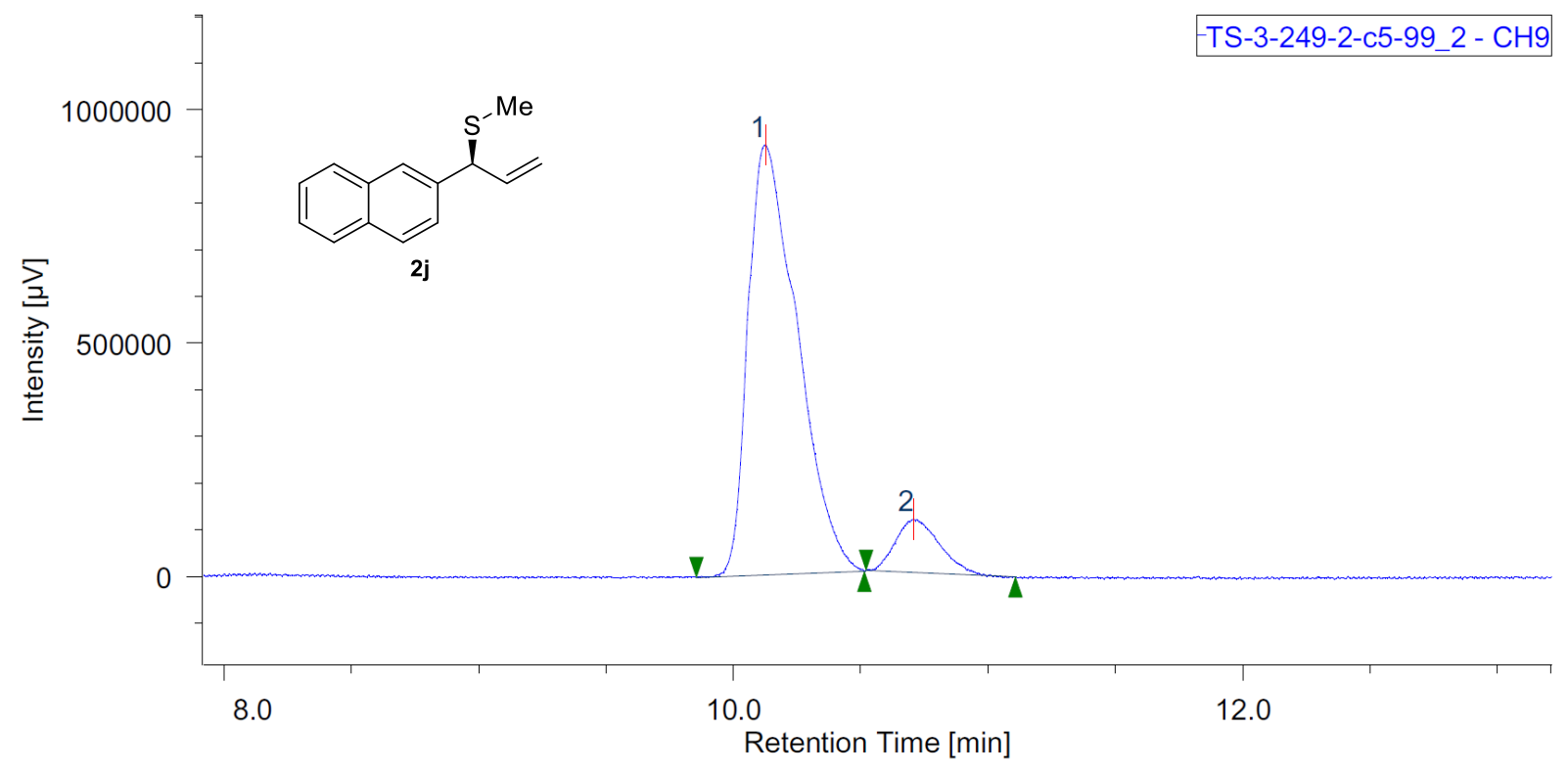

\begin{tabular}{|r|c|c|r|r|r|r|r|r|r|r|r|}
\hline$\#$ & Peak Name & $\mathrm{CH}$ & $\mathrm{tR}[\mathrm{min}]$ & \multicolumn{1}{|c|}{ Area $[\mu \mathrm{V} \cdot \mathrm{sec}]$} & Height $[\mu \mathrm{V}]$ & \multicolumn{1}{|c|}{ Area\% } & Height $\%$ & Quantity & NTP & Resolution & Symmetry Factor \\
\hline 1 & Unknown & 9 & 10.127 & 12446017 & 920938 & 90.108 & 89.025 & $\mathrm{~N} / \mathrm{A}$ & 11678 & 1.670 & 1.573 \\
\hline 2 & Unknown & 9 & 10.707 & 1366268 & 113532 & 9.892 & 10.975 & $\mathrm{~N} / \mathrm{A}$ & 17753 & $\mathrm{~N} / \mathrm{A}$ & 1.309 \\
\hline
\end{tabular}




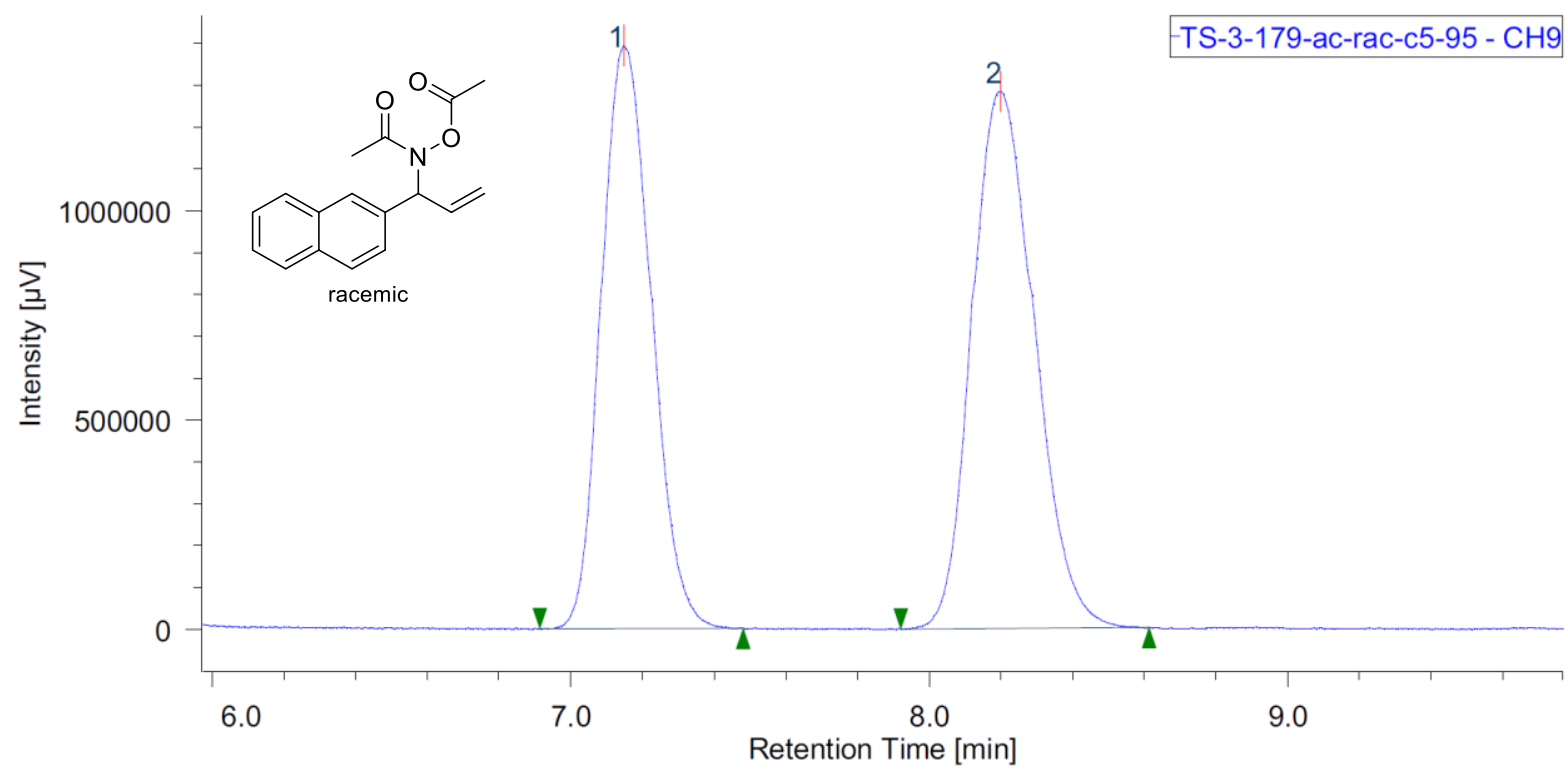

\begin{tabular}{|r|c|c|r|r|r|r|r|r|r|r|r|}
\hline$\#$ & Peak Name & $\mathrm{CH}$ & $\mathrm{tR}[\mathrm{min}]$ & \multicolumn{1}{|c|}{ Area $[\mu \mathrm{V} \cdot \mathrm{sec}]$} & Height $[\mu \mathrm{V}]$ & \multicolumn{1}{|c|}{ Area $\%$} & Height $\%$ & Quantity & \multicolumn{1}{c|}{ NTP } & Resolution & Symmetry Factor \\
\hline 1 & Unknown & 9 & 7.147 & 13524602 & 1391285 & 46.491 & 52.082 & $\mathrm{~N} / \mathrm{A}$ & 11308 & 3.512 & 1.186 \\
\hline 2 & Unknown & 9 & 8.200 & 15566342 & 1280046 & 53.509 & 47.918 & $\mathrm{~N} / \mathrm{A}$ & 9727 & $\mathrm{~N} / \mathrm{A}$ & 1.168 \\
\hline
\end{tabular}

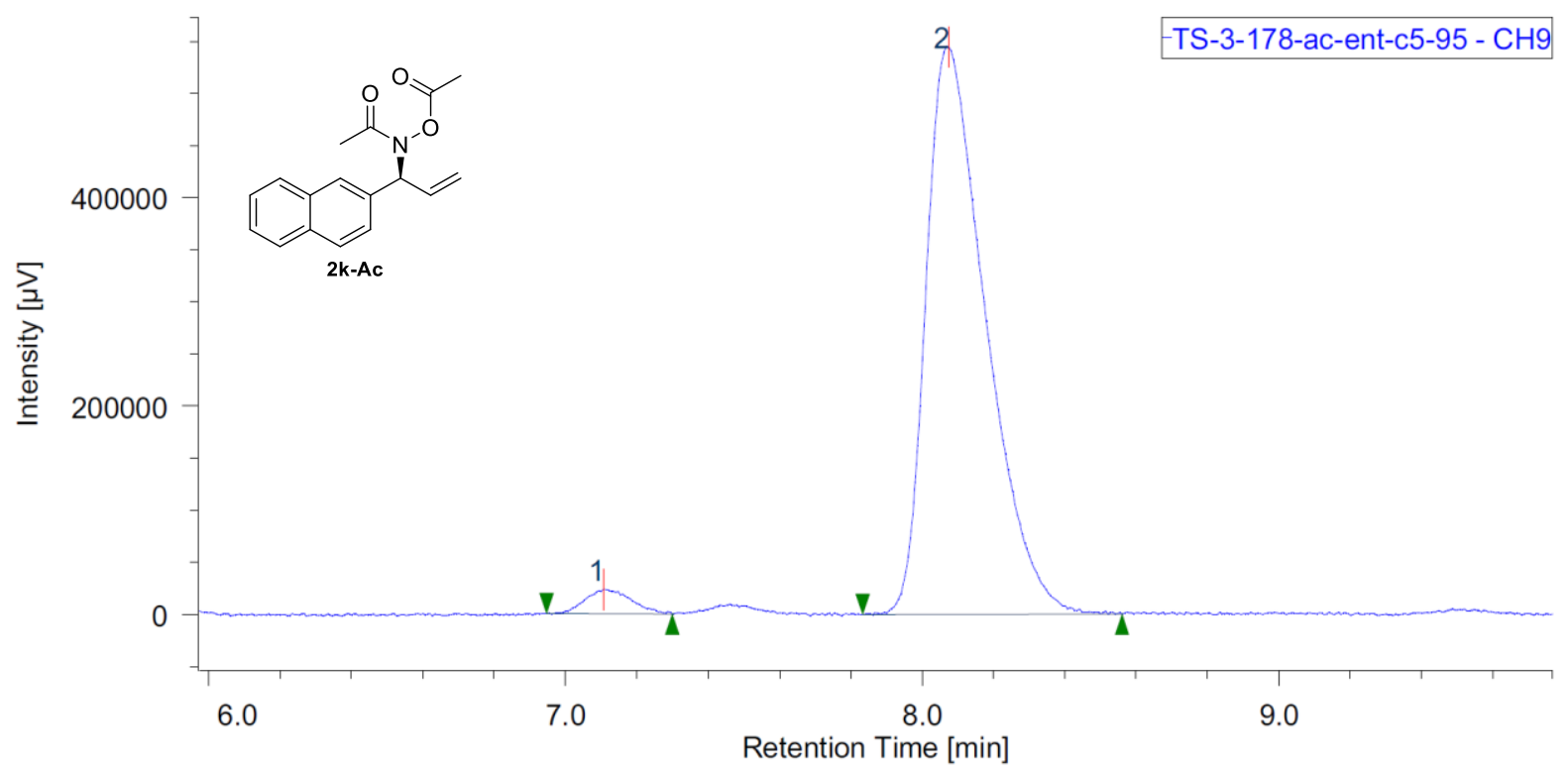

\begin{tabular}{|r|c|c|r|r|r|r|r|r|r|r|r|}
\hline$\#$ & Peak Name & $\mathrm{CH}$ & $\mathrm{tR}[\mathrm{min}]$ & Area $[\mu \mathrm{V} \cdot \mathrm{sec}]$ & Height $[\mu \mathrm{V}]$ & \multicolumn{1}{|c|}{ Area $\%$} & Height $\%$ & Quantity & \multicolumn{1}{c|}{ NTP } & Resolution & Symmetry Factor \\
\hline 1 & Unknown & 9 & 7.107 & 214232 & 23504 & 3.261 & 4.142 & $\mathrm{~N} / \mathrm{A}$ & 13686 & 3.540 & 1.298 \\
\hline 2 & Unknown & 9 & 8.073 & 6354580 & 543998 & 96.739 & 95.858 & $\mathrm{~N} / \mathrm{A}$ & 11246 & $\mathrm{~N} / \mathrm{A}$ & 1.541 \\
\hline
\end{tabular}




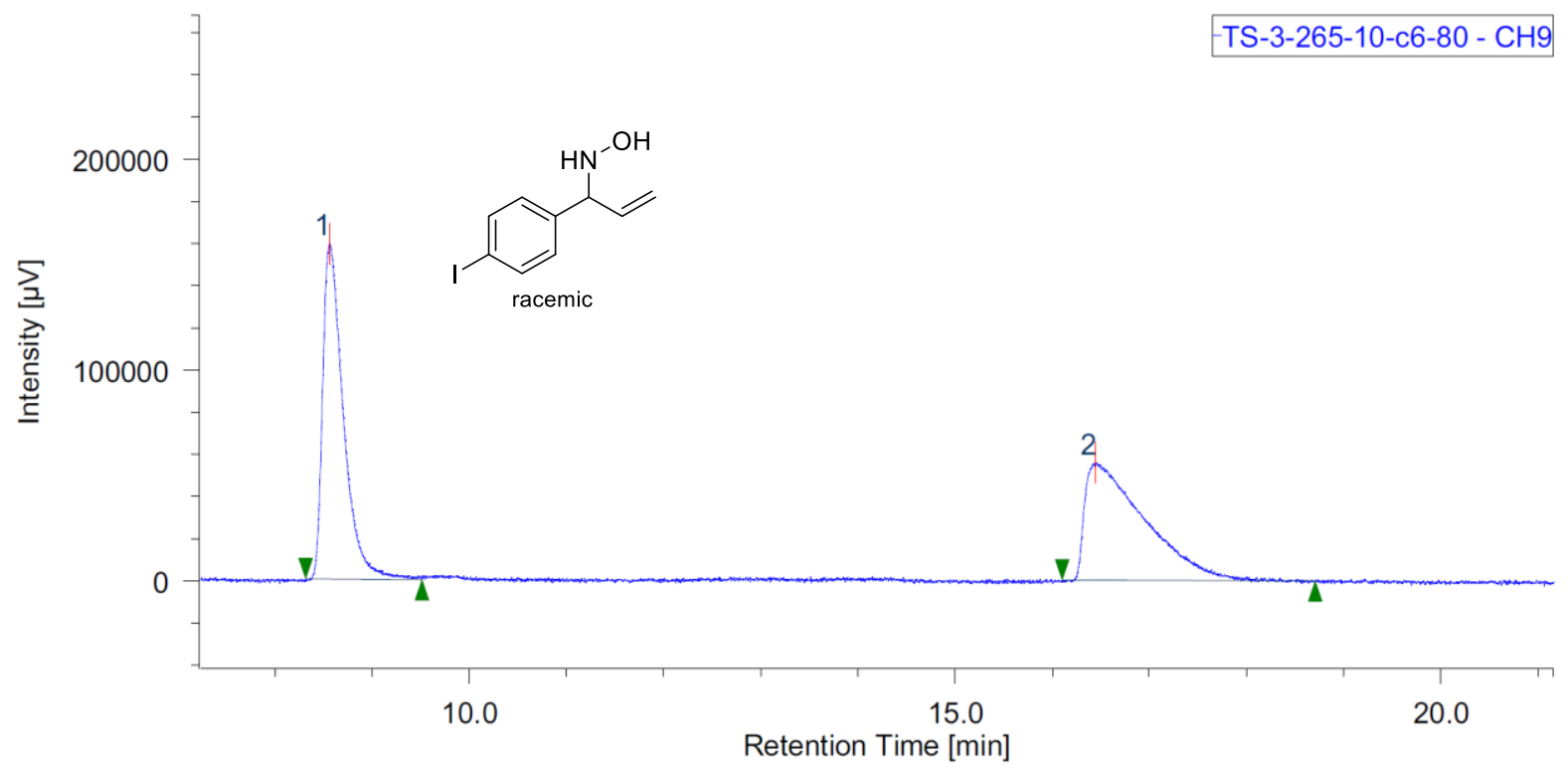

\begin{tabular}{|c|c|c|c|c|c|c|c|c|c|c|c|}
\hline$\#$ & Peak Name & CH & tR $[\mathrm{min}]$ & Area $[\mu \mathrm{V} \cdot \mathrm{sec}]$ & Height $[\mu \mathrm{V}]$ & Area\% & Height $\%$ & Quantity & NTP & Resolution & Symmetry Factor \\
\hline
\end{tabular}

\begin{tabular}{|c|c|c|c|c|c|c|c|c|c|c|}
\hline Unknown & 9 & 8.560 & 2371236 & 158903 & 49.937 & 74.046 & $\mathrm{~N} / \mathrm{A}$ & 8415 & 10.604 & 1.842 \\
\hline Unknown & 9 & 16.447 & 2377183 & 55699 & 50.063 & 25.954 & $\mathrm{~N} / \mathrm{A}$ & 3461 & $\mathrm{~N} / \mathrm{A}$ & \\
\hline
\end{tabular}

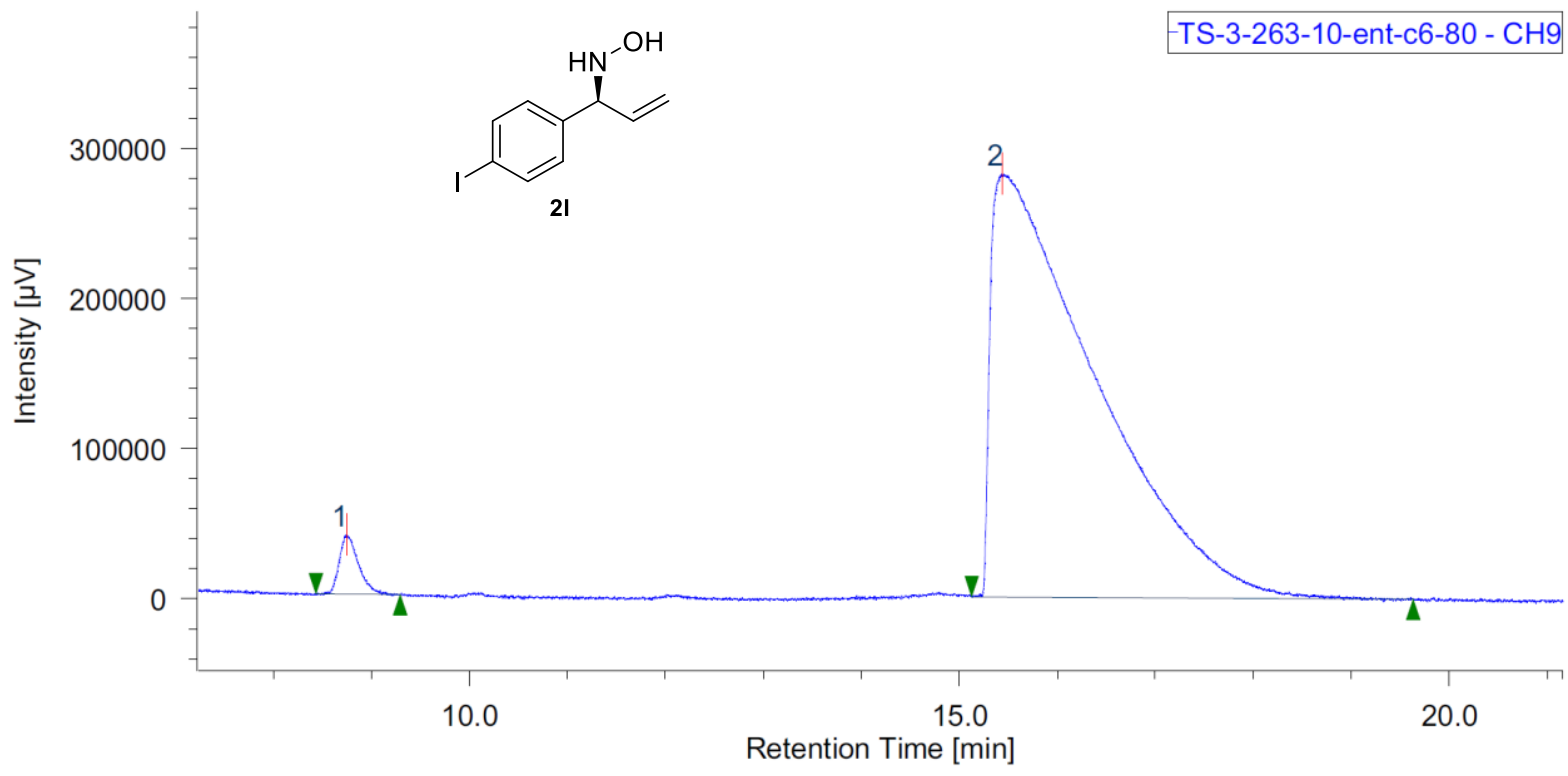

\begin{tabular}{|r|c|c|r|r|r|r|r|r|r|r|r|}
\hline$\#$ & Peak Name & CH & tR $[\mathrm{min}]$ & Area $[\mu \mathrm{V} \cdot \mathrm{sec}]$ & Height $[\mu \mathrm{V}]$ & \multicolumn{1}{|c|}{ Area $\%$} & Height $\%$ & Quantity & NTP & Resolution & Symmetry Factor \\
\hline 1 & Unknown & 9 & 8.747 & 533310 & 39433 & 2.496 & 12.274 & N/A & 9849 & 5.910 & 1.584 \\
\hline 2 & Unknown & 9 & 15.433 & 20832417 & 281851 & 97.504 & 87.726 & N/A & 1038 & N/A & 7.188 \\
\hline
\end{tabular}




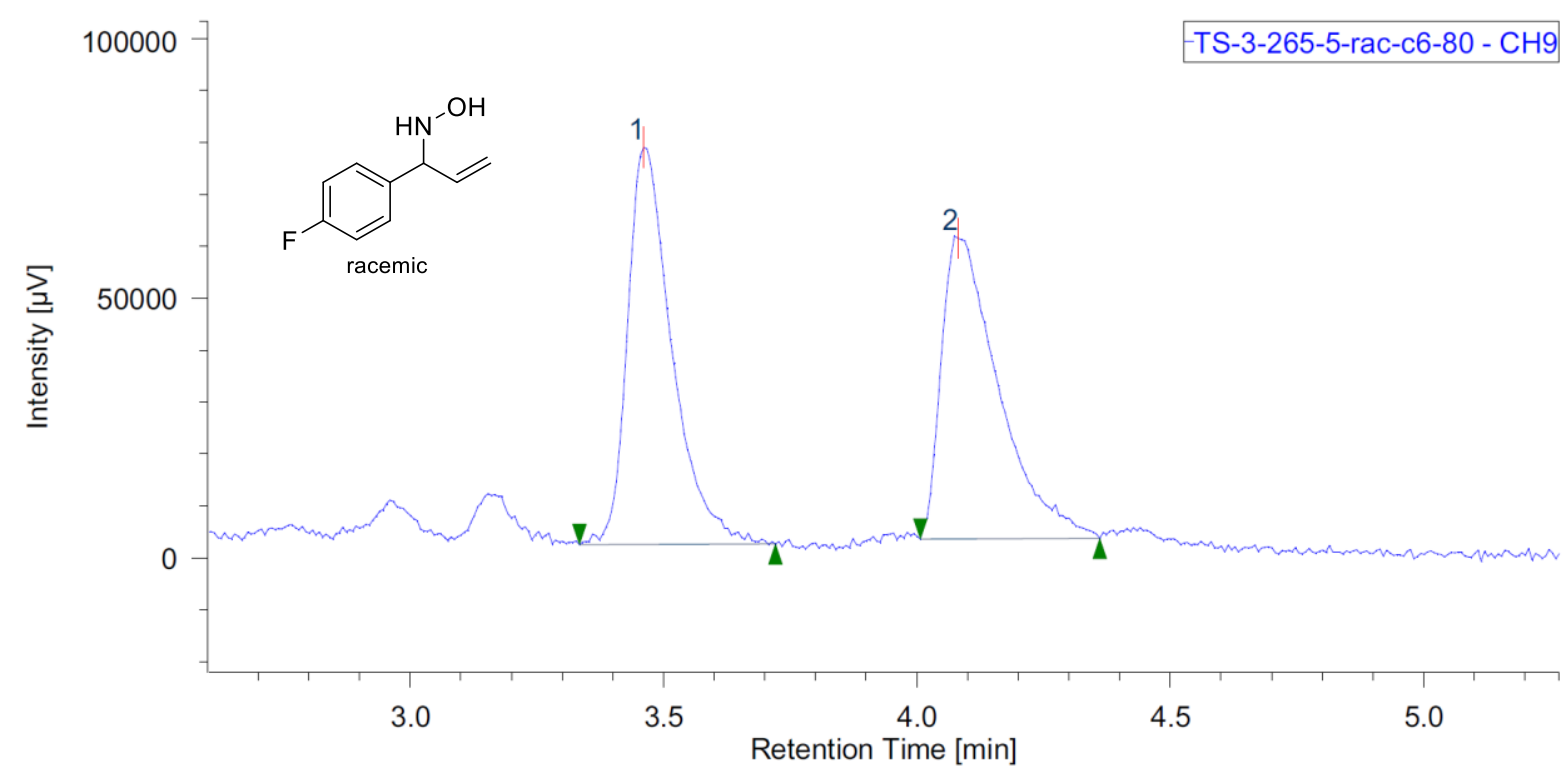

\begin{tabular}{|c|c|c|c|c|c|c|c|c|c|c|}
\hline Peak Name & $\overline{\mathrm{CH}}$ & $\mathrm{tR}[\mathrm{min}]$ & Area $[\mu \mathrm{V} \cdot \mathrm{sec}]$ & Height $[\mu \mathrm{V}]$ & Area $\%$ & Height $\%$ & Quantity & NTP & Resolution & Symmetry Factor \\
\hline Unknown & 9 & 3.460 & 468447 & 76476 & 50.544 & 56.902 & $\mathrm{~N} / \mathrm{A}$ & 8371 & 3.533 & 1.600 \\
\hline 2 Unknown & 9 & 4.080 & 458371 & 57925 & 49.456 & 43.098 & $\mathrm{~N} / \mathrm{A}$ & 6618 & $\mathrm{~N} / \mathrm{A}$ & 2.257 \\
\hline
\end{tabular}

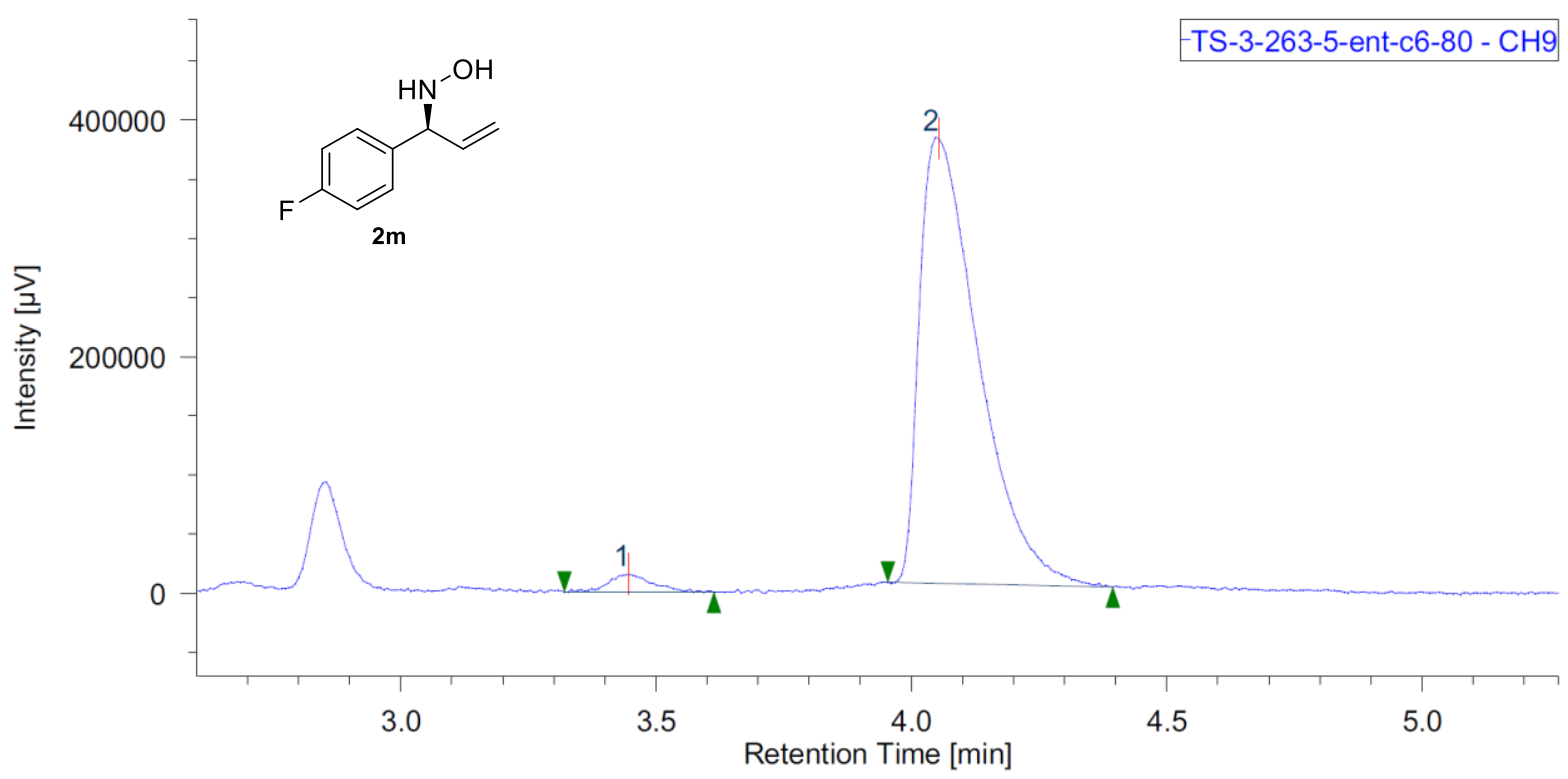

\begin{tabular}{|c|c|c|c|c|c|c|c|c|c|c|c|}
\hline & Peak Name & $\mathrm{CH}$ & $\mathrm{tR}[\mathrm{min}]$ & Area $[\mu \mathrm{V} \cdot \mathrm{sec}]$ & Height $[\mu \mathrm{V}]$ & Area $\%$ & Height $\%$ & Quantity & NTP & Resolution & Symmetry Factor \\
\hline & Unknown & 9 & 3.447 & 91438 & 15067 & 2.942 & 3.855 & $\mathrm{~N} / \mathrm{A}$ & 8703 & 3.420 & 1.193 \\
\hline & Unknown & 9 & 4.053 & 3016564 & 375822 & 97.058 & 96.145 & $\mathrm{~N} / \mathrm{A}$ & 6083 & $\mathrm{~N} / \mathrm{A}$ & 2.032 \\
\hline
\end{tabular}




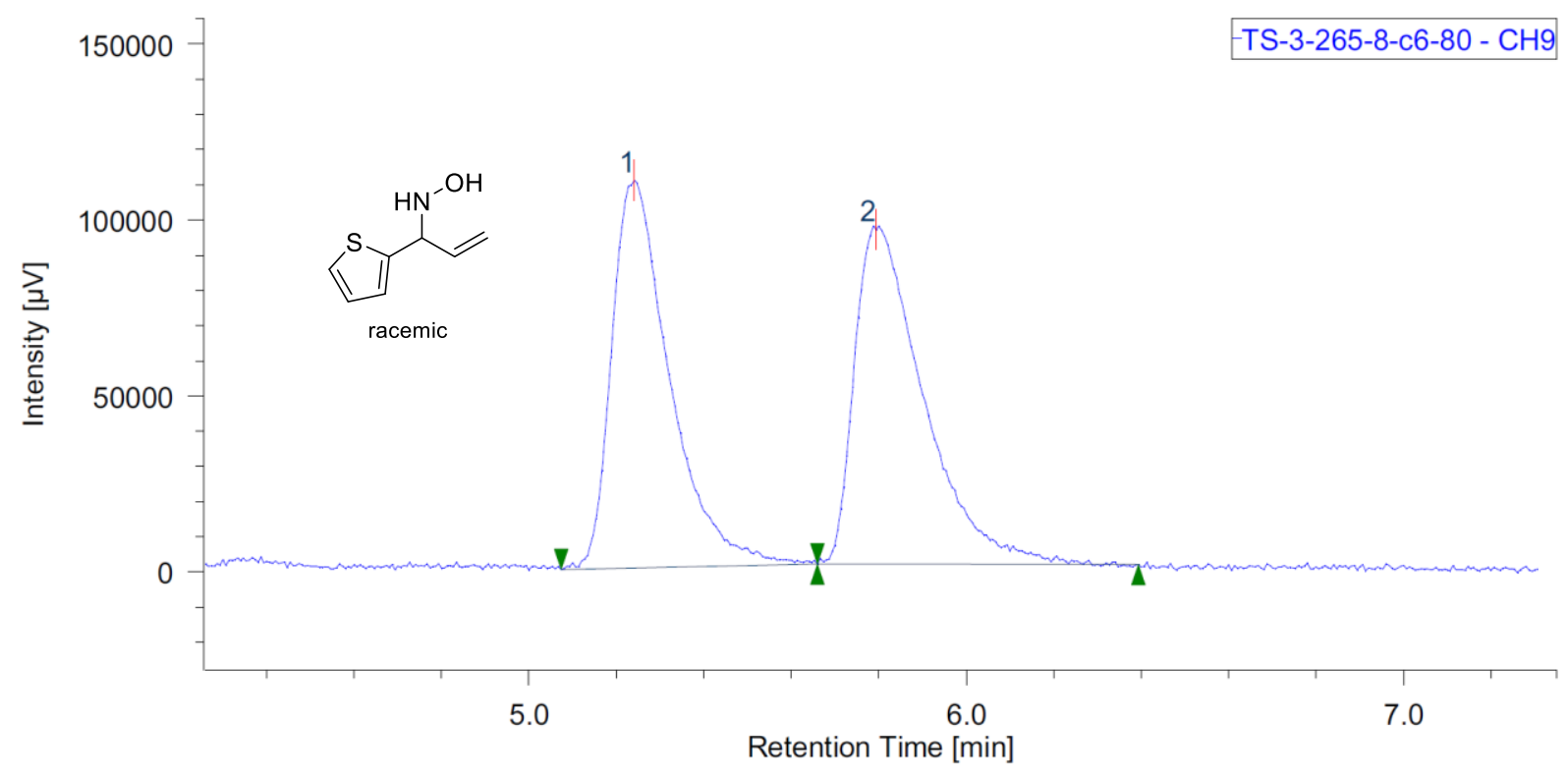

\begin{tabular}{|r|c|c|r|r|r|r|r|r|r|r|r|}
\hline$\#$ & Peak Name & $\mathrm{CH}$ & \multicolumn{1}{|c|}{$[\mathrm{min}]$} & Area $[\mu \mathrm{V} \cdot \mathrm{sec}]$ & Height $[\mu \mathrm{V}]$ & \multicolumn{1}{|c|}{ Area $\%$} & Height $\%$ & Quantity & NTP & Resolution & Symmetry Factor \\
\hline 1 & Unknown & 9 & 5.240 & 1018956 & 110160 & 49.256 & 53.676 & $\mathrm{~N} / \mathrm{A}$ & 8227 & 2.177 & 1.645 \\
\hline 2 & Unknown & 9 & 5.793 & 1049747 & 95070 & 50.744 & 46.324 & $\mathrm{~N} / \mathrm{A}$ & 6918 & $\mathrm{~N} / \mathrm{A}$ & 2.157 \\
\hline
\end{tabular}

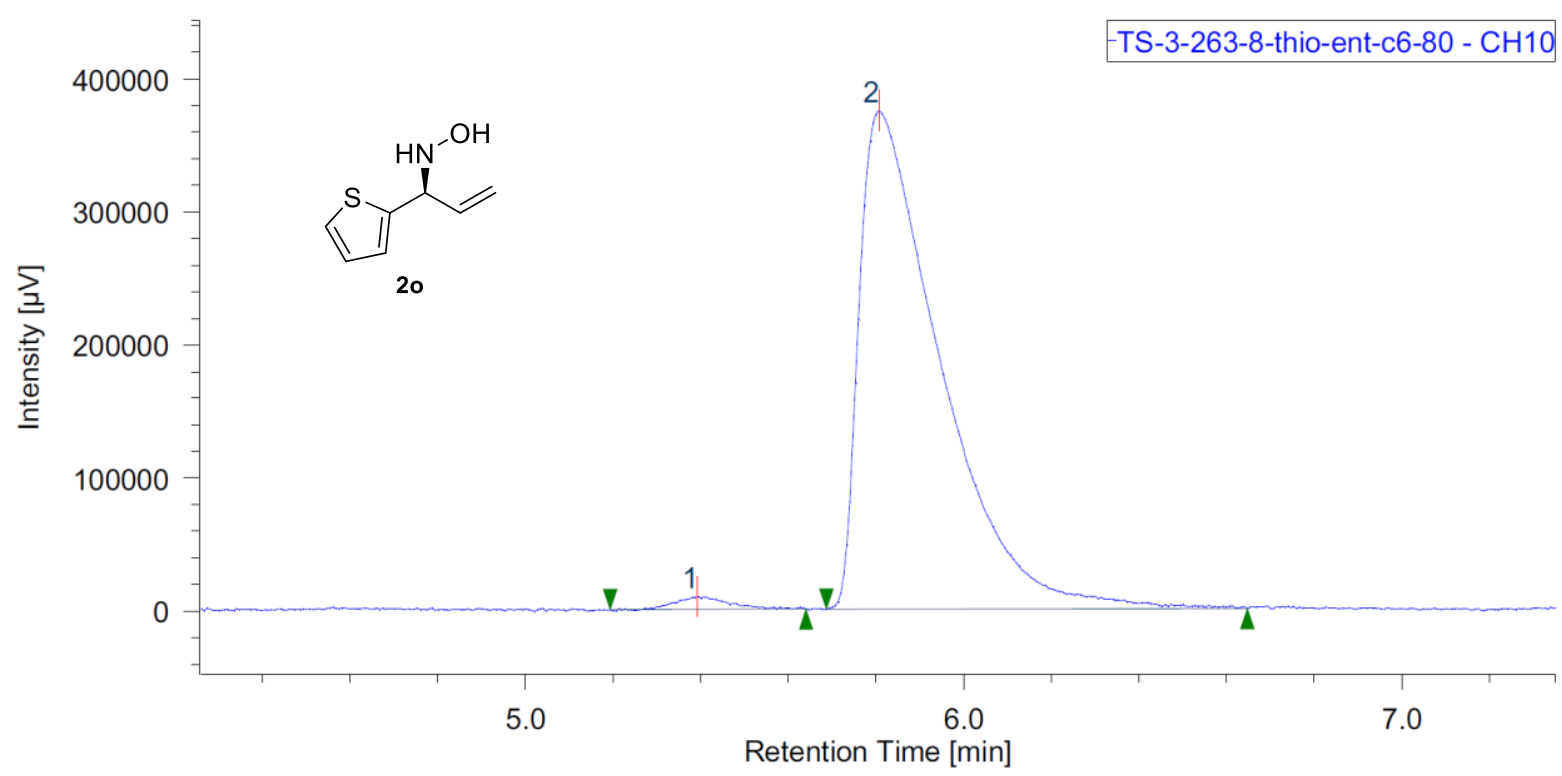

\begin{tabular}{|r|c|c|r|r|r|r|r|r|r|r|r|}
\hline$\#$ & Peak Name & $\mathrm{CH}$ & $\mathrm{tR}[\mathrm{min}]$ & Area $[\mu \mathrm{V} \cdot \mathrm{sec}]$ & Height $[\mu \mathrm{V}]$ & \multicolumn{1}{|c|}{ Area $\%$} & Height $\%$ & Quantity & NTP & Resolution & Symmetry Factor \\
\hline 1 & Unknown & 10 & 5.393 & 83230 & 9707 & 1.699 & 2.526 & $\mathrm{~N} / \mathrm{A}$ & 9651 & 1.526 & 1.164 \\
\hline 2 & Unknown & 10 & 5.807 & 4814379 & 374640 & 98.301 & 97.474 & $\mathrm{~N} / \mathrm{A}$ & 5153 & $\mathrm{~N} / \mathrm{A}$ & 2.705 \\
\hline
\end{tabular}




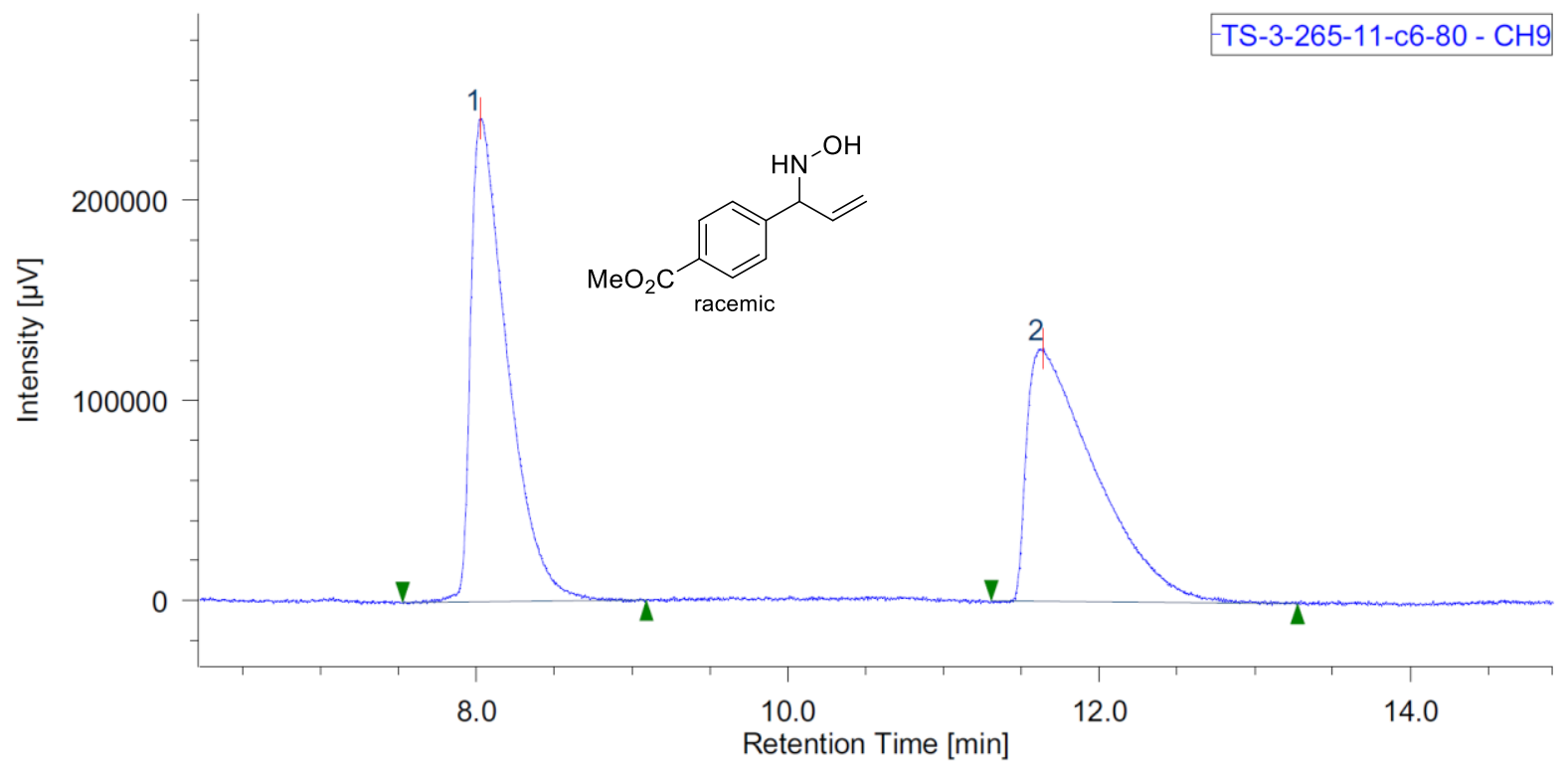

\begin{tabular}{|r|c|c|r|r|r|r|r|r|r|r|r|}
\hline$\#$ & Peak Name & $\mathrm{CH}$ & $\mathrm{tR}[\mathrm{min}]$ & Area $[\mu \mathrm{V} \cdot \mathrm{sec}]$ & Height $[\mu \mathrm{V}]$ & \multicolumn{1}{|c|}{ Area $\%$} & Height $\%$ & Quantity & NTP & Resolution & Symmetry Factor \\
\hline 1 & Unknown & 9 & 8.027 & 3973927 & 241564 & 50.814 & 65.637 & $\mathrm{~N} / \mathrm{A}$ & 5946 & 6.011 & 2.367 \\
\hline 2 & Unknown & 9 & 11.640 & 3846627 & 126464 & 49.186 & 34.363 & $\mathrm{~N} / \mathrm{A}$ & 3481 & $\mathrm{~N} / \mathrm{A}$ & 3.083 \\
\hline
\end{tabular}

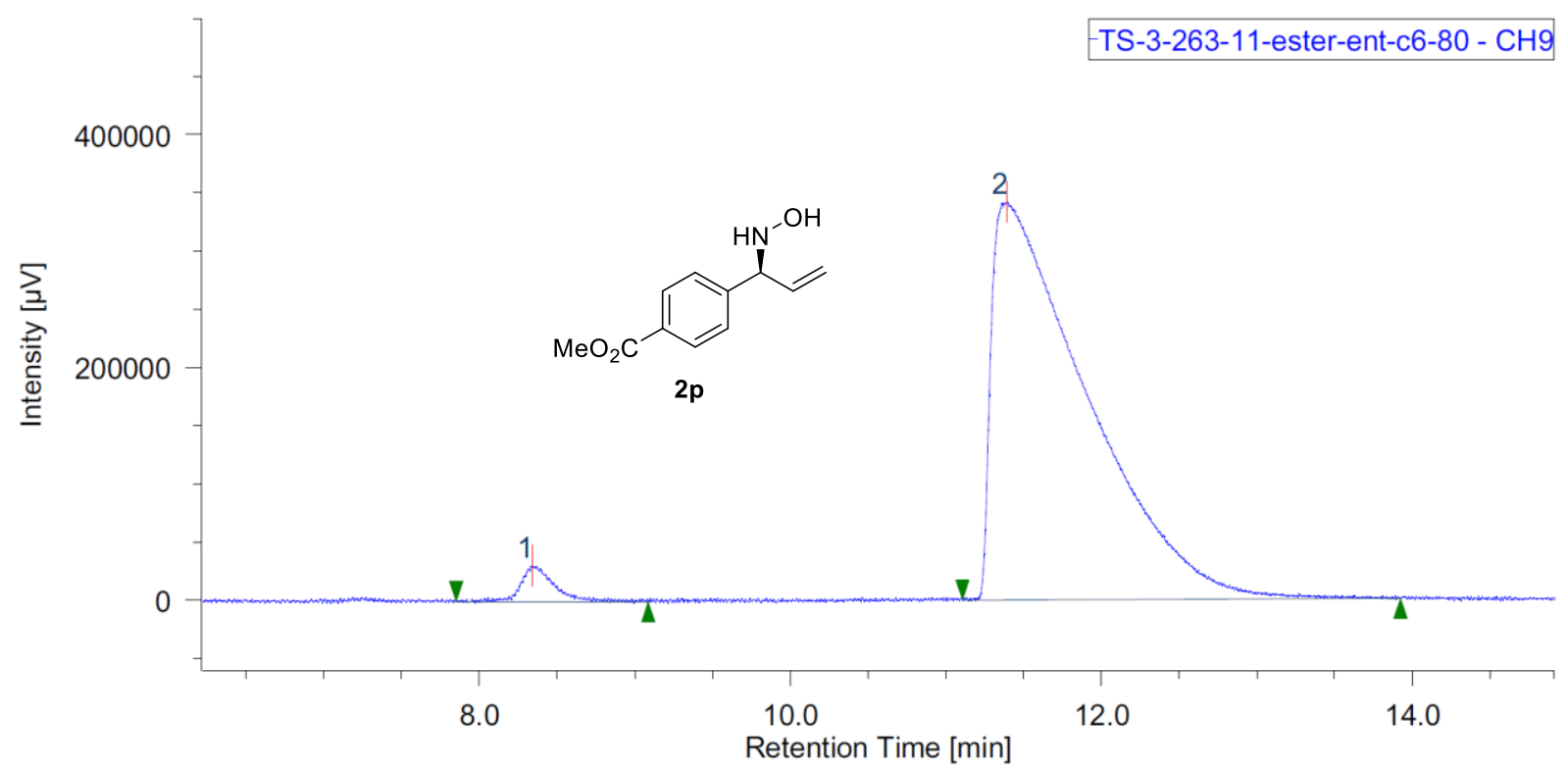

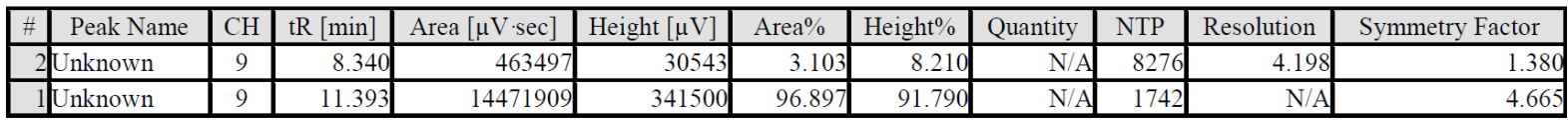




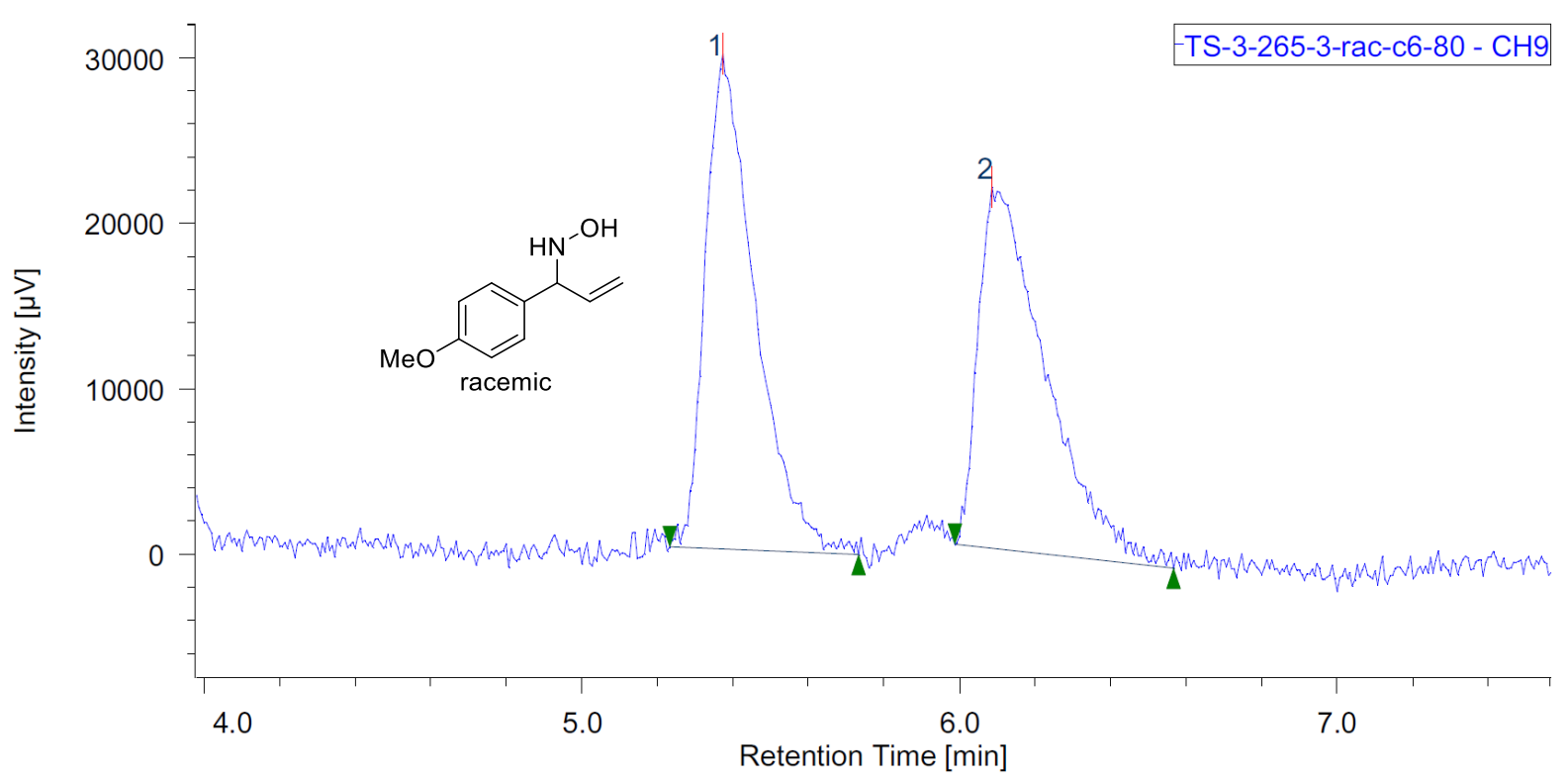

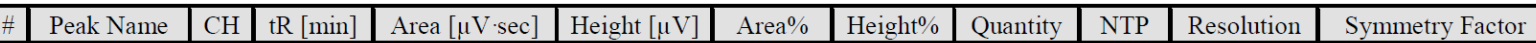

\begin{tabular}{|c|c|c|c|c|c|c|c|c|c|c|}
\hline Unknown & 9 & 5.373 & 272611 & 29931 & 50.185 & 57.788 & $\mathrm{~N} / \mathrm{A}$ & 8332 & 2.622 & 1.775 \\
\hline Unk & 9 & 6.087 & 270601 & 21863 & 49.815 & 42.212 & $\mathrm{~N} / \mathrm{A}$ & 6165 & $\mathrm{~N} / \mathrm{A}$ & 2.7 \\
\hline
\end{tabular}

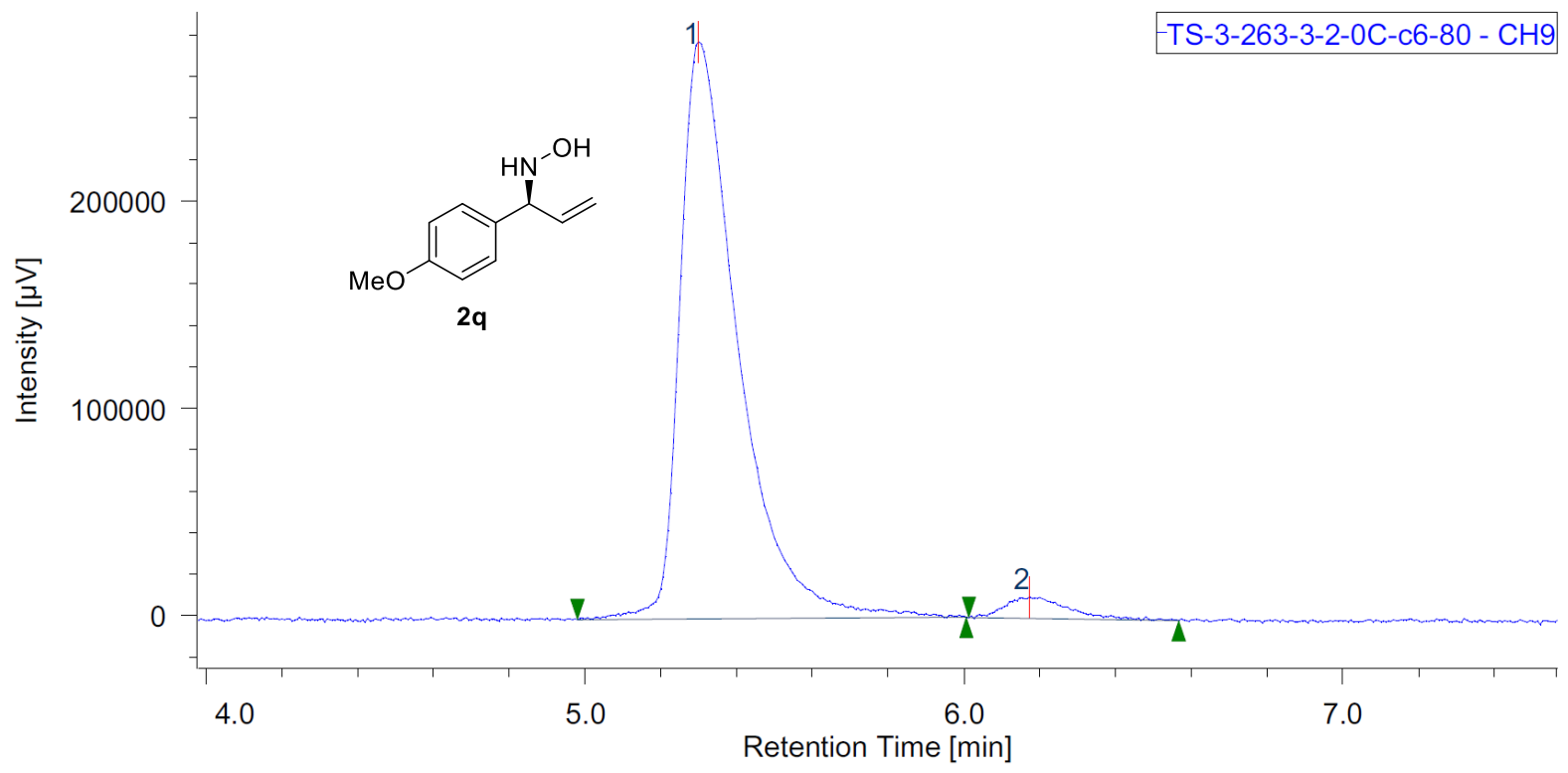

\begin{tabular}{|r|c|c|r|r|r|r|r|r|r|r|r|}
\hline$\#$ & Peak Name & CH & tR $[\mathrm{min}]$ & Area $[\mu \mathrm{V} \cdot \mathrm{sec}]$ & Height $[\mu \mathrm{V}]$ & \multicolumn{1}{|c|}{ Area $\%$} & Height $\%$ & Quantity & NTP & Resolution & Symmetry Factor \\
\hline 2 & Unknown & 9 & 5.300 & 2961273 & 278388 & 96.108 & 96.434 & N/A & 6730 & 3.165 & 1.919 \\
\hline 1 & Unknown & 9 & 6.173 & 119920 & 10295 & 3.892 & 3.566 & N/A & 7013 & N/A & 1.620 \\
\hline
\end{tabular}




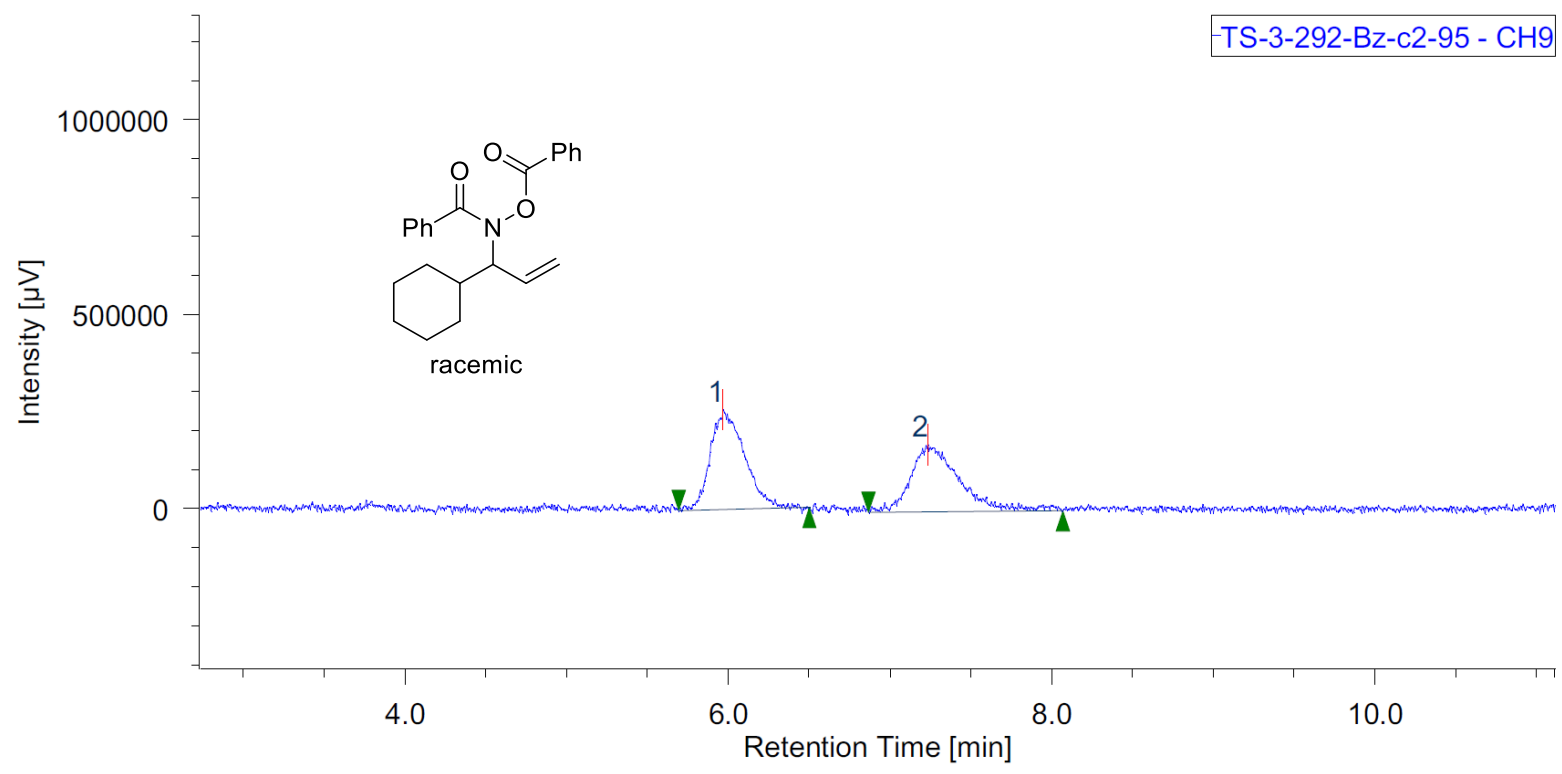

\begin{tabular}{|l|c|c|c|c|c|c|c|c|c|c|c|}
$\#$ & Peak Name & $\mathrm{CH}$ & tR $[\mathrm{min}]$ & Area $[\mu \mathrm{V}$-sec] & Height $[\mu \mathrm{V}]$ & Area $\%$ & Height $\%$ & Quantity & NTP & Resolution & Symmetry Factor \\
\hline
\end{tabular}

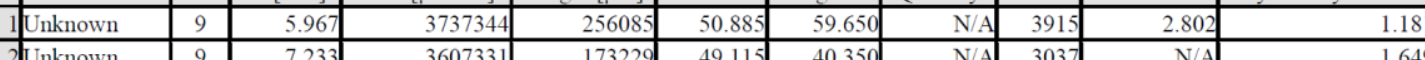

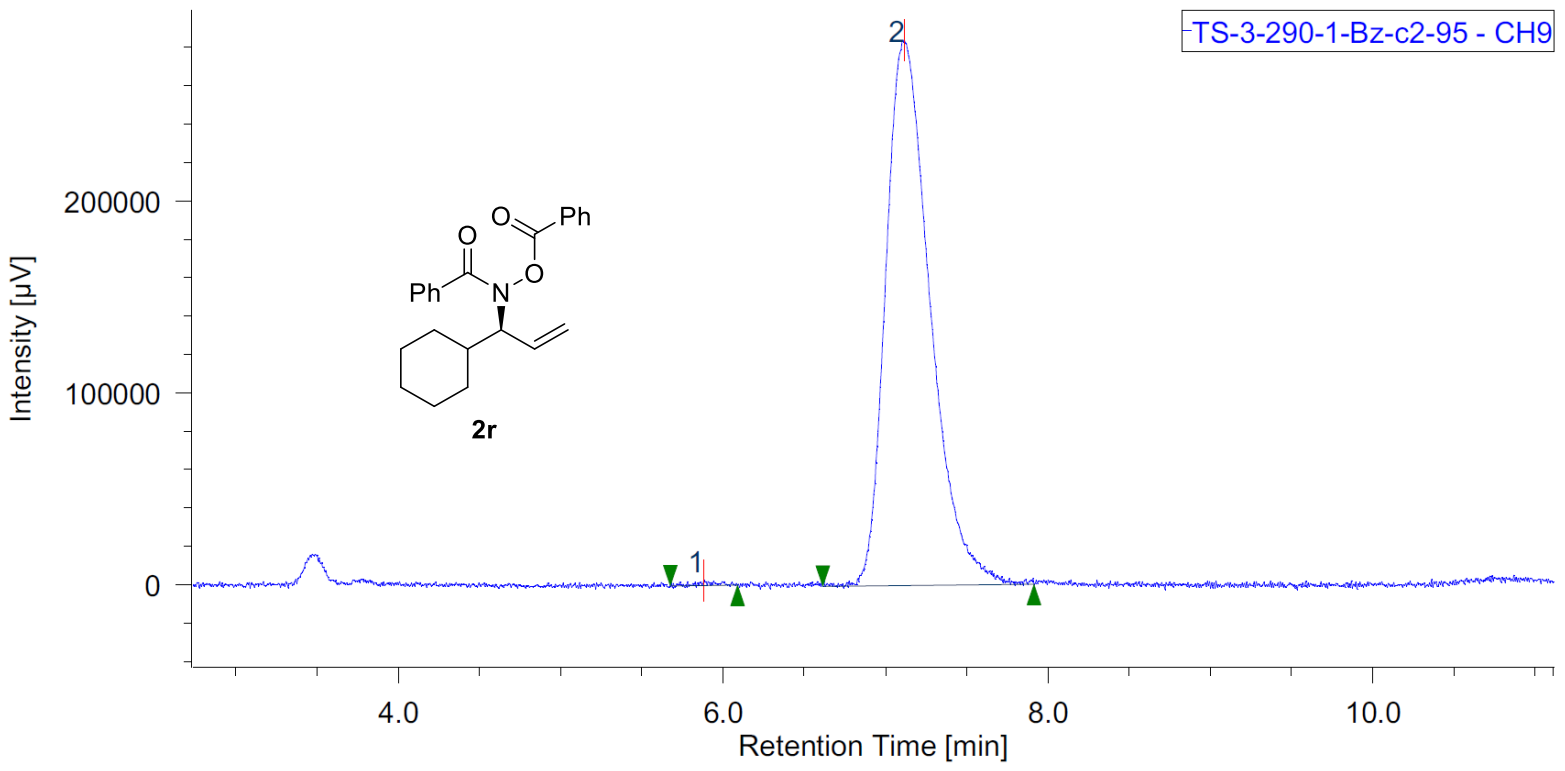

\begin{tabular}{|r|c|c|r|r|r|r|r|r|r|r|r|}
\hline$\#$ & Peak Name & CH & tR $[\mathrm{min}]$ & Area $[\mu \mathrm{V} \cdot \mathrm{sec}]$ & Height $[\mu \mathrm{V}]$ & \multicolumn{1}{|c|}{ Area $\%$} & Height $\%$ & Quantity & NTP & Resolution & Symmetry Factor \\
\hline 2 & Unknown & 9 & 5.880 & 20300 & 2615 & 0.374 & 0.911 & N/A & 1356 & 2.190 & 0.999 \\
\hline 1 & Unknown & 9 & 7.113 & 5401663 & 28422 & 99.626 & 99.089 & N/A & 3367 & N/A & 1.412 \\
\hline
\end{tabular}




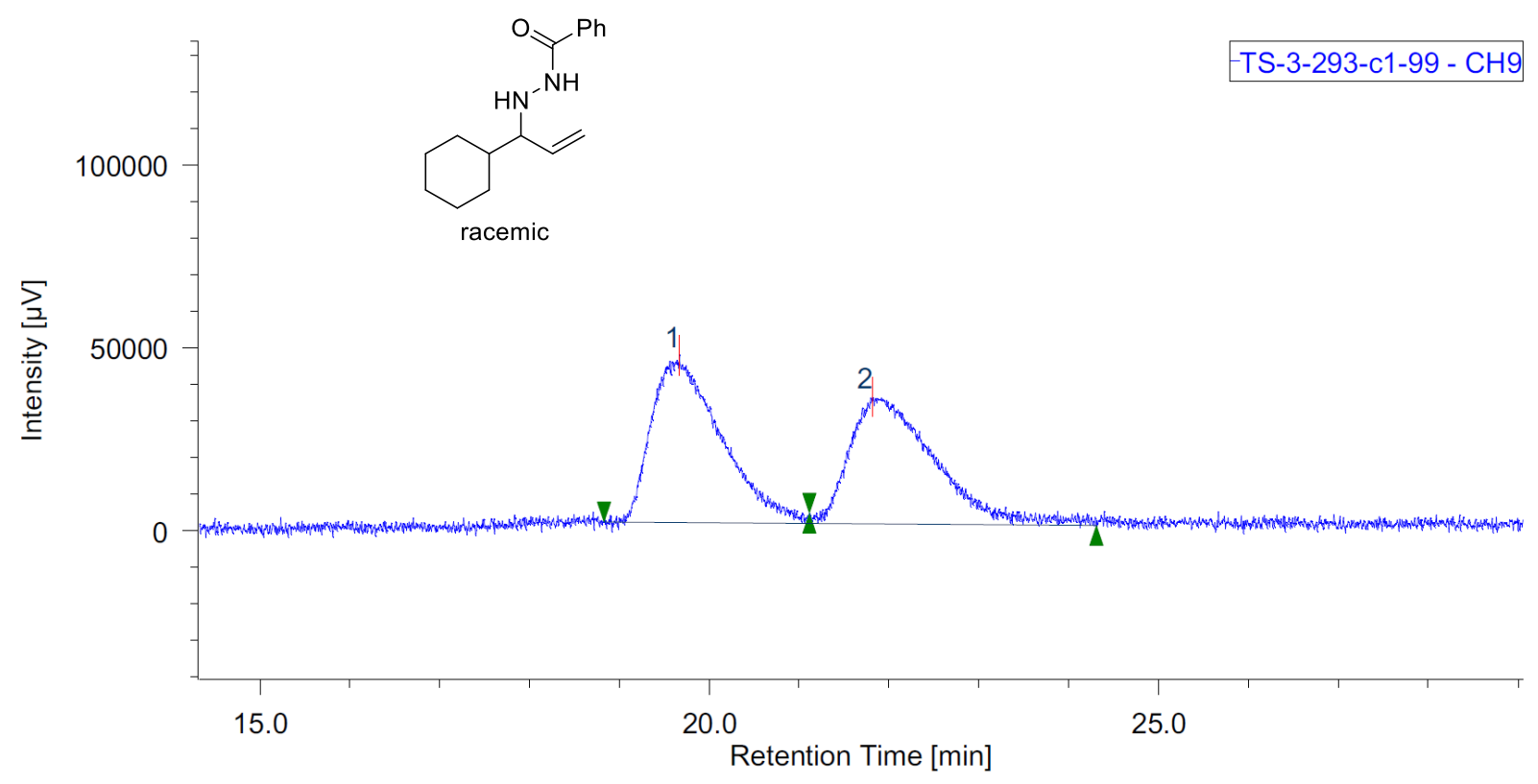

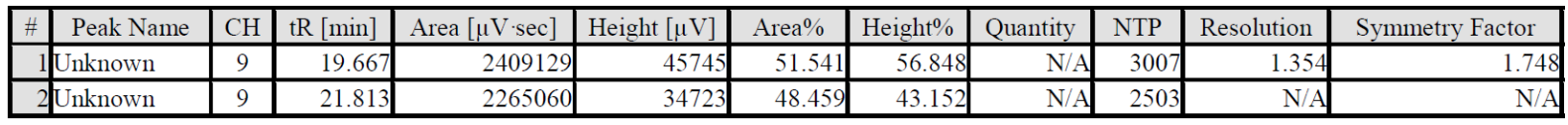

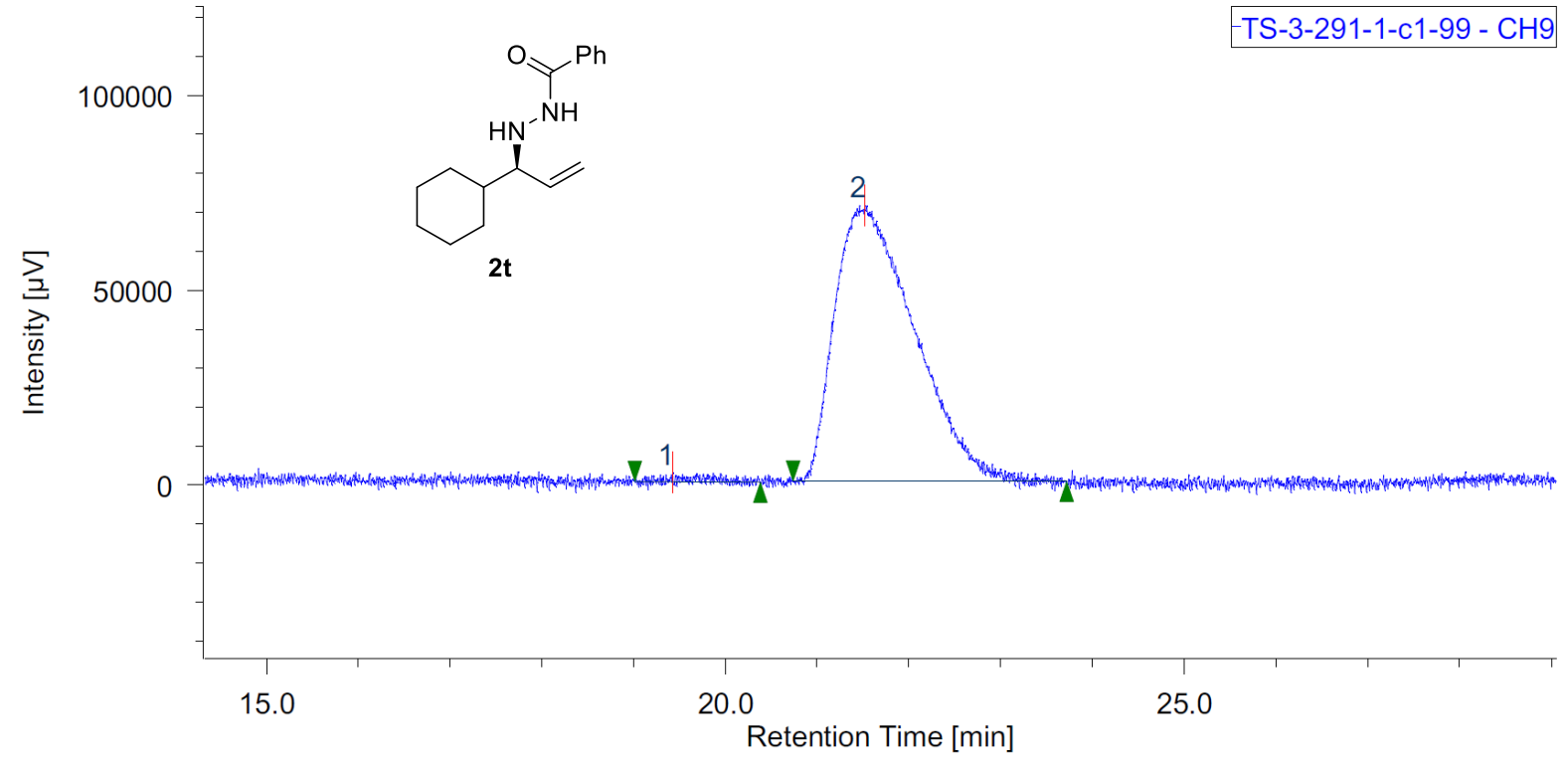

\begin{tabular}{|r|c|c|r|r|r|r|r|r|r|r|r|}
\hline$\#$ & Peak Name & $\mathrm{CH}$ & $\mathrm{tR}[\mathrm{min}]$ & Area $[\mu \mathrm{V} \cdot \mathrm{sec}]$ & Height $[\mu \mathrm{V}]$ & \multicolumn{1}{|c|}{ Area\% } & Height $\%$ & Quantity & NTP & Resolution & Symmetry Factor \\
\hline 2 & Unknown & 9 & 19.427 & 52167 & 2313 & 1.217 & 3.172 & $\mathrm{~N} / \mathrm{A}$ & 1166 & 1.070 & 1.644 \\
\hline 1 & Unknown & 9 & 21.520 & 4233866 & 70605 & 98.783 & 96.828 & $\mathrm{~N} / \mathrm{A}$ & 2726 & $\mathrm{~N} / \mathrm{A}$ & 1.687 \\
\hline
\end{tabular}




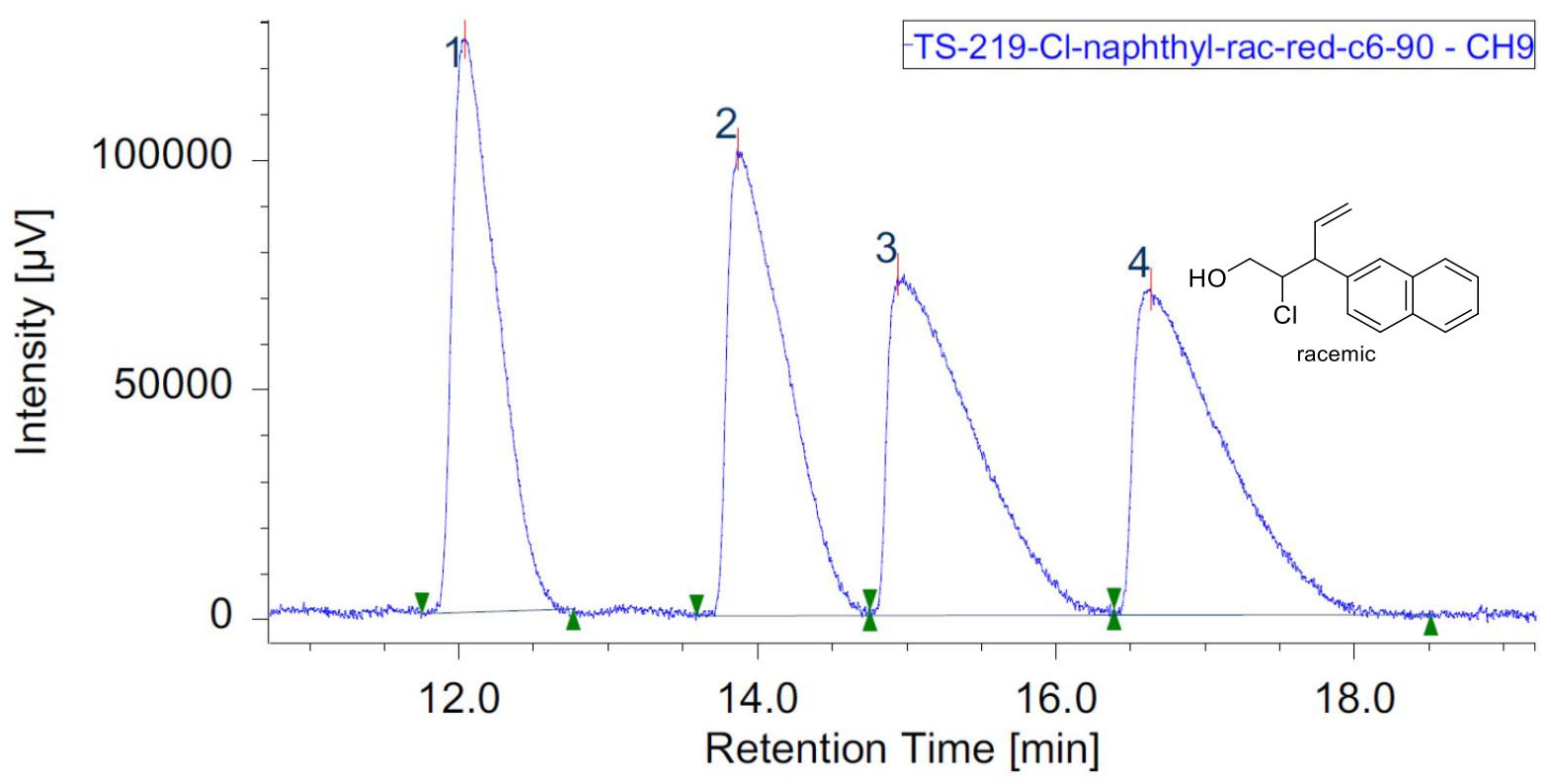

\begin{tabular}{|c|c|c|c|c|c|c|c|c|c|c|c|}
\hline$\#$ & Peak Name & $\mathrm{CH}$ & $\mathrm{tR}$ [min] & Area $[\mu \mathrm{V} \cdot \sec ]$ & Height $[\mu \mathrm{V}]$ & Area $\%$ & Height $\%$ & Quantity & NTP & Resolution & Symmetry Factor \\
\hline & Unknown & 9 & 12.040 & 2586346 & 125096 & 23.242 & 33.636 & $\mathrm{~N} / \mathrm{A}$ & 6991 & 2.798 & 2.132 \\
\hline & Unknown & 9 & 13.867 & 2706696 & 101562 & 24.324 & 27.308 & $\mathrm{~N} / \mathrm{A}$ & 5721 & 1.208 & 3.361 \\
\hline 3 & Unknown & 9 & 14.940 & 2912215 & 74255 & 26.171 & 19.966 & $\mathrm{~N} / \mathrm{A}$ & 3250 & 1.580 & 5.166 \\
\hline 4 & Unknown & 9 & 16.633 & 2922449 & 71001 & 26.263 & 19.091 & $\mathrm{~N} / \mathrm{A}$ & 3654 & N/A & 3.812 \\
\hline
\end{tabular}

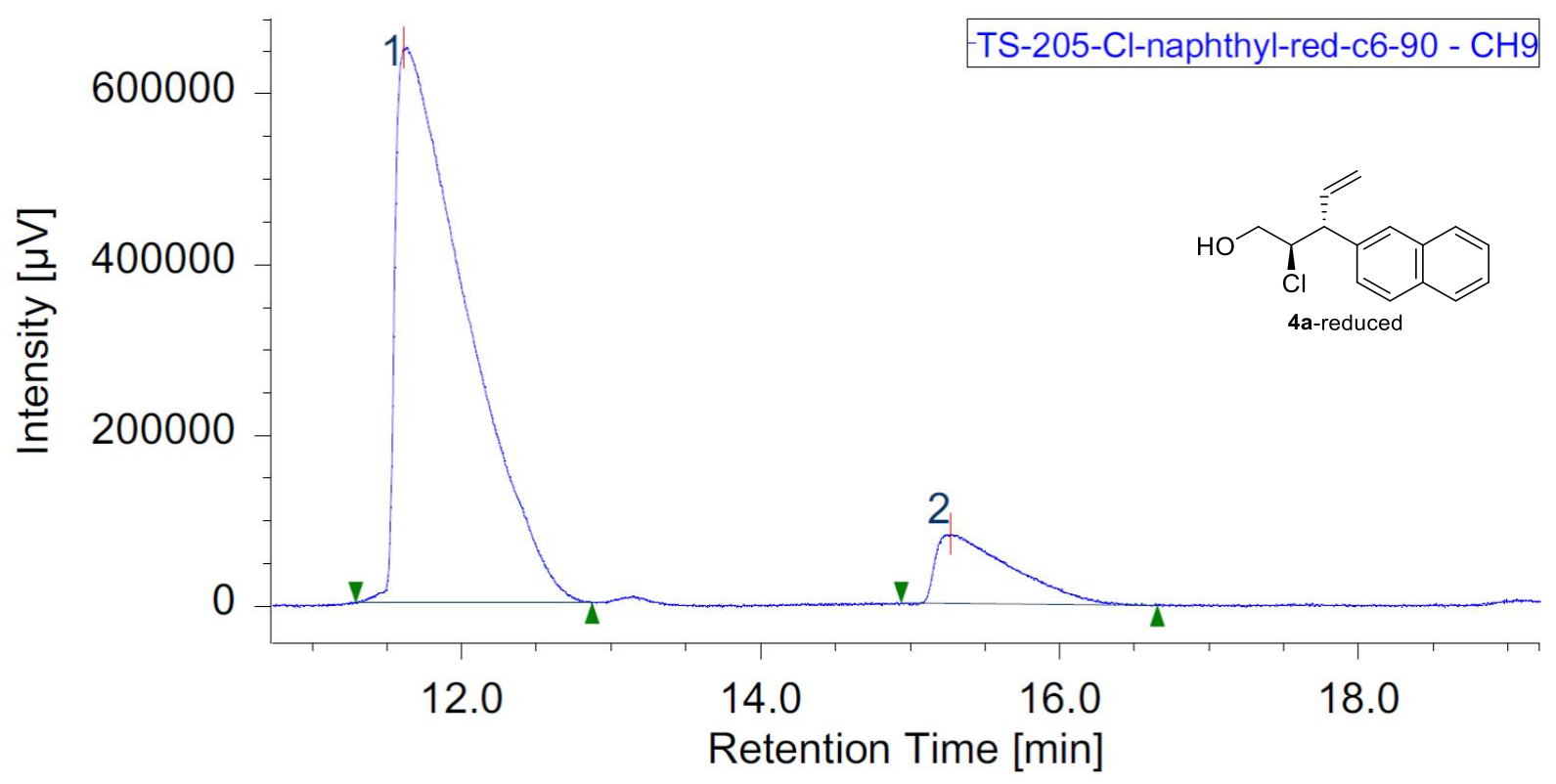

\begin{tabular}{|r|l|c|r|r|r|r|r|r|r|r|r|}
\hline$\#$ & Peak Name & CH & tR $[\mathrm{min}]$ & Area $[\mu \mathrm{V} \cdot \mathrm{sec}]$ & Height $[\mu \mathrm{V}]$ & Area $\%$ & Height $\%$ & Quantity & NTP & Resolution & Symmetry Factor \\
\hline 1 & Unknown & 9 & 11.613 & 21338934 & 650033 & 88.274 & 88.944 & N/A & 2861 & 4.085 & 5.215 \\
\hline 2 & Unknown & 9 & 15.26 & 2834546 & 80797 & 11.726 & 11.05 & N/A & 4357 & N/A & 3.533 \\
\hline
\end{tabular}




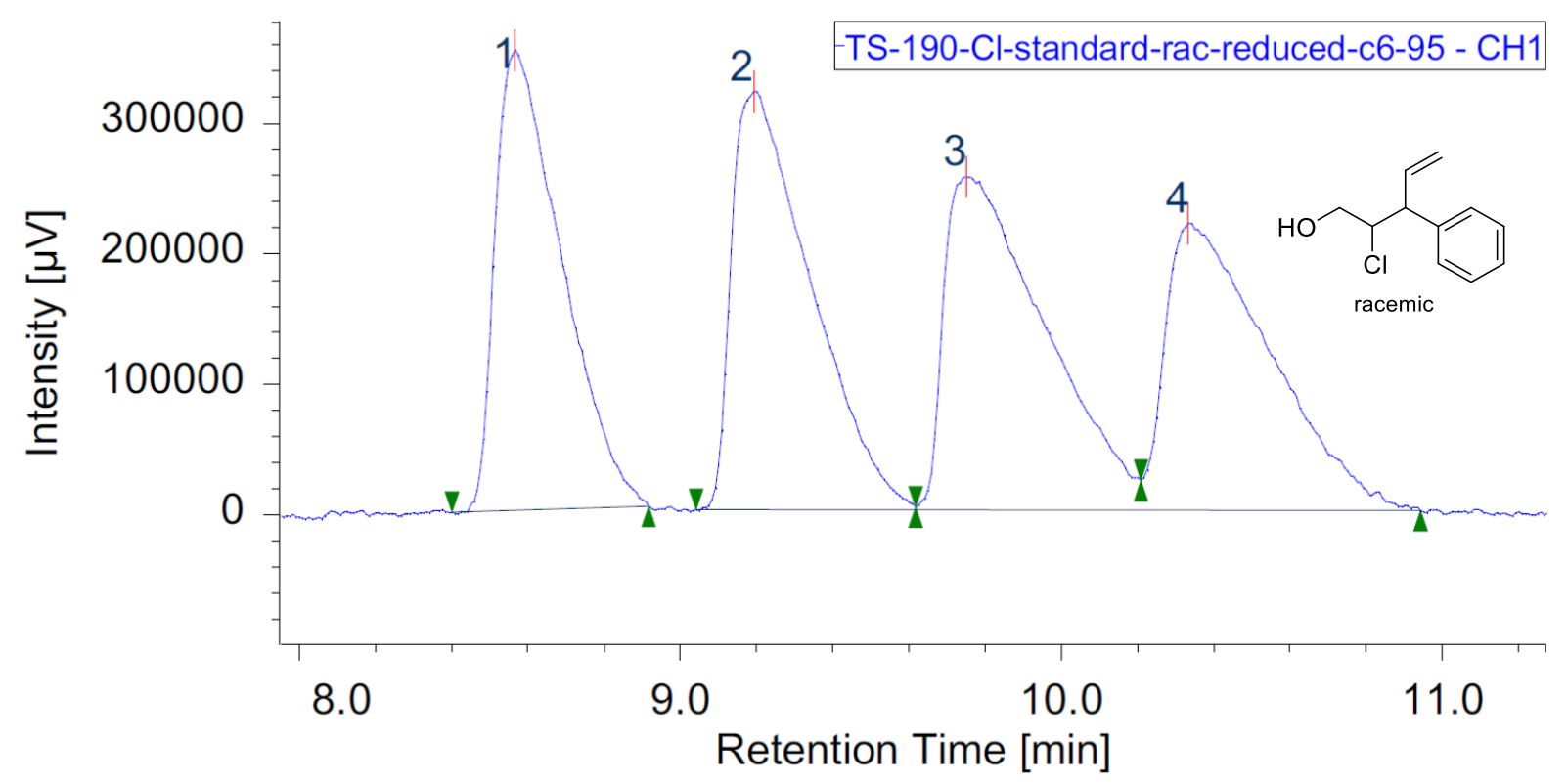

\begin{tabular}{|c|c|c|c|c|c|c|c|c|c|c|c|}
\hline \# & Peak Name & $\mathrm{CH}$ & {$[\mathrm{min}]$} & ea $[\mu \mathrm{V} \cdot \mathrm{sec}]$ & Height $[\mu \mathrm{V}]$ & Area $\%$ & Height $\%$ & Quantity & NTP & Resolution & Symmetry Factor \\
\hline & Unknown & 1 & 8.567 & 4259149 & $35248 \mathrm{~s}$ & 23.967 & 30.707 & $\mathrm{~N} / \mathrm{A}$ & 10823 & 1.747 & 1.985 \\
\hline & Unknown & 1 & 9.192 & 4624563 & 320203 & 26.024 & 27.894 & $\mathrm{~N} / \mathrm{A}$ & 8977 & 1.255 & 2.314 \\
\hline & Unknown & 1 & 9.750 & 4682858 & 255121 & 26.352 & 22.225 & $\mathrm{~N} / \mathrm{A}$ & 5989 & 1.145 & $\mathrm{~N} / \mathrm{A}$ \\
\hline & Unknown & 1 & 10.333 & 4204148 & 220110 & 23.658 & 19.175 & $\mathrm{~N} / \mathrm{A}$ & 6381 & $\mathrm{~N} / \mathrm{A}$ & $\mathrm{N} / \mathrm{A}$ \\
\hline
\end{tabular}

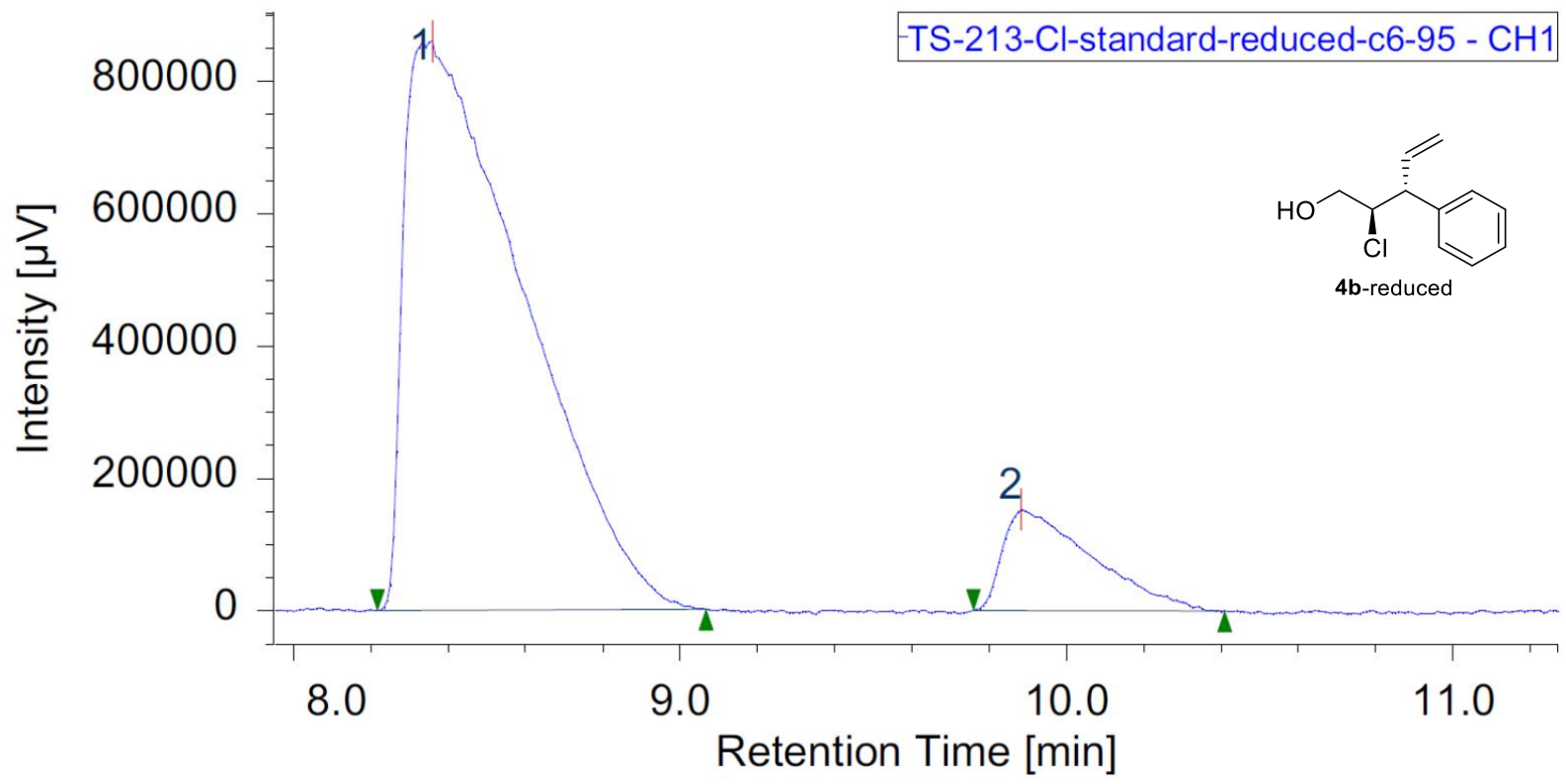

\begin{tabular}{|c|c|c|c|c|c|c|c|c|c|c|c|}
\hline \# & Peak Name & $\mathrm{CH}$ & $\mathrm{tR}$ [min] & Area $[\mu \mathrm{V} \cdot \sec ]$ & Height $[\mu \mathrm{V}]$ & Area $\%$ & Height $\%$ & Quantity & NTP & Resolution & try Factor \\
\hline & Unknown & 1 & 8.358 & 18447005 & 859932 & 88.355 & 84.962 & $\mathrm{~N} / \mathrm{A}$ & 3164 & 3.000 & 3.065 \\
\hline & Unknown & 1 & 9.883 & 2431232 & 152199 & 11.645 & 15.038 & $\mathrm{~N} / \mathrm{A}$ & 8652 & $\mathrm{~N} / \mathrm{A}$ & 2.706 \\
\hline
\end{tabular}




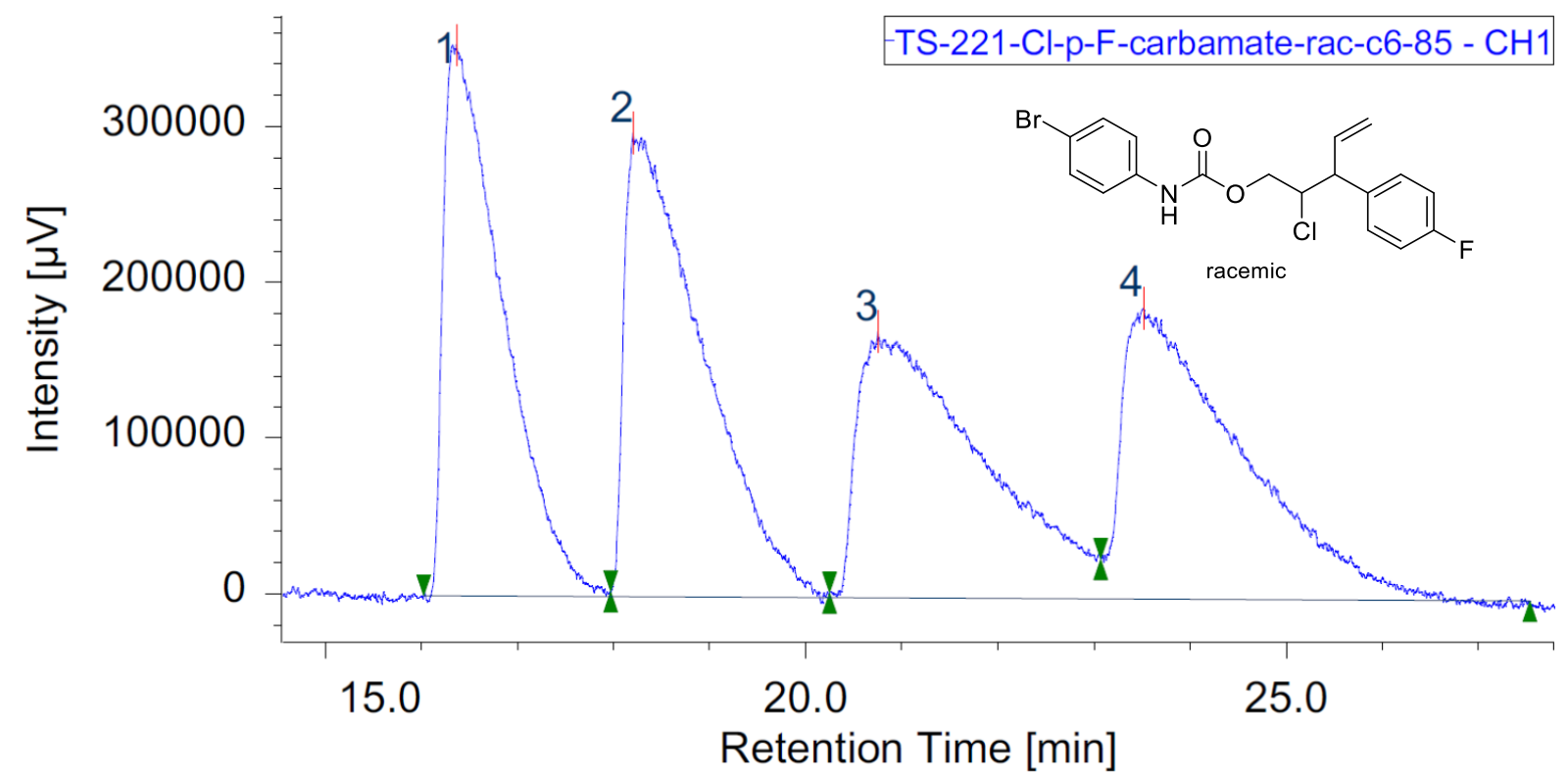

\begin{tabular}{|c|c|c|c|c|c|c|c|c|c|c|c|}
\hline$\#$ & Peak Name & $\mathrm{CH}$ & $\mathrm{tR}$ [min] & Area $[\mu \mathrm{V} \cdot \mathrm{sec}]$ & Height $[\mu \mathrm{V}]$ & Area $\%$ & Height $\%$ & Quantity & NTP & Resolution & Symmetry Factor \\
\hline 1 & Unknown & 1 & 16.367 & 15155913 & 353526 & 24.058 & 35.051 & $\mathrm{~N} / \mathrm{A}$ & 3232 & 1.386 & 3.125 \\
\hline 2 & Unknown & 1 & 18.200 & 16748922 & 297417 & 26.587 & 29.488 & $\mathrm{~N} / \mathrm{A}$ & 2351 & 1.325 & 5.048 \\
\hline 4 & Unknown & 1 & 20.750 & 15155399 & 170921 & 24.057 & 16.946 & $\mathrm{~N} / \mathrm{A}$ & 1239 & 1.215 & $\mathrm{~N} / \mathrm{A}$ \\
\hline
\end{tabular}

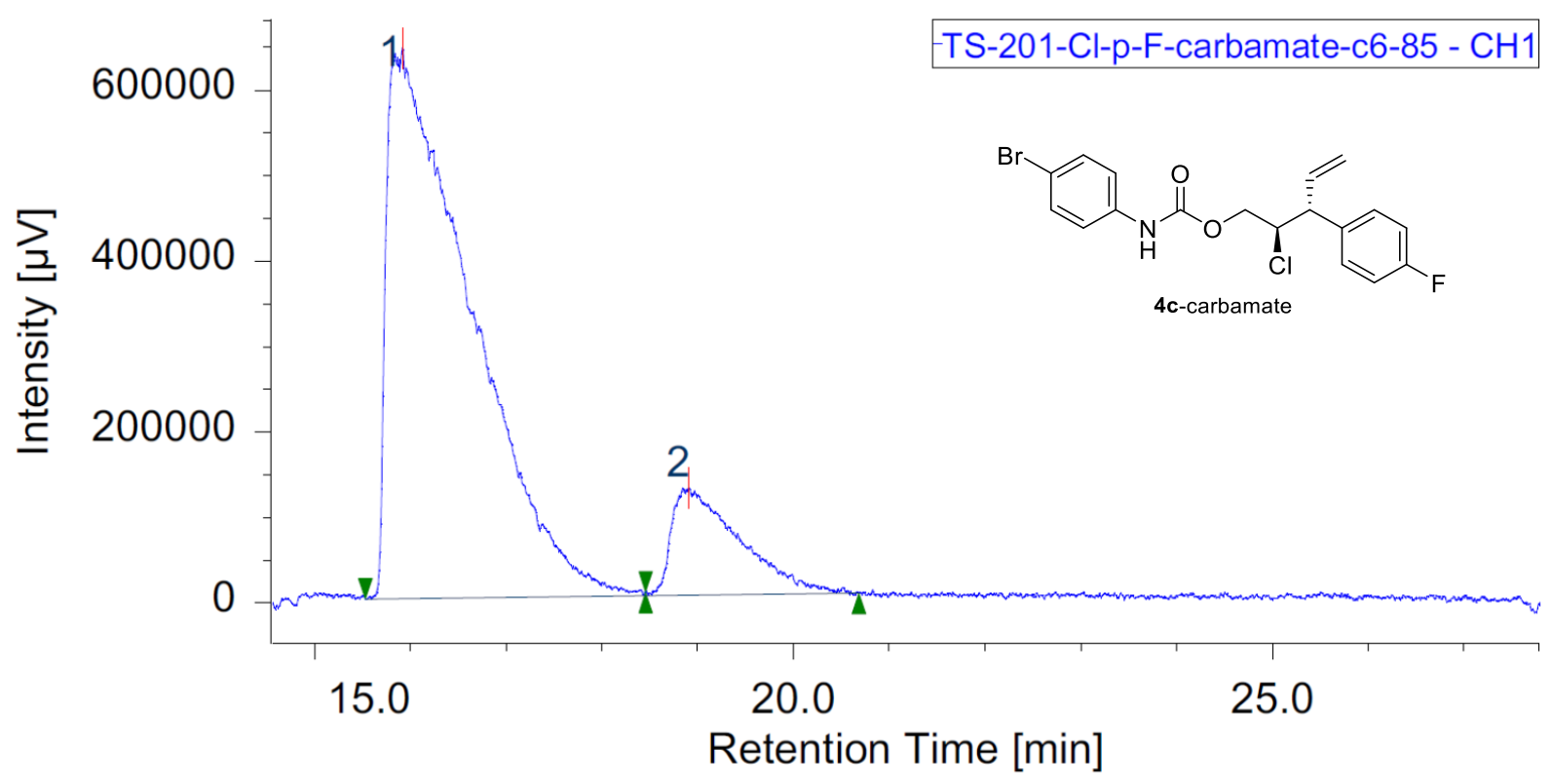

\begin{tabular}{|c|c|c|c|c|c|c|c|c|c|c|c|}
\hline 4 & Peak Name & $\mathrm{CH}$ & $\mathrm{tR}[\mathrm{min}]$ & Area $[\mu \mathrm{V} \cdot \mathrm{sec}]$ & Height $[\mu \mathrm{V}]$ & Area $\%$ & Height $\%$ & Quantity & NTP & Resolution & try Factor \\
\hline & Unknown & 1 & 15.917 & 3904125 & 644167 & 86.377 & 83.710 & $\mathrm{~N} / \mathrm{A}$ & 1545 & 2.070 & 4.257 \\
\hline & Unknown & 1 & 18.908 & 615764 & 125355 & 13.623 & 16.290 & $\mathrm{~N} / \mathrm{A}$ & 3496 & $\mathrm{~N} / \mathrm{A}$ & 2.879 \\
\hline
\end{tabular}




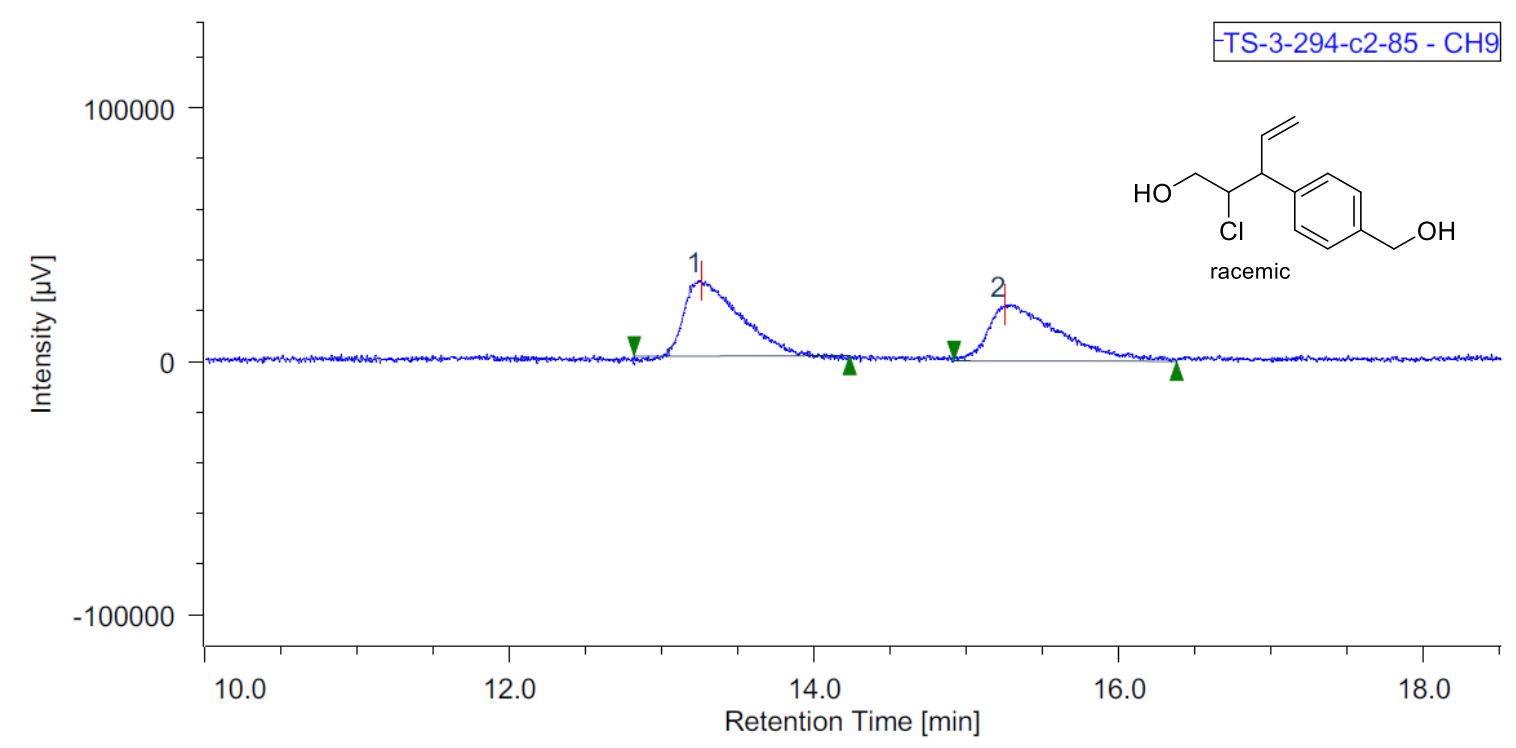

\begin{tabular}{|c|c|c|c|c|c|c|c|c|c|c|c|c|}
\hline$\# 1$ & Peak Name & $\mathrm{CH}$ & $\mathrm{tR}[\mathrm{min}]$ & Area $[\mu \mathrm{V} \cdot \mathrm{sec}]$ & Height $[\mu \mathrm{V}]$ & Area $\%$ & Height $\%$ & Quantity & NTP & Resolution & Symmetry Factor & Warning \\
\hline & Unknown & 9 & 13.260 & 752985 & 29734 & 50.899 & 57.181 & $\mathrm{~N} / \mathrm{A}$ & 5984 & 2.600 & 2.070 & \\
\hline & Unknown & 9 & 15.253 & 726395 & 22265 & 49.101 & 42.819 & $\mathrm{~N} / \mathrm{A}$ & 5133 & N/A & 2.176 & \\
\hline
\end{tabular}

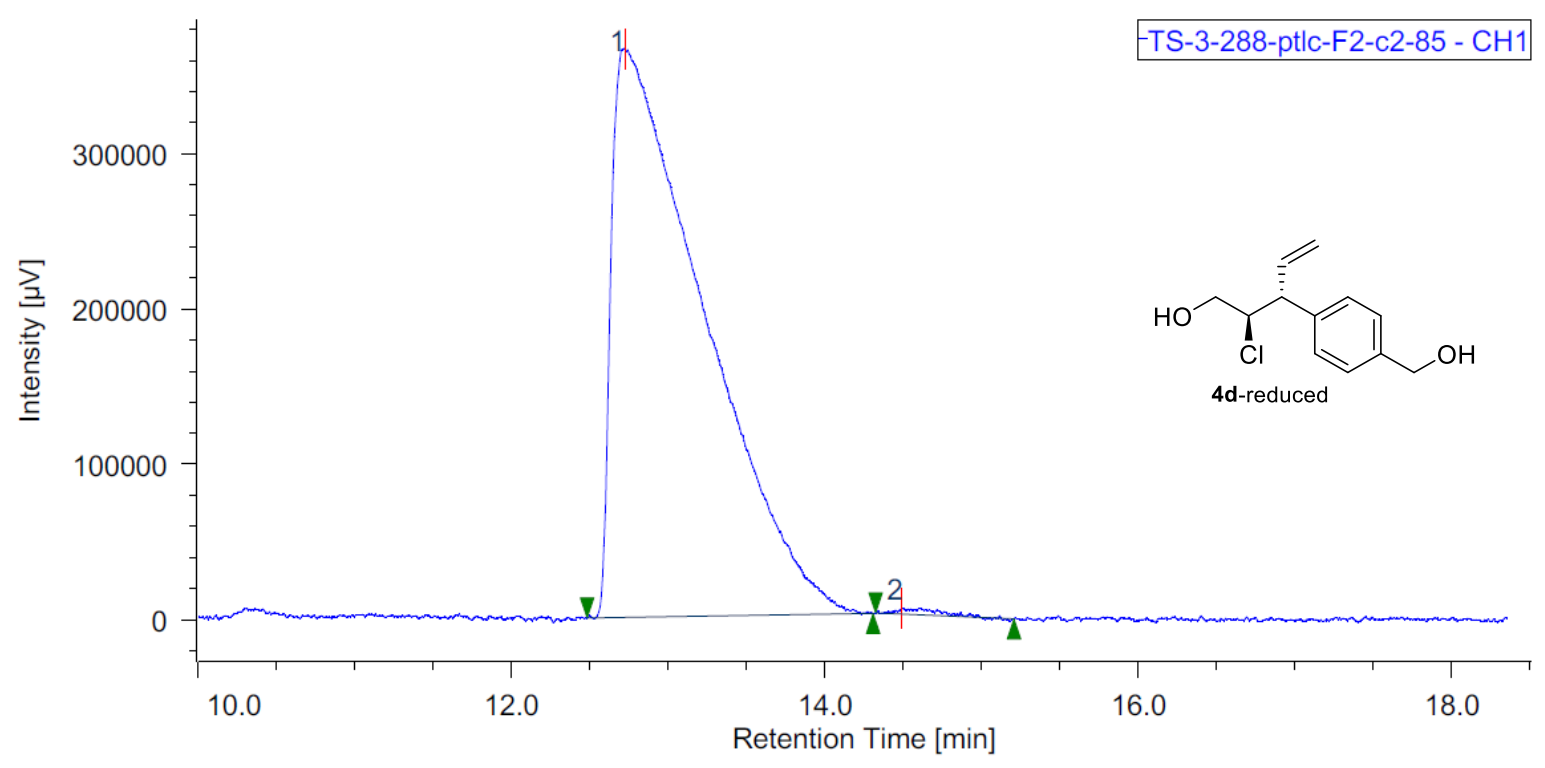

\begin{tabular}{|c|c|c|c|c|c|c|c|c|c|c|}
\hline \begin{tabular}{l|l}
$\#$ & Peak Name
\end{tabular} & $\mathrm{CH}$ & $\mathrm{tR}[\mathrm{min}]$ & Area $[\mu \mathrm{V} \cdot \mathrm{sec}]$ & Height $[\mu \mathrm{V}]$ & Area\% & Height $\%$ & Quantity & NTP & \begin{tabular}{l|} 
Resolution \\
\end{tabular} & Symmetry Factor \\
\hline Unknown & 1 & 12.725 & 14957029 & 366251 & 99.463 & 98.964 & $\mathrm{~N} / \mathrm{A}$ & 2201 & 1.862 & 4.595 \\
\hline Unknown & 1 & 14.492 & 80759 & 3836 & 0.537 & 1.036 & $\mathrm{~N} / \mathrm{A}$ & 5030 & N/A & 2.425 \\
\hline
\end{tabular}




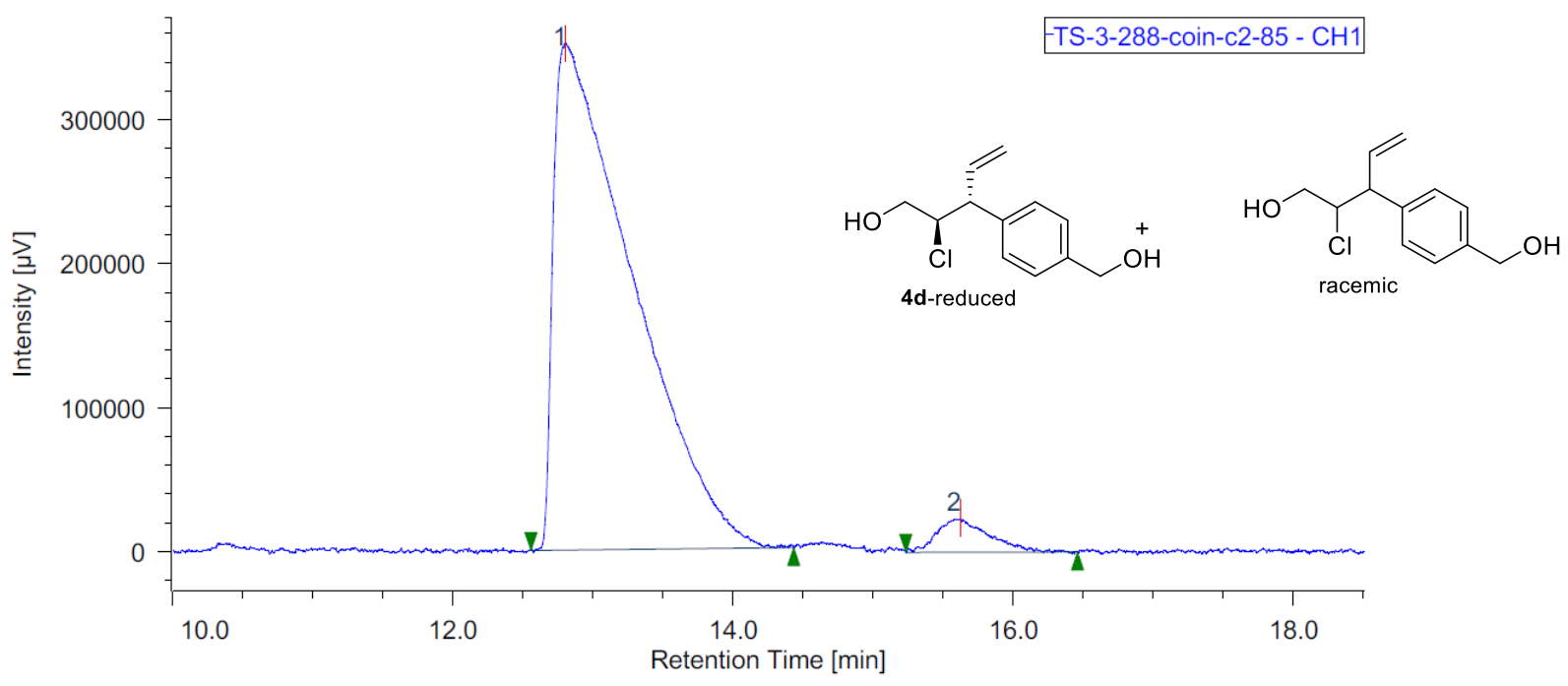

\begin{tabular}{|r|c|c|r|r|r|r|r|r|r|r|r|}
\hline$\#$ & Peak Name & $\mathrm{CH}$ & $\mathrm{tR}[\mathrm{min}]$ & Area $[\mu \mathrm{V} \cdot \mathrm{sec}]$ & Height $[\mu \mathrm{V}]$ & \multicolumn{1}{|c|}{ Area $\%$} & Height $\%$ & Quantity & NTP & Resolution & Symmetry Factor \\
\hline \multicolumn{1}{|c|}{} & Unknown & 1 & 12.808 & 13827841 & 352023 & 95.794 & 93.678 & $\mathrm{~N} / \mathrm{A}$ & 2389 & 3.319 & 4.347 \\
\hline 2 & Unknown & 1 & 15.625 & 607106 & 2375 & 4.206 & 6.322 & $\mathrm{~N} / \mathrm{A}$ & 9143 & $\mathrm{~N} / \mathrm{A}$ & 1.463 \\
\hline
\end{tabular}




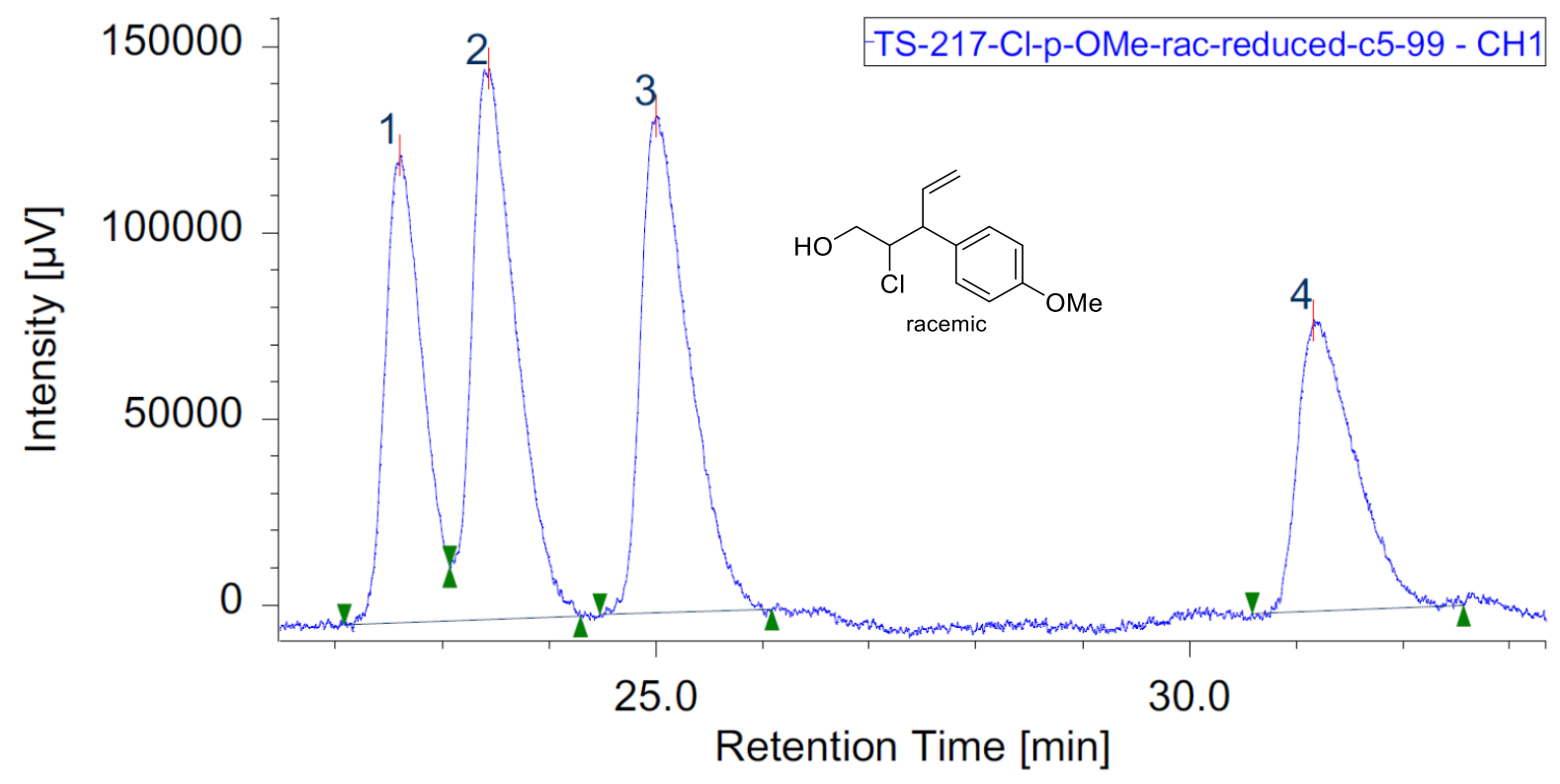

\begin{tabular}{|c|c|c|c|c|c|c|c|c|c|c|c|}
\hline \# & Peak Name & $\mathrm{CH}$ & $\mathrm{tR}[\mathrm{min}]$ & Area $[\mu \mathrm{V} \cdot \mathrm{sec}]$ & Height $[\mu \mathrm{V}]$ & Area $\%$ & Height $\%$ & Quantity & NTP & Resolution & Symmetry Factor \\
\hline & Unknown & 1 & 22.600 & 3049694 & 125521 & 21.170 & 25.881 & $\mathrm{~N} / \mathrm{A}$ & 19577 & 1.201 & $\mathrm{~N} / \mathrm{A}$ \\
\hline & Unknown & 1 & 23.433 & 4293116 & 147886 & 29.801 & 30.493 & $\mathrm{~N} / \mathrm{A}$ & 15805 & 2.022 & $\mathrm{~N} / \mathrm{A}$ \\
\hline & Unknown & 1 & 25.008 & 4171700 & 133402 & 28.959 & 27.506 & $\mathrm{~N} / \mathrm{A}$ & 15003 & 6.845 & 1.771 \\
\hline & Unknown & 1 & 31.158 & 2891210 & 78178 & 20.070 & 16.120 & $\mathrm{~N} / \mathrm{A}$ & 16013 & $\mathrm{~N} / \mathrm{A}$ & 2.056 \\
\hline
\end{tabular}

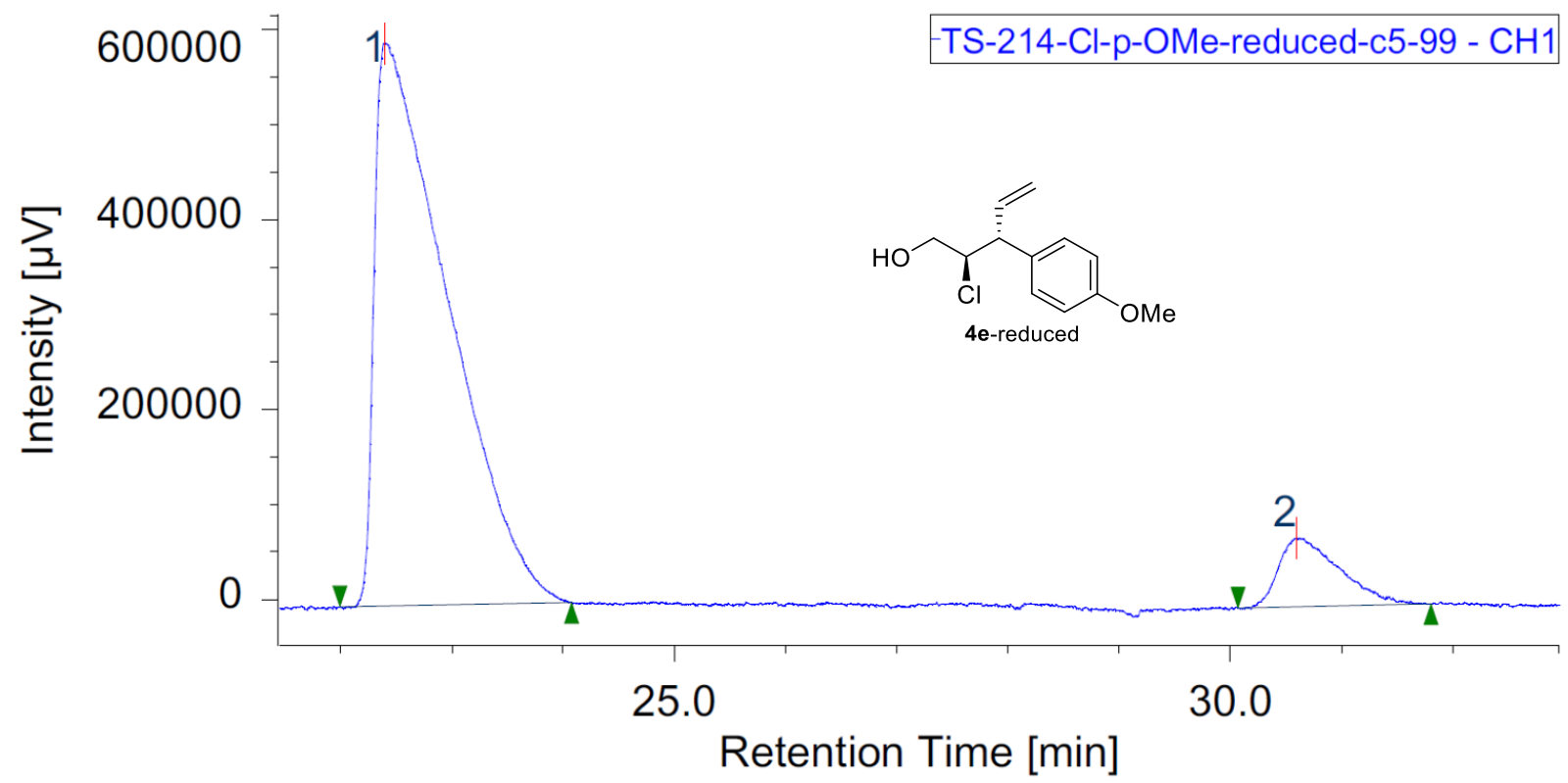

\begin{tabular}{|c|c|c|c|c|c|c|c|c|c|c|c|}
\hline$\pi$ & Peak Name & $\mathrm{CH}$ & $\mathrm{tR}$ [min] & Area $[\mu \mathrm{V} \cdot \mathrm{sec}]$ & Height $[\mu \mathrm{V}]$ & Area $\%$ & Height $\%$ & Quantity & NTP & Resolution & Symmetry Factor \\
\hline & Unknown & 1 & 22.400 & 26986628 & 592387 & 90.748 & 89.099 & $\mathrm{~N} / \mathrm{A}$ & 5338 & 7.283 & 4.020 \\
\hline & Unknown & 1 & 30.592 & 2751277 & 72473 & 9.252 & 10.901 & $\mathrm{~N} / \mathrm{A}$ & 14137 & $\mathrm{~N} / \mathrm{A}$ & 1.84. \\
\hline
\end{tabular}




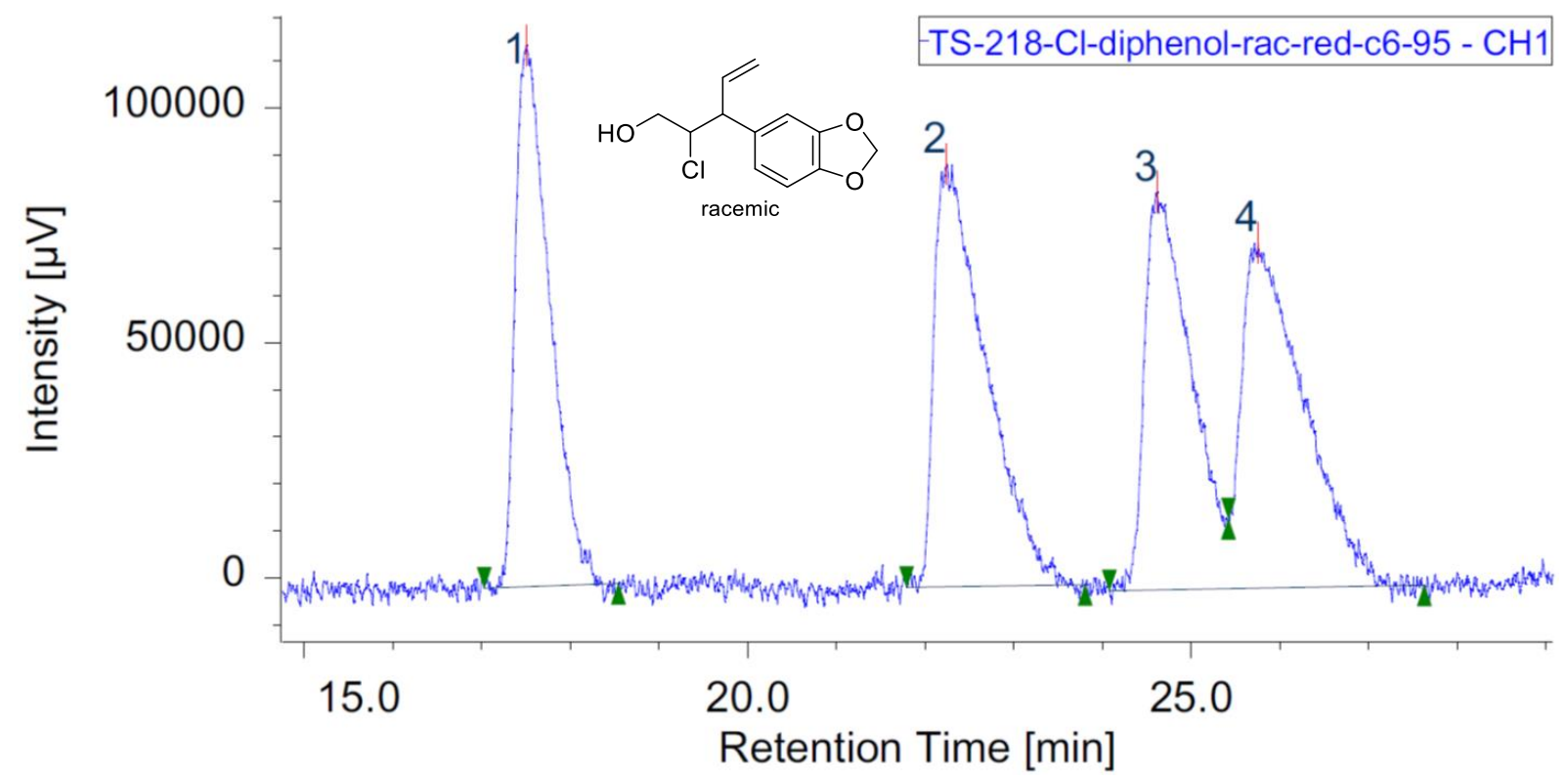

\begin{tabular}{|c|c|c|c|c|c|c|c|c|c|c|c|}
\hline \# & Peak Name & $\mathrm{CH}$ & $\mathrm{tR}[\mathrm{min}]$ & Area $[\mu \mathrm{V} \cdot \mathrm{sec}]$ & Height $[\mu \mathrm{V}]$ & Area\% & Height $\%$ & Quantity & NTP & Resolution & Symmetry Factor \\
\hline & Unknown & 1 & 17.508 & 3086918 & 115138 & 23.019 & 31.714 & $\mathrm{~N} / \mathrm{A}$ & 9676 & 5.272 & 2.016 \\
\hline & Unknown & 1 & 22.242 & 3640096 & 89884 & 27.144 & 24.758 & $\mathrm{~N} / \mathrm{A}$ & 6680 & 2.299 & 2.890 \\
\hline & Unknown & 1 & 24.617 & 3091216 & 84600 & 23.051 & 23.302 & $\mathrm{~N} / \mathrm{A}$ & 10037 & 0.974 & $\mathrm{~N} / \mathrm{A}$ \\
\hline & Unknown & 1 & 25.750 & 3591931 & 73429 & 26.785 & 20.226 & $\mathrm{~N} / \mathrm{A}$ & 5812 & $\mathrm{~N} / \mathrm{A}$ & $\mathrm{N} / \mathrm{A}$ \\
\hline
\end{tabular}

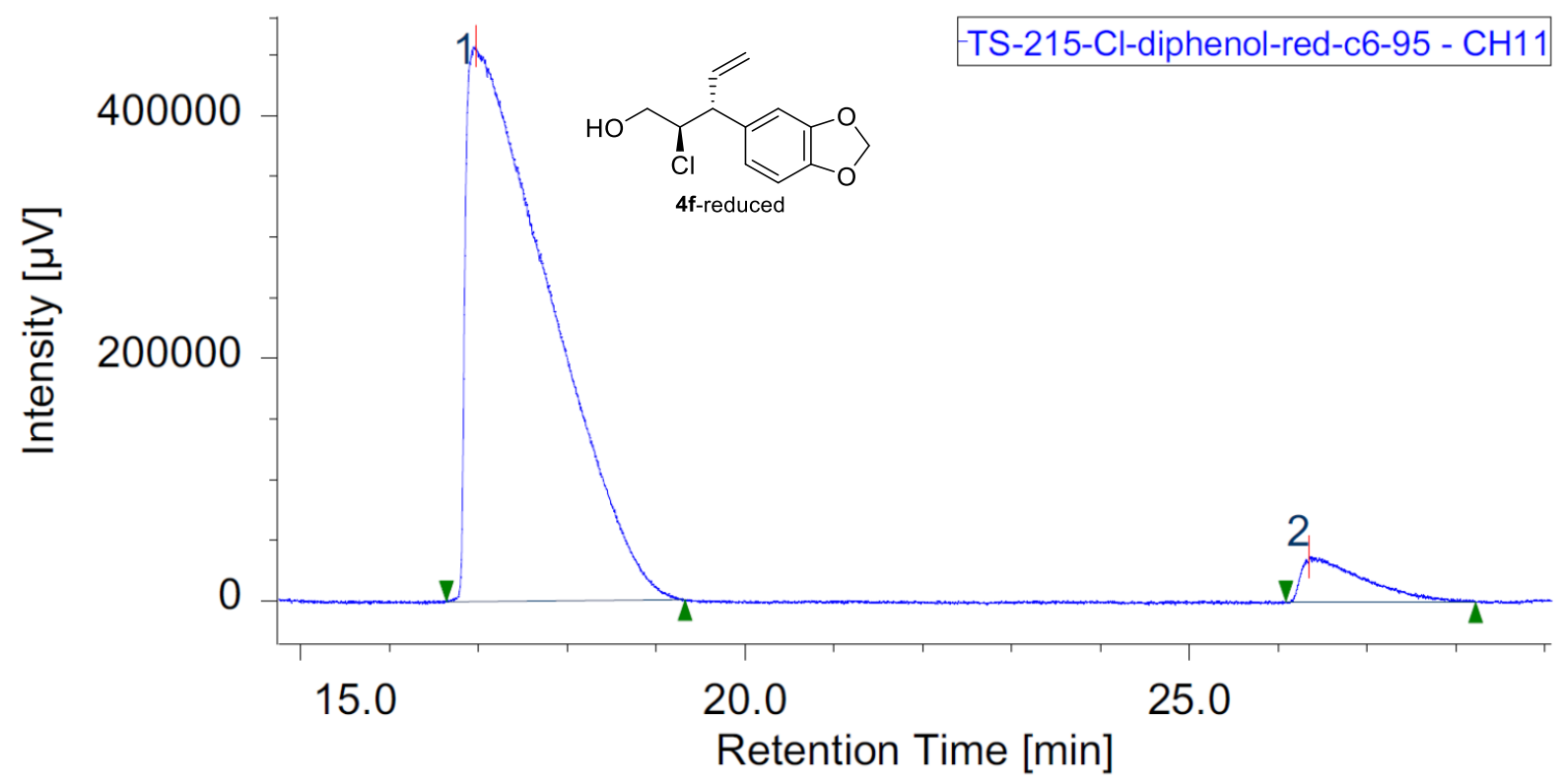

\begin{tabular}{|r|c|c|r|r|r|r|r|r|r|r|r|}
\hline$\#$ & Peak Name & $\mathrm{CH}$ & $\mathrm{tR}[\mathrm{min}]$ & Area $[\mu \mathrm{V} \cdot \mathrm{sec}]$ & Height $[\mu \mathrm{V}]$ & \multicolumn{1}{|c|}{ Area $\%$} & Height $\%$ & Quantity & NTP & Resolution & Symmetry Factor \\
\hline 1 & Unknown & 11 & 16.967 & 29707395 & 458003 & 94.380 & 92.554 & N/A & 1441 & 6.078 & 6.168 \\
\hline 2 & Unknown & 11 & 26.347 & 1769105 & 36849 & 5.620 & 7.446 & N/A & 6504 & N/A & 5.145 \\
\hline
\end{tabular}




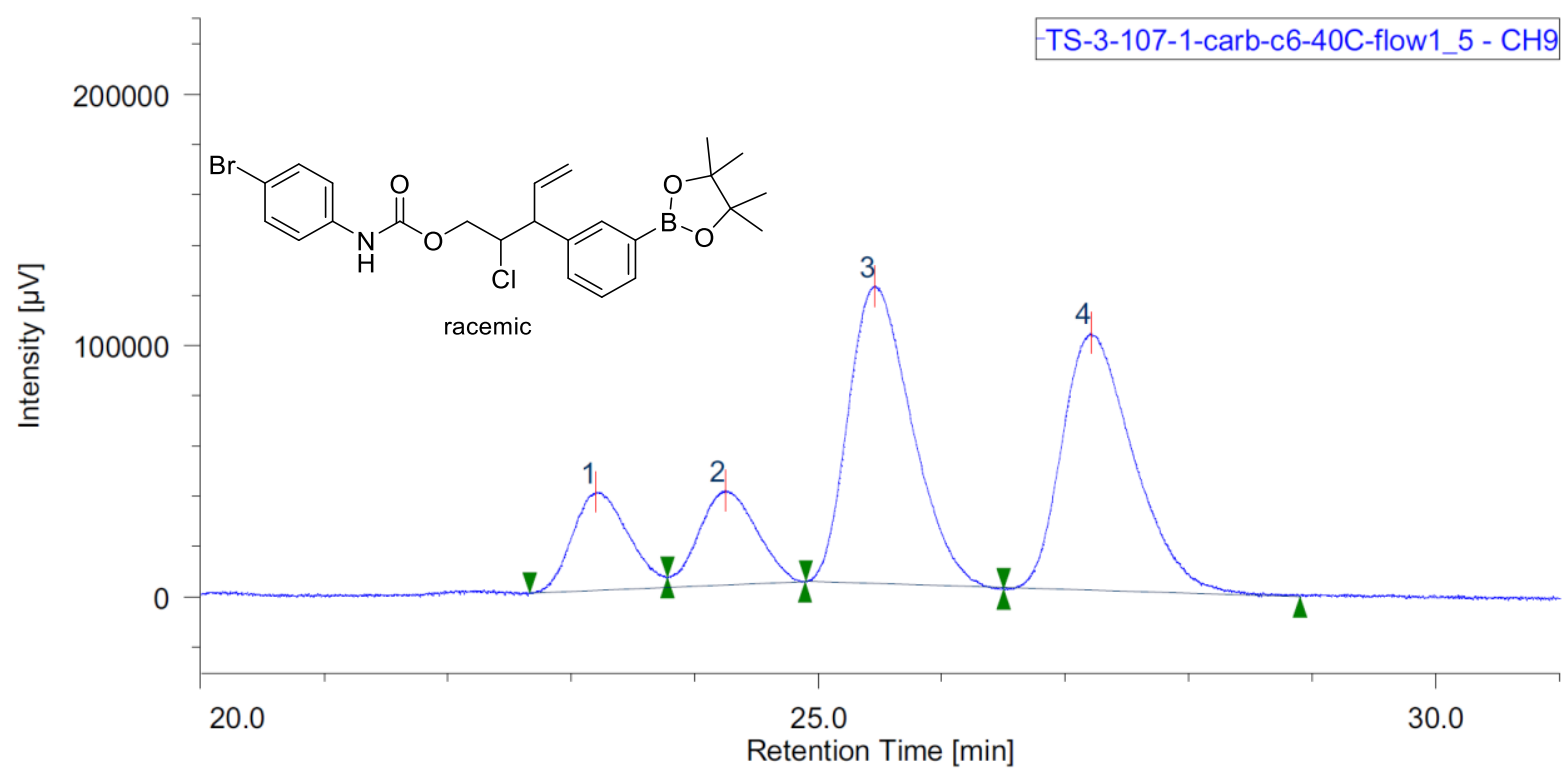

\begin{tabular}{|r|l|c|r|r|r|r|r|r|r|r|r|}
\hline$\#$ & Peak Name & CH & tR $[\mathrm{min}]$ & Area $[\mu \mathrm{V} \cdot \mathrm{sec}]$ & Height $[\mu \mathrm{V}]$ & \multicolumn{1}{|c|}{ Area $\%$} & Height $\%$ & Quantity & NTP & Resolution & Symmetry Factor \\
\hline 2 & Unknown & 9 & 23.200 & 1221345 & 39078 & 11.344 & 13.136 & $\mathrm{~N} / \mathrm{A}$ & 12101 & 1.222 & $\mathrm{~N} / \mathrm{A}$ \\
\hline 1 & Unknown & 9 & 24.247 & 1214262 & 37611 & 11.278 & 12.643 & $\mathrm{~N} / \mathrm{A}$ & 12305 & 1.336 & $\mathrm{~N} / \mathrm{A}$ \\
\hline 3 & Unknown & 9 & 25.460 & 4206644 & 118337 & 39.070 & 39.779 & $\mathrm{~N} / \mathrm{A}$ & 11553 & 1.756 & 1.303 \\
\hline 4 & Unknown & 9 & 27.207 & 4124615 & 102457 & 38.308 & 34.441 & $\mathrm{~N} / \mathrm{A}$ & 10797 & $\mathrm{~N} / \mathrm{A}$ & 1.382 \\
\hline
\end{tabular}

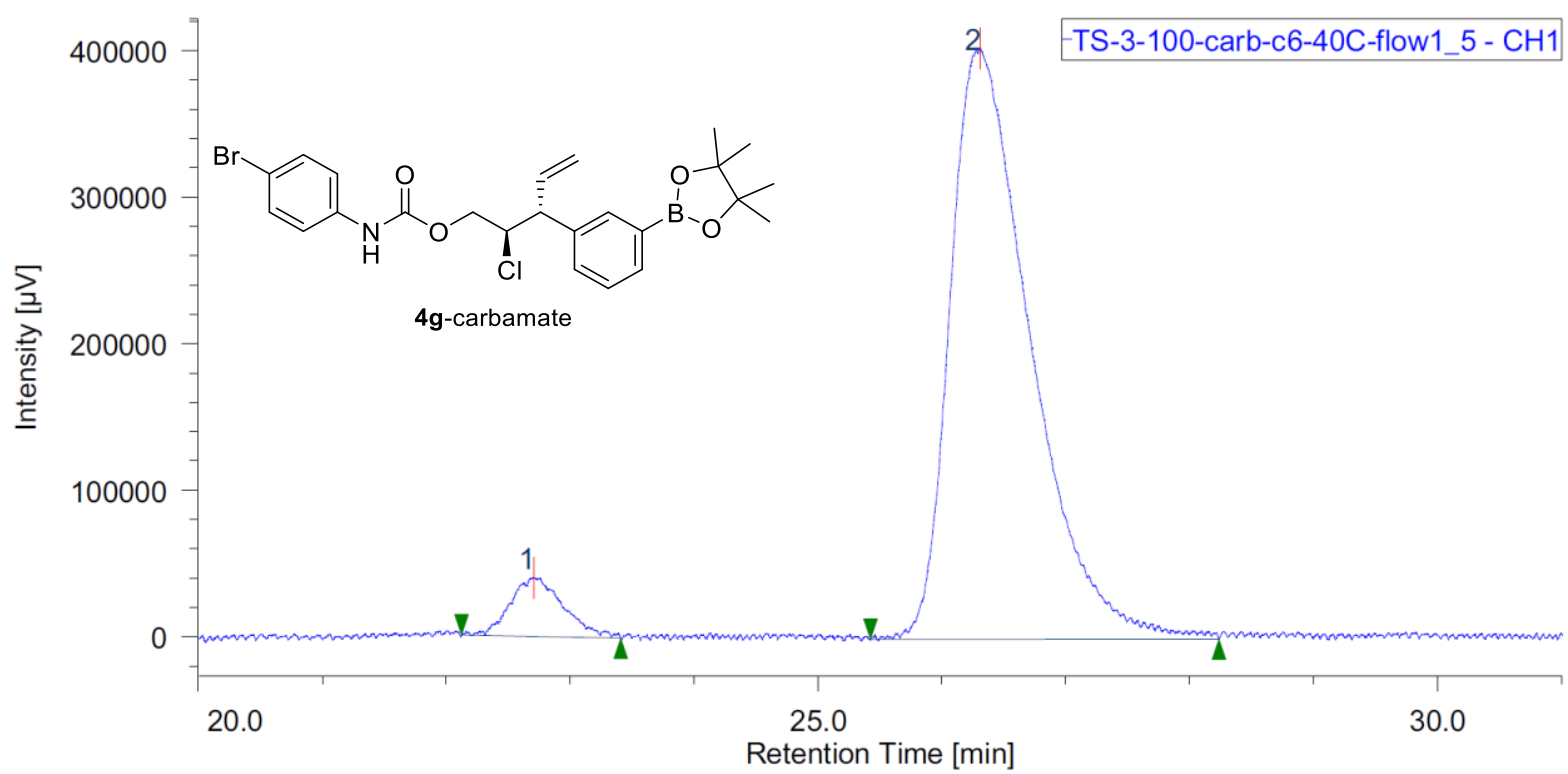

\begin{tabular}{|r|c|c|r|r|r|r|r|r|r|r|r|}
\hline$\#$ & Peak Name & $\mathrm{CH}$ & $\mathrm{tR}[\mathrm{min}]$ & \multicolumn{1}{|c|}{ Area $[\mu \mathrm{V} \cdot \mathrm{sec}]$} & Height $[\mu \mathrm{V}]$ & \multicolumn{1}{|c|}{ Area $\%$} & Height $\%$ & Quantity & \multicolumn{1}{|c|}{ NTP } & Resolution & \multicolumn{1}{c}{ Symmetry Factor } \\
\hline & Unknown & 1 & 22.708 & 1205432 & 40146 & 6.226 & 9.062 & $\mathrm{~N} / \mathrm{A}$ & 14107 & 3.765 & 1.121 \\
\hline 2 & Unknown & 1 & 26.308 & 18155431 & 402887 & 93.774 & 90.938 & $\mathrm{~N} / \mathrm{A}$ & 8335 & $\mathrm{~N} / \mathrm{A}$ & \\
\hline
\end{tabular}




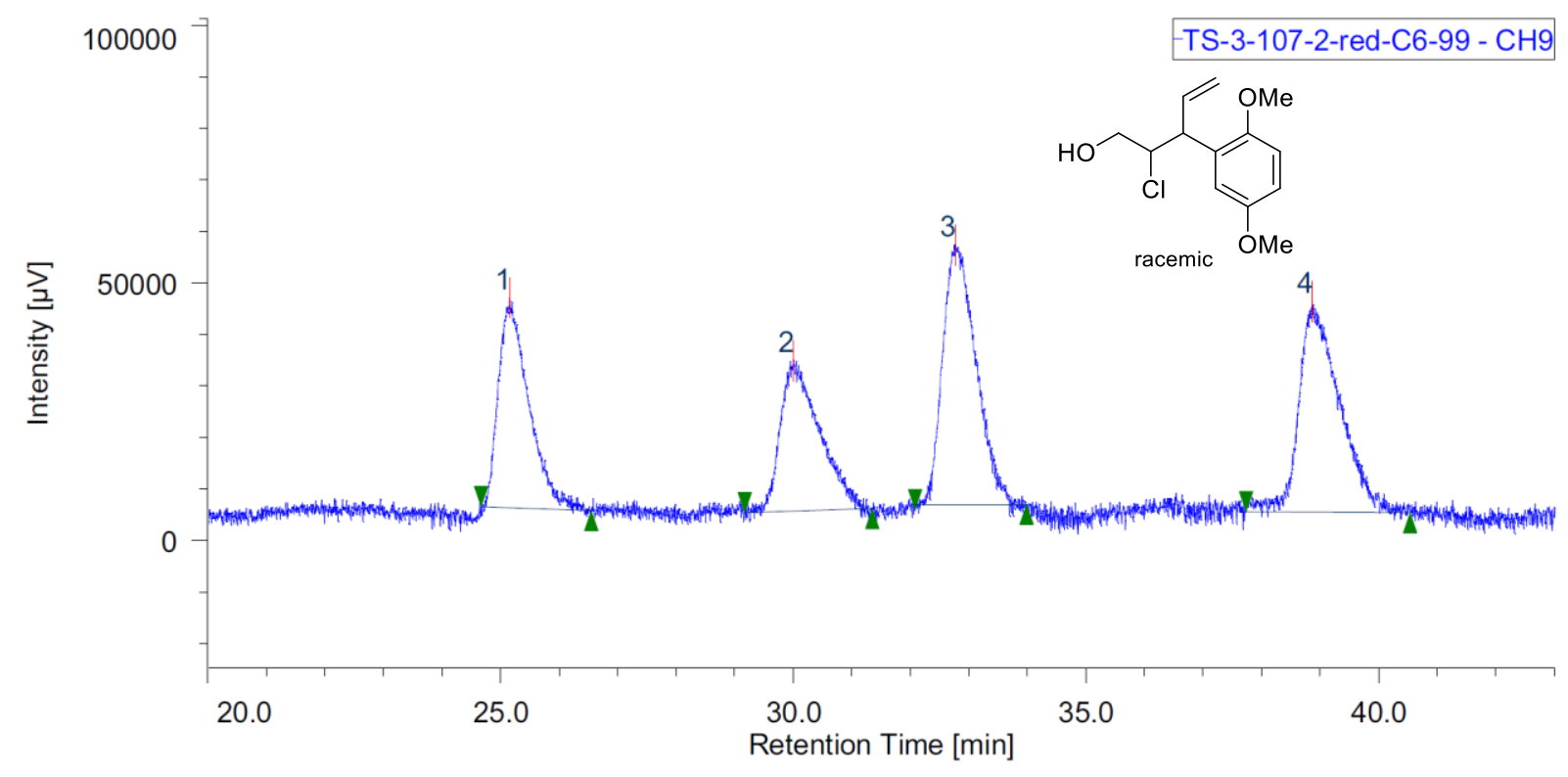

\begin{tabular}{|c|c|c|c|c|c|c|c|c|c|c|c|}
\hline \# & Peak Name & $\mathrm{CH}$ & $\mathrm{tR}[\mathrm{min}]$ & Area $[\mu \mathrm{V} \cdot \mathrm{sec}]$ & Height $[\mu \mathrm{V}]$ & Area $\%$ & Height $\%$ & Quantity & NTP & Resolution & Symmetry Factor \\
\hline & Unknown & 9 & 25.153 & 1446802 & 40722 & 21.409 & 25.272 & $\mathrm{~N} / \mathrm{A}$ & 12291 & 4.495 & 1.928 \\
\hline & Unknown & 9 & 30.000 & 1353874 & 29229 & 20.034 & 18.139 & $\mathrm{~N} / \mathrm{A}$ & 9145 & 2.368 & 1.299 \\
\hline & Unknown & 9 & 32.760 & 1995289 & 50326 & 29.526 & 31.232 & $\mathrm{~N} / \mathrm{A}$ & 14646 & 5.195 & 1.428 \\
\hline & Unknown & $\overline{9}$ & 38.867 & 1961802 & 40857 & 29.030 & 25.356 & $\mathrm{~N} / \mathrm{A}$ & 14884 & N/A & 1.059 \\
\hline
\end{tabular}

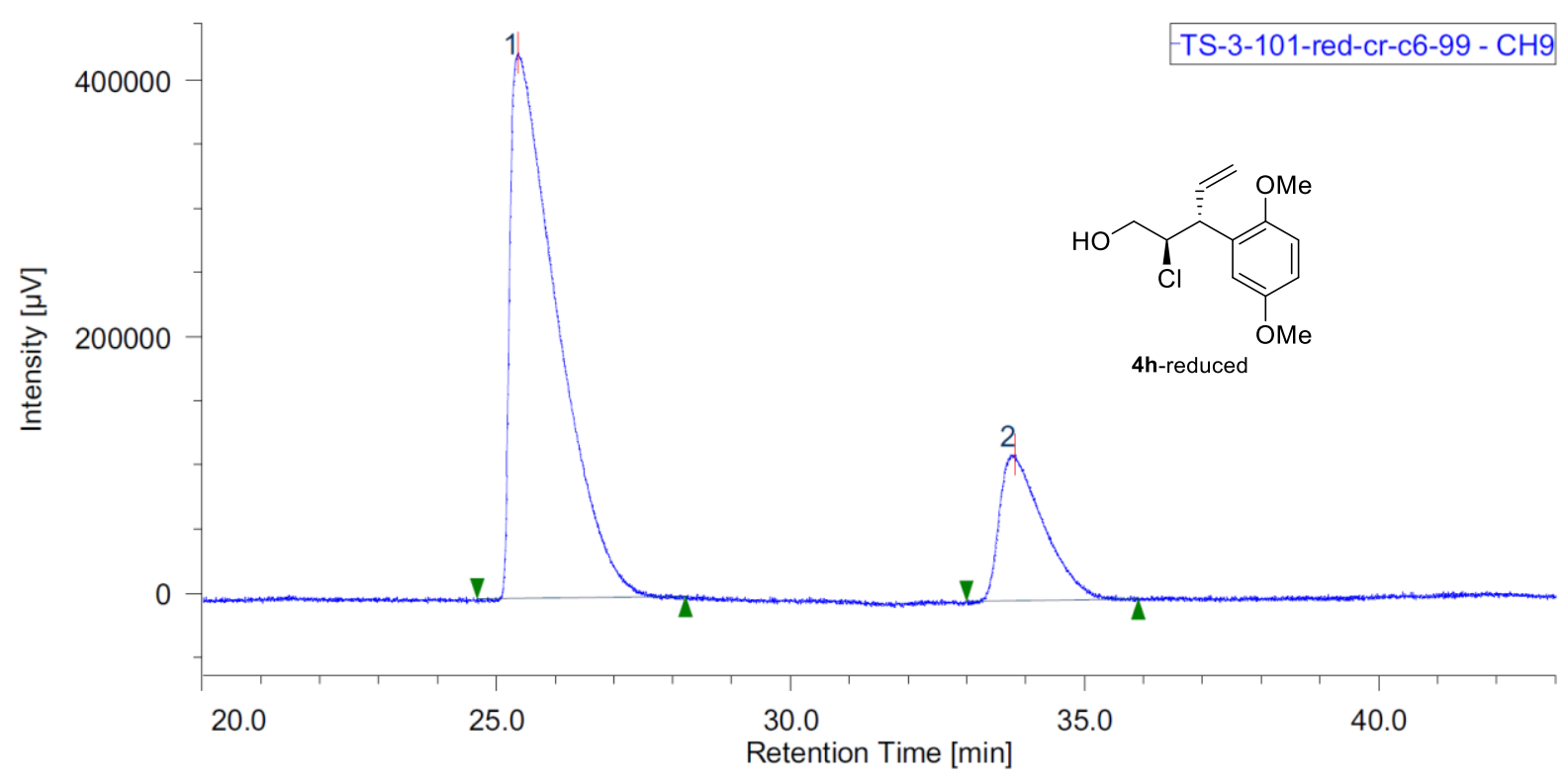

\begin{tabular}{|r|c|c|r|r|r|r|r|r|r|r|r|}
\hline$\#$ & Peak Name & CH & tR $[\mathrm{min}]$ & Area $[\mu \mathrm{V} \cdot \mathrm{sec}]$ & Height $[\mu \mathrm{V}]$ & \multicolumn{1}{|c|}{ Area $\%$} & Height $\%$ & Quantity & NTP & Resolution & Symmetry Factor \\
\hline 1 & Unknown & 9 & 25.373 & 23410081 & 425324 & 79.991 & 78.930 & N/A & 5068 & 6.092 & 4.012 \\
\hline 2 & Unknown & 9 & 33.807 & 5855885 & 113536 & 20.009 & 21.070 & N/A & 10028 & N/A & 1.839 \\
\hline
\end{tabular}




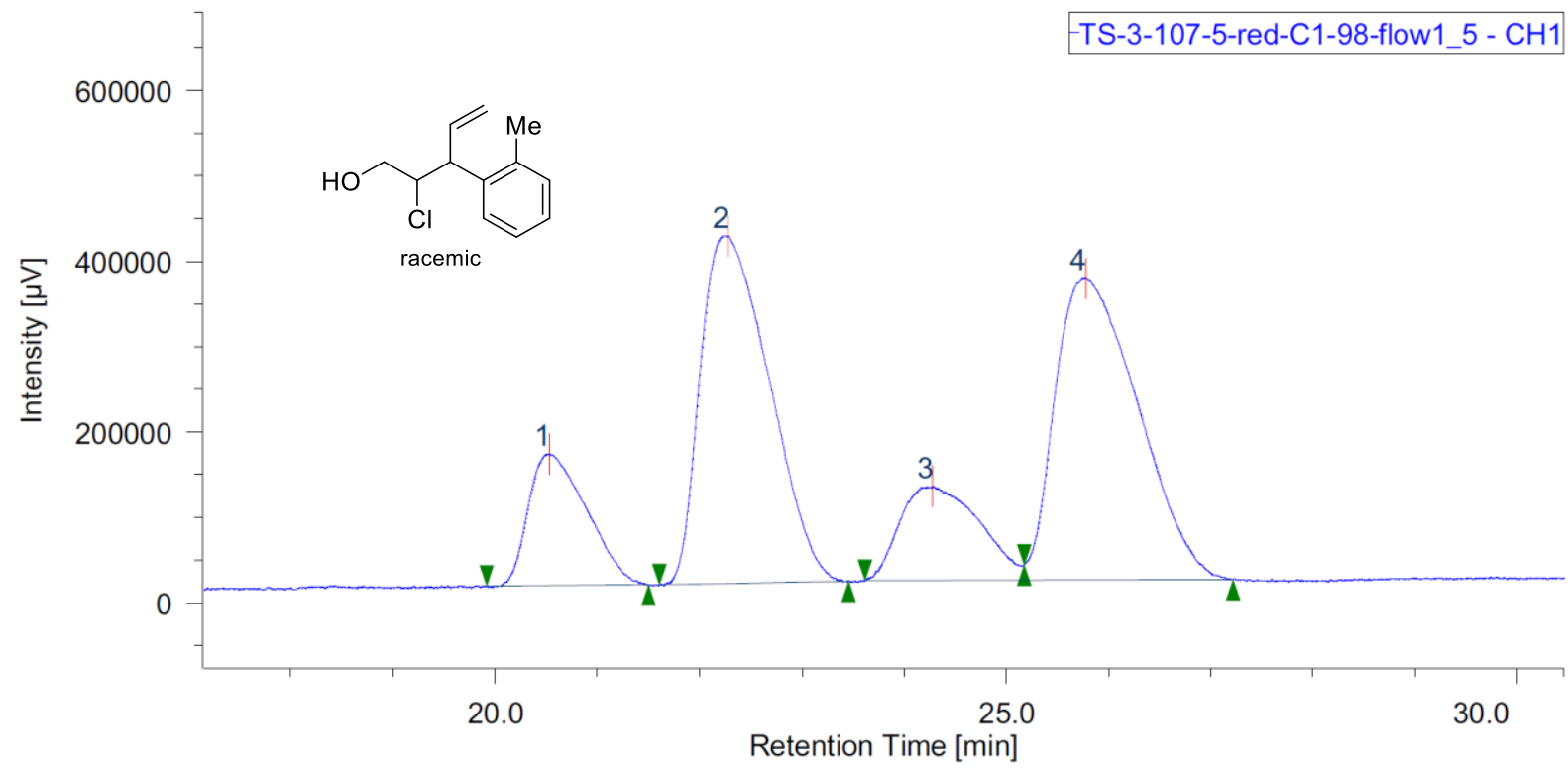

\begin{tabular}{|c|c|c|c|c|c|c|c|c|c|c|c|}
\hline \# & Peak Name & $\mathrm{CH}$ & $\mathrm{tR}[\mathrm{min}]$ & Area $[\mu \mathrm{V} \cdot \mathrm{sec}]$ & Height $[\mu \mathrm{V}]$ & Area $\%$ & Height $\%$ & Quantity & NTP & Resolution & Symmetry Factor \\
\hline & Unknown & 1 & 20.533 & 6056677 & 153725 & 11.914 & 15.017 & $\mathrm{~N} / \mathrm{A}$ & 5558 & 1.452 & 1.481 \\
\hline & Unknown & 1 & 22.275 & 18973770 & 406802 & 37.324 & 39.740 & $\mathrm{~N} / \mathrm{A}$ & 4673 & 1.401 & 1.433 \\
\hline & Unknown & 1 & 24.275 & 5981306 & 110004 & 11.766 & 10.746 & $\mathrm{~N} / \mathrm{A}$ & 3875 & 0.962 & $\mathrm{~N} / \mathrm{A}$ \\
\hline & Unknown & 1 & 25.775 & 19824004 & 353126 & 38.996 & 34.496 & N/A & 4326 & N/A & 1.437 \\
\hline
\end{tabular}

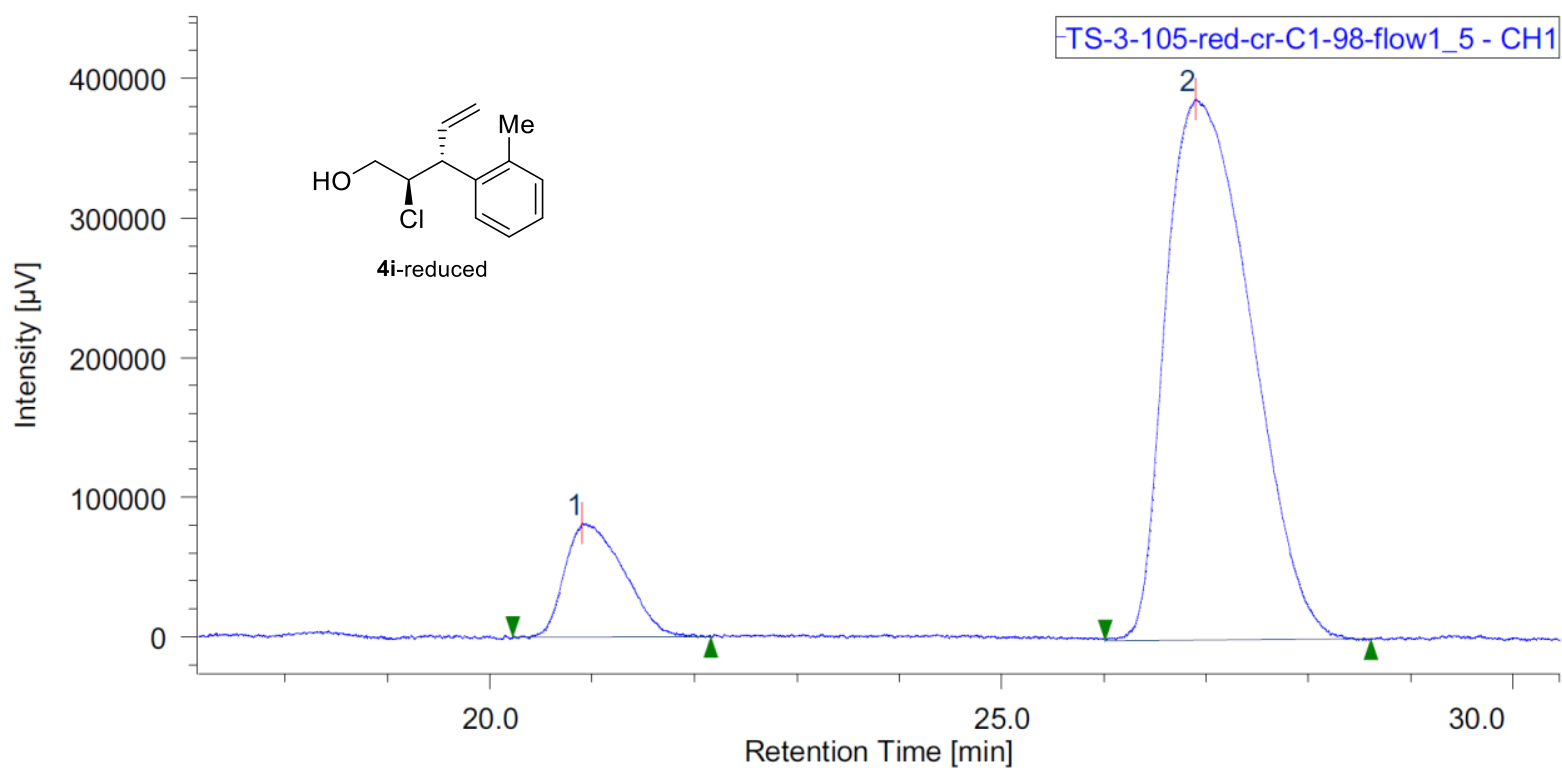

\begin{tabular}{|r|c|c|r|r|r|r|r|r|r|r|r|}
\hline$\#$ & Peak Name & CH & tR $[\mathrm{min}]$ & Area $[\mu \mathrm{V} \cdot \mathrm{sec}]$ & Height $[\mu \mathrm{V}]$ & \multicolumn{1}{|c|}{ Area $\%$} & Height $\%$ & Quantity & NTP & Resolution & Symmetry Factor \\
\hline 1 & Unknown & 1 & 20.900 & 3249111 & 81409 & 12.738 & 17.390 & N/A & 5639 & 4.432 & 1.599 \\
\hline 2 & Unknown & 1 & 26.892 & 22258408 & 386729 & 87.262 & 82.610 & N/A & 4531 & N/A & 1.520 \\
\hline
\end{tabular}




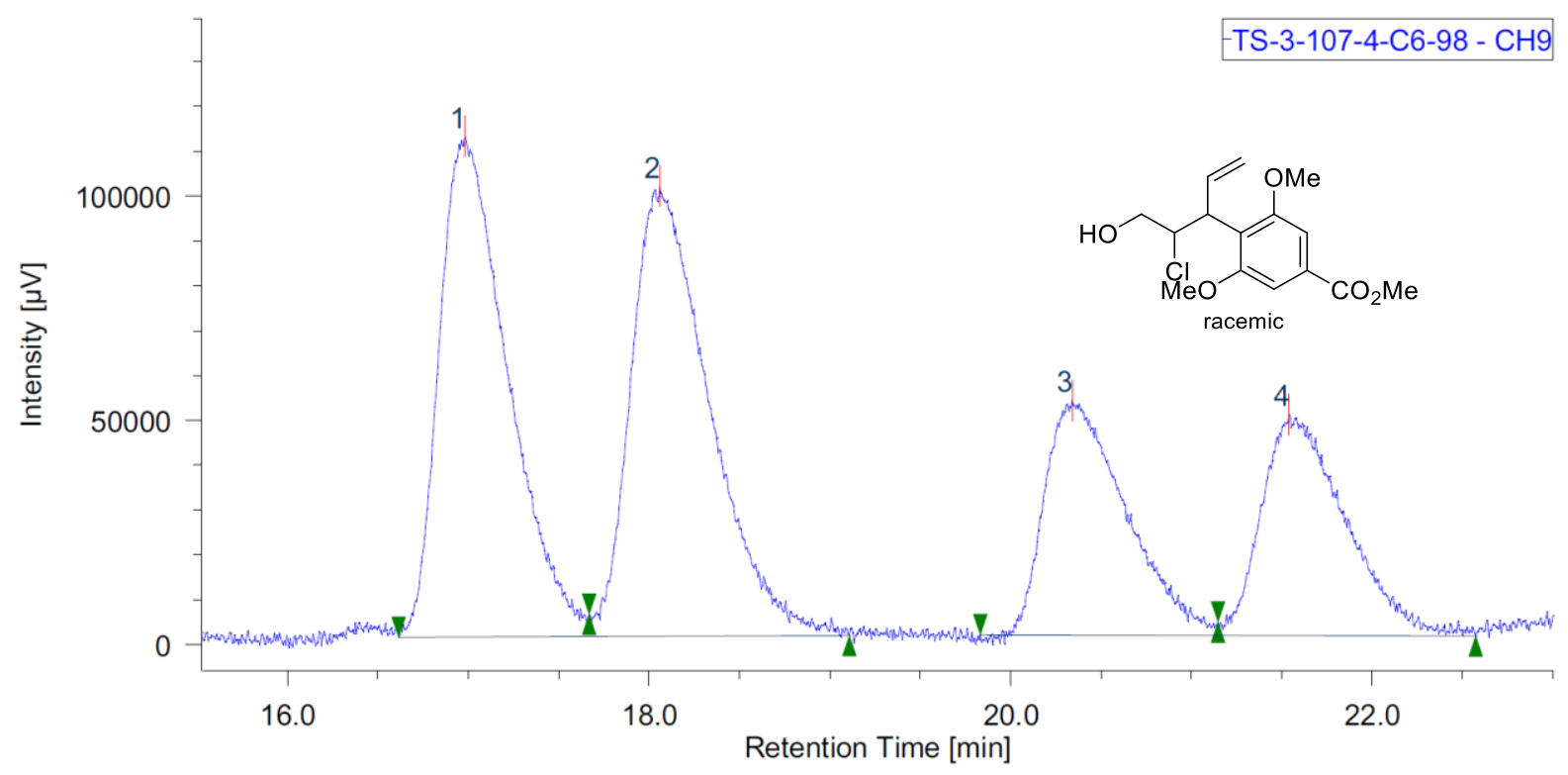

\begin{tabular}{|c|c|c|c|c|c|c|c|c|c|c|c|}
\hline \# & Peak Name & $\mathrm{CH}$ & $\mathrm{tR}$ [min] & Area $[\mu \mathrm{V} \cdot \mathrm{sec}]$ & Height $[\mu \mathrm{V}]$ & Area $\%$ & Height $\%$ & Quantity & NTP & Resolution & Symmetry Factor \\
\hline & Unknown & 9 & 16.980 & 2934063 & 111573 & 32.030 & 35.607 & $\mathrm{~N} / \mathrm{A}$ & 9717 & 1.478 & 1.628 \\
\hline & Unknown & 9 & 18.060 & 3018613 & 100222 & 32.953 & 31.985 & $\mathrm{~N} / \mathrm{A}$ & 8652 & 2.787 & 1.522 \\
\hline & Unknown & 9 & 20.340 & 1625686 & 52269 & 17.747 & 16.681 & $\mathrm{~N} / \mathrm{A}$ & 8864 & 1.395 & 1.805 \\
\hline 4 & Unknown & $\overline{9}$ & 21.540 & 1582077 & 49281 & 17.271 & 15.727 & $\mathrm{~N} / \mathrm{A}$ & 10010 & $\mathrm{~N} / \mathrm{A}$ & 1.676 \\
\hline
\end{tabular}

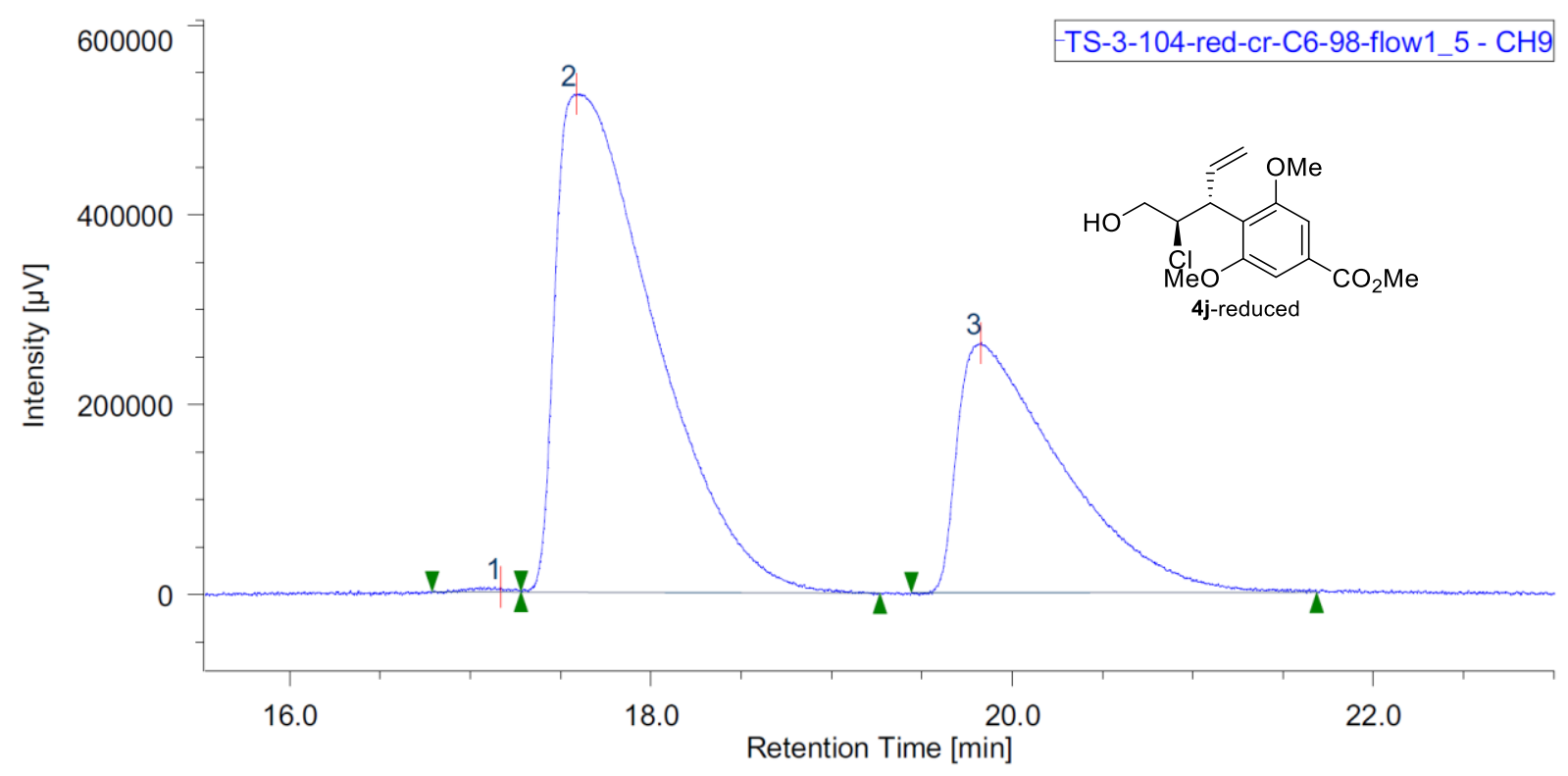

\begin{tabular}{|r|l|c|r|r|r|r|r|r|r|r|r|}
\hline$\#$ & Peak Name & CH & tR $[\mathrm{min}]$ & \multicolumn{1}{|c|}{ Area $[\mu \mathrm{V} \cdot \mathrm{sec}]$} & Height $[\mu \mathrm{V}]$ & \multicolumn{1}{|c|}{ Area\% } & Height\% & Quantity & NTP & Resolution & Symmetry Factor \\
\hline 2 & Unknown & 9 & 17.167 & 65645 & 5102 & 0.215 & 0.644 & N/A & 16699 & 0.550 & N/A \\
\hline 1 & Unknown & 9 & 17.587 & 19994753 & 524464 & 65.350 & 66.156 & N/A & 4944 & 2.211 & 3.015 \\
\hline 3 & Unknown & 9 & 19.827 & 10536075 & 263206 & 34.436 & 33.201 & N/A & 5912 & N/A & 3.111 \\
\hline
\end{tabular}




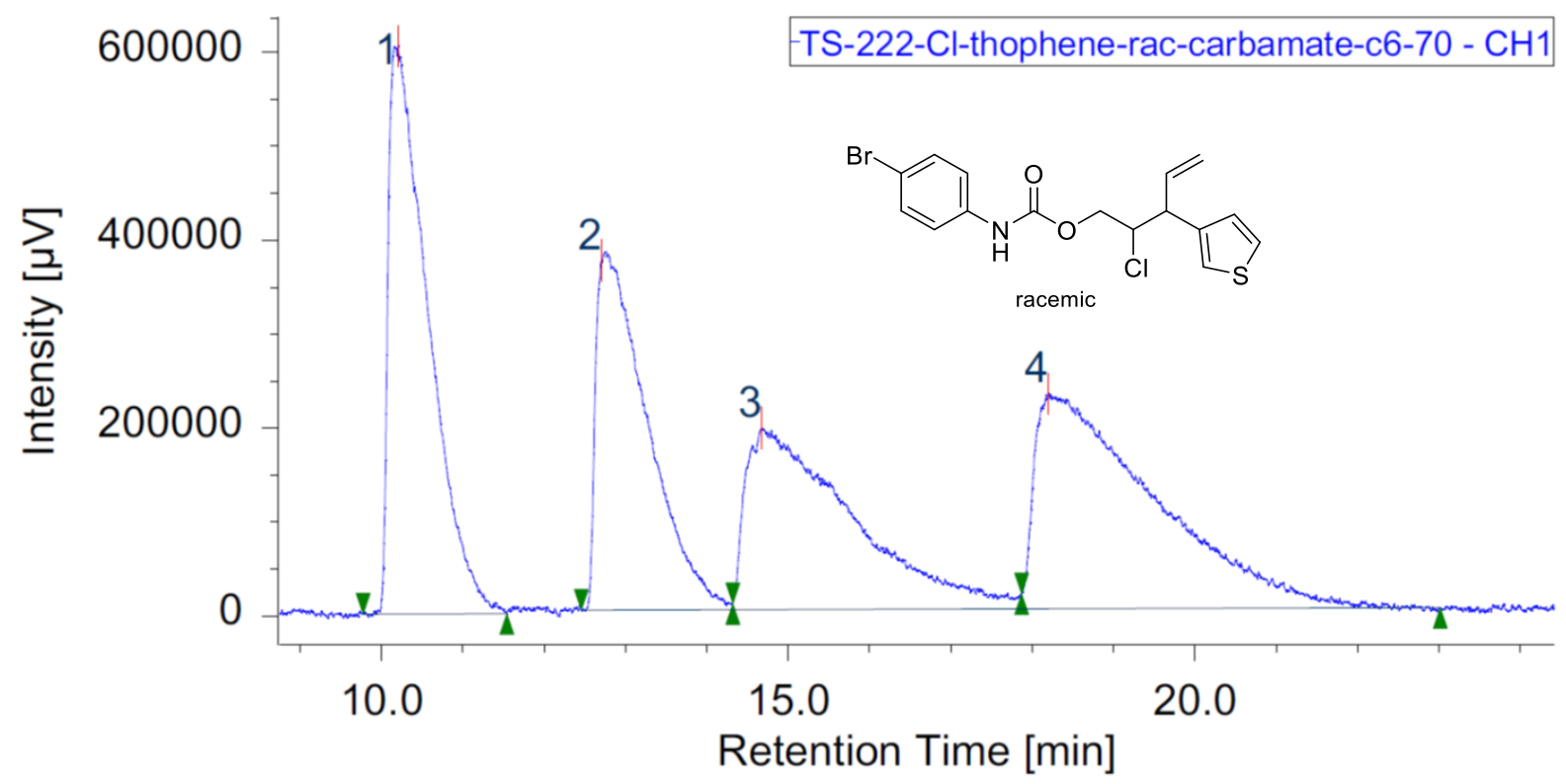

\begin{tabular}{|c|c|c|c|c|c|c|c|c|c|c|c|c|}
\hline \# & Peak Name & $\mathrm{CH}$ & tR [min] & Area $[\mu \mathrm{V} \cdot \mathrm{sec}]$ & Height $[\mu \mathrm{V}]$ & Area\% & Height $\%$ & Quantity & NTP & Resolution & Symmetry Factor & Warning \\
\hline & Unknown & 1 & 10.208 & 20469253 & 604439 & 26.209 & 43.220 & $\mathrm{~N} / \mathrm{A}$ & 2103 & 2.416 & 2.973 & \\
\hline & Unknown & 1 & 12.700 & 16712829 & 371617 & 21.400 & 26.572 & $\mathrm{~N} / \mathrm{A}$ & 1861 & 1.118 & 5.156 & \\
\hline & Unknown & 1 & 14.675 & 17543025 & 193333 & 22.463 & 13.824 & $\mathrm{~N} / \mathrm{A}$ & 615 & 1.422 & $\mathrm{~N} / \mathrm{A}$ & \\
\hline & Unknown & 1 & 18.200 & 23373871 & 229141 & 29.929 & 16.384 & $\mathrm{~N} / \mathrm{A}$ & 781 & N/A & $\mathrm{N} / \mathrm{A}$ & \\
\hline
\end{tabular}

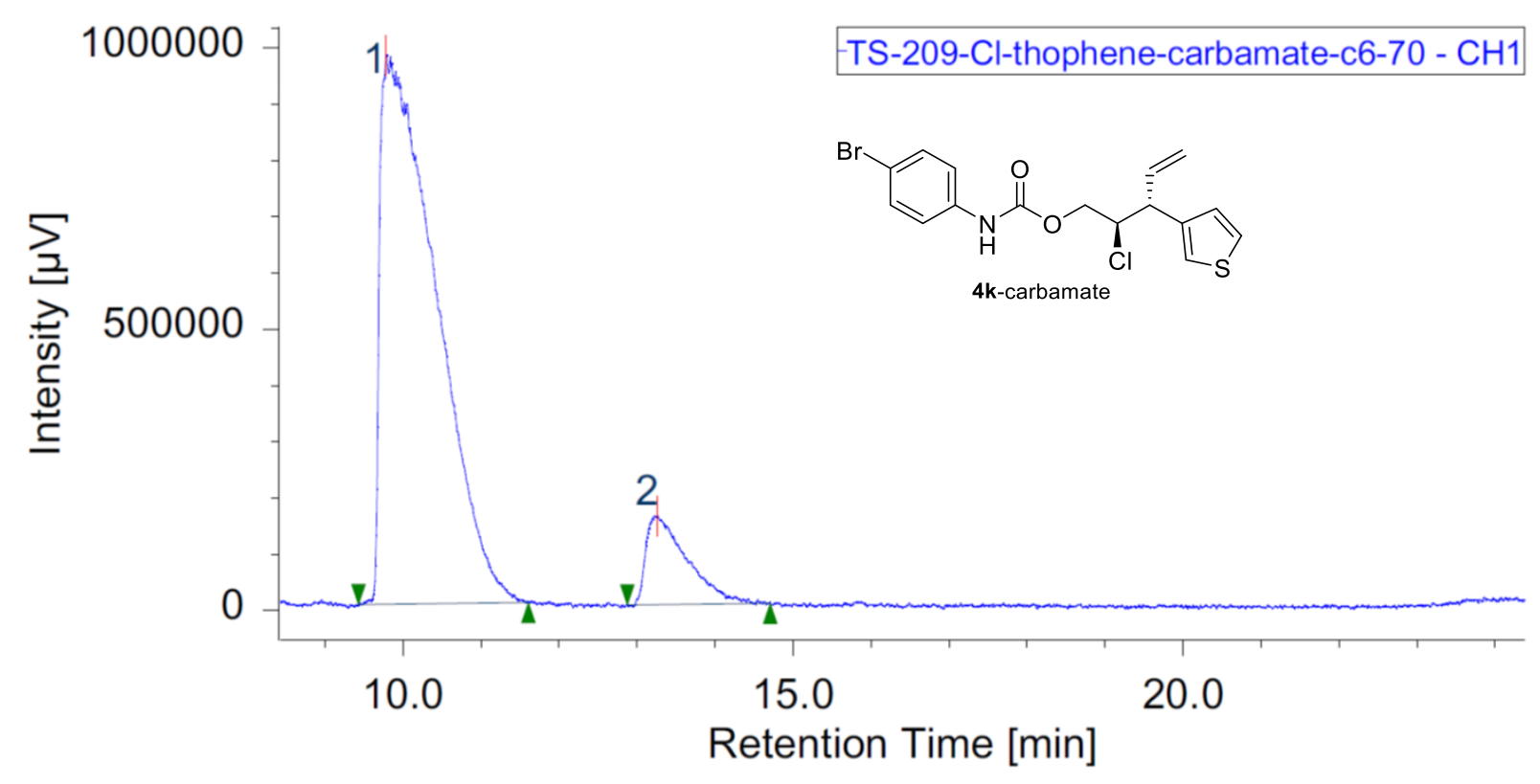




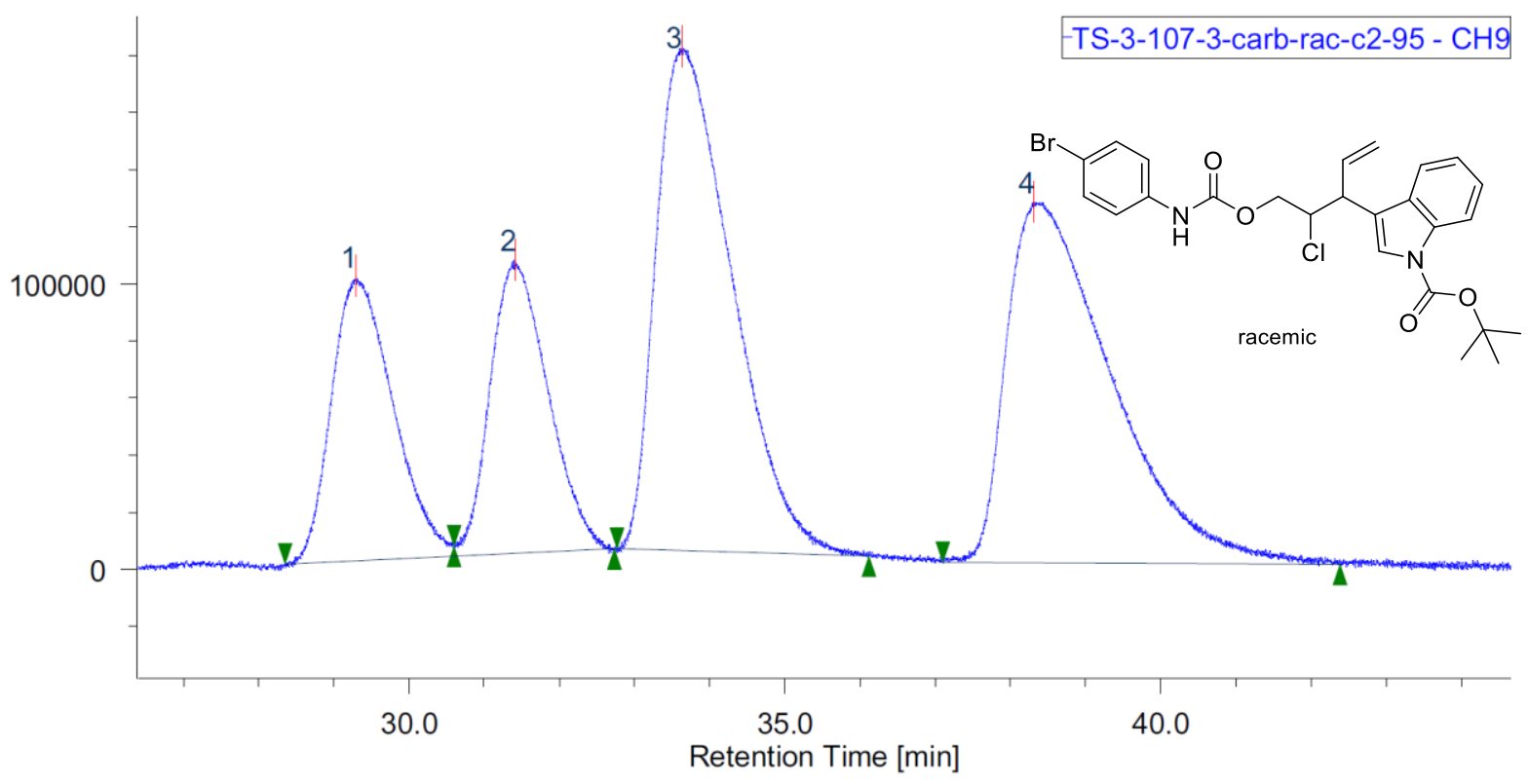

\begin{tabular}{|r|l|c|r|r|r|r|r|r|r|r|r|}
\hline$\#$ & Peak Name & $\mathrm{CH}$ & \multicolumn{1}{c}{$\mathrm{tR}[\mathrm{min}]$} & Area $[\mu \mathrm{V} \cdot \mathrm{sec}]$ & Height $[\mu \mathrm{V}]$ & Area $\%$ & Height $\%$ & Quantity & NTP & Resolution & \multicolumn{1}{c}{ Symmetry Factor } \\
\hline 3 & Unknown & 9 & 29.293 & 5634032 & 99809 & 15.755 & 19.742 & N/A & 6027 & 1.447 & 1.399 \\
\hline 1 & Unknown & 9 & 31.413 & 5459239 & 102796 & 15.267 & 20.333 & N/A & 7731 & 1.340 & 1.234 \\
\hline 4 & Unknown & 9 & 33.627 & 1238823 & 176587 & 34.643 & 34.929 & N/A & 5104 & 2.128 & 1.707 \\
\hline 2 & Unknown & 9 & 38.313 & 12278030 & 126367 & 34.335 & 24.995 & N/A & 3661 & N/A & 2.341 \\
\hline
\end{tabular}

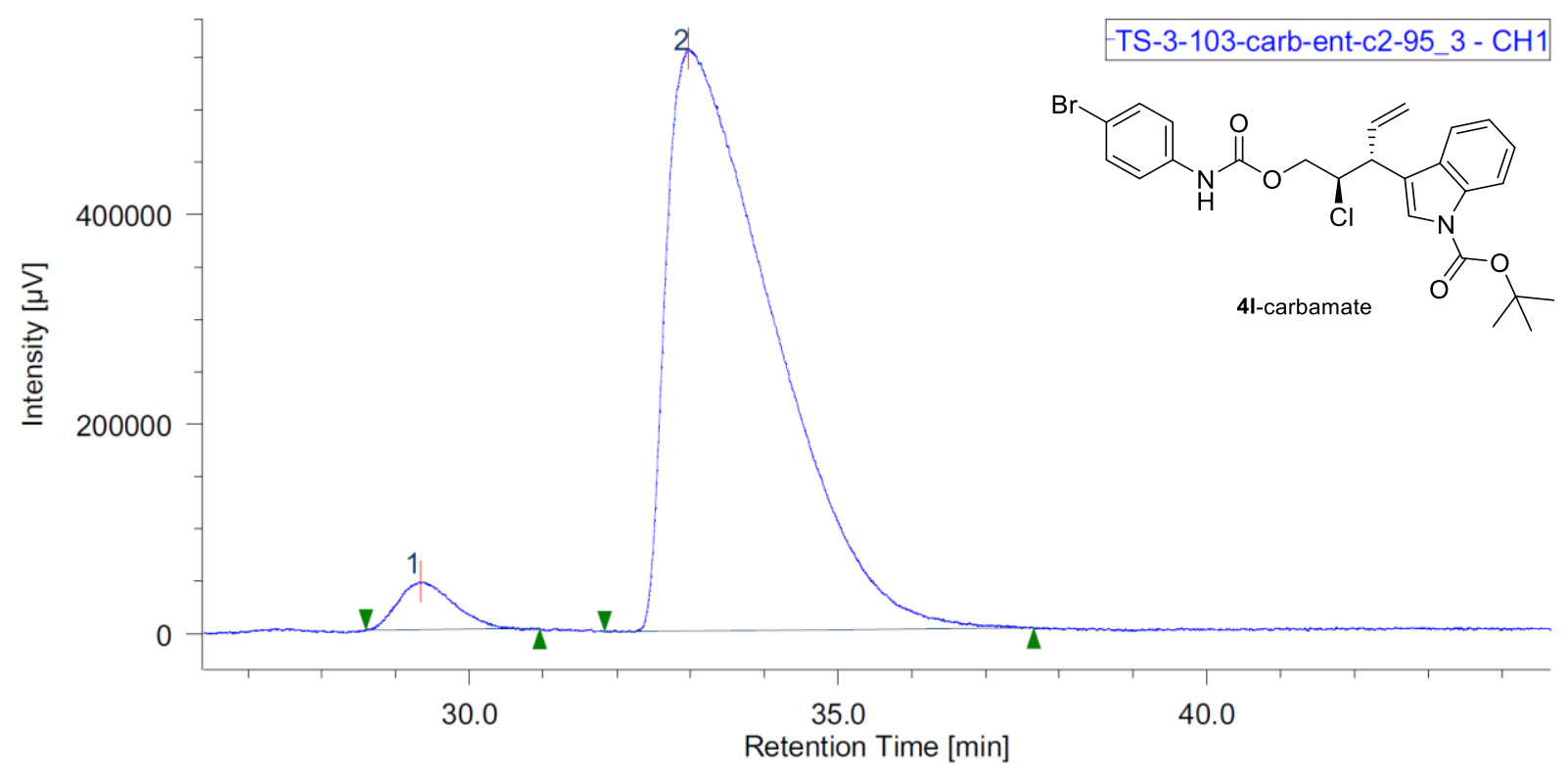

\begin{tabular}{|r|c|c|r|r|r|r|r|r|r|r|r|}
\hline$\#$ & Peak Name & CH & tR $[\mathrm{min}]$ & Area $[\mu \mathrm{V} \cdot \mathrm{sec}]$ & Height $[\mu \mathrm{V}]$ & \multicolumn{1}{|c|}{ Area $\%$} & Height $\%$ & Quantity & NTP & Resolution & Symmetry Factor \\
\hline 2 & Unknown & 1 & 29.333 & 2397841 & 45878 & 4.140 & 7.630 & $\mathrm{~N} / \mathrm{A}$ & 6895 & 1.786 & 1.329 \\
\hline 1 & Unknown & 1 & 32.975 & 55515904 & 555396 & 95.860 & 92.370 & $\mathrm{~N} / \mathrm{A}$ & 2432 & $\mathrm{~N} / \mathrm{A}$ & 3.008 \\
\hline
\end{tabular}




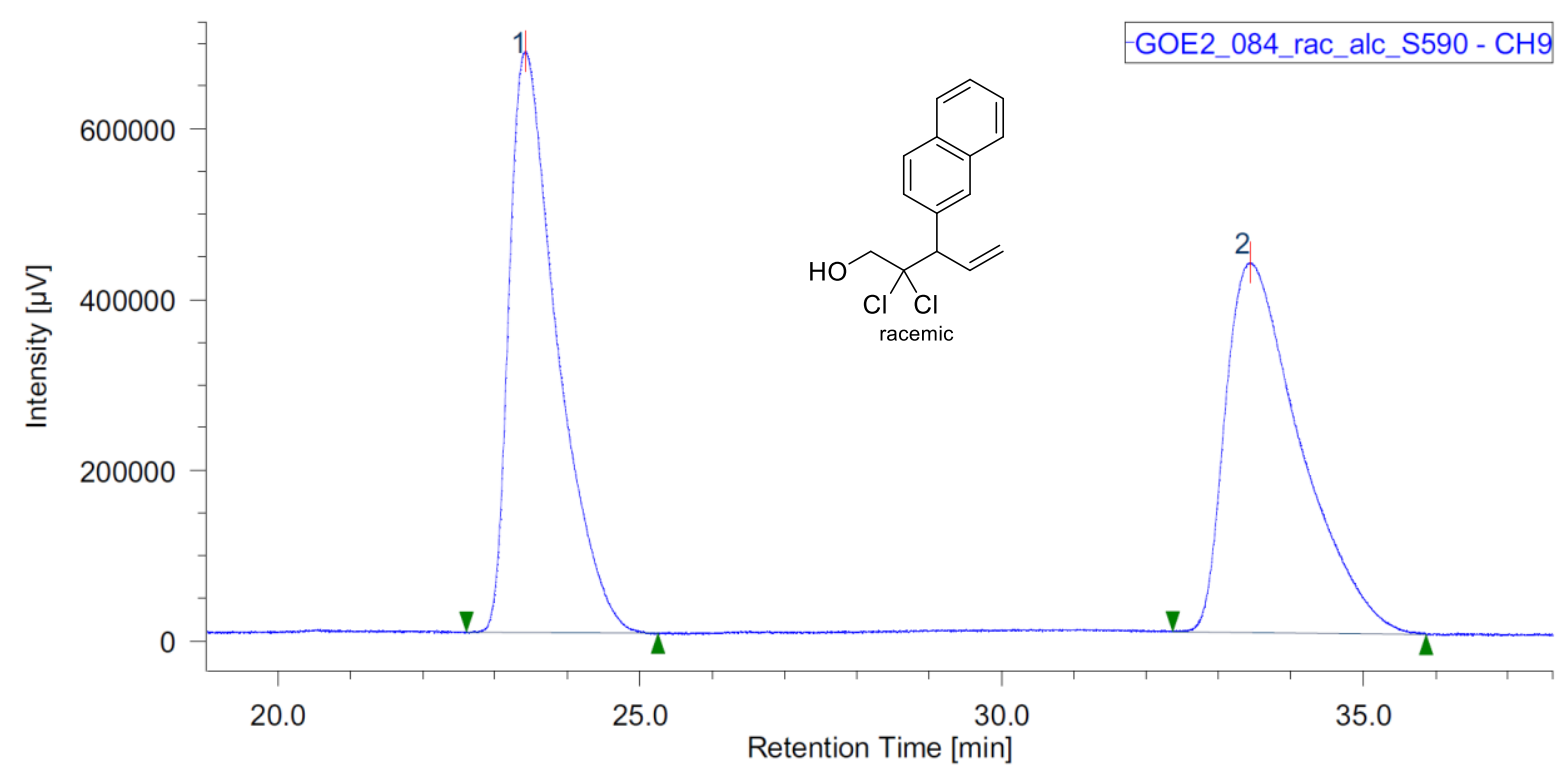

\begin{tabular}{|c|c|c|c|c|c|c|c|c|c|c|c|c|}
\hline & Peak Name & $\mathrm{CH}$ & $\mathrm{tR}[\mathrm{min}]$ & Area $[\mu \mathrm{V} \cdot \mathrm{sec}]$ & \begin{tabular}{|l} 
Height $[\mu \mathrm{V}]$ \\
\end{tabular} & Area $\%$ & Height $\%$ & Quantity & NTP & \begin{tabular}{|l} 
Resolution \\
\end{tabular} & Symmetry Factor & Warning \\
\hline & Unknown & 9 & 23.427 & 31282636 & 680666 & 50.036 & 61.094 & $\mathrm{~N} / \mathrm{A}$ & 6467 & 6.622 & 1.780 & \\
\hline & Unknown & 9 & 33.433 & 31237361 & 433463 & 49.964 & 38.906 & $\mathrm{~N} / \mathrm{A}$ & 5142 & $\mathrm{~N} / \mathrm{A}$ & 1.824 & \\
\hline
\end{tabular}

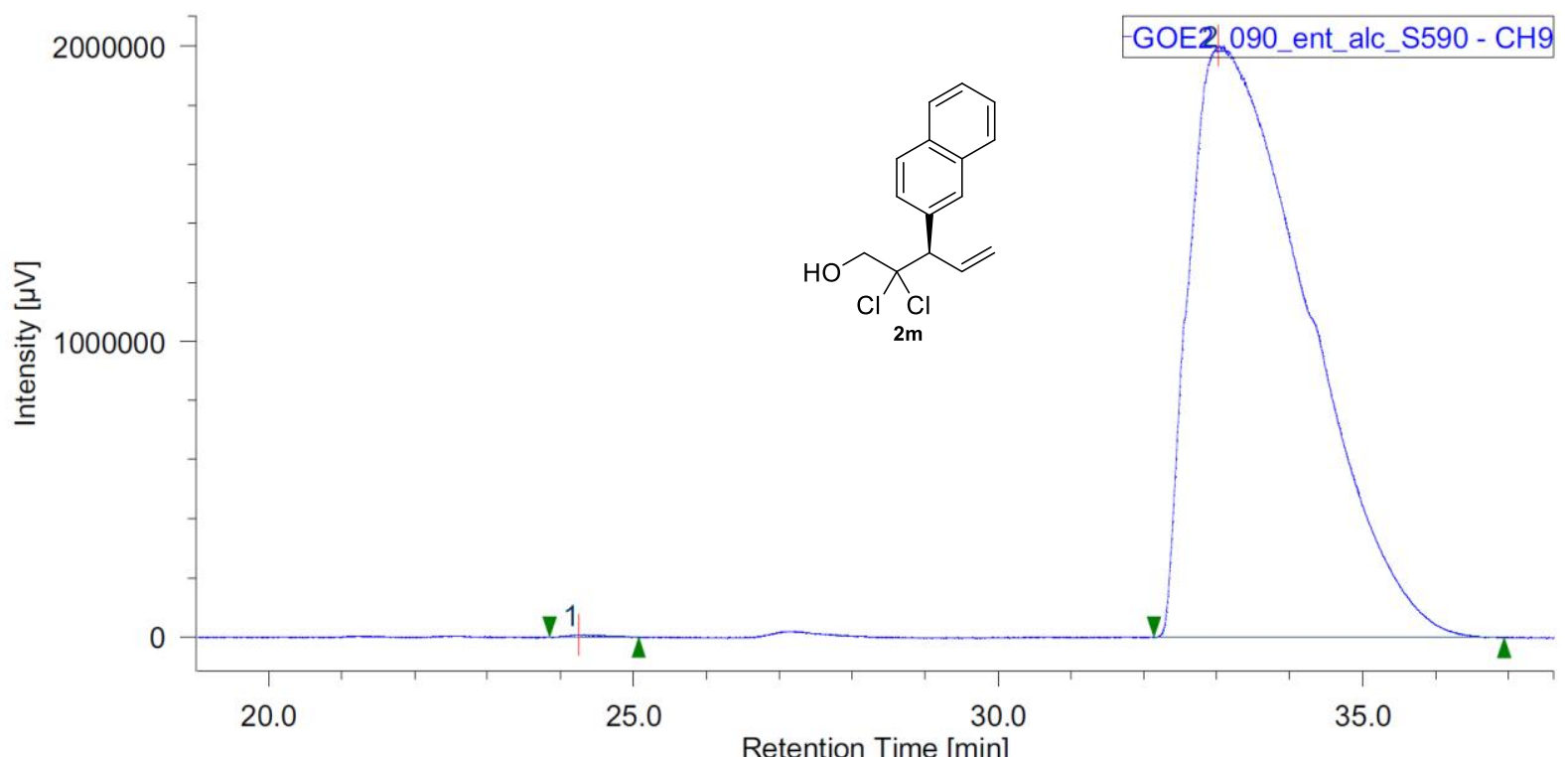

\begin{tabular}{|c|c|c|c|c|c|c|c|c|c|c|c|c|}
\hline \# & Peak Name & $\mathrm{CH}$ & $\mathrm{tR}[\mathrm{min}]$ & Area $[\mu \mathrm{V} \cdot \mathrm{sec}]$ & Height $[\mu \mathrm{V}]$ & Area $\%$ & Height $\%$ & Quantity & NTP & Resolution & Symmetry Factor & Warning \\
\hline & Unknown & 9 & 24.247 & 247375 & 763 & 0.111 & 0.380 & $\mathrm{~N} / \mathrm{A}$ & 9101 & 4.170 & 1.552 & \\
\hline 1 & Unknown & 9 & 33.013 & 223617029 & 2001952 & 99.889 & 99.620 & $\mathrm{~N} / \mathrm{A}$ & 1704 & $\mathrm{~N} / \mathrm{A}$ & 2.437 & \\
\hline
\end{tabular}




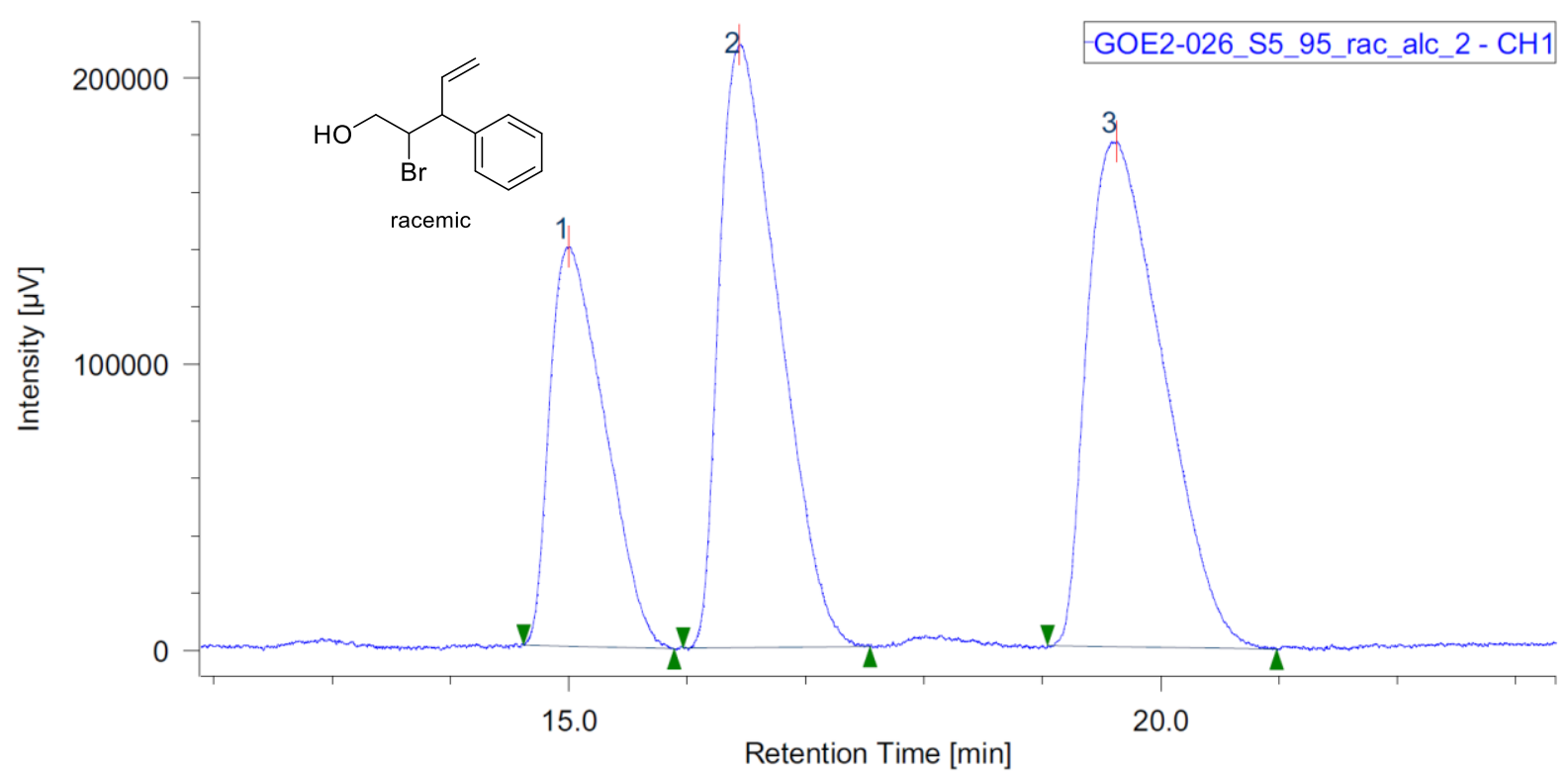

\begin{tabular}{|c|c|c|c|c|c|c|c|c|c|c|c|}
\hline$\#$ & Peak Name & $\mathrm{CH}$ & $\mathrm{tR}$ [min] & Area $[\mu \mathrm{V} \cdot \mathrm{sec}]$ & Height $[\mu \mathrm{V}]$ & Area $\%$ & Height $\%$ & Quantity & NTP & Resolution & Symmetry Factor \\
\hline & Unknown & 1 & 15.000 & 4482528 & 139485 & 22.762 & 26.493 & $\mathrm{~N} / \mathrm{A}$ & 4526 & 1.563 & 1.620 \\
\hline & Unknown & 1 & 16.442 & 7364102 & 210607 & 37.394 & 40.002 & $\mathrm{~N} / \mathrm{A}$ & 4719 & 2.918 & 1.689 \\
\hline & Unknown & 1 & 19.625 & 7846541 & 176401 & 39.844 & 33.505 & $\mathrm{~N} / \mathrm{A}$ & 4069 & $\mathrm{~N} / \mathrm{A}$ & 1.579 \\
\hline
\end{tabular}

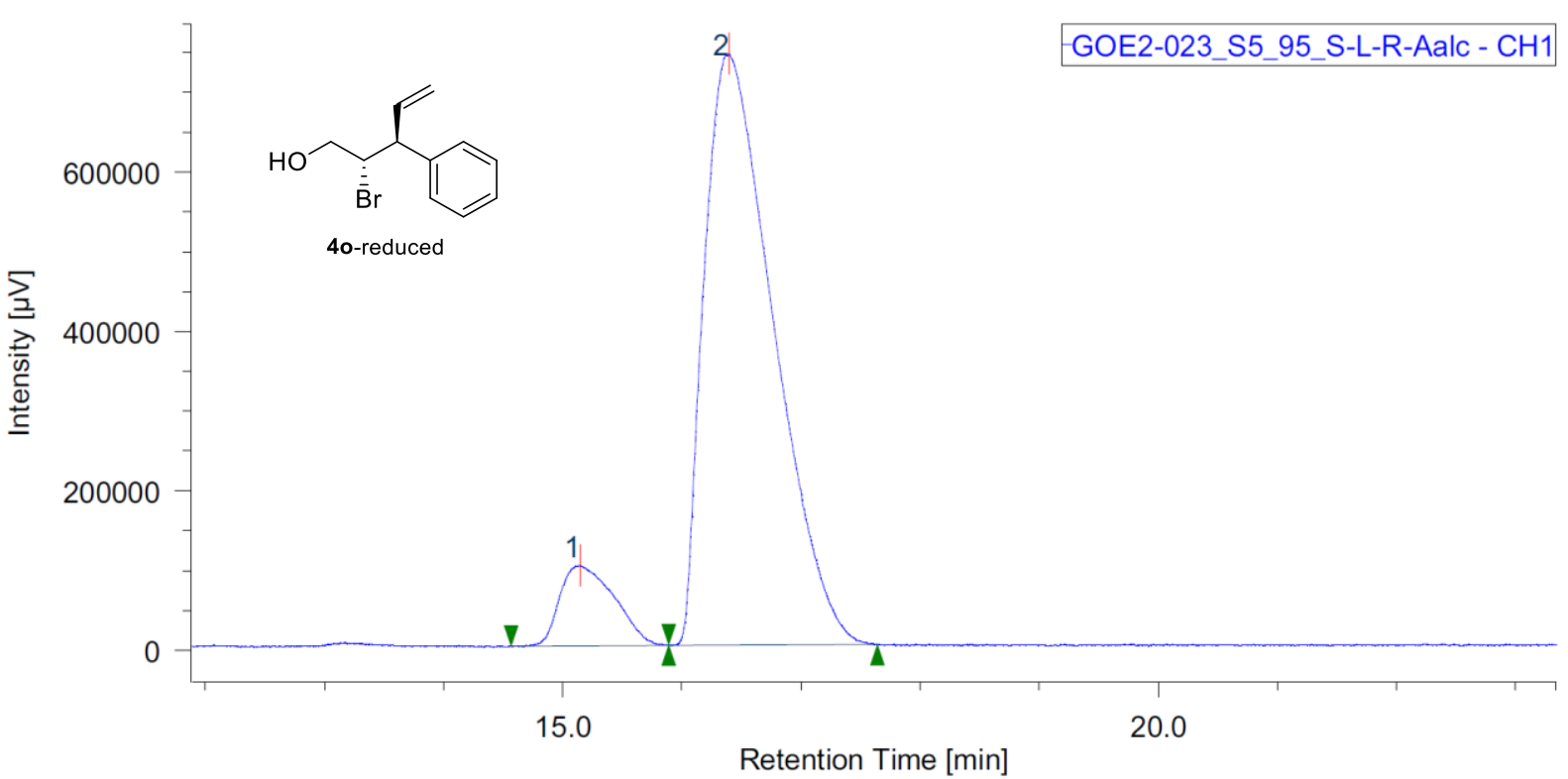

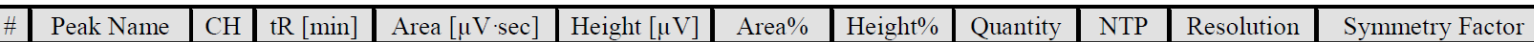

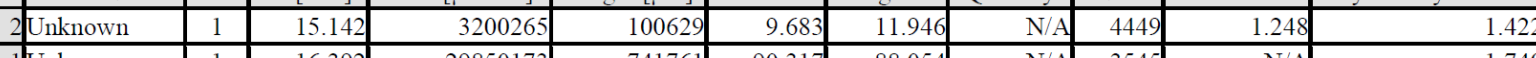

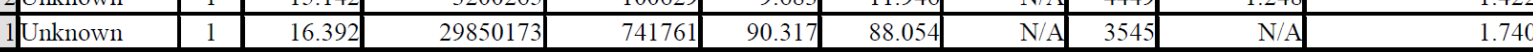




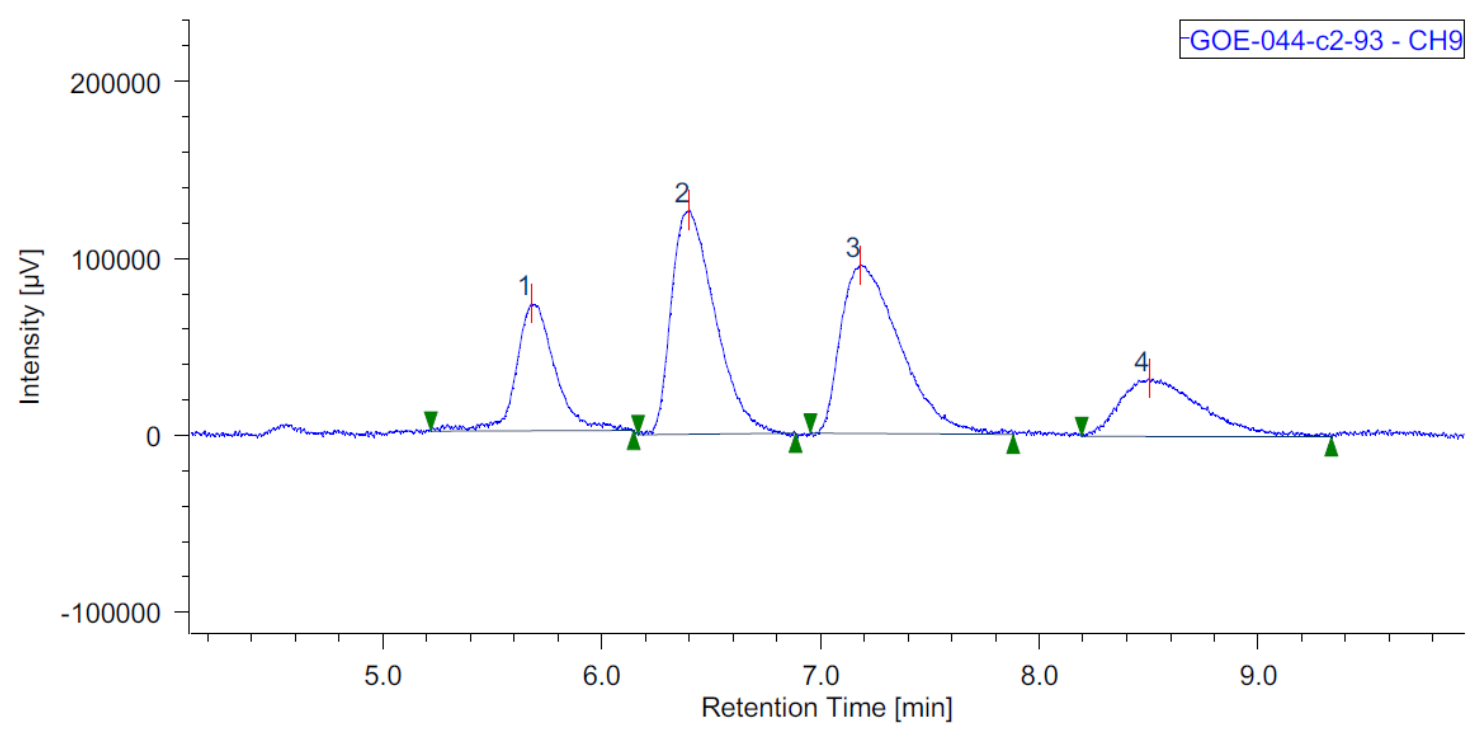

\begin{tabular}{|c|c|c|c|c|c|c|c|c|c|c|c|}
\hline \# & Peak Name & $\mathrm{CH}$ & $\mathrm{tR}$ [min] & Area $[\mu \mathrm{V} \cdot \mathrm{sec}]$ & Height $[\mu \mathrm{V}]$ & Area $\%$ & Height $\%$ & Quantity & NTP & Resolution & Symmetry Factor \\
\hline & Unknown & 9 & 5.680 & 893720 & 71964 & 16.905 & 22.028 & $\mathrm{~N} / \mathrm{A}$ & 5915 & 2.186 & 0.966 \\
\hline & Unknown & 9 & 6.400 & 1751770 & 126900 & 33.136 & 38.843 & $\mathrm{~N} / \mathrm{A}$ & 4915 & 1.812 & 1.502 \\
\hline & Unknown & 9 & 7.180 & 1791616 & 95140 & 33.890 & 29.121 & $\mathrm{~N} / \mathrm{A}$ & 3322 & 2.202 & 1.875 \\
\hline & Unknown & 9 & 8.500 & 849499 & 32696 & 16.069 & 10.008 & $\mathrm{~N} / \mathrm{A}$ & 2334 & $\mathrm{~N} / \mathrm{A}$ & 1.989 \\
\hline
\end{tabular}

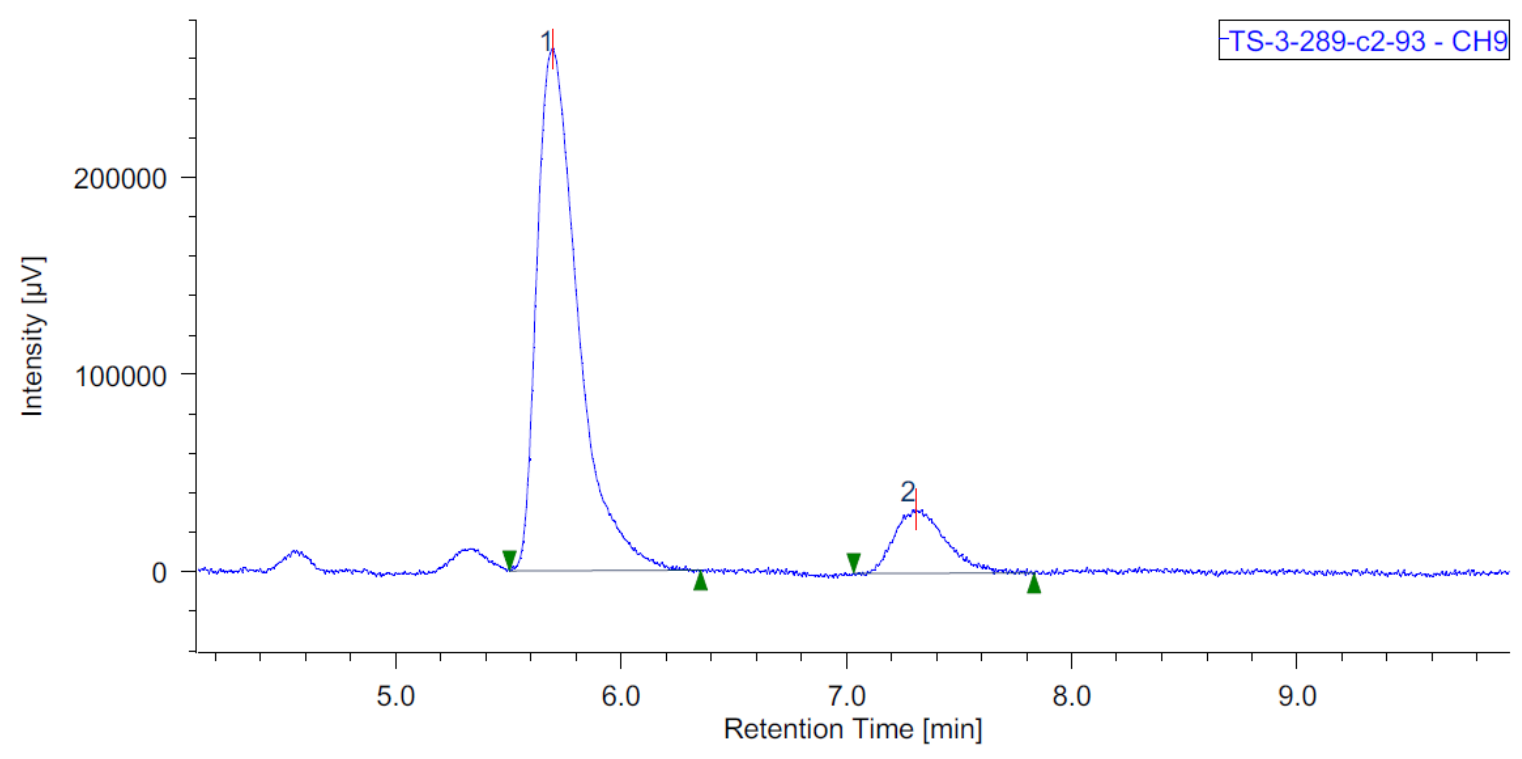

\begin{tabular}{|r|c|c|r|r|r|r|r|r|r|r|r|}
\hline$\#$ & Peak Name & $\mathrm{CH}$ & $\mathrm{tR}[\mathrm{min}]$ & Area $[\mu \mathrm{V} \cdot \mathrm{sec}]$ & Height $[\mu \mathrm{V}]$ & Area $\%$ & Height $\%$ & Quantity & NTP & Resolution & Symmetry Factor \\
\hline 1 & Unknown & 9 & 5.700 & 3346869 & 26471 & 86.604 & 89.099 & $\mathrm{~N} / \mathrm{A}$ & 5123 & 4.450 & 1.756 \\
\hline 2 & Unknown & 9 & 7.307 & 517695 & 32387 & 13.39 & 10.901 & $\mathrm{~N} / \mathrm{A}$ & 5196 & $\mathrm{~N} / \mathrm{A}$ & 1.769 \\
\hline
\end{tabular}




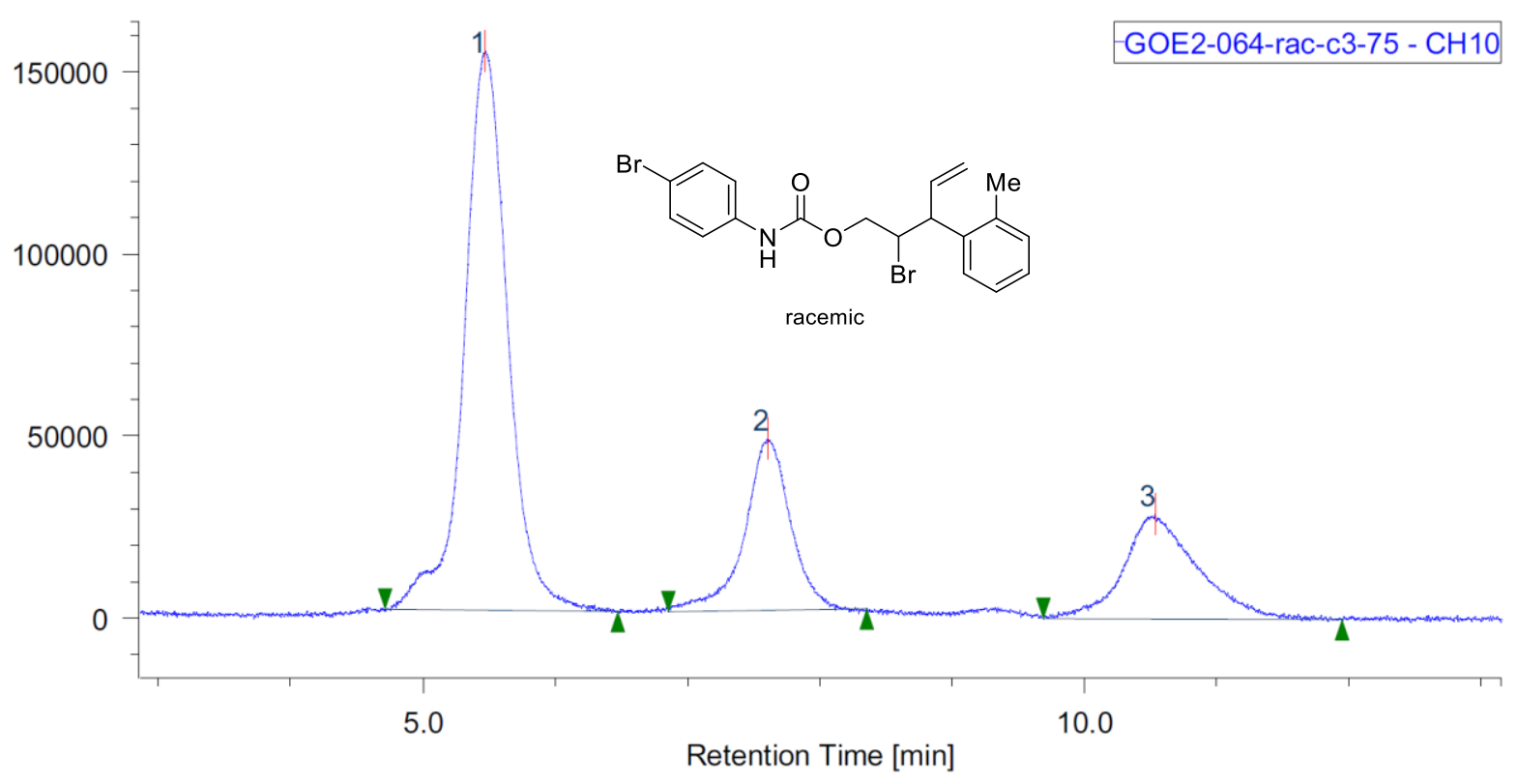

\begin{tabular}{|c|c|c|c|c|c|c|c|c|c|c|}
\hline Peak Name & $\mathrm{CH}$ & $t \mathrm{t}[\mathrm{min}]$ & Area $[\mu \mathrm{V} \cdot \mathrm{sec}]$ & Height $[\mu \mathrm{V}]$ & Area $\%$ & Height\% & Quantity & NTP & Resolution & Symmetry Factor \\
\hline 3 Unknown & 10 & 5.473 & 3518259 & 153415 & 60.958 & 66.924 & $\mathrm{~N} / \mathrm{A}$ & 1538 & 3.778 & 0.909 \\
\hline 2 Unknown & 10 & 7.607 & 1140271 & 47106 & 19.757 & 20.549 & $\mathrm{~N} / \mathrm{A}$ & 2809 & 3.867 & 0.852 \\
\hline 1 Unknown & 10 & 10.533 & 1113076 & 28716 & 19.285 & 12.527 & $\mathrm{~N} / \mathrm{A}$ & 1994 & $\mathrm{~N} / \mathrm{A}$ & 1.162 \\
\hline
\end{tabular}

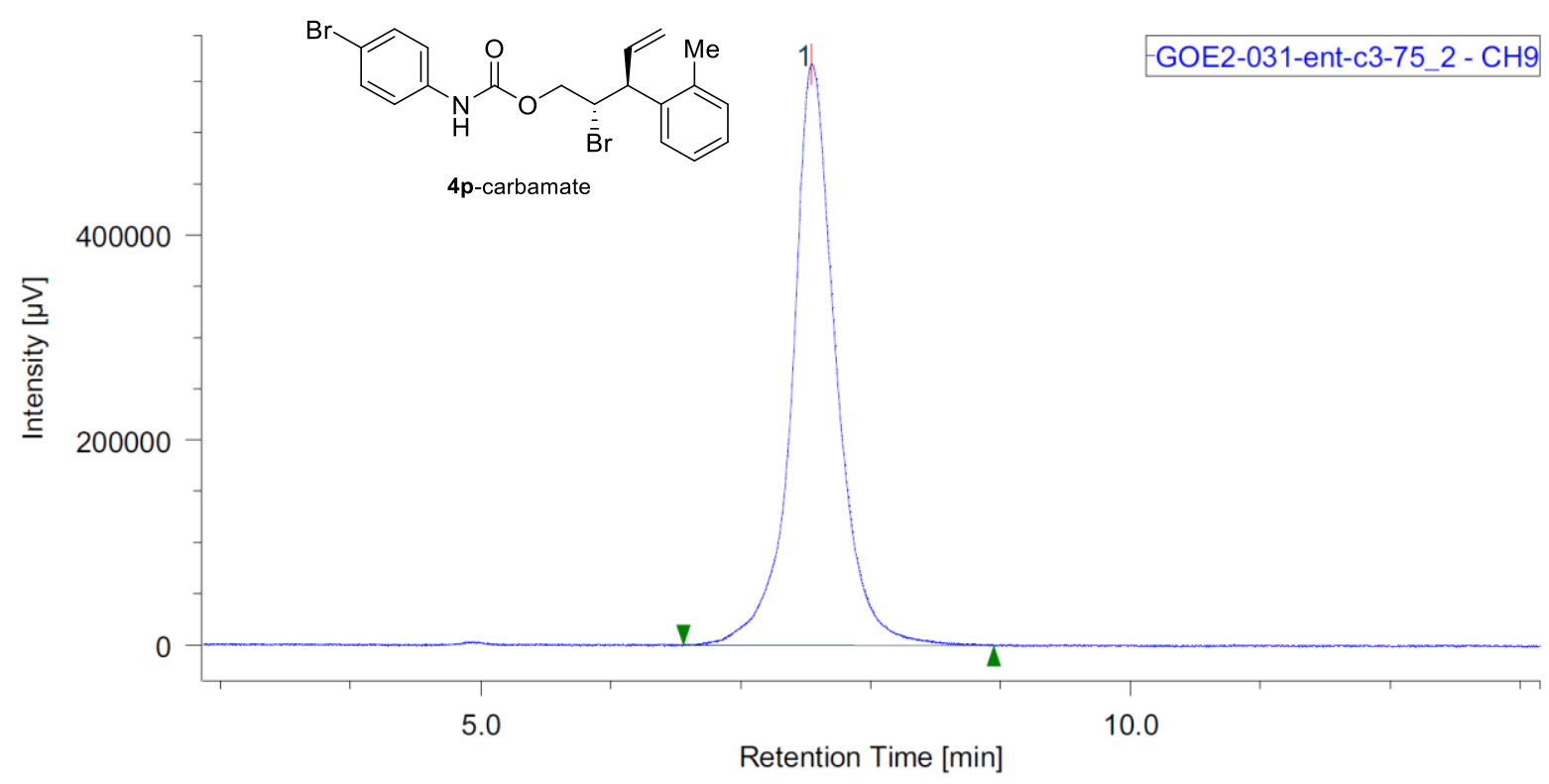

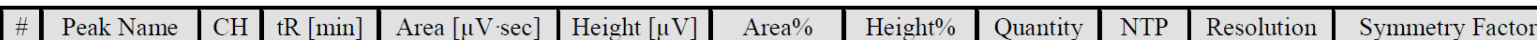

\begin{tabular}{|l|c|r|r|r|r|r|r|r|r|}
\hline 1 & Unknown & 9 & 7.547 & 13444078 & 566465 & 100.000 & 100.000 & $\mathrm{~N} / \mathrm{A}$ & 2959 \\
\hline
\end{tabular}




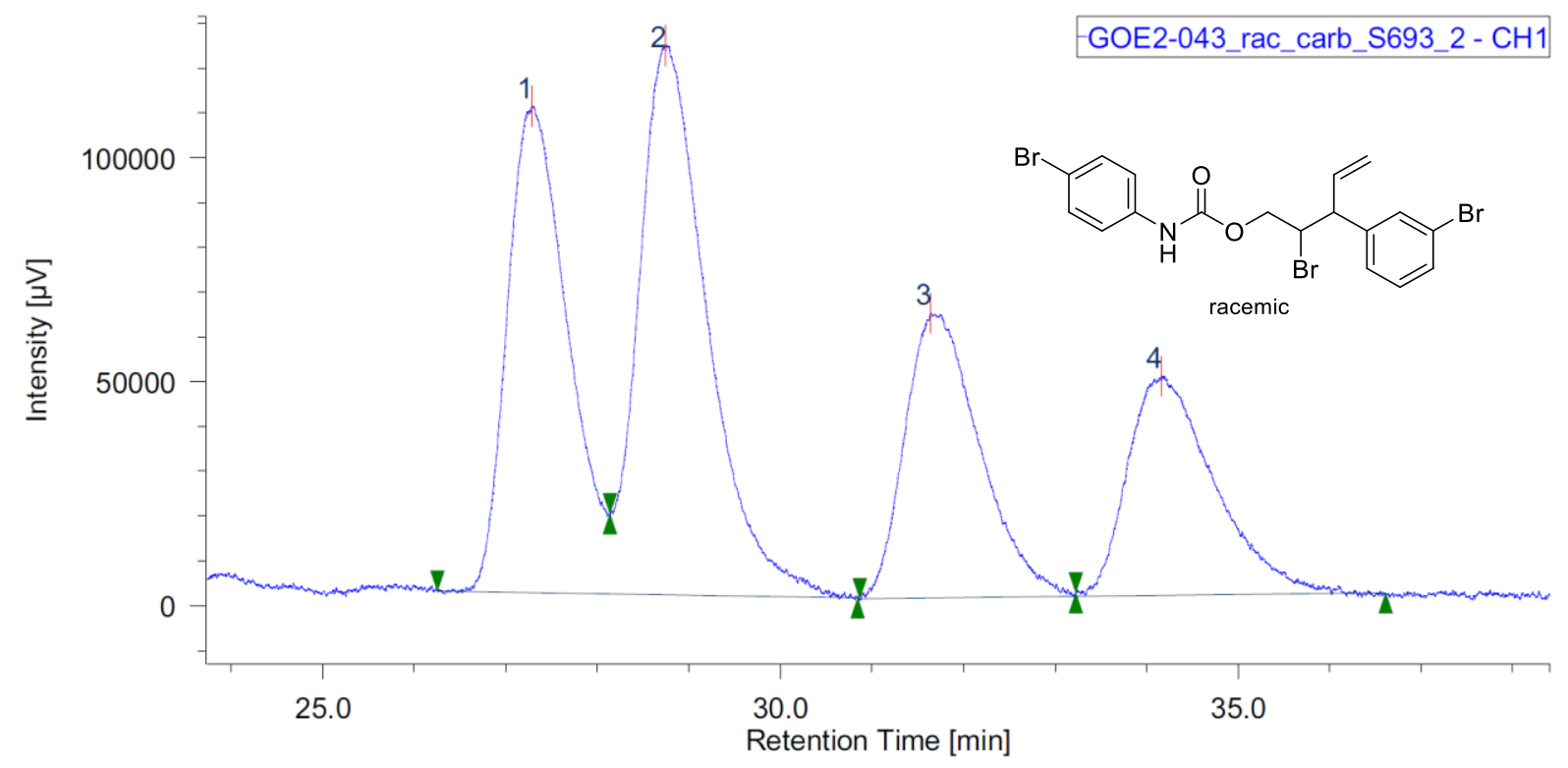

\begin{tabular}{|c|c|c|c|c|c|c|c|c|c|c|c|}
\hline \# & Peak Name & $\mathrm{CH}$ & $\mathrm{tR}$ [min] & Area $[\mu \mathrm{V} \cdot \mathrm{sec}]$ & Height $[\mu \mathrm{V}]$ & Area $\%$ & Height $\%$ & Quantity & NTP & Resolution & Symmetry Factor \\
\hline & Unknown & 1 & 27.283 & 4969425 & 108466 & 27.289 & 31.566 & $\mathrm{~N} / \mathrm{A}$ & 7921 & 1.152 & $\mathrm{~N} / \mathrm{A}$ \\
\hline & Unknown & 1 & 28.750 & 6414073 & 122580 & 35.223 & 35.673 & $\mathrm{~N} / \mathrm{A}$ & 7517 & 2.048 & $\mathrm{~N} / \mathrm{A}$ \\
\hline & Unknown & 1 & 31.642 & 3610485 & 63655 & 19.827 & 18.525 & $\mathrm{~N} / \mathrm{A}$ & 7078 & 1.573 & 1.524 \\
\hline & Unknown & 1 & 34.167 & 3216163 & 48921 & 17.661 & 14.237 & $\mathrm{~N} / \mathrm{A}$ & 6361 & N/A & 1.547 \\
\hline
\end{tabular}

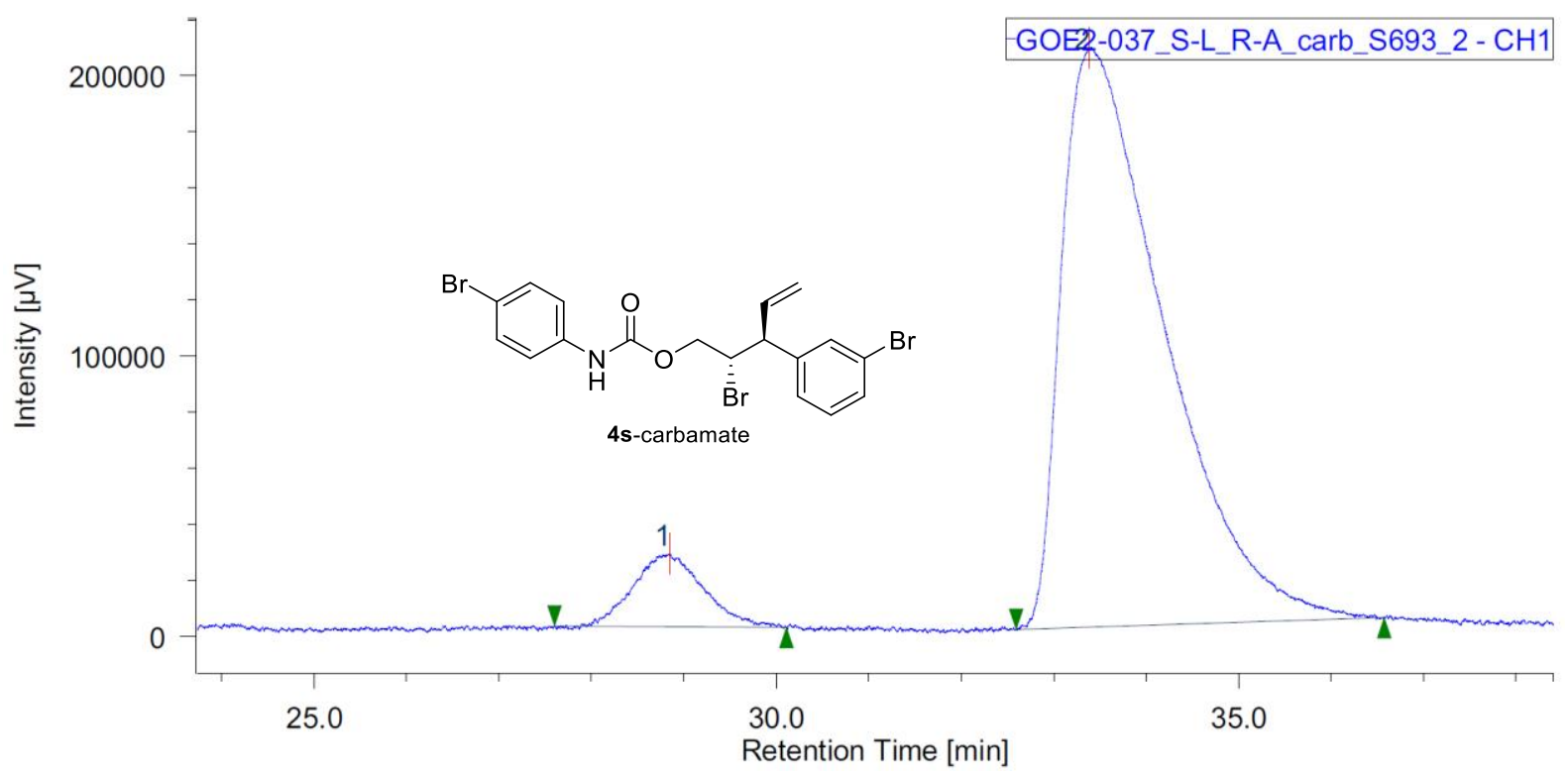

\begin{tabular}{|c|c|c|c|c|c|c|c|c|c|c|c|}
\hline$\#$ & Peak Name & $\overline{\mathrm{CH}}$ & $\mathrm{tR}$ [min] & Area $[\mu \mathrm{V} \cdot \mathrm{sec}]$ & Height $[\mu \mathrm{V}]$ & Area $\%$ & Height $\%$ & Quantity & NTP & Resolution & y Factor \\
\hline & Unknown & 1 & 28.850 & 1363866 & 25910 & 7.893 & 11.145 & $\mathrm{~N} / \mathrm{A}$ & 7142 & 2.697 & 1.162 \\
\hline & Unknown & 1 & 33.383 & 15916496 & 206566 & 92.107 & 88.855 & $\mathrm{~N} / \mathrm{A}$ & 4437 & $\mathrm{~N} / \mathrm{A}$ & 2.352 \\
\hline
\end{tabular}




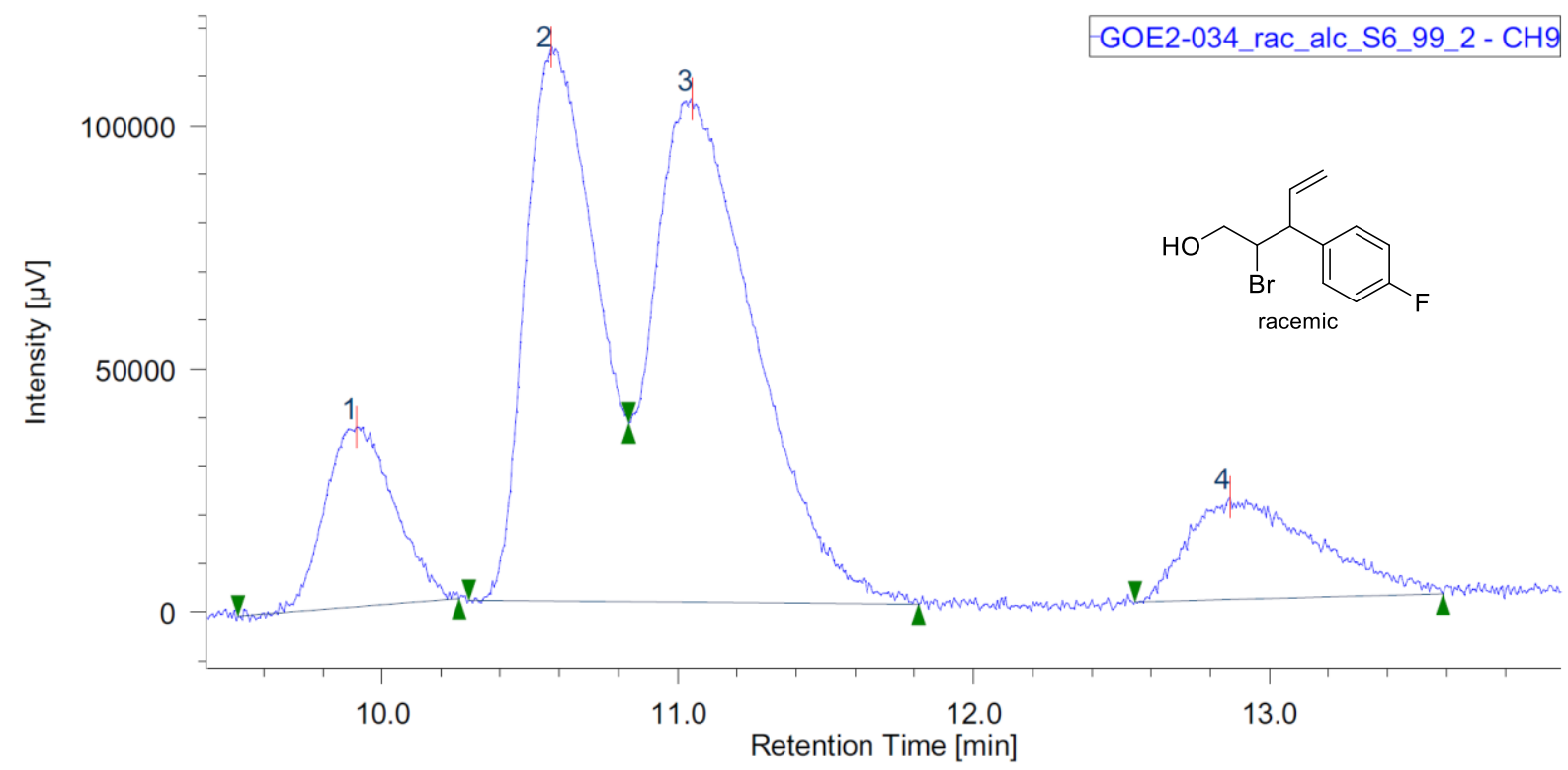

\begin{tabular}{|r|l|c|r|r|r|r|r|r|r|r|r|}
\hline$\#$ & Peak Name & $\mathrm{CH}$ & $\mathrm{tR}[\mathrm{min}]$ & Area $[\mu \mathrm{V} \cdot \mathrm{sec}]$ & Height $[\mu \mathrm{V}]$ & Area $\%$ & Height $\%$ & Quantity & NTP & Resolution & Symmetry Factor \\
\hline 1 & Unknown & 9 & 9.913 & 606834 & 36822 & 10.802 & 13.391 & $\mathrm{~N} / \mathrm{A}$ & 8070 & 1.442 & 1.058 \\
\hline 3 & Unknown & 9 & 10.573 & 1886731 & 113789 & 33.585 & 41.382 & $\mathrm{~N} / \mathrm{A}$ & 7876 & 0.847 & N/A \\
\hline & Unknown & 9 & 11.047 & 2503026 & 103360 & 44.555 & 37.589 & $\mathrm{~N} / \mathrm{A}$ & 4713 & 2.440 & N/A \\
\hline 2 & Unknown & 9 & 12.867 & 621248 & 21000 & 11.058 & 7.637 & N/A & 3649 & N/A & 1.806 \\
\hline
\end{tabular}

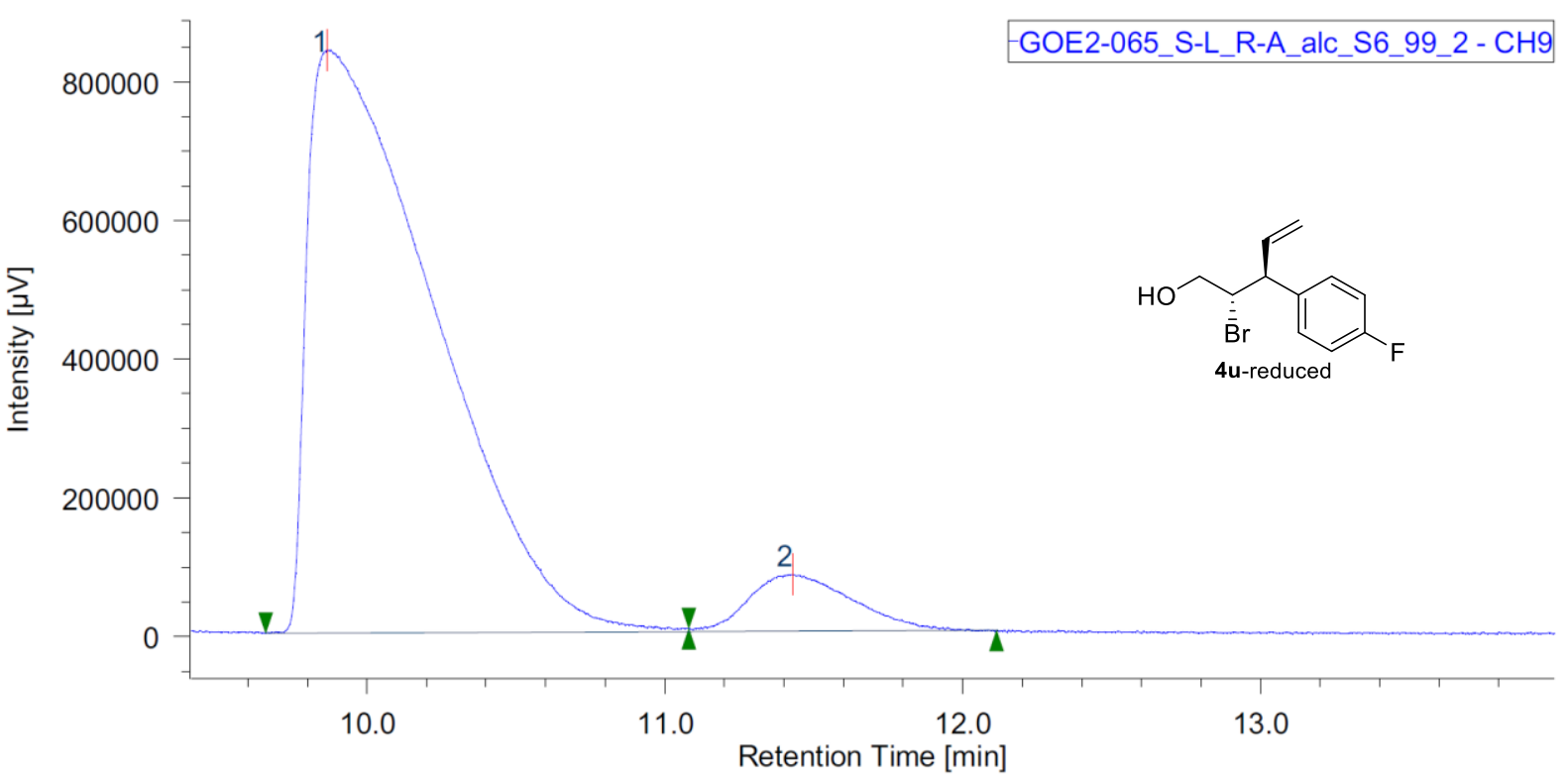

\begin{tabular}{|r|c|c|r|r|r|r|r|r|r|r|r|}
\hline$\#$ & Peak Name & $\mathrm{CH}$ & $\mathrm{tR}[\mathrm{min}]$ & Area $[\mu \mathrm{V} \cdot \mathrm{sec}]$ & Height $[\mu \mathrm{V}]$ & \multicolumn{1}{|c|}{ Area $\%$} & Height $\%$ & Quantity & NTP & Resolution & Symmetry Factor \\
\hline 1 & Unknown & 9 & 9.867 & 24941806 & 840997 & 92.713 & 91.138 & $\mathrm{~N} / \mathrm{A}$ & 2392 & 2.163 & 4.022 \\
\hline 2 & Unknown & 9 & 11.427 & 1960284 & 81780 & 7.287 & 8.862 & $\mathrm{~N} / \mathrm{A}$ & 5107 & $\mathrm{~N} / \mathrm{A}$ & \\
\hline
\end{tabular}




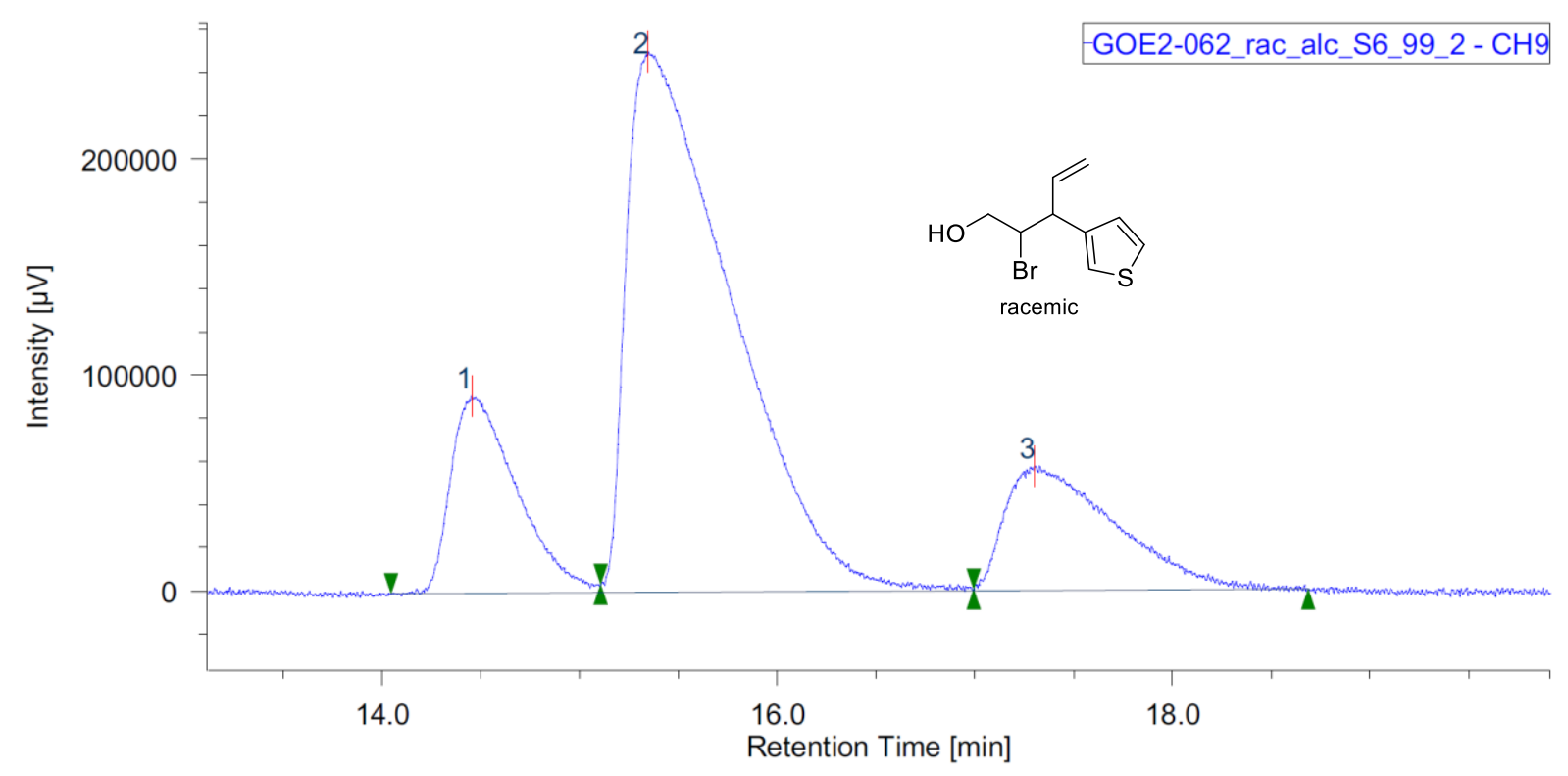

\begin{tabular}{|c|c|c|c|c|c|c|c|c|c|c|c|}
\hline$\#$ & Peak Name & $\overline{\mathrm{CH}}$ & $\mathrm{tR}[\mathrm{min}]$ & Area $[\mu \mathrm{V} \cdot \mathrm{sec}]$ & Height $[\mu \mathrm{V}]$ & Area $\%$ & Height $\%$ & Quantity & NTP & \begin{tabular}{l|} 
Resolution \\
\end{tabular} & Symmetry Factor \\
\hline & Unknown & 9 & 14.453 & 2111188 & 91181 & 15.617 & 22.864 & $\mathrm{~N} / \mathrm{A}$ & 8987 & 1.120 & 1.882 \\
\hline & Unknown & 9 & 15.347 & 9205433 & 250138 & 68.096 & 62.723 & N/A & 3850 & 1.934 & 3.016 \\
\hline & Unknown & 9 & 17.300 & 2201642 & 57477 & 16.286 & 14.413 & N/A & 4466 & $\mathrm{~N} / \mathrm{A}$ & 2.459 \\
\hline
\end{tabular}

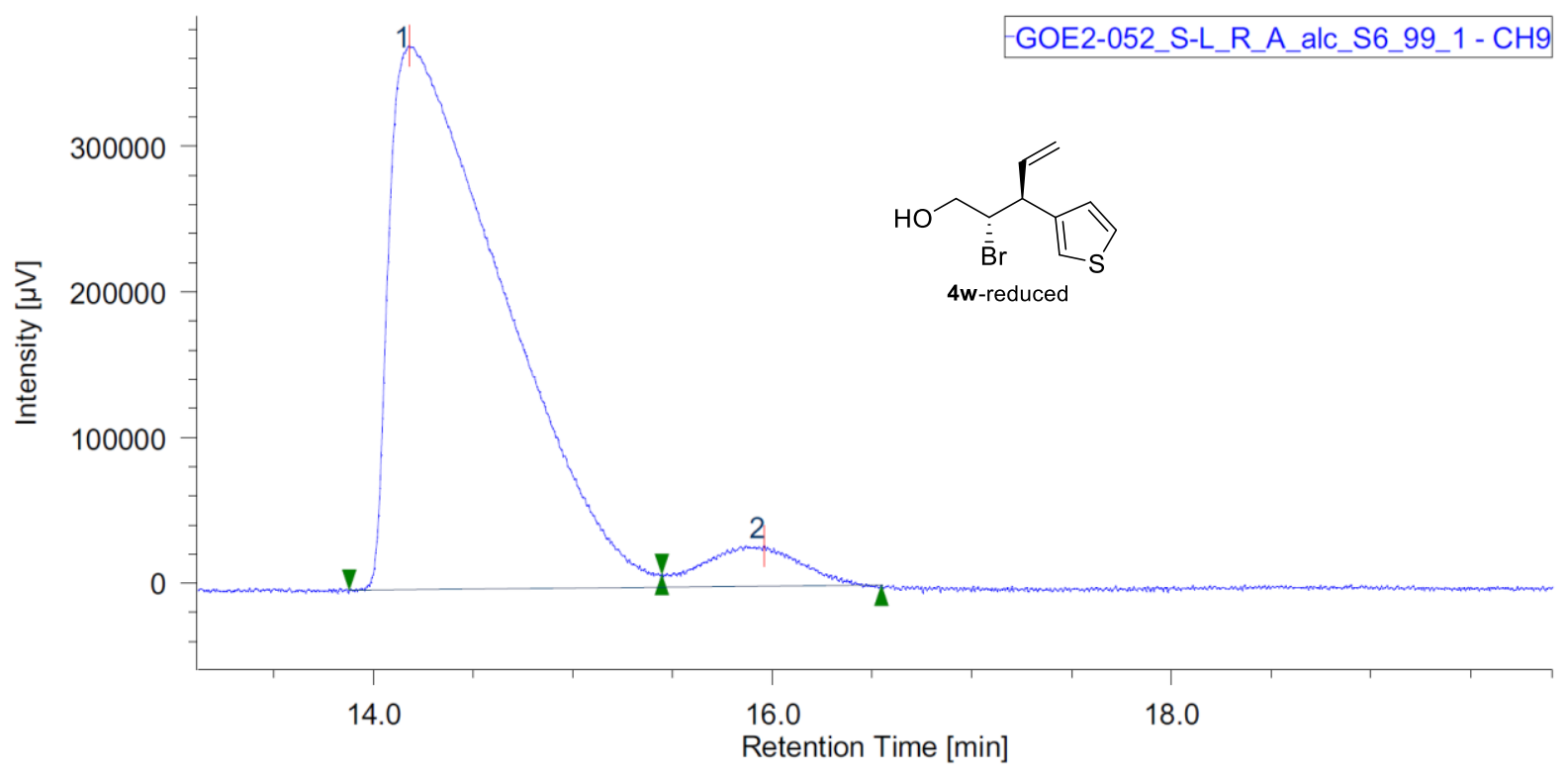

\begin{tabular}{|r|c|c|r|r|r|r|r|r|r|r|r|}
\hline$\#$ & Peak Name & CH & tR $[\mathrm{min}]$ & Area $[\mu \mathrm{V} \cdot \mathrm{sec}]$ & Height $[\mu \mathrm{V}]$ & \multicolumn{1}{|c|}{ Area $\%$} & Height $\%$ & Quantity & NTP & Resolution & Symmetry Factor \\
\hline 1 & Unknown & 9 & 14.180 & 14738925 & 373373 & 94.121 & 93.086 & N/A & 2793 & 1.759 & 3.720 \\
\hline 2 & Unknown & 9 & 15.960 & 920700 & 27733 & 5.879 & 6.914 & N/A & 4458 & N/A & N/A \\
\hline
\end{tabular}




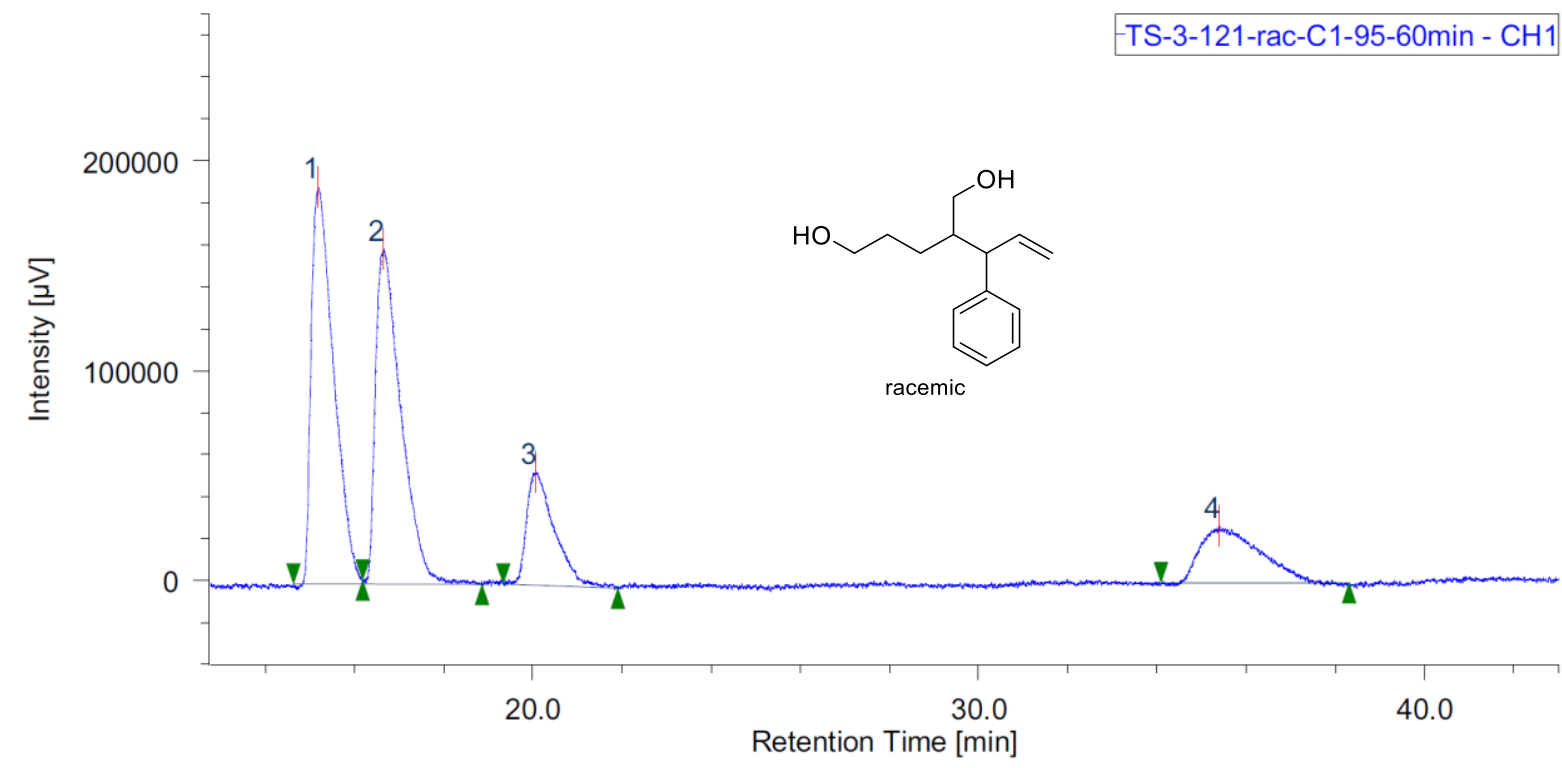

\begin{tabular}{|c|c|c|c|c|c|c|c|c|c|c|c|}
\hline \# & Peak Name & $\mathrm{CH}$ & $\mathrm{tR}$ [min] & Area $[\mu \mathrm{V} \cdot \mathrm{sec}]$ & Height $[\mu \mathrm{V}]$ & Area\% & Height $\%$ & Quantity & NTP & Resolution & Symmetry Factor \\
\hline & Unknown & 1 & 15.183 & 6600441 & 189207 & 37.269 & 44.037 & $\mathrm{~N} / \mathrm{A}$ & 4150 & 1.494 & 1.991 \\
\hline & Unknown & 1 & 16.650 & 6328873 & 159648 & 35.736 & 37.157 & $\mathrm{~N} / \mathrm{A}$ & 4219 & 3.266 & 1.994 \\
\hline & Unknown & 1 & 20.075 & 2348274 & 53818 & 13.259 & 12.526 & $\mathrm{~N} / \mathrm{A}$ & 5554 & 8.360 & 1.835 \\
\hline & Unknown & 1 & 35.392 & 2432683 & 26985 & 13.736 & 6.281 & $\mathrm{~N} / \mathrm{A}$ & 2972 & $\mathrm{~N} / \mathrm{A}$ & 1.755 \\
\hline
\end{tabular}

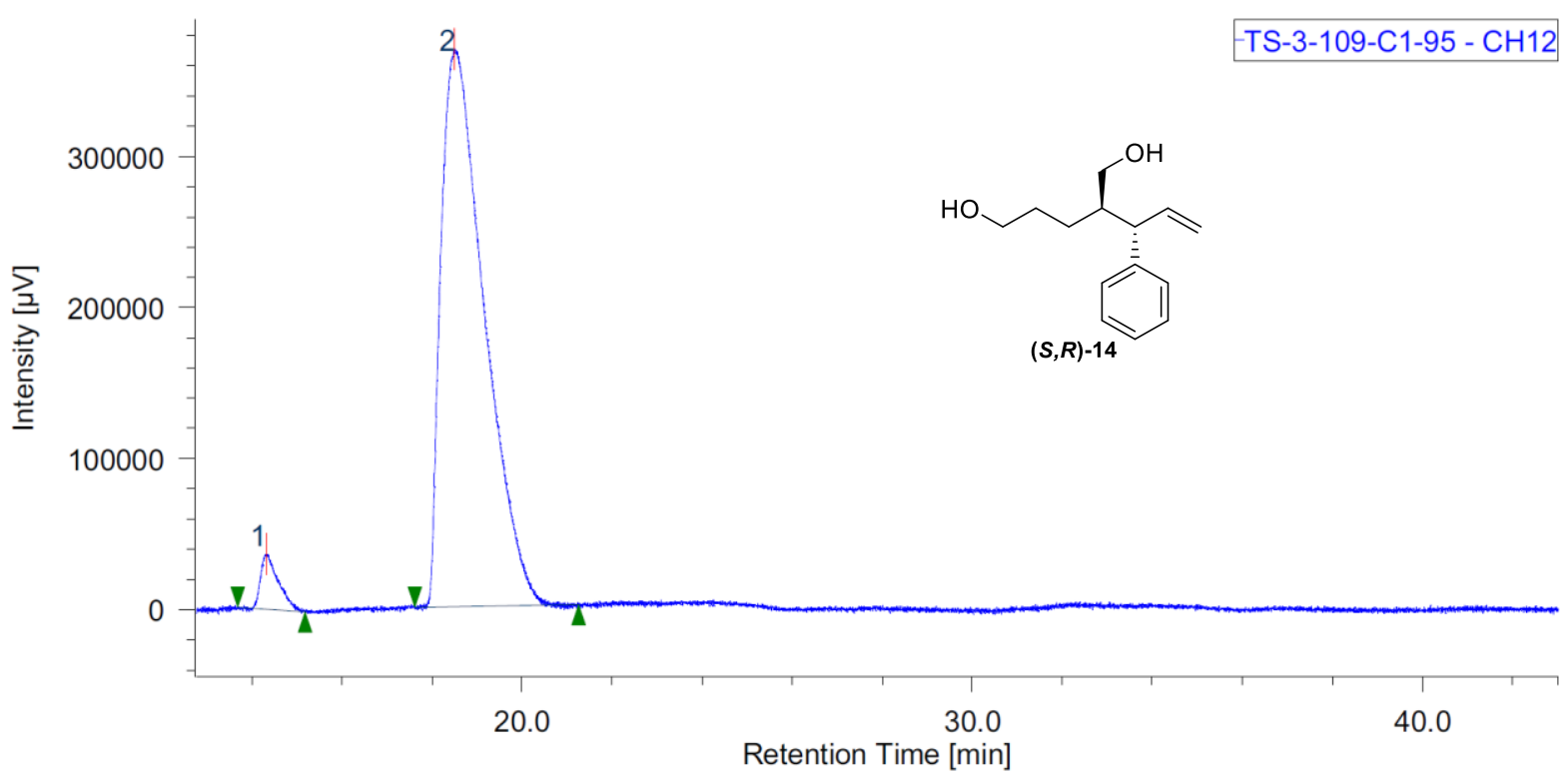

\begin{tabular}{|r|c|c|r|r|r|r|r|r|r|r|r|}
\hline$\#$ & Peak Name & $\mathrm{CH}$ & tR $[\mathrm{min}]$ & Area $[\mu \mathrm{V} \cdot \mathrm{sec}]$ & Height $[\mu \mathrm{V}]$ & \multicolumn{1}{|c|}{ Area\% } & Height\% & Quantity & NTP & Resolution & Symmetry Factor \\
\hline 1 & Unknown & 12 & 14.313 & 1006024 & 36251 & 3.922 & 8.947 & N/A & 6173 & 3.325 & 2.057 \\
\hline 2 & Unknown & 12 & 18.500 & 24644503 & 368940 & 96.078 & 91.053 & N/A & 1697 & N/A & 2.043 \\
\hline
\end{tabular}




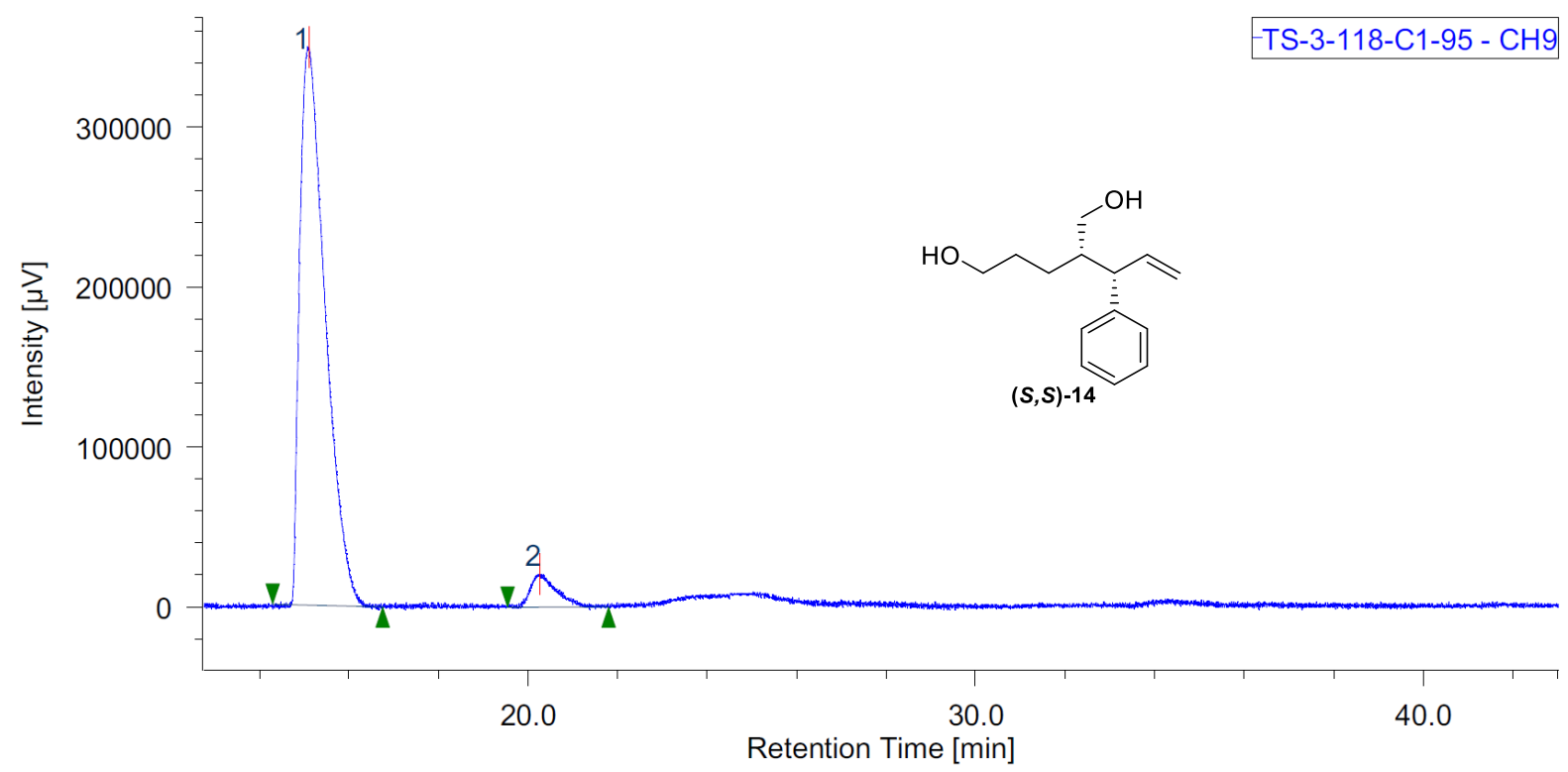

\begin{tabular}{|r|c|c|r|r|r|r|r|r|r|r|r|}
\hline$\#$ & Peak Name & CH & tR $[\mathrm{min}]$ & Area $[\mu \mathrm{V} \cdot \mathrm{sec}]$ & Height $[\mu \mathrm{V}]$ & \multicolumn{1}{|c|}{ Area $\%$} & Height\% & Quantity & NTP & Resolution & Symmetry Factor \\
\hline 1 & Unknown & 9 & 15.100 & 13831551 & 348698 & 94.244 & 94.389 & $\mathrm{~N} / \mathrm{A}$ & 3231 & 4.666 & 1.914 \\
\hline 2 & Unknown & 9 & 20.267 & 844802 & 20727 & 5.756 & 5.611 & $\mathrm{~N} / \mathrm{A}$ & 4903 & $\mathrm{~N} / \mathrm{A}$ & 1.585 \\
\hline
\end{tabular}




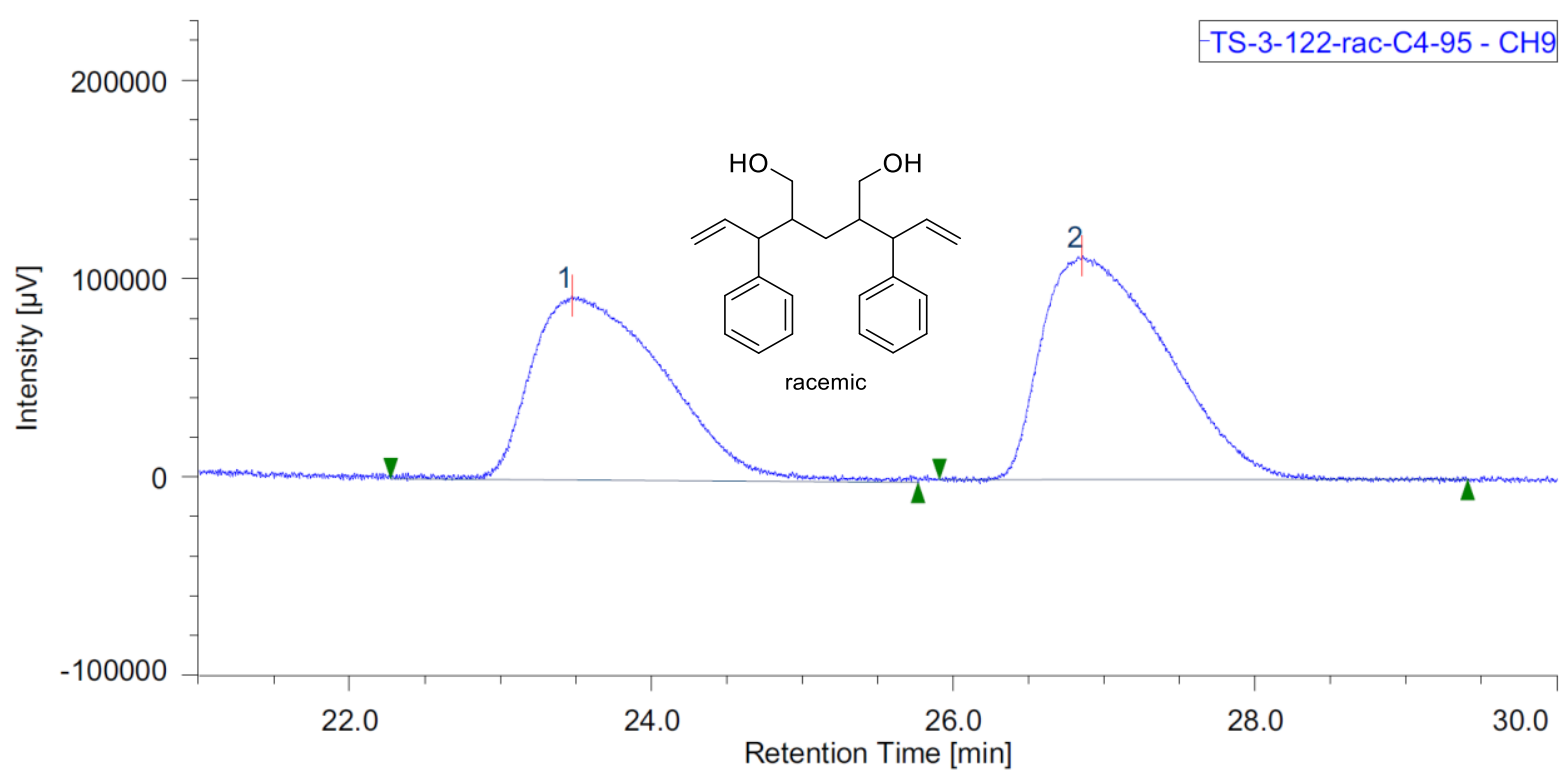

\begin{tabular}{|r|c|c|r|r|r|r|r|r|r|r|r|}
\hline$\#$ & Peak Name & $\mathrm{CH}$ & $\mathrm{tR}[\mathrm{min}]$ & Area $[\mu \mathrm{V} \cdot \mathrm{sec}]$ & Height $[\mu \mathrm{V}]$ & \multicolumn{1}{|c|}{ Area $\%$} & Height $\%$ & Quantity & NTP & Resolution & Symmetry Factor \\
\hline 1 & Unknown & 9 & 23.473 & 5710682 & 92860 & 47.021 & 45.111 & $\mathrm{~N} / \mathrm{A}$ & 3021 & 2.054 & 1.880 \\
\hline 2 & Unknown & 9 & 26.853 & 6434168 & 112986 & 52.979 & 54.889 & $\mathrm{~N} / \mathrm{A}$ & 4556 & $\mathrm{~N} / \mathrm{A}$ & 1.722 \\
\hline
\end{tabular}

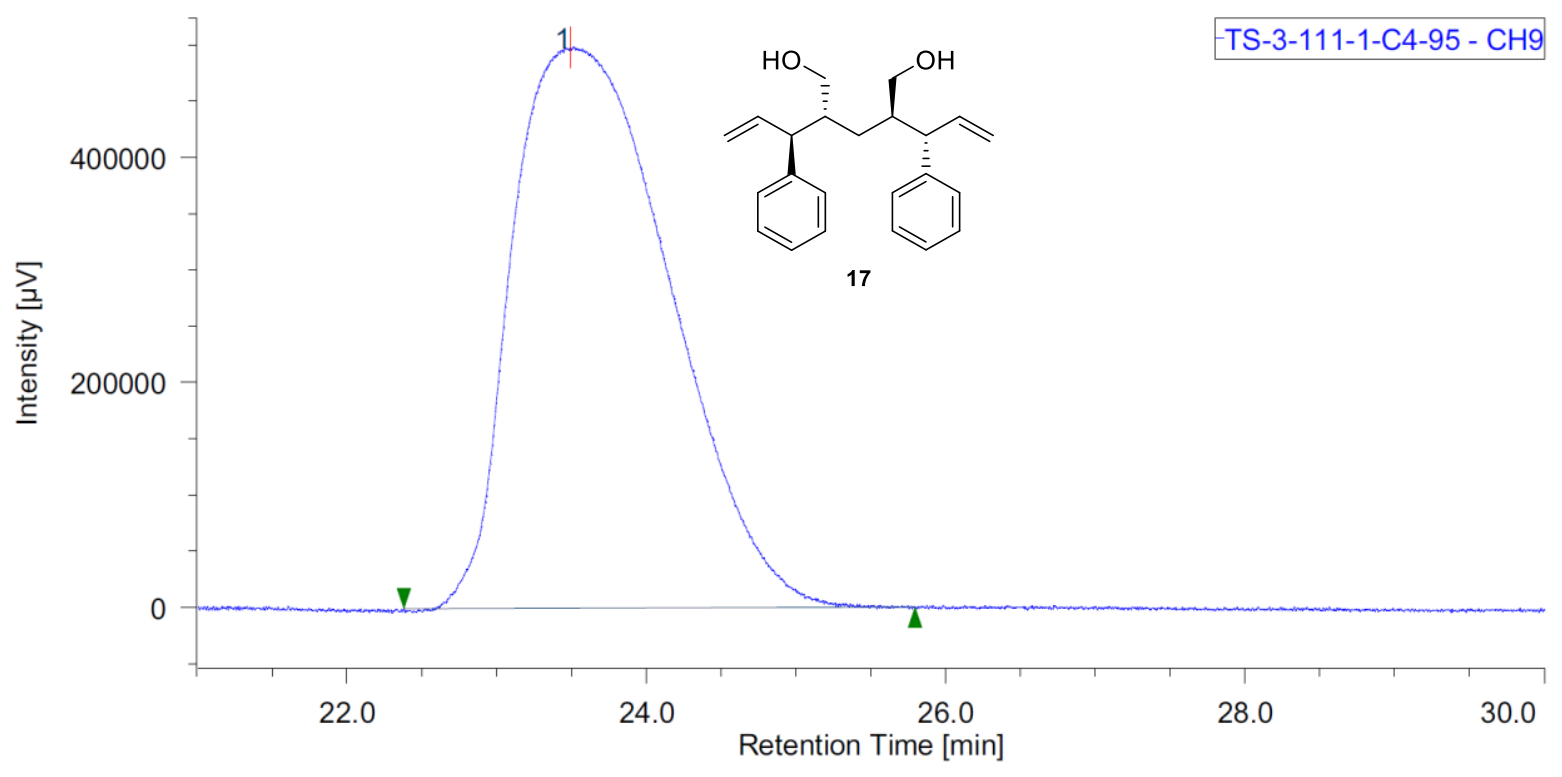

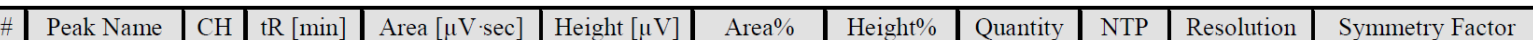

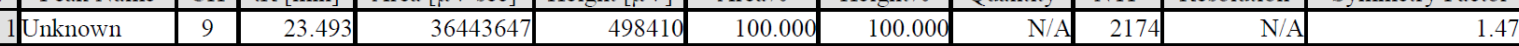




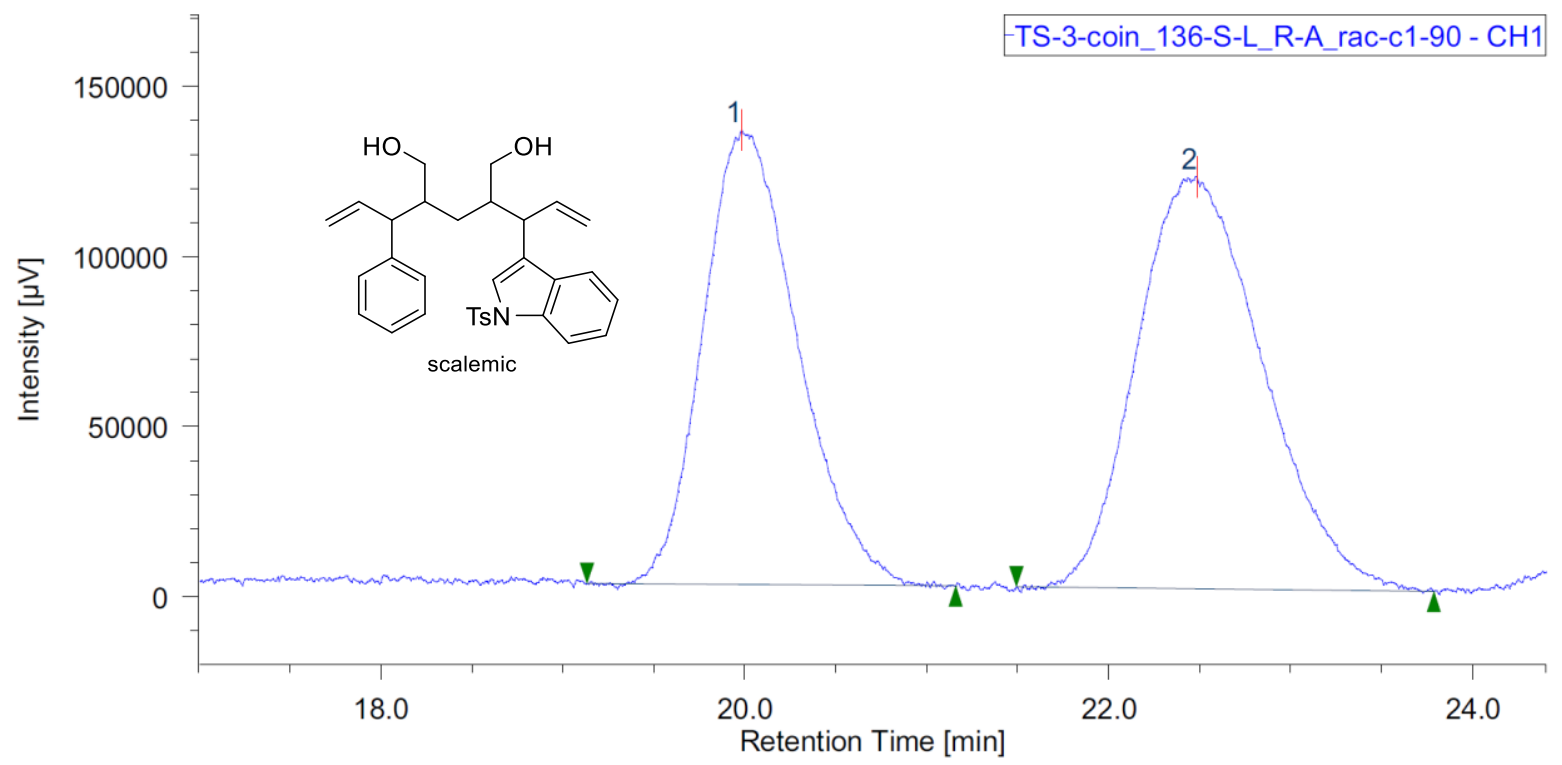

\begin{tabular}{|r|c|c|r|r|r|r|r|r|r|r|r|}
\hline$\#$ & Peak Name & $\mathrm{CH}$ & $\mathrm{tR}[\mathrm{min}]$ & Area $[\mu \mathrm{V} \cdot \mathrm{sec}]$ & Height $[\mu \mathrm{V}]$ & \multicolumn{1}{|c|}{ Area\% } & Height $\%$ & Quantity & NTP & Resolution & Symmetry Factor \\
\hline 1 & Unknown & 1 & 19.983 & 4819981 & 133409 & 45.291 & 52.456 & $\mathrm{~N} / \mathrm{A}$ & 7043 & 2.249 & 1.284 \\
\hline 2 & Unknown & 1 & 22.483 & 5822324 & 120918 & 54.709 & 47.544 & $\mathrm{~N} / \mathrm{A}$ & 4960 & $\mathrm{~N} / \mathrm{A}$ & 1.179 \\
\hline
\end{tabular}

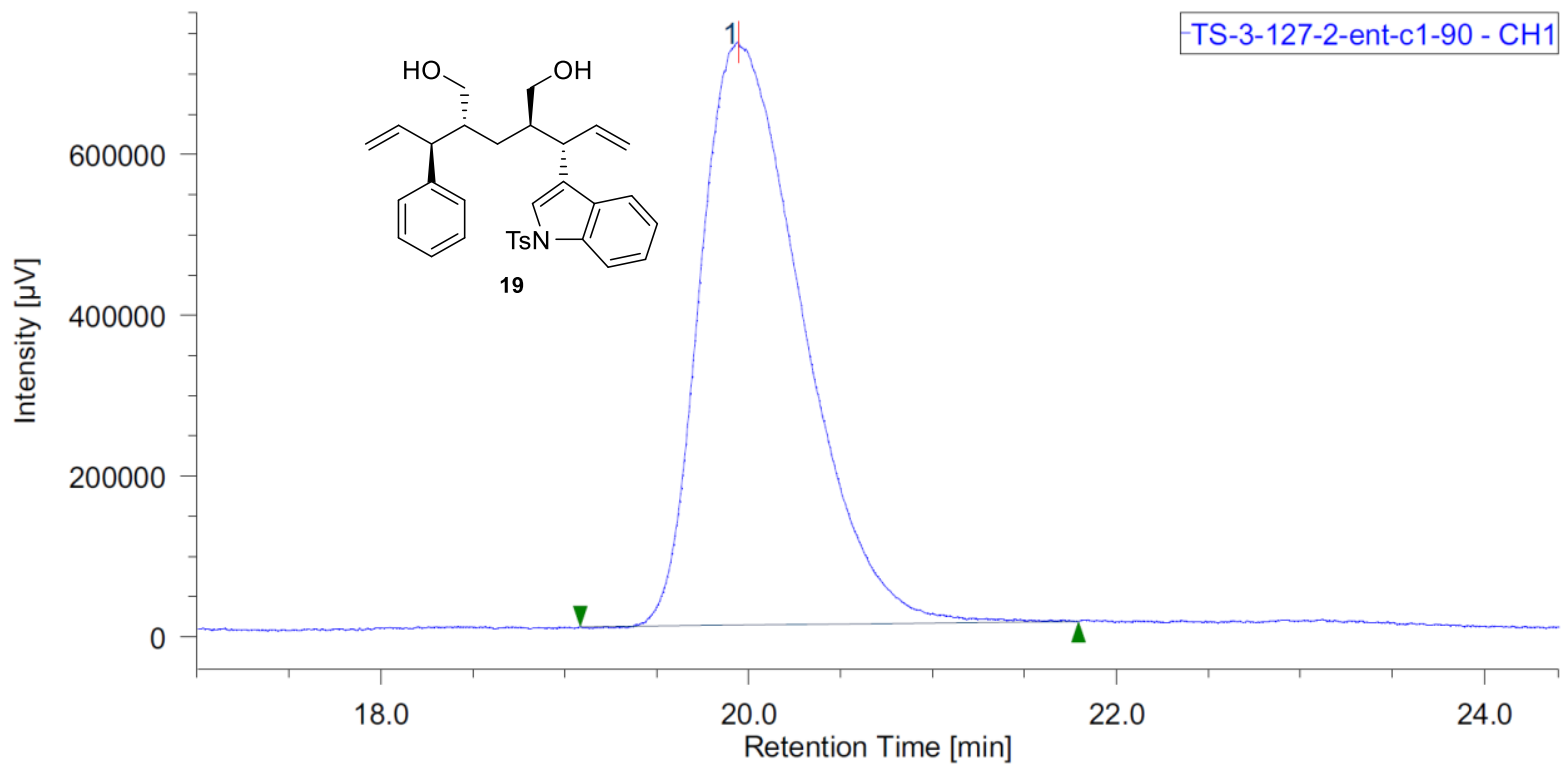

\begin{tabular}{|c|c|c|r|r|r|r|r|r|r|r|}
\hline$\#$ & Peak Name & $\mathrm{CH}$ & $\mathrm{tR}[\mathrm{min}]$ & Area $[\mu \mathrm{V}$ sec $]$ & Height $[\mu \mathrm{V}]$ & \multicolumn{1}{c|}{ Area $\%$} & Height $\%$ & Quantity & NTP & Resolution \\
\hline $\mathrm{1}$ & Unknown & 1 & 19.942 & 27963501 & 724896 & 100.000 & 100.000 & $\mathrm{~N} / \mathrm{A}$ & 6138 & N/A \\
\hline \hline
\end{tabular}




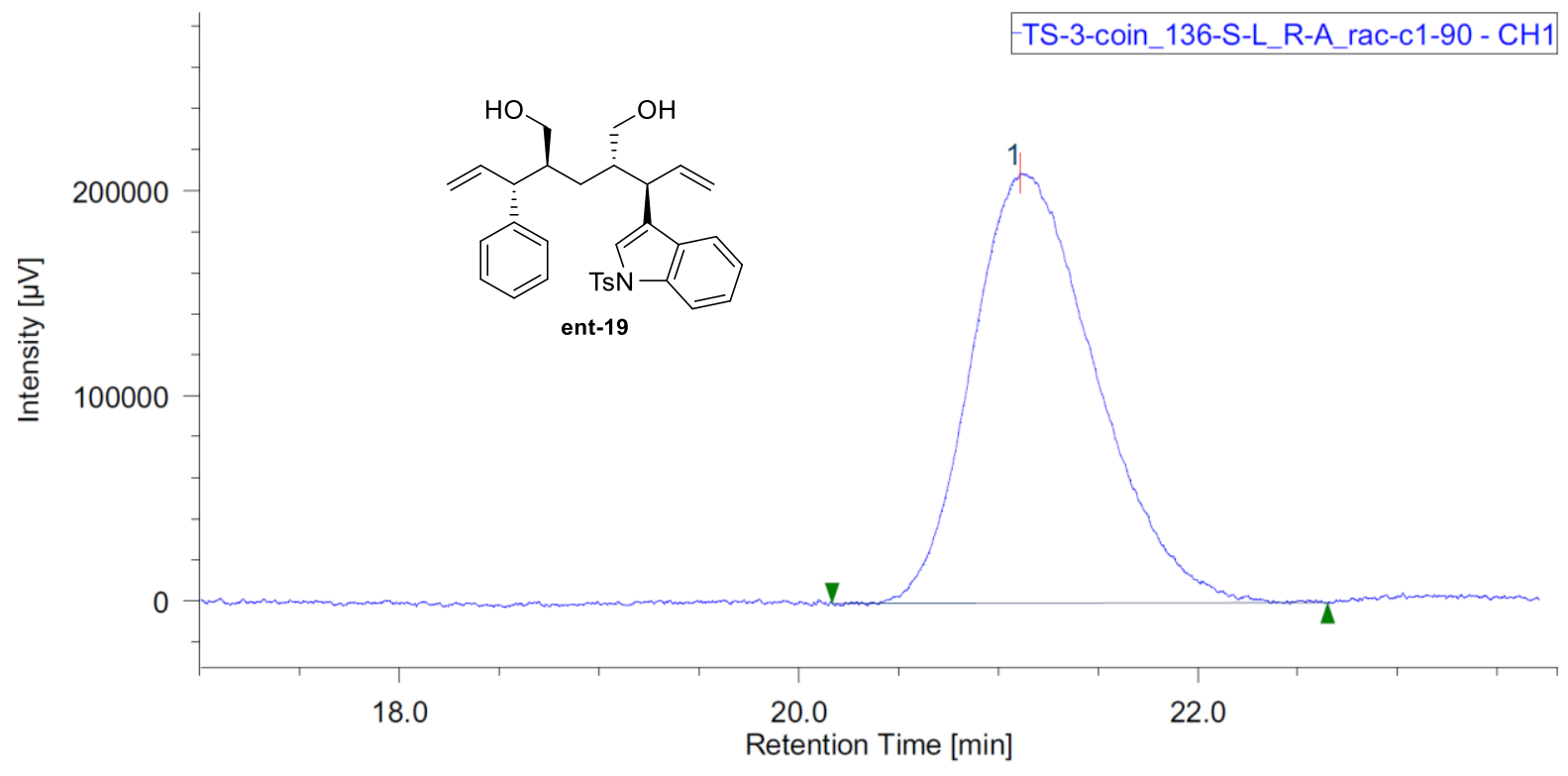

\begin{tabular}{|c|c|c|c|c|c|c|c|c|c|c|c|}
\hline$\#$ & Peak Name & $\mathrm{CH}$ & $\mathrm{tR}$ [min] & Area $[\mu \mathrm{V} \cdot \mathrm{sec}]$ & Height $[\mu \mathrm{V}]$ & Area $\%$ & Height $\%$ & Quantity & NTP & Resolution & Symmetry Factor \\
\hline & Unknown & 1 & 21.108 & 9036645 & 209351 & 100.000 & 100.000 & $\mathrm{~N} / \mathrm{A}$ & 5545 & $\mathrm{~N} / \mathrm{A}$ & 1.318 \\
\hline
\end{tabular}

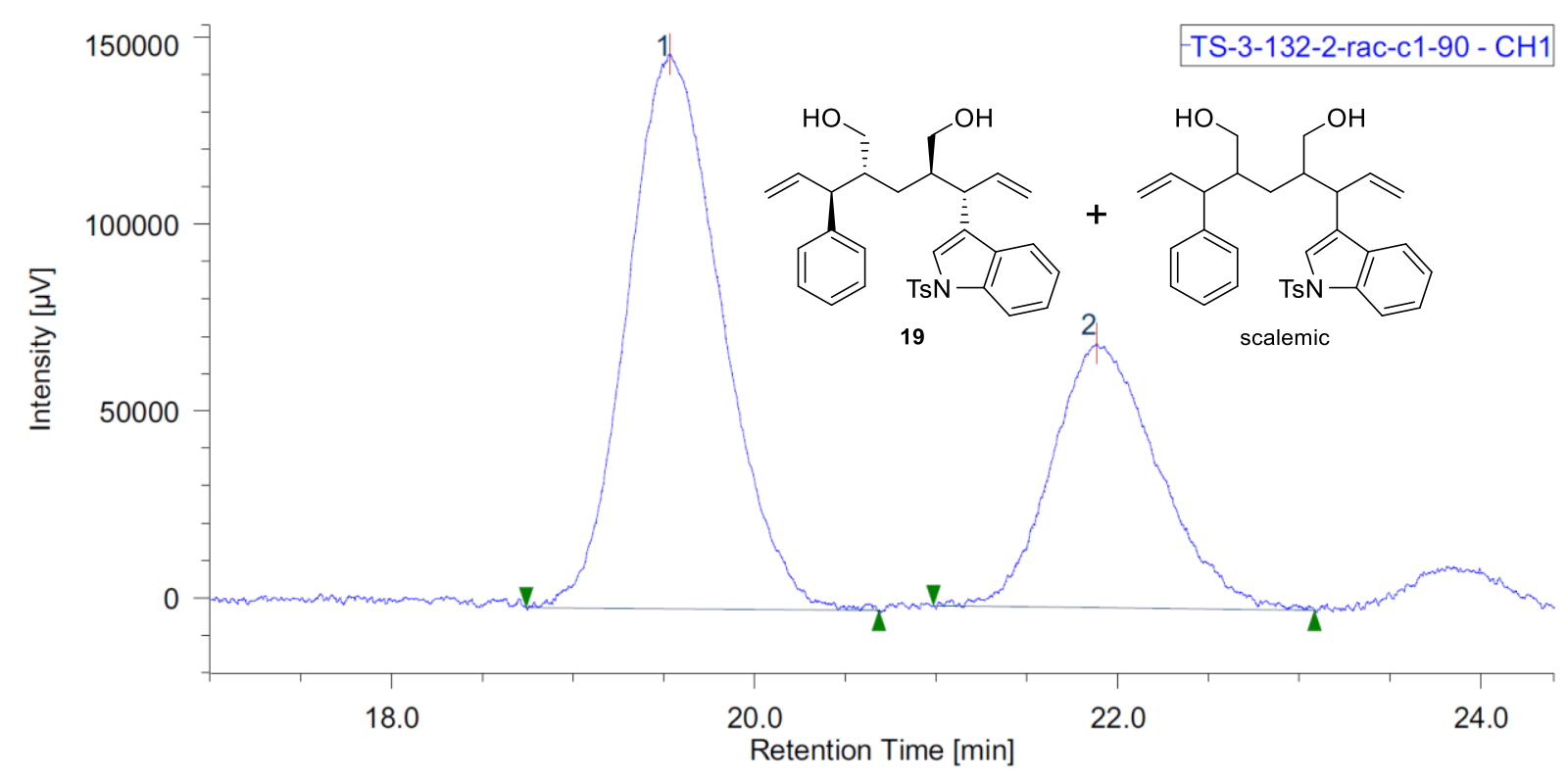

\begin{tabular}{|c|c|c|c|c|c|c|c|c|c|c|}
\hline Peak Name & $\mathrm{CH}$ & tR [min] & Area $[\mu \mathrm{V} \cdot \mathrm{sec}]$ & Height $[\mu \mathrm{V}]$ & Area $\%$ & Height $\%$ & Quantity & NTP & Resolution & Symmetry Factor \\
\hline Unknown & 1 & 19.533 & 5367830 & 148304 & 65.080 & 67.698 & $\mathrm{~N} / \mathrm{A}$ & 6791 & 2.338 & 1.160 \\
\hline Unknown & 1 & 21.883 & 2880265 & 70762 & 34.920 & 32.302 & $\mathrm{~N} / \mathrm{A}$ & 6721 & $\mathrm{~N} / \mathrm{A}$ & 1.250 \\
\hline
\end{tabular}




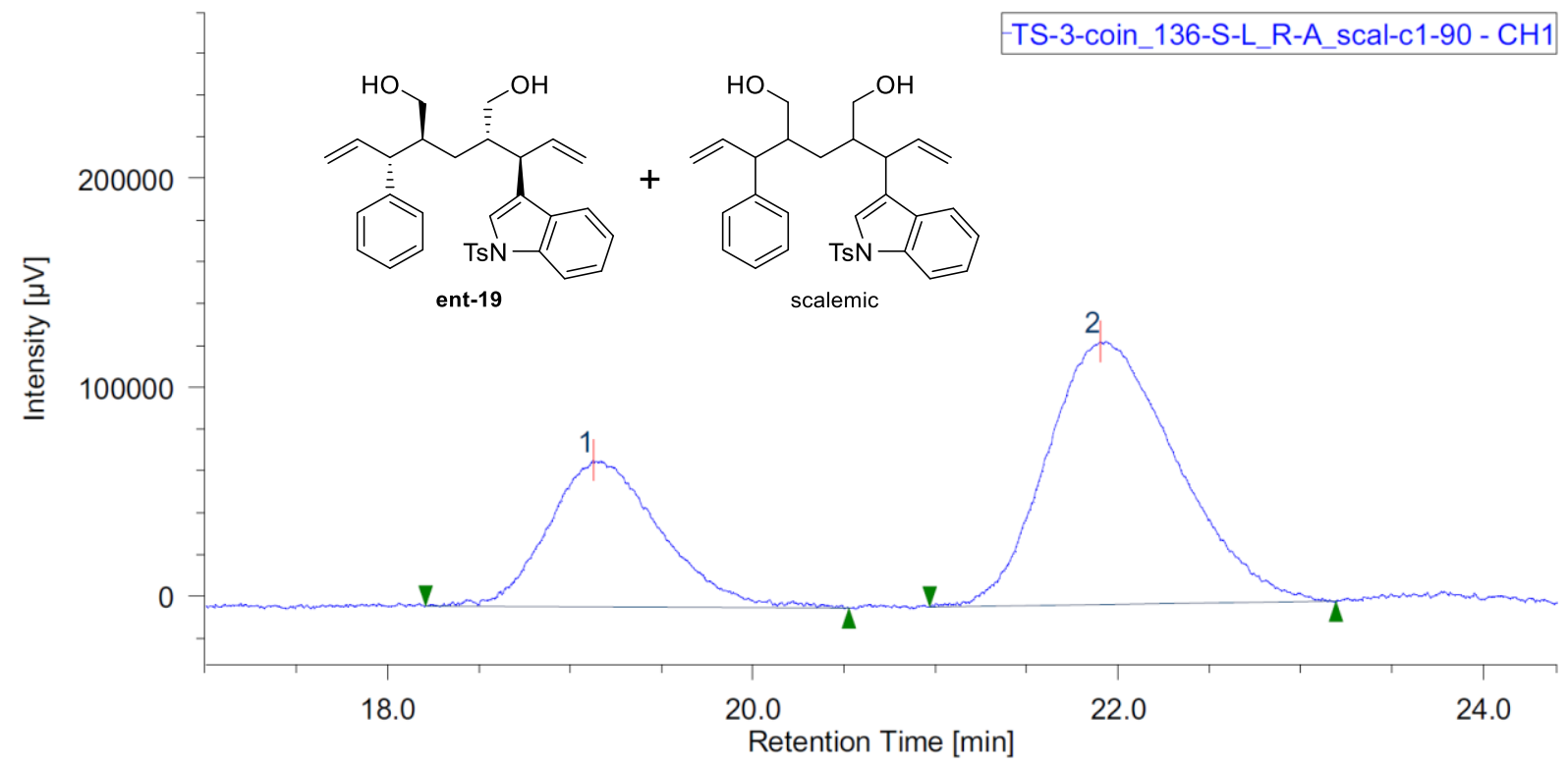

\begin{tabular}{|r|c|c|r|r|r|r|r|r|r|r|r|}
\hline$\#$ & Peak Name & CH & tR $[\mathrm{min}]$ & Area $[\mu \mathrm{V} \cdot \mathrm{sec}]$ & Height $[\mu \mathrm{V}]$ & \multicolumn{1}{|c|}{ Area $\%$} & Height $\%$ & Quantity & NTP & Resolution & Symmetry Factor \\
\hline 1 & Unknown & 1 & 19.125 & 2997711 & 70005 & 32.544 & 35.735 & N/A & 4751 & 2.306 & 1.369 \\
\hline 2 & Unknown & 1 & 21.900 & 6213462 & 125895 & 67.456 & 64.265 & N/A & 4518 & N/A & 1.251 \\
\hline
\end{tabular}




\section{HPLC Traces}

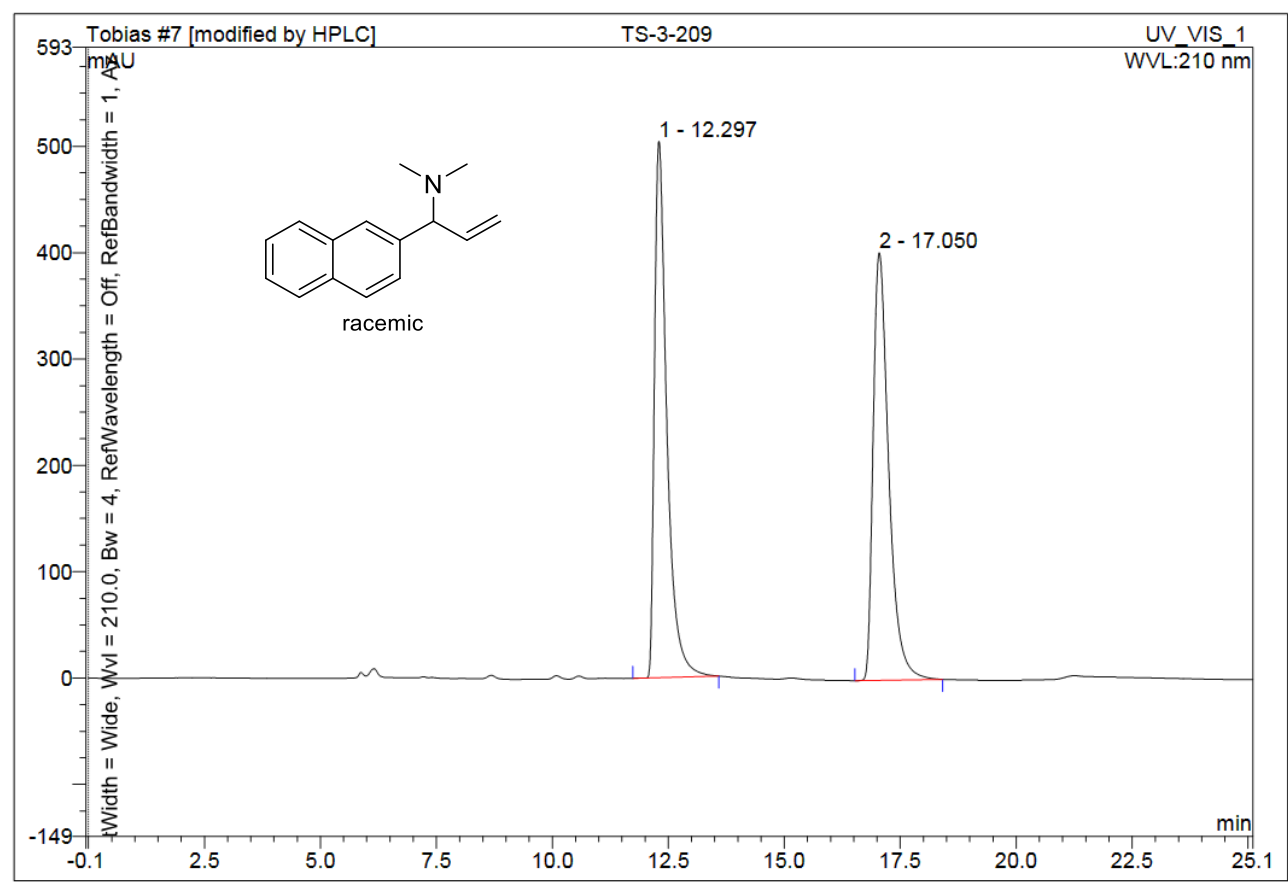

\begin{tabular}{|r|ccccccc|}
\hline No. & $\begin{array}{c}\text { Ret.Time } \\
\text { min }\end{array}$ & Peak Name & $\begin{array}{c}\text { Height } \\
\text { mAU }\end{array}$ & $\begin{array}{c}\text { Area } \\
\text { mAU*min }\end{array}$ & $\begin{array}{c}\text { Rel.Area } \\
\%\end{array}$ & Amount & Type \\
\hline 1 & 12.30 & n.a. & 504.225 & 155.687 & 49.87 & n.a. & BMB $^{*}$ \\
2 & 17.05 & n.a. & 401.994 & 156.514 & 50.13 & n.a. & BMB $^{*}$ \\
\hline Total: & & & 906.218 & 312.201 & 100.00 & 0.000 & \\
\hline
\end{tabular}

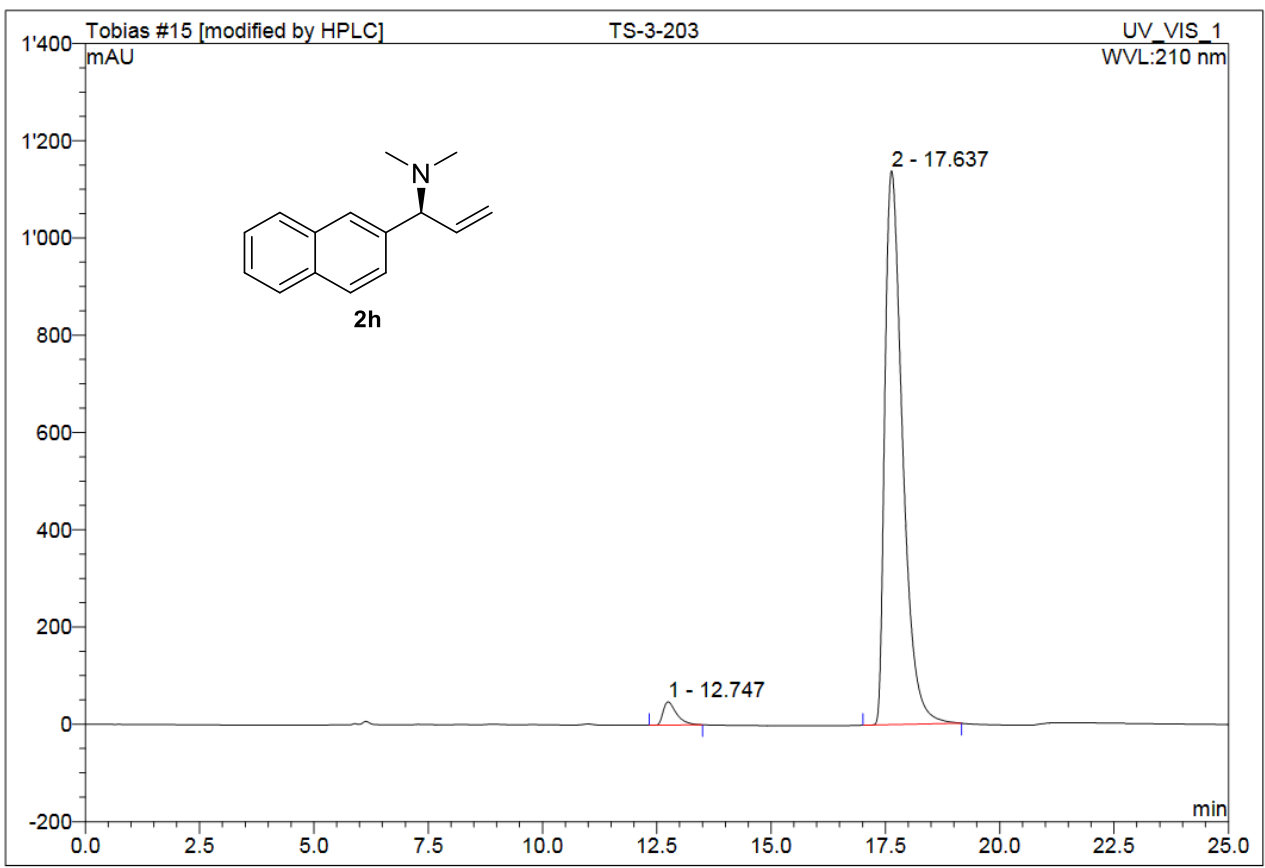

\begin{tabular}{|r|ccrrrrr|}
\hline No. & $\begin{array}{c}\text { Ret.Time } \\
\text { min }\end{array}$ & Peak Name & $\begin{array}{c}\text { Height } \\
\text { mAU }\end{array}$ & $\begin{array}{c}\text { Area } \\
\text { mAU*min }\end{array}$ & $\begin{array}{c}\text { Rel.Area } \\
\%\end{array}$ & Amount & Type \\
\hline 1 & 12.75 & n.a. & 47.730 & 16.287 & 3.08 & n.a. & BMB $^{*}$ \\
2 & 17.64 & n.a. & 1139.039 & 512.109 & 96.92 & n.a. & BMB $^{*}$ \\
\hline Total: & & & 1186.769 & 528.396 & 100.00 & 0.000 & \\
\hline
\end{tabular}




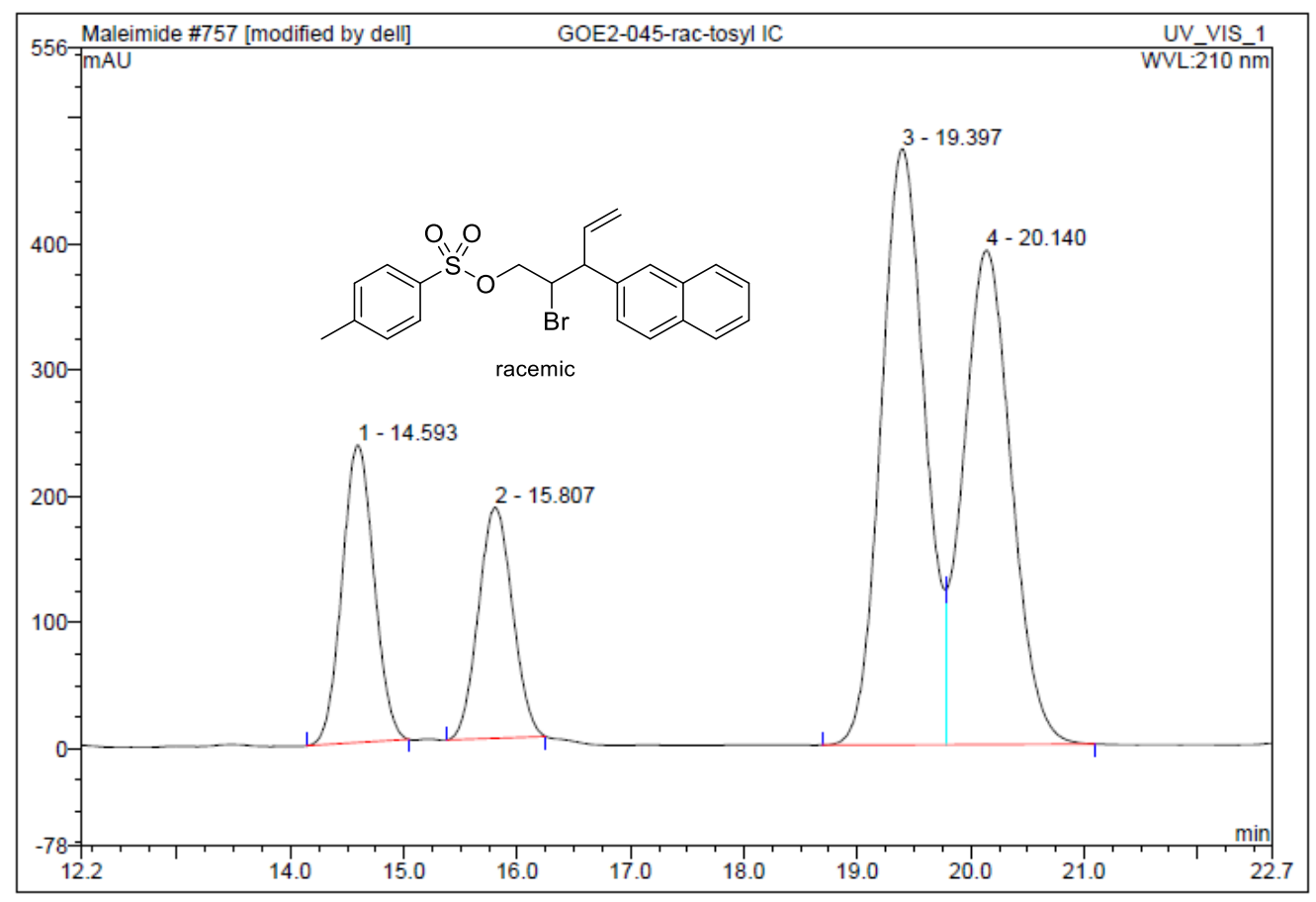

\begin{tabular}{|r|ccrrrrr|}
\hline No. & $\begin{array}{c}\text { Ret.Time } \\
\text { min }\end{array}$ & Peak Name & $\begin{array}{c}\text { Height } \\
\text { mAU }\end{array}$ & $\begin{array}{c}\text { Area } \\
\text { mAU*min }\end{array}$ & $\begin{array}{c}\text { Rel.Area } \\
\%\end{array}$ & Amount & Type \\
\hline 1 & 14.59 & n.a. & 236.174 & 77.213 & 14.12 & n.a. & BMB $^{*}$ \\
2 & 15.81 & n.a. & 183.224 & 63.975 & 11.70 & n.a. & BMB $^{*}$ \\
3 & 19.40 & n.a. & 472.860 & 211.628 & 38.69 & n.a. & BM $^{*}$ \\
4 & 20.14 & n.a. & 391.991 & 194.146 & 35.50 & n.a. & MB $^{*}$ \\
\hline Total: & & & 1284.249 & 546.962 & 100.00 & 0.000 & \\
\hline
\end{tabular}

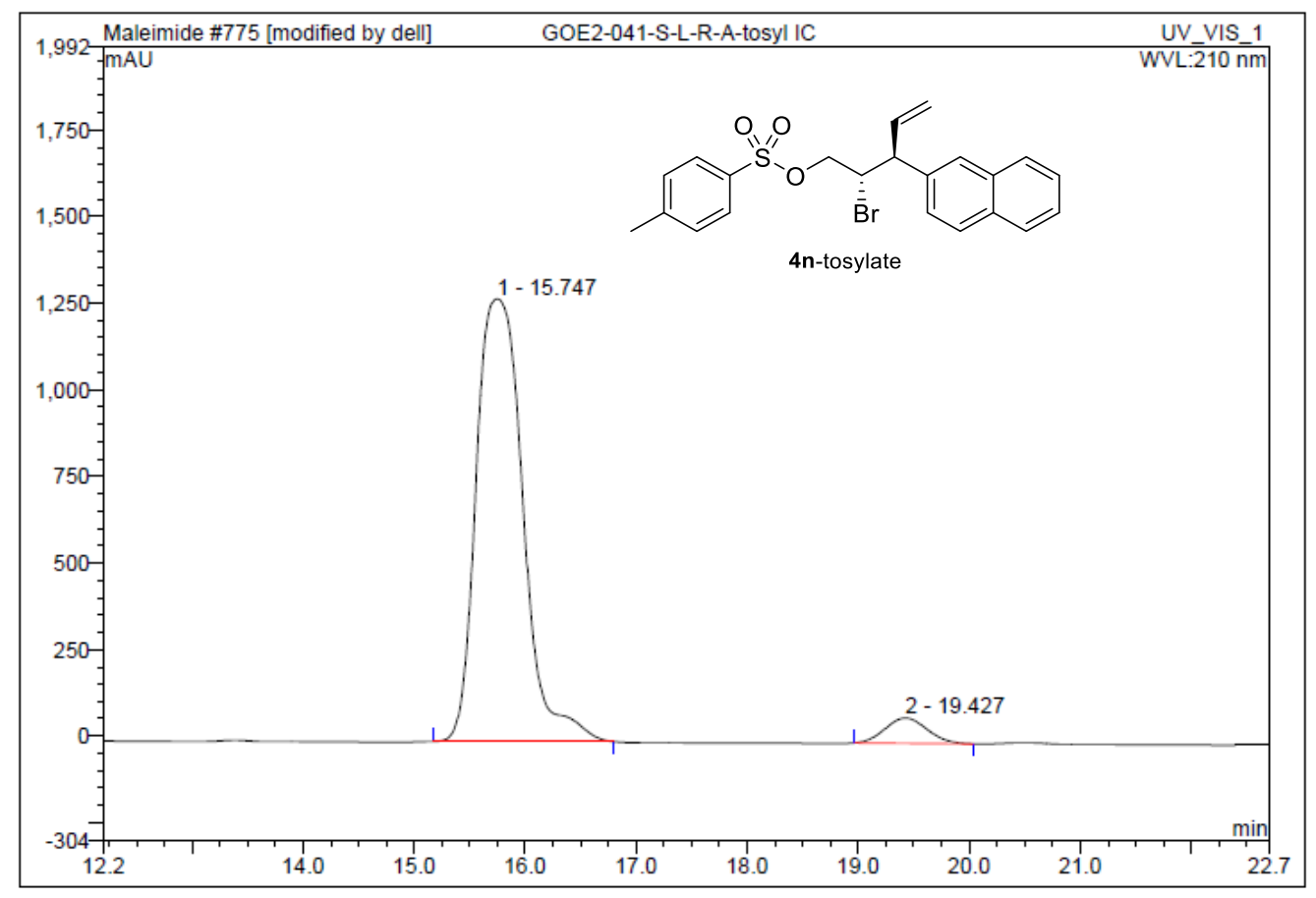

\begin{tabular}{|r|rrrrrrr|}
\hline No. & $\begin{array}{c}\text { Ret.Time } \\
\text { min }\end{array}$ & Peak Name & $\begin{array}{c}\text { Height } \\
\text { mAU }\end{array}$ & $\begin{array}{c}\text { Area } \\
\text { mAU*min }\end{array}$ & $\begin{array}{r}\text { Rel.Area } \\
\%\end{array}$ & Amount & Type \\
\hline 1 & 15.75 & n.a. & 1277.921 & 623.219 & 95.12 & n.a. & BMB $^{*}$ \\
2 & 19.43 & n.a. & 73.010 & 31.981 & 4.88 & n.a. & BMB $^{*}$ \\
\hline Total: & & & 1350.931 & 655.200 & 100.00 & 0.000 & \\
\hline
\end{tabular}

S204 

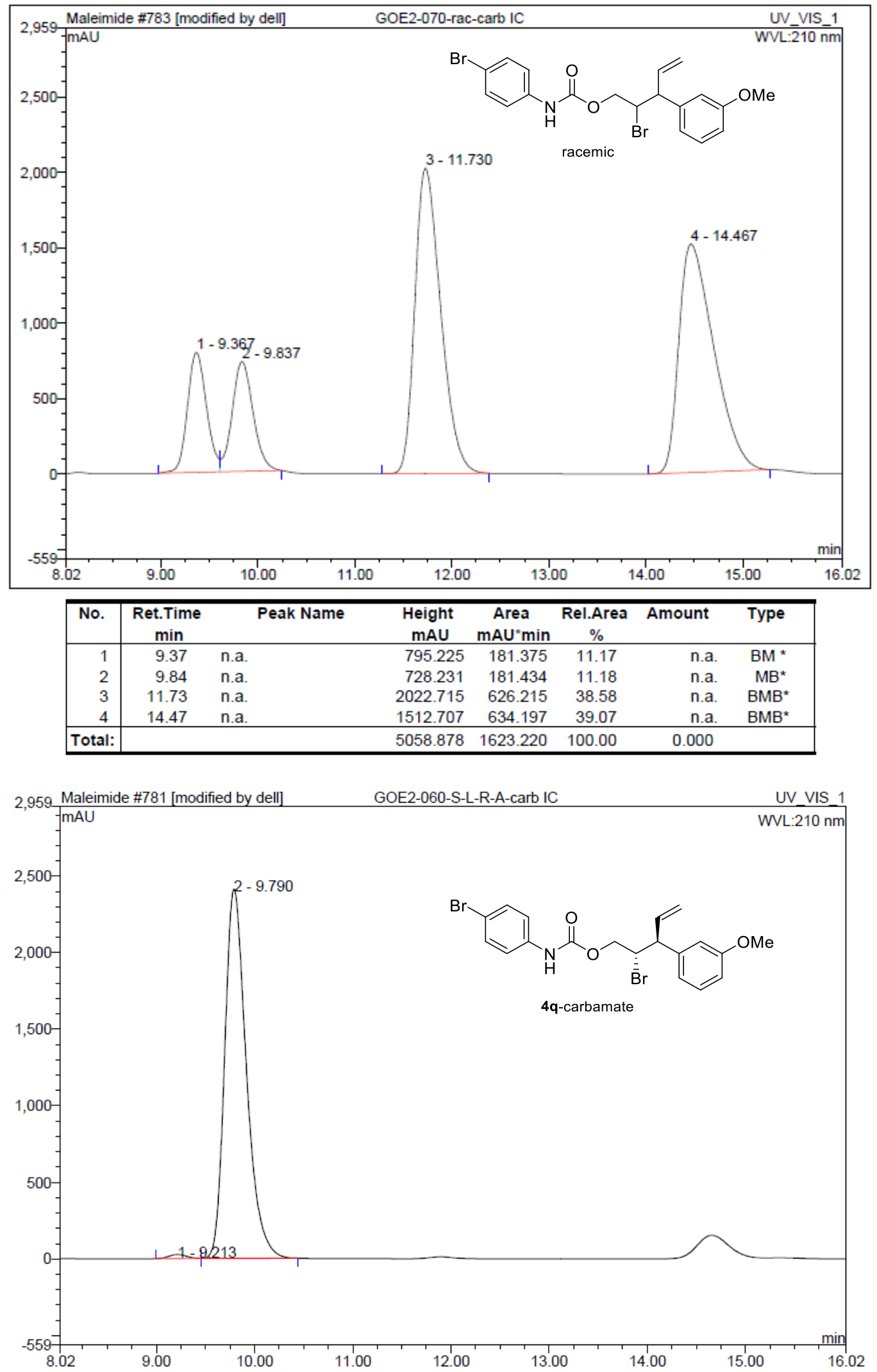

\begin{tabular}{|r|ccrrrrr|}
\hline No. & $\begin{array}{c}\text { Ret.Time } \\
\text { min }\end{array}$ & Peak Name & $\begin{array}{c}\text { Height } \\
\text { mAU }\end{array}$ & $\begin{array}{c}\text { Area } \\
\text { mAU*min }\end{array}$ & $\begin{array}{r}\text { Rel.Area } \\
\%\end{array}$ & Amount & Type \\
\hline 1 & 9.21 & n.a. & 26.653 & 5.731 & 0.92 & n.a. & BM $^{*}$ \\
2 & 9.79 & n.a. & 2413.040 & 616.911 & 99.08 & n.a. & MB $^{*}$ \\
\hline Total: & & & 2439.693 & 622.642 & 100.00 & 0.000 & \\
\hline
\end{tabular}




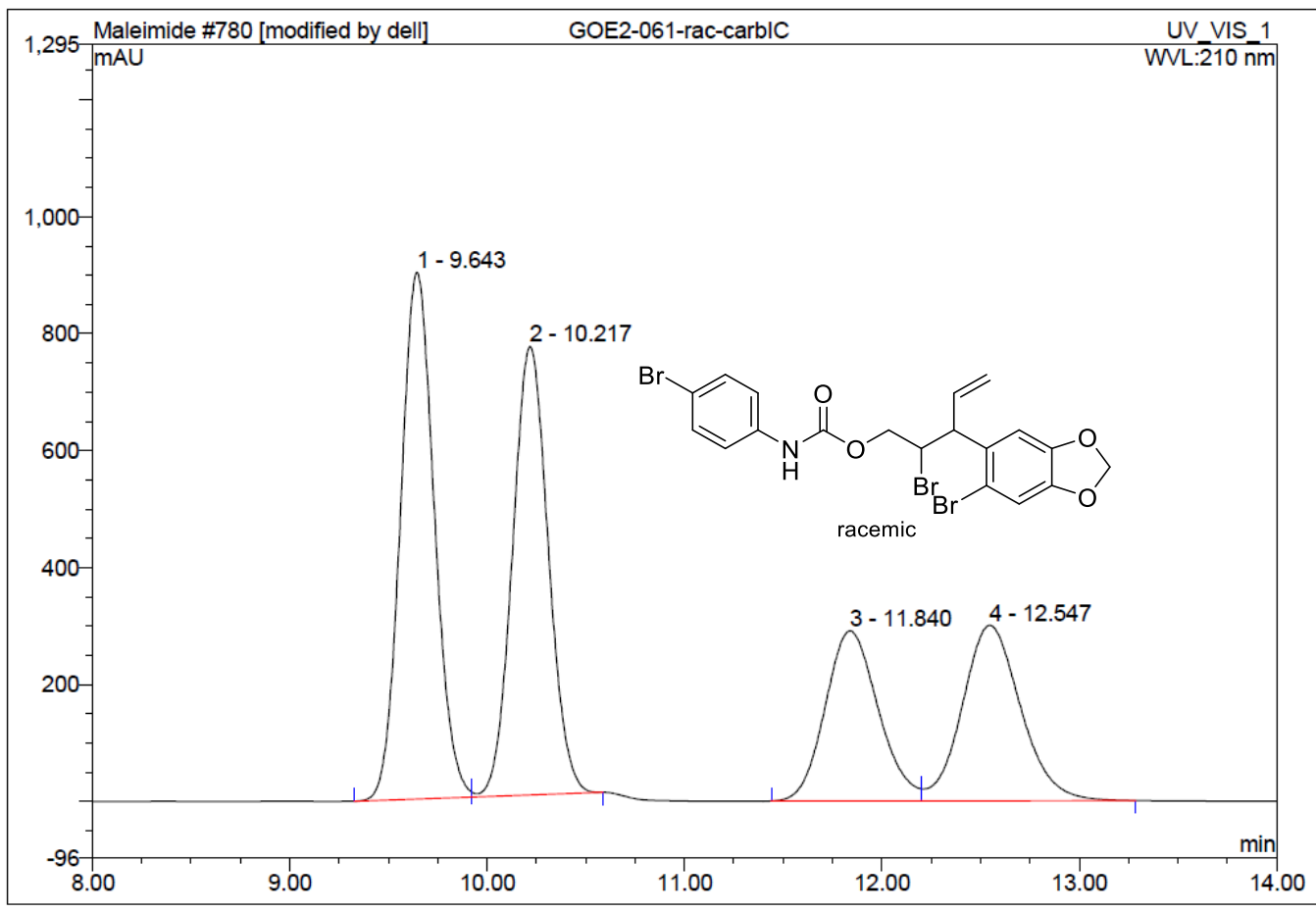

\begin{tabular}{|r|rrrrrrr|}
\hline No. & $\begin{array}{c}\text { Ret.Time } \\
\text { min }\end{array}$ & Peak Name & $\begin{array}{c}\text { Height } \\
\text { mAU }\end{array}$ & $\begin{array}{r}\text { Area } \\
\text { mAU*min }\end{array}$ & $\begin{array}{c}\text { Rel.Area } \\
\%\end{array}$ & Amount & Type \\
\hline 1 & 9.64 & n.a. & 900.504 & 178.289 & 33.47 & n.a. & BM $^{*}$ \\
2 & 10.22 & n.a. & 766.675 & 161.103 & 30.24 & n.a. & MB $^{*}$ \\
3 & 11.84 & n.a. & 291.037 & 90.568 & 17.00 & n.a. & BM * $^{*}$ \\
4 & 12.55 & n.a. & 300.322 & 102.763 & 19.29 & n.a. & MB $^{*}$ \\
\hline Total: & & & 2258.538 & 532.722 & 100.00 & 0.000 & \\
\hline
\end{tabular}

$\begin{array}{lll}4,000 & \text { Maleimide \#782 [modified by dell] } & \text { UV_VIS_1 } \\ -\mathrm{mAU} & & \text { WVL:210 } 2 \text { nm }\end{array}$

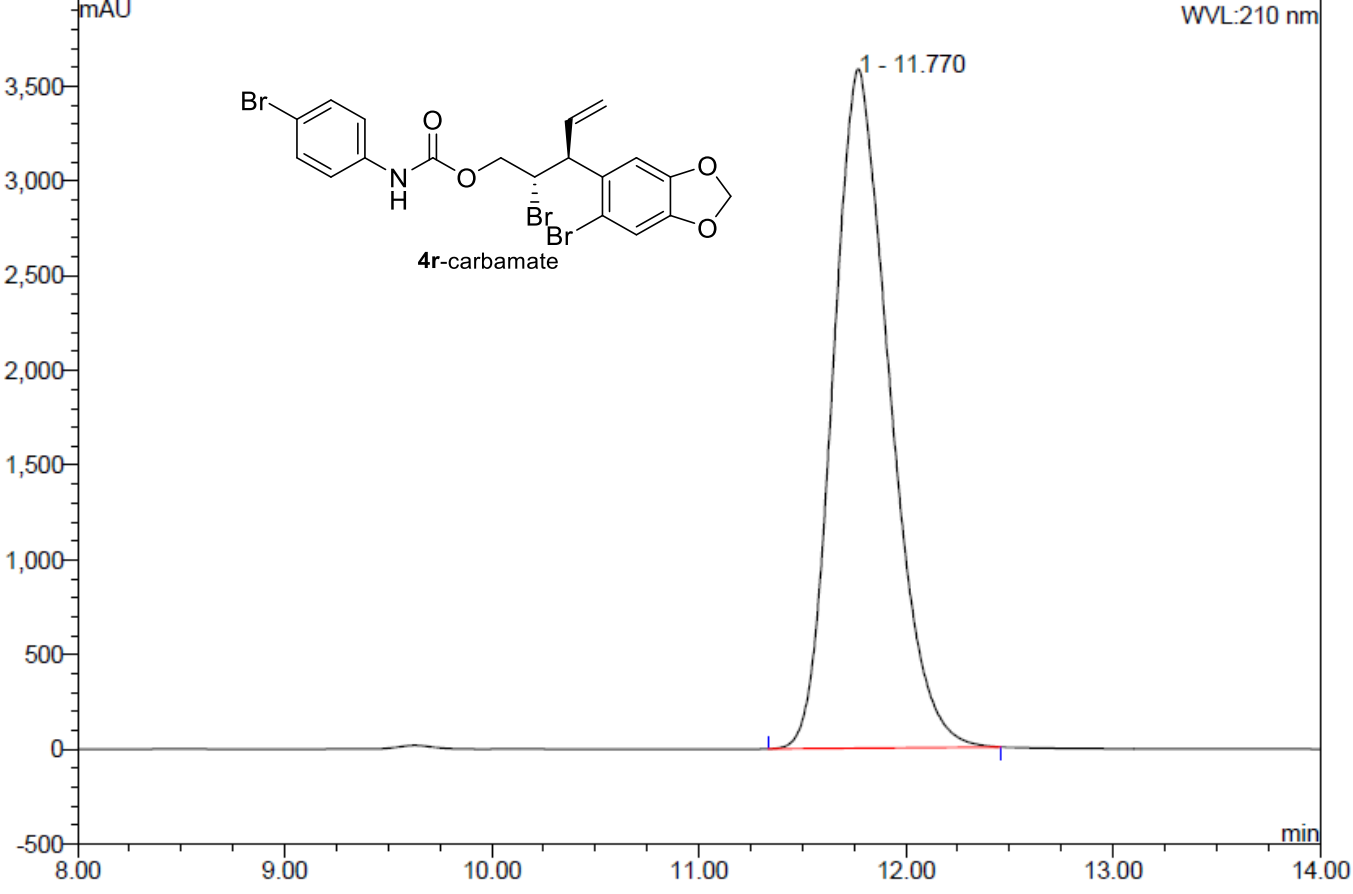

\begin{tabular}{|c|c|c|c|c|c|c|c|}
\hline No. & $\begin{array}{c}\text { Ret.Time } \\
\text { min }\end{array}$ & Peak Name & $\begin{array}{c}\text { Height } \\
\text { mAU }\end{array}$ & $\begin{array}{c}\text { Area } \\
\text { mAU*min }\end{array}$ & $\begin{array}{c}\text { Rel.Area } \\
\%\end{array}$ & Amount & Type \\
\hline 1 & 11.77 & n.a. & 3587.064 & 1133.970 & 100.00 & n.a. & $\mathrm{BMB}^{*}$ \\
\hline Total: & & & 3587.064 & 1133.970 & 100.00 & 0.000 & \\
\hline
\end{tabular}




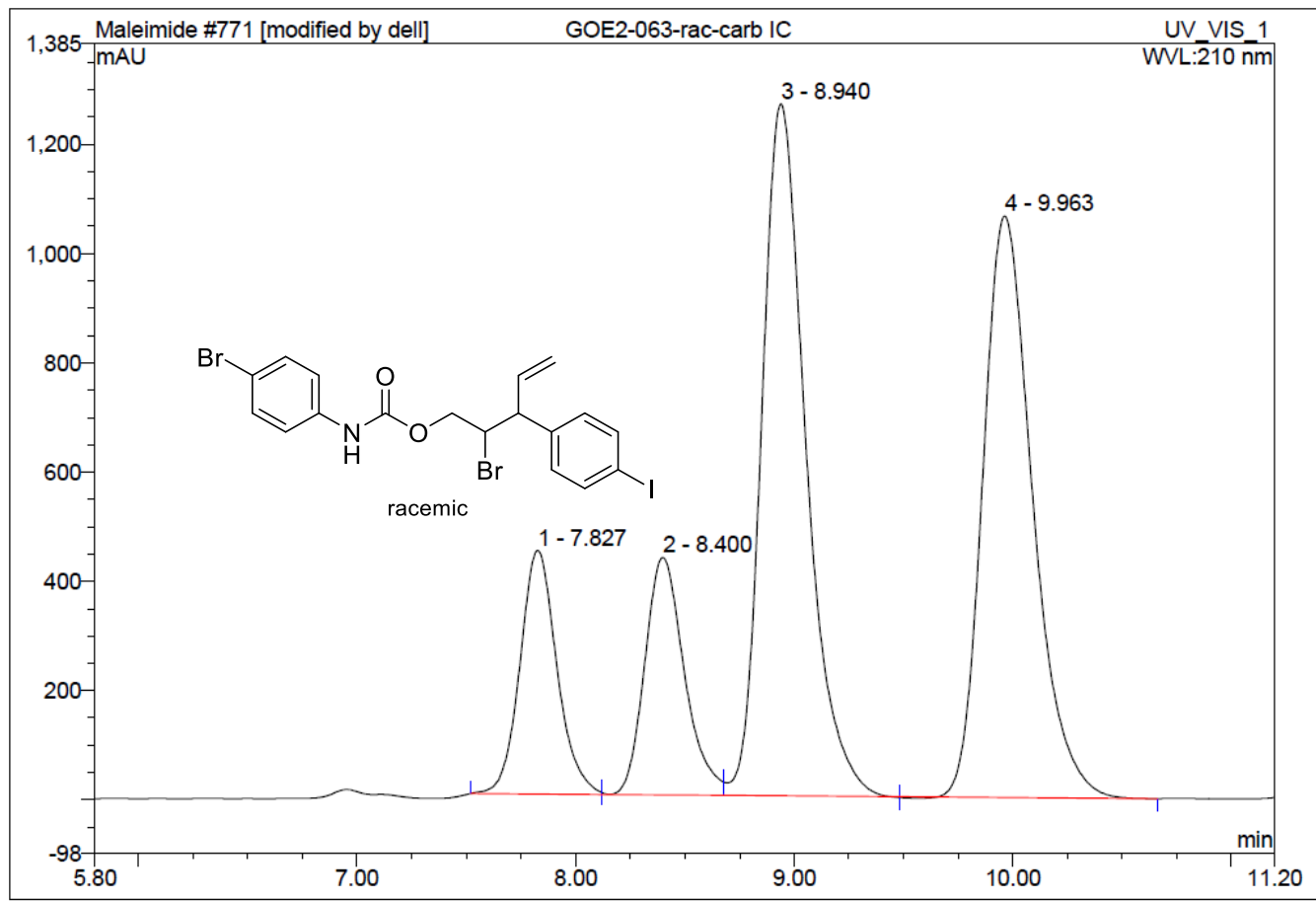

\begin{tabular}{|r|ccrrrrr|}
\hline No. & $\begin{array}{c}\text { Ret.Time } \\
\text { min }\end{array}$ & Peak Name & $\begin{array}{c}\text { Height } \\
\text { mAU }\end{array}$ & $\begin{array}{r}\text { Area } \\
\text { mAU*min }\end{array}$ & $\begin{array}{r}\text { Rel.Area } \\
\%\end{array}$ & Amount & Type \\
\hline 1 & 7.83 & n.a. & 446.493 & 87.303 & 11.59 & n.a. & BM $^{*}$ \\
2 & 8.40 & n.a. & 435.093 & 90.835 & 12.06 & n.a. & $M^{*}$ \\
3 & 8.94 & n.a. & 1266.911 & 295.457 & 39.23 & n.a. & M $^{*}$ \\
4 & 9.96 & n.a. & 1064.819 & 279.511 & 37.11 & n.a. & MB $^{*}$ \\
\hline Total: & & & 3213.315 & 753.106 & 100.00 & 0.000 & \\
\hline
\end{tabular}

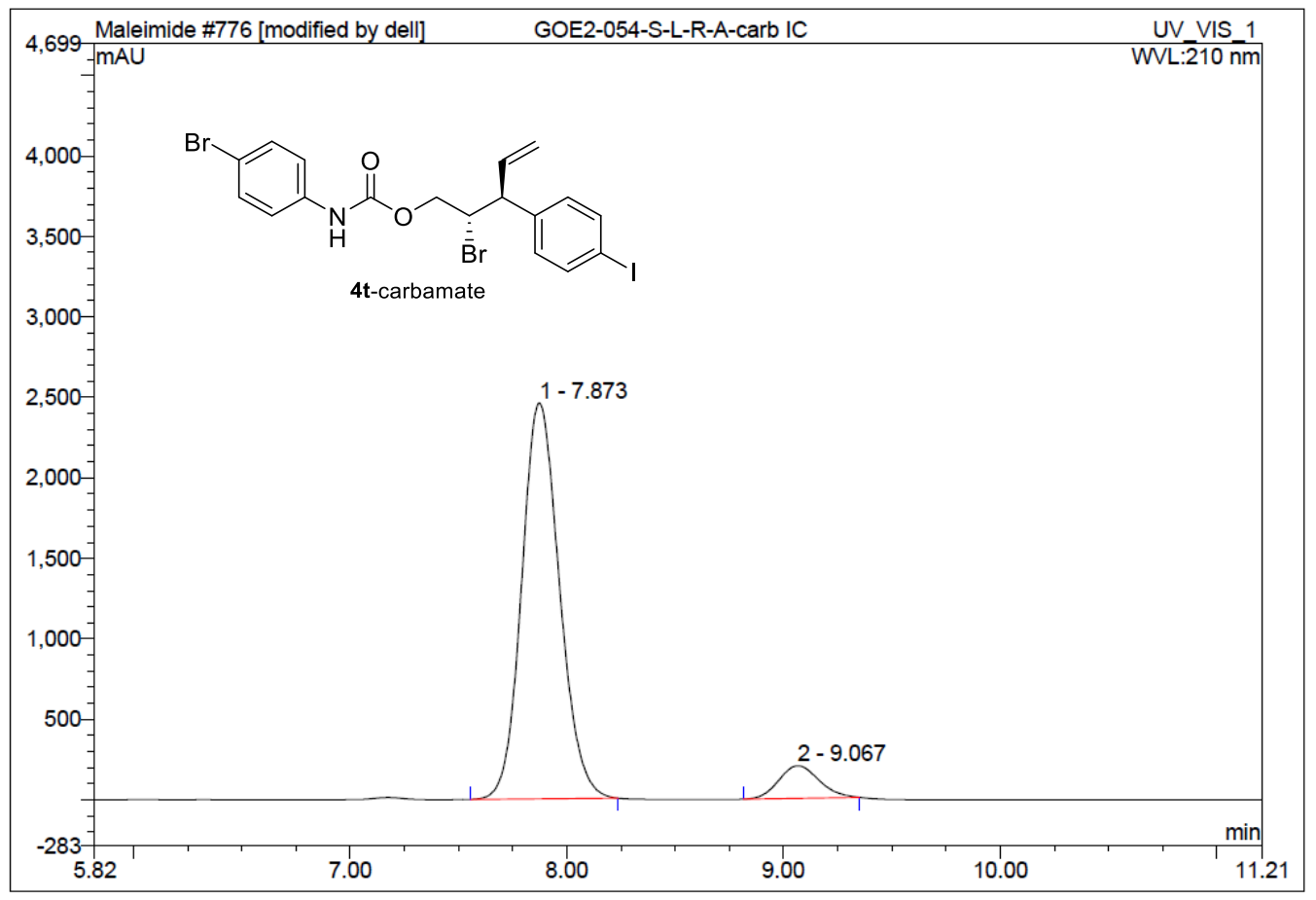

\begin{tabular}{|r|ccrrrrr|}
\hline No. & $\begin{array}{c}\text { Ret.Time } \\
\text { min }\end{array}$ & Peak Name & $\begin{array}{c}\text { Height } \\
\text { mAU }\end{array}$ & $\begin{array}{c}\text { Area } \\
\text { mAU*min }\end{array}$ & $\begin{array}{c}\text { Rel.Area } \\
\%\end{array}$ & Amount & Type \\
\hline 1 & 7.87 & n.a. & 2460.031 & 497.814 & 91.97 & n.a. & BMB $^{*}$ \\
2 & 9.07 & n.a. & 202.534 & 43.475 & 8.03 & n.a. & BMB $^{*}$ \\
\hline Total: & & & 2662.566 & 541.289 & 100.00 & 0.000 & \\
\hline
\end{tabular}




\section{GC Traces}

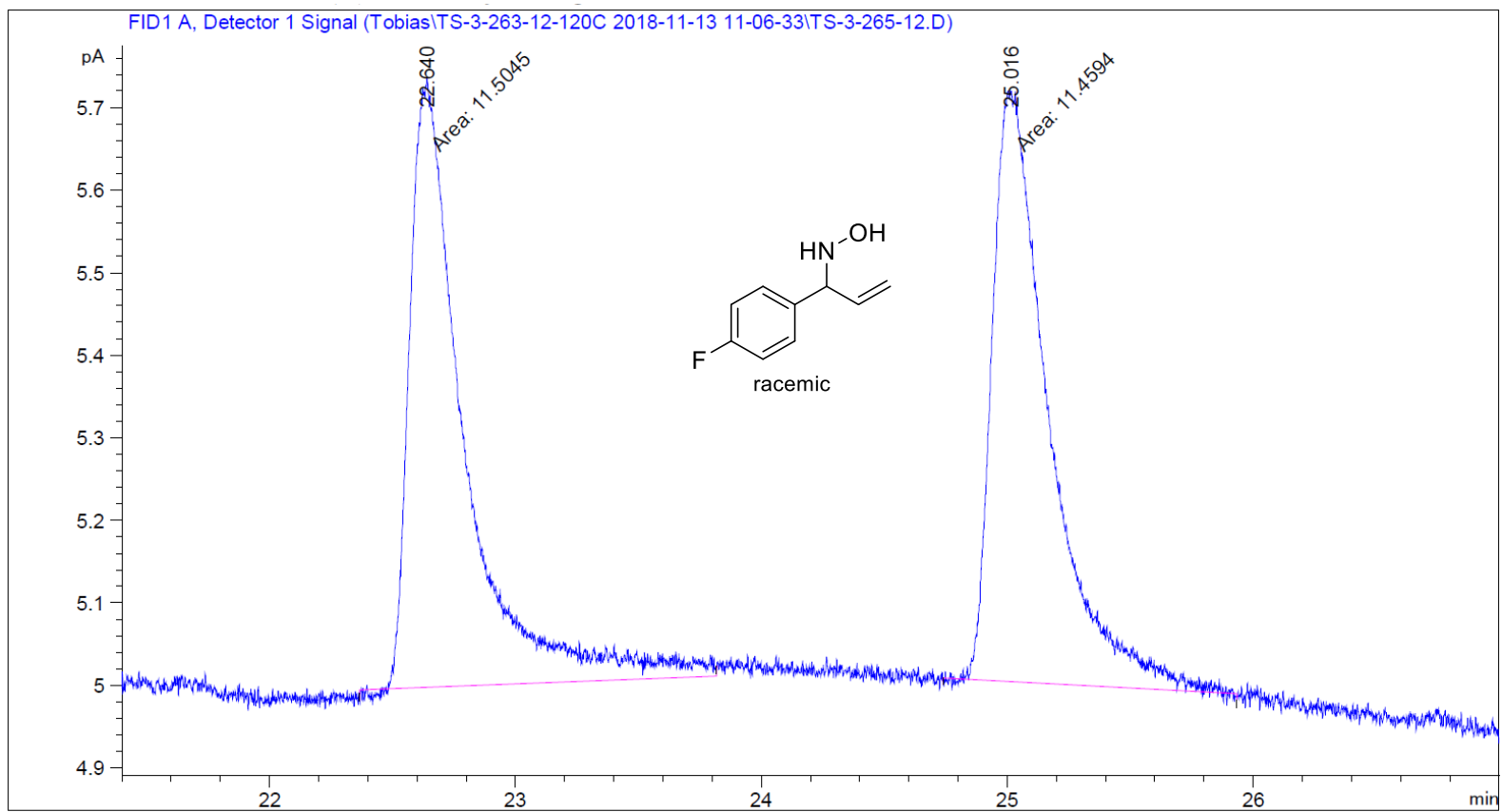

\begin{tabular}{cccccc}
$\begin{array}{c}\text { Peak RetTime Type } \\
\text { \# }\end{array}$ & $\begin{array}{c}\text { Width } \\
{[\mathrm{min}]}\end{array}$ & $\begin{array}{c}\text { Area } \\
{[\mathrm{min}]}\end{array}$ & $\begin{array}{c}\text { Height } \\
{[\mathrm{pA} \text { s }]}\end{array}$ & $\begin{array}{c}\text { Area } \\
{[\mathrm{pA}]}\end{array}$ & $\%$ \\
\hline 1 & 22.640 MM & 0.2600 & 11.50454 & $7.37405 \mathrm{e}-1$ & 50.09835 \\
2 & 25.016 MM & 0.2666 & 11.45937 & $7.16440 \mathrm{e}-1$ & 49.90165
\end{tabular}

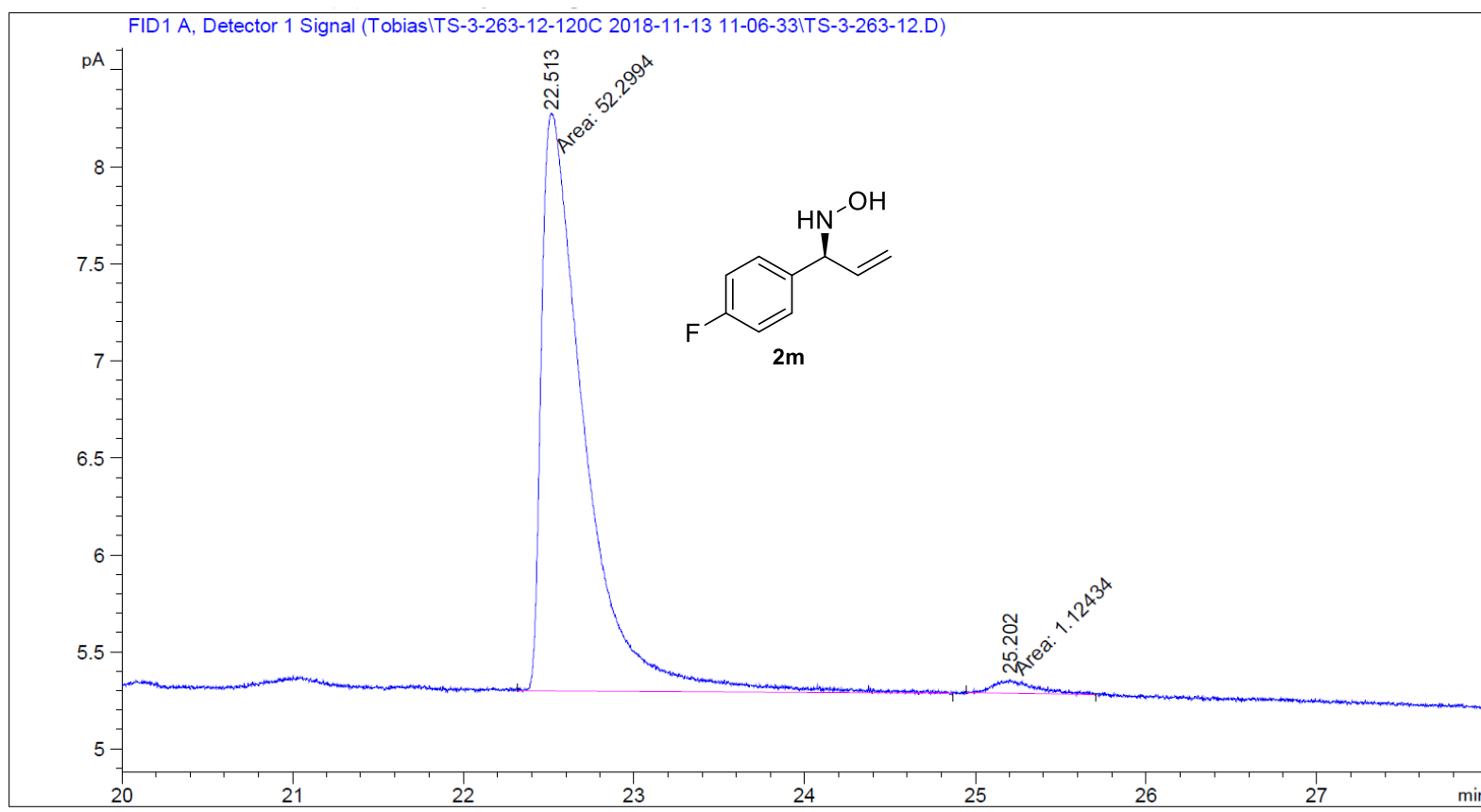

\begin{tabular}{|c|c|c|c|c|c|c|}
\hline $\begin{array}{c}\text { Peak } \\
\#\end{array}$ & $\begin{array}{c}\text { RetTime } \\
\text { [min] }\end{array}$ & Type & $\begin{array}{l}\text { Width } \\
\text { [min] }\end{array}$ & $\begin{array}{r}\text { Area } \\
{\left[\mathrm{pA}^{*} \mathrm{~s}\right]}\end{array}$ & $\begin{array}{l}\text { Height } \\
{[\mathrm{pA}]}\end{array}$ & $\begin{array}{c}\text { Area } \\
\%\end{array}$ \\
\hline & & & & & & \\
\hline 1 & 22 & 4 & 9 & 52.29944 & 621 & 97.89542 \\
\hline 2 & 2 & & & 434 & $e-2$ & \\
\hline
\end{tabular}

